\title{
IntechOpen
}

\section{Frontiers in Adaptive Control}

\author{
Edited by Shuang Cong
}

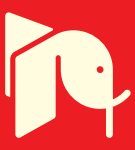



FRINTIERS IN ADAPTIVE CINTRLL

Edited by

SHUANG CaNG 


\section{Frontiers in Adaptive Control}

http://dx.doi.org/10.5772/96

Edited by Shuang Cong

\section{(c) The Editor(s) and the Author(s) 2009}

The moral rights of the and the author(s) have been asserted.

All rights to the book as a whole are reserved by INTECH. The book as a whole (compilation) cannot be reproduced, distributed or used for commercial or non-commercial purposes without INTECH's written permission.

Enquiries concerning the use of the book should be directed to INTECH rights and permissions department (permissions@intechopen.com).

Violations are liable to prosecution under the governing Copyright Law.

\section{(cc) BY}

Individual chapters of this publication are distributed under the terms of the Creative Commons Attribution 3.0 Unported License which permits commercial use, distribution and reproduction of the individual chapters, provided the original author(s) and source publication are appropriately acknowledged. If so indicated, certain images may not be included under the Creative Commons license. In such cases users will need to obtain permission from the license holder to reproduce the material. More details and guidelines concerning content reuse and adaptation can be foundat http://www.intechopen.com/copyright-policy.html.

\section{Notice}

Statements and opinions expressed in the chapters are these of the individual contributors and not necessarily those of the editors or publisher. No responsibility is accepted for the accuracy of information contained in the published chapters. The publisher assumes no responsibility for any damage or injury to persons or property arising out of the use of any materials, instructions, methods or ideas contained in the book.

First published in Croatia, 2009 by INTECH d.o.o.

eBook (PDF) Published by IN TECH d.o.o.

Place and year of publication of eBook (PDF): Rijeka, 2019.

IntechOpen is the global imprint of IN TECH d.o.o.

Printed in Croatia

Legal deposit, Croatia: National and University Library in Zagreb

Additional hard and PDF copies can be obtained from orders@intechopen.com

Frontiers in Adaptive Control

Edited by Shuang Cong

p. $\mathrm{cm}$.

ISBN 978-953-7619-43-5

eBook (PDF) ISBN 978-953-51-6404-3 


\section{We are IntechOpen, \\ the world's leading publisher of Open Access books}

\section{Built by scientists, for scientists}

\section{$4,400+$}

Open access books available

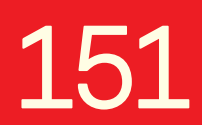

Countries delivered to

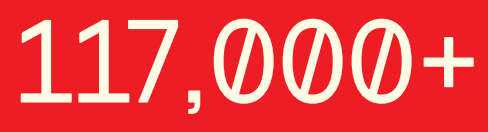

International authors and editors
$130 \mathrm{M}+$

Downloads

Our authors are among the

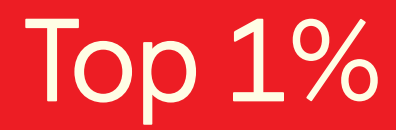

most cited scientists

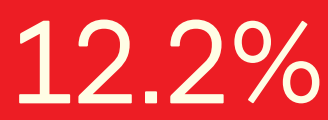

Contributors from top 500 universities

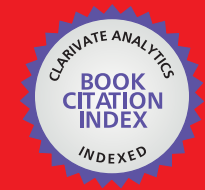

WEB OF SCIENCE ${ }^{\text {IM }}$

Selection of our books indexed in the Book Citation Index in Web of Science ${ }^{\mathrm{TM}}$ Core Collection (BKCI)

\section{Interested in publishing with us? \\ Contact book.department@intechopen.com}

Numbers displayed above are based on latest data collected.

For more information visit www.intechopen.com 



\section{Preface}

Starting in the early 1950s, the design of autopilots for high performance aircraft motivated an intense research activity in adaptive control. Today, adaptive control theory has grown to be a rigorous and mature discipline. Because it is good at dealing with uncertain quantities in dynamic systems in which exist unknown parameters and disturbances, adaptive control has become more popular in many fields of engineering and science in terms of algorithms, design techniques, analytical tools, and modifications. Nowadays, the presence of robotics in human-oriented applications demands control paradigms to face partly known, unstructured, and time-varying environments. Variety disturbances including applied external forces, higher order dynamics, nonlinearities and noise are always present in complex control systems such as robot manipulators and the human-machine ensemble. Adaptive control is required to be applied into new fields and more complex situations.

The objective of this book is to provide an up-to-date and state-of-the-art coverage of diverse aspects related to adaptive control theory, methodologies and applications. These include various robust techniques, performance enhancement techniques, techniques with less a-priori knowledge, nonlinear adaptive control techniques and intelligent adaptive techniques. There are several themes in this book which instance both the maturity and the novelty of the general adaptive control. The book consists of 17 Chapters. Each chapter is introduced by a brief preamble providing the background and objectives of subject matter. The experiment results are presented in considerable detail in order to facilitate the comprehension of the theoretical development, as well as to increase sensitivity of applications in practical problems. The outline of each chapter is as follows:

In Chapter 1, an adaptive control for a free-floating space robot is proposed by using the inverted chain approach, which is a unique formulation for a space robot compared with that for a ground-based manipulator system. Chapter 2 deals with introducing how to obtain models linear in parameters for real systems and then using observations from the system to estimate the parameters or to fit the models to the systems with a practical view. A new procedure for model validation in the frequency domain is presented in Chapter 3 . This procedure permits to validate or invalidate models over certain frequency ranges. The procedure is the translation of a time domain residual whiteness test to a frequency dependent residual whiteness test. The counterpart on the frequency domain of a time domain whiteness test is established.

In the methodologies, substantial progress of the Kalman filtering design for nonlinear stochastic systems made in the past decade offers promise for solving some long-standing control problems, which is considered in Chapter 4. In Chapter 5, a backstepping-like procedure incorporating the model reference adaptive control (MRAC) is employed to circumvent the difficulty introduced by its cascade structure and various uncertainties. A Lyapunov-like analysis is used to justify the closed-loop stability and boundedness of internal signals. In Chapter 6, a novel Takagi-Sugeno(TS) Feedforward fuzzy approximator(FFA)-based adaptive control scheme is proposed and applied to motion/force tracking 
control of holonomic systems. By integrating the feed-forward fuzzy compensation and error-feedback concepts, the proposed FFA-based control concept avoids heavy computation load and achieves global control results. In Chapter 7, two sliding mode adaptive control strategies have been proposed for single-input single-output(SISO) and single-input multiple-output(SIMO) systems with unknown bound time-varying uncertainty respectively. Chapter 8 introduces the Active Observer (AOB) algorithm for robotic manipulation. The $\mathrm{AOB}$ reformulates the classical Kalman filter (CKF) to accomplish MRAC. The AOB provides a methodology to achieve model-reference adaptive control through extra states and stochastic design in the framework of Kalman filters. In Chapter 9, the human-machine ensemble is regarded as an adaptive controller where both the environment and human cognition vary, the latter due to environmental and situational demands. Chapter 10 presents a parameter estimation routine that allows exact reconstruction of the unknown parameters in finite-time provided a given excitation condition is satisfied. The robustness of the routine to an unknown bounded disturbance or modeling error is also shown. In Chapter 11, a general scheme to construct adaptive policies in control models is to combine statistical estimation methods of the unknown distribution with control procedures. Such policies have optimality properties provided that the estimators are consistent in an appropriate sense. Chapter 12 develops a new adaptive control framework which applies to any nonlinearly parameterized system satisfying a general Lipschitzian property. This allows one to extend the scope of adaptive control to handle very general control problems of nonlinear parameterization since Lipschitzian parameterizations include as special cases convex/concave and smooth parameterizations. Chapter 13 presents a simple and straightforward adaptive controller strategy from the class of direct methods, based on reference models. The algorithm offers an alternative solution to the burden of process identification, and will present possibilities to tune both integer-and fractional-order controllers.

In the applications, Chapter 14 considers yaw dynamics of a vehicle operating under uncertain road conditions with unknown velocity and mass. Authors develop an adaptive control design technique motivated by the demand for a system capable of adjusting to deviations in vehicle parameters with almost negligible performance compromises. Chapter 15 proposes an indirect multiple -input multiple-output (MIMO) MRACS with structural estimation of the interactor. By using indirect method, unreasonable assumptions such as assuming the diagonal degrees of interactor can be avoided. Since the controller parameters are calculated based on the observability canonical realization of the estimated values, the proposed method is suitable for on-line calculations. Chapter 16 discourses on adaptive control for wireless local area networks introducing the Priority Oriented Adaptive Control with QoS Guarantee (POAC-QG) protocol for WLANs. It can be adapted into the Hybrid Control Function (HCF) protocol of the IEEE 802.11e standard in place of Hybrid Control Channel Access (HCCA). A Time Division Multiple Access (TDMA) scheme is adopted for the access mechanism. POAC-QG is designed to efficiently support all types of real-time traffic. Chapter 17 surveyed various topics in Very Large Scale Integrated (VLSI) technology in adaptive control perspective: The design margins in process and circuit level are considered to be headroom for power savings, and adaptive control schemes are used to figure out the margins automatically and to make adjustment without harming the system operation. An adaptive control is also used to optimize the circuit operation for time-varying circumstances. This type of scheme enables the chip to operate always in optimal condition for wide range of operation conditions.

I believe the new algorithms and adaptive control strategies presented in this book are very effective approaches to solve the problems in unknown parameter estimation, model- 
ing, analysis, adaptive controller design and some important research challenge. The book is also intended to be served as a reference for the researcher as well as the practitioner who wants to solve the problems caused by the uncertainty in the controlled systems. I hope that the reader will share my excitement to present this book on frontiers in adaptive control and will find it useful.

Finally, I would like to thanks all the authors of each Chapter for their contribution to make this book possible. My special thanks go to the publisher, In-Tech, for publishing this book.

\section{Shuang Cong}

University of Science and Technology of China

P. R. China

scong@ustc.edu.cn 



\section{Contents}

Preface VII

1. An Adaptive Control for a Free-Floating Space Robot by Using Inverted 001 Chain Approach

Satoko Abiko and Gerd Hirzinger

2. On-line Parameters Estimation with Application to Electrical Drives

Navid R. Abjadi, Javad Askari, Marzieh Kamali and Jafar Soltani

017

3. A New Frequency Dependent Approach to Model Validation

031

Pedro Balaguer and Ramon Vilanova

4. Fast Particle Filters and Their Applications to Adaptive Control

051

in Change-Point ARX Models and Robotics

Yuguo Chen, Tze Leung Lai and Bin Wu

5. An Adaptive Controller Design for Flexible-joint Electrically-driven Robots

071

With Consideration of Time-Varying Uncertainties

Ming-Chih Chien and An-Chyau Huang

6. Global Feed-forward Adaptive Fuzzy Control of Uncertain MIMO Nonlinear

097

Systems

Chian-Song Chiu and Kuang-Yow Lian

7. Function Approximation-based Sliding Mode Adaptive Control for

Time-varying Uncertain Nonlinear Systems

Shuang Cong, Yanyang Liang and Weiwei Shang

8. Model Reference Adaptive Control for Robotic Manipulation with Kalman

Active Observers

Rui Cortesão

9. Triggering Adaptive Automation in Naval Command and Control

165

Tjerk de Greef and Henryk Arciszewski

10. Advances in Parameter Estimation and Performance Improvement in 
11. Estimation and Control of Stochastic Systems under Discounted Criterion 209 Hilgert Nadine and Minjárez-Sosa J. Adolfo

12. Lipschitzian Parameterization-Based Approach for Adaptive Controls of

Nonlinear Dynamic Systems with Nonlinearly Parameterized Uncer-

tainties: A Theoretical Framework and Its Applications

N.V.Q. Hung, H.D. Tuan and T. Narikiyo

13. Model-free Adaptive Control in Frequency Domain:

Application to Mechanical Ventilation

Clara lonescu and Robin De Keyser

14. Adaptive Control Design for Uncertain and Constrained Vehicle Yaw

Dynamics

Nazli E. Kahveci

15. A Design of Discrete-Time Indirect Multivariable MRACS with Structural

Estimation of Interactor

Wataru Kase and Yasuhiko Mutoh

16. Adaptive Control in Wireless Networks

Thomas D. Lagkas, Pantelis Angelidis and Loukas Georgiadis

17. Adaptive Control Methodology for High-performance Low-power VLSI Design Se-Joong Lee 


\title{
An Adaptive Control for a Free-Floating Space Robot by Using Inverted Chain Approach
}

\author{
Satoko Abiko and Gerd Hirzinger \\ Institute of Robotics and Mechatronics, German Aerospace Center (DLR)
}

Germany

\section{Introduction}

On-orbit servicing space robots are one of the challenging fields in the robotics and space technology. The space robots are expected to perform various tasks including capturing a target, constructing a large structure and autonomous maintenance of on-orbit systems. In these space missions, one of the main tasks with the robotic system would be the tracking, the grasping and the positioning of a target in operational space. In this chapter, we address the task of following a desired trajectory in operational space while the space robot grasps a target with unknown dynamic properties. The dynamic uncertainty leads to a tracking problem, where a given nominal trajectory has to be tracked, while accounting for the parameter uncertainty.

In ground-based manipulator systems, the dynamic parameter uncertainty affects only dynamic equations. In free-floating space robots, however, the parameter uncertainty appears not only in the dynamic equations but also in kinematic mapping from the joint space to the Cartesian space due to the absence of a fixed base. Therefore, the model inaccuracies lead to the deviation of operational space trajectory provided by the kinematic mapping.

One method to deal with this issue can be found in an adaptive control. $\mathrm{Xu}$ and $\mathrm{Gu}$ proposed an adaptive control scheme for space robots in both joint space and operational space [Xu et al., 1992, Gu \& Xu, 1993]. However, the adaptive control proposed in [Xu et al., 1992] requires perfect attitude control and the adaptive control in [Gu \& $\mathrm{Xu}, 1993$ ] is developed based on an under-actuated system on the assumption that the acceleration of the base-satellite is measurable.

In this chapter, we propose an adaptive control for a fully free-floating space robot in operational space. This chapter particularly focuses on the uncertainty of kinematic mapping, which includes the dynamic parameters of the system. To achieve the desired input torque, it is assumed here that the velocity-based closed-loop servo controller is used as noted in [Konno et al., 1997].

In the modeling of the space robot, we consider the system switched around since a freefloating space robot does not have any fixed base, and then the robotic system is modeled from the end-effector to the base-satellite. This approach was termed the inverted chain approach in [Abiko et al., 2006]. The inverted chain approach explicitly explains coupled dynamics between the end-effector and the robot arm. A proposed adaptive control for 
operational space trajectory tracking is developed based on the inverted chain approach. The control method is verified in simulation for a realistic three-dimensional scenario (See Fig. 1).

The chapter is organized as follows. Section 2 describes the dynamic model of a space robot by the inverted chain approach. Section 3 discusses the operational space motion control for the space robot based on the passivity theorem. Section 4 proposes an adaptive control for trajectory tracking in operational space against parameter uncertainties. Section 5 derives an alternative adaptive control for performance improvement. Section 6 illustrates the simulation results with a three-dimensional realistic model. The conclusions are summarized in Section 7.

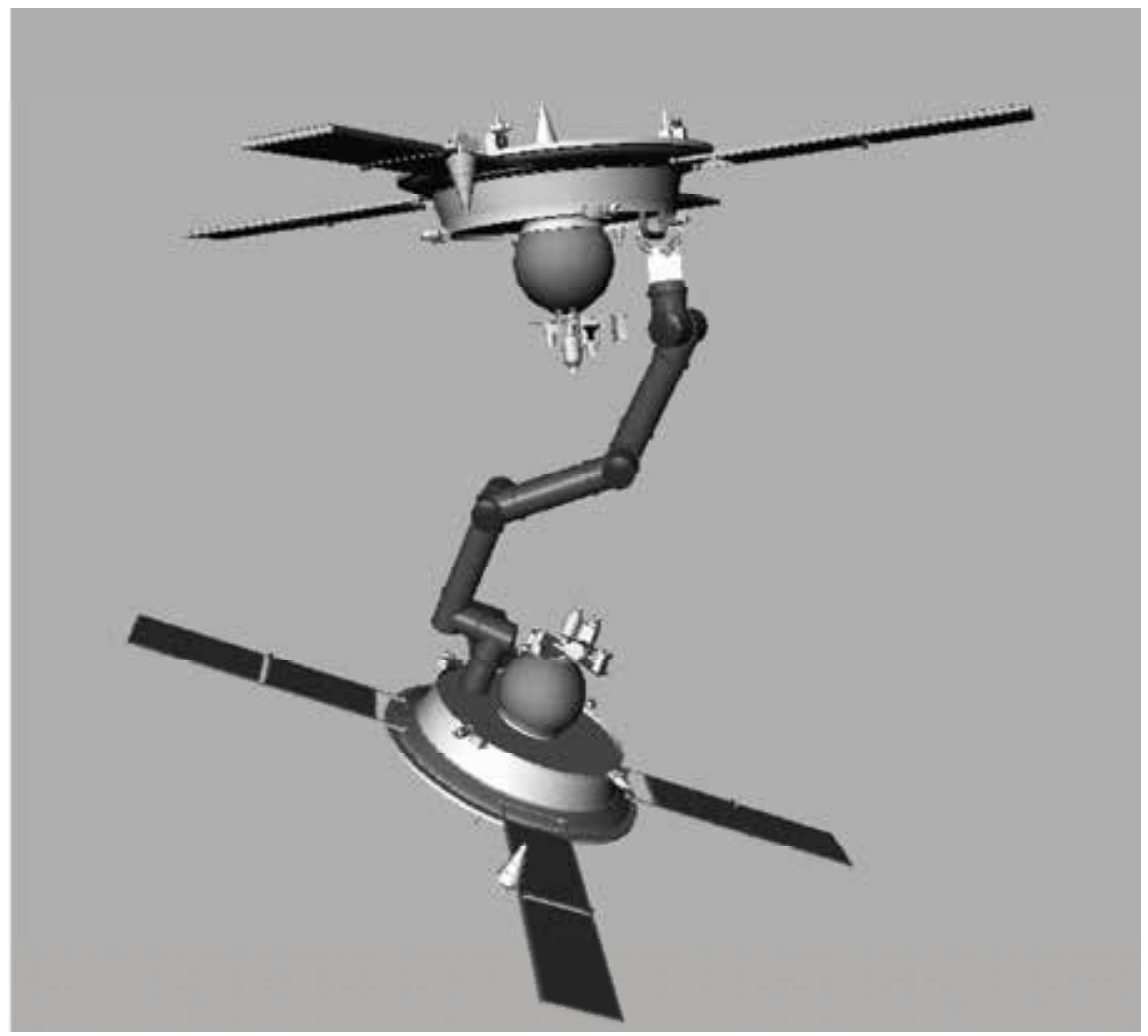

Figure 1. Chaser-robot and target scenario

\section{Modeling and Equations of Motion}

This section introduces the model of a space robot. Since the focus of this research is on following a desired trajectory in operational space, it is convenient to refer to operational space formulation.

Due to the lack of a fixed base, one can model a free-floating space robot with two approaches. The general dynamic expressions of the free-floating robot use linear and angular velocities of the base-satellite and the motion rate of each joint as the generalized coordinates [ $\mathrm{Xu} \&$ Kanade, 1993]. However, by considering the system switched around, modeled from the end-effector to the base, it can be represented by the motion of the end 
effector and that of the joints in the same structure as in the conventional expression. This scheme is termed the inverted chain approach.

The following subsections explain the dynamic equations of the system in the inverted chain approach, for a serial rigid-link manipulator attached to a floating base, as shown in Fig. 2. The main notations used in this section are listed in Table 1.

\subsection{Equations of motion - Inverted chain approach}

Let us consider the linear and angular velocities of the end-effector, $\dot{x}_{e}=\left(v_{e}^{T}, \omega_{e}^{T}\right)^{T} \in R^{6 \times 1}$, and the motion rate of the joints, $\dot{\phi} \in R^{n \times 1}$ as the generalized coordinates. The equations of motion are expressed in the following form:

$$
\left[\begin{array}{cc}
\boldsymbol{H}_{e} & \boldsymbol{H}_{e m} \\
\boldsymbol{H}_{e m}^{T} & \boldsymbol{H}_{m}
\end{array}\right]\left[\begin{array}{c}
\ddot{\boldsymbol{x}}_{e} \\
\ddot{\boldsymbol{\phi}}
\end{array}\right]+\left[\begin{array}{cc}
\boldsymbol{c}_{e} & \boldsymbol{c}_{e m} \\
\boldsymbol{c}_{e m}^{T} & \boldsymbol{c}_{m}
\end{array}\right]\left[\begin{array}{c}
\dot{\boldsymbol{x}}_{e} \\
\dot{\boldsymbol{\phi}}
\end{array}\right]=\left[\begin{array}{c}
\mathcal{F}_{e} \\
\boldsymbol{\tau}
\end{array}\right]+\left[\begin{array}{c}
\boldsymbol{J}_{e}^{T} \\
\boldsymbol{J}_{m}^{T}
\end{array}\right] \mathcal{F}_{b} .
$$

In the case that $\mathcal{F}_{b}$ is generated actively (e.g. jet thrusters or reaction wheels etc.), the system is called a free-flying robot. On the other hand, if no active actuators are applied on the base, the system is termed a free-floating robot. In this chapter, we consider the free-floating robot. The dynamic equation (1) possesses following important properties.

Property 1: The inerta matrices $\boldsymbol{H}_{e} \in R^{6 \times 6}, \boldsymbol{H}_{\mathrm{m}} \in R^{n \times n}$ and $\boldsymbol{H}=\left[\begin{array}{cc}\boldsymbol{H}_{e} & \boldsymbol{H}_{e m} \\ \boldsymbol{H}_{e m}^{T} & \boldsymbol{H}_{m}\end{array}\right] \in R^{(6+n) \times(6+n]}$ are symmetric and uniformly positive-definite for all $\boldsymbol{x}_{e} \in R^{6 \times 1}, \phi \in R^{n \times 1}$.

$n$

$v_{\mathrm{t}}$

$\omega_{\epsilon}$

$\dot{\boldsymbol{x}}_{e}=\left[\boldsymbol{v}_{\mathrm{c}}^{T} \omega_{\mathrm{c}}^{T}\right]^{T}$

$\phi$

$\boldsymbol{H}_{c}\left(\boldsymbol{x}_{c}, \phi\right)$

$H_{\mathrm{m}}\left(x_{c}, \phi\right)$

$\boldsymbol{H}_{e m}\left(\boldsymbol{x}_{\epsilon}, \phi\right)$

$c_{e}\left(x_{e}, \dot{x}_{e}, \dot{\phi}, \dot{\phi}\right)$

$c_{m}\left(x_{c}, \dot{x}_{c:} \phi, \phi\right)$

$c_{\mathrm{em}}\left(x_{c}, \dot{x}_{\mathrm{e}}, \phi, \phi\right)$ number of the joints.

$\in R^{3 \times 1}$ : linear velocity of the end-effector.

$\in R^{3 \times 1}$ : angular velocity of the end-effector.

$\in R^{6 \times 1}$ : spatial velocity of the end-effector.

$\in R^{n \times 1}$ : vector for the joint angle of the arm.

$\in R^{6 \times 6}$ : inertia matrix of the end-effector.

$\in R^{n \times n}$ : inertia matrix of the robot arm.

$\in R^{6 \times n}$ : coupling inertia matrix between the end-effector and the arm.

$\in R^{6 \times 6}$ : non-linear velocity dependent term on the end-effector.

$\in R^{n \times n}$ : non-linear velocity dependent term of the arm.

$\in R^{6 \times n}$ : coupling non-linear velocity dependent term between the end-effector and the arm.

$\in R^{6 \times 1}$ : force and moment exerted on the end-effector.

$\in R^{6 \times 1}$ : force and moment exerted on the base.

$\mathcal{F}_{b}$

$\mathcal{F}_{i}=-\boldsymbol{H}_{m, m} \ddot{\phi}-c_{e m} \dot{\phi}$

$\in \in R^{6 \times 1}$ : reaction force and moment due to the motion of the robot arm.

$\in R^{n \times 1}:$ torque on the joints.

$\tau$

$J_{t}$

$\boldsymbol{J}_{m}$ $\in R^{6 \times 1}$ : total linear and angular momentum around the end-effector.

$\in R^{6 \times 6}$ : Jacobian matrix related to the end-effector and the base.

$\in R^{6 \times n}$ : Jacobian matrix related to the arm and the base.

Table 1. Main notations in dynamic equations 


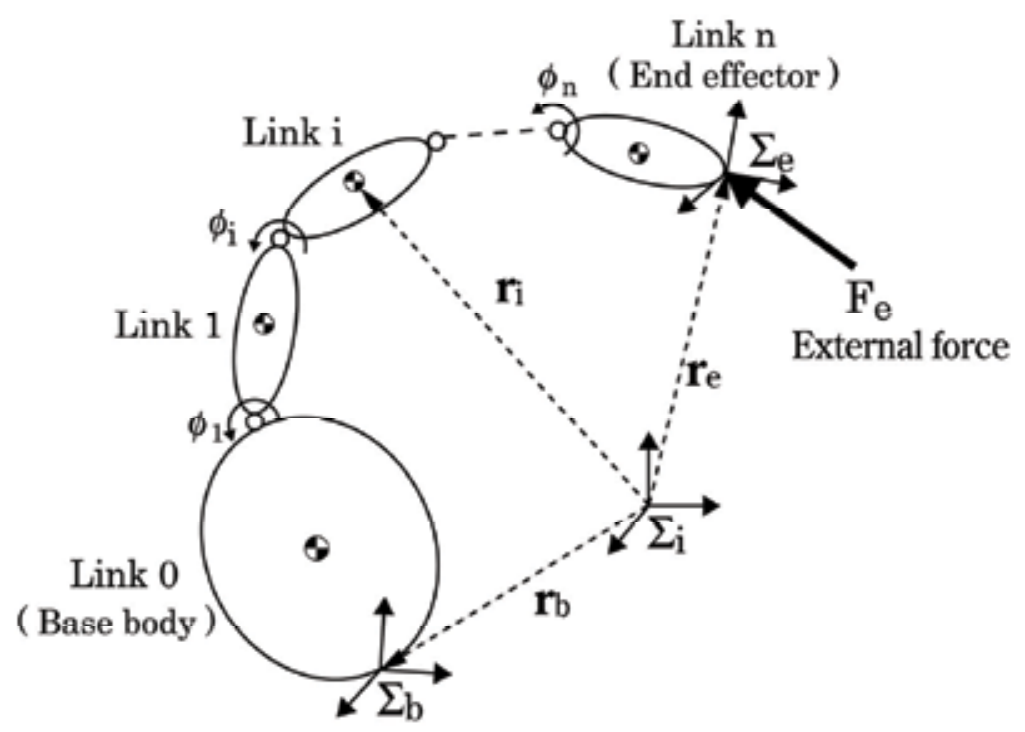

Figure 2. General model for a space robot

Property 2: The following matrices are skew-symmetric :

$$
\boldsymbol{S}_{e}=\boldsymbol{c}_{e}-\frac{1}{2} \dot{\boldsymbol{H}}_{e}, \quad \boldsymbol{S}_{m}=\boldsymbol{c}_{m}-\frac{1}{2} \dot{\boldsymbol{H}}_{m}, \quad \boldsymbol{S}=\boldsymbol{c}-\frac{1}{2} \dot{\boldsymbol{H}},
$$

so that:

$$
\nu_{c}^{T} \boldsymbol{S}_{\mathrm{e}} \nu_{\varepsilon}=0, \nu_{m}^{T} \boldsymbol{S}_{m} \nu_{m}=0, \nu^{T} \boldsymbol{S} \nu=0
$$

for all $\nu_{e} \in R^{n \times 1}, \nu_{m} \in R^{n \times 1}$ and $\nu \in R^{(6+x) \times 1}$, respectively. $c=\left[\begin{array}{cc}c_{\mathrm{c}} & c_{\mathrm{cm}} \\ c_{\mathrm{c} m}^{T} & c_{m}\end{array}\right] \in R^{(6+n) \times(f i+n)}$,

\subsection{Equations of motion in operational space}

The upper part of (1) clearly describes the equation of motion in operational space:

$$
\boldsymbol{H}_{e} \ddot{x}_{e}+\boldsymbol{H}_{e m} \ddot{\phi}+\boldsymbol{c}_{e} \dot{x}_{e}+\boldsymbol{c}_{e m} \dot{\phi}=F_{e}+J_{c}^{T} F_{b}
$$

In the free-floating space robot, only the joint motion can be considered as the generalized coordinate.

$$
\begin{aligned}
\boldsymbol{H}_{e} \ddot{x}_{e}+c_{e} \dot{x}_{e} & =-\boldsymbol{H}_{e m} \ddot{\phi}-\boldsymbol{c}_{\mathrm{e} m} \dot{\phi}+\mathcal{F}_{e}+\boldsymbol{J}_{e}^{T} \mathcal{F}_{b} \\
& =\mathcal{F}_{i}+\mathcal{F}_{e}+\boldsymbol{J}_{\mathrm{e}}^{T} \mathcal{F}_{b}
\end{aligned}
$$

where $\mathcal{F}_{i}=-H_{\mathrm{cm}} \ddot{\phi}-\boldsymbol{c}_{\mathrm{cm}} \dot{\phi} \in l^{2 \times 1}$ stands for a reaction force onto the end-effector due to the robot arm motion.

\section{Remark 1: Input command for the operational space dynamics}

The right-hand side in (3) apparently shows the reaction or coupling effect due to the motion of the robot arm with joint acceleration expression. The torque control input does not appear explicitly in (3). Joint acceleration, however, can be achieved by velocity-based 
closed-loop servo controller straightforwardly as noted in [Konno et al., 1997]. Therefore, eq. (3) are convenient formulation for constructing a control strategy. Hereafter, $\ddot{\phi}$ is considered as an input command to the system and the appropriate joint acceleration for proper control law is computed. Then, one can refer $\mathcal{F}_{i}=-\boldsymbol{H}_{\mathrm{cm}} \ddot{\phi}-c_{\mathrm{cm}} \dot{\phi}$ as a reaction force due to the motion of the robot arm, which can be used to analyze the influence of the parameter errors in Section 4.

\section{Remark 2: Linearity in the Dynamic Parameters}

The linearity of eq. (2) is one of the significant features in the articulated-body system. This characteristic plays a key role in the derivation of an adaptive control. The integral of eq. (2) represents the total linear and angular momentum around the center of mass of the endeffector. Then, on the assumption that no active force and torque are applied on the base (e.g. $\mathcal{F}_{b}=0$ ), eq. (2) can be described as the time-derivative of the momentum $\mathcal{L}_{e} \in R^{6 \times 1}$ as follows:

$$
\begin{aligned}
\mathcal{F}_{e}= & \frac{d}{d t} \mathcal{L}_{e}=\boldsymbol{H}_{e} \ddot{x}_{e}+\boldsymbol{H}_{e m} \ddot{\boldsymbol{\phi}}+\boldsymbol{c}_{e} \dot{\boldsymbol{x}}_{e}+\boldsymbol{c}_{e m} \dot{\boldsymbol{\phi}}, \\
\mathcal{L}_{e} & =\boldsymbol{H}_{e} \dot{\boldsymbol{x}}_{e}+\boldsymbol{H}_{e m} \dot{\boldsymbol{\phi}} \\
& =\sum_{i=0}^{n}\left[\begin{array}{c}
m_{i} \dot{\boldsymbol{r}}_{i} \\
\boldsymbol{I}_{i} \boldsymbol{\omega}_{i}+\left(\boldsymbol{r}_{i}-\boldsymbol{r}_{e}\right) \times m_{i} \dot{\boldsymbol{r}}_{i}
\end{array}\right],
\end{aligned}
$$

where $\boldsymbol{I}_{i}, \boldsymbol{\omega}_{i}$ and $m_{i}$ stand for the inertia matrix, angular velocity and mass of the link $i$, respectively, $r_{i}$ and $r_{e}$ denote the vector from the inertial frame to the center of mass of the link $i$ and that from the inertial frame to the center of mass of the end-effector, respectively (see Fig. 2). Once eq. (5) can be linearized with respect to a suitable set of dynamic parameters, eq. (4) can be linear in terms of the dynamic parameters since the dynamic parameters are independent on the motion of the system.

Through some calculations, eq. (5) is linearized in terms of a set of arbitrary dynamic parameters $\boldsymbol{a}$.

$$
\mathcal{L}_{e}=\boldsymbol{y}\left(\boldsymbol{x}_{e}, \dot{\boldsymbol{x}}_{e}, \boldsymbol{\phi}, \dot{\boldsymbol{\phi}}\right) \boldsymbol{a}
$$

Then eq. (4) can be expressed as a function of $\boldsymbol{a}$.

$$
\mathcal{F}_{e}=\boldsymbol{H}_{e} \ddot{x}_{e}+\boldsymbol{H}_{e m} \ddot{\boldsymbol{\phi}}+c_{e} \dot{\boldsymbol{x}}_{e}+c_{e m} \dot{\boldsymbol{\phi}}=\boldsymbol{Y}\left(\boldsymbol{x}_{e}, \dot{\boldsymbol{x}}_{e}, \ddot{\boldsymbol{x}}_{e}, \phi, \dot{\phi}, \ddot{\phi}\right) a
$$

where $\boldsymbol{Y}$ stands for the time-derivative of $\boldsymbol{y}$, which is a function of state values and is called the regressor. The choice of the regressor $\boldsymbol{Y}$ and the dynamic parameter vector $\boldsymbol{a}$, is generally arbitrary. In this chapter, we assume that only a grasped target, attached on the end-effector, includes unknown dynamic parameters. The dynamic parameters of the rest of the system and the kinematic parameters are supposed to be well-identified in advance. Therefore, the unknown dynamic parameter vector $\boldsymbol{a}$ is defined as a $p$-dimensional vector containing the mass, center of mass, moment of inertia and product of inertia of the target. Note that $\boldsymbol{a}$. defined here is constant.

$$
\boldsymbol{a}=\left(m, r_{y, z}, r_{y y y}, r_{y z}, I_{\mathrm{ax}:}, I_{y y}, I_{z z}, I_{\mathrm{a}: 3 y}, I_{y, z}, I_{z x}\right)^{T}(p=10) .
$$




\section{Trajectory Control in Operational Space}

This section shows the trajectory controller in operational space for a free-floating space robot. The control law shown in this section is derived based on the passivity theorem [van der Schaf, 2000].

\subsection{Passivity based trajectory tracking control}

Let us define a reference output velocity $\eta$ and a reference output acceleration $\dot{\eta}$ as follows:

$$
\begin{aligned}
& \eta=\dot{x}_{\epsilon}^{d}+\boldsymbol{K}_{v} \widetilde{x}_{e}, \\
& \dot{\eta}=\ddot{x}_{\epsilon}^{j}+\boldsymbol{K}_{z} \dot{\tilde{x}}_{r},
\end{aligned}
$$

where $K_{v} \in R^{b \times 6}$ is a strictly positive definite matrix. $\dot{x}_{t:}^{d t}=\left(v_{t}^{d T}, \omega_{v}^{d T}\right)^{I^{\prime}} \in R^{6 \times 1}$ represents the desired velocity in operational space. $\tilde{x}_{c}=\left(e_{p}^{T}, e_{O}^{T}\right)^{T} \subset R^{\phi \times 1}$ depicts the operational space error consisting of the position error $e_{p} \in R^{3 \times 1}$ and the orientation error $\varepsilon_{0} \in R^{3 \times 1}$. The position error $e_{p}$ is expressed as:

$$
e_{p}=r_{c}^{d}-r_{e}
$$

The orientation error $\boldsymbol{e}_{Q}$ is expressed by means of the quaternion expression $\mathcal{Q}=\left[\xi, \epsilon^{T}\right]$ where $\xi$ and $\epsilon$ are the scalar and vector part of the quaternion:

$$
e_{0}=\Delta \epsilon=\xi \epsilon^{d}-\xi^{d} \epsilon-\epsilon^{d} \times \epsilon,
$$

where the operator $x$ denotes the cross-product operator.

The reference error $s$ between the reference output $\eta$ and the actual velocity $\dot{x}_{c}$ can be described by:

$$
\boldsymbol{s}=\eta-\dot{\boldsymbol{x}}_{r}=\dot{\tilde{\boldsymbol{x}}}_{r}+\boldsymbol{K}_{n} \ddot{\boldsymbol{x}}_{r}
$$

In the case without any parameter errors, the trajectory tracking control law can be determined by using the feedback linearization as follows:

$$
\ddot{\phi}^{\alpha}=-\boldsymbol{H}_{e m}^{+}\left(\boldsymbol{H}_{e} \dot{\eta}+\boldsymbol{c}_{e} \eta+\boldsymbol{c}_{e m} \dot{\phi}+\boldsymbol{A} s\right)
$$

where $\boldsymbol{\Lambda} \in I^{i \times 6}$ denotes a positive definite symmetric constant matrix. $\{\cdot\}^{u}$ stands for the input command and \{\}$^{+}$denotes the pseudo-inverse operator. Note that the control law (9) can be achieved under the condition when $\boldsymbol{H}_{e m}$ is nonsingular. Since several researches have already been proposed the treatment of the singularity problem [Nenchev et al., 2000,Tsumaki et al., 2001, Senft \& Hirzinger, 1995, Nakamura \& Hanafusa, 1986], it is out of focus in this chapter.

\subsection{Stability analysis}

The stability of the control law (9) can be analyzed by means of the Lyapunov direct method. The following reference error energy is considered as a Lyapunov function: 


$$
E(l)=\frac{1}{2} s^{T} H_{e} s .
$$

The time-derivative of $E$ is given as:

$$
\begin{aligned}
\dot{E}(t) & =s^{T}\left(\boldsymbol{H}_{\mathrm{c}} \dot{s}+\frac{1}{2} \dot{\boldsymbol{H}}_{\mathrm{c}} \boldsymbol{s}\right) \\
& =\boldsymbol{s}^{T}\left(\boldsymbol{H}_{\mathrm{E}} \dot{\boldsymbol{\eta}}-\boldsymbol{H}_{\mathrm{e}} \ddot{x}_{\mathrm{e}}+\frac{1}{2} \dot{\boldsymbol{H}}_{\mathrm{e}} \boldsymbol{s}\right) \\
& =\boldsymbol{s}^{T}\left(\boldsymbol{H}_{\mathrm{t}} \dot{\boldsymbol{\eta}}+\boldsymbol{H}_{e m} \ddot{\boldsymbol{\phi}}+\boldsymbol{c}_{\mathrm{r}} \boldsymbol{\eta}+\boldsymbol{c}_{\mathrm{e} m} \dot{\phi}\right),
\end{aligned}
$$

where Property 2 in Section 2 is used. Since the control command is expressed in eq. (9), $\left(\ddot{\phi}=\ddot{\phi}^{u}\right.$ noted in Remark 1 in Section 2), the time-derivative of $E(t)$ results in:

$$
\dot{E}(t)=-s^{T} \Lambda s \leq 0
$$

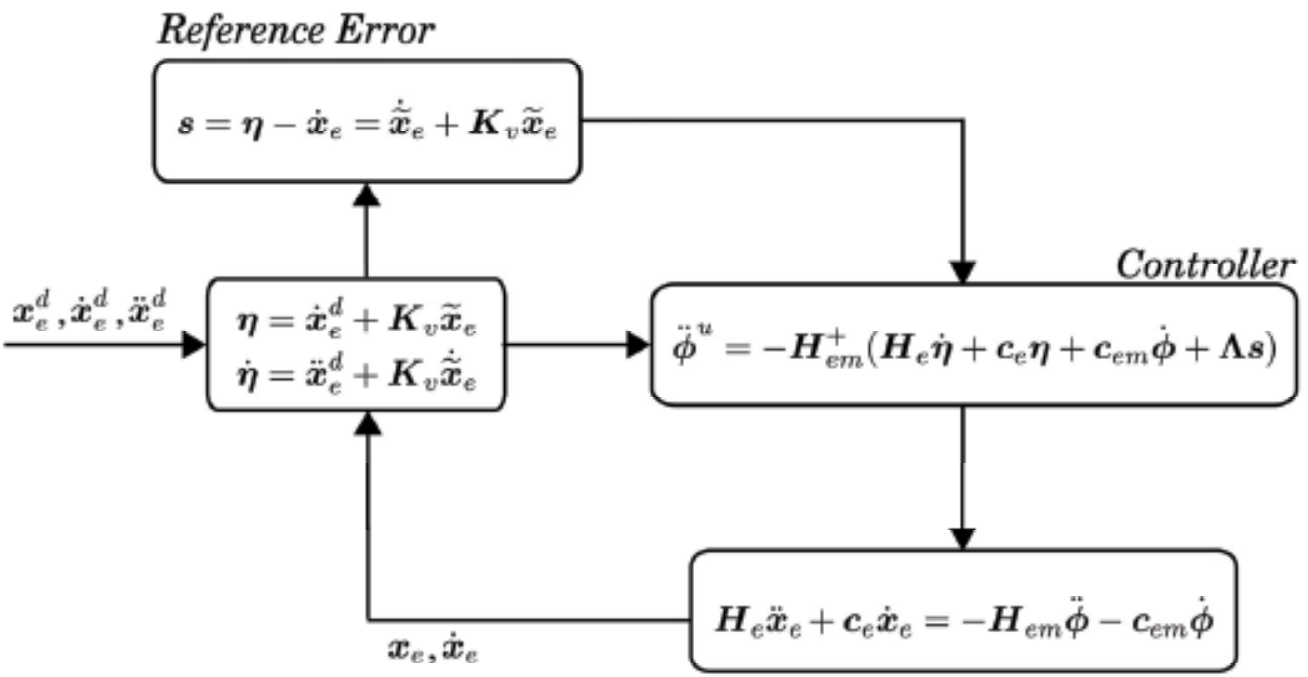

Robot Plant

Figure 3. Control diagram for trajectory tracking

Consequently, the result of $\dot{F}_{:}$holds always semi-negative and the closed-loop system (2) with (9) is guaranteed to be asymptotically stable. The inequality (12) implies that the steady-state reference error $s$ converges asymptotically to zero, which leads to the convergence of the steady-state position. The control diagram for operational tracking control is shown in Fig. 3.

\section{Adaptive Control}

The previous section explained the trajectory control for a free-floating space robot based on the inverted chain approach on the assumption of no dynamic parameter errors. In practical situations, however, the robot arm handles various components whose dynamic properties 
are not known in advance. Those model inaccuracies may lead to the degradation of the control performance and the deviation of the trajectory tracking from the desired one.

This section proposes an adaptive control for a free-floating space robot against the parameter uncertainties.

\subsection{Influence of the dynamic parameter errors}

In the presence of dynamic parameter inaccuracies, the dynamic model in operational space can be described as follows:

$$
\widehat{\boldsymbol{H}}_{\mathrm{c}} \ddot{x}_{\mathrm{c}}+\widehat{\boldsymbol{\epsilon}}_{\mathrm{c}} \dot{x}_{\mathrm{c}}=-\widehat{\boldsymbol{H}}_{\mathrm{cm}} \ddot{\phi}-\hat{\boldsymbol{c}}_{\mathrm{cm}} \dot{\phi}=\hat{\mathcal{F}}_{i}
$$

where $\{\cdot\}$ stands for the matrix including dynamic parameter errors. In analogy with (9), the control law derived from the dynamic model (13) becomes:

$$
\ddot{\phi}^{u}=-\widehat{\boldsymbol{H}}_{\mathrm{cm}}^{l}\left(\widehat{\boldsymbol{H}}_{\epsilon} \dot{\boldsymbol{\eta}}+\widehat{\boldsymbol{c}}_{\epsilon} \boldsymbol{\eta}+\widehat{\boldsymbol{c}}_{e m} \dot{\boldsymbol{\phi}}+\boldsymbol{\Lambda} s\right)
$$

In the implementation of the input command (14) to the dynamic system (2), the reaction force due to the motion of the robot $\operatorname{arm} \mathcal{F}_{;}$and the corresponding expected reaction force $\tilde{\mathcal{F}}_{i}$ has error $\ddot{\mathcal{F}}_{i}$.

$$
\begin{aligned}
\widetilde{\mathcal{F}}_{\dot{i}} & =\mathcal{F}_{i}-\widehat{\mathcal{F}}_{i} \\
& =-\boldsymbol{H}_{\mathrm{cm}} \ddot{\phi}^{u}+\widehat{\boldsymbol{H}}_{\mathrm{em}} \ddot{\phi}^{u}-c_{\mathrm{em}} \dot{\phi}+\hat{\boldsymbol{c}}_{\mathrm{cm}} \dot{\phi} \\
& =-\widetilde{\boldsymbol{H}}_{\mathrm{m} m} \ddot{\phi}^{u}-\widehat{\boldsymbol{c}}_{\mathrm{m} m} \dot{\phi},
\end{aligned}
$$

where $\{r\}$ stands for the error matrix. With the input acceleration (14), the reaction force $\mathcal{F}_{i}$ can be described by the corresponding expected force $\widehat{\mathcal{F}}_{i}$ and the error $\widetilde{\mathcal{F}}_{i}$ as follows:

$$
\begin{aligned}
\mathcal{F}_{i} & =\widehat{\mathcal{F}}_{i}+\widetilde{\mathcal{F}}_{i} \\
& =\widehat{\boldsymbol{H}}_{b} \dot{\boldsymbol{\eta}}+\widehat{\boldsymbol{c}}_{n} \eta+\boldsymbol{\Lambda} s-\widetilde{\boldsymbol{H}}_{c m} \ddot{\phi}^{\mu}-\widetilde{\boldsymbol{c}}_{c m} \dot{\phi} .
\end{aligned}
$$

Let us analyze here the stability of the system containing the dynamic parameter errors by using the Lyapunov function (10). In the closed-loop system (2) with the controller (14), the time-derivative of the Lyapunov function (10) is given by:

$$
\begin{aligned}
\dot{E}(t) & =s^{T}\left(\boldsymbol{H}_{\mathrm{c}} \dot{\boldsymbol{\eta}}+\boldsymbol{H}_{\mathrm{cm}} \ddot{\phi}+\boldsymbol{c}_{\mathrm{c}} \boldsymbol{\eta}+\boldsymbol{c}_{\mathrm{cm}} \dot{\phi}\right) \\
& =\boldsymbol{s}^{T}\left(\boldsymbol{H}_{e} \dot{\boldsymbol{\eta}}+\widetilde{\boldsymbol{H}}_{\mathrm{c} m} \ddot{\phi}^{\mathrm{w}}+\widetilde{\boldsymbol{c}}_{e} \boldsymbol{\eta}+\widetilde{\boldsymbol{c}}_{\mathrm{em}} \dot{\phi}-\boldsymbol{\Lambda} \boldsymbol{s}\right)
\end{aligned}
$$

where Remark 1 is used, namely $\ddot{\phi}=\ddot{\phi}^{u t}$.As mentioned in Remark 2 , the dynamic system is linearized with the vector of dynamic parameters $\boldsymbol{a}$ and the regressor $\boldsymbol{Y}$. Then, the above time-derivative can be rewritten as:

$$
\dot{E}(t)=s^{T}(\boldsymbol{Y} \ddot{a}-\Lambda s)
$$


where $\ddot{a}=\boldsymbol{a}-\widehat{a}$ denotes the parameter estimation error vector, $\boldsymbol{a}$ is a $p$-dimensional vector including the unknown dynamic parameters and $\boldsymbol{a}$ is its estimate. The above equality indicates that each component $\Lambda_{i}$ in the gain matrix $\Lambda$ needs to meet the following condition in order to obtain the robust system against the model inaccuracies:

$$
\Lambda_{i} \geq[\mathbf{Y a}]_{\dot{x}}+\mu_{i},(i=1 \cdots 6)
$$

where the constant $\mu_{i}$ is strictly positive. As long as the above condition holds, the controller (14) is robust against the parameter inaccuracies and the tracking error converges to zero.

\subsection{Adaptive controller design}

Equation (17) suggests two solutions to compensate the parameter uncertainty in the system. One is the improvement of the robustness in the control law (14) with proper design of the gain matrix as shown in (19). The other is to adjust the dynamic parameter itself during the operation, which is called an adaptive control [Slotine \& Li, 1987] [Slotine \& Li, 1988].

This section proposes an adaptive control in the case without any knowledge of the dynamic parameters in advance, such that the space robot grasps a target whose dynamic parameters are unknown.

Let us consider the following Lyapunov function described with the sum of the reference error energy of the system (10) and the potential energy due to the model uncertainties:

$$
V(l)=E(l)+\frac{1}{2} \tilde{a}^{\prime \prime} \Gamma \tilde{a}
$$

where $\Gamma \in R^{p \times p}$ is a positive definite matrix. The time-derivative of (20) becomes:

$$
\dot{V}(t)=-s^{T} \boldsymbol{\Lambda} s+\tilde{\boldsymbol{a}}^{T}\left(\boldsymbol{Y}^{Y^{\prime}} s+\Gamma \dot{\tilde{a}}\right) .
$$

This suggests the following condition should be met to guarantee the system stability,

$$
\boldsymbol{Y}^{T} s+\Gamma \dot{\tilde{a}}=0
$$

Then, the following adaptive control law is derived as:

$$
\dot{\hat{a}}=-\Gamma^{-1} Y^{T} s,
$$

where $\ddot{a}=\boldsymbol{a}-\widehat{a}$ and the parameter vector $\boldsymbol{a}$ is constant.

Consequently, the time-derivative of the Lyapunov function results in:

$$
\dot{V}(t)=-s^{T} \Lambda s \leq 0
$$

The inequality (24) indicates the reference error $s$ converges asymptotically to zero if and only if $\dot{\vec{x}}_{e} \rightarrow \mathbf{0}$ and $\ddot{x}_{\mathrm{z}} \rightarrow \mathbf{0}$. Accordingly, the control law for the trajectory tracking in operational space (14) and the adaptation law (23) yield a stable adaptive controller. Fig. 4 shows the control diagram for the proposed adaptive control. 


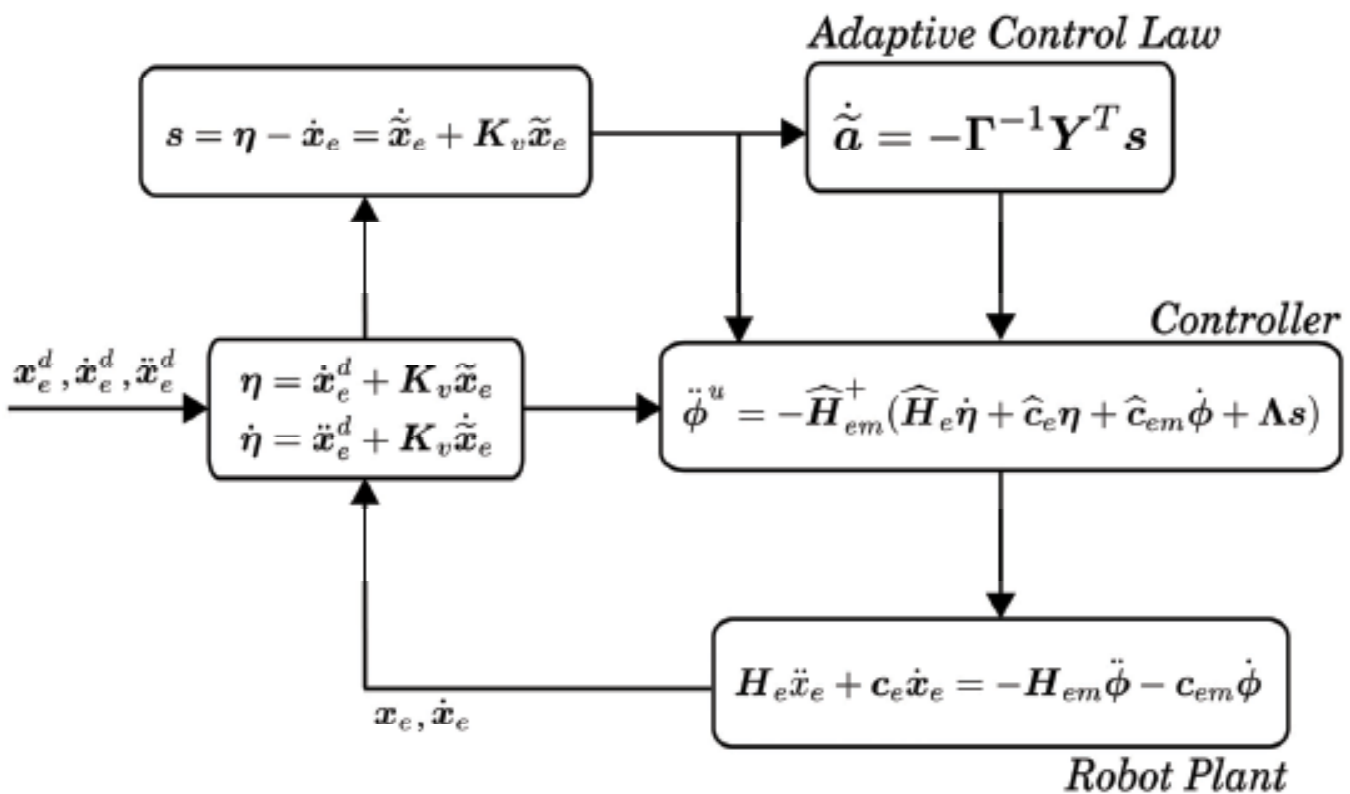

Figure 4. Control diagram for an adaptive trajectory tracking control in operational space

\section{Composite Adaptive Control}

The adaptive controller developed in the previous section exploits the tracking error to extract the parameter information. To obtain the parameter information, however, one can find various candidates [Slotine \& Li, 1991]. One possible candidate is the prediction error, which is generally used for parameter estimation. In this section, an alternative adaptive control law is developed with the combination of the tracking error and the reaction force error. The reaction forces due to the motion of the robot-arm are assumed to be measured by the force/torque sensor attached on the end-effector, to which the target is attached. The measurement values are used for parameter adaptation together with the nominal adaptive control law (23).

\subsection{Composite adaptive controller design}

In analogy with Section 2, the reaction forces on the end-effector are able to be linearized with a proper set of the dynamic parameters $a$ as $F_{\varepsilon}^{l / T}=W a$ and its prediction error can be described as $\hat{\mathcal{F}}_{*}^{F T}=\boldsymbol{W} \tilde{\boldsymbol{a}}$, where $\boldsymbol{W}$ stands for the regressor. The detail derivation is omitted in this chapter.

The adaptive control law (23) is extended to the following expression combined with the tracking error and the predicted reaction force error:

$$
\dot{a}=-\Gamma^{-1}\left\{\boldsymbol{Y}^{T} s+\boldsymbol{W}^{T} \boldsymbol{R} \mathcal{F}_{e}^{+\gamma l}\right\}
$$

where $R \in R^{\mathrm{b} \times 6}$ is a uniformly weighting matrix. Eq. (25) can be rewritten as: 


$$
\dot{\hat{\boldsymbol{a}}}+\Gamma^{-1} \boldsymbol{W}^{T} \boldsymbol{R} \boldsymbol{W} \tilde{\boldsymbol{a}}=-\Gamma^{-1} \boldsymbol{Y}^{T} s
$$

which indicates a time-varying low-pass filter and that parameter and tracking error convergence in composite adaptive control can be smoother and faster than in the nominal adaptive control only.

To analyze the stability of the system applied the above composite adaptive control law and the trajectory tracking control, the Lyapunov function (20) is considered again. The timederivative of (20) is derived as (21). Since the adaptive control law is determined by (25), substitution of (25) into (21) leads to the following inequality:

$$
\dot{V}(b)=-s^{\top} \Lambda s-\tilde{a}^{T} W^{T} R \boldsymbol{W} \tilde{a} \leq 0
$$

which describes that the reference error $s$ and the prediction error $\pi^{T}$ globally converge to zero if the desired trajectories are bounded. If the trajectories are persistently exciting and uniformly continuous, the estimated parameters converge asymptotically to the real ones. Fig. 5 shows the control diagram for the proposed composite adaptive control.

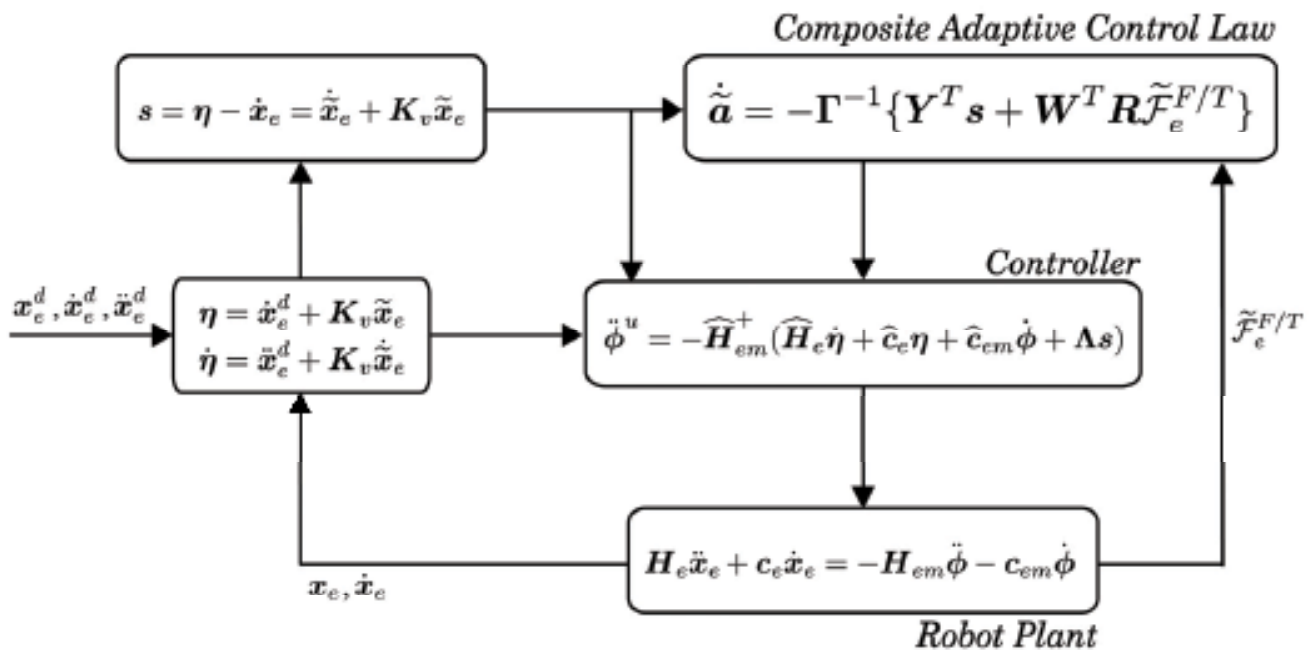

Figure 5. Control diagram for a composite adaptive trajectory tracking control in operational space

\section{Simulation Study}

This section presents the numerical simulation results of a realistic three-dimensional model as shown in Fig. 1. In this simulation, the chaser-robot is assumed to track a given trajectory while it grasps firmly a target including unknown dynamic properties. The dynamic parameters of the rest of the system and the kinematic parameters are supposed to be well-identified in advance. The initial total linear and angular momentum for whole system are zero in the simulation. During the tracking phase, no external force is applied. The chaser robot has a 7DOF manipulator mounted on the base satellite, whose dynamic parameters are shown in Table 2. The robot arm has one redundancy with respect to the 
end-effector motion, then the null-space can be used for an additional task. In the simulation examples, the target parameters of the planned motion are supposed to be zero, while those of the controlled motion are in Table 3, giving the extent of uncertainty introduced in the system. As mentioned in Section 2, the vector of the unknown dynamic parameters $\boldsymbol{a}$ is defined as follows:

$$
\boldsymbol{a}=\left(m, r_{g x}, r_{g y}, r_{g z}, I_{x x}, I_{y y}, T_{z z}, I_{x y}, I_{y z}, I_{z x}\right)^{\gamma \prime}(\mu=10) .
$$

The adaptation gain $\mathbf{\Gamma}^{-1}$ in eq. (23) is determined as:

$$
\boldsymbol{\Gamma}^{-1}=\operatorname{diag}\left(\left[5 \times 10^{3}, 10,10,10,5 \times 10^{2}, 5 \times 10^{2}: 5 \times 10^{2}: 5 \times 10^{-1}, 5 \times 10^{-1}: 5 \times 10^{-1}\right]\right)
$$

The control gains $\boldsymbol{\Lambda}$ and $\boldsymbol{K}_{v}$ in eq. (14) are set to be:

$$
\begin{aligned}
\boldsymbol{\Lambda} & =\operatorname{diog}([20,20,20,3000,3000,3000]), \\
\boldsymbol{K}_{i} & =\operatorname{diag}([10,10,10,1000,1000,1000]) .
\end{aligned}
$$

The weighing matrix $\boldsymbol{R}$ in the composite adaptive control (25) is determined as:

$$
\boldsymbol{R}=\operatorname{diag}([0.5,0.5,0.5,0.5,0.5,0.5]) \text {. }
$$

\begin{tabular}{|l|c|c|c|c|}
\hline & mass $[\mathrm{kg}]$ & $I_{x x}\left[\mathrm{kgm}^{2}\right]$ & $I_{y y}\left[\mathrm{kgm}^{2}\right]$ & $I_{z z}\left[\mathrm{kgm}^{2}\right]$ \\
\hline Base & 140 & 18.0 & 20.0 & 22.0 \\
\hline
\end{tabular}

\begin{tabular}{|l|c|c|c|c|}
\hline & mass $[\mathrm{kg}]$ & $I_{x x}\left[\mathrm{kgm}^{2}\right]$ & $I_{y y}\left[\mathrm{kgm}^{2}\right]$ & $I z z\left[\mathrm{kgm}^{2}\right]$ \\
\hline Each Link & 3.3 & 0.0056 & 0.0056 & 0.0056 \\
\hline
\end{tabular}

Table 2. Dynamic parameters for a chaser-robot

\begin{tabular}{|c|c|c|c|}
\hline mass $[\mathrm{kg}]$ & $I_{x x}\left[\mathrm{kgm}^{2}\right]$ & $I_{y y}\left[\mathrm{kgm}^{2}\right]$ & $I_{z z}\left[\mathrm{kgm}^{2}\right]$ \\
\hline 87.5 & 11.25 & 12.5 & 12.5 \\
\hline
\end{tabular}

Table 3. Dynamic parameters for a target

\begin{tabular}{|l|l|l|c|}
\hline & w/oAC & with AC & with CAC \\
\hline RMS error & 0.0141 & 0.0048 & 0.0032 \\
\hline
\end{tabular}

Table 4. Root Mean Square error for tracking error

Figs. 6 and 7 illustrate the desired and actual trajectories in Cartesian space. Fig. 6 shows the case with parameter deviations but without adaptive control. Fig. 7 shows the case 
with adaptive control (23). The left graphs depict the trajectory in xy plane and the right graphs show the trajectory in $\mathrm{xz}$ plane in Cartesian space. In the graphs, the solid line depicts the desired trajectory and the dashed line depicts the actual trajectory, respectively. It is clearly observed that the end effector follows the trajectory when the adaptive control is activated, even though the parameter deviations exist, while in the case without adaptive control law, the end effector deviates the desired trajectory due to the model errors. Fig. 8 depicts the typical examples for the parameter adaptation process when the adaptive control law is applied. In the figure, the adaptation processes of the mass, moment of inertia of each axis are shown. Note here that the adjusted dynamic parameters do not have to converge to the real ones since the demanded task is to follow a given trajectory. If one would like to identify real values, the persistent excitation of the input command is required.

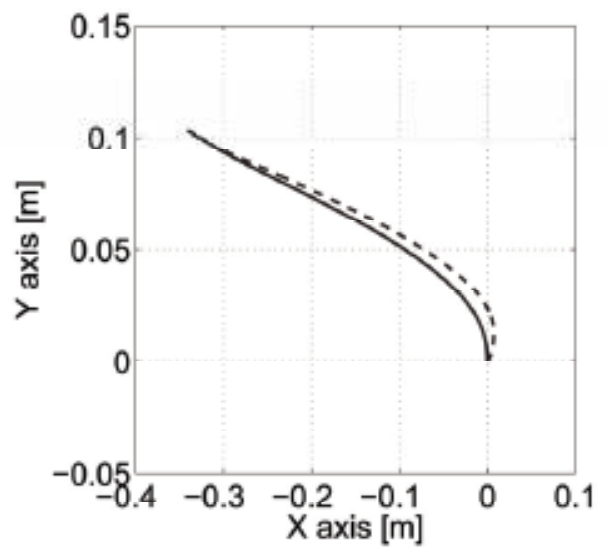

(a) XY Plan:

Figure 6. Trajectory without adaptive control

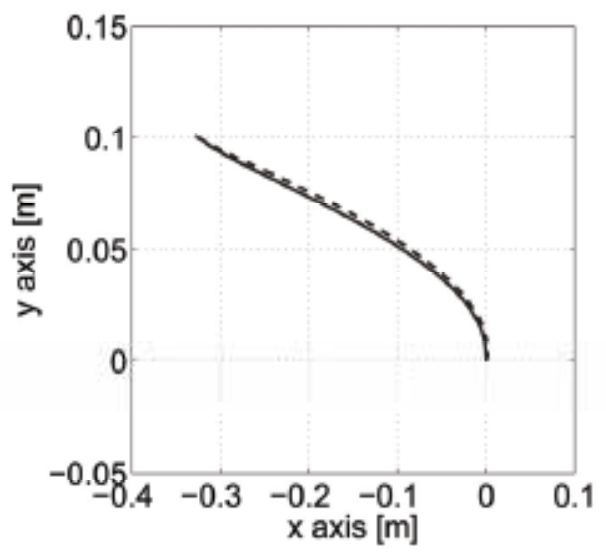

(d) XY Plane

Figure 7. Trajectory with adaptive control

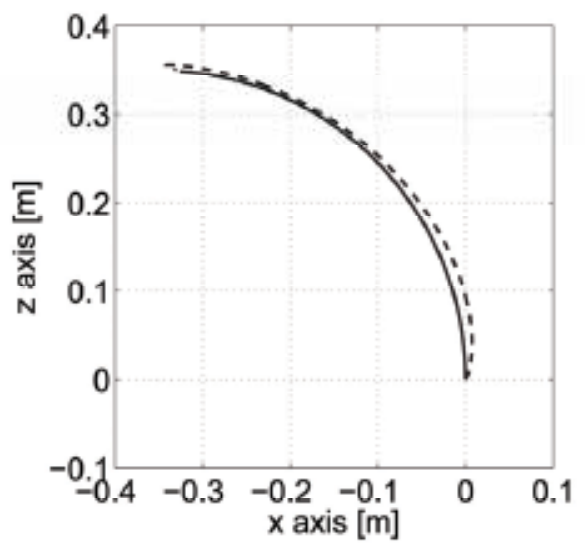

(b) X7. Planc

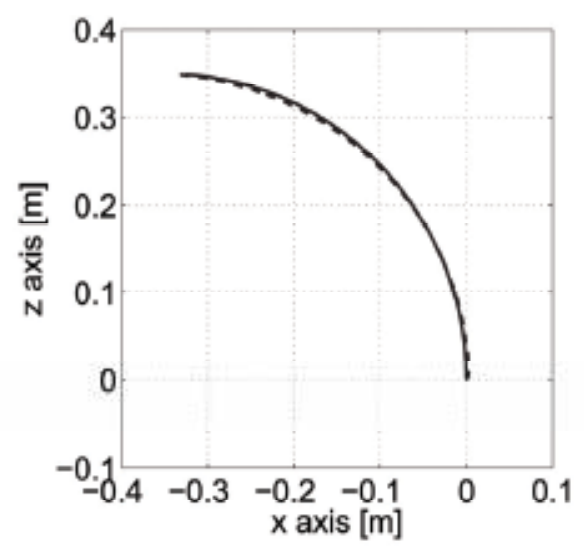

(b) X7. Plane 

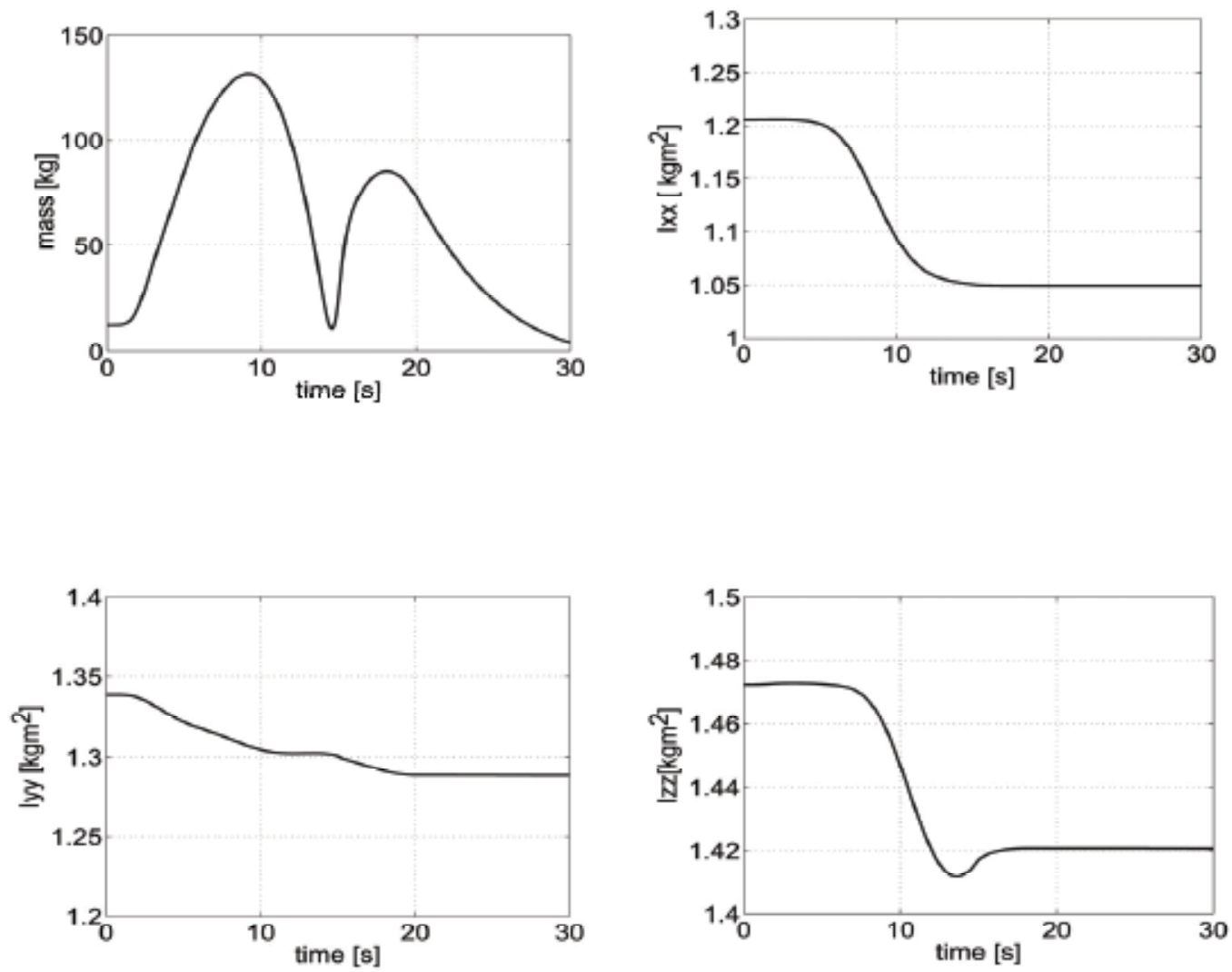

Figure 8. Adaptation process of the parameters

Furthermore, the composite adaptive control (25) is verified in the same condition. The actual trajectory follows the desired one with less tracking error than the normal adaptive control (23) since not only the tracking reference error but also the reaction force error are utilized to extract more information for the unknown dynamic parameters. Table 4 shows the root mean square error(RMSE) of the tracking error for each case. The root mean square error is calculated as follows:

$$
\text { R.MS error }=\sqrt{\frac{\sum_{i=1}^{m}\left\{\boldsymbol{\epsilon}_{p}^{T}(i) \boldsymbol{\epsilon}_{p}(i)\right\}}{m}}
$$

where $m$ denotes the number of data points in the simulation. The vector $\boldsymbol{e}_{p}$ depicts the position error of the end-effector described in Section 3. In Table 4, "w/o AC", "with AC" and "with CAC" stand for the case without adaptive control, with adaptive control and the case with composite adaptive control, respectively.

The simulations verify that the proposed adaptive controls are effective to achieve the trajectory tracking against the parameter uncertainties. 


\section{Conclusions}

In this chapter, we proposed an adaptive control for a free-floating space robot by using the inverted chain approach, which is a unique formulation for a space robot compared with that for a ground-based manipulator system. This gives the explicit description of the coupled dynamics between the end-effector and the robot arm, and provides the advantage of linearity with respect to the inertial parameters for the operational space formulation.

In a free-floating space robot, the dynamic parameters affect not only its dynamics but also its kinematics. By paying attention to the internal dynamics between the end-effector motion and the joint motion, we developed an adaptive control for operational space trajectory tracking in the presence of model uncertainties. To improve the adaptive control performance, a composite adaptive control by using the information of the tracking error and the reaction force is further discussed. The proposed control methods are verified by realistic numerical simulations. The simulation results clearly show that the proposed adaptive controls are effective against the dynamic parameter errors.

\section{References}

Abiko, S.; Lampariello, R. \& Hirzinger, G. (2006). Impedance Control for a Free-Floating Robot in the Grasping of a Tumbling Target with Parameter Uncertainty, Proc. of the 2006 IEEE/RSJ Int. Conf. on Intelligent Robots and Systems, pp. 1020 - 1025, Beijing, China, Oct. 2006.

Gu, Y. L. \& Xu, Y. (1993). A Normal Form Augmentation Approach to Adaptive Control of Space Robot Systems, Proc. of the 1993 IEEE Int. Conf. on Robotics and Automation, vol. 2, pp. 731 - 737, Atlanta, USA, May 1993.

Konno, A.; Uchiyama, M.; Kito, Y. \& Murakami, M. (1997). Configuration-Dependent Vibration Controllability of Flexible-Link Manipulators, Int. Journal Robot. Research, vol. 16, no. 4, pp. 567 - 576, 1997.

Nakamura, Y.; \& Hanafusa, H. (1986). Inverse Kinematic Solutions with Singularity Robustness for Robot Manipulator Control, Journal of Dynamic Systems, Measurement, and Cotnrol. vol. 108, pp.163 - 171, 1986.

Nenchev, D. N.; Tsumaki, Y. \& Uchiyama, M. (2000). Singularity-Consistent Parameterization of Robot Motion and Control, Int. Journal of Robotics Research, vol. 19, no. 2, pp. 159 - 182, 2000.

Senft, V. \& Hirzinger, G. (1995). Redundant Motions of Non Redundant Robots - A New Approach to Singularity Treatment, Proc. of the 1995 IEEE Int. Conf. on Robotics and Automation, pp. 1553 - 1558, Nagoya, Japan, May 1995.

Slotine, J. J. E. \& Li, W. (1987). On the Adaptive Control of Robot Manipulators, The Int. Journal of Robotics Research, vol. 6, no. 3, pp. 49 - 59, 1987.

Slotine, J. J. E. \& Li, W. (1988). Adaptive Manipulator Control: A Case Study, IEEE Transactions on Automatic Control, vol. 33, no. 11, pp. 995 - 1003, 1988.

Slotine, J. J. E. \& Li, W. (1991). Applied Nonlinear Control: Prentice Hall, ISBN 9780130408907.

Tsumaki, Y; Fiorini, P.; Chalfant, G. \& Seraji, H. (2001). A Numerical SC Approach for A Teleoperated 7-DOF Manipulator, Proc. of the 2001 IEEE Int. Conf. on Robotics and Automation, pp. 1039 - 1044, Seoul, Korea, May 2001. 
van der Schaf, A. (2000). L2-Gain and Passivity Techniques in Nonlinear Control: SprigerVerlag, ISBN 978-1852330736.

Xu, Y. \& Kanade, T. (1993). Space Robotics: Dynamics and Control: Kluwer Academic Publishers, ISBN 978-0792392651.

Xu, Y; Shum, H.-Y; Lee, J.-J. \& Kanade, T. (1992). Adaptive Control of Space Robot System with an Attitude Controlled Base, Proc. of the 1992 Int. Conf. on Robotics and Automation, pp. 2005 - 2011, Nice, France, May 1992. 


\title{
On-line Parameters Estimation with Application to Electrical Drives
}

\author{
Navid R. Abjadi ${ }^{1}$, Javad Askari ${ }^{1}$, Marzieh Kamali ${ }^{1}$ and Jafar Soltani ${ }^{2}$ \\ ${ }^{1}$ Isfahan University of Tech., ${ }^{2}$ Islamic Azad University- Khomeinishar Branch \\ Iran
}

\section{Introduction}

The main part of this chapter deals with introducing how to obtain models linear in parameters for real systems and then using observations from the system to estimate the parameters or to fit the models to the systems with a practical view.

Karl Friedrich Gauss formulated the principle of least squares at the end of the eighteenth century and used it to determine the orbits of planets and asteroids (Astrom \& Wittenmark, 1995).

One of the main applications of on-line parameters estimation is self-tuning regulator in adaptive control; nevertheless other applications such as load monitoring or failure detection, estimation of some states to omit corresponding sensors and etc. also have great importance.

\section{Models linear in parameters}

A system is a collection of objects whose properties we want to study and a model of a system is a tool we use to answer questions about a system without having to do an experiment (Ljung \& Glad, 1994). The models we work in this chapter are mathematical models, relationships between quantities.

There are different mathematical models categories such as (Ljung \& Glad, 1994)

\section{Deterministic-Stochastic}

Stochastic models despite deterministic models contain stochastic variables or processes. Deterministic models are exact relationships between variables without uncertainty.

Dynamic-Static

The variables of a system usually change with time. If there is a direct, instantaneous relationship between these variables, the system or model is called static; otherwise the system is called dynamic. For example a resistor is a static system, but a series connection of a resistor and a capacitor is a dynamic system. In this chapter we interest dynamic systems which are described by differential or difference equations.

\section{Continuous Time- Discrete Time}

If the signals used in a model are continuous signals, the model is a continuous time model; which is described by differential equations. If the signals used in a model are sampled signals, the model is a discrete time model; which is described by difference equations. 


\section{Lumped-Distributed}

Many physical systems are described by partial differential equations; the events in such systems are dispersed over the space variables. These systems are called distributed parameters systems. If a system is described by ordinary differential equations or a finite number of changing variables, it is a lumped system or model.

\section{Change Oriented-Discrete Event Driven}

The physical world and the laws of nature are usually described in continuous signals and variables, even discrete time systems obey the same basics. These systems are known as change oriented systems. For systems constructed by human, the changes take place in terms of discrete event, examples of such systems are queuing system and production system, which are called discrete event driven systems.

Models linear in parameters or linear regressions are among the most common models in statistics. The statistical theory of regression is concerned with the prediction of a variable $y$, on the basis of information provided by other measured variables $\varphi_{1}, \ldots, \varphi_{n}$ called the regression variables or regressors. The regressors can be functions of other measured variables. A model linear in parameters can be represented in the following form

$$
y(t)=\varphi_{1}(t) \theta_{1}+\ldots+\varphi_{n}(t) \theta_{n}=\varphi^{T}(t) \theta
$$

where

$\varphi^{T}(t)=\left[\begin{array}{llll}\varphi_{1}(t) & \ldots & \varphi_{n}(t)\end{array}\right], \quad \theta=\left[\begin{array}{lll}\theta_{1} & \ldots & \theta_{n}\end{array}\right]^{T}$ is the vector of parameters to be determined.

There are many systems whose models can be transformed to (1); including finite-impulse response (FIR) models, transfer function models, some nonlinear models and etc.

In some cases to attain (1), the time derivatives of some variables are needed. To avoid the noises in measurement data and to avoid the direct differentiation wich amplifies these noises, some filters may be applied on system dynamics.

Example: The $\mathrm{d}$ and $\mathrm{q}$ axis equivalent circuits of a rotor surface permanent magnet synchronous motor (SPMSM) drive are shown in Fig. 1. In these circuits the iron loss resistance is taken into account. From Fig. 1, the SPMSM mathematical model is obtained as (Abjadi et al., 2005)

$$
\begin{aligned}
& \frac{d i_{d m}}{d t}=-\frac{R}{K} i_{d m}+P i_{q m} \omega_{r}+\frac{1}{K} v_{d} \\
& \frac{d i_{q m}}{d t}=-\frac{R}{K} i_{q m}-P i_{d m} \omega_{r}-\frac{P K_{\phi}}{K} \omega_{r}+\frac{1}{K} v_{q}
\end{aligned}
$$

where $R, B, J, P$ and $T_{L}$ are stator resistance, friction coefficient, momentum of inertia, number of pole pairs and load torque, also $K$ and $K_{\phi}$ are defined by

$$
K=\left(1+\frac{R}{R_{i}}\right) L \quad, \quad K_{\phi}=\left(1+\frac{R}{R_{i}}\right) \phi
$$

here $R_{i}, \phi$ and $L$ are respectively the motor iron loss resistance, rotor permanent magnet flux and stator inductance. 


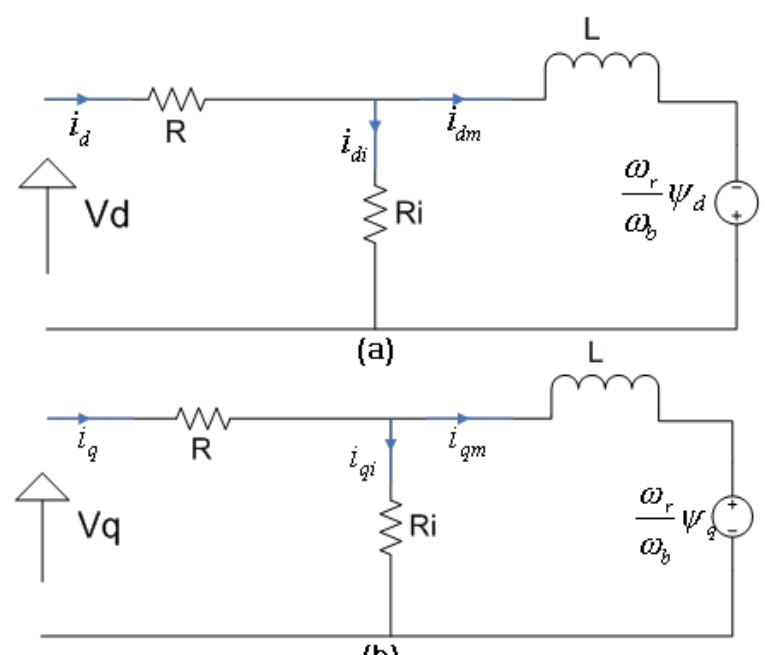

(b)

Figure 1. The $d$ and $q$ axis equivalent circuits of a SPMSM

From Fig. 1-b, the $q$ axis voltage equation of SPMSM can be obtained as

$$
K p i_{q}=-R i_{q}-K P \omega_{r} i_{d}-P \phi \omega_{r}+v_{q}+\frac{L}{R_{i}} p_{v_{q}}+\frac{L}{R_{i}} P \omega_{r} v_{d}
$$

where $p=\frac{d}{d t}$

Multiplying both sides of (3) by $\frac{1}{p+a}$, (3) becomes

$$
\begin{aligned}
K \frac{p}{p+a} i_{q}= & -R \frac{1}{p+a} i_{q}-K \frac{1}{p+a}\left(P \omega_{r} i_{d}\right)-\phi \frac{1}{p+a} P \omega_{r}+\frac{1}{p+a} v_{q} \\
& +\frac{L}{R_{i}} \frac{p}{p+a} v_{q}+\frac{L}{R_{i}} \frac{1}{p+a}\left(P \omega_{r} v_{d}\right)
\end{aligned}
$$

Assume

$$
\begin{aligned}
& i_{q f}=\frac{1}{p+a} i_{q}, v d f=\frac{1}{p+a} v_{d}, \omega_{r f}=\frac{1}{p+a} \omega_{r} \\
& \omega i d f=\frac{1}{p+a} \omega_{r} i_{d}, \omega v d f=\frac{1}{p+a} \omega_{r} v_{d}
\end{aligned}
$$

then

$$
\frac{p}{p+a} i_{q}=i_{q}-a i_{q f}, \frac{p}{p+a} v_{q}=v_{q}-a v_{q f}
$$


Linking (4), (5) and (6), yields

$$
v_{q f}=K\left(i_{q}-a i_{q f}+P \omega i_{d f}\right)+R i_{q f}+\phi P \omega_{r f}-\frac{L}{R_{i}}\left(v_{q}-a_{v} q f+P \omega v d f\right)
$$

Comparing (7) by (1), $y=v_{q f}, \theta=\left[\begin{array}{lll}K & R & \frac{L}{R_{i}}\end{array}\right]^{T}$,

$\varphi^{T}=\left[i_{q}-a_{q f}+P \omega i d f \quad i_{q f} \quad P \omega_{r f} \quad-\left(v_{q}-a_{v} q f+P \omega v d f\right)\right]$

\section{Prediction Error Algorithms}

In some parameters estimation algorithms, parameters are estimated such that the error between the observed data and the model output is minimized; these algorithms called prediction error algorithms. One of the prediction error algorithms is least squares estimation; which is an off-line algorithm. Changing this estimation algorithm to a recursive form, it can be used for on-line parameters estimation.

\subsection{Least-Squares Estimation}

In least square estimation, the unknown parameters are chosen in such a way that the sum of the squares of the differences between the actually observed and the computed (predicted) values, multiplied by some numbers, is a minimum (Astrom \& Wittenmark, 1995).

Consider the models linear in parameters or linear regressions in (1), base on the least squares estimation the parameter $\theta$ are chosen to minimize the following loss function

$$
J=\frac{1}{2} \sum_{t=1}^{N} w(t)\left[y(t)-\varphi^{T}(t) \hat{\theta}\right]^{2}
$$

where $\hat{\theta}$ is the estimation of $\theta$ and $w(t)$ are positive weights.

There are several methods in literatures to obtain $\theta$ such that (8) becomes minimized, the first one is to expand (8), then separate it in two terms, one including $\theta$ (it can be shown this term is positive or equal to zero) the other independent of $\theta$; by equating the first term to zero, (8) is minimized. In other approach the least squares problem is interpreted as a geometric problem. The observations vector is projected in the vector space spanned by regression vectors and then the parameters are obtained such that this projected vector is produced by a linear combination of regressors (Astrom \& Wittenmark, 1995). The last approach which is used here to obtain estimated parameters is to determine the gradient of (8), since (8) is in a quadratic form by equating the gradient to zero, one can obtain an analytic solution as follow.

To simplify the solution assume

$$
Y=\left[\begin{array}{lll}
y(1) & y(2) \ldots y(N)
\end{array}\right]^{T}, E=\left[\begin{array}{lll}
e(1) & e(2) \ldots e(N)
\end{array}\right]^{T}, \Phi=\left[\begin{array}{c}
\varphi^{T}(1) \\
\vdots \\
\varphi^{T}(N)
\end{array}\right]^{T}
$$

where $e(t)=y(t)-\varphi^{T}(t) \hat{\theta}$ 
Using these notations on can obtain

$$
E=Y-\Phi \hat{\theta}
$$

then (8) can be rewritten as

$$
J=\frac{1}{2} E^{T} W E
$$

where $W$ is a diagonal matrix of weights.

Substitute for $E$ in (10)

$$
J=\frac{1}{2}(Y-\Phi \hat{\theta})^{T} W(Y-\Phi \hat{\theta})
$$

Expand (11) and calculate its gradient with respect to $\hat{\theta}$

$$
\begin{gathered}
J=\frac{1}{2} Y^{T} W Y-Y^{T} W \Phi \hat{\theta}-\hat{\theta}^{T} \Phi^{T} W Y+\hat{\theta}^{T} \Phi^{T} W \Phi \hat{\theta} \\
\frac{\partial J}{\partial \hat{\theta}}=-Y^{T} W \Phi+\hat{\theta}^{T} \Phi^{T} W \Phi
\end{gathered}
$$

Equating gradient to zero

$$
\begin{aligned}
\hat{\theta} & =\hat{\theta}(N)=\left(\Phi^{T} W \Phi\right)^{-1} \Phi^{T} W Y \\
& =\left[\sum_{t=1}^{N} w(t) \varphi(t) \varphi^{T}(t)\right] \sum_{t=1}^{-1} w(t) \varphi(t) y(t)
\end{aligned}
$$

provided that the inverse is existed; this condition is called an excitation condition.

\section{Bias and Variance}

There are two different source cause model inadequacy. One is the model error that arises because of the measurement noise and system noise. This causes model variations called variance errors. The other source is model deficiency, that means the model is not capable of describing the system. Such errors are called systematic errors or bias errors (Ljung \& Glad, 1994).

The least-squares method can be interpreted in statistical terms. Assume the data are generated by

$$
y(t)=\varphi^{T}(t) \theta+e(t)
$$

where $\{e(t), t=1,2, \ldots\}$ is a sequence of independent, equally distributed random variables with zero mean. $e(t)$ is also assumed independent of $\varphi(t)$. The least-squares estimates are unbiased, that is, $E(\hat{\theta}(t))=\theta$ and an estimate converges to the true parameter value as the number of observations increases toward infinity. This property is called consistency (Astrom \& Wittenmark, 1995). 


\section{Recursive Least-Squares (RLS)}

In adaptive controller such as self-tuning regulator the estimated parameters are needed online. The least-squares estimation in (14) is not suitable for real-time purposes. It is more convenient to convert (14) to a recursive form.

Define

$$
\begin{aligned}
P^{-1}(t) & =\Phi^{T}(t) W(t) \Phi(t)=\sum_{i=1}^{t} w(i) \varphi(i) \varphi^{T}(i) \\
& =P^{-1}(t-1)+w(t) \varphi(t) \varphi^{T}(t)
\end{aligned}
$$

From (14)

$$
\hat{\theta}(t-1)=P(t-1) \sum_{i=1}^{t-1} w(i) \varphi(i) y(i)
$$

Expanding (14) and substituting for $\sum_{i=1}^{t-1} w(i) \varphi(i) y(i)$ from (17)

$$
\begin{gathered}
\hat{\theta}(t)=P(t)\left(\sum_{i=1}^{t-1} w(i) \varphi(i) y(i)+w(t) \varphi(t) y(t)\right) \\
=P(t)\left(P^{-1}(t-1) \hat{\theta}(t-1)+w(t) \varphi(t) y(t)\right)
\end{gathered}
$$

From (16) it follows that

$$
\begin{aligned}
\hat{\theta}(t) & =P(t)\left(\left(P^{-1}(t)-w(t) \varphi(t) \varphi^{T}(t)\right) \hat{\theta}(t-1)+w(t) \varphi(t) y(t)\right) \\
& =\hat{\theta}(t-1)+P(t) w(t) \varphi(t)\left(y(t)-\varphi^{T}(t) \hat{\theta}(t-1)\right)
\end{aligned}
$$

Using (16) and (19) together establish a recursive least-squares (RLS) algorithm. The major difficulty is the need of matrix inversion in (16) which can be solved by using matrix inversion lemma.

Matrix inversion lemma. Let $A, C$ and $C^{-1}+D A^{-1} B$ be non-singular square matrices. Then

$$
(A+B C D)^{-1}=A^{-1}-A^{-1} B\left(C^{-1}+D A^{-1} B\right)^{-1} D A^{-1}
$$

For the proof see (Ljung \& Soderstrom, 1985) or (Astrom \& Wittenmark, 1995).

Applying this lemma to (16)

$$
\begin{aligned}
P(t) & =\left[P^{-1}(t-1)+w(t) \varphi(t) \varphi^{T}(t)\right]^{-1} \\
& =P(t-1)-P(t-1) \varphi(t)\left[\frac{1}{w(t)} I+\varphi^{T}(t) P(t-1) \varphi(t)\right]^{-1} \varphi^{T}(t) P(t-1)
\end{aligned}
$$


Thus the formulas of RLS algorithm can be written as

$$
\begin{aligned}
& \hat{\theta}(t)=\hat{\theta}(t-1)+P(t) w(t) \varphi(t)\left(y(t)-\varphi^{T}(t) \hat{\theta}(t-1)\right) \\
& P(t)=P(t-1)-P(t-1) \varphi(t)\left[\frac{1}{w(t)} I+\varphi^{T}(t) P(t-1) \varphi(t)\right]^{-1} \varphi^{T}(t) P(t-1)
\end{aligned}
$$

It is worthwhile to note that if $y$ is a scalar, $\frac{1}{w(t)} I+\varphi^{T}(t) P(t-1) \varphi(t)$ will be a scalar too and there is no need to any matrix inversion in RLS algorithm.

In model (1), the vector of parameters is assumed to be constant, but in several cases parameters may vary. To overcome this problem, two methods have been suggested. First is to use a discount factor or a forgetting factor; by choosing the weights in (8) one can discount the effect of old data in parameters estimation. Second is to reset the matrix $P(t)$ alternatively with a diagonal matrix with large elements; this causes the parameters are estimated with larger steps in (22); for more details see (Astrom \&\& Wittenmark, 1995).

Example: For a doubly-fed induction machine (DFIM) drive the following models linear in parameters can be obtained without and with considering iron loss resistance respectively (abjadi, et all, 2006)

Model 1.

$$
\begin{aligned}
y= & v d s-v d r \\
\varphi^{T}= & {\left[i_{d s}, p_{i d s}-\omega_{r} i_{q s},-i_{d r},-p i_{d r},-\omega_{r} i_{q s}-\omega_{r} i_{q r}\right] } \\
\theta^{T}= & {\left[R_{S}, L l_{s}, R_{r}, L l_{r}, L_{m}\right] } \\
y= & v_{d s}-v_{d r} \\
\varphi^{T}= & {\left[-p\left(v_{d s}-v_{d r}\right), p_{i d s}, i_{d s}, p^{2} i_{d s}-p \omega_{r} i_{q s},\right.} \\
& \left.p i_{d s}-\omega_{r} i_{q s},-\omega_{r}\left(i_{q s}+i_{q r}\right),-p i_{d r},-i_{d r},-p^{2} i_{d r}\right] \\
\theta^{T}= & {\left[\frac{L_{m}}{R_{i}}, \frac{R_{S} L_{m}}{R_{i}}, R_{S}, \frac{L_{l s} L_{m}}{R_{i}}, L l_{s}, L_{m}, \frac{R_{r} L_{m}}{R_{i}}+L l_{r}, R_{r}, \frac{L_{l r} L_{m}}{R_{i}}\right] }
\end{aligned}
$$

Model 2. $\quad y=v_{d s}-v d r$

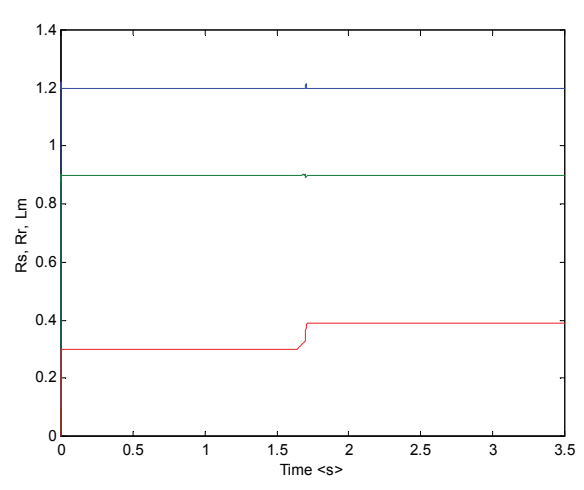

(a) model 1

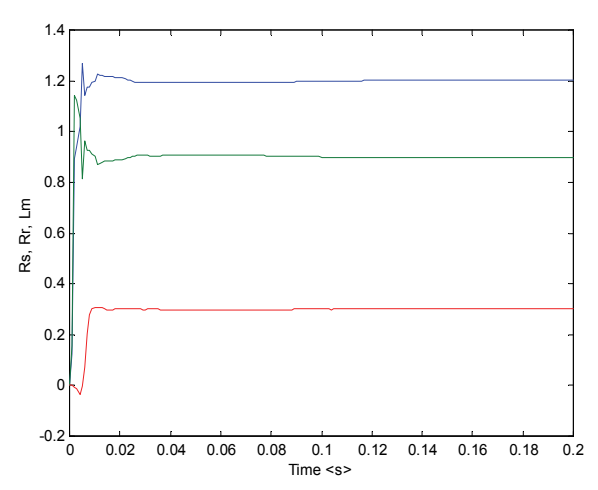

(b) model 2

Figure 2. Estimated parameters for DFIM 
To solve the problem of derivatives $\left(p_{i_{d s}}, p_{i_{d r}}\right)$ in model 1 , a first order filter is used and in order to solve the problem caused by second derivatives in model 2 , a second order filter is used.

The true parameters of the machine are given in Table 1. Using RLS algorithm, the estimated values of parameters are shown in Fig. 2. In Fig. 2.a. at the time $t=1.65 \mathrm{~s}$ the value of the magnetizing inductance $\left(L_{m}\right)$ increases $30 \%$. In this simulation the matrix $P(t)$ has been reset each $0.1 \mathrm{~s}$ with a diagonal matrix.

\begin{tabular}{|l|l|}
\hline$P_{n}=5.5 \mathrm{~kW}$ & $L_{m}=300 \mathrm{mH}$ \\
\hline$R_{S}=1.2 \Omega$ & $L_{l s}=14 \mathrm{mH}$ \\
\hline$R_{r}=0.9 \Omega$ & $L_{l r}=12 \mathrm{mH}$ \\
\hline
\end{tabular}

Table 1. Machine parameters

\section{Simplified algorithms}

There are simplified algorithms with less computation than RLS. Kaczmarz's projection algorithm is one of these algorithms. In this algorithm the following cost function is considered

$$
J=\frac{1}{2}(\hat{\theta}(t)-\hat{\theta}(t-1))^{T}(\hat{\theta}(t)-\hat{\theta}(t-1))+\alpha\left(y(t)-\varphi^{T}(t) \hat{\theta}(t)\right)
$$

In fact in this algorithm $\hat{\theta}(t)$ is chosen such that $\|\hat{\theta}(t)-\hat{\theta}(t-1)\|$ is minimized subject to the constraint $y(t)=\varphi^{T}(t) \hat{\theta}(t) . \quad \alpha$ is a Lagrangian multiplier in (23), taking derivatives with respect to $\hat{\theta}(t)$ and $\alpha$ the following parameters estimation law is obtained (Astrom \& Wittenmark, 1995)

$$
\hat{\theta}(t)=\hat{\theta}(t-1)+\frac{\varphi(t)}{\varphi^{T}(t) \varphi(t)}\left(y(t)-\varphi^{T}(t) \hat{\theta}(t-1)\right)
$$

To change the step length of the parameters adjustment and to avoid zero denominator in (24) the following modified estimation law is introduced

$$
\hat{\theta}(t)=\hat{\theta}(t-1)+\frac{\gamma \varphi(t)}{\lambda+\varphi^{T}(t) \varphi(t)}\left(y(t)-\varphi^{T}(t) \hat{\theta}(t-1)\right)
$$

where $\lambda>0$ and $0<\gamma<2$.

This algorithm is called normalized projection algorithm.

\section{Iterative Search for Minimum}

For many model structures the function $J=J(\hat{\theta})$ in (8) is a rather complicated function of $\hat{\theta}$, and the minimizing value must then be computed by computer numerical search for the minimum. The most common method to solve this problem is Newton-Raphson method (Ljung \& Glad, 1994).

To minimize $J(\hat{\theta})$ its gradient should be equated to zero

$$
\frac{\partial J(\hat{\theta})}{\partial \hat{\theta}}=0
$$


It is achieved by the following recursive estimation

$$
\hat{\theta}(t)=\hat{\theta}(t-1)-\mu(t-1)\left[J^{\prime \prime}(\hat{\theta}(t-1))\right]^{-1} J^{\prime}(\hat{\theta}(t-1))
$$

\section{Continuous-Time Estimation}

Instead of considering the discrete framework to estimate parameters, one can consider continuous framework. Using analogue procedure similar parameter estimation laws can be obtained. For continuous gradient estimator and RLS see (Slotine \& Weiping, 1991).

\section{Model-Reference Estimation Techniques}

Model-reference estimation techniques can be categorizes as techniques analog regression methods and techniques using Lyapunove or Passivity Theorem. For a detail discuss on techniques analog regression methods see (Ljung \& Soderstrom, 1985) and for examples on Lyapunove or passivity theorem based techniques see (Soltani \& Abjadi, 2002) \& (Elbuluk, et all, 1998).

In model-reference techniques two models are considered; one contains the parameters to be determined (adaptive model) and the other is free or independent from those parameters (reference model). The two models have same kind output; a mechanism is used to estimate the parameters in such a way that the error between these models outputs becomes minimized or converges to zero.

\subsection{Other Algorithms}

\section{Maximum Likelihood Estimation}

In prior sections it was assumed that the observations are deterministic and reliable. But in stochastic studies, observations are supposed to be unreliable and are assumed as random variables. In this section we mention a method for estimating a parameter vector $\theta$ using random variables.

Consider the random variable $y=\left(y_{1}, y_{2}, \ldots, y_{N}\right) \in \Re^{N}$ as observations of the system. The probability that the realization indeed should take value $y$ is described as $f(\theta ; y)$, where $\theta \in \mathfrak{R}^{d}$ is the unknown parameter vector. A reasonable estimator for the vector $\theta$ is to determine it so that the function $f(\theta ; y)$ takes it maximum (Ljung, 1999), i.e. the observed event becomes as likely as possible. So we can see that

$$
\hat{\theta}_{M L}(y)=\underset{\theta}{\arg \max } f(\theta ; y)
$$

The function $f(\theta ; y)$ is called the likelihood function and the maximizing vector $\hat{\theta}_{M L}(y)$ is known as the maximum likelihood. For a resistance maximum likelihood estimator and recursive maximum likelihood estimator see (Ljung \& Soderstorm, 1985).

Instrumental Variable Method

Instrumental variable method, is a modification of the least squares method designed to overcome the convergence problems.

Consider the linear system

$$
y(t)=\varphi^{T}(t) \theta+v(t)
$$


In the least squares method, $\hat{\theta}(N)$ will not converge to $\theta$, if there exists correlation between $\varphi(t)$ and $v(t)$ (Ljung, 1999). A solution for this problem is to replace $\varphi(t)$ by a vector $\zeta(t)$ that is uncorrelated with $v(t)$. The elements of $\zeta(t)$ are called instrumental variables and the estimation method is called instrumental variable method.

By replacing $\varphi(t)$ by $\zeta(t)$ in the least squares method we have

$$
\hat{\theta}(N)=\left[\sum_{i=1}^{N} \zeta(t) \varphi^{T}(t)\right]^{-1} \sum_{i=1}^{N} \zeta(t) y(t)
$$

for the off-line case and

$$
\begin{aligned}
& \hat{\theta}(t)=\hat{\vartheta}(t-1)+L(t)\left[y(t)-\hat{\theta}^{T}(t-1) \varphi(t)\right] \\
& L(t)=\frac{P(t-1) \zeta(t)}{1+\varphi^{T}(t) P(t-1) \zeta(t)}=P(t) \zeta(t), \\
& P(t)=P(t-1)-\frac{P(t-1) \zeta(t) \varphi^{T}(t) P(t-1)}{1+\varphi^{T}(t) P(t-1) \zeta(t)}
\end{aligned}
$$

for recursive fashion.

The instrumental variables should be chosen such that

1. $\zeta(t)$ and ${ }^{v(t)}$ be uncorrelated,

2. The matrix $\lim _{N \rightarrow \infty} \frac{1}{N} \sum_{i=1}^{N} \zeta(t) \varphi^{T}(t)$ be invertible.

under these conditions and if $v(t)$ has zero mean, $\hat{\theta}(N)$ will converge to $\theta$. A common choice of instrumental variables is (Ljung \& Soderstorm, 1985)

$$
\zeta^{T}(t)=\left(-y_{M}(t-1) \ldots-y_{M}(t-n) u(t-1) \ldots u(t-m)\right)
$$

where $y_{M}(t)$ is the output of the system

$$
y_{M}(t)+\bar{a}_{1} y_{M}^{(t-1)+\ldots+\bar{a}_{n} y_{M}(t-n)=\bar{b}_{1} u(t-1)+\ldots+\bar{b}_{M} u(t-m)}
$$

For the recursive fashion it is common to let $\bar{a}_{i}$ and $\bar{b}_{i}$ be time-dependent.

\section{Bayesian Method}

In the Bayesian method, in addition to observations, parameter is considered as a random variable too. In this method, parameter vector $\theta$ is considered to be a random vector with a certain prior distribution. The value of this parameter is determined using the observations $u^{t}$ and $y^{t}$ (input and output of the system until time $t$ ) of random variables that are correlated with it. 
The posterior probability density function for $\theta$ is considered as $p\left(\theta \mid u^{t}, y^{t}\right)$. There are several ways to determine the parameter estimation $\hat{\theta}(t)$ from the posterior distribution. This is a very difficult problem in general to find the estimate $\hat{\theta}(t)$ and only approximate solutions can be found. But under the specific conditions mentioned in the following lemma, there exists an exact solution.

Lemma. (Ljung \& Soderstorm, 1985) Suppose that the data is generated according to

$$
y(t)=\varphi^{T}(t) \theta+e(t)
$$

where the vector $\varphi(t)$ is a function of $u^{t-1}, y^{t-1}$ and $\{e(t)\}$ is a sequence of independent Gaussian variable with $E e(t)=0$ and $E e^{2}(t)=r_{2}(t)$. Suppose also that the prior distribution of $\theta$ is Gaussian with mean $\theta_{0}$ and covariance matrix $P_{0}$. Then the posterior distribution $p\left(\theta \mid u^{t}, y^{t}\right)$ is also Gaussian with mean $\hat{\theta}(t)$ and covariance matrix $P(t)$, where $\hat{\theta}(t)$ and $P(t)$ are determined according to

$$
\begin{aligned}
& \hat{\theta}(t)=\hat{\theta}(t-1)+L(t)\left[y(t)-\hat{\theta}^{T}(t-1) \varphi(t)\right], \\
& L(t)=\frac{1}{r_{2}(t)} P(t) \varphi(t)=\frac{P(t-1) \varphi(t)}{r_{2}(t)+\varphi^{T}(t) P(t-1) \varphi(t)}, \\
& P(t)=P(t-1)-\frac{P(t-1) \varphi(t) \varphi^{T}(t) P(t-1)}{r_{2}(t)+\varphi^{T}(t) P(t-1) \varphi(t)}, \\
& \hat{\theta}(0)=\theta_{0}, \quad P(0)=P_{0}
\end{aligned}
$$

For the proof see (Ljung, 1985).

\section{Nonlinear models}

There are many applications that linear in parameters models dose not suffice to describe the system. Systems with nonlinearities are very common in real world; in this section some models suitable for such systems are introduced.

\section{Wiener and Hammerstein System}

Some especial cases of nonlinearities in system are static nonlinearities at the input or the output or both of them. In other words there are systems with dynamics with a linear nature, but there are static nonlinearities at the input or the output or both of them. Example for static nonlinearity at the input is saturation in the actuators and static nonlinearity at the output is sensors characteristics (Ljung, 1999).

A model with a static nonlinearity at the input is called a Hammerstein model while a model with a static nonlinearity at the output is called a Wiener model. Fig. 3 shows these models. 


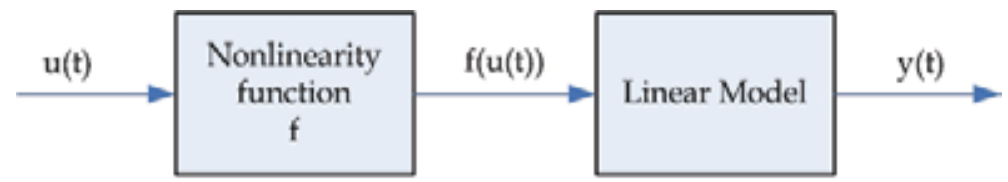

Hammerstein model

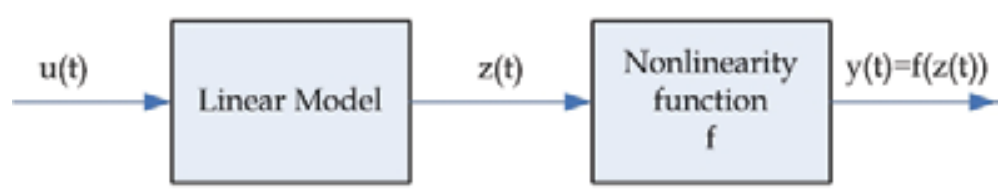

Wiener model

Figure 3. Hammerstein and Wiener models

\section{Fuzzy System}

Fuzzy Systems or knowledge-based systems are a type of nonlinear systems that can be used to approximate nonlinear behavior of many practical systems (Wang, 1997).

Certain types of fuzzy systems can be written as compact nonlinear formulas. In this section we will consider Takagi-Sugeno fuzzy systems that are a common used type of fuzzy systems.

Consider a multi input-single output Takagi-Sugeno fuzzy system given by (Passino \& Yurkovich, 1998)

$$
y=\frac{\sum_{i=1}^{R} g_{i}(x) \mu_{i}(x)}{\sum_{i=1}^{R} \mu_{i}(x)}
$$

Where, $\mu_{i}(x)$ is the certainty of the premise of the i-th rule (Trabelsi \& Lafont, 2004) and $g_{i}(x)=a_{i, 0}+a_{i, 1} x_{1}+\ldots+a_{i, n} x_{n}$ is the consequent of the $\mathrm{i}$-th rule

With extending (36) we have

$$
y=\frac{\sum_{i=1}^{R} a_{i, 0} \mu_{i}(x)}{\sum_{i=1}^{R} \mu_{i}(x)}+\frac{\sum_{i=1}^{R} a_{i, 1} x_{1} \mu_{i}(x)}{\sum_{i=1}^{R} \mu_{i}(x)}+\ldots+\frac{\sum_{i=1}^{R} a_{i, n} x_{n} \mu_{i}(x)}{\sum_{i=1}^{R} \mu_{i}(x)}
$$

If we define

$$
\begin{gathered}
\xi_{i}(x)=\frac{\mu_{i}(x)}{\sum_{i=1}^{R} \mu_{i}(x)} \\
\xi(x)=\left[\xi_{1}, \xi_{2}, \ldots, \xi_{R}, x_{1} \xi_{1}, x_{1} \xi_{2}, \ldots, x_{1} \xi_{R}, \ldots, x_{n} \xi_{1}, x_{n} \xi_{2}, \ldots, x_{n} \xi_{R}\right]^{T}
\end{gathered}
$$




$$
\theta=\left[a_{1,0}, a_{2,0}, \ldots, a_{R, 0}, a_{1,1}, a_{2,1}, \ldots, a_{R, 1}, \ldots, a_{1, n^{\prime}} a_{2, n^{\prime}, \ldots, a_{R, n}}\right]^{T}
$$

We can write (36) as

$$
y=\xi(x)^{T} \theta
$$

We see that (41) is in the same form as we defined for linear systems and is linear versus $\theta$. Thus it is possible to use mentioned Estimators like recursive least square and Gradient Estimators to estimate parameter vector $\theta$.

Neural Network System

Another common method to model systems is using artificial neural network for the details see (Ljung, 1999).

\section{Conclusion}

In this chapter, some parameters estimation algorithms are presented; among them RLS is one of the most common parameters estimation algorithms, which is discussed in details. The main key to use this algorithm or similar ones is the model must be linear in parameters.

Two practical examples from the field of electrical motor drives are introduced to show that even from nonlinear complex systems one may obtain models linear in parameters.

There are several ways to model a real system. Some of the models used to predict the behaviour of systems are presented.

\section{References}

Abjadi, N. R.; Soltani, J.; Pahlavaninezhad, M. \& Askari, J. (2005). A Nonlinear Adaptive Controller for Speed Sensorless PMSM Taking The Iron Loss Resistance Into Account, Proceedings of The Eighth International Conference on Electrical Machines and Systems (ICEMS), pp. 188-193, Nanjing, China, September 2005, Southeast University, Nanjing

Abjadi, N. R.; Askari, J. \& Soltani, J. (2006). Adaptive Control of Doubly Fed Field-Oriented Induction Machine Based On Recursive Least Squares Method Taking the Iron Loss Into account, Proceedings of CES/IEEE $5^{\text {th }}$ International Power Electronics and Motion Control Conference (IPEMC), pp. 1923-1927, ISBN 1-4244-0448-7, Shanghai, China, August 2006, Shanghai Jiao Tong University, Shanghai

Astrom, K. J. \& Wittenmark, B. (1995). Adaptive control, Addison-Wesley Longman, Inc., Californian

Elbuluk, M.; Langovsky, N. \& Kankam, D. (1998). Design and Implementation of a ClosedLoop Observer and Adaptive Controller for Induction Motor Drives, IEEE TRANSACTIONS ON INDUSTRY APPLICATIONS, pp. 435-443, Vol. 34, No. 3, MAY/JUNE 1998

Ljung, L. \& Soderstrom, T. (1985). Theory and Practice of Recursive Identification, MIT Press, Massachusetts

Ljung, L. \& Glad, T. (1994). Modeling of dynamic systems, Prentice-Hall, Inc., ISBN 0-13597097-0, NJ 
Ljung, L. (1999). System identification: Theory for the user, Prentice-Hall, Inc., NJ

Passino, K. M. \& Yurkovich S. (1998). Fuzzy control, Addison-Wesley Longman, Inc., Californian

Slotine, J. J. E. \& Weiping, L. (1991). Applied nonlinear control, Prentice-Hall, Inc., NJ

Soltani, J. \& Abjadi, N. R. (2002). A Modified Sliding Mode Speed Controller for an Induction Motor Drive without Speed Sensor Using the Feedback Linearization Theory, Proceedings of EPE-PEMC 10 th Power Electronics and Motion Control Conference, Dubrovnik, Croatia, 2002, Zagreb University, Dubrovnik

Trabelsi, A.; Lafont F.; Kamoun M. \& Enea G. (2004). Identification of nonlinear multivariable system by adaptive fuzzy Takagi-Sugeno model, Int. J. Computational Cognition, vol. 2, no. 3, pp. 137-153

Wang L. X. (1997). A Course in Fuzzy Systems and Control, Prentice-Hall, NJ 


\title{
A New Frequency Dependent Approach to Model Validation
}

\author{
Pedro Balaguer and Ramon Vilanova \\ Autonomous University of Barcelona \\ Spain
}

\section{Introduction}

In order to have confidence in a model it is necessary to validate it. Different model validation approaches exist. Their difference is based upon the assumptions about the plant and models. Classical validation methods, based on classical model identification (Ljung, 1994; Soderstrom and Stoica, 1989) rely on statistical uncertainty assumptions due to stochastic noise only. On the other hand, control oriented identification methods (Chen and $\mathrm{Gu}, 200)$ (i.e. $\mathcal{H}_{\infty}$ identification, stochastic embedding, set membership identification, etc.) lead to validation assumptions based on bounded noise and bounded model undermodelling. However in both cases the output of the validation process is just a "validated/invalidated" result.

Regarding classical validation methods, the problem of assessing the validity of an identified model has been traditionally linked with the problem of model order selection. The classical model validation literature (Ljung, 1994; Soderstrom and Stoica, 1989) has approached the problem in two ways:

- use of plots and common sense.

- $\quad$ use of statistical tests on the residuals $\xi(t)$ (i.e. the difference between the real output and the model output $\xi(t)=y(t)-\hat{y}(t))$.

The first approach is basically based on the comparison of experimental data with the model output. If both are similar then the model can be considered a good one. However there are two unavoidable reasons that prevent the model output to fit data perfectly: the modelling errors and perturbations. The second approach is to apply a hypothesis test over the residual $\xi(t)$, which follows from the assumptions on the disturbance. The more common assumptions over the residual are (Soderstrom and Stoica, 1989):

- $\mathrm{h} 1: \xi(t)$ is a zero mean white noise.

- $\mathrm{h} 2: \xi(t)$ has a symmetric distribution.

- h3: $\xi(t)$ is independent of past inputs (i.e. $E[\xi(t) u(\tau)]=0, t>\tau)$.

- $\quad$ h4: $\xi(t)$ is independent of all inputs (i.e. $E[\xi(t) u(\tau)]=0, \forall t, \tau)$. 
The above assumptions lead to check two main residual properties, the whiteness of the residuals (i.e. h1, h2) and the cross-correlation between residuals $\xi(t)$ and control actions $u(t)$ (i.e. h3, h4).

Hence classical validation tests can be classified as follows

1. Whiteness Test.

- $\quad$ Autocorrelation test (Assumption h1).

- $\quad$ Testing changes of sign (Assumptions h1 and h2).

2. Independence between residuals and inputs.

- Cross-correlation test of past inputs (Assumption h3 or Assumptions h3 and h1).

- Cross-correlation test of all inputs (Assumption h4 or Assumptions h4 and h1).

The rationale of the tests is to detect causes of variation on the residual distinct than the ones assumed. For example if the residual is assumed to be white noise and the test shows that the whiteness statistical hypothesis is violated then we assume that there is a distinct cause producing the mismatch (i.e. model error).

The result of the statistical tests above reviewed is a binary one. In fact the test either validates or invalidates the model. No further information is provided by the test. As a result, two important drawbacks are

1. There is no information on important model aspects such as (Balaguer and Vilanova, 2006a):

- The reasons why the model is invalidate.

- How to improve the model.

- The model usefulness degree.

2. In control design, undermodelling (i.e. use of low order models) is normally present (Balaguer and Vilanova, 2006b). In fact as stated in (Ljung, 1994) "For such a model (a model simpler that the one that minimizes the total error) typically the bias error is the dominating contribution to the total error. Consequently, such models would normally be falsified during model validation".

Thus, as a conclusion, although the theory of classical validation methods is well developed and plenty of successful applications it has limitations when a more informative validation procedure is required.

In this chapter we present a new view to the model validation procedure. The main characteristic of the presented approach is that the validation is frequency dependent. As a result the validation procedure is more informative as:

1. The same model can result validated for a frequency range and invalidated for a distinct frequency band. Thus the result of the validation procedure is no longer binary but frequency dependent.

2. The algorithm provides the frequency range for which the model is validated.

3. The validation procedure is suited to be used with residuals generate in a control oriented fashion (i.e. in closed loop).

The application areas of this new, more informative, validation procedure are wide. The applicability potential hinges on the frequency dependence of the validation result. In particular we focus on the application for model validation on Iterative Identification and Control Design Schemes. In Iterative Identification and Control Design Schemes (Albertos and Sala, 2002), a new model is identified at each iteration. Thus it is necessary to validate the new model. Moreover these iterative schemes have some requirements, such as input 
experiment design, model order selection, etc. The proposed frequency dependent algorithm gives information in order to i) select controller bandwidth, ii) select model order and iii) design the input experiment for the next iteration.

A distinct application area of the algorithm presented here is the validation of controllers. The objective is to validate the behaviour of a possibly low order controller (e.g. PID) against a more complex controller (e.g. optimal controller). The procedure presented here can be applied to ascertain for what frequency ranges both controllers are behaving in an equivalent manner. Moreover the validation procedure also suggests a tuning method by means of minimizing the residual generated by the comparison of both controllers. First results can be found in (Balaguer et al., 2008).

Summing up, in this chapter we present a new model validation algorithm in which the validation result is no longer "validated/invalidated" but frequency dependent. Thus the validation result is more informative, providing new features of applicability in several areas of control theory.

The chapter contributions are organized as follows

1. A new procedure for model validation in the frequency domain is presented (Section 2). This procedure permits to validate or invalidate models over certain frequency ranges. The procedure is the translation of a time domain residual whiteness test to a frequency dependent residual whiteness test. The counterpart on the frequency domain of a time domain whiteness test is established. This leads to stating the statistical properties of each spectrum frequency component if the time domain transformed signal is white noise. It is shown that the normalized spectrum is a random variable with a $\chi^{2}$ distribution of 2 degrees of freedom (Theorem 2). The validation/invalidation step is based on a hypothesis test applied to each frequency component. This determines if certain frequency components have an unusual content that discards the model validity for this frequency value. The acceptance/rejection decision of the frequency component validity comes with a probability measure.

2. The intended use of the model (e.g. open loop or closed loop) is taken into account by considering the structure from which the residuals are generated. In Section 3 a study of the residuals information content and its statistical properties for different structures (e.g. open loop Vs closed loop) is conducted. As the validation procedure is based on a whiteness test, the residual should be white noise whenever the model fits accurately the plant. This however is not the general case when residuals coming from closed loop comparisons are used. In order to overcome this difficulty, a novel structure is proposed in Section 3.3 which is able to provide white residuals in closed loop.

3. The application of the presented validation procedure on Iterative Identification and Control Design Schemes is discussed in Section 4. It is shown that the validation procedure shifts from a classical static test (i.e. validation/invalidation) to a dynamic one which gives frequency domain information useful for improvement of identification and control design on iterative schemes.

4. The chapter ends in Section 5 stating the conclusions and the possible extensions of the frequency dependent validation algorithm. 


\section{Frequency Dependent Model Validation}

The main objective of the methodology presented is to validate a model on the frequency domain. To this end a time domain validation procedure based on testing the residual whiteness is modified to achieve the pursued objectives. The idea is as follows. It is assumed that if the residual is white noise the model is validated because the residual contains no further useful information that could be used to improve the model accuracy. This test is usually performed in the time domain by studying the residual autocorrelation, the number of sign changes, etc (Ljung, 1994).

We translate the time domain residual to the frequency domain by its discrete Fourier transform. Moreover, the statistical properties of the spectrum of a white noise signal are calculated. The objective is to test if the spectrum calculated from the residual has properties of white noise. As a result, one unique test in the time domain has been translated to $\mathrm{N}$ different tests in the frequency domain. We check if the $k^{\text {th }}$ frequency component of the spectrum has the properties of a typical frequency component of a white noise. In the affirmative case we have no reason to believe that the model is invalid on that frequency component. On the other hand, if there are certain frequency components that clearly do not behave accordingly with the statistical properties of white noise then it is likely that at this frequency range there is an important mismatch between the model and the plant. As a result the model is invalid for that frequency range.

\subsection{Whiteness Test on the Frequency Domain}

In this section the statistical time domain properties of a white noise are translated to the frequency domain. This is accomplished by means of two theorems. The first one is an intermediate result that is used by the second one which describes the frequency domain distribution of the spectrum of a white noise.

\section{Theorem 1}

Let $\xi(n)$ be a sequence of independent identically distributed (IID) samples of normal distribution $N\left(\mu_{\xi}, \sigma_{\xi}^{2}\right)$. If we express the discrete Fourier transform by its real and imaginary part, that is

$$
\xi_{k}=R_{k}+j I_{k}=\frac{1}{N} \sum_{n=0}^{N-1} \xi(n) e^{-j \Omega_{0} k n}
$$

then the real part $R_{k}$ is a random variable normally distributed $R_{k} \in N\left(\mu_{R_{k}}, \sigma_{R_{k}}^{2}\right)$ with mean $\mu_{R_{k}}$ and variance $\sigma_{R_{k}}^{2}$ given by

$$
\begin{gathered}
\mu_{R_{k}}=\mu_{\xi} \frac{1}{N} \sum_{n=0}^{N-1} \operatorname{Cos}\left(\Omega_{0} k n\right) \\
\sigma_{R_{k}}^{2}=\sigma_{\xi}^{2} \frac{1}{N^{2}} \sum_{n=0}^{N-1} \operatorname{Cos}^{2}\left(\Omega_{0} k n\right)
\end{gathered}
$$


Similarly the Imaginary part $I_{k}$ is a random variable normally distributed $I_{k} \in N\left(\mu_{I_{k}}, \sigma_{I_{k}}^{2}\right)$ with mean $\mu_{I_{k}}$ and variance $\sigma_{I_{k}}^{2}$ given by

$$
\begin{gathered}
\mu_{I_{k}}=\mu_{\xi} \frac{1}{N} \sum_{n=0}^{N-1} \operatorname{Sin}\left(\Omega_{0} k n\right) \\
\sigma_{I_{k}}^{2}=\sigma_{\xi}^{2} \frac{1}{N^{2}} \sum_{n=0}^{N-1} \operatorname{Sin}^{2}\left(\Omega_{0} k n\right)
\end{gathered}
$$

Proof:

The discrete Fourier transform of a discrete time signal $\xi_{k}$ is given by

$$
\xi_{k}=\frac{1}{N} \sum_{n=0}^{N-1} \xi(n) e^{-j \Omega_{0} k n}
$$

where $\Omega_{0}=\frac{2 \pi}{N}$ is the fundamental frequency. Decomposing the equation (5) into its real part and its imaginary part gives

$$
\begin{aligned}
\xi_{k} & =\frac{1}{N} \sum_{n=0}^{N-1} \xi(n) e^{-j \Omega_{0} k n} \\
& =\frac{1}{N} \sum_{n=0}^{N-1} \xi(n)\left(\operatorname{Cos}\left(\Omega_{0} k n\right)-j \operatorname{Sin}\left(\Omega_{0} k n\right)\right) \\
& =\frac{1}{N} \sum_{n=0}^{N-1} \xi(n) \operatorname{Cos}\left(\Omega_{0} k n\right)-j \frac{1}{N} \sum_{n=0}^{N-1} \xi(n) \operatorname{Sin}\left(\Omega_{0} k n\right) \\
& =R_{k}-j I_{k}
\end{aligned}
$$

where the second equality comes from Euler's identity. Hence the real and imaginary parts are linear combinations of normally distributed random variables

$$
\begin{aligned}
& R_{k}=\frac{1}{N} \sum_{n=0}^{N-1} \xi(n) \operatorname{Cos}\left(\Omega_{0} k n\right) \\
& I_{k}=\frac{1}{N} \sum_{n=0}^{N-1} \xi(n) \operatorname{Sin}\left(\Omega_{0} k n\right)
\end{aligned}
$$

As a result, it follows that $R_{k}$ and $I_{k}$ are also normally distributed random variables. The distribution parameters for the stochastic variable $R_{k}$ are calculated as follows (see, for example, (Box et al., 1978), page 87)

$$
\begin{gathered}
\mu_{R_{k}}=\mu_{\xi} \frac{1}{N} \sum_{n=0}^{N-1} \operatorname{Cos}\left(\Omega_{0} k n\right) \\
\sigma_{R_{k}}^{2}=\sigma_{\xi}^{2} \frac{1}{N^{2}} \sum_{n=0}^{N-1} \operatorname{Cos}^{2}\left(\Omega_{0} k n\right)
\end{gathered}
$$

The same reasoning is applicable to the imaginary part $I_{k}$. 


\section{Remark 1}

$\mu_{R_{k}}$ is equal to zero for $k \in\{1,2, \ldots, N-1\}$ and $\mu_{R_{0}}$ equals the mean value of the residual (i.e. $\left.\mu_{R_{0}}=\mu_{\xi}\right) . \mu_{I_{k}}$ is always equal to zero for $k \in\{0,1,2, \ldots, N-1\}$.

\section{Theorem 2}

The normalized squared gain $M_{k}^{2}$ defined as

$$
M_{k}^{2}=\left(\frac{R_{k}-\mu_{R_{k}}}{\sigma_{R_{k}}}\right)^{2}+\left(\frac{I_{k}-\mu_{I_{k}}}{\sigma_{I_{k}}}\right)^{2}
$$

has a $\chi^{2}$ distribution of 2 degrees of freedom.

Proof:

By definition the sum of $r$ independent squared random normal variables $N(0,1)$ has a $\chi^{2}$ distribution of $r$ degrees of freedom. Due to the normalization of $R_{k}$ and $I_{k}$, it follows that $\frac{R_{k}-\mu_{R_{k}}}{\sigma_{R_{k}}} \in N(0,1)$ and $\frac{I_{k}-\mu_{I_{k}}}{\sigma_{I_{k}}} \in N(0,1)$.

\subsection{Procedure}

The frequency domain model validation procedure is as follows

1. Calculate the residual as the difference of the real output and the model estimated output $(\xi(n)=y(n)-\hat{y}(n))$.

2. Calculate the discrete Fourier transform of the residual $\xi_{k}$.

3. Decompose each frequency component on its real part and imaginary part $\xi_{k}=R_{k}+j I_{k}$.

4. Calculate distribution parameters of the Real $\left(R_{k}\right)$ and Imaginary $\left(I_{k}\right)$ part for each frequency component $k$, as shown in Theorem 1 , that is:

- $\quad$ Real part $\left(R_{k}\right)$ : Calculate $\mu_{R_{0}}$ and $\sigma_{R_{k}}^{2}$ for $k \in\{0,1,2, \ldots, N-1\}$ (Equations (1) and (2)).

- Imaginary part $\left(I_{k}\right)$ : Calculate $\sigma_{I_{k}}^{2}$, for $k \in\{0,1,2, \ldots, N-1\}$ (Equations (3) and (4)).

5. Calculate the normalized magnitude spectrum for each frequency $k \in\{0,1,2, \ldots, N-1\}$ as follows:

$$
M_{k}^{2}=\left(\frac{R_{k}-\mu_{R_{k}}}{\sigma_{R_{k}}}\right)^{2}+\left(\frac{I_{k}-\mu_{I_{k}}}{\sigma_{I_{k}}}\right)^{2}
$$

6. Perform a hypothesis tests over each one of the normalized magnitude spectrum $M_{k}^{2}$, indexed by $k \in\{0,1,2, \ldots, N-1\}$, as follows:

- $H_{0}: M_{k}^{2} \in \chi_{2}^{2}$

- $H_{1}: M_{k}^{2} \notin \chi_{2}^{2}$ 
See next section for a deeper discussion on the hypothesis test procedure.
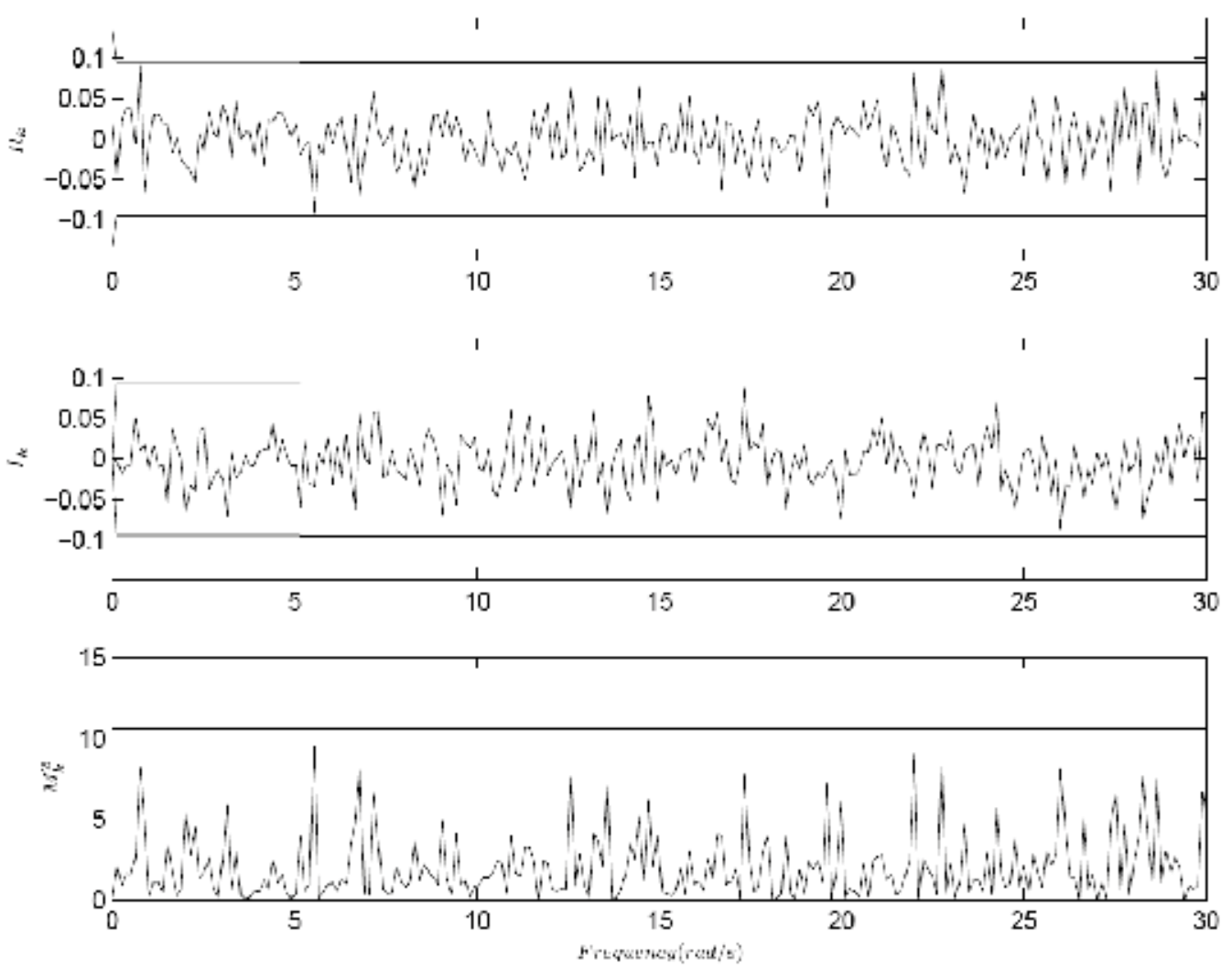

Figure 1. White noise example

The above steps are materialized in the following example. A realization of a normally distributed random variable of zero mean and unity variance is performed with 500 samples. The discrete Fourier transform of the realization is calculated and decomposed into its real and imaginary parts (i.e. $R_{k}, I_{k}$ ). The values of $R_{k}$ and $I_{k}$ are shown in figure 1 . They follow a normal variable distribution with parameters given by equations (1), (2), (3) and (4) (i.e. $R_{k} \in N\left(\mu_{R_{k}}=0, \sigma_{R_{k}}^{2}=0.001\right)$ and $I_{k} \in N\left(\mu_{I_{k}}=0, \sigma_{I_{k}}^{2}=0.001\right)$. On figure 1 the realizations of $R_{k}$ and $I_{k}$ are plotted together with the 3 sigma limits of their distribution (i.e. the $99.73 \%$ of the samples fall between the plotted limits). It can be seen that all points fall inside this range.

Finally the normalized magnitude spectrum is calculated following equation (6). The magnitude spectrum can be seen in figure 1 together with the $99.5 \%$ confidence limit of the $\chi_{2}^{2}$ distribution (i.e. the $99.5 \%$ of the samples fall between 0 and 10.6). All the magnitude frequency components remain below of the confidence limit so there are no reasons to invalidate the model. The whiteness test has passed. In the sequent section the hypothesis test features are described more thoroughly. 


\subsection{Hypothesis Test}

A hypothesis test is a statement about a random variable. This statement is expressed by means of two hypothesis $H_{0}$ and $H_{1} . H_{0}$ is called the null hypothesis and $H_{1}$ is the alternative hypothesis. In order to decide the validity of either the null hypothesis $H_{0}$ or the alternative hypothesis $H_{1}$, an estimation of a population parameter (e.g. mean or variance) is computed from a population sample and it is compared against the assumed population parameters. These population parameters are random variables too with certain mean and variance. If it is likely that the computed statistic is inside the population parameters distribution then $H_{0}$ is accepted, otherwise $H_{0}$ is rejected in favour of $H_{1}$. As a result, two errors are possible: to reject $H_{0}$ when it is true (type I error or $\alpha$ error), or to fail to reject $H_{0}$ when $H_{0}$ is false (type II error or $\beta$ error).

The hypothesis test is the last step of the presented procedure, where the decision of validation/invalidation of certain frequency component is taken. The hypothesis test to be applied in the proposed procedure is:

$$
\begin{aligned}
& H_{0}: M_{k}^{2} \in \chi_{2}^{2} \\
& H_{1}: M_{k}^{2} \notin \chi_{2}^{2}
\end{aligned}
$$

The hypothesis $H_{0}$ states that the normalized modulus $M_{k}^{2}$ of the $k$ frequency component is $\chi_{2}^{2}$ distributed. On the other hand the hypothesis $H_{1}$ states that the normalized modulus $M_{k}^{2}$ of the $k$ frequency component is not $\chi_{2}^{2}$ distributed.

\section{Remark 2}

The hypothesis test stated in (7) is applied to each frequency component, from $0 \mathrm{rad} / \mathrm{sec}$ up to the Nyquist frequency (i.e. $\frac{\pi}{T_{s}}$, where $T_{s}$ is the sample time).

In order to decide the validity of either the null hypothesis $H_{0}$ or the alternative hypothesis $H_{1}, M_{k}^{2}$ is computed. If it is "likely" that the value of $M_{k}^{2}$ lies inside the $\chi_{2}^{2}$ distribution then $H_{0}$ is accepted, otherwise $H_{0}$ is rejected in favour of $H_{1}$. As a result, two errors are possible as discussed above, to reject $H_{0}$ when it is true, or to fail to reject $H_{0}$ when $H_{0}$ is false.

The probability of rejecting $H_{0}$ when it is true is set by the user by choosing the confidence limit. For example, if the confidence limit is chosen to be 10.6 then the $99.5 \%$ of samples of a $\chi_{2}^{2}$ distribution fall inside the limit. This confidence limit sets the type I error. Following the example presented in the preceding section, the type I error was of $0.5 \%$, that is the $0.5 \%$ of the samples of a $\chi_{2}^{2}$ must be greater than 10.6. The type II error is more difficult to be calculated as it depends on knowledge of of the actual distribution followed by $M_{k}^{2}$.

The hypothesis test is then simply a check that any magnitude of the normalized spectrum is less than the test limit. If the value is greater then it is very unlikely and the model results invalidate for this frequency. 


\subsection{Open Loop Model Validation Example}

The proposed validation procedure in the frequency domain is applied to a stable plant in open loop (see figure 2). The real plant $G$ and the model $\hat{G}$ chosen to approximate it are:

$$
\begin{aligned}
& G=\frac{10}{(s+1)(s+10)} \\
& \hat{G}=\frac{1}{s+1}
\end{aligned}
$$

The Bode diagram comparing the real plant with the model is shown in figure 3.

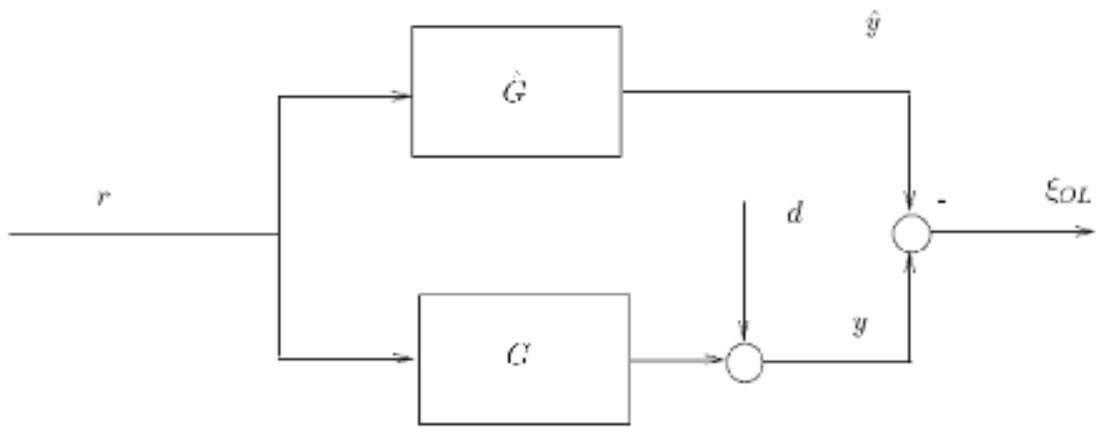

Figure 2. Open loop structure

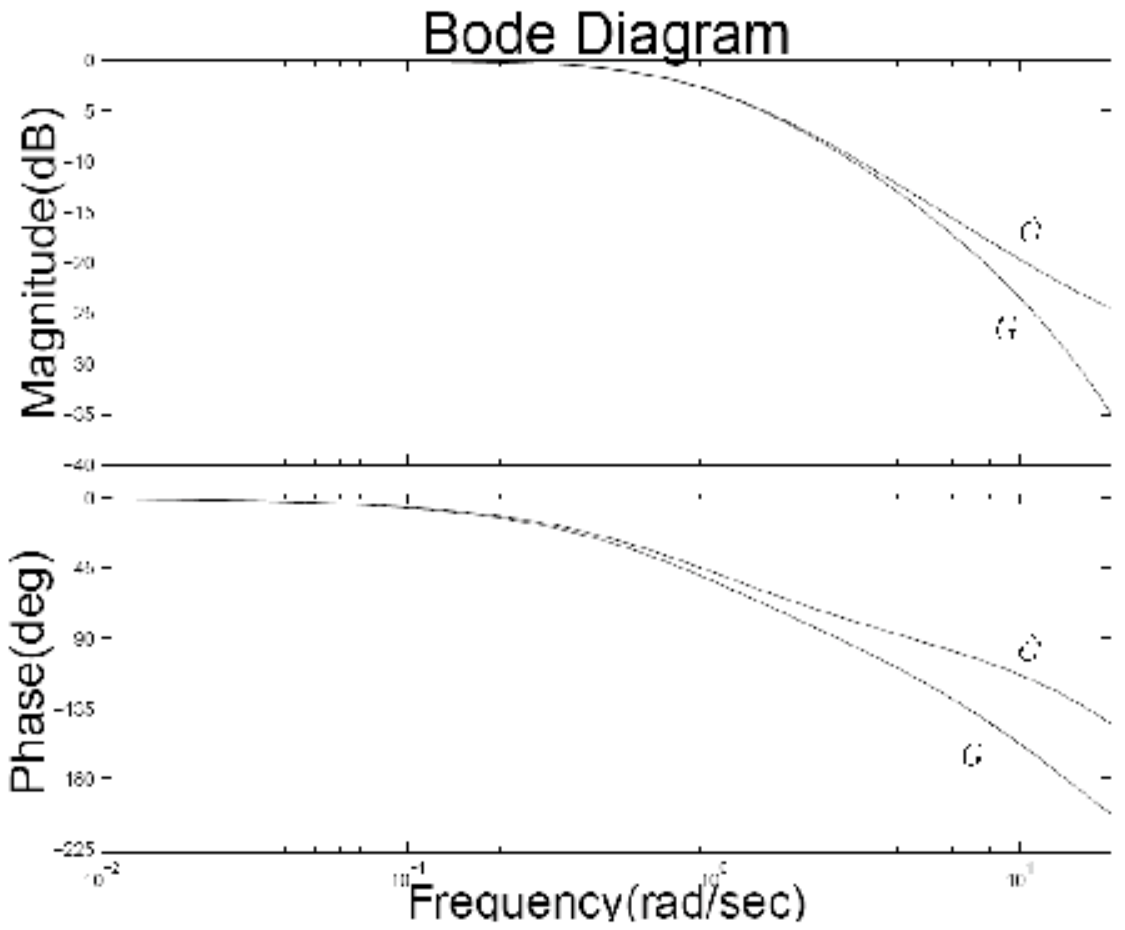

Figure 3. Comparison of Bode Diagram of plant and model 
The experimental setup is as follows. The residuals generated by the open loop structure of figure 2 are given by:

$$
\xi_{O L}=d+(G-\hat{G}) r
$$

Hence the residual $\xi_{O L}$ is just the noise $d$ if the model and the plant are equal (i.e. $\hat{G}=G$ ). Hence the residual has the same stochastic properties than the noise. The perturbation $d$ is assumed to be white noise with $\sigma=1$. The reference input $r$ is a train of sinusoids up to frequency $3 \mathrm{rad} / \mathrm{sec}$.

The validation procedure results can be seen in figure 4 . The model $\hat{G}$ shows no invalidation signs up to $1.4 \mathrm{rad} / \mathrm{sec}$. However for higher frequencies the hypothesis test fails to validate the model. As a conclusion we can state that, for the input applied, the model is correct for frequencies below to $1.4 \mathrm{rad} / \mathrm{sec}$. It is worth to mention that although between $1.7 \mathrm{rad} / \mathrm{sec}$ and $2.3 \mathrm{rad} / \mathrm{sec}$ there are no spikes out of the confidence limits, a deeper examination reveals that several consecutive spikes are abnormally high to belong a $\chi_{2}^{2}$ distribution. In order to detect this situation further probabilities should be checked (e.g. the probability that two consecutive points of a $\chi_{2}^{2}$ distribution be higher than some given value.)

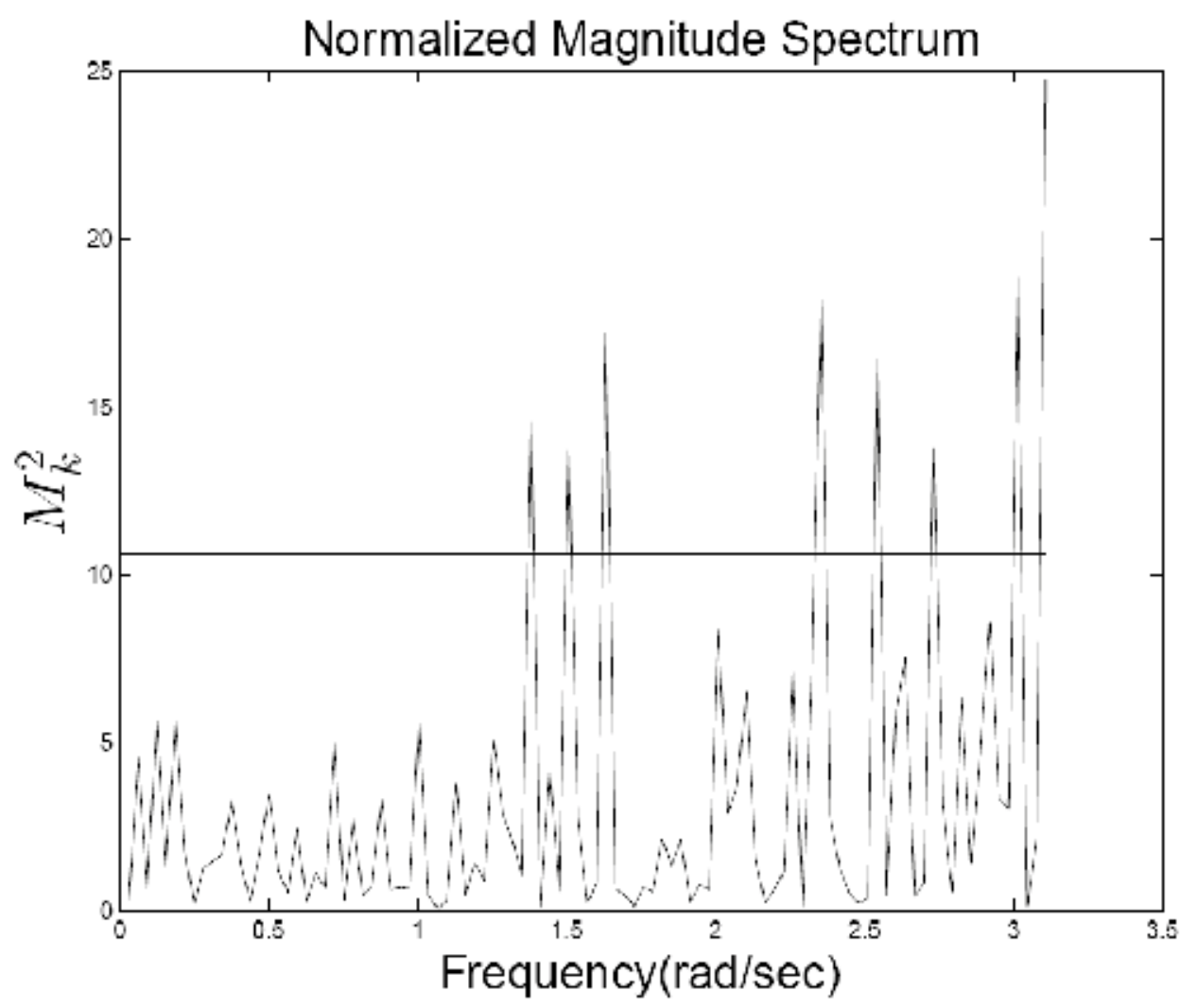

Figure 4. Normalized Magnitude Spectrum and Confidence Limit 
As a conclusion, the model $\hat{G}$ can be accepted as a good approximation of the plant $G$ up to frequency $1.4 \mathrm{rad} / \mathrm{sec}$. For higher frequencies the mismatch between model and plant is present up to the input bandwidth (i.e. $3 \mathrm{rad} / \mathrm{sec}$ ). It should be mention that this result is input dependent. However the results obtained up to now can serve as a guideline to design new input signals with suitable frequency contents for new identification steps (e.g. high energy around the frequencies were a significant error exists, that is between $1.4 \mathrm{rad} / \mathrm{sec}$ and $3 \mathrm{rad} / \mathrm{sec})$.

\section{Control Oriented Model Validation}

Model validation theory is aimed towards checking the model usefulness for some intended use. Thus the model validation procedure should take into account the model use, for example control design or prediction purposes. It is recognized in (Skelton, 1989) that arbitrary small model errors in open loop can lead to bad closed loop performance. On the other hand large open loop modelling errors do not necessarily lead to bad closed loop performance. As a result the model accuracy should be checked in such a way that the intended model use is taken into account in the model validation procedure.

An important aspect in the validation procedure to take into account is the intended model use and the validation conditions. In fact validation from open loop data can provide a different result that validation with closed loop data. Furthermore it is completely different to validate an open loop model than to compare two closed loops, the one with the model and the real one (See for example (Gevers et al., 1999)). This result points out the importance of the information that is being validated.

In order to consider the model intended use in the validation procedure, the conditions for data generation must be considered. In the following subsections different structures are proposed in order to compute the residuals and it is shown that they have considerable importance on the actual information that is validated. Its statistical properties are reviewed as the residuals must be statistically white under perfect model matching in order to apply the proposed algorithm. It is shown that the new model validation procedure introduced in this article can be endowed with the control oriented property by generating the residual using the structure presented in section 3.3.

\subsection{Open Loop Validation (Stable Plants)}

The model validation procedure is in open loop when there is no controller closing the loop. In that case, the structure used to validate the model is shown in figure 5 . In open loop validation it is required that both, the plant $P$ and the plant model $\hat{P}$ be stable in order to obtain a bounded residual $\xi_{O L}$.

The residual $\xi_{O L}$ is given by the following expression:

$$
\xi_{O L}=d+(P-\hat{P}) r
$$

Now we analyze the residual characteristics when the model equals the plant and when there is a model plant mismatch. The residual $\xi_{O L}$ given by equation (8) is just the noise $d$ if the model and the plant are equal (i.e. $P=\hat{P}$ ). Hence the residual has the same stochastic properties than the noise. As a result, under white noise assumption, the residual $\xi_{O L}$ is also 
white noise and then will pass the frequency dependent validation procedure. On the other hand if there exist a discrepancy between the model and the plant, a new term $(P-\hat{P}) r$ appears in the residual. This term makes that the residual $\xi_{O L}$ is no longer white noise, hence the residual will not pass the frequency dependent test. It should be remarked however that the model-plant error which will be detected is deeply dependent on the reference signal $r$.

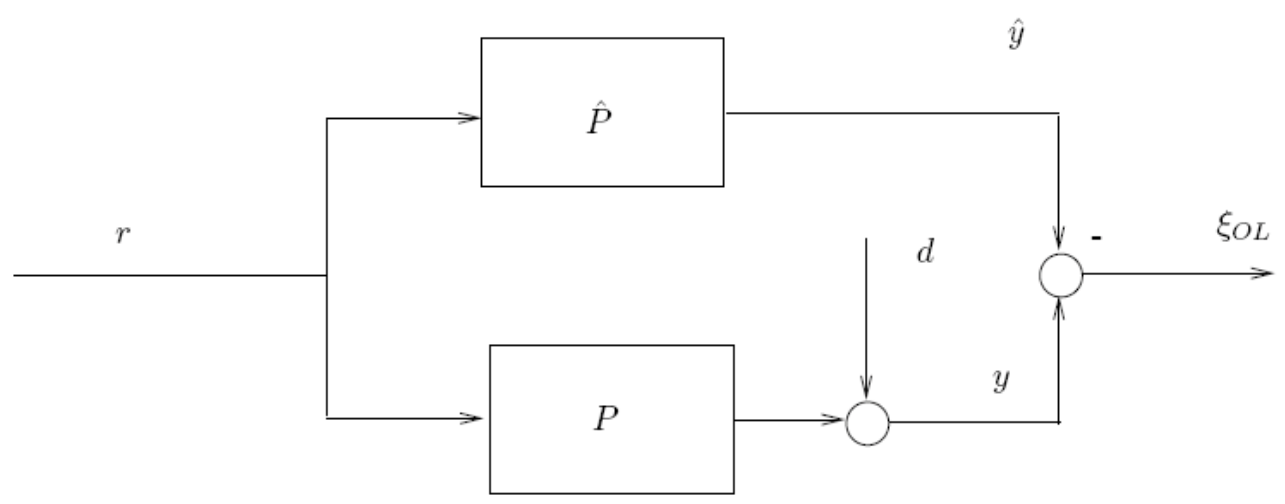

Figure 5. Open loop residual generation

\subsection{Closed Loop Validation (Unstable Plants)}

In the general closed loop validation case, the residual is generated as the comparison of two closed loops. On the one hand the closed loop formed by the controlled plant and on the other hand the closed loop formed by the controlled model (See figure 6). The main advantage of this configuration is that it permits validation of unstable models of unstable plants. Moreover, as we discuss below, the model-plant error is weighted.
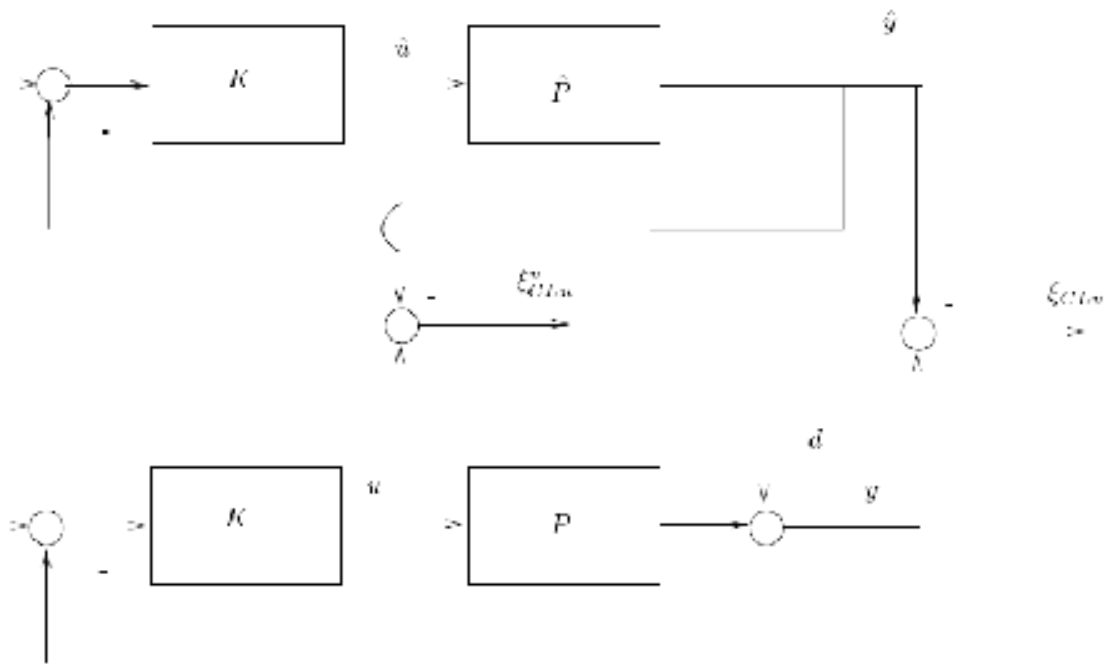

Figure 6. Closed loop residual generation (Unstable plants and models) 
The residual at the output $\xi_{C L u}$ (at the input $\xi_{C L u}^{u}$ ) of figure 6 is:

$$
\begin{aligned}
& \xi_{C L u}=S d+K S \hat{S}(P-\hat{P}) r \\
& \xi_{C L u}^{u}=-K S d+K K S \hat{S}(P-\hat{P}) r
\end{aligned}
$$

where $K$ is the controller, $S$ is the real sensitivity function (i.e. $\left.S=(1+P K)^{-1}\right)$ and $\hat{S}$ is the model sensitivity function (i.e. $\left.\hat{S}=(1+\hat{P} K)^{-1}\right)$. In the case there is a perfect model-plant match, that is when $\hat{P}=P$, the residual $\xi_{C L u}\left(\xi_{C L u}^{u}\right)$ yields $S d(-K S d)$. As a result, independently of the noise characteristics, the residual is always autocorrelated, as the noise is filtered by $S(-K S)$. Hence it is not possible to perform the frequency dependent whiteness test in order to validate the model.

If there is a model-plant mismatch (i.e. $\hat{P} \neq P$ ), a new term arises in residual $\xi_{C L u}\left(\xi_{C L u}^{u}\right)$. This term is $K S \hat{S}(P-\hat{P}) r \quad(K K S \hat{S}(P-\hat{P}) r)$, that is the model plant error weighted by $K S \hat{S}(K K S \hat{S})$. As a result, the relative importance of the model plant error is weighted, in such a way that if the gain of term $K S \hat{S}$ ( $K K S \hat{S})$ is "low" the error is not important but when the term gain $K S \hat{S}$ ( $K K S \hat{S})$ is "high" then the error is amplified. Thus we can see how the closed loop validation takes into account the model errors for control design purposes.

Summing up, although the closed loop validation structure presented in figure 6 is control oriented and allows the validation of unstable models, the residual generated by this structure is not suited for performing the frequency dependent validation procedure. In the next section we present a structure that allows performing the frequency dependent model validation on residuals generated in a control oriented way.

\subsection{Closed Loop Validation (Stable Plants)}

In this section we present a structure for generating the residual in such a way that first, it is control oriented and secondly it is suitable for the frequency dependent control oriented procedure proposed. The structure is shown in figure 7 .

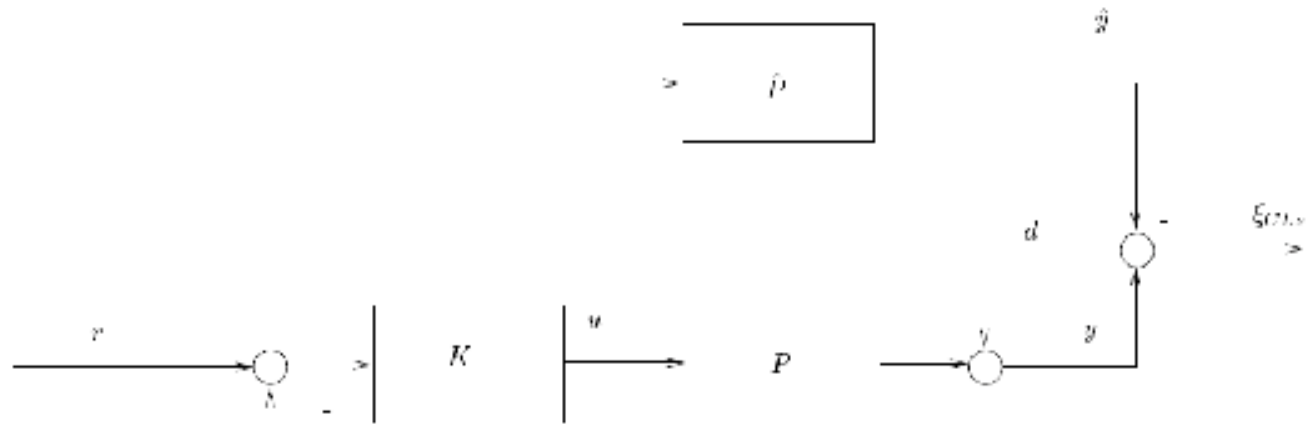

Figure 7. Closed loop residual generation (Stable models) 
In this case, the residual is given by:

$$
\xi_{C L S}=\frac{S}{\hat{S}} d+K S(P-\hat{P}) r
$$

where $K$ is the controller, $S$ is the real sensitivity function (i.e. $\left.S=(1+P K)^{-1}\right)$ and $\hat{S}$ is the model sensitivity function (i.e. $\hat{S}=(1+\hat{P} K)^{-1}$ ). The residual $\xi_{C L S}$ given by equation (10) is the noise $d$ filtered by the fraction of the real Sensitivity function $S=(1+P K)^{-1}$ and the Sensitivity function of the model $\hat{S}=(1+\hat{P} K)^{-1}$ plus a term that is the discrepancy of the plants weighted by the control sensitivity function (i.e. $K S$ ). If the model and the plant are equal (i.e. $\hat{P}=P$ ) then the real sensitivity function $S$ and the model sensitivity function $\hat{S}$ are equal so the first term of equation (10) yields the noise $d$. Moreover the second term, under the same perfect model-plant matching assumption, is zero. Hence in this case the residuals are again the noise $d$, thus it is suitable for our proposed frequency dependent validation algorithm.

On the other hand, if a discrepancy exists between the model $\hat{P}$ and the plant $P$, the division of $S$ by $\hat{S}$ is no longer unity but equals a transfer function resulting from the noise $d$ filtered by $S / \hat{S}$ (i.e. autocorrelated). Additionally the second term of equation (10) gives a signal proportional to the model-plant error weighted by the control sensitivity function (i.e. $K S$ ).

The presented structure is then suited to generate the residual in order to be used by the proposed validation algorithm.

\section{Application of the Frequency Dependent Model Validation to Iterative Identification and Control Schemes}

Iterative identification and control design schemes improve performance by designing new controllers on the basis of new identified models (Albertos and Sala, 2002). The procedure is as follows: an experiment is performed in closed loop with the current designed controller. A new model is identified with the experimental data and a new controller is designed using the new model. The procedure is repeated until satisfactory performance is achieved. The rationale behind iterative control is that if iteratively "better" models are identified, hence "better" performing controllers can be designed. However the meaning of "better" model needs some clarification. The idea of modelling the "true" plant has proven to be bogus (Hjalmarsson, 2005). Instead a good model for control is one that captures accurately the interesting frequency range for control purposes. In fact the model has no other use than to design a controller, thus the use of the model is instrumental (Lee et al., 1995). Hence, once a model is obtained it is necessary to validate it. On the iterative identification and control schemes this should be done each time a new model is identified (i.e. at each iteration).

The main problem of the validation methods reviewed is that the answer is a binary result (i.e. validated/invalidated). However models are neither good nor bad but have a certain valid frequency range (e.g. normally models are good at capturing low frequency behaviour 
but their accuracy degrades at higher frequencies). Moreover the iterative identification and control procedures have their own particular requirements

- Is it possible to improve an existing model? Is the data informative enough to attempt a new identification?

- How can the model be improved? Is the model order/structure rich enough to capture the interesting features of the plant?

- How authoritative can be the controller designed on the basis of the new model? Which is the validity frequency range of my model?

The above requirements for iterative control can not be provided by the classical model validation approaches above introduced because

- No indication on the possibility to improve an existing model. This problem is solved in (Lee et al., 1995) by the use of classical validation methods (i.e. cross-correlation test) together with the visual comparison of two power spectra.

- In iterative identification and control approaches a low order model is fitted to capture the frequency range of interest for control. Hence undermodelling is always present. This fact makes it difficult to apply traditional model validation schemes as the output of the validation procedure is a binary answer (i.e. validated/no validated) (Ljung, 1994).

- No indication on how to improve the model on the next iteration (i.e. model order selection and/or input experiment design).

- No indication on the model validity range for control design (i.e. controller bandwidth selection).

In the next section we present the benefits on the proposed validation algorithm on the iterative identification and control schemes.

\subsection{Model Validation on Iterative Identification and Control Schemes}

The benefits of the frequency dependent model validation for the iterative identification and control schemes hinge on the frequency domain information produced by the algorithm. It is possible to assess for what frequency range a new model should be identified (perhaps increasing the model order) and what frequency content should contain the input of the experiment. Moreover we have information over the frequency range for which the model is validated, thus it is possible to choose the proper controller bandwidth.

The benefits of the frequency dependent model validation approach over iterative identification and control (see figure 8) are:

- Designing the input experiment for the next identification step. It is well known that the identified model quality hinges on the experiment designed to obtain the data. The experiment should contain high energy components on the frequency range where the model is being validated if informative data are pursued for a new identification in the following step.

- $\quad$ Detecting model undermodelling and/or choosing model order. A higher order model can be fitted over the frequency range where the current model is being invalidated. It can be done even inside the current iteration step without the need of performing a new experiment. In (Balaguer et al., 2006c) a methodology to add poles and zeroes to an existing model can be found.

- Selecting controller bandwidth on the controller design step. Once a frequency range of the model has been validated, if no further improvement of the model is sought, the final controller designed should respect the allowable bandwidths of the model. 
These issues are shown by means of the next section illustrative example.

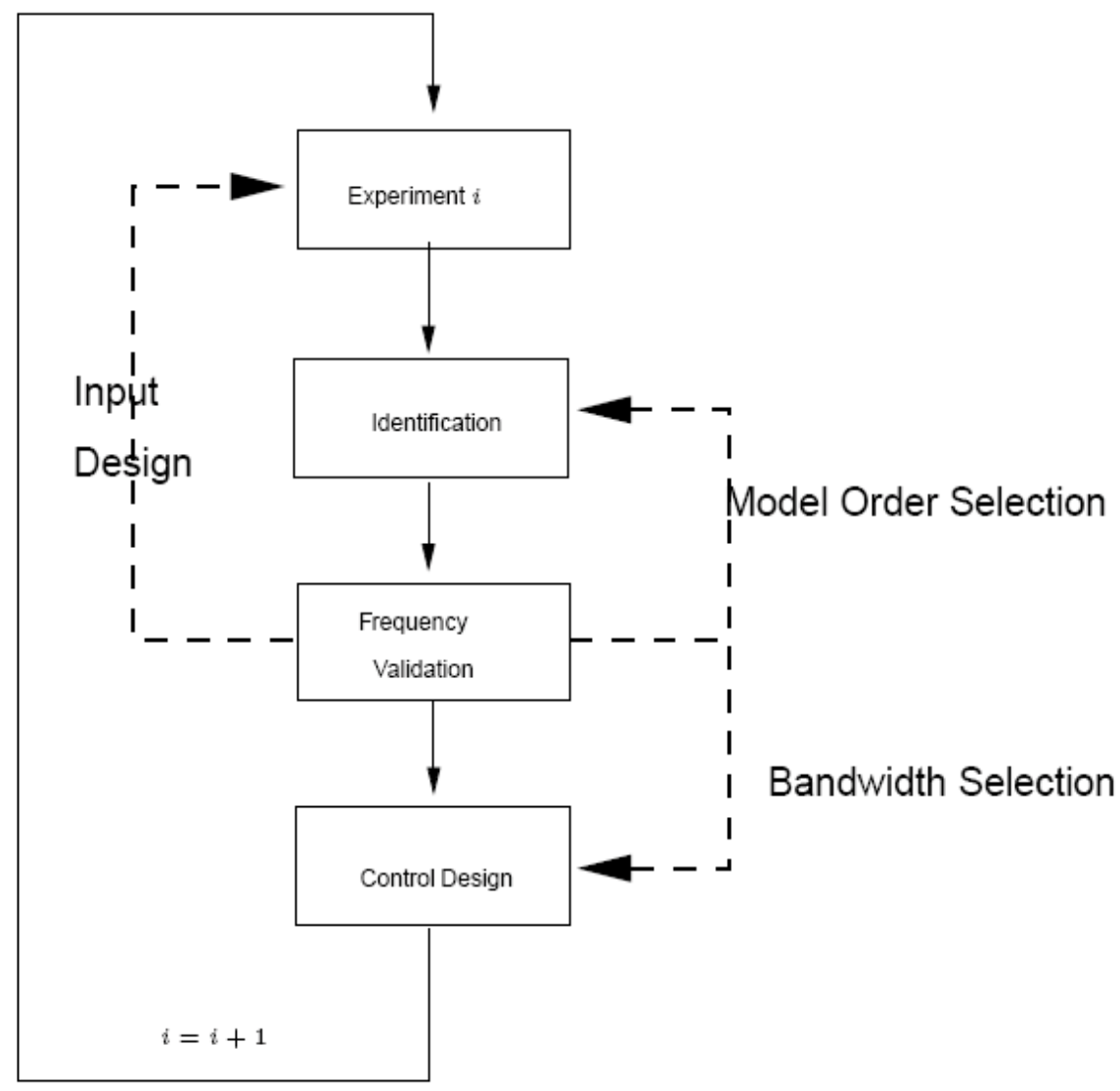

Figure 8. Frequency dependent model validation on iterative control

\subsection{Illustrative Example}

The present example is the application of the proposed frequency domain model validation to an iterative identification and control design. As baseline we take the Iterative Control Design example presented in (Albertos and Sala, 2002), page 126, where a stable plant with highfrequency resonant modes is controlled by successive plant identification (e.g. step response) and the subsequent controller design (e.g. model matching and cancellation controller). We apply to the successive models and controllers given in the example our frequency domain model validation procedure. Moreover we propose a customized structure in order to generate adequate residuals to claim for a control oriented model validation.

The proposed structure to generate the residuals is in closed loop, as shown in figure 7. The residual is given by equation (10), which is repeated here, following the example notation, for the sake of clarity:

$$
\xi_{C L S}=\frac{S}{\hat{S}} d+K S(G-\hat{G}) r
$$


The experimental setup is as follows. First a model of the plant $\hat{G}$ is obtained by a step response identification. For this model successive controllers $K$ are designed by imposing more stringent reference models $M$. When the closed loop step response is unsatisfactory, a new model is identified and the controller design steps repeated. The measurement noise $d$ is white noise with $\sigma=10^{-2}$. The reference input $r$ is a train of sinusoids up to frequency $200 \mathrm{rad} / \mathrm{sec}$. Finally, the plant $G$ to be controlled is sixth order, given by

$$
G=\frac{10^{6}(s+1000)}{\left(s^{2}+0.002 s+1000^{2}\right)\left(s^{2}+0.1 s+50^{2}\right)(s+0.1)(s+0.2)}
$$
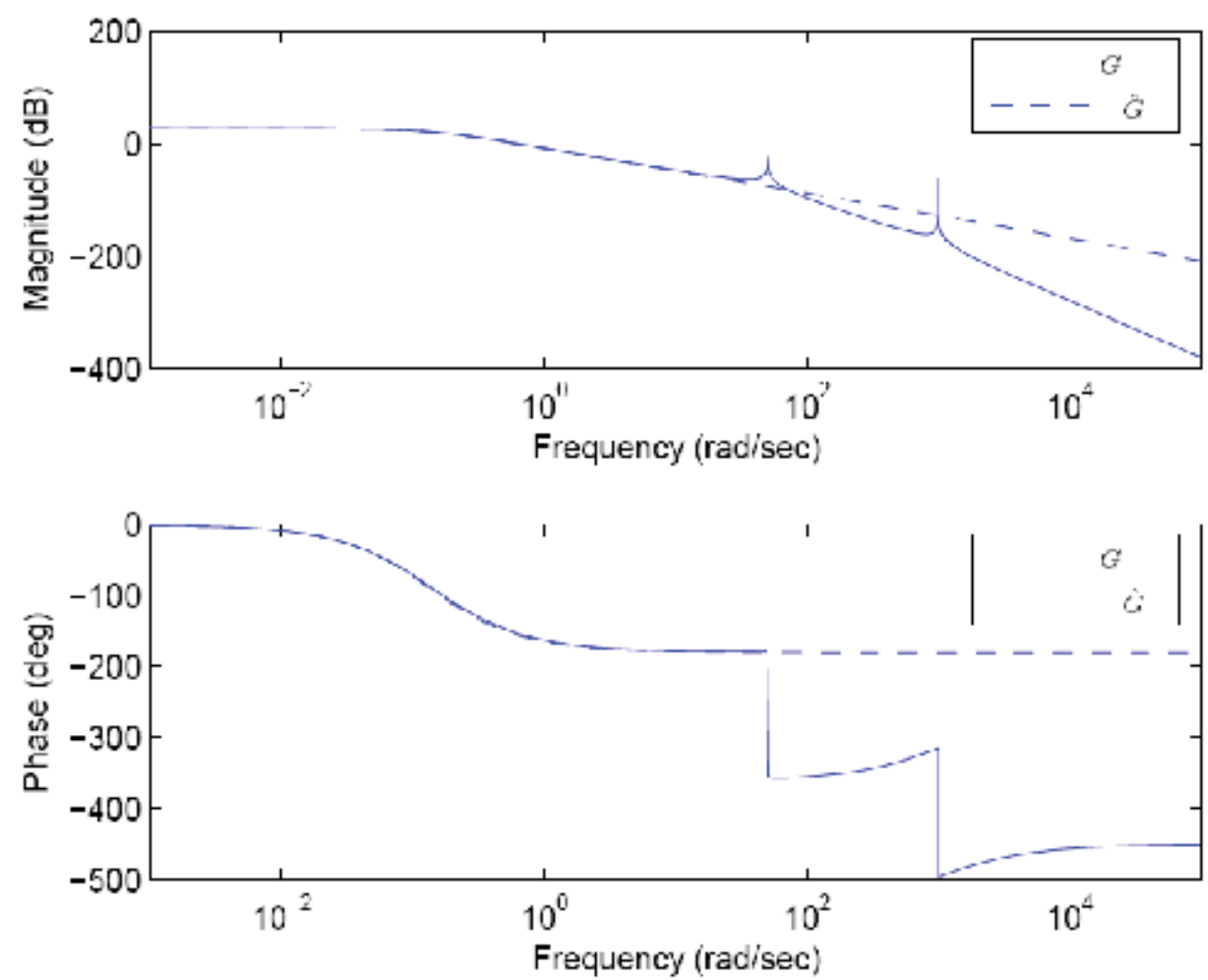

Figure 9. Bode diagrams of the plant and the model

\section{First Iteration}

The first identified model $\hat{G}_{0}$ and the model reference $M_{01}$ used for controller design are:

$$
\begin{aligned}
& \hat{G}_{0}=\frac{20}{(1+7.4 s)^{2}} \\
& M_{01}=\frac{0.5^{2}}{(s+0.5)^{2}}
\end{aligned}
$$


The bode plot of the real plant $G$ and the first model $\hat{G}_{0}$ are shown in figure 9 . The frequency domain validation is applied, given a positive validation result, as can be seen in the first plot of figure 10 .
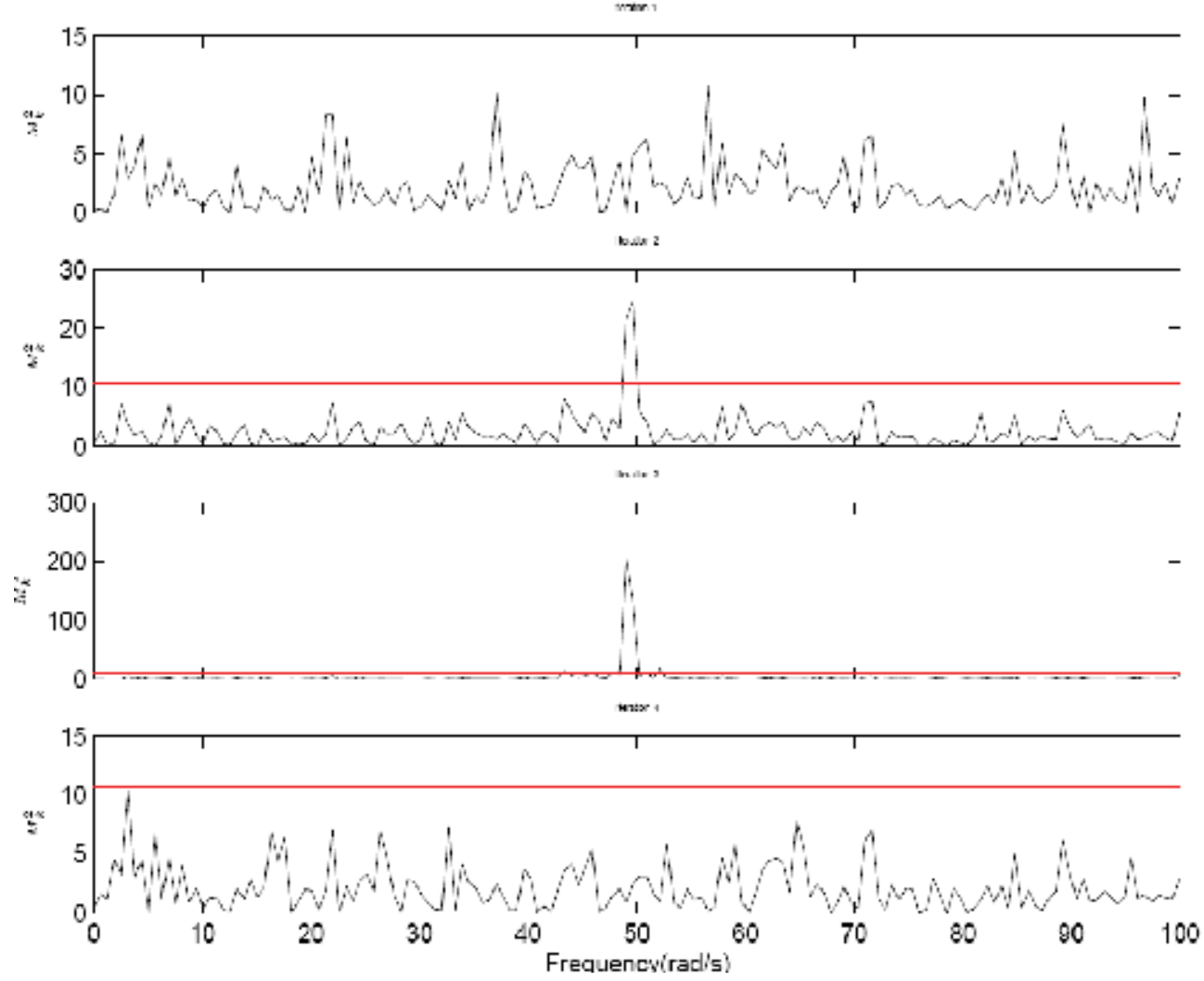

Figure 10. Frequency dependent validation result at each iteration

\section{Second Iteration}

Following the positive validation result of the first iteration the same model is kept as valid and the performance is pushed forward by a new, more stringent, reference model $M_{02}$ :

$$
\begin{aligned}
& \hat{G}_{0}=\frac{20}{(1+7.4 s)^{2}} \\
& M_{02}=\frac{3^{2}}{(s+3)^{2}}
\end{aligned}
$$

The validation test invalidate the model for frequencies around $50 \mathrm{rad} / \mathrm{sec}$ (see plot 2 of figure 10. This is due to the non modelled resonance peak as can be seen in the bode diagram of figure 9 .

\section{Third Iteration}

In (Albertos and Sala, 2002), the new identification step is taken after pushing even forward the desired reference model $M_{03}$ : 


$$
\begin{aligned}
& \hat{G}_{0}=\frac{20}{(1+7.4 s)^{2}} \\
& M_{03}=\frac{5^{2}}{(s+5)^{2}}
\end{aligned}
$$

The invalidation of the model for frequencies around $50 \mathrm{rad} / \mathrm{sec}$ for this controller is evident (plot 3 of figure 10).

\section{Fourth Iteration}

In (Albertos and Sala, 2002) a new model plant is identified due to the unacceptable closed loop behaviour for the controller designed with the reference model $M_{03}$. The new identified plant $G_{1}$ captures the first resonance peak of the plant. The reference model is $M_{11}$ which keeps the same time constant as the former reference model $M_{03}$.

$$
\begin{aligned}
& \hat{G}_{1}=\hat{G}_{{ }_{00}} \frac{0.01^{2}+50^{2}}{(s+0.01+50 j)(s+0.01-50 j)} \\
& M_{11}=\frac{5^{4}}{(s+5)^{4}}
\end{aligned}
$$

The model validation result shows that now, the model is validated for all the frequency range covered by the input (plot 4 of figure 10).

Summarizing the example results, we have shown how the frequency dependent model validation scheme can be helpful to guide the identification step by aiming towards the interesting frequencies content that an identification experiment should excite. The procedure is also helpful to choose the appropriate controller bandwidth suitable for the actual model accuracy. Moreover it has been proven that the proposed methodology can be applied in iterative identification and control design schemes and the validation can be control oriented.

\section{Conclusion}

In this paper a new algorithm for model validation has been presented. The originality of the approach is that it validates the model in the frequency domain rather than in the time domain. The procedure of validating a model in the frequency domain has proven to be more informative for control identification and design purposes than classical validation methods.

- Firstly, the model is neither validated nor invalidated. Instead valid/invalid frequency ranges are given.

- Secondly, the invalidated frequency range is useful in order to determine the new experiment to identified better models in those frequency ranges.

- Thirdly, the model validity frequency range establishes a maximum controller bandwidth allowable for the model quality.

Our model validation procedure is of interest for Iterative Identification and Control schemes. Normally these schemes start with a low quality model and low authoritative controller which are improved iteratively. As a result poor models must be improved. This 
raises the questions on model validation and controller bandwidth that our approach helps to solve. Classical validation methods would invalidate the first low quality model meanwhile it is of use for future improvements.

Another application area of the proposed frequency dependent model validation is the tuning and validation of controllers. In this way it is possible to find low order controllers that behave similarly to high order ones in some frequency band.

Summing up the major advantage of the proposed algorithm is the frequency viewpoint which enables a richer validation result than the binary answer of the existing algorithms.

\section{References}

Albertos, P. \& Sala, A. (2002). Iterative Identification and Control, Springer

Balaguer, P. \& Vilanova, R. (2006a). Model Validation on Iterative Identification and Control Schemes, Proceedings of 7 th Portuguese Conference on Automatic Control, pp. 14-17, Lisbon

Balaguer, P. \& Vilanova, R. (2006b). Quality assessment of models for iterative/adaptive control, Proceedings of the $4^{\text {th }}$ Conference on Decision and Control, pp. 14-17, San Diego

Balaguer, P., Vilanova, R \& A. Ibeas. (2006c). Validation and improvement of models in the frequency domain, Computational Engineering in System Applications, pp. 14-17, Beijing

Balaguer, P., Wahab, N.A., Katebi, R. \& Vilanova, R. (2008). Multivariable PID control tuning: a controller validation approach, Emerging Technologies and Factory Automation, pp. 14-17, Hamburg

Box, G., Hunter W. \& Hunter, J. (1978). Statistics for Experimenters. An Introduction to Design, Data Analisis and Model Building, John Wiley E Sons, Inc.

Chen, J. \& Gu, G. (2000). Control Oriented System Identification. An $H_{\infty}$ Approach, John Wiley $\mathcal{E}$ Sons, Inc.

Gevers, M.; Codrons, B. \& Bruyne, F. (1999). Model Validation in Closed Loop, Proceedings of the American Control Conference

Hjalmarsson, H. (2005). From Experiment Design to Closed-Loop Control. Automatica, Vol. 41, page numbers (393-438)

Lee, W., Anderson, B., Mareels, I. and Kosut, R. (1995). On Some Key Issues in the Windsurfer Approach to Adaptive Robust Control. Automatica, Vol. 31, page numbers (1619-1636)

Ljung, L. (1994). System Identification. Theory for the User, Prentice-Hall

Skelton, R. (1989). Model Error Concepts in Control Design. International Journal of Control, Vol. 49, No. 5, page numbers (1725-1753)

Soderstrom, T. \& Stoica, P. (1989). System Identification, Prentice Hall International Series in Systems and Control Engineering. 


\title{
Fast Particle Filters and Their Applications to Adaptive Control in Change-Point ARX Models and Robotics
}

\author{
Yuguo Chen, Tze Leung Lai and Bin $\mathrm{Wu}$ \\ University of Illinois at Urbana-Champaign \& Stanford University
}

USA

\section{Introduction}

The Kalman filter has provided an efficient and elegant solution to control problems in linear stochastic systems. For nonlinear stochastic systems, control problems become much more difficult and a large part of the literature resorts to linear approximations so that an "extended Kalman filter" or a "mixture of Kalman filters" can be used in place of the Kalman filter for linear systems. Since these linear approximations are local expansions around the estimated states, they may perform poorly when the true state differs substantially from its estimate. Substantial progress was made in the past decade for the filtering problem with the development of particle filters. This development offers promise for solving some long-standing control problems which we consider in this chapter.

As noted by Ljung \& Gunnarsson (1990), a parameterized description of a dynamic system that is convenient for identification is to specify the model's prediction of the output $y_{t}$ as a function of the parameter vector $\theta$ and past inputs and outputs $u_{s}$ and $y_{\mathrm{s}}$, respectively, for $s$ $<t$. When the function is linear in $\theta$, this yields the regression model $y_{t}=\theta^{T} \varphi_{t}+\epsilon_{t}$, which includes as a special case the ARX model (autoregressive model with exogenous inputs) that is widely used in control and signal processing. Here the regressor vector is

$$
\varphi_{t}=\left(-y_{t-1}, \ldots,-y_{t-k}, u_{t-1}, \ldots, u_{t-h}\right)^{T},
$$

consisting of lagged inputs and outputs. Whereas a comprehensive methodology has been developed for identification and control of ARX systems with time-invariant parameters (see e.g. Goodwin et al., 1981; Ljung \& Soderstrom, 1983; Goodwin \& Sin, 1984; Lai \& Wei, 1987; Guo \& Chen, 1991), the case of time-varying parameters in system identification and adaptive control still awaits definitive treatment despite a number of major advances during the past decade (Meyn \& Brown, 1993; Guo \& Ljung, 1993a, b). In Section 3 we show how particle filters can be used to resolve some of the long-standing difficulties due to the nonlinear interactions between the dynamics of the regressor vector (1) and of the parameter changes in the model $y_{t}=\theta_{t}^{T} \varphi_{t}+\epsilon_{t}$. Unlike continually fluctuating parameters modeled by a random walk in Meyn \& Brown (1993) and Guo \& Ljung (1993a, b), we consider here the parameter jump model similar to that in Eq. (21)-(22) of Ljung \& Gunnarsson (1990). As reviewed in Ljung \& Gunnarsson (1990, p. 11), an obvious way to 
handle parameter jumps is to apply carefully designed on-line change detection algorithms to segment the data. Another approach, called AFMM (adaptive forgetting through multiple models), is to use Bayesian updating formulas to calculate the posterior probability of each member in a family of models locating the change-points. To keep a fixed number of such models at every stage, the model with the lowest posterior probability is deleted while that with the highest posterior probability gives birth to a new model by allowing for a possible change-point from it. The fast particle filters introduced by Chen \& Lai (2007) enable them to develop a much more precise implementation of the Bayesian approach than AFMM, with little increase in computational cost, and to come up with more efficient adaptive control schemes, as shown in Section 3.

Another area where particle filters have been recognized to offer promising solutions to important and difficult control problems is probabilistic robotics. Section 4 provides a brief summary of the applications of particle filters to estimate the position and orientation of a robot in an unknown environment from sensor measurements. It also reviews previous work and ongoing work on using these particle filters to tackle the difficult stochastic control problems in robotics.

The stochastic models in Sections 3 and 4 are special cases of hidden Markov models. Section 2 gives a brief introduction to hidden Markov models and particle filters, which are sequential estimates of the hidden states by using Monte Carlo methods that involve sequential importance sampling and resampling. The basic idea underlying these sequential Monte Carlo filters is to represent the posterior distribution of the hidden state at time $t$ given the observations up to time $t$ by a large number of simulated samples ("particles"). Simulating a large number of samples, however, makes the Monte Carlo approach impractical for on-line identification and control applications. We show in Section 3 that by choosing appropriate resampling schemes and proposal distributions for importance sampling, we can arrive at good approximations to the optimal filter by using a manageable number (as small as 50) of simulated samples for on-line identification and adaptive control. This point is discussed further in Section 5 where we consider related issues and give some concluding remarks.

\section{Particle Filters in Hidden Markov Models}

A hidden Markov model (HMM) is a stochastic process $\left(x_{t}, y_{t}\right)$ in which (i) $\left\{x_{t}\right\}$ is an unobservable Markov process with transition probability density function $f(\cdot \mid)$ with respect to some measure $\nu$ on the state space, and (ii) given $\left\{x_{t}\right\}$, the observable random variables $y_{t}$ are conditionally independent such that $y_{s}$ has density function $g\left(\cdot \mid x_{s}\right)$ with respect to some measure. The filtering problem for HMM is to find the posterior distribution of the hidden state $x_{t}$ given the current and past observations $y_{1}, \ldots, y_{t}$. In particular, the optimal filter with respect to squared error loss is $E\left(x_{t} \mid y_{1}, \ldots, y_{t}\right)$. In engineering applications, there are often computational constraints for on-line updating of the filter and recursive algorithms are particularly attractive. For infinite state spaces, direct computation of the optimal filters is not feasible except in linear Gaussian state-space models, for which Kalman filtering provides explicit recursive filters. Analytic approximations or Monte Carlo methods are therefore used instead. Although Markov chain Monte Carlo has provided a versatile simulation-based tool to calculate the posterior distributions of hidden states in HMMs, it is cumbersome for updating and is too slow for 
on-line filtering problems. Sequential Monte Carlo methods represent the posterior distributions by a large number $M$ of random samples that are sequentially generated over time by using a combination of sequential importance sampling and resampling steps.

\subsection{Proposal Distribution for Sequential Importance Sampling}

Let $X_{1: n}=\left(x_{1}, \ldots, x_{n}\right), Y_{1: n}=\left(y_{1}, \ldots, y_{n}\right)$, and $p(\cdot \mid \cdot)$ and $p(\cdot)$ denote the conditional and the joint density functions (under the measure $P$ ) of the random variables indicated. Given $Y_{1: n}$, the conditional distribution of $X_{1: n}$ is that of an inhomogeneous Markov chain with transition probability density

$$
p\left(x_{t} \mid x_{t-1}, Y_{1: n}\right) \propto f\left(x_{t} \mid x_{t-1}\right) g\left(y_{t} \mid x_{t}\right) \int \prod_{i=t+1}^{n} f\left(x_{i} \mid x_{i-1}\right) g\left(y_{i} \mid x_{i}\right) d \nu\left(x_{i}\right),
$$

in which the constant of proportionality is the normalizing constant that makes the left hand side of (2) integrate to 1.

It is often difficult to sample directly from this Markov chain for Monte Carlo evaluation of the posterior distribution of $x_{n}$ given $Y_{1: n}$, which is used to estimate the optimal filter $E\left\{h\left(x_{n}\right) \mid Y_{1: n}\right\}$. Instead we sample from an alternative distribution $Q$ under which $X_{1: n}$ is an inhomogeneous Markov chain with transition density

$$
q_{t}\left(x_{t} \mid x_{t-1}\right) \propto f\left(x_{t} \mid x_{t-1}\right) g\left(y_{t} \mid x_{t}\right),
$$

which is tantamount to replacing $p\left(x_{t} \mid x_{t-1}, Y_{1: n}\right)$ in (2) by $p\left(x_{t} \mid x_{t-1}, Y_{1: t}\right)$. The optimal filter can be expressed in terms of $Q$ via

$$
E\left\{h\left(x_{n}\right) \mid Y_{1: n}\right\}=E_{Q}\left\{h\left(x_{n}\right) \prod_{t=1}^{n}\left[p\left(x_{t} \mid x_{t-1}, Y_{1: n}\right) / q_{t}\left(x_{t} \mid x_{t-1}\right)\right]\right\},
$$

where $E_{Q}$ denotes expectation under the measure $Q$. Therefore, instead of drawing $M$ samples $X_{1: n}^{(1)}, \ldots, X_{1: n}^{(M)}$ from (2) and using $M^{-1} \sum_{j=1}^{M} h\left(x_{n}^{(j)}\right)$ to estimate the optimal filter (4), we can draw $M$ samples from (3) and estimate the optimal filter by

$$
\hat{h}_{n}=\left\{\sum_{j=1}^{M} h\left(x_{n}^{(j)}\right) w_{n}^{(j)}\right\} / \sum_{j=1}^{M} w_{n}^{(j)},
$$

where $w_{n}^{(j)}$ are the importance weights given recursively by

$$
w_{1}^{(j)}=1, \quad w_{t}^{(j)}=w_{t-1}^{(j)} p\left(y_{t} \mid x_{t-1}^{(j)}\right),
$$

noting that $p\left(y_{t} \mid x_{t-1}\right)=\int f\left(x \mid x_{t-1}\right) g\left(y_{t} \mid x\right) d \nu(x), p\left(y_{1} \mid x_{0}\right) \quad$ and $\quad p\left(Y_{1: n} \mid x_{0}\right)$ are proportionality constants and 


$$
\begin{gathered}
\prod_{t=1}^{n} p\left(x_{t} \mid x_{t-1}, Y_{1: n}\right)=p\left(x_{1: n} \mid x_{0}, Y_{1: n}\right)=\left\{\prod_{t=1}^{n} f\left(x_{t} \mid x_{t-1}\right) g\left(y_{t} \mid x_{t}\right)\right\} / p\left(Y_{1: n} \mid x_{0}\right), \\
\prod_{t=1}^{n} q_{t}\left(x_{t} \mid x_{t-1}\right)=\prod_{t=1}^{n}\left\{f\left(x_{t} \mid x_{t-1}\right) g\left(y_{t} \mid x_{t}\right) / p\left(y_{t} \mid x_{t-1}\right)\right\} .
\end{gathered}
$$

In the case where $x_{0}$ is specified by an initial distribution $\pi_{0}$, we replace $x_{0}$ above by $x_{0}^{(j)}$ drawn from $\pi_{0}(j=1, \ldots, M)$.

In situations where the normalizing constant $\int f\left(x \mid x_{t-1}\right) g\left(y_{t} \mid x\right) d \nu(x)$ in (3) does not have a closed-form expression, sampling from $Q$ defined by (3) can still be carried out by rejection sampling or other methods, but the importance weights (6) do not have explicit formulas and rejection sampling slows down the procedure considerably. A better idea is to choose another $Q$ which is easier to sample from and has explicit formulas for the importance weights, and which approximates (3) in some sense. One way to do this is to use a finite mixture of Gaussian distributions to approximate (3), with suitably chosen mixing proportions, means and covariance matrices. Using (3) or more convenient approximations thereof as the proposal distribution for sequential importance sampling provides substantial improvement over the original particle filter of Gordon et al. (1993) who simply use $q_{t}\left(x_{t} \mid x_{t-1}\right)=f\left(x_{t} \mid x_{t-1}\right)$, not adapting to the observed data $Y_{1: n}$. Whereas the adaptive transition probability density (2) is non-recursive (because $Y_{1: n}$ and $Y_{1: n+1}$ result in different transition probabilities $p\left(x_{t} \mid x_{t-1}, Y_{1: n}\right)$ and $\left.p\left(x_{t} \mid x_{t-1}, Y_{1: n+1}\right)\right)$, the proposal distribution (3) is adaptive and recursive.

\subsection{Periodic Rejuvenation via Resampling}

The particle filter of Gordon et al. (1993) is often called a "bootstrap filter" because, besides sampling $\hat{x}_{t}^{(j)}$ from $f\left(\cdot \mid x_{t-1}^{(j)}\right)$ to form $\hat{X}_{1: t}^{(j)}=\left(x_{1}^{(j)}, \ldots, x_{t-1}^{(j)}, \hat{x}_{t}^{(j)}\right)$, it also resamples from $\left\{\hat{X}_{1: t}^{(1)}, \ldots, \hat{X}_{1: t}^{(M)}\right\}$ with probabilities proportional to the importance weights $w_{t}^{(j)}\left(=w_{t-1}^{(j)} g\left(y_{t} \mid \hat{x}_{t}^{(j)}\right)\right)$, thereby generating the particles (trajectories) $X_{1: t}^{(1)}, \ldots, X_{1: t}^{(M)}$. In other words, at every $t$ there is an importance sampling step followed by a resampling step. We can think of importance sampling as generating a weighted representation $\left(q\left(x_{1}, \ldots, x_{t}\right), w_{t}\right)$ of $p\left(x_{1}, \ldots, x_{t} \mid Y_{1: t}\right)$ and resampling as transforming the weighted representation to an unweighted approximation of $p\left(x_{1}, \ldots, x_{t} \mid Y_{1: t}\right)$. For the bootstrap filter, since resampling introduces additional variability, resampling at every $t$ may result in substantial loss in statistical efficiency. In addition, the computational cost of resampling at every $t$ also accumulates over time.

If we forgo resampling altogether, then we have a weighted representation $\left(q\left(x_{1}, \ldots, x_{n}\right), w_{n}\right)$ of $p\left(x_{1}, \ldots, x_{n} \mid Y_{1: n}\right)$ at stage $n$. In view of (4), if we use the normalized weights

$$
w_{t}=\prod_{i=1}^{t}\left[p\left(x_{i} \mid x_{i-1}, Y_{1: n}\right) / q_{i}\left(x_{i} \mid x_{i-1}\right)\right]
$$


then $M^{-1} \sum_{j=1}^{M} h\left(x_{n}^{(j)}\right) w_{n}^{(j)}$ is an unbiased estimate of $E\left\{h\left(x_{n}\right) \mid Y_{1: n}\right\}$. However, for large $n$, sequential importance sampling without resampling also has difficulties because of the large variance of $w_{n}$. In particular, for the special cases (i) $q_{t}\left(x_{t} \mid x_{t-1}\right)=f\left(x_{t} \mid x_{t-1}\right)$ and (ii) $q_{t}\left(x_{t} \mid x_{t-1}\right)=f\left(x_{t} \mid x_{t-1}\right) g\left(y_{t} \mid x_{t}\right), \quad n^{-1} \log w_{n}$ converges almost surely under certain integrability assumptions and $E\left\{\operatorname{var}_{Q}\left(w_{n} \mid Y_{1: n}\right)\right\} \rightarrow \infty$ as $n \rightarrow \infty$; see Chan \& Lai (2008) where an asymptotic theory of particle filters in HMMs, including consistent estimation of their standard errors, is given.

A compromise between forgoing resampling altogether and resampling at every stage $t$ is to resample periodically. The motivation for resampling is to make multiple copies of the trajectories with large weights and to prune away those with small weights. The trajectories with small weights contribute little to the final estimate and it is a waste to carry many trajectories with very small weights. In particular, Kong et al. (1994) propose to monitor the coefficient of variation $(\mathrm{cv})$ of the importance weights $w_{t}$, defined by

$$
\mathrm{cv}^{2}=\operatorname{var}_{Q}\left(w_{t}\right) / E_{Q}^{2}\left(w_{t}\right),
$$

and to perform resampling if the $\mathrm{cv}^{2}$ of the current weights $w_{t}$ is greater than or equal to a certain bound. Specifically the procedure can be described as follows, starting with $M$ samples $X_{1: t-1}^{(1)}, \ldots, X_{1: t-1}^{(M)}$ having weights $w_{t-1}^{(1)}, \ldots, w_{t-1}^{(M)}$ at time $t-1$.

a. Draw $\hat{x}_{t}^{(j)}$ from $q_{t}\left(\cdot \mid x_{t-1}^{(j)}\right)$ and update the weight $w_{t}^{(j)}, j=1, \ldots, M$.

b. If the $\mathrm{cv}^{2}$ of $\left\{w_{t}^{(1)}, \ldots, w_{t}^{(M)}\right\}$ exceeds or equals a certain bound, resample from $\left\{\hat{x}_{t}^{(1)}, \ldots, \hat{x}_{t}^{(M)}\right\}$ with probabilities proportional to $\left\{w_{t}^{(1)}, \ldots, w_{t}^{(M)}\right\}$ to produce a random sample $\left\{x_{t}^{(1)}, \ldots, x_{t}^{(M)}\right\}$ with equal weights. Otherwise let $\left\{x_{t}^{(1)}, \ldots, x_{t}^{(M)}\right\}=\left\{\hat{x}_{t}^{(1)}, \ldots, \hat{x}_{t}^{(M)}\right\}$ and return to step a.

Strictly speaking, since the weight $w_{t}^{(j)}$ is associated with the entire path $\hat{X}_{1: t}^{(j)}=\left(x_{1}^{(j)}, \ldots, x_{t-1}^{(j)}, \hat{x}_{t}^{(j)}\right)$, resampling should be performed on $\left\{\hat{X}_{1: t}^{(1)}, \ldots, \hat{X}_{1: t}^{(M)}\right\}$. However, because of the Markovian structure, the past observations $x_{1}, \ldots, x_{s-1}$ can be discarded after generating the current state $x_{s}$. This explains why $X_{1: t-1}^{(1)}, \ldots, X_{1: t-1}^{(M)}$ are discarded in Step b above. In the second paragraph of Section 3.1, since the sequential importance sampling with resampling (SISR) filter is defined via certain functions of the Markov chain (instead of the Markov chain itself), resampling has to be performed on the sample of $M$ trajectories.

\section{Fast Particle Filters in Change-Point ARX Models}

\subsection{Preliminaries: Normal Mean Shift Model}

Before considering the more general change-point regression model $y_{t}=\theta_{t}^{T} \varphi_{t}+\epsilon_{t}$, we find it helpful to explain some important ideas for constructing fast particle filters in the simple case of univariate $\varphi_{t} \equiv 1$, dating back to Yao's (1984) simple mean shift model, in which the observations $y_{t}$ are independent normal with variance 1 and means $\mu_{t}$ such that at time $t, \mu_{t}$ equals $\mu_{t-1}$ (i.e. undergoes no change) with probability $1-\rho$ and assumes a new value, which is normally distributed with mean 0 and variance $\sigma^{2}$, with probability $\rho$. Note that $\left\{\left(\mu_{t}, y_{t}\right), t \geq 1\right\}$ forms a HMM, with $g(\cdot \mid \mu)$ being the normal density function 
with mean $\mu$ and variance 1 and such that the transition probability distribution of $\mu_{t}$ has (i) a discrete component putting mass $1-\rho$ at $\mu_{t-1}$ and (ii) an absolutely continuous component having density function $\rho \sigma^{-1} \phi(\cdot / \sigma)$, where $\phi$ denotes the standard normal density function. The proposal distribution (3) (with $x_{t}=\mu_{t}$ ) for $\mu_{t} \mid \mu_{t-1}$ is a mixture of a degenerate distribution at $\mu_{t-1}$ and a normal distribution with mean $y_{t} /\left(1+\sigma^{-2}\right)$ and variance $1 /\left(1+\sigma^{-2}\right)$, with mixing probabilities proportional to $(1-\rho) \phi\left(y_{t}-\mu_{t-1}\right)$ and $\rho\left(\sigma^{2}+1\right)^{-1 / 2} \phi\left(y_{t} /\left(\sigma^{2}+1\right)^{1 / 2}\right)$, respectively. It is, therefore, easy to sample from this proposal distribution. Because of the discrete and absolutely continuous components of the transition probability distribution, the importance weights (see (6)) $w_{t}$ are now given recursively by $w_{1}=1$ and

$$
w_{t}=w_{t-1}\left\{\rho\left(\sigma^{2}+1\right)^{-1 / 2} \phi\left(y_{t} /\left(\sigma^{2}+1\right)^{1 / 2}\right)+(1-\rho) \phi\left(y_{t}-\mu_{t-1}\right)\right\} .
$$

Instead of working with the unobserved Markov chain $\left\{\mu_{t}\right\}$, it is more efficient to consider the latent variable $I_{t}=1_{\left\{\mu_{t} \neq \mu_{t-1}\right\}}$, indicating whether $t$ is a change-point. The reason is that given $Y_{1: n}$ the $I_{t}$ are Bernoulli random variables that can be generated recursively and $E\left(\mu_{n} \mid I_{1: n}, Y_{1: n}\right)$ can be easily computed by a closed-form formula, where $I_{1: n}=\left\{I_{1}, \ldots, I_{n}\right\}$. Following Yao (1984), we rewrite the optimal filter as

$$
E\left(\mu_{n} \mid Y_{1: n}\right)=E\left\{E\left[\mu_{n} \mid I_{1: n}, Y_{1: n}\right] \mid Y_{1: n}\right\}=E\left\{\left(n-C_{n}+1+\sigma^{-2}\right)^{-1} \sum_{j=C_{n}}^{n} y_{j} \mid Y_{1: n}\right\},
$$

where $C_{t}=\max \left\{j \leq t: I_{j}=1\right\}(\max \emptyset=1)$, i.e., $C_{t}$ is the most recent change-point up to time $t$. Consider the proposal distribution $Q$ for which $I_{t} \mid I_{1: t-1}$ has the same distribution as $P\left(I_{t}=\cdot \mid I_{1: t-1}, Y_{1: t}\right)$. It is easy to sample $I_{1}, \ldots, I_{n}$ sequentially from $Q$, under which $I_{t} \mid I_{1: t-1}$ is Bernoulli assuming the values 1 and 0 with probabilities in the proportion

$$
\frac{\rho}{\left(1+\sigma^{2}\right)^{1 / 2}} \phi\left(\frac{y_{t}}{\left(1+\sigma^{2}\right)^{1 / 2}}\right): \frac{1-\rho}{\left(1+v_{t}\right)^{1 / 2}} \phi\left(\frac{y_{t}-v_{t} \sum_{j=C_{t-1}}^{t-1} y_{j}}{\left(1+v_{t}\right)^{1 / 2}}\right),
$$

where $v_{t}=\left(t-C_{t-1}+\sigma^{-2}\right)^{-1}$. Letting $a_{t}(\rho)$ and $b_{t}(\rho)$ denote the two terms in (10), note that $p\left(y_{t} \mid I_{1: t-1}, Y_{1: t-1}\right)=a_{t}(\rho)+b_{t}(\rho)$. Combining this with

$$
\frac{p\left(I_{1: n} \mid Y_{1: n}\right)}{q\left(I_{1: n}\right)}=\frac{p\left(y_{1}\right)}{p\left(Y_{1: n}\right)} \prod_{t=2}^{n} \frac{p\left(I_{1: t-1}, Y_{1: t}\right)}{p\left(I_{1: t-1}, Y_{1: t-1}\right)} \propto \prod_{t=2}^{n} p\left(y_{t} \mid I_{1: t-1}, Y_{1: t-1}\right)
$$

yields the following recursive formula for the importance weights $w_{t}$.

$$
w_{t}=w_{t-1}\left\{a_{t}(\rho)+b_{t}(\rho)\right\}, t \geq 2 ; w_{1}=1 .
$$

When $\rho$ is small, change-points occur very infrequently and many sequences $I_{1: n}^{(j)}$ sampled from $Q$ may contain no change-points. We can modify $Q$ by increasing $\rho$ in $(10)$ to $\tilde{\rho}$, thereby picking up more change-points, and adjust the importance weights accordingly. 
Specifically, take $\tilde{\rho}>\rho$ and choose the proposal distribution $\tilde{Q}$ for which $I_{t} \mid I_{1: t-1}$ is a Bernoulli random variable with success probability $a_{t}(\tilde{\rho}) /\left(a_{t}(\tilde{\rho})+b_{t}(\tilde{\rho})\right)$. Since

$$
p\left(I_{1: n} \mid Y_{1: n}\right) / \tilde{q}\left(I_{1: n}\right)=\left\{p\left(I_{1: n} \mid Y_{1: n}\right) / q\left(I_{1: n}\right)\right\}\left\{q\left(I_{1: n}\right) / \tilde{q}\left(I_{1: n}\right)\right\},
$$

the importance weights $\tilde{w}_{t}$ can be determined recursively by

$$
\tilde{w}_{t}=\left\{\begin{array}{cc}
\tilde{w}_{t-1}\left\{a_{t}(\tilde{\rho})+b_{t}(\tilde{\rho})\right\} a_{t}(\rho) / a_{t}(\tilde{\rho}) & \text { if } I_{t}=1 \\
\tilde{w}_{t-1}\left\{a_{t}(\tilde{\rho})+b_{t}(\tilde{\rho})\right\} b_{t}(\rho) / b_{t}(\tilde{\rho}) & \text { if } I_{t}=0
\end{array}\right.
$$

with $\tilde{w}_{1}=(\rho / \tilde{\rho}) 1_{\left\{I_{1}=1\right\}}+1_{\left\{I_{1}=0\right\}}(1-\rho) /(1-\tilde{\rho})$, assuming $\mu_{0} \sim N\left(0, \sigma^{2}\right)$.

\subsection{A Numerical Example}

Table 1 studies how the $\mathrm{cv}^{2}$ bound for resampling affects performance, using the sum of squared error criterion $\operatorname{SSE}=E\left\{\sum_{i=1}^{n}\left(\hat{\mu}_{i}-\mu_{i}\right)^{2}\right\}$ to evaluate the performance of a procedure. For $\rho=0.001,100$ sequences of observations, each of length $n=10000$, were generated. We applied SISR $(M=50)$ with $\tilde{\rho}=\rho$ to each sequence, using different $\mathrm{cv}^{2}$ bounds. As pointed out in the paragraph preceding Section 3, resampling is performed at time $t$ with the entire vector $I_{1: t}$ (instead of $I_{t}$ ) so that we can keep track of the most recent change-point. Table 1 displays the average number of resamplings (Resampling \#) used for each $\mathrm{cv}^{2}$ bound, together with the SSE and its standard error (s.e.) based on 100 simulation runs. It shows the best value of SSE around 188 when we choose 1 as the $\mathrm{cv}^{2}$ bound, involving an average of 51 resamplings.

\begin{tabular}{cccccccccc}
\hline $\mathrm{cv}^{2}$ bound & 0 & 0.5 & 1 & 2 & 4 & 10 & 20 & 30 & $\infty$ \\
Resampling \# & 9999 & 85 & 51 & 32 & 22 & 14 & 10 & 8 & 0 \\
SSE & 240.38 & 205.24 & 188.06 & 193.24 & 196.19 & 206.01 & 217.51 & 208.75 & 603.94 \\
s.e. & 9.65 & 6.53 & 6.73 & 6.31 & 6.53 & 6.69 & 8.27 & 7.28 & 28.11 \\
\hline
\end{tabular}

Table 1. Effect of $\mathrm{cv}^{2}$ bound on performance of SISR for mean shift model

We have also computed the SSE of the SISR filter based on $\mu_{n}$ and have found over $50 \%$ reduction in SSE by working with $I_{n}$ instead of $\mu_{n}$. In addition, we have studied how SISR performs when different $\tilde{\rho}^{\prime}$ 's are used in the sampling distribution by simulating data from the same setting as Table 1, but with the $\mathrm{cv}^{2}$ bound fixed at 1 . Our results in Table 2 show that for $\rho(=0.001)<\tilde{\rho}<100 \rho$, the SSE is always smaller than that of $\tilde{\rho}=\rho$, with the smallest SSE at $\tilde{\rho}=5 \rho$, which shows the benefits of tilting.

\begin{tabular}{ccccccccccc}
\hline $\bar{\rho}$ & $\rho$ & $2 \rho$ & $4 \rho$ & $5 \rho$ & $6 \rho$ & $7 \rho$ & $10 \rho$ & $15 \rho$ & $50 \rho$ & $100 \rho$ \\
$\mathrm{SSE}$ & 188.06 & 169.12 & 159.58 & 150.82 & 152.07 & 151.50 & 158.80 & 159.84 & 169.86 & 185.42 \\
s.e. & 6.73 & 5.39 & 5.30 & 4.63 & 4.37 & 4.61 & 4.60 & 5.07 & 5.15 & 5.00 \\
\hline
\end{tabular}

Table 2. Effect of $\tilde{\rho}$ on performance of SISR for mean shift model with $\rho=0.001$

\subsection{Change-Point ARX Models}

Letting $\theta_{t}=\left(a_{1, t}, \ldots, a_{k, t}, b_{1, t}, \ldots, b_{h, t}\right)^{T}$, we can write the ARX model 


$$
y_{t}+a_{1, t} y_{t-1}+\ldots+a_{k, t} y_{t-k}=b_{1, t} u_{t-1}+\ldots+b_{h, t} u_{t-h}+\epsilon_{t}
$$

in the regression form $y_{t}=\theta_{t}^{T} \varphi_{t}+\epsilon_{t}, t \geq t_{0} \triangleq \max (k, h)+1$. Suppose that the changetimes of $\theta_{t}$ form a discrete renewal process with parameter $\rho$, or equivalently, that $I_{t} \triangleq 1_{\left\{\theta_{t} \neq \theta_{t-1}\right\}}$ are independent Bernoulli random variables with $P\left(I_{t}=1\right)=\rho$ for $t \geq t_{0}+1$, assuming $I_{t_{0}}=1$. At a change-point, $\theta_{t}$ takes a new value which is assumed to have the multivariate normal distribution with mean $\mu$ and covariance matrix $V$. Assume also that the $\epsilon_{t}$ are independent normal with mean 0 and variance $v_{\epsilon}$, which is chosen to be 1 in the following for simplicity.

Let $C_{t}$ be the most recent change-time up to time $t$. The conditional distribution of $\theta_{t}$ given $C_{t}, y_{t}$ and $\varphi_{s}, s \leq t$, is normal with mean $\mu_{C_{t}, t}$ and covariance matrix $V_{C_{t}, t}$, where for $t_{0}<s \leq t$,

$$
V_{s, t}=\left(V^{-1}+\sum_{j=s}^{t} \varphi_{j} \varphi_{j}^{T}\right)^{-1}, \mu_{s, t}=V_{s, t}\left(V^{-1} \mu+\sum_{j=s}^{t} y_{j} \varphi_{j}\right),
$$

which can be computed by standard recursions that follow from the matrix inversion lemma:

$$
\begin{aligned}
V_{t, t} & =V-V \varphi_{t} \varphi_{t}^{T} V /\left(1+\varphi_{t}^{T} V \varphi_{t}\right), \\
V_{s, t} & =V_{s, t-1}-V_{s, t-1} \varphi_{t} \varphi_{t}^{T} V_{s, t-1} /\left(1+\varphi_{t}^{T} V_{s, t-1} \varphi_{t}\right) \text { if } s<t, \\
\mu_{s, t} & =\mu_{s, t-1}+V_{s, t-1} \varphi_{t}\left(y_{t}-\varphi_{t}^{T} \mu_{s, t-1}\right) /\left(1+\varphi_{t}^{T} V_{s, t-1} \varphi_{t}\right) .
\end{aligned}
$$

Therefore, analogous to (9), the optimal filter is given by

$$
\begin{aligned}
E\left(\theta_{n} \mid y_{n}, \varphi_{t}, t \leq n\right) & =E\left\{E\left(\theta_{n} \mid C_{n}, y_{n}, \varphi_{t}, t \leq n\right) \mid y_{n}, \varphi_{t}, t \leq n\right\} \\
& =E\left\{\mu_{C_{n}, n} \mid y_{n}, \varphi_{t}, t \leq n\right\} .
\end{aligned}
$$

We can compute (15) by simulating $M$ trajectories $I_{1: n}^{(j)} \quad(j=1, \ldots, M)$ via sequential importance sampling with resampling. The proposal distribution $Q$ is similar to that in Section 3.1. Analogous to (10), the conditional distribution of $I_{t}$ given $I_{1: t-1}$ is Bernoulli assuming the values 1 and 0 with probabilities in the proportion

$$
\begin{aligned}
& \frac{\rho}{\left(1+\varphi_{t}^{T} V \varphi_{t}\right)^{1 / 2}} \phi\left(\frac{y_{t}}{\left(1+\varphi_{t}^{T} V \varphi_{t}\right)^{1 / 2}}\right) \\
: & \frac{1-\rho}{\left(1+\varphi_{t}^{T} V_{C_{t-1}, t-1} \varphi_{t}\right)^{1 / 2}} \phi\left(\frac{y_{t}-\mu_{C_{t-1}, t-1}^{T} \varphi_{t}}{\left(1+\varphi_{t}^{T} V_{C_{t-1}, t-1} \varphi_{t}\right)^{1 / 2}}\right) .
\end{aligned}
$$

Letting $a_{t}(\rho)$ and $b_{t}(\rho)$ denote the two terms in (16), we can define the importance weights $w_{t}$ recursively by (11). Resampling is performed when the squared coefficient of variation 
of the importance weights $w_{t}^{(j)}$ exceeds some threshold, which we can choose as 1 that usually works quite well. When $\rho$ is small, we can modify $Q$ by increasing $\rho$ in (16) to $\tilde{\rho}$ and adjusting the importance weights accordingly.

Chen and Lai (2007, Section IIIA) have applied the above particle filter, with $M=50$ sequentially generated trajectories to an open-loop change-point ARX model with $k=2, h=$ $1, \rho=0.001, V=$ identity matrix. The actual autoregressive parameters are assumed to belong to the stability region $\left\{\left(a_{1, t}, a_{2, t}\right):\left|a_{1, t}\right|+\left|a_{2, t}\right|<1\right\}$, and the inputs are assumed to be independent standard normal random variables. They carry out simulation studies of the Bayes estimate (particle filter) $\hat{\theta}_{t}^{(B)}$ of $\theta_{t}$ given by (14)-(15) that ignores, for computational and analytic tractability, the stability constraint on the prior distribution $\theta_{t}$. These studies also compare $\hat{\theta}_{t}^{(B)}$ with the following two modifications $\hat{\theta}_{t}^{(W)}$ and $\hat{\theta}_{t}^{(F)}$ of the usual least squares estimate which have been commonly used to handle occasional jumps in $\theta_{t}$ (cf. Benveniste et al., 1987, pp. 140, 161):

a. Sliding window estimate $\hat{\theta}_{t}^{(W)}$ : The least squares estimate is applied only to data in the immediate past, i.e., to the data set $\left\{\left(y_{s}, \varphi_{s}\right): t-k<s \leq t\right\}$, where $k$ is the window size.

b. Forgetting factor estimate $\hat{\theta}_{t}^{(F)}$ : A weighted least squares estimate is used, with weight $p^{t-s}$ for $\left(y_{s}, \varphi_{s}\right)$, i.e., the estimate at time $t$ minimizes $\Sigma_{s=1}^{t} p^{t-s}\left(y_{s}-\theta^{T} \varphi_{s}\right)^{2}$; where $0<p<1$ is the "forgetting factor" to discount past observations.

They use the following two performance measures

$$
\operatorname{SSE}_{1}=E\left\{\sum_{t=n^{\prime}}^{n^{\prime \prime}}\left[\left(\hat{\theta}_{t}-\theta_{t}\right)^{T} \varphi_{t}\right]^{2}\right\}, \quad \operatorname{SSE}_{2}=E\left\{\sum_{t=n^{\prime}}^{n^{\prime \prime}}\left\|\hat{\theta}_{t}-\theta_{t}\right\|^{2}\right\}
$$

to compare these estimates. The second measure considers how well $\hat{\theta}_{t}$ estimates $\theta_{t}$, whereas the first measure evaluates how well $\hat{\theta}_{t}^{T} \varphi_{t}$ estimates the minimum variance predictor $\theta_{t}^{T} \varphi_{t}$ of $y_{t+1}$. The results reported in their Table 2, which chooses 1-500, 501-1000, 1001-2000 and 2001-3000 as the ranges from $n^{\prime}$ to $n^{\prime \prime}$, show substantial improvements of $\hat{\theta}_{t}^{(B)}$ over $\hat{\theta}_{t}^{(W)}$ and $\hat{\theta}_{t}^{(F)}$, especially for $n^{\prime}$ exceeding 1000 .

\subsection{Application of Fast Particle Filters to Adaptive Control}

Section IIIB of Chen and Lai (2007) considers the control problem of choosing the inputs $u_{t}$ in the ARX model (13) so that the outputs $y_{t+1}$ are as close as possible (in $L^{2}$ ) to $y_{t+1}^{*}$, some reference signal such that $\left\{y_{n}^{*}\right\}$ and $\left\{\theta_{n}, \epsilon_{n}\right\}$ are independent. In the case of known $\theta_{t+1}$, the optimal input is defined by $\theta_{t+1}^{T} \varphi_{t+1}=y_{t+1}^{*}$, assuming that $b_{1, t+1} \neq 0$. When $\theta_{t+1}$ is unknown, the certainty equivalence rule replaces $\theta_{t+1}$ in the optimal input by an estimate $\hat{\theta}_{t+1 \mid t}$ based on the observations up to time $t$ so that $u_{t}$ is given by $\hat{\theta}_{t+1 \mid t}^{T} \varphi_{t+1}=y_{t+1}^{*}$ if $\hat{b}_{1, t+1 \mid t} \neq 0$. Letting 


$$
\begin{aligned}
\gamma_{t+1} & =\left(a_{1, t+1}, \ldots, a_{k, t+1}, b_{2, t+1}, \ldots, b_{h, t+1}\right)^{T}, \\
\hat{\gamma}_{t+1 \mid t} & =\left(\hat{a}_{1, t+1 \mid t}, \ldots, \hat{a}_{k, t+1 \mid t}, \hat{b}_{2, t+1 \mid t}, \ldots, \hat{b}_{h, t+1 \mid t}\right)^{T}, \\
\psi_{t} & =\left(-y_{t}, \ldots,-y_{t-k+1}, u_{t-1}, \ldots, u_{t-h+1}\right)^{T},
\end{aligned}
$$

they modify the certainty equivalence rule by

$$
u_{t}=\left\{\begin{array}{lll}
\left(y_{t+1}^{*}-\hat{\gamma}_{t+1 \mid t}^{T} \psi_{t}\right) / \hat{b}_{1, t+1 \mid t} & \text { if } & \left|\hat{b}_{1, t+1 \mid t}\right| \geq \delta_{t}, \\
y_{t+1}^{*}+\omega_{t} & \text { if } & \left|\hat{b}_{1, t+1 \mid t}\right|<\delta_{t}
\end{array}\right.
$$

where $\delta_{t}$ is some small prespecified number and $\omega_{t}$ is extraneous noise used to enhance the information content of the reference signal (including the case $\omega_{t}=0$ if the reference signal is already persistently exciting); see Caines \& Lafortune (1984).

Chen and Lai (2007) also consider an alternative approximation to the optimal control in the case of unknown $\theta_{t+1}$ by using the one-step ahead error $y_{t+1}-y_{t+1}^{*}$ without making use of dynamic programming to determine how the current control $u_{t}$ impacts on the information content of the estimates $\hat{\theta}_{s+1 \mid s}$ for the future errors $y_{s+1}-y_{s+1}^{*}(s>t)$. Noting that

$$
\begin{aligned}
& \arg \min _{u} E\left\{\left(b_{1, t+1} u+\gamma_{t+1}^{T} \psi_{t}-y_{t+1}^{*}\right)^{2} \mid y_{t}, y_{t-1}, u_{t-1}, \ldots, y_{1}, u_{1}\right\} \\
= & \left\{y_{t+1}^{*} E_{t}\left(b_{1, t+1}\right)-E_{t}\left(b_{1, t+1} \gamma_{t+1}^{T}\right) \psi_{t}\right\} / E_{t}\left(b_{1, t+1}^{2}\right),
\end{aligned}
$$

where $E_{t}$ denotes conditional expectation given $\left\{y_{t}, y_{t-1}, u_{t-1}, \ldots, y_{1}, u_{1}\right\}$, they define the following variant of (18) that incorporates uncertainty adjustments due to unknown parameters into the optimal rule $\theta_{t}^{T} \varphi_{t+1}=y_{t+1}^{*}$ assuming known $\theta_{t}$ :

$$
u_{t}= \begin{cases}\left\{y_{t+1}^{*} E_{t}\left(b_{1, t+1}\right)-E_{t}\left(b_{1, t+1} \gamma_{t+1}^{T}\right) \psi_{t}\right\} / E_{t}\left(b_{1, t+1}^{2}\right) & \text { if } \sqrt{E_{t}\left(b_{1, t+1}^{2}\right)} \geq \delta_{t}, \\ y_{t+1}^{*}+\omega_{t} & \text { if } \sqrt{E_{t}\left(b_{1, t+1}^{2}\right)}<\delta_{t} .\end{cases}
$$

To implement this adaptive control rule, one needs to compute the one-step ahead predictors $E_{t}\left(b_{1, t+1}\right), E_{t}\left(b_{1, t+1}^{2}\right)$ and $E_{t}\left(b_{1, t+1} \gamma_{t+1}^{T}\right)$. Note that

$$
\begin{aligned}
& p\left(\theta_{t+1} \mid y_{t}, y_{t-1}, u_{t-1}, \ldots, y_{1}, u_{1}\right) \\
= & (1-\rho) p\left(\theta_{t} \mid y_{t}, y_{t-1}, u_{t-1}, \ldots, y_{1}, u_{1}\right)+\rho p\left(\theta_{t+1}\right) .
\end{aligned}
$$

The first term on the right hand side of (21) can be approximated by fast particle filters, whereas the second term corresponds to a change-point at time $t+1$. Note that replacing $\left(E_{t}\left(b_{1, t+1}\right), E_{t}\left(b_{1, t+1}^{2}\right), E\left(b_{1, t+1} \gamma_{t+1}^{T}\right)\right)$ by $\left(\hat{b}_{1, t+1 \mid t}, \hat{b}_{1, t+1 \mid t}^{2}, \hat{b}_{1, t+1 \mid t} \hat{\gamma}_{t+1 \mid t}^{T}\right)$ in (20) reduces it to the certainty equivalence rule (18), which simply uses the estimates $\left(\hat{b}_{1, t+1 \mid t}, \hat{\gamma}_{t+1 \mid t}^{T}\right)$ to substitute for $\left(b_{1, t+1}, \gamma_{t+1}^{T}\right)$ in the optimal control assuming known $\theta_{t+1}$. The rule (20) introduces uncertainty adjustments for the unknown $\theta_{t+1}$ by considering the expected onestep ahead control error $E_{t}\left(y_{t+1}-y_{t+1}^{*}\right)^{2}$ that leads to (19), and by introducing extraneous 
white noise to enhance the information content of the control for future estimates of $\theta_{s}$ whenever (19) has a small denominator that may lead to a large (and numerically unstable) control action. The choice of $\delta_{t}$ depends on whether $\theta_{t}$ is known to belong to some stability region. If the $\theta_{t}$ are restricted to a stability region, then white noise perturbations do not destabilize the system while improving the experimental design for future estimates of $\theta_{s}$. On the other hand, without such a priori stability assumption on the $\theta_{t}$, small $\delta_{t}$ should be used in (20) because the perturbations can have an explosive effect.

Table III of Chen and Lai reports the results of a simulation study for an ARX model (13) in which $k=2, h=1$ and the inputs $u_{t}$ are determined by the certainty equivalence rule (18) or the uncertainty-adjusted certainty equivalence rule (20), in which $\delta_{t}=1 / 4$ and the $\omega_{t}$ are independent and identically distributed normal random variables. The $\left(b_{1, t}, b_{2, t}\right)$ are assumed to belong to a stability region. The table shows that the certainty equivalence rules based on $\hat{\theta}_{t}^{(W)}$ or $\hat{\theta}_{t}^{(F)}$ perform much worse than those based on $\hat{\theta}_{t}^{(B)}$ implemented by fast particle filters, and that the modification (20) of the certainty equivalence (18) based on $\hat{\theta}_{t}^{(B)}$ outperforms (18).

Chen and Lai (2007, Table IV) also consider the case where the $\left(b_{1, t}, b_{2, t}\right)$ do not belong to a stability region. They show that by weakening the extraneous perturbations (specifically choosing $\delta_{t}=\operatorname{var}\left(\omega_{t}\right)=0.04$, instead of $1 / 4$ for which the system with inputs (20) becomes unstable), the adaptive rule (20) can stabilize the system and still performs well.

\subsection{Extensions to Hammerstein and Nonlinear ARX Systems}

The particle filter described in (14) - (16) can be applied to estimate the piecewise constant $\theta_{t}$ in the general stochastic regression model

$$
y_{t}=\theta_{t}^{T} \varphi_{t}+\epsilon_{t}
$$

in which $\varphi_{t}$ is a vector-valued function of past lagged outputs and inputs and the changepoints of $\theta_{t}$ form a discrete renewal process with parameter $\nu$, with $\theta_{t}$ taking a new value from the $N(\mu, V)$ distribution at each change-point. The ARX model (13) is only a special case of (22) with $\varphi_{t}$ given by (1). Another important special case is the Hammerstein system that has a static nonlinearity on the input side, replacing $u_{t}$ in (13) (and therefore (1) accordingly) by some nonlinear transformation $f\left(u_{t}\right)$. When $f$ is unknown, it is usually approximated by a polynomial $f(u)=c_{1} u+\cdots+c_{q} u^{l}$ (Ljung, 1987). To identify the Hammerstein system, we express it in the form of (22) with

$$
\varphi_{t}=\left(-y_{t-1}, \ldots,-y_{t-k} ; u_{t-1}, \ldots, u_{t-h}, \ldots, u_{t-1}^{l}, \ldots, u_{t-h}^{l}\right)^{T} .
$$

Instead of using a polynomial to approximate $f$, we can use other basis functions (e.g., splines), yielding the representation $f(u)=\sum_{i=1}^{l} c_{i} \psi_{i}(u)$. Moreover, we can allow nonlinear interactions among the lagged outputs by making use of basis function approximations, and thereby express nonlinear ARX models with occasionally changing parameters in the form of (22) with

$$
\begin{aligned}
\varphi_{t}= & \left(\tilde{\psi}_{1}\left(y_{t-1}, \ldots, y_{t-k}\right), \ldots, \tilde{\psi}_{m}\left(y_{t-1}, \ldots, y_{t-k}\right)\right. \\
& \left.\psi_{1}\left(u_{t-1}\right), \ldots, \psi_{l}\left(u_{t-1}\right), \ldots, \psi_{1}\left(u_{t-h}\right), \ldots, \psi_{l}\left(u_{t-h}\right)\right)^{T} .
\end{aligned}
$$




\section{Particle Filters in Robotic Control and Planning}

The monograph by Thrun et al. (2005) gives a comprehensive treatment of the emerging field of probabilistic robotics. Here we summarize several basic concepts that are related to particle filters, referring to the monograph and other papers for details, and describe some ongoing work in this area.

\subsection{Robot Motion Models}

As in Thrun et al. (2005), we restrict to mobile robots in planar environments for which the pose $x_{t}$ of a robot at time $t$ is represented by $\left(x_{t 1}, x_{t 2}, \theta_{t}\right)^{T}$, where $\left(x_{t 1}, x_{t 2}\right)^{T}$ represents the robot's position in the plane and $\theta_{t}$ its angular orientation. If we drop the restriction of planar motion, then $x_{t}$ is a 6-dimensional vector in which the first three components are the Cartesian coordinates and $\theta_{t}$ consists of the three Euler angles relative to the coordinate frame. The velocity motion model of a probabilistic robot is specified by the conditional density $p\left(x_{t} \mid u_{t-1}, x_{t-1}\right)$, in which $u_{s}$ is a motion command that depends on all observations up to stage $s$ and controls the robot through a translational velocity $v_{s}$ and a rotational velocity $w_{s}$, i.e., $u_{s}=\left(v_{s}, w_{s}\right)$; see Thrun et al. (2005, pp. 127-132) for concrete examples. An alternative to the use of the robot's velocities to evaluate its motion over time is to use its odometry measurements for $u_{t}$ in $p\left(x_{t} \mid u_{t-1}, x_{t-1}\right)$, leading to the odometry motion model; see Thrun et al. (2005, pp. 133-139).

The preceding description of robot motion does not incorporate the nature of the environment. In practice, there is also a map $m$, which contains information pertaining to the places that the robot can navigate; for example, the robot's pose can only be in "free" space, which is the complement of space already occupied. A map-based motion model is specified by $p\left(x_{t} \mid u_{t-1}, x_{t-1}, m\right)$. A simple way to build such models is to combine $p\left(x_{t} \mid u_{t-1}, x_{t-1}\right)$ and $p\left(x_{t} \mid m\right)$ by

$$
p\left(x_{t} \mid u_{t-1}, x_{t-1}, m\right) \propto p\left(x_{t} \mid u_{t-1}, x_{t-1}\right) p\left(x_{t} \mid m\right) / p\left(x_{t}\right) ;
$$

see Thrun et al. (2005, pp. 140-143). Typical maps can be classified as feature-based or location-based. A feature-based map is a list of objects, called landmarks, in the environment along with the features. A prototypical location-based map is the occupancy grid map which assigns to any location a binary label that specifies whether the location is occupied by an object.

\subsection{Environment Measurement Models}

Mobile robots use their sensors to perceive their environment. Range finders, which are among the most popular sensors in robotics, measure the range to nearby objects along a beam (laser range finders) or within a cone (ultrasonic sensors). The sensor measurements $y_{t}$ are typically vectors since many sensors generate more than one numerical measurement; e.g., range finders usually give entire scans of ranges. Sections 6.3 and 6.4 of Thrun et al. (2005) describe the beam model and an alternative model, called likelihood field, to model $p\left(y_{t} \mid x_{t}, m\right)$ for range finders. Instead of using raw sensor measurements, an alternative approach is to extract features from the measurements and it is particularly suited to feature-based maps; see Section 6.6 of Thrun et al. (2005). 


\subsection{Pose Estimation, Mapping and SLAM}

The problem of estimating the pose of a robot relative to a given map of the environment is often called localization. It is a fundamental problem in robotics since nearly all tasks of a robot require knowledge of its location in the environment. In view of the hidden Markov model defined by $p\left(x_{t} \mid u_{t-1}, x_{t-1}, m\right)$ and $p\left(y_{t} \mid x_{t}, m\right)$ in Sections 4.1 and 4.2, estimation of the pose $x_{t}$ from the measurements $y_{1}, \ldots, y_{t}$ for a given map representation is a filtering problem. Extended Kalman filters are often used because of their simplicity and fast updating capability; see Thrun et al. (2005, Section 7.4) for details. The most popular localization filters to date are particle filters, and Section 8.3 of Thrun et al. (2005) describes these filters and their computational issues.

The preceding paragraph assumes that the robot has a map that is given in advance. Acquiring such an a priori map is often a difficult problem in practice. Mapping, which is the task of a robot to learn the map from scratch, not only circumvents this difficulty but also enables the robot to adapt to changes in the environment. To see how the robot can learn a map, first consider occupancy grid mapping in the case where the poses are known exactly. An occupancy grid map $m$ partitions the space into finitely many grid cells $m_{1}, \ldots$, $m_{I}$, where $m_{i}=1$ (if occupied) or 0 (if free) for the $i$ th cell. Putting a prior distribution on $\left(m_{1}, \ldots, m_{I}\right)$, Chapter 9 of Thrun et al. (2005) considers the posterior density $p\left(m \mid y_{1: t}, x_{1: t}\right)$ since the poses are assumed known, and describes how the MAP (maximum a posteriori) estimate of $\mathrm{m}$ can be evaluated.

The ideas in the preceding two paragraphs can be combined and modified to address the actual problem of simultaneous localization and mappling (SLAM), which involves the posterior density $p\left(x_{t}, m \mid y_{1: t}, u_{1: t-1}\right)$. A convenient way to learn the map is to use a feature-based approach involving landmarks. Typically, the robot has some uncertainty in identifying landmarks, especially those it has not observed previously. To incorporate this uncertainty in the data association decision, a correspondence variable $c_{t}^{j}$ can be introduced to give the true identity of the $j$ th observed feature (i.e., $c_{t}^{j}=i$ if the $j$ th feature corresponds to the ith landmark). In this case, SLAM involves the posterior density $p\left(x_{t}, m, c_{t} \mid y_{1: t}, u_{1: t-1}\right)$. This is sometimes called the "on-line SLAM posterior" to be distinguished from the "full SLAM posterior" $p\left(x_{1: t}, m, c_{1: t} \mid y_{1: t}, u_{1: t-1}\right)$. Chapter 10 of Thrun et al. (2005) uses extended Kalman filters to approximate the on-line SLAM posteriors, while Chapter 11 describes an alternative linearization technique that builds a sparse graph of soft constraints to approximate the full SLAM posteriors. Chapter 12 modifies the off-line full SLAM approximation of Chapter 11 into an on-line approximation to $p\left(x_{t}, m, c_{t} \mid y_{1: t}, u_{1: t-1}\right)$.

\subsection{The FastSLAM Algorithm}

FastSLAM uses particle filters to estimate the robot path and extended Kalman filters to estimate the map features. A key insight of FastSLAM is the factorization

$$
p\left(x_{1: t}, m \mid y_{1: t}, u_{1: t-1}, c_{1: t}\right)=p\left(x_{1: t} \mid y_{1: t}, u_{1: t-1}, c_{1: t}\right) \prod_{i=1}^{I} p\left(m_{i} \mid x_{1: t}, u_{1: t-1}, c_{1: t}\right),
$$

where $m$ consists of $I$ features $m_{1}, \ldots, m_{I}$ whose mapping errors are conditionally independent; see Section 13.2.1 of Thrun et al. (2005) for the derivation. As noted on p. 
438 of Thrun et al. (2005), an important advantage of FastSLAM "stems from the fact that data association decisions can be made on a per-particle basis," and consequently, "the filter maintains posteriors over multiple data associations, not just the most likely one." Moreover, "FastSLAM is formulated to calculate the full path posterior - only the full path renders feature locations conditionally independent." While it solves the full SLAM problem, it is also an on-line algorithm "because particle filters estimate one pose at-atime." Details of the development and implementation of FastSLAM are given in Sections 13.3-13.10 of Thrun et al. (2005). An important idea underlying FastSLAM is to use RaoBlackwellized particle filters for certain state variables and Gaussian posteriors to represent all other state variables. Recent papers by Grisetti et al. $(2005,2007)$ use adaptive proposal distributions and selective resampling to improve the RaoBlackwellized particle filters for learning grid maps, and provide a compact map model in which individual particles can share large parts of the model for the environment.

\subsection{Path Planning for Robot Movement}

Given an environment, the path planning problem for a robot is to choose the best path to reach a target location, starting from its initial pose. The traditional approach to robot motion planning is deterministic in nature, assuming that there is no uncertainty in the robot's pose over time and focusing on the complexities of the state space in the optimization problem. Chapter 14 of Thrun et al. (2005) incorporates uncertainty in the controls on the robot's motion by using methods from Markov decision processes (MDP) to solve the stochastic optimization problem, assuming that the robot's poses are fully observable or well approximated with negligible error. In MDP, $x_{t+1}$ does not evolve deterministically from $x_{t}$ and $u_{t}$, but is governed by a transition probability density function $p\left(x_{t+1} \mid x_{t}, u_{t}\right)$ with respect to some measure $\mu$. A Markov policy uses control $u_{t}$ that is a function of $x_{t}$ at every stage $t$. More generally, a policy can choose $u_{t}$ based on $\left(x_{1: t}, u_{1: t-1}\right)$. However, because of Markovian transitions, it suffices to restrict to Markov policies in maximizing the total discounted reward

$$
E\left\{\sum_{i=0}^{T} \alpha^{i} R\left(x_{t+i}, u_{t+i}\right) \mid x_{t}\right\}
$$

over all policies, where $0<\alpha \leq 1$ is the discount factor, $R(\cdot)$ is the payoff function and $T$ is the planning horizon (which may be infinite).

For the case $T=1$, the myopic policy that chooses $u_{t}$ to maximize $R\left(x_{t}, u_{t}\right)+\alpha E\left\{R\left(x_{t+1}, u_{t+1}\right) \mid x_{t}, u_{t}\right\}$ is optimal. With longer planning horizons, one has to strike an optimal balance between the next-stage reward and the evolution of future rewards. The optimal policy can be determined by dynamic programming as follows. The value $V_{T}(x)$ of (24) for the optimal policy is called the value function, and it satisfies the Bellman equation

$$
V_{T}(x)=\max _{u}\left\{R(x, u)+\alpha \int V_{T-1}\left(x^{\prime}\right) p\left(x^{\prime} \mid x, u\right) d \mu\left(x^{\prime}\right)\right\} .
$$

The optimal policy chooses the control $u=u_{T}(x)$ that maximizes the right-hand side of (25). 
Unless the state space is finite or of dimension 1 or 2 , direct computation of (25) via discretization of the state space is prohibitively difficult. One approach is to use a lowdimensional state approximation that assumes the value function to be relatively constant in the complementary state variables; see Thrun et al. (2005, pp. 505-507) who also note that "in higher dimensional situations it is common to introduce learning algorithms to represent the value function." Instead of working directly with the value function, it is more convenient to use the functions $Q_{n}$ defined by backward induction via $Q_{T}(x)=\max _{u}$ $R(x, u)$ and

$$
Q_{n}(x)=\max _{u}\left\{R(x, u)+\alpha E\left[Q_{n+1}\left(x_{n+1}\right) \mid x_{n}=x, u_{n}=u\right]\right\} \text { for } T-1 \geq n \geq 1,
$$

noting that $V_{T}=Q_{1}$. Since conditional expectation is a regression function, one can approximate $Q_{n+1}$ in (26) by using ideas from nonparametric regression, which basically uses certain basis functions to approximate $Q_{n+1}$ and estimates the coefficients of the basis functions by the method of least squares from simulated samples drawn from the conditional distribution of $x_{n+1}$ given $x_{n}$ and $u_{n}=u$; see Bertsekas and Tsitsiklis (1996) and Tsitsiklis and Van Roy (2001) for details.

\subsection{Robotic Control via Approximate Dynamic Programming}

Whereas path planning is usually carried out off-line before the robot is in motion, robotic control is concerned with on-line control of the motion of the robot to maximize a total discounted reward. It has to address the uncertainties in both the robot's poses and the control effects, which are incorporated in $p\left(x_{t} \mid u_{t-1}, x_{t-1}, m\right)$ and $p\left(y_{t} \mid x_{t}, m\right)$ in Sections 4.1 and 4.2. Accordingly Thrun et al. (2005, Chapter 15) use methods from partially observable Markov decision processes (POMDP) to address the corresponding stochastic control problem of maximizing (24) over control policies that are functions of the posterior distribution $\pi_{n}$ of $x_{n}$ given $y_{1: n}$ and $u_{1: n-1}$, instead of functions of $x_{n}$ as in MDP because the $x_{n}$ cannot be fully observed. Calling these posterior distributions beliefs, Thrun et al. (2005, p. 514) extend the Bellman equation (25) formally to

$$
V_{T}(\pi)=\max _{u}\left\{R(\pi, u)+\alpha \int V_{T-1}\left(\pi^{\prime}\right) p\left(\pi^{\prime} \mid \pi, u\right) d \mu(\pi)\right\},
$$

where $\mu$ is a measure on the space $\mathcal{B}$ of beliefs and $p\left(\pi^{\prime} \mid \pi, u\right)$ is the one-step transition probability density function of the belief $\pi$ to $\pi^{\prime}$ when control $u$ is taken. The optimal control chooses the maximizer $u$ in (27) when one's current belief is $\pi$. This is tantamount to working with the Markov chain $\left(x_{t}, \pi_{t}\right)$ on the state space $\mathcal{X} \times \mathcal{B}$, where $\mathcal{X}$ is the state space of the poses $x_{t}$. Since $\mathcal{B}$ is a set of probability measures, $\mu$ is a measure on the space of probability measures and the existence of the transition density function in (27) is "problematic". Moreover, "given the complex nature of the belief, it is not at all obvious that the integration can be carried out exactly, or that effective approximation can be found" (Thrun et al., 2005, p. 514).

Because of the inherent complexity of POMDP problems, the literature has focused almost exclusively on the infinite-horizon case $(T=\infty)$ so that the value function in the Bellman equation (27) does not depend on $T$ and is a function of the posterior distribution (belief) only. Thrun et al. (2005, Sections 15.3 and 15.4) consider the case where the state space $\mathcal{X}$, 
the observation space and the control set are all finite. If $\mathcal{X}$ has $k$ elements, then any probability distribution $\pi$ on $\mathcal{X}$ can be represented by $\left(p_{1}, \ldots, p_{k}\right)$ such that $0 \leq p_{i} \leq 1$ and $\sum_{i=1}^{k} p_{i}=1$. In this case, the value function (27) can be expressed as a convex, piecewise linear function of $\left(p_{1}, \ldots, p_{k}\right)$.

For more general state spaces $\mathcal{X}$, Thrun (2000) has proposed a Monte Carlo procedure, called MC-POMDP, involving particle filters to perform approximate value iteration in $\mathcal{B}$. MC-POMDP uses a finite particle set to approximate a probability distribution $\pi$. Specifically, this iterative procedure updates the value function $V$ at $\pi$ by simulating for each applicable control $u$ a sample of possible subsequent beliefs $\pi^{\prime}$ and then averaging over the simulated sample:

$$
Q(\pi, u)=R(\pi, u)+\alpha E\left\{V\left(\pi^{\prime}\right) \mid \pi, u\right\},
$$

so that $V$ is updated by $V(\pi)=\max _{u} Q(\pi, u)$ in the iterative procedure. The basic idea is taken from model-based reinforcement learning (Gordon, 1995; Kaebling et al., 1996; Sutton \& Barto, 1998), in which function approximations such as neural networks, decision trees and spline basis functions are used to represent the value function $V$ in MDPs. To extend the idea to POMDPs, the challenge lies in how to represent $V$ since it is a function of a probability distribution on the state space, instead of the state itself. Thrun (2000) uses a "nearest neighbor" approximation to represent $V\left(\pi^{\prime}\right)$ in (28). His MCPOMDP algorithm keeps a set (database) of reference beliefs $\pi_{i}$, and associated values $V_{i}$. When a new belief state $\pi^{\prime}$ (not in the database) is generated, its $V$ value is obtained by finding the $k$ nearest neighbors in the database and taking a weighted average of the corresponding $V_{i}$ values. To measure the distance of $\pi_{i}$ from $\pi^{\prime}$, he convolves each particle with a normal $N(0, v)$ distribution having a small variance $v$ so that $\pi_{i}$ and $\pi^{\prime}$ can be represented by Gaussian mixtures, and then uses the Kullback-Leibler divergence to measure the distance (divergence) $d_{i}$ of $\pi_{i}$ from $\pi^{\prime}$. Denoting the $k$ nearest neighbors of $\pi^{\prime}$ by $\pi_{1}, \ldots, \pi_{k}, V\left(\pi^{\prime}\right)$ in (28) is approximated by $\left(\sum_{i=1}^{k} d_{i}^{-1} V_{i}\right) /\left(\sum_{i=1}^{k} d_{i}^{-1}\right)$; see Section 16.4 of Thrun et al. (2005) for further details and refinements of the MC-POMDP algorithm. Because of the complexity of the actual value function, how well the above nearest neighbor method approximates $V$ is formidable to explore. Although the valuefunction approximation approach has been the "dominant approach" in reinforcement learning, Sutton et al. (2000) have pointed out various limitations of this approach and have proposed an alternative approach that uses function approximations for the optimal policy to carry out approximate policy iteration in MDPs. Instead of extending this approximate policy iteration approach to POMDPs whose optimal policies are prohibitively difficult to visualize, Lai and $\mathrm{Wu}$ (2008) propose to begin with a good (albeit sub-optimal) policy that captures the essence of the control objective and quantifies the uncertainties concerning the current state reflected by the particle filter, and to use it as the base policy in a rollout algorithm (Bayard, 1991; Bertsekas, 2000; Han et al., 2006) that successively improves the base policy into a new base policy, iterating till convergence to the optimal policy. 


\section{Concluding Remarks}

In this section we describe some issues that have not been addressed in the preceding sections and summarize the connections between the two control problems in Sections 3 and 4 .

\subsection{Adaptive Particle Filters}

In the previous sections, we have assumed that the hidden Markov model has specified parameters. However, in practice, the HMM usually has unknown parameters that need to be estimated besides the unobservable states. We consider here a Bayesian formulation in which the unknown parameter vector $\theta$ has a prior distribution, so that $\theta$ can be incorporated into the state vector at the expense of increasing the dimension. Such augmentation of the state vector does not pose additional difficulties if it can still be conveniently simulated. Here we show that it is sometimes even possible to integrate out the unknown parameter vector $\theta$, with respect to a posterior distribution, in the SISR filter. Whenever this is possible, integrating out $\theta$ can improve substantially the performance of the Monte Carlo method; this principle is called marginalization (Kong et al., 1994).

As an illustration, suppose that in the normal mean shift model, the probability $\rho$ of change is unknown and is specified by a prior $\operatorname{Beta}(\alpha, \beta)$ distribution with mean $\alpha /(\alpha+\beta)$, where $\alpha$ and $\beta$ are positive integers. It turns out that when $\rho$ in $(10)$ is unknown but has a Beta prior distribution, we can follow the same arguments to come up with an analogous proposal distribution $Q$ from which $I_{1}, \ldots, I_{t}$ are sampled sequentially. Using the closed-form expression for $P\left(I_{t}=\cdot \mid I_{1: t-1}, Y_{1: t}\right)$, it can be shown that under $Q$, $I_{t} \mid I_{1: t-1}$ is Bernoulli assuming the values 1 and 0 with probabilities in the proportion

$$
\begin{aligned}
& \frac{n_{t-1,1}+\alpha}{(t-1+\alpha+\beta)\left(1+\sigma^{2}\right)^{1 / 2}} \phi\left(\frac{Y_{t}}{\left(1+\sigma^{2}\right)^{1 / 2}}\right) \\
: & \frac{n_{t-1,0}+\beta}{(t-1+\alpha+\beta)\left(1+v_{t}\right)^{1 / 2}} \phi\left(\frac{Y_{t}-v_{t} \sum_{j=C_{t-1}}^{t-1} Y_{j}}{\left(1+v_{t}\right)^{1 / 2}}\right),
\end{aligned}
$$

where $v_{t}=\left(t-C_{t-1}+\sigma^{-2}\right)^{-1}, n_{t-1,1}$ is the number of 1 's in $\left\{I_{1}, I_{2}, \ldots, I_{t-1}\right\}$ and $n_{t-1,0}=t-1-n_{t-1,1}$ is the number of 0 's in $\left\{I_{1}, I_{2}, \ldots, I_{t-1}\right\}$. Note how closely (29) resembles (10). Accordingly, letting $a_{t}$ and $b_{t}$ denote the two terms in (29), the importance weights $w_{t}$ are given recursively by $w_{t}=w_{t-1}\left(a_{t}+b_{t}\right), t \geq 1$, with $w_{0}=1$.

Parallel to Table 1 that studies the performance of the SISR filter (with $M=50$ and various $\mathrm{cv}^{2}$ bounds) when $\rho=0.001$ is known, Table 3 considers the performance of the adaptive filter when $\rho$ is assumed to have the $\operatorname{Beta}(1,999)$ distribution (with mean 0.001). Performance is measured by the $\operatorname{SSE}(\nu)=E_{\rho}\left\{\sum_{i=1}^{n}\left(\hat{\mu}_{i}-\mu_{i}\right)^{2}\right\}$ at $\rho=0.001$, and also by the Bayes SSE, which is the expected value of $\operatorname{SSE}(\nu)$ when $\rho$ is regarded as a Beta $(1,999)$ random variable. Each result is based on 100 sequences of $n=10000$ observations. As in Table $1, \sigma$ is assumed to be 1 . Comparison of SSE $(\nu)$ with the SSE values in Table 1 shows that the performance of the adaptive filter is comparable to that of the optimal filter that assumes $\rho$ to be known. Table 3 shows a minimal value of $\operatorname{SSE}(\nu)$ and the best Bayes performance when the $\mathrm{cv}^{2}$ bound is 2 . 


\begin{tabular}{cccccccccc}
\hline CV $^{2}$ Bound & 0 & 0.5 & 1 & 2 & 4 & 10 & 20 & 30 & $\infty$ \\
Resampling \# & 9999 & 100 & 50 & 35 & 30 & 15 & 12 & 9 & 0 \\
SSE $(\rho)$ & 288.52 & 200.82 & 201.92 & 200.55 & 205.57 & 206.72 & 207.43 & 215.75 & 478.93 \\
Bayes SSE & 240.52 & 174.73 & 158.16 & 153.56 & 184.35 & 188.47 & 190.20 & 186.31 & 501.01 \\
Resampling \#B & 9999 & 80 & 50 & 30 & 20 & 15 & 10 & 7 & 0 \\
\hline
\end{tabular}

Table 3. Effect of $\mathrm{cv}^{2}$ bound on performance of SISR for mean shift model with unknown $\rho$ Note: $\operatorname{SSE}(\nu)=$ Expected sum of squared errors at $\rho=0.001$; Bayes SSE $=$ Expected sum of squared errors when $\rho \sim \operatorname{Beta}(1,999)$; Resampling \# = Average number of resamplings when $\rho=0.001$; Resampling \#B= Expected number of resamplings when $\rho \sim \operatorname{Beta}(1,999)$

\subsection{Control Law Heuristics, Approximate Policy Iteration and On-Line Implementation} In Section 3.4 we have addressed the adaptive control problem by using simple heuristics like certainty equivalence and uncertainty adjustment. The treatment of robotic control reviewed in Section 4.6 uses more general and formal tools like approximate value iteration, reinforcement learning and MC-POMDP. Although these tools are powerful, they may be difficult to implement, as noted in Section 4.6. A major difficulty is that since the form of the value function is hard to guess, it is not clear what basis functions should be chosen to approximate the value function in carrying out approximate value iteration. This difficulty is compounded for POMDPs because the value function is a function of a probability distribution on the state space, as we have explained in Section 4.6. An alternative approach is to use control law heuristics to come up with a good practical policy as in Section 3.4, and to study its performance by Monte Carlo simulations and refine it by using the rollout method as in Lai and $\mathrm{Wu}(2008)$. Another challenge is the realtime computational requirement for on-line control, under which one can only afford to perform few iterations, especially when there is a Monte Carlo inner loop involving particle filters. This is why fast particle filters, which use efficient proposal distributions and resampling schemes like those in Section 3, or which use conditional independence of feature locations to perform data association on a per-particle basis as in FastSLAM in Section 4.4, are of particular importance for applications of particle filters to control of HMMs.

\section{References}

Bayard, D. S. (1991). A forward method for optimal stochastic nonlinear and adaptive control. IEEE Trans. Automat. Contr., Vol 36, pp. 1046-1053.

Benveniste, A.; Metivier, M. \& Priouret, P. (1987). Adaptive Algorithms and Stochastic Approximations, New York: Springer-Verlag.

Bertsekas, D. P. (2000). Dynamic Programming and Optimal Control, Athena Scientific, Belmont, MA.

Bertsekas, D. P. \& Tsitsiklis, J. N. (1996). Neuro-Dynamic Programming. Belmont, MA: Athena Scientific.

Caines, P. E. \& Lafortune, S. (1984). Adaptive control with recursive identification for stochastic linear systems. IEEE Trans. Automat. Contr., Vol. 29, pp. 312-321.

Chan, H. P. \& Lai, T. L. (2008). A general theory of particle filters in hidden Markov models and some applications. Tech. Report, Department of Statistics, Stanford University. 
Chen, Y. \& Lai, T. L. (2007). Identification and adaptive control of change-point ARX models via Rao-Blackwellized particle filters. IEEE Trans. Automat. Contr., Vol 52, pp. 67-72.

Goodwin, G. C.; Ramadge, P. J. \& Caines, P. E. (1981). Discrete time stochastic adaptive control. SI AM J. Contr. Optimiz., Vol. 19, pp. 829-853.

Goodwin, G. C. \& Sin, K. S. (1984). Adaptive Filtering, Prediction and Control. Englewood Cliffs, NJ: Prentice Hall.

Gordon, G. J. (1995). Stable function approximation in dynamic programming. Proc. 12th Internal. Conf. Machine Learning, pp. 261-268, Morgan Kaufmann.

Gordon, N. J.; Salmond, D. J. \& Smith, A. F. M. (1993). A novel approach to non-linear and non-Gaussian Bayesian state estimation. IEEE Proc. Radar \& Signal Processing, Vol. 140, pp. 107-113.

Grisetti, G.; Stachniss, C. \& Burgard, W. (2005). Improving grid-based SLAM with RaoBlackwellized particle filters by adaptive proposals and selective resampling. Proceedings of the 2005 IEEE International Conference on Robotics and Automation, pp. 2432-2437, ISBN: 0-7803-8914-X.

Grisetti, G.; Tipaldi, G. D.; Stachniss, C.; Burgard, W. \& Nardi, D. (2007). Fast and accurate SLAM with Rao-Blackwellized particle filters. Robotics and Autonomous Systems archive, Vol. 55 , No. 1, (January 2007) pp. 30-38, ISSN:0921-8890.

Guo, L. \& Chen, H. F. (1991). The Astrom-Wittenmark self-tuning regulator revisited and ELS-based adaptive trackers. IEEE Trans. Automat. Contr., Vol. 36, pp. 802-812.

Guo, L. \& Ljung, L. (1993a). Exponential stability of general tracking algorithms. IEEE Trans. Automat. Contr., Vol. 40, pp. 1376-1387.

Guo, L. \& Ljung, L. (1993b). Performance analysis of general tracking algorithms. IEEE Trans. Automat. Contr., Vol. 40, pp. 1388-1402.

Han, J.; Lai, T. L. \& Spivakovsky, V. (2006). Approximate policy optimization and adaptive control in regression models. Comput. Econ., Vol. 27, 433-452.

Kaebling, L. P.; Littman, M. L. \& Moore, A., W. (1996). Reinforcement learning: A survey. J. Articial Intelligence Research, Vol. 4, pp. 237-285.

Kong, A.; Liu, J. S. \& Wong, W. H. (1994). Sequential imputations and Bayesian missing data problems. J. Amer. Statist. Assoc., Vol. 89, pp. 278-288.

Lai, T. L. \& Wei, C. Z. (1987). Asymptotically efficient self-tuning regulators. SIAM J. Contr. Optimiz, Vol. 25, pp. 466-481.

Lai, T. L. \& Wu, B. (2008). Approximate policy iteration in partially observable Markov decision processes with applications to robot control. Tech. Report, Department of Statistics, Stanford University.

Ljung, L. (1987). System Identification: Theory for the User, Englewood Cliffs, NJ: Prentice Hall.

Ljung, L. \& Gunnarsson, S. (1990). Adaptation and tracking in system identification - a survey. Automatica, Vol. 26, pp. 7-21.

Ljung, L. \& Soderstrom, T. (1983). Theory and Practice of Recursive Identification. MIT Press.

Meyn, S. P. \& Brown, L. J. (1993). Model reference adaptive control of time varying and stochastic systems. IEEE Trans. Automat. Contr., Vol. 38, pp. 1738-1753.

Sutton, R. S. \& Barto, A. G. (1998). Reinforcement Learning: An Introduction. MIT Press. 
Sutton, R. S.; McAllester, S. S. \& Mansour, Y. (2000). Policy gradient methods for reinforcement learning with function approximation. In: Adv. Neural Information Processing Systems, Vol. 12, pp. 1057-1063, MIT press.

Thrun, S. (2000). Monte Carlo POMDPs. In: Advances in Neural Information Processing Systems 12, S.A. Solla, T.K. Leen, and K.-R. Mller (Ed.), pp 1064-1070. MIT Press.

Thrun, S.; Burgard, W. \& Fox, D. (2005). Probabilistic Robotics. MIT Press, Cambridge, MA.

Tsitsiklis, J. N. \& Van Roy, B. (2001). Regression methods for pricing complex Americanstyle options. IEEE Trans. Neural Networks, Vol. 12, pp. 694-703.

Yao, Y. (1984). Estimation of a noisy discrete-time step function: Bayes and empirical Bayes approaches. Ann. Statist., Vol. 12, pp. 1434-1447. 


\title{
An Adaptive Controller Design for Flexible-joint Electrically-driven Robots With Consideration of Time-Varying Uncertainties
}

\author{
Ming-Chih Chien ${ }^{1}$ and An-Chyau Huang ${ }^{2}$ \\ ${ }^{1}$ Mechanical and Systems Research Laboratories, Industrial Technology Research Institute, \\ 2Dept. of Mechanical Engineering, National Taiwan University of Science and Technology \\ Taiwan, R.O.C.
}

\section{Abstract}

Almost all present control strategies for electrically-driven robots are under the rigid robot assumption. Few results can be found for the control of electrically driven robots with joint flexibility. This is because the presence of the joint flexibility greatly increases the complexity of the system dynamics. What is worse is when some system dynamics are not available and a good performance controller is required. In this paper, an adaptive design is proposed to this challenging problem. A backstepping-like procedure incorporating the model reference adaptive control is employed to circumvent the difficulty introduced by its cascade structure and various uncertainties. A Lyapunov-like analysis is used to justify the closed-loop stability and boundedness of internal signals. Moreover, the upper bounds of tracking errors in the transient state are also derived. Computer simulation results are presented to demonstrate the usefulness of the proposed scheme.

Keywords: Adaptive control; Flexible-joint electrically-driven robot; FAT

\section{Introduction}

Control of rigid robots has been well understood in recent years, but most of the schemes ignore the dynamics coming from electric motors and harmonic drivers that are widely implemented in the industrial robots. However, actuator dynamics constitute an important part of the complete robot dynamics, especially in the cases of high-velocity movement and highly varying loads[1],[2]. The main reason for using a reduced model is to simplify complexity of controller design. For each joint, consideration of the flexibility from the

\footnotetext{
${ }^{1}$ M. C. Chien was with the Department of Mechanical Engineering, National Taiwan University of Science and Technology. He is now with the Mechanical and Systems Research Laboratories, Industrial Technology Research Institute, No. 195, Sec. 4, Chung-Hsing Rd., Chutung, Hsinchu, 310, Taiwan, R.O.C. (e-mail: D9203401@mail.ntust.edu.tw).

2 A. C. Huang is with the Department of Mechanical Engineering, National Taiwan University of Science and Technology. No. 43, Keelung Rd., Sec. 4, Taipei, Taiwan, ROC. (Tel:+886-2-27376490, Fax: +886-2-37376460, E-mail: achuang@mail.ntust.edu.tw). (A. C. Huang provides phone number because he is the corresponding author.)
} 
harmonic driver results in an additional $2^{\text {nd }}$ order dynamics. If the motor dynamics is also included, a totally $5^{\text {th }}$ order dynamics should be considered. It is well-known that a multiDOF rigid robot is a highly nonlinear and coupled system. If we consider the motor dynamics and joint flexibility for all joints, the controller design problem would become extremely difficult. In this paper, we would like to design a controller for a flexible-joint electrically-driven robot under more challenging conditions, that is, the robot system contains various uncertainties.

Better motion control performance was obtained by Tarn et. al.[2] under experimental verification for a rigid robot when considering the motor dynamics. For the robust control of rigid robots with consideration of actuator dynamics can be found in [3]-[9]. Important developments for the adaptive control of electrically-driven rigid robots can be seen in [10][18].

The above mentioned schemes are all for the control of rigid robots. For the control of flexible-joint electrically driven robots, few results can be found. Some robust designs were presented in [19]-[22]. However, to our best knowledge, no work has been reported on adaptive control of flexible-joint robot manipulators incorporating motor dynamics. The main contribution of the present paper is to propose an adaptive controller for this system. The controller does not need to calculate the regressor[23] which is required in conventional robot adaptive control. The design follows a backstepping-like procedure with the support of the model reference adaptive control. The function approximation technique (FAT)[24][35] is employed to deal with the system uncertainties. A Lyapunov-like analysis is used to justify the closed-loop stability and boundedness of internal signals.

This paper is organized as follows. Section 2 derives the proposed adaptive motion controller in detail. Section 3 presents simulation results of the motion control of a 2-D robot using the proposed controller. Section 4 concludes the paper.

\section{Main Results}

The dynamics of a rigid-link flexible-joint electrically-driven (RLFJED) robot can be described by

$$
\begin{gathered}
\mathbf{D}(\mathbf{q}) \ddot{\mathbf{q}}+\mathbf{C}(\mathbf{q}, \dot{\mathbf{q}}) \dot{\mathbf{q}}+\mathbf{g}(\mathbf{q})=\mathbf{K}(\boldsymbol{\theta}-\mathbf{q}) \\
\mathbf{J} \boldsymbol{\theta}+\mathbf{B} \dot{\boldsymbol{\theta}}+\mathbf{K}(\boldsymbol{\theta}-\mathbf{q})=\mathbf{H i} \\
\mathbf{L i}+\mathbf{R}(\mathbf{i}, \dot{\boldsymbol{\theta}})=\mathbf{u}
\end{gathered}
$$

where $\mathbf{q} \in \mathfrak{R}^{n}$ is the vector of link angles, $\boldsymbol{\theta} \in \mathfrak{R}^{n}$ is the vector of actuator angles, $\mathbf{i} \in \mathfrak{R}^{n}$ is the motor armature currents, $\mathbf{u} \in \mathfrak{R}^{n}$ is the control input voltage, $\mathbf{D}(\mathbf{q})$ is the $n \times n$ inertia matrix, $\mathbf{C}(\mathbf{q}, \dot{\mathbf{q}}) \dot{\mathbf{q}}$ is an $n$-vector of centrifugal and Coriolis forces, and $\mathbf{g}(\mathbf{q})$ is the gravity vector. $\mathbf{J}, \mathbf{B}$ and $\mathbf{K}$ are $n \times n$ constant diagonal matrices of actuator inertia, damping and joint stiffness, respectively. $\mathbf{H} \in \mathfrak{R}^{n \times n}$ is an invertible constant diagonal matrix which characterizes the electro-mechanical conversion between current and torque, $\mathbf{L} \in \mathfrak{R}^{n \times n}$ is the constant diagonal matrix of electrical inductance, $\mathbf{R}(\mathbf{i}, \dot{\boldsymbol{\theta}}) \in \mathfrak{R}^{n}$ 
represents the effect of the electrical resistance and the motor back-emf. Here, we would like to consider the case when the precise forms of $\mathbf{D}(\mathbf{q}), \mathbf{C}(\mathbf{q}, \dot{\mathbf{q}}) \dot{\mathbf{q}}, \mathbf{g}(\mathbf{q}), \mathbf{L}$ and $\mathbf{R}(\mathbf{i}, \dot{\boldsymbol{\theta}})$ are not available and their variation bounds are not given. This implies that traditional adaptive control and robust control cannot be applicable. In the following, we would like to use the FAT to design an adaptive controller for the robot. Moreover, it is well-known that derivation of the regressor matrix for the adaptive control of high DOF rigid robot is generally tedious. For the RLFJED robot in (1), (2), and (3) its dynamics is much more complex than that of its rigid-joint counterpart. Therefore, the computation of the regressor matrix becomes extremely difficult. One of the contributions of the present paper is to propose an adaptive controller which does not need to calculate the regressor matrix needed in the conventional robot adaptive control.

Define $\boldsymbol{\tau}_{t}=\mathbf{K}(\boldsymbol{\theta}-\mathbf{q})[36,37]$ to be the vector of transmission torques, so (1) and (2) becomes

$$
\begin{gathered}
\mathbf{D}(\mathbf{q}) \ddot{\mathbf{q}}+\mathbf{C}(\mathbf{q}, \dot{\mathbf{q}}) \dot{\mathbf{q}}+\mathbf{g}(\mathbf{q})=\boldsymbol{\tau}_{t} \\
\mathbf{J}_{t} \ddot{\boldsymbol{\tau}}_{t}+\mathbf{B}_{t} \dot{\boldsymbol{\tau}}_{t}+\boldsymbol{\tau}_{t}=\mathbf{H i}-\overline{\mathbf{q}}(\dot{\mathbf{q}}, \ddot{\mathbf{q}})
\end{gathered}
$$

where $\mathbf{J}_{t}=\mathbf{J K}^{-1}, \quad \mathbf{B}_{t}=\mathbf{B K}^{-1}$ and $\overline{\mathbf{q}}(\dot{\mathbf{q}}, \ddot{\mathbf{q}})=\mathbf{J} \ddot{\mathbf{q}}+\mathbf{B} \dot{\mathbf{q}}$. Define signal vector $\mathbf{s}=\dot{\mathbf{e}}+\boldsymbol{\Lambda} \mathbf{e}$ and $\mathbf{v}=\dot{\mathbf{q}}_{d}-\boldsymbol{\Lambda} \mathbf{e}$, where $\mathbf{q}_{d} \in \mathfrak{R}^{n}$ is the vector of desired states, $\mathbf{e}=\mathbf{q}-\mathbf{q}_{d}$ is the state error, and $\boldsymbol{\Lambda}=\operatorname{diag}\left(\lambda_{1}, \lambda_{2}, \ldots, \lambda_{n}\right)$ with $\lambda_{i}>0$ for all $i=1, \ldots$ $n$. Rewrite (4) in the form

$$
\mathbf{D} \dot{\mathbf{s}}+\mathbf{C s}+\mathbf{g}+\mathbf{D} \dot{\mathbf{v}}+\mathbf{C v}=\boldsymbol{\tau}_{t}
$$

\section{A. Controller Design for Known Robot}

Suppose $\mathbf{D}(\mathbf{q}), \mathbf{C}(\mathbf{q}, \dot{\mathbf{q}}) \dot{\mathbf{q}}$ and $\mathbf{g}(\mathbf{q})$ are known, and we may design a proper control law such that $\boldsymbol{\tau}$ follows the trajectory below

$$
\boldsymbol{\tau}_{t}=\mathbf{g}+\mathbf{D} \dot{\mathbf{v}}+\mathbf{C v}-\mathbf{K}_{d} \mathbf{s}
$$

where $\mathbf{K}_{d}$ is a positive definite matrix. Substituting (7) into (6), the closed loop dynamics becomes $\mathbf{D} \dot{\mathbf{s}}+\mathbf{C s}+\mathbf{K}_{d} \mathbf{s}=\mathbf{0}$. Define a Lyapunov function candidate as $V=\frac{1}{2} \mathbf{s}^{T} \mathbf{D s}$. Its time derivative along the trajectory of the closed loop dynamics can be computed as $\dot{V}=-\mathbf{s}^{T} \mathbf{K}_{d} \mathbf{s}+\mathbf{s}^{T}(\dot{\mathbf{D}}-2 \mathbf{C}) \mathbf{s}$. Since $\dot{\mathbf{D}}-2 \mathbf{C}$ can be proved to be skew-symmetric, the above equation becomes $\dot{V}=-\mathbf{s}^{T} \mathbf{K}_{d} \mathbf{s} \leq 0$. It is easy to prove that $\mathbf{s}$ is uniformly bounded and square integrable, and $\dot{\mathbf{s}}$ is also uniformly bounded. Hence, $\mathbf{s} \rightarrow \mathbf{0}$ as $t \rightarrow \infty$, or we may say $\mathbf{e} \rightarrow \mathbf{0}$ as $t \rightarrow \infty$. To make the actual $\boldsymbol{\tau}$ converge to the perfect $\boldsymbol{\tau}$ in (7), let us consider the reference model 


$$
\mathbf{J}_{r} \ddot{\boldsymbol{\tau}}_{r}+\mathbf{B}_{r} \dot{\boldsymbol{\tau}}_{r}+\mathbf{K}_{r} \boldsymbol{\tau}_{r}=\mathbf{J}_{r} \ddot{\boldsymbol{\tau}}_{t d}+\mathbf{B}_{r} \dot{\boldsymbol{\tau}}_{t d}+\mathbf{K}_{r} \boldsymbol{\tau}_{t d}
$$

where $\boldsymbol{\tau}_{r} \in \mathfrak{N}^{n}$ is the state vector of the reference model and $\boldsymbol{\tau}_{t d} \in \mathfrak{R}^{n}$ is the vector of desired states. Matrices $\mathbf{J}_{r} \in \mathfrak{R}^{n \times n}, \mathbf{B}_{r} \in \mathfrak{R}^{n \times n}$ and $\mathbf{K}_{r} \in \mathfrak{R}^{n \times n}$ are selected such that $\boldsymbol{\tau}_{r} \rightarrow \boldsymbol{\tau}_{t d}$ exponentially. Define $\overline{\boldsymbol{\tau}}_{t d}\left(\dot{\boldsymbol{\tau}}_{t d}, \ddot{\boldsymbol{\tau}}_{t d}\right)=\mathbf{K}_{r}^{-1}\left(\mathbf{B}_{r} \dot{\boldsymbol{\tau}}_{t d}+\mathbf{J}_{r} \ddot{\boldsymbol{\tau}}_{t d}\right)$, we may rewrite (5) and (8) in the state space form as

$$
\begin{array}{r}
\dot{\mathbf{x}}_{p}=\mathbf{A}_{p} \mathbf{x}_{p}+\mathbf{B}_{p} \mathbf{H i}-\mathbf{B}_{p} \overline{\mathbf{q}} \\
\dot{\mathbf{x}}_{m}=\mathbf{A}_{m} \mathbf{x}_{m}+\mathbf{B}_{m}\left(\boldsymbol{\tau}_{t d}+\overline{\boldsymbol{\tau}}_{t d}\right)
\end{array}
$$

where $\mathbf{x}_{p}=\left[\begin{array}{ll}\boldsymbol{\tau}_{t} & \dot{\boldsymbol{\tau}}_{t}\end{array}\right]^{T} \in \mathfrak{R}^{2 n} \quad$ and $\quad \mathbf{x}_{m}=\left[\begin{array}{ll}\boldsymbol{\tau}_{r} & \dot{\boldsymbol{\tau}}_{r}\end{array}\right]^{T} \in \mathfrak{R}^{2 n}$ are augmented state vectors. $\mathbf{A}_{p}=\left[\begin{array}{cc}\mathbf{0} & \mathbf{I}_{n \times n} \\ -\mathbf{J}_{t}^{-1} & -\mathbf{J}_{t}^{-1} \mathbf{B}_{t}\end{array}\right] \in \mathfrak{R}^{2 n \times 2 n}$

and $\mathbf{A}_{m}=\left[\begin{array}{cc}\mathbf{0} & \mathbf{I}_{n \times n} \\ -\mathbf{J}_{r}^{-1} \mathbf{K}_{r} & -\mathbf{J}_{r}^{-1} \mathbf{B}_{r}\end{array}\right] \in \mathfrak{R}^{2 n \times 2 n}$ are $\quad$ augmented $\quad$ system matrices. $\mathbf{B}_{p}=\left[\begin{array}{c}\mathbf{0} \\ \mathbf{J}_{t}^{-1}\end{array}\right] \in \mathfrak{N}^{2 n \times n}$ and $\mathbf{B}_{m}=\left[\begin{array}{c}\mathbf{0} \\ \mathbf{J}_{r}^{-1} \mathbf{K}_{r}\end{array}\right] \in \mathfrak{R}^{2 n \times n}$ are augmented input gain matrices, and the pair $\left(\mathbf{A}_{m}, \mathbf{B}_{m}\right)$ is controllable. Since all system parameters are assumed to be available at the present stage, we may select a perfect current trajectory in the form[38]

$$
\mathbf{i}=\mathbf{H}^{-1}\left[\Theta \mathbf{x}_{p}+\Phi \boldsymbol{\tau}_{t d}+\mathbf{h}\left(\overline{\boldsymbol{\tau}}_{t d}, \overline{\mathbf{q}}\right)\right]
$$

where $\Theta \in \mathfrak{R}^{n \times 2 n}$ and $\Phi \in \mathfrak{R}^{n \times n} \quad$ satisfy $\quad \mathbf{A}_{p}+\mathbf{B}_{p} \Theta=\mathbf{A}_{m} \quad$ and $\quad \mathbf{B}_{p} \Phi=\mathbf{B}_{m}$ ， respectively, and $\mathbf{h}\left(\overline{\boldsymbol{\tau}}_{t d}, \overline{\mathbf{q}}\right)=\Phi \overline{\boldsymbol{\tau}}_{t d}+\overline{\mathbf{q}}$. Substituting (11) into (9) and after some rearrangements, we may have the system dynamics

$$
\dot{\mathbf{x}}_{p}=\mathbf{A}_{m} \mathbf{x}_{p}+\mathbf{B}_{m}\left(\boldsymbol{\tau}_{t d}+\overline{\boldsymbol{\tau}}_{t d}\right)
$$

Define $\mathbf{e}_{m}=\mathbf{x}_{p}-\mathbf{x}_{m}$ and we may have the error dynamics directly from (10) and (12)

$$
\dot{\mathbf{e}}_{m}=\mathbf{A}_{m} \mathbf{e}_{m}
$$

Let $\mathbf{e}_{\tau}=\boldsymbol{\tau}_{t}-\boldsymbol{\tau}_{r}$ be the output vector of the error dynamics (13) as

$$
\mathbf{e}_{\tau}=\mathbf{C}_{m} \mathbf{e}_{m}
$$


where $\mathbf{C}_{m} \in \mathfrak{R}^{n \times 2 n}$ is the augmented output matrix such that the pair $\left(\mathbf{A}_{m}, \mathbf{C}_{m}\right)$ is observable and the transfer function $\mathbf{C}_{m}\left(s \mathbf{I}-\mathbf{A}_{m}\right)^{-1} \mathbf{B}_{m}$ is strictly positive real. Since $\mathbf{A}_{m}$ is stable, (13) implies $\mathbf{e}_{m} \rightarrow 0$ as $t \rightarrow \infty$. This further gives $\boldsymbol{\tau}_{t} \rightarrow \boldsymbol{\tau}_{t d}$ as $t \rightarrow \infty$. To ensure the actual $\mathbf{i}$ to converge to the perfect $\mathbf{i}$ in (11), let us select the control input in (3) as

$$
\mathbf{u}=\mathbf{L} \dot{\mathbf{i}}_{d}+\mathbf{R}(\mathbf{i}, \dot{\boldsymbol{\theta}})-\mathbf{K}_{c} \mathbf{e}_{i}
$$

where $\mathbf{e}_{i}=\mathbf{i}-\mathbf{i}_{d}$ is the current error, $\mathbf{i}_{d} \in \mathfrak{R}^{n}$ is the desired current which is equivalent to the perfect current trajectory $\mathbf{i}$ in (11), and $\mathbf{K}_{c} \in \mathfrak{R}^{n \times n}$ is a positive definite matrix. Substituting (15) into (3), the closed loop dynamics becomes $\mathbf{L} \dot{\mathbf{e}}_{i}+\mathbf{K}_{c} \mathbf{e}_{i}=\mathbf{0}$. According to this, it is easy to prove that $\mathbf{i} \rightarrow \mathbf{i}_{d}$ as $t \rightarrow \infty$ with proper selection of $\mathbf{K}_{c}$.

In summary, if all parameters in the RLFJED robot (1), (2), and (3) are available, the desired transmission torque (7), the desired current (11), the control input (15) can give asymptotic convergence tracking performance.

\section{B. Controller Design for Uncertain Robot}

Suppose $\mathbf{D}(\mathbf{q}), \mathbf{C}(\mathbf{q}, \dot{\mathbf{q}}) \dot{\mathbf{q}}, \mathbf{g}(\mathbf{q}), \mathbf{L}$ and $\mathbf{R}(\mathbf{i}, \dot{\boldsymbol{\theta}})$ are not available, and $\ddot{\mathbf{q}}, \ddot{\boldsymbol{\theta}}$ are not easy to measure, we would like to design a desired transmission torque $\boldsymbol{\tau}_{d}$ so that a proper controller $\mathbf{u}$ can be constructed to have $\boldsymbol{\tau}_{t} \rightarrow \boldsymbol{\tau}_{t d}$. Instead of (7), let us design a desired transmission torque $\boldsymbol{\tau}_{t d}$ as

$$
\boldsymbol{\tau}_{t d}=\hat{\mathbf{g}}+\hat{\mathbf{D}} \dot{\mathbf{v}}+\hat{\mathbf{C}} \mathbf{v}-\mathbf{K}_{d} \mathbf{s}
$$

where $\hat{\mathbf{D}}, \hat{\mathbf{C}}$ and $\hat{\mathbf{g}}$ are estimates of $\mathbf{D}(\mathbf{q}), \mathbf{C}(\mathbf{q}, \dot{\mathbf{q}})$ and $\mathbf{g}(\mathbf{q})$, respectively. Using (16), we may have the closed loop dynamics

$$
\mathbf{D} \dot{\mathbf{s}}+\mathbf{C s}+\mathbf{K}_{d} \mathbf{s}=-\widetilde{\mathbf{D}} \dot{\mathbf{v}}-\widetilde{\mathbf{C}} \mathbf{v}-\widetilde{\mathbf{g}}+\left(\boldsymbol{\tau}_{t}-\boldsymbol{\tau}_{t d}\right)
$$

where $\widetilde{\mathbf{D}}=\mathbf{D}-\hat{\mathbf{D}}, \widetilde{\mathbf{C}}=\mathbf{C}-\hat{\mathbf{C}}$ and $\widetilde{\mathbf{g}}=\mathbf{g}-\hat{\mathbf{g}}$. If a proper controller and update laws for $\hat{\mathbf{D}}, \hat{\mathbf{C}}$ and $\hat{\mathbf{g}}$ can be designed, we may have $\boldsymbol{\tau}_{t} \rightarrow \boldsymbol{\tau}_{t d}, \hat{\mathbf{D}} \rightarrow \mathbf{D}, \hat{\mathbf{C}} \rightarrow \mathbf{C}$ and $\hat{\mathbf{g}} \rightarrow \mathbf{g}$ so that (17) can give desired performance. Let us consider the desired current $\mathbf{i}_{d}$ instead of (11)

$$
\mathbf{i}_{d}=\mathbf{H}^{-1}\left(\Theta \mathbf{x}_{p}+\Phi \boldsymbol{\tau}_{d}+\hat{\mathbf{h}}\right)
$$


where $\hat{\mathbf{h}}$ is an estimate of $\mathbf{h}$. By (18), we may have the system dynamics

$$
\dot{\mathbf{x}}_{p}=\mathbf{A}_{m} \mathbf{x}_{p}+\mathbf{B}_{m}\left(\boldsymbol{\tau}_{d}+\overline{\boldsymbol{\tau}}_{d}\right)+\mathbf{B}_{p} \mathbf{H}\left(\mathbf{i}-\mathbf{i}_{d}\right)+\mathbf{B}_{p}(\hat{\mathbf{h}}-\mathbf{h})
$$

Together with (10), we may have the error dynamics

$$
\begin{aligned}
& \dot{\mathbf{e}}_{m}=\mathbf{A}_{m} \mathbf{e}_{m}+ \mathbf{B}_{p}\left[\mathbf{H e} \mathbf{e}_{i}+(\hat{\mathbf{h}}-\mathbf{h})\right] \\
& \mathbf{e}_{\tau}=\mathbf{C}_{m} \mathbf{e}_{m}
\end{aligned}
$$

If we may design a control input $u$ and an appropriate update law such that $\mathbf{i} \rightarrow \mathbf{i}_{d}$ and $\hat{\mathbf{h}} \rightarrow \mathbf{h}$, then (20) implies $\mathbf{e}_{m} \rightarrow 0$ as $t \rightarrow \infty$. This further implies $\boldsymbol{\tau}_{t} \rightarrow \boldsymbol{\tau}_{t d}$ as $t \rightarrow \infty$. Here, according to (15), let us select the control input in (3) as

$$
\mathbf{u}=\hat{\mathbf{f}}-\mathbf{K}_{c} \mathbf{e}_{i}
$$

where $\hat{\mathbf{f}}$ is an estimate of $\mathbf{f}\left(\dot{\mathbf{i}}_{d}, \mathbf{i}, \dot{\boldsymbol{\theta}}\right)=\mathbf{L} \dot{\mathbf{i}}_{d}+\mathbf{R}(\mathbf{i}, \dot{\boldsymbol{\theta}})$. Substituting (22) into (3), we may have the system dynamics

$$
\mathbf{L}_{i}+\mathbf{K}_{c} \mathbf{e}_{i}=\hat{\mathbf{f}}-\mathbf{f}
$$

If an appropriate update law for $\hat{\mathbf{f}}$ can be selected, we may have $\mathbf{i} \rightarrow \mathbf{i}_{d}$. Since $\mathbf{D}, \mathbf{C}, \mathbf{g}, \mathbf{h}$ and $\mathbf{f}$ are functions of time, traditional adaptive controllers are not directly applicable. To design the update laws, let us apply the function approximation representation

$$
\begin{array}{ll}
\mathbf{D}=\mathbf{W}_{\mathbf{D}}^{T} \mathbf{Z}_{\mathbf{D}}+\boldsymbol{\varepsilon}_{\mathbf{D}}, & \mathbf{C}=\mathbf{W}_{\mathbf{C}}^{T} \mathbf{Z}_{\mathbf{C}}+\boldsymbol{\varepsilon}_{\mathbf{C}}, \quad \mathbf{g}=\mathbf{W}_{\mathrm{g}}^{T} \mathbf{Z}_{\mathrm{g}}+\boldsymbol{\varepsilon}_{\mathrm{g}}, \\
\mathbf{h}=\mathbf{W}_{\mathbf{h}}^{T} \mathbf{Z}_{\mathbf{h}}+\boldsymbol{\varepsilon}_{\mathbf{h}}, \quad \mathbf{f}=\mathbf{W}_{\mathbf{f}}^{T} \mathbf{Z}_{\mathbf{f}}+\boldsymbol{\varepsilon}_{\mathrm{f}}
\end{array}
$$

where $\mathbf{W}_{\mathbf{D}} \in \mathfrak{R}^{n^{2} \beta_{D} \times n}, \quad \mathbf{W}_{\mathbf{C}} \in \mathfrak{R}^{n^{2} \beta_{\mathbf{C}} \times n}, \quad \mathbf{W}_{\mathbf{g}} \in \mathfrak{R}^{n \beta_{g} \times n}, \quad \mathbf{W}_{\mathbf{h}} \in \mathfrak{R}^{n \beta_{h} \times n}, \quad$ and $\mathbf{W}_{\mathbf{f}} \in \mathfrak{R}^{n \beta_{f} \times n}$ are weighting matrices, $\mathbf{Z}_{\mathbf{D}} \in \mathfrak{R}^{n^{2} \beta_{D} \times n}, \mathbf{Z}_{\mathbf{C}} \in \mathfrak{R}^{n^{2} \beta_{\mathbf{C}} \times n}, \mathbf{Z}_{\mathbf{g}} \in \mathfrak{R}^{n \beta_{g} \times 1}$, $\mathbf{Z}_{\mathbf{h}} \in \mathfrak{R}^{n \beta_{h} \times 1}$ and $\mathbf{Z}_{\mathbf{f}} \in \mathfrak{R}^{n \beta_{f} \times 1}$ are matrices of basis functions, and $\boldsymbol{\varepsilon}_{(\cdot)}$ are approximation error matrices. The number $\beta_{(\cdot)}$ represents the number of basis functions used. Using the same set of basis functions, the corresponding estimates can also be represented as

$$
\begin{array}{ll}
\hat{\mathbf{D}}=\hat{\mathbf{W}}_{\mathbf{D}}^{T} \mathbf{Z}_{\mathbf{D}}, & \hat{\mathbf{C}}=\hat{\mathbf{W}}_{\mathbf{C}}^{T} \mathbf{Z}_{\mathbf{C}}, \hat{\mathbf{g}}=\hat{\mathbf{W}}_{\mathbf{g}}^{T} \mathbf{Z}_{\mathbf{g}}, \\
\hat{\mathbf{h}}=\hat{\mathbf{W}}_{\mathbf{h}}^{T} \mathbf{Z}_{\mathbf{h}}, \quad \hat{\mathbf{f}}=\hat{\mathbf{W}}_{\mathbf{f}}^{T} \mathbf{Z}_{\mathbf{f}}
\end{array}
$$


Define $\widetilde{\mathbf{W}}_{(\cdot)}=\mathbf{W}_{(\cdot)}-\hat{\mathbf{W}}_{(\cdot)}$, then equation (17), (20) and (23) becomes

$$
\begin{gathered}
\mathbf{D} \dot{\mathbf{s}}+\mathbf{C s}+\mathbf{K}_{d} \mathbf{s}=\left(\boldsymbol{\tau}_{t}-\boldsymbol{\tau}_{t d}\right)-\widetilde{\mathbf{W}}_{\mathbf{D}}^{T} \mathbf{Z}_{\mathbf{D}} \dot{\mathbf{v}}-\widetilde{\mathbf{W}}_{\mathbf{C}}^{T} \mathbf{Z}_{\mathbf{C}} \mathbf{v}-\widetilde{\mathbf{W}}_{\mathbf{g}}^{T} \mathbf{Z}_{\mathbf{g}}+\boldsymbol{\varepsilon}_{1} \\
\dot{\mathbf{e}}_{m}=\mathbf{A}_{m} \mathbf{e}_{m}-\mathbf{B}_{p} \widetilde{\mathbf{W}}_{\mathbf{h}}^{T} \mathbf{Z}_{\mathbf{h}}+\mathbf{B}_{p} \mathbf{H} \mathbf{e}_{i}+\mathbf{B}_{p} \boldsymbol{\varepsilon}_{2} \\
\mathbf{L}_{i}+\mathbf{K}_{c} \mathbf{e}_{i}=-\widetilde{\mathbf{W}}_{\mathbf{f}}^{T} \mathbf{Z}_{\mathbf{f}}+\boldsymbol{\varepsilon}_{3}
\end{gathered}
$$

where $\boldsymbol{\varepsilon}_{1}=\boldsymbol{\varepsilon}_{1}\left(\boldsymbol{\varepsilon}_{\mathbf{D}}, \boldsymbol{\varepsilon}_{\mathbf{C}}, \boldsymbol{\varepsilon}_{\mathbf{g}}, \mathbf{s}, \ddot{\mathbf{q}}_{d}\right), \boldsymbol{\varepsilon}_{2}=\boldsymbol{\varepsilon}_{2}\left(\boldsymbol{\varepsilon}_{\mathbf{h}}, \mathbf{e}_{m}\right)$ and $\boldsymbol{\varepsilon}_{3}=\boldsymbol{\varepsilon}_{3}\left(\boldsymbol{\varepsilon}_{\mathbf{f}}, \mathbf{e}_{i}\right)$ are lumped approximation errors. Since $\mathbf{W}_{(\cdot)}$ are constant matrices, their update laws can be easily found by proper selection of the Lyapunov-like function. Let us consider a candidate

$$
\begin{aligned}
& V\left(\mathbf{s}, \mathbf{e}_{m}, \mathbf{e}_{i}, \widetilde{\mathbf{W}}_{\mathbf{D}}, \widetilde{\mathbf{W}}_{\mathbf{C}}, \widetilde{\mathbf{W}}_{\mathbf{g}}, \widetilde{\mathbf{W}}_{\mathbf{h}}, \widetilde{\mathbf{W}}_{\mathbf{f}}\right)=\frac{1}{2} \mathbf{s}^{T} \mathbf{D} \mathbf{s}+\mathbf{e}_{m}^{T} \mathbf{P}_{t} \mathbf{e}_{m}+\frac{1}{2} \mathbf{e}_{i}^{T} \mathbf{L e}_{i} \\
& \quad+\frac{1}{2} \operatorname{Tr}\left(\widetilde{\mathbf{W}}_{\mathbf{D}}^{T} \mathbf{Q}_{\mathbf{D}} \widetilde{\mathbf{W}}_{\mathbf{D}}+\widetilde{\mathbf{W}}_{\mathbf{C}}^{T} \mathbf{Q}_{\mathbf{C}} \widetilde{\mathbf{W}}_{\mathbf{C}}+\widetilde{\mathbf{W}}_{\mathbf{g}}^{T} \mathbf{Q}_{\mathbf{g}} \widetilde{\mathbf{W}}_{\mathbf{g}}+\widetilde{\mathbf{W}}_{\mathbf{h}}^{T} \mathbf{Q}_{\mathbf{h}} \widetilde{\mathbf{W}}_{\mathbf{h}}+\widetilde{\mathbf{W}}_{\mathbf{f}}^{T} \mathbf{Q}_{\mathbf{f}} \widetilde{\mathbf{W}}_{\mathbf{f}}\right)
\end{aligned}
$$

where $\mathbf{P}_{t}=\mathbf{P}_{t}^{T} \in \mathfrak{R}^{2 n \times 2 n}$ is a positive definite matrix satisfying the Lyapunov equation $\mathbf{A}_{m}^{T} \mathbf{P}_{t}+\mathbf{P}_{t} \mathbf{A}_{m}=-\mathbf{C}_{m}^{T} \mathbf{C}_{m}$. The matrices $\mathbf{Q}_{\mathbf{D}} \in \mathfrak{R}^{n^{2} \beta_{\mathbf{D}} \times n^{2} \beta_{\mathbf{D}}}, \quad \mathbf{Q}_{\mathbf{C}} \in \mathfrak{R}^{n^{2} \beta_{C} \times n^{2} \beta_{C}}$, $\mathbf{Q}_{\mathbf{g}} \in \mathfrak{R}^{n \beta_{g} \times n \beta_{g}}, \quad \mathbf{Q}_{\mathbf{h}} \in \mathfrak{R}^{n \beta_{h} \times n \beta_{h}}$ and $\mathbf{Q}_{\mathbf{f}} \in \mathfrak{R}^{n \beta_{f} \times n \beta_{f}}$ are positive definite. The notation $\operatorname{Tr}($.$) denotes the trace operation of matrices. The time derivative of V$ along the trajectory of (25), (26), and (27) can be computed as

$$
\begin{aligned}
& \dot{V}=\mathbf{s}^{T} \mathbf{D} \dot{\mathbf{s}}+\frac{1}{2} \mathbf{s}^{T} \dot{\mathbf{D}} \mathbf{s}+\dot{\mathbf{e}}_{m}^{T} \mathbf{P}_{t} \mathbf{e}_{m}+\mathbf{e}_{m}^{T} \mathbf{P}_{t} \dot{\mathbf{e}}_{m}+\mathbf{e}_{i}^{T} \mathbf{L} \dot{\mathbf{e}}_{i} \\
& -\operatorname{Tr}\left(\widetilde{\mathbf{W}}_{\mathbf{D}}^{T} \mathbf{Q}_{\mathbf{D}} \dot{\hat{\mathbf{W}}}_{\mathbf{D}}+\widetilde{\mathbf{W}}_{\mathbf{C}}^{T} \mathbf{Q}_{\mathbf{C}} \dot{\hat{\mathbf{W}}}_{\mathbf{C}}+\widetilde{\mathbf{W}}_{\mathrm{g}}^{T} \mathbf{Q}_{\mathbf{g}} \dot{\hat{\mathbf{W}}}_{\mathrm{g}}+\widetilde{\mathbf{W}}_{\mathbf{h}}^{T} \mathbf{Q}_{\mathbf{h}} \dot{\hat{\mathbf{W}}}_{\mathbf{h}}+\widetilde{\mathbf{W}}_{\mathbf{f}}^{T} \mathbf{Q}_{\mathbf{f}} \dot{\hat{\mathbf{W}}}_{\mathbf{f}}\right) \\
& =-\mathbf{s}^{T} \mathbf{K}_{d} \mathbf{s}+\mathbf{s}^{T} \mathbf{e}_{\tau}-\mathbf{e}_{\tau}^{T} \mathbf{e}_{\tau}+\mathbf{e}_{m}^{T} \mathbf{P}_{t} \mathbf{B}_{p} \mathbf{H e} \mathbf{e}_{i}-\mathbf{e}_{i}^{T} \mathbf{K}_{c} \mathbf{e}_{i}+\mathbf{s}^{T} \boldsymbol{\varepsilon}_{1}+\mathbf{e}_{m}^{T} \mathbf{P}_{t} \mathbf{B}_{p} \boldsymbol{\varepsilon}_{2}+\mathbf{e}_{i}^{T} \boldsymbol{\varepsilon}_{3} \\
& -\operatorname{Tr}\left[\tilde{\mathbf{W}}_{\mathbf{D}}^{T}\left(\mathbf{Z}_{\mathbf{D}} \dot{\mathbf{v}} \mathbf{s}^{T}+\mathbf{Q}_{\mathbf{D}} \dot{\hat{\mathbf{W}}} \mathbf{D}\right)+\tilde{\mathbf{W}}_{\mathbf{C}}^{T}\left(\mathbf{Z}_{\mathbf{C}} \mathbf{v} \mathbf{s}^{T}+\mathbf{Q}_{\mathbf{C}} \dot{\hat{\mathbf{W}}}_{\mathbf{C}}\right)\right] \\
& -\operatorname{Tr}\left[\tilde{\mathbf{W}}_{\mathbf{g}}^{T}\left(\mathbf{Z}_{\mathbf{g}} \mathbf{s}^{T}+\mathbf{Q}_{\mathbf{g}} \dot{\hat{\mathbf{W}}} \mathbf{g}\right)+\widetilde{\mathbf{W}}_{\mathbf{h}}^{T}\left(\mathbf{Z}_{\mathbf{h}} \mathbf{e}_{m}^{T} \mathbf{P}_{t} \mathbf{B}_{p}+\mathbf{Q}_{\mathbf{h}} \dot{\hat{\mathbf{W}}}_{\mathbf{h}}\right)\right] \\
& -\operatorname{Tr}\left[\tilde{\mathbf{W}}_{\mathbf{f}}^{T}\left(\mathbf{Z}_{\mathbf{f}} \mathbf{e}_{i}^{T}+\mathbf{Q}_{\mathbf{f}} \dot{\hat{\mathbf{W}}}_{\mathbf{f}}\right)\right]
\end{aligned}
$$

According to the Kalman-Yakubovic Lemma, we have $\mathbf{e}_{m}^{T} \mathbf{P}_{t} \mathbf{B}_{p}=\mathbf{e}_{\tau}^{T}$ by picking $\mathbf{B}_{m}=\mathbf{B}_{p}$ [39]. According to (29), the update laws can be selected as 


$$
\begin{array}{ll}
\dot{\hat{\mathbf{W}}}_{\mathbf{D}}=-\mathbf{Q}_{\mathbf{D}}^{-1} \mathbf{Z}_{\mathbf{D}} \dot{\mathbf{V}}^{T}-\sigma_{\mathbf{D}} \hat{\mathbf{W}}_{\mathbf{D}}, & \dot{\hat{\mathbf{W}}}_{\mathbf{C}}=-\mathbf{Q}_{\mathbf{C}}^{-1} \mathbf{Z}_{\mathbf{C}} \mathbf{V} \mathbf{s}^{T}-\sigma_{\mathbf{C}} \hat{\mathbf{W}}_{\mathbf{C}}, \\
\dot{\hat{\mathbf{W}}}_{\mathbf{g}}=-\mathbf{Q}_{\mathbf{g}}^{-1} \mathbf{Z}_{\mathbf{g}} \mathbf{s}^{T}-\sigma_{\mathbf{g}} \hat{\mathbf{W}}_{\mathbf{g}}, & \dot{\hat{\mathbf{W}}}_{\mathbf{h}}=-\mathbf{Q}_{\mathbf{h}}^{-1} \mathbf{Z}_{\mathbf{h}} \mathbf{e}_{\tau}^{T}-\sigma_{\mathbf{h}} \hat{\mathbf{W}}_{\mathbf{h}}, \\
\dot{\hat{\mathbf{W}}}_{\mathbf{f}}=-\mathbf{Q}_{\mathbf{f}}^{-1} \mathbf{Z}_{\mathbf{f}} \mathbf{e}_{i}^{T}-\sigma_{\mathbf{f}} \hat{\mathbf{W}}_{\mathbf{f}} &
\end{array}
$$

where $\sigma_{(\cdot)}$ are positive numbers. Then (29) becomes

$$
\begin{aligned}
\dot{V}= & -\left[\begin{array}{lll}
\mathbf{s}^{T} & \mathbf{e}_{\tau}^{T} & \mathbf{e}_{i}^{T}
\end{array}\right] \mathbf{Q}\left[\begin{array}{c}
\mathbf{s} \\
\mathbf{e}_{\tau} \\
\mathbf{e}_{i}
\end{array}\right]+\left[\begin{array}{lll}
\mathbf{s}^{T} & \mathbf{e}_{\tau}^{T} & \mathbf{e}_{i}^{T}
\end{array}\right]\left[\begin{array}{c}
\boldsymbol{\varepsilon}_{1} \\
\boldsymbol{\varepsilon}_{2} \\
\boldsymbol{\varepsilon}_{3}
\end{array}\right] \\
& +\sigma_{\mathbf{D}} \operatorname{Tr}\left(\widetilde{\mathbf{W}}_{\mathbf{D}}^{T} \hat{\mathbf{W}}_{\mathbf{D}}\right)+\sigma_{\mathbf{C}} \operatorname{Tr}\left(\widetilde{\mathbf{W}}_{\mathbf{C}}^{T} \hat{\mathbf{W}}_{\mathbf{C}}\right)+\sigma_{\mathbf{g}} \operatorname{Tr}\left(\widetilde{\mathbf{W}}_{\mathbf{g}}^{T} \hat{\mathbf{W}}_{\mathbf{g}}\right) \\
& +\sigma_{\mathbf{h}} \operatorname{Tr}\left(\tilde{\mathbf{W}}_{\mathbf{h}}^{T} \hat{\mathbf{W}}_{\mathbf{h}}\right)+\sigma_{\mathbf{f}} \operatorname{Tr}\left(\widetilde{\mathbf{W}}_{\mathbf{f}}^{T} \hat{\mathbf{W}}_{\mathbf{f}}\right)
\end{aligned}
$$

where $\mathbf{Q}=\left[\begin{array}{ccc}\mathbf{K}_{d} & -\frac{1}{2} \mathbf{I}_{n \times n} & \mathbf{0} \\ -\frac{1}{2} \mathbf{I}_{n \times n} & \mathbf{I}_{n \times n} & -\frac{1}{2} \mathbf{H} \\ \mathbf{0} & -\frac{1}{2} \mathbf{H} & \mathbf{K}_{c}\end{array}\right]$ is positive definite due to proper selections

of $\mathbf{K}_{d}$ and $\mathbf{K}_{c}$. Owing to the existence of $\boldsymbol{\varepsilon}_{1}, \boldsymbol{\varepsilon}_{2}$, and $\boldsymbol{\varepsilon}_{3}$ the definiteness of $\dot{V}$ cannot be determined. According to Appendix Lemma A.1、Lemma A.4 and Lemma A.7, the right hand side of (31) can be divided into two parts to derive following inequalities

$$
\begin{aligned}
& -\left[\begin{array}{lll}
\mathbf{s}^{T} & \mathbf{e}_{\tau}^{T} & \mathbf{e}_{i}^{T}
\end{array}\right] \mathbf{Q}\left[\begin{array}{c}
\mathbf{s} \\
\mathbf{e}_{\tau} \\
\mathbf{e}_{i}
\end{array}\right]+\left[\begin{array}{lll}
\mathbf{s}^{T} & \mathbf{e}_{\tau}^{T} & \mathbf{e}_{i}^{T}
\end{array}\right]\left[\begin{array}{l}
\boldsymbol{\varepsilon}_{1} \\
\boldsymbol{\varepsilon}_{2} \\
\boldsymbol{\varepsilon}_{3}
\end{array}\right] \\
& \leq-\frac{1}{2}\left(\lambda_{\min }(\mathbf{Q})\left\|\left[\begin{array}{l}
\mathbf{s} \\
\mathbf{e}_{\tau} \\
\mathbf{e}_{i}
\end{array}\right]\right\|^{2}-\frac{1}{\lambda_{\min }(\mathbf{Q})}\left\|\left[\begin{array}{l}
\boldsymbol{\varepsilon}_{1} \\
\boldsymbol{\varepsilon}_{2} \\
\boldsymbol{\varepsilon}_{3}
\end{array}\right]\right\|^{2}\right) \\
& \operatorname{Tr}\left(\widetilde{\mathbf{W}}_{\mathbf{D}}^{T} \hat{\mathbf{W}}_{\mathbf{D}}\right) \leq \frac{1}{2} \operatorname{Tr}\left(\mathbf{W}_{\mathbf{D}}^{T} \mathbf{W}_{\mathbf{D}}\right)-\frac{1}{2} \operatorname{Tr}\left(\tilde{\mathbf{W}}_{\mathbf{D}}^{T} \widetilde{\mathbf{W}}_{\mathbf{D}}\right)
\end{aligned}
$$




$$
\begin{aligned}
& \operatorname{Tr}\left(\widetilde{\mathbf{W}}_{\mathbf{C}}^{T} \hat{\mathbf{W}}_{\mathbf{C}}\right) \leq \frac{1}{2} \operatorname{Tr}\left(\mathbf{W}_{\mathbf{C}}^{T} \mathbf{W}_{\mathbf{C}}\right)-\frac{1}{2} \operatorname{Tr}\left(\widetilde{\mathbf{W}}_{\mathbf{C}}^{T} \widetilde{\mathbf{W}}_{\mathbf{C}}\right) \\
& \operatorname{Tr}\left(\widetilde{\mathbf{W}}_{\mathbf{g}}^{T} \hat{\mathbf{W}}_{\mathbf{g}}\right) \leq \frac{1}{2} \operatorname{Tr}\left(\mathbf{W}_{\mathbf{g}}^{T} \mathbf{W}_{\mathbf{g}}\right)-\frac{1}{2} \operatorname{Tr}\left(\widetilde{\mathbf{W}}_{\mathbf{g}}^{T} \widetilde{\mathbf{W}}_{\mathbf{g}}\right) \\
& \operatorname{Tr}\left(\widetilde{\mathbf{W}}_{\mathbf{h}}^{T} \hat{\mathbf{W}}_{\mathbf{h}}\right) \leq \frac{1}{2} \operatorname{Tr}\left(\mathbf{W}_{\mathbf{h}}^{T} \mathbf{W}_{\mathbf{h}}\right)-\frac{1}{2} \operatorname{Tr}\left(\widetilde{\mathbf{W}}_{\mathbf{h}}^{T} \widetilde{\mathbf{W}}_{\mathbf{h}}\right) \\
& \operatorname{Tr}\left(\widetilde{\mathbf{W}}_{\mathbf{f}}^{T} \hat{\mathbf{W}}_{\mathbf{f}}\right) \leq \frac{1}{2} \operatorname{Tr}\left(\mathbf{W}_{\mathbf{f}}^{T} \mathbf{W}_{\mathbf{f}}\right)-\frac{1}{2} \operatorname{Tr}\left(\widetilde{\mathbf{W}}_{\mathbf{f}}^{T} \widetilde{\mathbf{W}}_{\mathbf{f}}\right)
\end{aligned}
$$

According to (28), we hav

$$
\begin{aligned}
& V=\frac{1}{2}\left[\mathbf{s}^{T} \mathbf{D s}+\mathbf{e}_{i}^{T} \mathbf{L} \mathbf{e}_{i}+2 \mathbf{e}_{m}^{T} \mathbf{P}_{t} \mathbf{e}_{m}\right. \\
& \left.\left.+\operatorname{Tr}\left(\widetilde{\mathbf{W}}_{\mathbf{D}}^{T} \mathbf{Q}_{\mathbf{D}} \widetilde{\mathbf{W}}_{\mathbf{D}}+\widetilde{\mathbf{W}}_{\mathbf{C}}^{T} \mathbf{Q}_{\mathbf{C}} \widetilde{\mathbf{W}}_{\mathbf{C}}+\widetilde{\mathbf{W}}_{\mathrm{g}}^{T} \mathbf{Q}_{\mathrm{g}} \tilde{\mathbf{W}}_{\mathbf{g}}+\widetilde{\mathbf{W}}_{\mathbf{h}}^{T} \mathbf{Q}_{\mathbf{h}} \widetilde{\mathbf{W}}_{\mathbf{h}}\right)+\widetilde{\mathbf{W}}_{\mathbf{f}}^{T} \mathbf{Q}_{\mathbf{f}} \widetilde{\mathbf{W}}_{\mathbf{f}}\right)\right] \\
& \leq \frac{1}{2}\left[\lambda_{\max }(\mathbf{A})\left\|\left[\begin{array}{c}
\mathbf{s} \\
\mathbf{e}_{\tau} \\
\mathbf{e}_{i}
\end{array}\right]\right\|^{2}+\lambda_{\max }\left(\mathbf{Q}_{\mathbf{D}}\right) \operatorname{Tr}\left(\tilde{\mathbf{W}}_{\mathbf{D}}^{T} \widetilde{\mathbf{W}}_{\mathbf{D}}\right)+\lambda_{\max }\left(\mathbf{Q}_{\mathbf{C}}\right) \operatorname{Tr}\left(\tilde{\mathbf{W}}_{\mathbf{C}}^{T} \widetilde{\mathbf{W}}_{\mathbf{C}}\right)\right. \\
& \left.+\lambda_{\max }\left(\mathbf{Q}_{\mathrm{g}}\right) \operatorname{Tr}\left(\widetilde{\mathbf{W}}_{\mathrm{g}}^{T} \widetilde{\mathbf{W}}_{\mathrm{g}}\right)+\lambda_{\max }\left(\mathbf{Q}_{\mathbf{h}}\right) \operatorname{Tr}\left(\widetilde{\mathbf{W}}_{\mathbf{h}}^{T} \widetilde{\mathbf{W}}_{\mathbf{h}}\right)+\lambda_{\max }\left(\mathbf{Q}_{\mathbf{f}}\right) \operatorname{Tr}\left(\widetilde{\mathbf{W}}_{\mathbf{f}}^{T} \widetilde{\mathbf{W}}_{\mathbf{f}}\right)\right]
\end{aligned}
$$

where $\mathbf{A}=\left[\begin{array}{ccc}\mathbf{D} & \mathbf{0} & \mathbf{0} \\ \mathbf{0} & 2 \mathbf{C}_{m}^{T} \mathbf{P}_{t} \mathbf{C}_{m} & \mathbf{0} \\ \mathbf{0} & \mathbf{0} & \mathbf{L}\end{array}\right]$. With (32) and (33), (31) can be further written as

$$
\begin{aligned}
& \dot{V} \leq-\alpha V+\frac{1}{2}\left\{\left[\alpha \lambda_{\text {max }}(\mathbf{A})-\lambda_{\min }(\mathbf{Q})\right]\left\|\left[\begin{array}{c}
\mathbf{s} \\
\mathbf{e}_{\tau} \\
\mathbf{e}_{i}
\end{array}\right]\right\|^{2}+\left[\alpha \lambda_{\max }\left(\mathbf{Q}_{\mathbf{D}}\right)-\sigma_{\mathbf{D}}\right] \operatorname{Tr}\left(\tilde{\mathbf{W}}_{\mathbf{D}}^{T} \widetilde{\mathbf{W}}_{\mathbf{D}}\right)\right. \\
& +\left[\alpha \lambda_{\max }\left(\mathbf{Q}_{\mathbf{C}}\right)-\sigma_{\mathbf{C}}\right] \operatorname{Tr}\left(\widetilde{\mathbf{W}}_{\mathbf{C}}^{T} \widetilde{\mathbf{W}}_{\mathbf{C}}\right)+\left[\alpha \lambda_{\max }\left(\mathbf{Q}_{\mathbf{g}}\right)-\sigma_{\mathbf{g}}\right] \operatorname{Tr}\left(\widetilde{\mathbf{W}}_{\mathbf{g}}^{T} \widetilde{\mathbf{W}}_{\mathbf{g}}\right) \\
& +\left[\alpha \lambda_{\max }\left(\mathbf{Q}_{\mathbf{h}}\right)-\sigma_{\mathbf{h}}\right] \operatorname{Tr}\left(\tilde{\mathbf{W}}_{\mathbf{h}}^{T} \tilde{\mathbf{W}}_{\mathbf{h}}\right)+\left[\alpha \lambda_{\max }\left(\mathbf{Q}_{\mathbf{f}}\right)-\sigma_{\mathbf{f}}\right] \operatorname{Tr}\left(\tilde{\mathbf{W}}_{\mathbf{f}}^{T} \tilde{\mathbf{W}}_{\mathbf{f}}\right)
\end{aligned}
$$

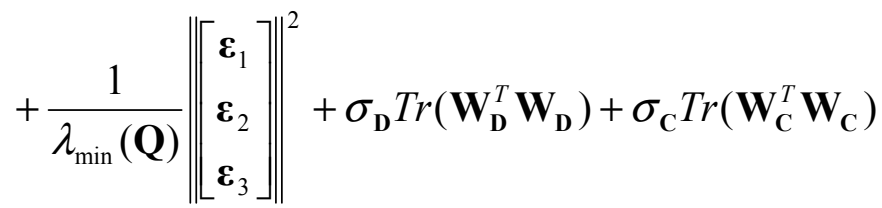

$$
\begin{aligned}
& \left.+\sigma_{\mathrm{g}} \operatorname{Tr}\left(\mathbf{W}_{\mathrm{g}}^{T} \mathbf{W}_{\mathrm{g}}\right)+\sigma_{\mathbf{h}} \operatorname{Tr}\left(\mathbf{W}_{\mathbf{h}}^{T} \mathbf{W}_{\mathbf{h}}\right)+\sigma_{\mathbf{f}} \operatorname{Tr}\left(\mathbf{W}_{\mathbf{f}}^{T} \mathbf{W}_{\mathbf{f}}\right)\right\}
\end{aligned}
$$


Although $\mathbf{D}$ and $\mathbf{L}$ are unknown, we know that $\exists \bar{D}$ and $\underline{D}$ s.t. $\underline{D} \leq\|\mathbf{D}\| \leq \bar{D}, \exists \bar{L}$ and $\underline{L} \quad$ s.t. $\quad \underline{L} \leq\|\mathbf{L}\| \leq \bar{L}, \quad \exists \bar{\eta}_{A}, \underline{\eta}_{A}>0 \quad$ s.t. $\quad \lambda_{\max }(\mathbf{A}) \leq \bar{\eta}_{A} \quad$ and $\lambda_{\min }(\mathbf{A}) \geq \underline{\eta}_{A}[40]$. Picking $\alpha \leq \min \left\{\frac{\lambda_{\min }(\mathbf{Q})}{\bar{\eta}_{A}}, \frac{\sigma_{\mathbf{D}}}{\lambda_{\max }\left(\mathbf{Q}_{\mathbf{D}}\right)}, \frac{\sigma_{\mathbf{C}}}{\lambda_{\max }\left(\mathbf{Q}_{\mathbf{C}}\right)}, \frac{\sigma_{\mathbf{g}}}{\lambda_{\max }\left(\mathbf{Q}_{\mathbf{g}}\right)}, \frac{\sigma_{\mathbf{h}}}{\lambda_{\max }\left(\mathbf{Q}_{\mathbf{h}}\right)}, \frac{\sigma_{\mathbf{f}}}{\lambda_{\max }\left(\mathbf{Q}_{\mathbf{f}}\right)}\right\}$,

then we have

$$
\begin{array}{r}
\dot{V} \leq-\alpha V+\frac{1}{2 \lambda_{\min }(\mathbf{Q}) \|}\left\|\left[\begin{array}{c}
\boldsymbol{\varepsilon}_{1} \\
\boldsymbol{\varepsilon}_{2} \\
\boldsymbol{\varepsilon}_{3}
\end{array}\right]\right\| \|^{2}+\frac{1}{2}\left[\sigma_{\mathbf{D}} \operatorname{Tr}\left(\mathbf{W}_{\mathbf{D}}^{T} \mathbf{W}_{\mathbf{D}}\right)+\sigma_{\mathbf{C}} \operatorname{Tr}\left(\mathbf{W}_{\mathbf{C}}^{T} \mathbf{W}_{\mathbf{C}}\right)\right. \\
\left.+\sigma_{\mathbf{g}} \operatorname{Tr}\left(\mathbf{W}_{\mathbf{g}}^{T} \mathbf{W}_{\mathbf{g}}\right)+\sigma_{\mathbf{h}} \operatorname{Tr}\left(\mathbf{W}_{\mathbf{h}}^{T} \mathbf{W}_{\mathbf{h}}\right)+\sigma_{\mathbf{f}} \operatorname{Tr}\left(\mathbf{W}_{\mathbf{f}}^{T} \mathbf{W}_{\mathbf{f}}\right)\right]
\end{array}
$$

Hence, $\dot{V}<0$ whenever

$$
\begin{gathered}
\left(\mathbf{s}, \mathbf{e}_{\tau}, \mathbf{e}_{i}, \widetilde{\mathbf{W}}_{\mathbf{D}}, \widetilde{\mathbf{W}}_{\mathbf{C}}, \widetilde{\mathbf{W}}_{\mathbf{g}}, \widetilde{\mathbf{W}}_{\mathbf{h}}, \widetilde{\mathbf{W}}_{\mathbf{f}}\right) \in\left\{\left(\mathbf{s}, \mathbf{e}_{\tau}, \mathbf{e}_{i}, \widetilde{\mathbf{W}}_{\mathbf{D}}, \widetilde{\mathbf{W}}_{\mathbf{C}}, \widetilde{\mathbf{W}}_{\mathbf{g}}, \widetilde{\mathbf{W}}_{\mathbf{h}}, \widetilde{\mathbf{W}}_{\mathbf{f}}\right) \mid V>\right. \\
\frac{1}{2 \alpha}\left[\begin{array}{c}
\frac{1}{\lambda_{\min }(\mathbf{Q})} \sup _{\tau \geq t_{0}}\left\|\left[\begin{array}{l}
\boldsymbol{\varepsilon}_{1}(\tau) \\
\boldsymbol{\varepsilon}_{2}(\tau) \\
\boldsymbol{\varepsilon}_{3}(\tau)
\end{array}\right]\right\| \|^{2}+\sigma_{\mathbf{D}} \operatorname{Tr}\left(\mathbf{W}_{\mathbf{D}}^{T} \mathbf{W}_{\mathbf{D}}\right)+\sigma_{\mathbf{C}} \operatorname{Tr}\left(\mathbf{W}_{\mathbf{C}}^{T} \mathbf{W}_{\mathbf{C}}\right) \\
\left.\left.+\sigma_{\mathbf{g}} \operatorname{Tr}\left(\mathbf{W}_{\mathbf{g}}^{T} \mathbf{W}_{\mathbf{g}}\right)+\sigma_{\mathbf{h}} \operatorname{Tr}\left(\mathbf{W}_{\mathbf{h}}^{T} \mathbf{W}_{\mathbf{h}}\right)+\sigma_{\mathbf{f}} \operatorname{Tr}\left(\mathbf{W}_{\mathbf{f}}^{T} \mathbf{W}_{\mathbf{f}}\right)\right]\right\}
\end{array}\right.
\end{gathered}
$$

This further concludes that $\mathbf{s}, \mathbf{e}_{\tau}, \mathbf{e}_{i}, \widetilde{\mathbf{W}}_{\mathbf{D}}, \widetilde{\mathbf{W}}_{\mathbf{C}}, \widetilde{\mathbf{W}}_{\mathbf{g}}, \widetilde{\mathbf{W}}_{\mathbf{h}}$, and $\widetilde{\mathbf{W}}_{\mathbf{f}}$ are uniformly ultimately bounded(u.u.b.). The implementation of the desired transmission torque (16), desired current (18), control input (22) and update law (30) does not need to calculate the regressor matrix which is required in most adaptive designs for robot manipulators. The convergence of the parameters, however, can be proved to depend on the persistent excitation condition of the input.

The above derivation only demonstrates the boundedness of the closed loop system, but in practical applications the transient performance is also of great importance. For further development, we may apply the comparison lemma[40] to (35) to have the upper bound for $V$ as

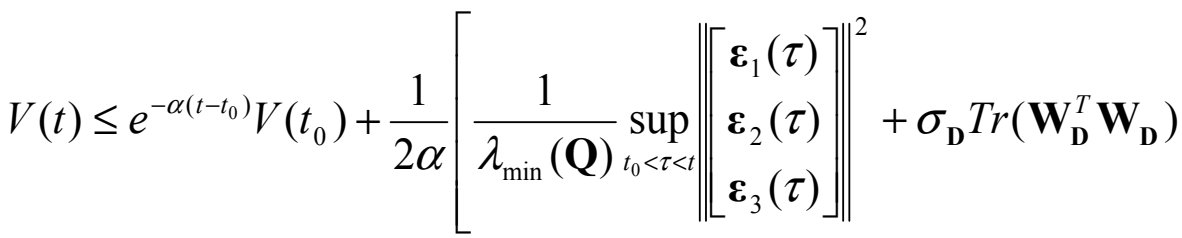

$$
\begin{aligned}
& \left.+\sigma_{\mathbf{C}} \operatorname{Tr}\left(\mathbf{W}_{\mathbf{C}}^{T} \mathbf{W}_{\mathbf{C}}\right)+\sigma_{\mathbf{g}} \operatorname{Tr}\left(\mathbf{W}_{\mathrm{g}}^{T} \mathbf{W}_{\mathbf{g}}\right)+\sigma_{\mathbf{h}} \operatorname{Tr}\left(\mathbf{W}_{\mathbf{h}}^{T} \mathbf{W}_{\mathbf{h}}\right)+\sigma_{\mathbf{f}} \operatorname{Tr}\left(\mathbf{W}_{\mathbf{f}}^{T} \mathbf{W}_{\mathbf{f}}\right)\right]
\end{aligned}
$$


From (28), we obtain

$$
\begin{aligned}
& V \geq \frac{1}{2}\left[\lambda_{\min }(\mathbf{A})\left\|\left[\begin{array}{c}
\mathbf{s} \\
\mathbf{e}_{\tau} \\
\mathbf{e}_{i}
\end{array}\right]\right\|^{2}+\lambda_{\min }\left(\mathbf{Q}_{\mathbf{D}}\right) \operatorname{Tr}\left(\widetilde{\mathbf{W}}_{\mathbf{D}}^{T} \widetilde{\mathbf{W}}_{\mathbf{D}}\right)+\lambda_{\min }\left(\mathbf{Q}_{\mathbf{C}}\right) \operatorname{Tr}\left(\widetilde{\mathbf{W}}_{\mathbf{C}}^{T} \widetilde{\mathbf{W}}_{\mathbf{C}}\right)\right. \\
& \left.\quad+\lambda_{\min }\left(\mathbf{Q}_{\mathbf{g}}\right) \operatorname{Tr}\left(\tilde{\mathbf{W}}_{\mathbf{g}}^{T} \tilde{\mathbf{W}}_{\mathbf{g}}\right)+\lambda_{\min }\left(\mathbf{Q}_{\mathbf{h}}\right) \operatorname{Tr}\left(\tilde{\mathbf{W}}_{\mathbf{h}}^{T} \tilde{\mathbf{W}}_{\mathbf{h}}\right)+\lambda_{\min }\left(\mathbf{Q}_{\mathbf{f}}\right) \operatorname{Tr}\left(\tilde{\mathbf{W}}_{\mathbf{f}}^{T} \tilde{\mathbf{W}}_{\mathbf{f}}\right)\right]
\end{aligned}
$$

Thus, the bound of $\left\|\left[\begin{array}{lll}\mathbf{S}^{T} & \mathbf{e}_{\tau}^{T} & \mathbf{e}_{i}^{T}\end{array}\right]^{T}\right\|^{2}$ for $t \geq t_{0}$ can be derived from (36) and (37) as

$$
\begin{aligned}
& \left\|\left[\begin{array}{c}
\mathbf{s} \\
\mathbf{e}_{\tau} \\
\mathbf{e}_{i}
\end{array}\right]\right\|^{2} \leq \frac{1}{\underline{\eta}_{A}}\left[V-\lambda_{\min }\left(\mathbf{Q}_{\mathbf{D}}\right) \operatorname{Tr}\left(\widetilde{\mathbf{W}}_{\mathbf{D}}^{T} \widetilde{\mathbf{W}}_{\mathbf{D}}\right)\right. \\
& -\lambda_{\min }\left(\mathbf{Q}_{\mathbf{C}}\right) \operatorname{Tr}\left(\tilde{\mathbf{W}}_{\mathbf{C}}^{T} \tilde{\mathbf{W}}_{\mathbf{C}}\right)-\lambda_{\min }\left(\mathbf{Q}_{\mathrm{g}}\right) \operatorname{Tr}\left(\tilde{\mathbf{W}}_{\mathbf{g}}^{T} \tilde{\mathbf{W}}_{\mathrm{g}}\right) \\
& \left.-\lambda_{\min }\left(\mathbf{Q}_{\mathbf{h}}\right) \operatorname{Tr}\left(\tilde{\mathbf{W}}_{\mathbf{h}}^{T} \tilde{\mathbf{W}}_{\mathbf{h}}\right)-\lambda_{\min }\left(\mathbf{Q}_{\mathbf{f}}\right) \operatorname{Tr}\left(\tilde{\mathbf{W}}_{\mathbf{f}}^{T} \tilde{\mathbf{W}}_{\mathbf{f}}\right)\right]
\end{aligned}
$$

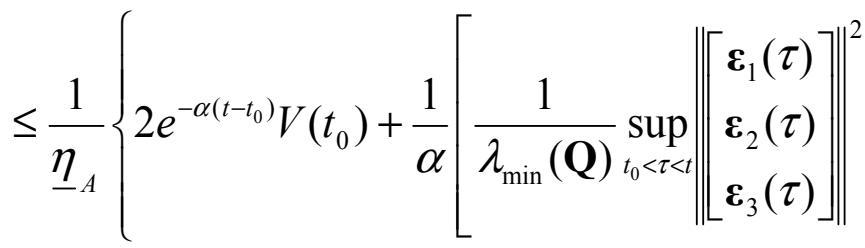

$$
\begin{aligned}
& +\sigma_{\mathbf{D}} \operatorname{Tr}\left(\mathbf{W}_{\mathbf{D}}^{T} \mathbf{W}_{\mathbf{D}}\right)+\sigma_{\mathbf{C}} \operatorname{Tr}\left(\mathbf{W}_{\mathbf{C}}^{T} \mathbf{W}_{\mathbf{C}}\right)+\sigma_{\mathbf{g}} \operatorname{Tr}\left(\mathbf{W}_{\mathbf{g}}^{T} \mathbf{W}_{\mathrm{g}}\right) \\
& \left.+\sigma_{\mathbf{h}} \operatorname{Tr}\left(\mathbf{W}_{\mathbf{h}}^{T} \mathbf{W}_{\mathbf{h}}\right)+\sigma_{\mathbf{f}} \operatorname{Tr}\left(\mathbf{W}_{\mathbf{f}}^{T} \mathbf{W}_{\mathbf{f}}\right)\right]-\lambda_{\min }\left(\mathbf{Q}_{\mathbf{D}}\right) \operatorname{Tr}\left(\tilde{\mathbf{W}}_{\mathbf{D}}^{T} \tilde{\mathbf{W}}_{\mathbf{D}}\right) \\
& -\lambda_{\text {min }}\left(\mathbf{Q}_{\mathbf{C}}\right) \operatorname{Tr}\left(\tilde{\mathbf{W}}_{\mathbf{C}}^{T} \tilde{\mathbf{W}}_{\mathbf{C}}\right)-\lambda_{\min }\left(\mathbf{Q}_{\mathrm{g}}\right) \operatorname{Tr}\left(\tilde{\mathbf{W}}_{\mathbf{g}}^{T} \widetilde{\mathbf{W}}_{\mathrm{g}}\right) \\
& \left.-\lambda_{\min }\left(\mathbf{Q}_{\mathbf{h}}\right) \operatorname{Tr}\left(\tilde{\mathbf{W}}_{\mathbf{h}}^{T} \tilde{\mathbf{W}}_{\mathbf{h}}\right)-\lambda_{\min }\left(\mathbf{Q}_{\mathbf{f}}\right) \operatorname{Tr}\left(\tilde{\mathbf{W}}_{\mathbf{f}}^{T} \tilde{\mathbf{W}}_{\mathbf{f}}\right)\right\}
\end{aligned}
$$

From the derivations above, we can conclude that the proposed design is able to give bounded tracking with guaranteed transient performance. The following theorem is a summary of the above results.

Theorem 1: Consider the RLFJED robot (1)-(3) with unknown parameters D, C, g, L and $\mathbf{R}$, then desired transmission torque (16), desired current (18), control input (22) and update law (30) ensure that

(i) error signals s, $\mathbf{e}_{\tau}, \mathbf{e}_{i}, \tilde{\mathbf{W}}_{\mathbf{D}}, \tilde{\mathbf{W}}_{\mathbf{C}}, \tilde{\mathbf{W}}_{\mathbf{g}}, \tilde{\mathbf{W}}_{\mathbf{h}}$, and $\tilde{\mathbf{W}}_{\mathbf{f}}$ are u.u.b.

(ii) the bound of the tracking error vectors for $t \geq t_{0}$ can be derived as the form of (38), if the Lyapunov-like function candidates are chosen as (28). 
Remark 1: The term with $\sigma_{(\cdot)}$ in (30) is to modify the update law to robust the closed-loop system for the effect of the approximation error[26]. Suppose a sufficient number of basis functions $\beta_{(\cdot)}$ is selected so that the approximation error can be neglected then we may have $\sigma_{(\cdot)}=0$, and (31) becomes

$$
\dot{V}=-\left[\begin{array}{lll}
\mathbf{s}^{T} & \mathbf{e}_{\tau}^{T} & \mathbf{e}_{i}^{T}
\end{array}\right] \mathbf{Q}\left[\begin{array}{c}
\mathbf{s} \\
\mathbf{e}_{\tau} \\
\mathbf{e}_{i}
\end{array}\right] \leq 0
$$

It is easy to prove that $\mathbf{s}, \mathbf{e}_{\tau}$, and $\mathbf{e}_{i}$ are also square integrable. From (25), (26) and (27), $\dot{\mathbf{s}}$, $\dot{\mathbf{e}}_{\tau}$ and $\dot{\mathbf{e}}_{i}$ are bounded; as a result, asymptotic convergence of $\mathbf{s}, \mathbf{e}_{\tau}$ and $\mathbf{e}_{i}$ can easily be shown by Barbalat's lemma. This further implies that $\mathbf{i} \rightarrow \mathbf{i}_{d}, \boldsymbol{\tau}_{t} \rightarrow \boldsymbol{\tau}_{t d}$ and $\mathbf{q} \rightarrow \mathbf{q}_{d}$ even though $\mathbf{D}, \mathbf{C}, \mathbf{g}, \mathbf{h}, \mathbf{L}$, and $\mathbf{f}$ are all unknown.

Remark 2: Suppose $\varepsilon_{1}, \varepsilon_{2}$, and $\varepsilon_{3}$ cannot be ignored but their variation bounds are available[25,26] i.e. there exists positive constants $\delta_{1}, \delta_{2}$ and $\delta_{3}$ such that $\left\|\boldsymbol{\varepsilon}_{1}\right\| \leq \delta_{1}$, $\left\|\boldsymbol{\varepsilon}_{2}\right\| \leq \delta_{2}$ and $\left\|\boldsymbol{\varepsilon}_{3}\right\| \leq \delta_{3}$. To cover the effect of these bounded approximation errors, the desired transmission torque (16), the desired current (18), and the control input (22) are modified to be

$$
\begin{gathered}
\boldsymbol{\tau}_{t d}=\hat{\mathbf{g}}+\hat{\mathbf{D}} \dot{\mathbf{v}}+\hat{\mathbf{C}} \mathbf{v}-\mathbf{K}_{d} \mathbf{s}+\boldsymbol{\tau}_{\text {robust } 1} \\
\mathbf{i}_{d}=\mathbf{H}^{-1}\left[\boldsymbol{\Theta} \mathbf{x}_{p}+\boldsymbol{\Phi} \boldsymbol{\tau}_{t d}+\hat{\mathbf{h}}+\boldsymbol{\tau}_{\text {robust } 2}\right] \\
\mathbf{u}=\hat{\mathbf{f}}-\mathbf{K}_{c} \mathbf{e}_{i}+\boldsymbol{\tau}_{\text {robust } 3}, \mathbf{e}_{i}=\mathbf{i}-\mathbf{i}_{d}
\end{gathered}
$$

where $\tau_{\text {robust } 1,}, \tau_{\text {robust } 2}$ and $\tau_{\text {robust } 3}$ are robust terms to be designed. Let us consider the Lyapunov-like function candidate (28) and the update law (30) again. The time derivative of $V$ can be computed as

$$
\begin{aligned}
\dot{V}= & -\left[\begin{array}{lll}
\mathbf{s}^{T} & \mathbf{e}_{\tau}^{T} & \mathbf{e}_{i}^{T}
\end{array}\right] \mathbf{Q}\left[\begin{array}{c}
\mathbf{s} \\
\mathbf{e}_{\tau} \\
\mathbf{e}_{i}
\end{array}\right]+\delta_{1}\|\mathbf{s}\|+\delta_{2}\left\|\mathbf{e}_{\tau}\right\|+\delta_{3}\left\|\mathbf{e}_{i}\right\| \\
& +\mathbf{s}^{T} \boldsymbol{\tau}_{\text {robust } 1}+\mathbf{e}_{\tau}^{T} \boldsymbol{\tau}_{\text {robust } 2}+\mathbf{e}_{i}^{T} \boldsymbol{\tau}_{\text {robust } 3}
\end{aligned}
$$

By picking $\boldsymbol{\tau}_{\text {robust } 1}=-\delta_{1}\left[\operatorname{sgn}\left(s_{1}\right) \quad \cdots \quad \operatorname{sgn}\left(s_{n}\right)\right]^{T}$, where $s_{k}, k=1, \ldots, n$ is the $k$-th element of $\mathbf{s}, \boldsymbol{\tau}_{\text {robust } 2}=-\delta_{2}\left[\operatorname{sgn}\left(e_{\tau_{1}}\right) \quad \cdots \quad \operatorname{sgn}\left(e_{\tau_{n}}\right)\right]^{T}$ where $e_{\tau_{k}}, k=1, \ldots, 2 n$ is the $k$-th 
element of $\mathbf{e}_{\tau}$ and $\boldsymbol{\tau}_{\text {robust } 3}=-\delta_{3}\left[\operatorname{sgn}\left(e_{i_{1}}\right) \quad \cdots \quad \operatorname{sgn}\left(e_{i_{n}}\right)\right]^{T}$, where $e_{i_{k}}, k=1, \ldots, n$ is the $k$-th element of $\mathbf{e}_{i}$, we may have $\dot{V} \leq 0$, and asymptotic convergence of the state error can be concluded by Barbalat's lemma.

\section{Simulation Study}

Consider a 2-DOF planar robot (Fig.1) represented by the differential equation (1), (2) and (3). The quantities $m_{i}, l_{i}, l_{c i}$ and $I_{i}$ are mass, length, gravity center distance and inertia of link $i$, respectively. Actual values of link parameters in the simulation[34] are $m_{1}=0.5 \mathrm{~kg}, m_{2}=0.5 \mathrm{~kg}$, $l_{1}=l_{2}=0.75 \mathrm{~m}, l_{c 1}=l_{c 2}=0.375 \mathrm{~m}, I_{1}=0.09375 \mathrm{~kg}-\mathrm{m}^{2}$, and $I_{2}=0.046975 \mathrm{~kg}-\mathrm{m}^{2}$. The actuator inertias, damping, and joint stiffness are $\mathbf{J}=\operatorname{diag}(0.02,0.01)\left(\mathrm{kg} \cdot \mathrm{m}^{2}\right), \quad \mathbf{B}=\operatorname{diag}(5,4)(\mathrm{Nm} \cdot \mathrm{sec} / \mathrm{rad})$ and $\mathbf{K}=\operatorname{diag}(100,100)(\mathrm{Nm} / \mathrm{rad})$ respectively. The motor parameters are: $\mathbf{L}=\operatorname{diag}(0.025,0.025)(H), \mathbf{H}=\operatorname{diag}(10,10)(N-m / A)$. Considering the cases of highvelocity movement, we would like the end-point to track a $0.2 m$-radius circle centered at $(0.8$ $m, 1.0 \mathrm{~m})$ in 2 seconds without knowing its precise model. The initial conditions of the link angles and the motor angles are $\mathbf{q}=\boldsymbol{\theta}=\left[\begin{array}{llll}0.0022 & 1.5019 & 0 & 0\end{array}\right]^{\mathrm{T}}$. The initial value of the reference model state vector is $\boldsymbol{\tau}_{r}=\left[\begin{array}{llll}15.97 & -47.26 & 0 & 0\end{array}\right]^{\mathrm{T}}$ which is the same as the initial value of the desired reference input $\boldsymbol{\tau}_{d}$. The initial condition of the motor armature currents is $\mathbf{i}=\left[\begin{array}{ll}77.49 & -83.92\end{array}\right]^{\mathrm{T}}$ which is the same as the initial value of the desired reference current $\mathbf{i}_{d}$. The controller gains are selected as $\mathbf{K}_{d}=\operatorname{diag}(20,20)$, $\mathbf{\Lambda}=\operatorname{diag}(10,10)$, and $\mathbf{K}_{c}=\operatorname{diag}(50,50)$. Each element of $\mathbf{D}, \mathbf{C}, \mathbf{g}, \mathbf{h}$, and $\mathbf{f}$ is approximated by the first 11 terms of the Fourier series. The simulation results are shown in Fig. 2 to 9. Fig. 2 shows the tracking performance of the end-point and the desired trajectory in the Cartesian space. It is observed that the end-point trajectory converges nicely to the desired trajectory, although the initial position error is quite large. Fig. 3 is the joint space tracking performance. It shows that the transient response vanishes very quickly. Fig. 4 is the control inputs in voltage. Fig. 5 to 9 are the performance of function approximation for $\mathbf{D}, \mathbf{C}, \mathbf{g}, \mathbf{h}$, and $\mathbf{f}$ respectively. Since the reference input does not satisfy the persistent excitation condition, some estimates do not converge to their actual values but remain bounded as desired. It is worth to note that in designing the controller we do not need much knowledge for the system. All we have to do is to pick some controller parameters and some initial weighting matrices.

\section{Conclusions}

An adaptive controller is proposed for RLFJED robots containing time-varying uncertainties. A backstepping-like procedure is developed to deal with the cascade structure in its dynamic equations. The function approximation technique is employed to cope with the time-varying uncertainties. The closed loop stability is proved by using the Lyapunovlike analysis. The realization of the proposed controller does not need to calculate the regressor which is required in most adaptive designs for robot manipulators. Simulation 
results justify the performance of the proposed controller in fast tracking operations although most of the robot parameters are not available.

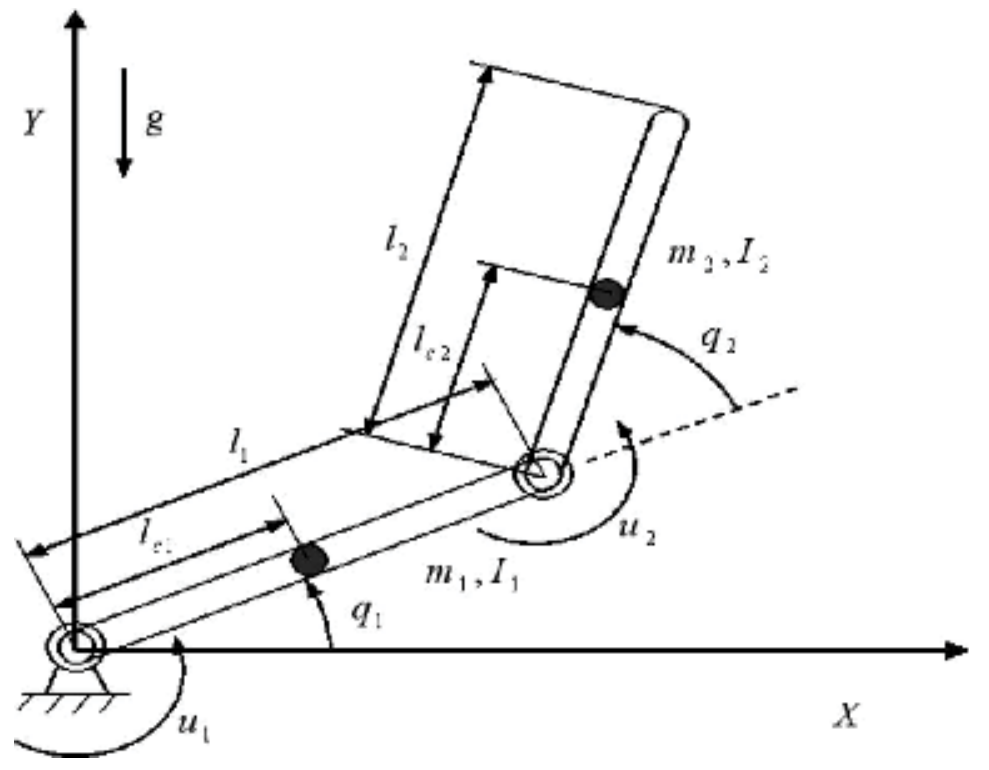

Figure 1. 2-DOF planar robot

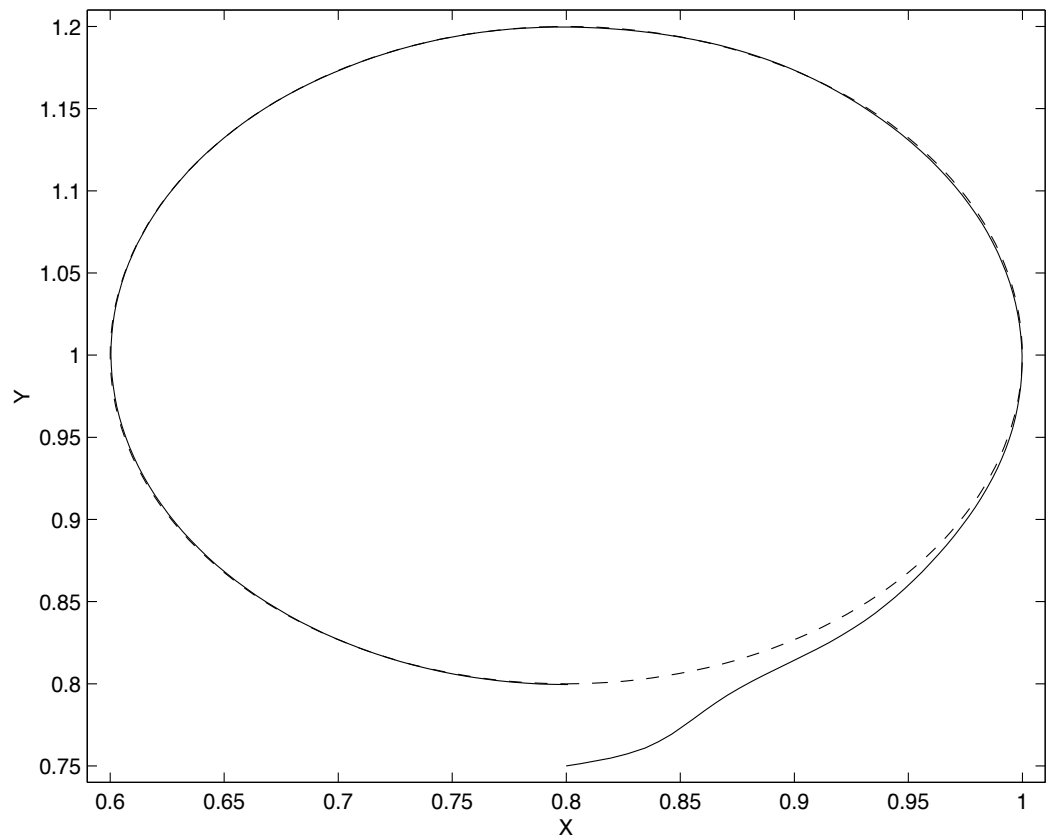

Figure 2. Tracking performance of end-point in the $X-Y$ space ( - actual; --- desired). The end-point is initialized at the point $(0.8 \mathrm{~m}, 0.75 \mathrm{~m})$ and is required to track a $0.2 \mathrm{~m}$-radius circle in 2 seconds. After some transient, the tracking error is very small, although we do not know precise dynamics of the robot 

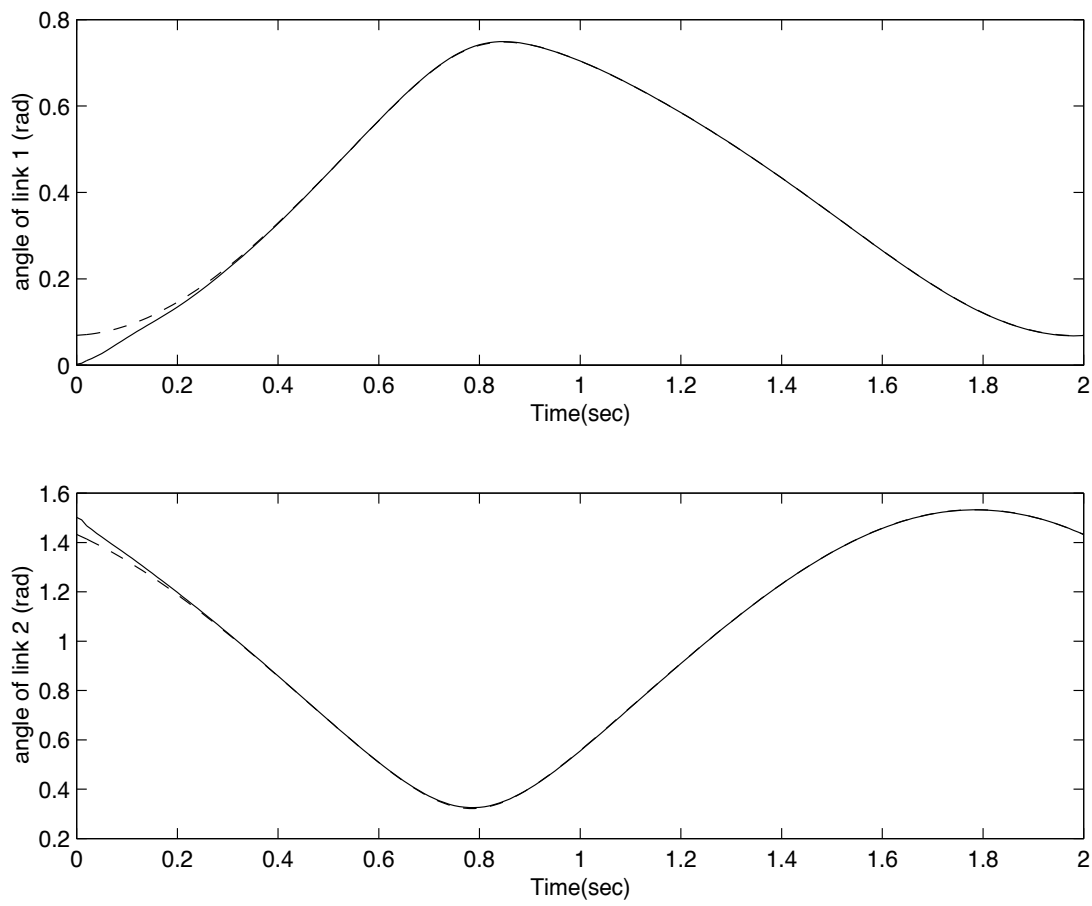

Figure 3. The joint space tracking performance (- actual; --- desired). The real trajectory converges very quickly
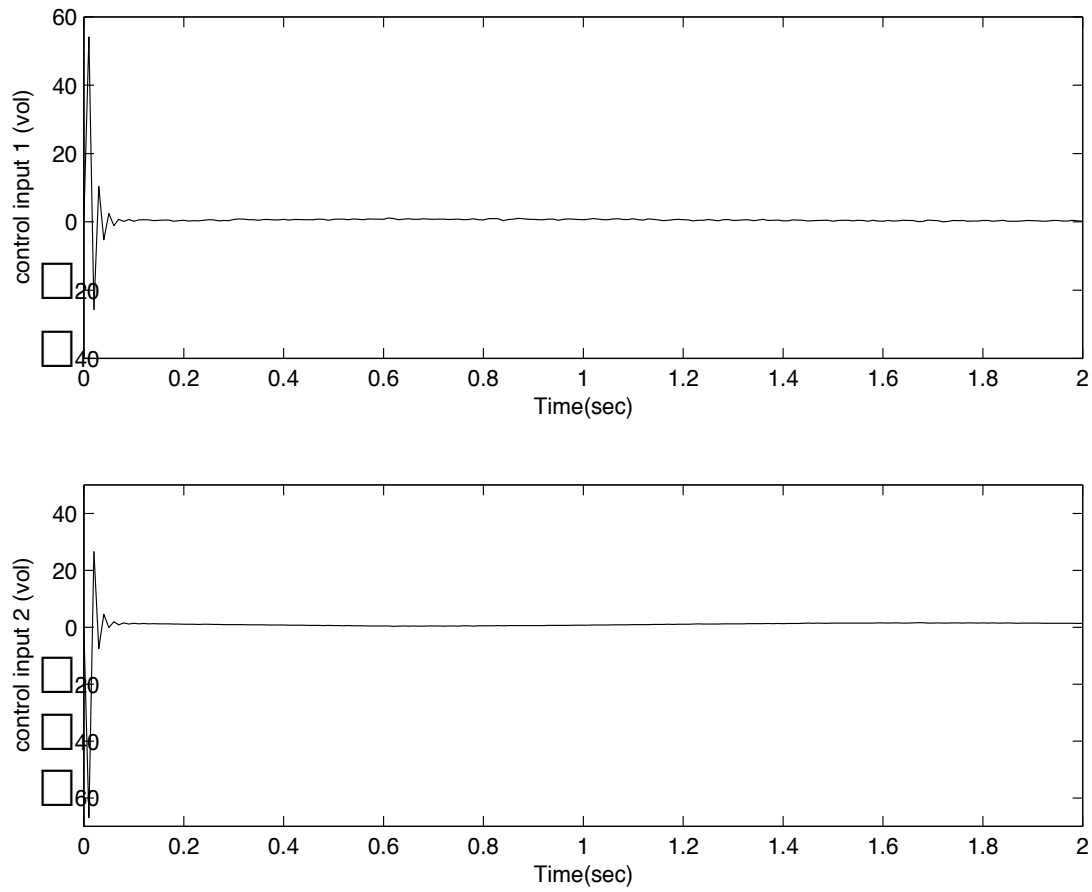

Figure 4. Control input voltage 

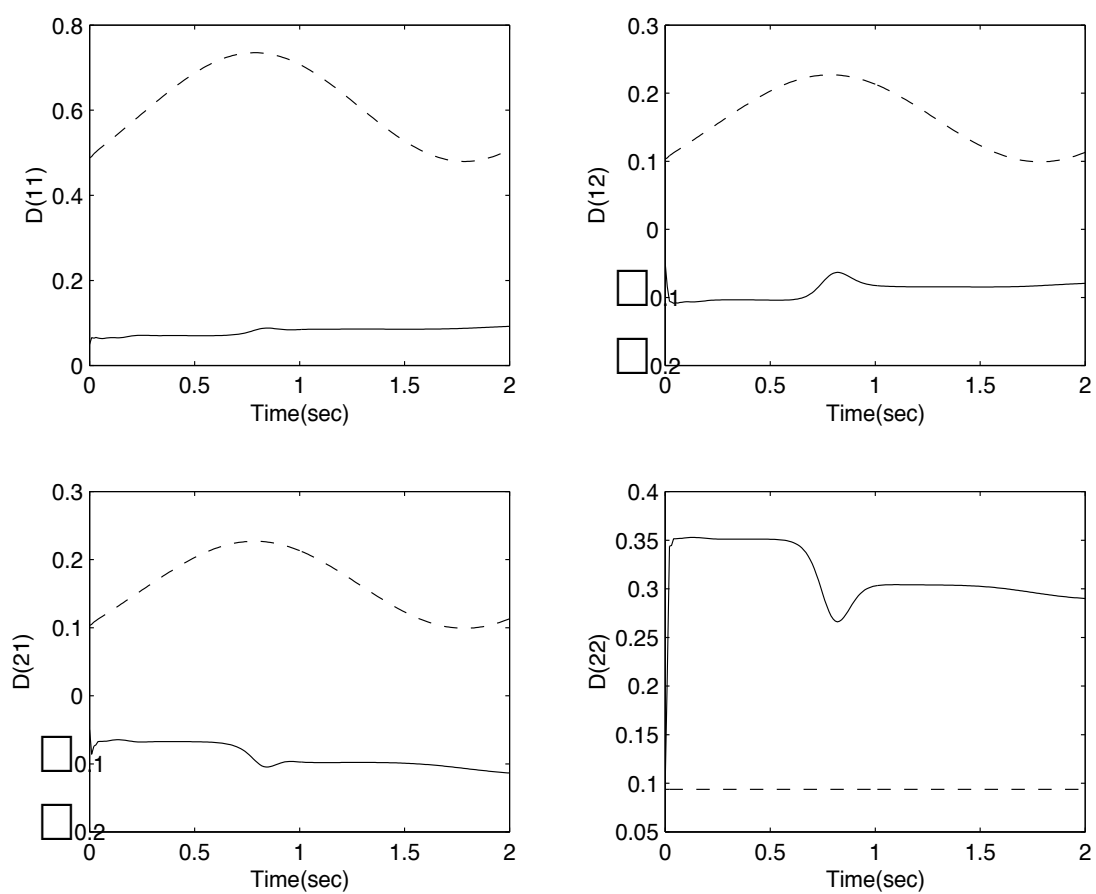

Figure 5. Approximation of $\mathbf{D}$ matrix ( - estimate; --- real). Although the estimated values do not converge to the true values, they are bounded and small
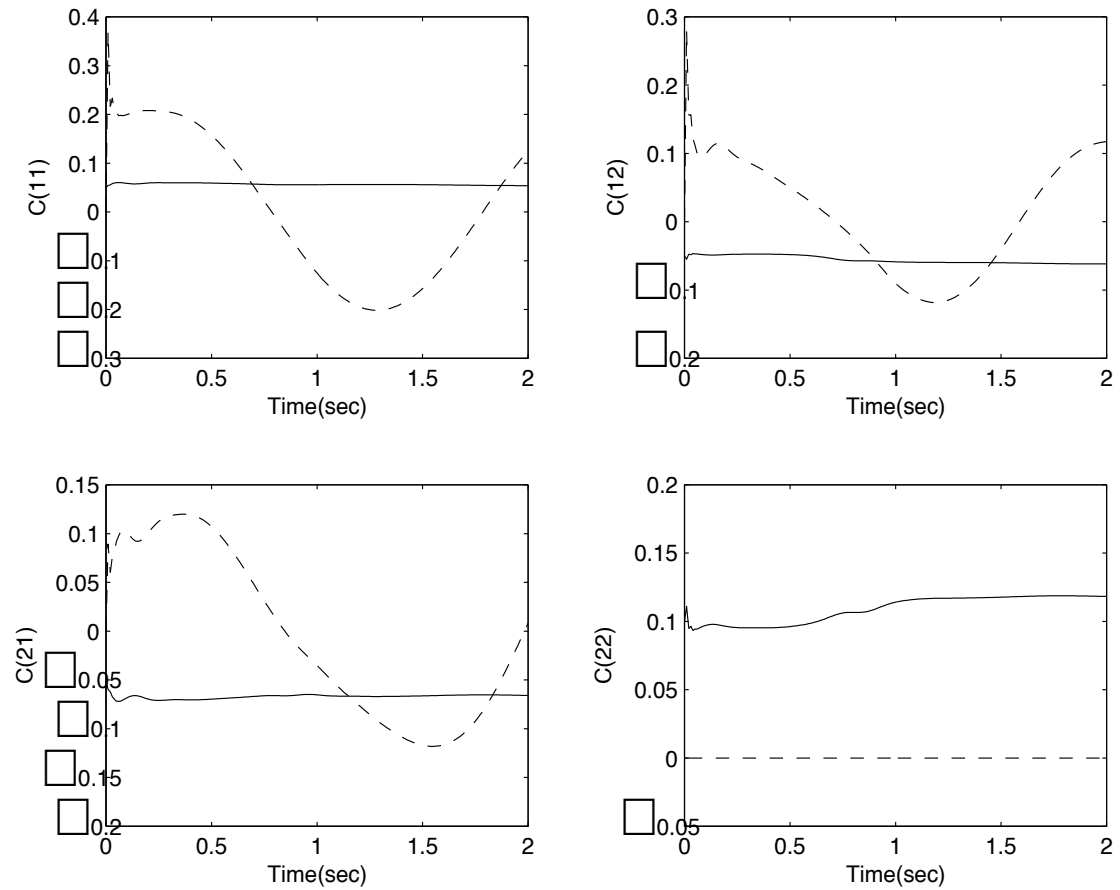

Figure 6. Approximation of $\mathbf{C}$ matrix(- estimate; --- real) 

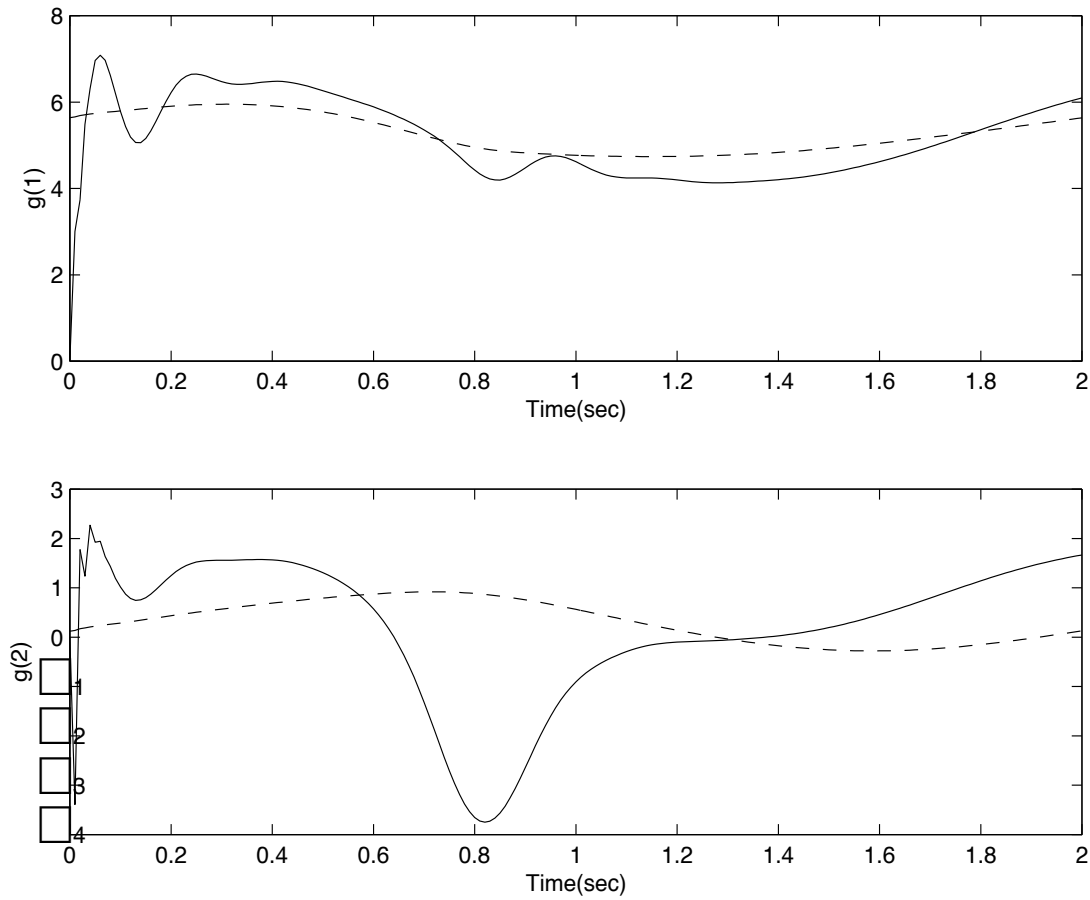

Figure 7. Approximation of vector $\mathbf{g}$ (- estimate; --- real)
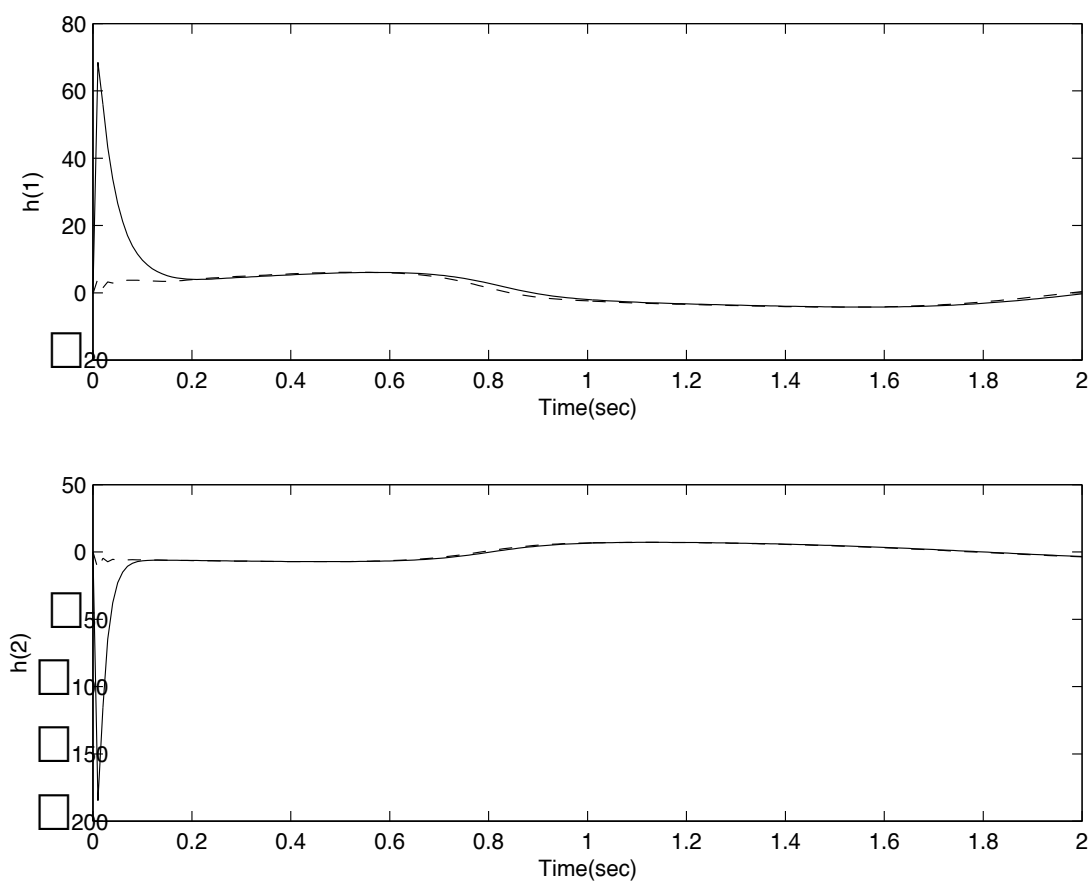

Figure 8. Approximation of vector $\mathbf{h}(-$ estimate; --- real) 

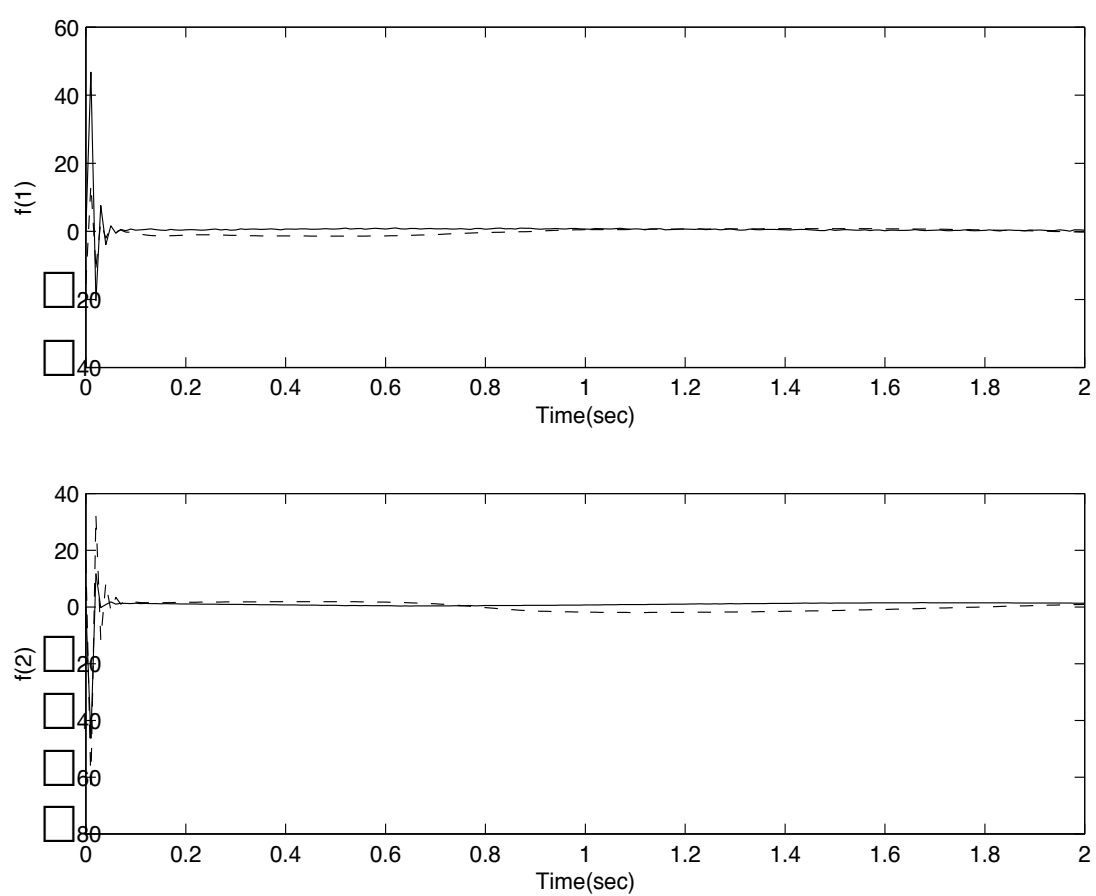

Figure 9. Approximation of vector $\mathbf{f}(-$ estimate; --- real $)$

\section{Reference}

M. C. Good, L. M. Sweet, and K. L. Strobel, Dynamic model for control system design of integrated robot and drive systems, J. Dynamic Systems, Measurement and Control, vol. 107, no.1, pp. 53-59, 1985. [1]

T. J. Tarn, A. K. Bejczy, X. Yun and Z. Li, Effect of motor dynamics on nonlinear feedback robot arm control, IEEE Trans. on Robotics and Automation, vol. 7, no. 1, pp.114-122, Feb. 1991. [2]

G. Liu and A. A. Goldenberg, Robust control of robot manipulators incorporating motor dynamics, in Proc. IEEE/RSJ Int. Conf. on Intelligent Robots and Systems, Yokohama, Japan, pp.68-75, 1993. [3]

M. D. Leviner and D. M. Dawson, Hybrid adaptive tracking control of rigid-link electrically driven robots actuated by switched reluctance motors, System Theory, Proc. SSST '93., Twenty-Fifth Southeastern Symposium, pp. 53-57, 1993. [4]

C. Y. Su and Y. Stepanenko, Guaranteed stability based control of robot manipulators incorporating motor dynamics, IEEE Int. Symposium on Industrial Electronics, pp. 345-350, 1994. [5]

C. Kwan, F. L. Lewis, and D. M. Dawson, Robust neural-network control of rigid-link electrically driven robots, IEEE Trans. on Neural Networks, vol. 9, no. 4, pp.581-588, July 1998. [6]

B. S. Chen, H. J. Uang and C. S. Tseng, Robust tracking enhancement of robot systems including motor dynamics: a fuzzy-based dynamics game approach, IEEE Trans. on Fuzzy Systems, vol. 6, no. 4, pp. 538-552, Nov. 1998. [7] 
M. Oya, C. Y. Su, and T. Kobayashi, State observer-based robust control scheme for electrically driven robot manipulators, IEEE Trans. on Robotics, vol. 20, no. 4, pp. 796-804, Aug. 2004. [8]

R. J. Wai, C. Y. Tu and P. C. Chen, Robust neural-fuzzy-network control for rigid-link electrically driven robot manipulator, in The 30th Annual Conf. of the IEEE Industrial Electronics Society, pp. 1763-1768, Busan, Korea, Nov. 2004. [9]

J. Hu, D. M. Dawson and Y. Ou, A global adaptive link position tracking controller for robot manipulators driven by induction motors, in Proc. IEEE Conf. on Decision and Control, pp.33-38, New Orleans, LA, December, 1995. [10]

M. M. Bridges and D. M. Dawson, Adaptive control of rigid-link electrically-driven robots actuated with switched reluctance motors, in Proc. American Control Conf., pp. 13921396, Seattle, Washington, June, 1995. [11]

J. Yuan and B. Yuan, A new adaptive robot controller taking account of motor dynamics, in Proc. American Control Conf., pp.1403-1407, 1995. [12]

H. Yu and S. Lloyd, Adaptive control of robot manipulators including motor dynamics, in Proc. American Control Conf., pp.3803-3807, Seattle, Washington, June, 1995. [13]

C. Y. Su and Y. Stepanenko, Redesign of Hybrid adaptive/robust motion control of rigidlink electrically-driven robot manipulators, IEEE Trans. on Robotics and Automation, vol. 14, no. 4, pp. 651-655, Aug. 1998. [14]

C. Ishii, T. Shen, and Z. Qu, Lyapunov recursive design of robot adaptive tracking control with $\mathrm{L}_{2}$-gain performance for electrically-driven robot manipulators, Int. J. Control, vol. 74, no. 8, pp. 811-828, 2001. [15]

Y. C. Chang, Adaptive tracking control for electrically-driven robots without overparametrization, Int. J. of Adaptive Control and Signal Processing, vol. 16, pp. 123150, 2002. [16]

G. G. Ramirez and Y. Tang, Adaptive nonlinear control of rigid robots driven by current-fed induction motors, in Proc. IEEE Conf. on Decision and Control, pp. 2038-2043, Las Vegas, Nevada, Dec. 2002. [17]

S. H. Hsu and L. C. Fu, Adaptive decentralized control of robot manipulators driven by current-fed induction motors, IEEE/ASME Trans. on Mechatronics, vol. 10, no. 4, pp. 465-468, Aug. 2005. [18]

A. Ailon, R. Lozano, and M. I. Gil', Point-to-point regulation of a robot with flexible joints including electrical effects of actuator dynamics, IEEE Trans. on Automatic Control, vol. 42, no. 4, pp. 559-564, April, 1997. [19]

A. Ailon, M. I. Gil', E. S. Choi and B. H. Ahn, Stabilizing robots with uncertain parameters actuated by DC motors with flexible coupling shafts, in Proc. IEEE Int. Conf. on Control Applications, pp. 877-881, Trieste, Italy, Sept. 1998. [20]

A. Ailon, M. I. Gil' and E. S. Choi, A simple linear stabilizing controller for a RLED robot with uncertain parameters and some related applications, Int. J. Control, vol. 72, no. 6, pp.546-559, 1999. [21]

A. Ailon, R. Lozano and M. I. Gil', Iterative regulation of an electrically driven flexible-joint robot with model uncertainties, IEEE Trans. on Robotics and Automation, vol. 16, no. 6, pp. 863-870, Dec. 2000. [22]

R. Ortega and M. W. Spong, Adaptive motion control of rigid robots: a tutorial, Automatica, vol. 25, pp. 509-519, 1989. [23] 
A. C. Huang and Y. S. Kuo (2001). Sliding control of nonlinear systems containing timevarying uncertainties with unknown bounds, Int. J. Control, vol.74, pp.252-264, 2001. [24]

S. S. Ge, C. C. Hang, T. H. Lee and T. Zang, Stable Adaptive Neural Network Control, Boston: Kluwer Academic, 2001. [25]

J. T. Spooner, M. Maggiore, R. Ordonez and K. M. Passino, Stable Adaptive Control and Estimation for Nonlinear Systems - Neural and Fuzzy Approximator Techniques, NY: John Wiley \& Sons, 2002 [26]

M. C. Chien and A. C. Huang, Adaptive impedance control of robot manipulators based on function approximation technique, Robotica, vol. 22, pp. 395-403, 2004. [27]

A. C. Huang and Y. C. Chen, Adaptive multiple-surface sliding control for non-autonomous systems with mismatched uncertainties, Automatica, vol. 40, pp. 1939-1945, 2004. [28]

A. C. Huang and Y. C. Chen (2004). Adaptive sliding control for single-link flexible-joint robot with mismatched uncertainties, IEEE Trans. on Control Systems Technology, vol. 12, pp. 770-775, 2004. [29]

P. C. Chen and A. C. Huang, Adaptive sliding control of non-autonomous active suspension systems with time-vary loadings, J. of Sound and Vibration, vol. 282, no. 3-5, pp. 1119-1135, 2005. [30]

P. C. Chen and A. C. Huang, Adaptive Sliding Control of Active Suspension Systems with Uncertain Hydraulic Actuator Dynamics, Vehicle System Dynamics, vol. 44, no. 5, pp357-368, 2006. [31]

A. C. Huang, S. C. Wu, and W. F. Ting, An FAT-based Adaptive Controller for Robot Manipulators without Regressor Matrix: Theory and Experiments, Robotica, vol. 24, pp. 205-210, 2006. [32]

A. C. Huang and K. K. Liao, FAT-based Adaptive Sliding Control for Flexible Arms, Theory and Experiments, Journal of Sound and Vibration, vol. 298, issue 1-2, pp. 194-205, 2006. [33]

M. C. Chien and A. C. Huang, Adaptive control of flexible-joint electrically-driven robot with time-varying uncertainties, IEEE Trans. Industrial Electronics, vol.54, no.2, pp.1032-1038, 2007. [34]

M. C. Chien and A. C. Huang, Adaptive Control of Electrically-driven Robot without Computation of Regressor Matrix, J. Chinese Institute of Engineers, vol.30, no.5, pp.855-862, 2007. [35]

M. W. Spong, Modeling and control of elastic joint robots, ASME J. Dynamic Systems, Measurement, and Control, vol.109, pp.310-319, 1987. [36]

T. Lin and A. A. Goldenberg, Robust adaptive control of flexible joint robots with joint torque feedback, in Proc. IEEE Conf. Robotics and Automation, pp.1229-1234, 1995. [37]

K. S. Narendra and A. M. Annaswamy, Stable Adaptive System, Prentice Hall, 1989. [38]

J-J. E. Slotine and W. Li, Applied Nonlinear Control, Prentice Hall, NJ, 1991. [39]

Khalil, H.K., Nonlinear Systems, Prentice-Hall, New Jersey, 2002. [40] 


\section{Appendix}

Lemma A.1:

Let $\mathbf{S} \in \mathfrak{R}^{n}, \boldsymbol{\varepsilon} \in \mathfrak{R}^{n}$ and $\mathbf{K}$ is the $n \times n$ positive definite matrix. Then,

$$
-\mathbf{s}^{T} \mathbf{K} \mathbf{s}+\mathbf{s}^{T} \boldsymbol{\varepsilon} \leq \frac{1}{2}\left[\lambda_{\min }(\mathbf{K})\|\mathbf{s}\|^{2}-\frac{\|\boldsymbol{\varepsilon}\|^{2}}{\lambda_{\min }(\mathbf{K})}\right] .
$$

Proof:

$$
\begin{aligned}
& -\mathbf{s}^{T} \mathbf{K s}+\mathbf{s}^{T} \boldsymbol{\varepsilon} \leq\left[-\lambda_{\text {min }}(\mathbf{K})\|\mathbf{s}\|+\|\boldsymbol{\varepsilon}\|\right]\|\mathbf{s}\| \\
& =-\frac{1}{2}\left[\sqrt{\lambda_{\min }(\mathbf{K})}\|\mathbf{s}\|-\frac{\|\boldsymbol{\varepsilon}\|}{\sqrt{\lambda_{\text {min }}(\mathbf{K})}}\right]^{2} \\
& -\frac{1}{2}\left[\lambda_{\min }(\mathbf{K})\|\mathbf{s}\|^{2}-\frac{\|\boldsymbol{\varepsilon}\|^{2}}{\lambda_{\text {min }}(\mathbf{K})}\right] \\
& \leq-\frac{1}{2}\left[\lambda_{\text {min }}(\mathbf{K})\|\mathbf{s}\|^{2}-\frac{\|\boldsymbol{\varepsilon}\|^{2}}{\lambda_{\text {min }}(\mathbf{K})}\right]
\end{aligned}
$$

\section{Lemma A.2:}

Let $\mathbf{w}_{i}^{T}=\left[\begin{array}{llll}w_{i 1} & w_{i 2} & \cdots & w_{i n}\end{array}\right] \in \mathfrak{R}^{1 \times n}, i=1, \ldots, m$ and $\mathbf{W}$ is a block diagonal matrix defined as $\mathbf{W}=\operatorname{diag}\left\{\mathbf{w}_{1}, \mathbf{w}_{2}, \cdots, \mathbf{w}_{m}\right\} \in \mathfrak{R}^{m n \times m}$. Then,

$$
\operatorname{Tr}\left(\mathbf{W}^{T} \mathbf{W}\right)=\sum_{i=1}^{m}\left\|\mathbf{w}_{i}\right\|^{2} .
$$

The notation $\operatorname{Tr}($.$) denotes the trace operation.$

Proof: The proof is straightforward as below: 


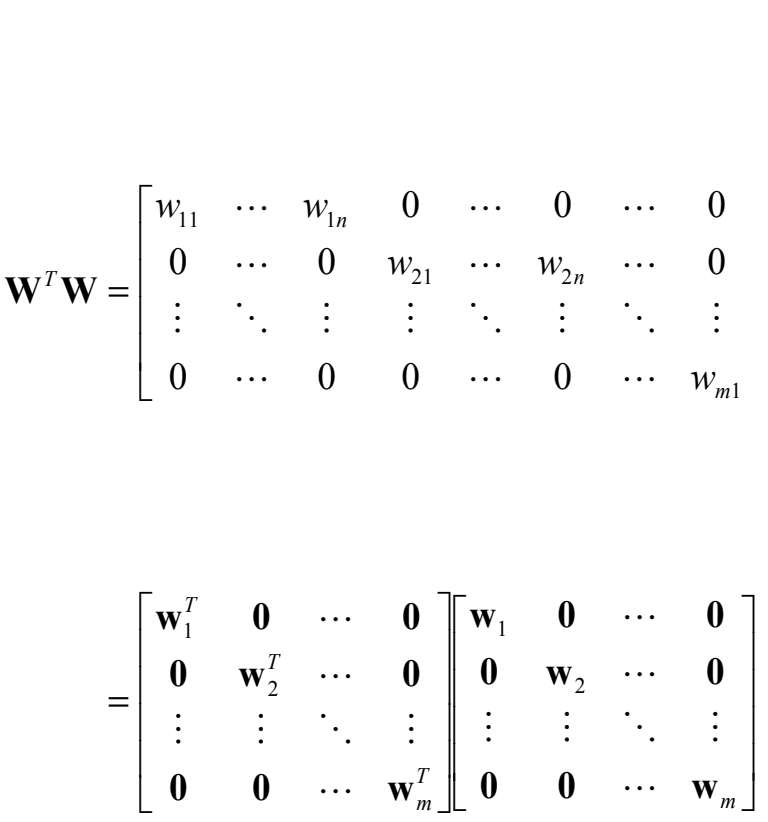

$$
\begin{aligned}
& =\left[\begin{array}{cccc}
\mathbf{w}_{1}^{T} \mathbf{w}_{1} & \mathbf{0} & \cdots & \mathbf{0} \\
\mathbf{0} & \mathbf{w}_{2}^{T} \mathbf{w}_{2} & \cdots & \mathbf{0} \\
\vdots & \vdots & \ddots & \vdots \\
\mathbf{0} & \mathbf{0} & \cdots & \mathbf{w}_{m}^{T} \mathbf{w}_{m}
\end{array}\right] \\
& =\left[\begin{array}{cccc}
\left\|\mathbf{w}_{1}\right\|^{2} & \mathbf{0} & \cdots & \mathbf{0} \\
\mathbf{0} & \left\|\mathbf{w}_{2}\right\|^{2} & \cdots & \mathbf{0} \\
\vdots & \vdots & \ddots & \vdots \\
\mathbf{0} & \mathbf{0} & \cdots & \left\|\mathbf{w}_{m}\right\|^{2}
\end{array}\right]
\end{aligned}
$$

The last equality holds because by definition $\mathbf{w}_{i}^{T} \mathbf{w}_{i}=w_{i 1}^{2}+w_{i 2}^{2}+\ldots+w_{i m}^{2}=\left\|\mathbf{w}_{i}\right\|^{2}$.

Therefore, we have

$$
\operatorname{Tr}=\left(\mathbf{W}^{T} \mathbf{W}\right)=\sum_{i=1}^{m}\left\|\mathbf{w}_{i}\right\|^{2}
$$

Lemma A.3:

Suppose $\mathbf{w}_{i}^{T}=\left[\begin{array}{llll}w_{i 1} & w_{i 2} & \cdots & w_{i n}\end{array}\right] \in \mathfrak{R}^{1 \times n}$ and $\mathbf{v}_{i}^{T}=\left[\begin{array}{llll}v_{i 1} & v_{i 2} & \cdots & v_{i n}\end{array}\right] \in \mathfrak{R}^{1 \times n}$, $i=1, \ldots, m$. Let $\mathbf{W}$ and $\mathbf{V}$ be block diagonal matrices that are defined as $\mathbf{W}=\operatorname{diag}\left\{\mathbf{w}_{1}, \mathbf{w}_{2}, \cdots, \mathbf{w}_{m}\right\} \in \mathfrak{R}^{m n \times m} \quad$ and $\quad \mathbf{V}=\operatorname{diag}\left\{\mathbf{v}_{1}, \mathbf{v}_{2}, \cdots, \mathbf{v}_{m}\right\} \in \mathfrak{R}^{m n \times m}$, respectively. Then, 


$$
\operatorname{Tr}\left(\mathbf{V}^{T} \mathbf{W}\right) \leq \sum_{i=1}^{m}\left\|\mathbf{v}_{i}\right\|\left\|\mathbf{w}_{i}\right\|
$$

Proof: The proof is also straightforward:

$$
\begin{aligned}
\mathbf{V}^{T} \mathbf{W} & =\left[\begin{array}{cccc}
\mathbf{v}_{1}^{T} & \mathbf{0} & \cdots & \mathbf{0} \\
\mathbf{0} & \mathbf{v}_{2}^{T} & \cdots & \mathbf{0} \\
\vdots & \vdots & \ddots & \vdots \\
\mathbf{0} & \mathbf{0} & \cdots & \mathbf{v}_{m}^{T}
\end{array}\right]\left[\begin{array}{cccc}
\mathbf{w}_{1} & \mathbf{0} & \cdots & \mathbf{0} \\
\mathbf{0} & \mathbf{w}_{2} & \cdots & \mathbf{0} \\
\vdots & \vdots & \ddots & \vdots \\
\mathbf{0} & \mathbf{0} & \cdots & \mathbf{w}_{m}
\end{array}\right] \\
& =\left[\begin{array}{cccc}
\mathbf{v}_{1}^{T} \mathbf{w}_{1} & \mathbf{0} & \cdots & \mathbf{0} \\
\mathbf{0} & \mathbf{v}_{2}^{T} \mathbf{w}_{2} & \cdots & \mathbf{0} \\
\vdots & \vdots & \ddots & \vdots \\
\mathbf{0} & \mathbf{0} & \cdots & \mathbf{v}_{m}^{T} \mathbf{w}_{m}
\end{array}\right]
\end{aligned}
$$

Hence,

$$
\begin{aligned}
\operatorname{Tr}\left(\mathbf{V}^{T} \mathbf{W}\right) & =\mathbf{v}_{1}^{T} \mathbf{w}_{1}+\mathbf{v}_{2}^{T} \mathbf{w}_{2}+\ldots+\mathbf{v}_{m}^{T} \mathbf{w}_{m} \\
& \leq\left\|\mathbf{v}_{1}\right\|\left\|\mathbf{w}_{1}\right\|+\left\|\mathbf{v}_{2}\right\|\left\|\mathbf{w}_{2}\right\|+\ldots+\left\|\mathbf{v}_{m}\right\|\left\|\mathbf{w}_{m}\right\| \\
& =\sum_{i=1}^{m}\left\|\mathbf{v}_{i} \mid\right\| \mathbf{w}_{i} \|
\end{aligned}
$$

Lemma A.4:

Let $\mathbf{W}$ be defined as in Lemma $A .2$, and $\widetilde{\mathbf{W}}$ is a matrix defined as $\widetilde{\mathbf{W}}=\mathbf{W}-\hat{\mathbf{W}}$, where $\hat{\mathbf{W}}$ is a matrix with proper dimension. Then

$$
\operatorname{Tr}\left(\widetilde{\mathbf{W}}^{T} \hat{\mathbf{W}}\right) \leq \frac{1}{2} \operatorname{Tr}\left(\mathbf{W}^{T} \mathbf{W}\right)-\frac{1}{2} \operatorname{Tr}\left(\widetilde{\mathbf{W}}^{T} \widetilde{\mathbf{W}}\right) .
$$

Proof: 


$$
\begin{aligned}
\operatorname{Tr}\left(\widetilde{\mathbf{W}}^{T} \hat{\mathbf{W}}\right) & =\operatorname{Tr}\left(\widetilde{\mathbf{W}}^{T} \mathbf{W}\right)-\operatorname{Tr}\left(\widetilde{\mathbf{W}}^{T} \widetilde{\mathbf{W}}\right) \\
& \leq \sum_{i=1}^{m}\left(\left\|\widetilde{\mathbf{w}}_{i}\right\|\left\|\mathbf{w}_{i}\right\|-\left\|\widetilde{\mathbf{w}}_{i}\right\|^{2}\right) \quad(\text { by Lemma A.2 and A.3) } \\
& =\frac{1}{2} \sum_{i=1}^{m}\left[\left\|\mathbf{w}_{i}\right\|^{2}-\left\|\widetilde{\mathbf{w}}_{i}\right\|^{2}-\left(\left\|\widetilde{\mathbf{w}}_{i}\right\|-\left\|\mathbf{w}_{i}\right\|\right)^{2}\right] \\
& \leq \frac{1}{2} \sum_{i=1}^{m}\left(\left\|\mathbf{w}_{i}\right\|^{2}-\left\|\widetilde{\mathbf{w}}_{i}\right\|^{2}\right) \\
& =\frac{1}{2} \operatorname{Tr}\left(\mathbf{W}^{T} \mathbf{W}\right)-\frac{1}{2} \operatorname{Tr}\left(\widetilde{\mathbf{W}}^{T} \tilde{\mathbf{W}}\right) \quad(\text { by Lemma A.2) }
\end{aligned}
$$

Q.E.D.

In the above lemmas, we consider properties of a block diagonal matrix. In the following, we would like to extend the analysis to a class of more general matrices.

Lemma A.5:

Let $\mathbf{W}$ be a matrix in the form $\mathbf{W}^{T}=\left[\begin{array}{lllll}\mathbf{W}_{1}^{T} & \mathbf{W}_{2}^{T} & \cdots & \mathbf{W}_{p}^{T}\end{array}\right] \in \mathfrak{R}^{p m n \times m}$ where $\mathbf{W}_{i}=\operatorname{diag}\left\{\mathbf{w}_{i 1}, \mathbf{w}_{i 2}, \cdots, \mathbf{w}_{i m}\right\} \in \mathfrak{R}^{m n \times m}, i=1, \ldots, p$, are block diagonal matrices with the entries of vectors $\mathbf{w}_{i j}^{T}=\left[\begin{array}{llll}w_{i j 1} & w_{i j 2} & \cdots & w_{i j n}\end{array}\right] \in \mathfrak{R}^{1 \times n}, j=1, \ldots, m$. Then, we may have

$$
\operatorname{Tr}\left(\mathbf{W}^{T} \mathbf{W}\right)=\sum_{i=1}^{p} \sum_{j=1}^{m}\left\|\mathbf{W}_{i j}\right\|^{2}
$$

Proof:

$$
\begin{aligned}
\mathbf{W}^{T} \mathbf{W} & =\left[\begin{array}{lll}
\mathbf{W}_{1}^{T} & \cdots & \mathbf{W}_{p}^{T}
\end{array}\right]\left[\begin{array}{c}
\mathbf{W}_{1} \\
\vdots \\
\mathbf{W}_{p}
\end{array}\right] \\
& =\mathbf{W}_{1}^{T} \mathbf{W}_{1}+\cdots+\mathbf{W}_{p}^{T} \mathbf{W}_{p}
\end{aligned}
$$

Hence, we may calculate the trace as 


$$
\begin{aligned}
\operatorname{Tr}\left(\mathbf{W}^{T} \mathbf{W}\right) & =\operatorname{Tr}\left(\mathbf{W}_{1}^{T} \mathbf{W}_{1}\right)+\cdots+\operatorname{Tr}\left(\mathbf{W}_{p}^{T} \mathbf{W}_{p}\right) \\
& =\sum_{j=1}^{m}\left\|\mathbf{w}_{1 j}\right\|^{2}+\cdots+\sum_{j=1}^{m}\left\|\mathbf{w}_{p j}\right\|^{2} \\
& =\sum_{i=1}^{p} \sum_{j=1}^{m}\left\|\mathbf{W}_{i j}\right\|^{2}
\end{aligned}
$$

Lemma A.6:

Q.E.D.

Let $\mathbf{V}$ and $\mathbf{W}$ be matrices defined in Lemma A.5, Then, $\operatorname{Tr}\left(\mathbf{V}^{T} \mathbf{W}\right) \leq \sum_{i=1}^{p} \sum_{j=1}^{m}\left\|\mathbf{V}_{i j}\right\|\left\|\mathbf{w}_{i j}\right\|$.

(A.6)

Proof:

$$
\begin{aligned}
\operatorname{Tr}\left(\mathbf{V}^{T} \mathbf{W}\right) & =\operatorname{Tr}\left(\mathbf{V}_{1}^{T} \mathbf{W}_{1}\right)+\cdots+\operatorname{Tr}\left(\mathbf{V}_{p}^{T} \mathbf{W}_{p}\right) \\
& \leq \sum_{j=1}^{m}\left\|\mathbf{v}_{1 j}\right\|\left\|\mathbf{W}_{1 j}\right\|+\cdots+\sum_{j=1}^{m}\left\|\mathbf{v}_{p j}\right\|\left\|\mathbf{w}_{p j}\right\| \\
& =\sum_{i=1}^{p} \sum_{j=1}^{m}\left\|\mathbf{v}_{i j} \mid\right\| \mathbf{w}_{i j} \|
\end{aligned}
$$

\section{Lemma A.7:}

Q.E.D.

Let $\mathbf{W}$ be defined as in Lemma A.5, and $\widetilde{\mathbf{W}}$ is a matrix defined as $\widetilde{\mathbf{W}}=\mathbf{W}-\hat{\mathbf{W}}$, where $\hat{\mathbf{W}}$ is a matrix with proper dimension. Then

$$
\operatorname{Tr}\left(\widetilde{\mathbf{W}}^{T} \hat{\mathbf{W}}\right) \leq \frac{1}{2} \operatorname{Tr}\left(\mathbf{W}^{T} \mathbf{W}\right)-\frac{1}{2} \operatorname{Tr}\left(\widetilde{\mathbf{W}}^{T} \tilde{\mathbf{W}}\right) .
$$


Proof:

$$
\begin{aligned}
\operatorname{Tr}\left(\widetilde{\mathbf{W}}^{T} \hat{\mathbf{W}}\right) & =\operatorname{Tr}\left(\widetilde{\mathbf{W}}^{T} \mathbf{W}\right)-\operatorname{Tr}\left(\widetilde{\mathbf{W}}^{T} \widetilde{\mathbf{W}}\right) \\
& \leq \sum_{i=1}^{p} \sum_{j=1}^{m}\left(\left\|\widetilde{\mathbf{w}}_{i j}\right\|\left\|\mathbf{w}_{i j}\right\|-\left\|\widetilde{\mathbf{w}}_{i j}\right\|^{2}\right) \quad(\text { by Lemma A.5 and A.6) } \\
& =\frac{1}{2} \sum_{i=1}^{p} \sum_{j=1}^{m}\left[\left\|\mathbf{w}_{i j}\right\|^{2}-\left\|\widetilde{\mathbf{w}}_{i j}\right\|^{2}-\left(\left\|\widetilde{\mathbf{w}}_{i j}\right\|-\left\|\mathbf{w}_{i j}\right\|\right)^{2}\right] \\
& \leq \frac{1}{2} \sum_{i=1}^{p} \sum_{j=1}^{m}\left(\left\|\mathbf{w}_{i j}\right\|^{2}-\left\|\widetilde{\mathbf{w}}_{i j}\right\|^{2}\right) \\
& =\frac{1}{2} \operatorname{Tr}\left(\mathbf{W}^{T} \mathbf{W}\right)-\frac{1}{2} \operatorname{Tr}\left(\widetilde{\mathbf{W}}^{T} \widetilde{\mathbf{W}}\right) \quad(\text { by Lemma A.5 })
\end{aligned}
$$




\title{
Global Feed-forward Adaptive Fuzzy Control of Uncertain MIMO Nonlinear Systems
}

\author{
Chian-Song Chiu ${ }^{1}$, and Kuang-Yow Lian ${ }^{2}$ \\ ${ }^{1}$ Chung-Yuan Christian University, ${ }^{2}$ National Taipei University of Technology \\ Taiwan, R.O.C.
}

\section{Abstract}

This study proposes a novel adaptive control approach using a feedforward Takagi-Sugeno (TS) fuzzy approximator for a class of highly unknown multi-input multi-output (MIMO) nonlinear plants. First of all, the design concept, namely, feedforward fuzzy approximator (FFA) based control, is introduced to compensate the unknown feedforward terms required during steady state via a forward TS fuzzy system which takes the desired commands as the input variables. Different from the traditional fuzzy approximation approaches, this scheme allows easier implementation and drops the boundedness assumption on fuzzy universal approximation errors. Furthermore, the controller is synthesized to assure either the disturbance attenuation or the attenuation of both disturbances and estimated fuzzy parameter errors or globally asymptotic stable tracking. In addition, all the stability is guaranteed from a feasible gain solution of the derived linear matrix inequality (LMI). Meanwhile, the highly uncertain holonomic constrained systems are taken as applications with either guaranteed robust tracking performances or asymptotic stability in a global sense. It is demonstrated that the proposed adaptive control is easily and straightforwardly extended to the robust TS FFA-based motion/force tracking controller. Finally, two planar robots transporting a common object is taken as an application example to show the expected performance. The comparison between the proposed and traditional adaptive fuzzy control schemes is also performed in numerical simulations.

Keywords: Adaptive control; Takagi-Sugeno (TS) fuzzy system; holonomic systems; motion/force control.

\section{Introduction}

In recent years, plenty of adaptive fuzzy control methods (Wang \& Mendel, 1992)-(Alata et al., 2001) have been proposed to deal with the control problem of poorly modeled plants. All these researches are based on the fuzzy universal approximator (first proposed by Wang \& Mendel, 1992), which is properly adjusted to compensate the uncertainties as close as possible. Due to the use of states as the inputs of the fuzzy system, we call this approach as the state-feedback fuzzy approximator (SFA) based control. In details, this methodology can be further classified into two types: i) Mamdani fuzzy approximator (Wang \& Mendel, 1992;

*Email: acs.chiu@gmail.com 
Chen et al., 1996; Lee \& Tomizuka, 2000; Lin \& Chen, 2002); and ii) Takagi-Sugeno (TS) fuzzy approximator (Ying, 1998; Tsay et al., 1999; Chen \& Wong, 2000; Alata et al., 2001). The first type approach constructs the consequent part only via tunable fuzzy sets, but a good enough approximation usually requires a large number of fuzzy rules. In contrast, the TS SFA-based controller uses the linear/nonlinear combination of states in consequent part such that fewer rules are required. Without loss of generality, the configuration of these controllers is shown in Fig. 1. The SFA-based control contains the following disadvantages: i) numerous fuzzy rules and tuning parameters are required, especially for multivariable systems; ii) the fuzzy approximation error is assumed a priori to be upper bounded although the bound depends on state variables; and iii) the consequent part of TS fuzzy approximator will become complex for dealing with multivariable nonlinear systems, i.e., needing a complicated consequent part.

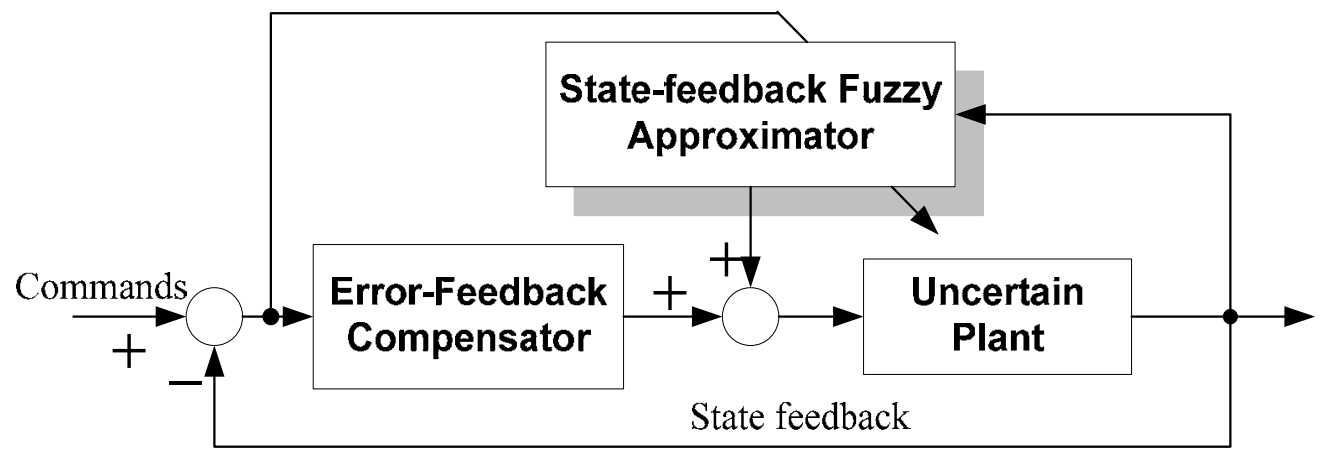

Figure 1. Configuration of SFA-based adaptive controller

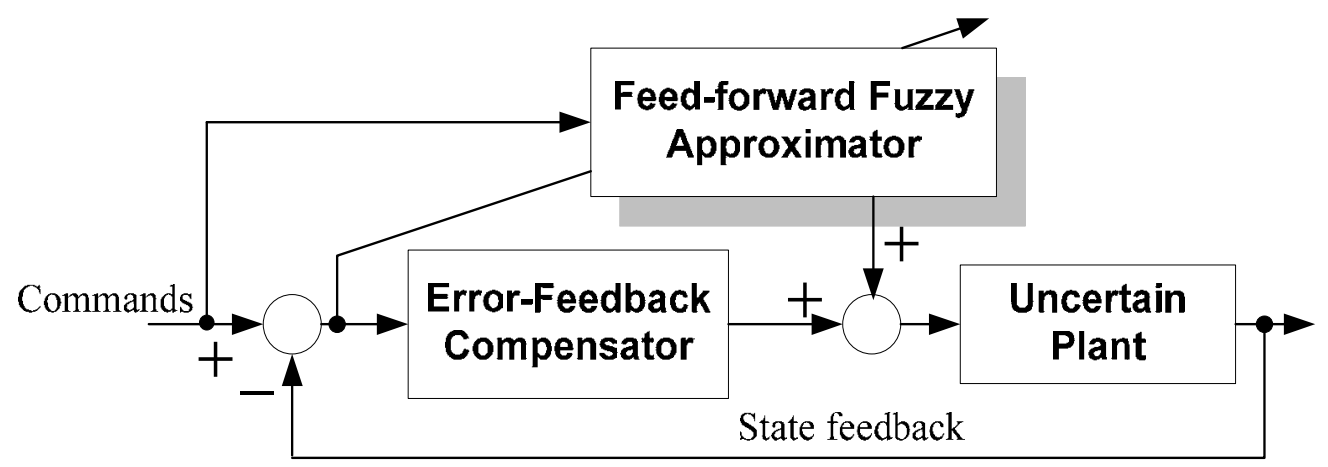

Figure 2. Configuration of FFA-based adaptive controller

To remove the above limitations, this study introduces the feed-forward fuzzy approximator (FFA) based control which takes the desired commands as the premise variables of fuzzy rules and approximately compensates an unknown feed-forward term required during steady state (note that the configuration is illustrated in Fig. 2). At the first glance, the SFA and FFA based control methods have a common adaptive learning concept, that is the feedback-error is used for tuning parameters of the compensator. But, a closer investigation reveals the differences on: i) the type of training signals, ii) the process of taming dynamic uncertainties; and iii) the 
type of error feedback terms. Especially, compared to SFA-based approaches (shown in Fig. 1), the FFA-based adaptive controller needs a nonlinear damping term. However, omitting feedback information in the fuzzy approximator leads to a less complex implementation (i.e., a simpler architecture compared to traditional SFA-based controllers). Furthermore, the fuzzy approximation error of FFA is always bounded, such that the synthesized controller assures global stability. In addition, the number of fuzzy rules can be further reduced by using a TStype FFA. In other words, the FFA-based adaptive controller has better advantages than the SFA-based adaptive controller.

To demonstrate the high application potential of the FFA-based adaptive control method to complicated and high-dimension systems, the FFA-based motion/force tracking controller is constructed for holonomic mechanical systems with an environmental constraint (McClamroch \& Wang, 1988) or a set of closed kinematic chains (Tarn et al., 1987; Li et al., 1989). Holonomic systems represent numerous industrial plants - two for example, are constrained robots and cooperative multi-robot systems. From the pioneering work (McClamroch \& Wang, 1988), a reduced-state-based approach is utilized in most researches (Tarn et al., 1987; Li et al., 1989; Wang et al., 1997). When considering parametric uncertainties, adaptive control schemes were introduced in (Jean \& Fu, 1993; Liu et al., 1997; Yu \& Lloyd, 1997; Zhu \& Schutter, 1999). Unfortunately, the reducedstate-based approach usually has a force tracking residual error proportional to estimated parameter errors. Thus, a high gain force feedback or acceleration feedback is needed (e.g., Jean \& Fu, 1993; Yu \& Lloyd, 1997). An alternative hybrid motion/force control stated in (Yuan, 1997) has assured both motion and force tracking errors to be zero. To deal with unstructured uncertainties, several robust control strategies (Chiu et al., 2004; Zhen \& Goldenberg, 1996; Gueaieb et al., 2003) provide asymptotic motion tracking and an ultimate bounded force error. In contrast to discontinuous control laws, the works (Chang \& Chen, 2000; Lian et al., 2002) apply adaptive fuzzy control to compensate unmodeled uncertainties and achieve $H^{\infty}$ tracking performance. However, their applications are limited due to high computation load arising from the numerous fuzzy rules and tuning parameters. All these points motivate the further research on improving the control of holonomic systems by using the FFA-based control.

As a result, the proposed adaptive controller is no longer with the disadvantages of the traditional SFA-based adaptive controllers mentioned above. In detail, the stability is guaranteed in a rigorous analysis via Lyapunov's method. The attenuation of both disturbances and estimated fuzzy parameter errors is achieved in an $L_{2}$-gain sense, while the LMI techniques (Boyd et al., 1994) are used to simplify the gain design. If applying the sliding mode control, the controlled system can further achieve asymptotic stability of tracking errors. Notice that the proposed approach assures global stability for controlling general MIMO uncertain systems in a straightforward manner. Compared to the mainly relative works (Chang \& Chen, 2000; Lian et al., 2002), the proposed scheme achieves both robust motion and force tracking control (but the work (Lian et al., 2002) does not) for more general holonomic systems. Meanwhile, the scheme has a novel architecture which can be easily implemented.

The remainder of this chapter is organized as follows. First, the TS FFA-based adaptive control method is introduced in Sec. 3. Then, the proposed control method is modified to motion/force tracking controller for holonomic constrained systems in Sec. 4 . Section 5 shows the simulation results of controlling a cooperative multi-robot system transporting a common object. Finally, some concluding remarks are made in Sec. 6. 


\section{TS FFA-based Adaptive Fuzzy Control}

\subsection{FFA-based Compensation Concept}

Without loss of generality, let us consider an $n$-th order multivariable nonlinear system

$$
G(\underline{x}(t)) x^{(n)}(t)=f(\underline{x}(t))+u(t)+w(t)
$$

where $n \geq 2 ; x \in R^{m}$ is a part of the state vector $\underline{x}$ defined as $\underline{x}(t)=\left[\begin{array}{lll}x^{T}(t) \quad \dot{x}^{T}(t) \quad \cdots & \cdots\end{array}\right.$ $\left.\left(x^{(n-1)}(t)\right)^{T}\right]^{T} \in R^{n m} ; f(\underline{x}(t)) \in R^{m}$ is an unknown nonlinear function which satisfies $f\left(\underline{x}_{d}(t)\right) \in L_{\infty}$ for an appropriate bounded desired tracking command $\underline{x}_{d}(t)=\left[\begin{array}{lll}x_{d}^{T}(t) & \dot{x}_{d}^{T}(t) \quad \cdots\end{array}\right.$ $\left.\left(x_{d}^{(n-1)}(t)\right)^{T}\right]^{T} ; G(\underline{x}(t)) \in R^{m \times m}$ is an unknown positive-definite symmetric matrix which satisfies $G\left(\underline{x}_{d}(t)\right), \dot{G}\left(\underline{x}_{d}(t)\right) \in L_{\infty} ; u(t) \in R^{m}$ is the control input; and $w(t) \in R^{m}$ is an external disturbance assumed to be bounded. Clearly, if the terms $f(\underline{x}(t))$ and $G(\underline{x}(t))$ are exactly known and no disturbance exists, we are able to apply the feedback linearization concept and set the control law as

$$
u=-f(\underline{x})+G(\underline{x}) \dot{q}_{a}(t)+\frac{1}{2} \dot{G}(\underline{x}) s+K s
$$

where the notations are given as $e(t)=x_{d}(t)-x(t), s(t)=q_{a}(t)-x^{(n-1)}(t), \quad q_{a}(t)=x_{d}^{(n-1)}(t)$ $+\Lambda_{n-1} e^{(n-2)}(t)+\cdots+\Lambda_{2} \dot{e}(t)+\Lambda_{1} e(t) ; \Lambda_{v} \in R^{m \times m}$, for $v=1,2, \ldots,(n-1)$, is a positive-definite diagonal matrix; and $K \in R^{m \times m}$ is a symmetric positive-definite matrix. This renders to the error dynamics $G(\underline{x}) \dot{s}=-\frac{1}{2} \dot{G}(\underline{x}) s-K s-w(t)$, which is exponentially stable once there is no disturbance. However, the state feedback term $u_{b}=-f(\underline{x})+G(\underline{x}) \dot{q}_{a}(t)+\frac{1}{2} \dot{G}(\underline{x}) s$ is often poorly understood such that the fuzzy approximator is considered to realize the ideal control law (2) in conventional SFA-based control methods. Nevertheless, when the tracking goal is achieved, terms $f(\underline{x}(t))$ and $G(\underline{x}(t))$ accordingly converge to functions $f\left(\underline{x}_{d}(t)\right)$ and $G\left(\underline{x}_{d}(t)\right)$. The state feedback term $u_{b}$ converges to

$$
u_{f}=-f\left(\underline{x}_{d}\right)+G\left(\underline{x}_{d}\right) x_{d}^{(n)}
$$

which is only dependent on the pre-planned desired command $\underline{x}_{d}$. In other words, the state feedback control law becomes a feedforward compensation law during steady state. Therefore, different to traditional works (Wang \& Mendel, 1992)-(Alata et al., 2001), here we use the universal fuzzy approximator to closely obtain the feed-forward compensation law (3), while the effect of omitting transient dynamics is compensated by error feedback. Since the pre-planned desired commands would be taken as the inputs of the fuzzy approximator, the so-called feed-forward fuzzy approximator (FFA) arises. By this way, we assume that there exist positive constants $\psi_{1}, \ldots, \psi_{p}$ and positive-semidefinite symmetric matrices $\Psi_{s}, \Psi_{e}$ such that the error between $u_{b}(\underline{x})$ and $u_{f}\left(\underline{x}_{d}\right)$ is shaped by

$$
s^{T}\left(u_{b}(\underline{x})-u_{f}\left(\underline{x}_{d}\right)\right) \leq \sum_{\kappa=1}^{p} \psi_{\kappa}\left\|e_{o}\right\|^{2 \kappa}\|s\|^{2}+s^{T} \Psi_{s} s+e_{o}^{T} \Psi_{e} e_{o}
$$


with the tracking error $e_{o}=\underline{x}-\underline{x}_{d}$. Then the design idea can be realized by combining both FFA and error-feedback based compensations later. Note that the above inequality is often held for most physical systems, such as robotic systems, dc motors, etc... Moreover, $p=1$ is often held. The similar property as (4) for nonlinear systems can be found in (Sadegh \& Horowitz, 1990; Chiu et al., 2006; Chiu, 2006).

From the definition of $u_{f}$ in (3), the TS-type FFA consists of the following rules:

Rule $l$ : If $z_{1}(t)$ is $\mathbf{X}_{1}^{l}$ and $\ldots$ and $z_{h}(t)$ is $\mathbf{X}_{h}^{l}$. Then

$$
\hat{u}_{f i}=\theta_{0 i}^{l}+\theta_{1 i}^{l} \chi\left(\underline{x}_{d}\right), l=1,2, \ldots, r
$$

where $z_{1}(t), \ldots, z_{h}(t)$ are the premise variables composed of the desired commands $x_{d}(t)$, $\dot{x}_{d}(t), \ldots, x_{d}^{(n-1)}(t)$ since $u_{f}\left(\underline{x}_{d}(t)\right)$ is functional of $\underline{x}_{d}(t) ; l=1,2, \ldots, r$ with $r$ denoting the total number of rules; $\mathbf{X}_{1}^{l}, \ldots, \mathbf{X}_{h}^{l}$ are proper fuzzy sets determined by the known behavior of the desired signals; $\hat{u}_{f i}$ is the $i$-th element of approximation of $u_{f} ; \chi \in R^{8}$ is a basis vector functional of $\underline{x}_{d}(t)$ to be chosen from the nonlinearity of $u_{f} ; \theta_{0 i}^{l} \in R$ and $\theta_{1 i}^{l} \in R^{1 \times g}$ are fuzzy parameters. Using the singleton fuzzifier, product fuzzy inference and weighted average defuzzifier, the inferred output of the fuzzy system (5) is

$$
\hat{u}_{f i}\left(z_{d}, \Theta_{u_{f i}}\right)=\xi^{T}\left(z_{d}\right) \Theta_{u_{f}} \bar{\chi}\left(z_{d}\right)
$$

where $\quad z_{d}(t) \equiv\left[z_{1}(t) \quad z_{2}(t) \quad \ldots \quad z_{h}(t)\right]^{T} \quad ; \quad \Theta_{u_{f i}} \equiv\left[\begin{array}{lllll}\theta_{u_{f i}}^{1} & \theta_{u_{f i}}^{2} & \ldots & \theta_{u_{f i}}^{r}\end{array}\right]^{T} \in R^{r \times(g+1)} \quad$ with $\quad \theta_{u_{f i}}^{l}=\left[\begin{array}{ll}\theta_{0 i}^{l} \\ \theta^{\prime}\end{array}\right.$ $\left.\theta_{1 i}^{l}\right]^{T} \in R^{g+1} ; \bar{\chi}=\left[\begin{array}{ll}1 & \chi^{T}\end{array}\right]^{T} \in R^{g+1} ;$ and $\xi\left(z_{d}(t)\right) \equiv\left[\begin{array}{llll}\xi_{1} & \xi_{2} & \ldots & \xi_{r}\end{array}\right]^{T} \in R^{r}$ is a fuzzy basis function vector consisting of $\xi_{l}\left(z_{d}(t)\right)=\mu_{l}\left(z_{d}(t)\right) / \sum_{l=1}^{r} \mu_{l}\left(z_{d}(t)\right)$ with $\mu_{l}\left(z_{d}(t)\right)=\prod_{\zeta=1}^{h} \mathbf{X}_{\zeta}^{l}\left(z_{\zeta}(t)\right) \geq 0$ for all $l$. Note that the form of (6) is a TS type of fuzzy representation. When we let $\chi=0$, the fuzzy system (5) is reduced to the special case with a Mamdani fuzzy representation, i.e., $\hat{u}_{f i}=\xi^{T}\left(z_{d}\right) \Theta_{u_{f i}}$ for $\Theta_{u_{f i}} \in R^{r}$ and $\bar{\chi}=1$. Based on the above fuzzy approximator (6), the overall approximation of $u_{f}$ is obtained as

$$
\hat{u}_{f}\left(z_{d}(t), \Theta_{u_{f}}\right)=\left[\hat{u}_{f i}\left(z_{d}(t), \Theta_{u_{f i}}\right)\right]_{m \times 1}=Y_{d}\left(z_{d}(t)\right) \Theta_{u_{f}} \bar{\chi}
$$

where $\Theta_{u_{f}}=\left[\begin{array}{llll}\Theta_{u_{f 1}}^{T} & \Theta_{u_{f 2}}^{T} & \cdots & \Theta_{u_{f n}}^{T}\end{array}\right]^{T} \in R^{m r \times(g+1)}$; and $Y_{d}=\operatorname{block-diag}\left\{\xi^{T}, \ldots, \xi^{T}\right\} \in R^{m \times m r}$ is a regression matrix. From the observation on (7), if $\Theta_{u_{f}}$ is bounded, then $\hat{u}_{f} \in L_{\infty}$ for all $t$ (due to $Y_{d}\left(z_{d}(t)\right) \in L_{\infty}$ and $\bar{\chi} \in L_{\infty}$ for all bounded $\underline{x}_{d}(t)$ ). In light of this, we limit the tunable fuzzy parameter $\Theta_{u_{f}}$ to a specified region

$$
\Omega_{\theta_{u}} \equiv\left\{\Theta_{u_{f}} \in R^{m r \times(g+1)} \mid \operatorname{tr}\left(\Theta_{u_{f}}^{T} \Theta_{u_{f}}\right) \leq \bar{\theta}_{u}, \bar{\theta}_{u}>0\right\}
$$

with an adjustable parameter $\bar{\theta}_{u}$. Meanwhile, an appropriate projection algorithm will be applied later to keep the tuned fuzzy parameters within the bounded region. Inside the 
specified set, there exists an optimal approximation parameter $\Theta_{u_{f}}^{*}$ defined as (for $U_{z}$ is a discussed space of $z_{d}$ )

$$
\Theta_{u_{f}}^{*} \equiv \operatorname{argmin}_{\Theta_{u_{f} \in \Omega_{\theta_{u}}}}\left(\sup _{z_{d} \in U_{z}} \mid u_{f}-\hat{u}_{f}\left(z_{d}, \Theta_{u_{f}}\right)\right)
$$

which leads to the minimum approximation error for $u_{f}$. This means that the minimum approximation error is

$$
W_{u_{f}}=u_{f}\left(z_{d}\right)-Y_{d}\left(z_{d}(t)\right) \Theta_{u_{f}}^{*} \bar{\chi}
$$

Note that if the parametric constraint is removed, the optimal approximation parameter $\Theta_{u_{f}}^{*}$ is still upper bounded (cf. Wang \& Mendel, 1992). Due to $\hat{u}_{f}\left(z_{d}, \Theta_{u_{f}}^{*}\right) \in L_{\infty}$ and $u_{f}\left(\underline{x}_{d}\right) \in L_{\infty}$, it is reasonably concluded that $W_{u_{f}}$ is upper bounded for all $t$. Moreover, based on the universal approximation theorem (Wang \& Mendel, 1992), $\left\|W_{u_{f}}\right\|$ can be arbitrarily small. In addition, special characteristics of the feedforward fuzzy approximator are summarized below.

Next, according to the FFA (7) and the bounded fashion of $u_{b}(\underline{x})-u_{f}\left(\underline{x}_{d}\right)$ as (4), the overall controller with an adaptively tuned FFA is given as follows:

$$
\begin{aligned}
& u=\hat{u}_{f}\left(z_{d}, \Theta_{u_{f}}\right)+\sum_{\kappa=1}^{p} \psi_{\kappa}\left\|e_{o}\right\|^{2 \kappa} s+K s \\
& \dot{\Theta}_{u_{f}}=\left\{\begin{array}{cc}
\gamma_{0} Y_{d}^{T} s \bar{\chi}^{T}-\gamma_{0} c_{u}\left(\Theta_{u_{f}}\right) \frac{\operatorname{tr}\left(\bar{\chi}^{T} Y_{d} \Theta_{u_{f}}\right)}{\operatorname{tr}\left(\Theta_{u_{f}}^{T} \Theta_{u_{f}}\right)} \Theta_{u_{f}}, & \text { if }\left(c_{u}\left(\Theta_{u_{f}}\right) \geq 0\right. \text { and } \\
\gamma_{0} E_{d}^{T} s \bar{\chi}^{T}, & \left.\operatorname{tr}\left(\bar{\chi}^{T} Y_{d} \Theta_{u_{f}}\right)>0\right)
\end{array}\right.
\end{aligned}
$$

where $\gamma_{0}>0 ; c_{u}\left(\Theta_{u_{f}}\right)=\left(\operatorname{tr}\left(\Theta_{u_{f}}^{T} \Theta_{u_{f}}\right)-\bar{\theta}_{u}+\varepsilon_{u}\right) / \varepsilon_{u}$ with $c_{u}\left(\Theta_{u_{f}}\left(t_{0}\right)\right)<0$ and $\bar{\theta}_{u}>\varepsilon_{u}>0$. Note that the above update law is an application of the smooth projection algorithm developed in the work (Pomet \& Praly, 1992). The update law assures the following properties: (a) $\operatorname{tr}\left(\Theta_{u_{f}}^{T} \Theta_{u_{f}}\right) \leq \bar{\theta}_{u}$ for all $t \geq t_{0}$ and (b) $\gamma_{0} \operatorname{tr}\left(\bar{\chi} s^{T} Y_{d} \tilde{\Theta}_{u_{f}}\right)-\operatorname{tr}\left(\dot{\Theta}_{u_{f}}^{T} \tilde{\Theta}_{u_{f}}\right) \leq 0$ for $\tilde{\Theta}_{u_{f}}=\Theta_{u_{f}}^{*}-\Theta_{u_{f}}$. Then, the controller (9) results in the overall error system

$$
\begin{gathered}
G(\underline{x}) \dot{s}=-\frac{1}{2} \dot{G}(\underline{x}) s-\sum_{k=1}^{p} \psi_{k}\left\|e_{o}\right\|^{2 \kappa} s-K s+Y_{d} \tilde{\Theta}_{u_{f}} \bar{\chi}+\Delta u+w_{a}(t) \\
\underline{\dot{e}}=\left[\begin{array}{cccc}
0 & I_{m} & \cdots & 0 \\
\vdots & \ddots & \ddots & \vdots \\
0 & \cdots & 0 & I_{m} \\
-\Lambda_{1} & \cdots & -\Lambda_{n-2} & -\Lambda_{n-1}
\end{array}\right]\left[\begin{array}{c}
e \\
\vdots \\
e^{(n-3)} \\
e^{(n-2)}
\end{array}\right]+\left[\begin{array}{c}
0 \\
\vdots \\
0 \\
I_{m}
\end{array}\right] s
\end{gathered}
$$




$$
\equiv \Lambda \underline{e}+B s
$$

where $\tilde{\Theta}_{u_{f}}=\Theta_{u_{f}}^{*}-\Theta_{u_{f}} ; w_{a}(t)=W_{u_{f}}-w(t) ; \Delta u=u_{b}(\underline{x})-u_{f}\left(\underline{x}_{d}\right)$; the definition of $W_{u_{f}}$ as (8) has been used; $\underline{e}=\left[\begin{array}{llll}e^{T} & \dot{e}^{T} & \cdots & \left(e^{(n-2)}\right)^{T}\end{array}\right]^{T} \in R^{m(n-1)} ; \Lambda \in R^{m(n-1) \times m(n-1)}$ and $B \in R^{m(n-1) \times m}$ are defined from the above associated components. Since the error system (11) is only perturbed by the bounded approximation error $w_{a}(t)$, the globally uniform ultimate bound of $e_{o}$ is assured straightforwardly. The detailed stability analysis will be carried out in the next subsection.

\subsection{Robustness Design}

To further enhance the robustness of the controlled system, three modified FFA-based adaptive controllers are developed in this subsection. First, the robust gain design is performed here. Let us consider the Lyapunov function candidate

$$
V_{1}(t)=\frac{1}{2} s^{T} G(\underline{x}) s+\underline{e}^{T} P \underline{e}+\frac{1}{2 \gamma_{0}} \operatorname{tr}\left(\tilde{\Theta}_{u_{f}}^{T} \tilde{\Theta}_{u_{f}}\right)
$$

with a positive-definite symmetric matrix $P$. The time derivative of $V$ along the error dynamics (11) and (12) is

$$
\begin{aligned}
\dot{V}_{1}= & -s^{T} K s+\underline{e}^{T}\left(\Lambda^{T} P+P \Lambda\right) \underline{e}+s^{T} B^{T} P \underline{e}+\underline{e}^{T} P B s+s^{T} \Delta u+s^{T} w_{a} \\
& -\sum_{\kappa=1}^{p} \psi_{\kappa}\left\|e_{o}\right\|^{2 \kappa} s^{T} s+\operatorname{tr}\left(s^{T} Y_{d} \tilde{\Theta}_{u_{f}} \bar{\chi}\right)-\frac{1}{\gamma_{0}} \operatorname{tr}\left(\dot{\Theta}_{u_{f}}^{T} \tilde{\Theta}_{u_{f}}\right) \\
\leq & -s^{T}\left(K-\Psi_{s}\right) s+\underline{e}^{T}\left(\Lambda^{T} P+P \Lambda\right) \underline{e}+s^{T} B^{T} P \underline{e}+\underline{e}^{T} P B s \\
& +e_{o}^{T} \Psi_{e} e_{o}+s^{T} w_{a}
\end{aligned}
$$

where the facts $\operatorname{tr}\left(s^{T} Y_{d} \tilde{\Theta}_{u_{f}} \bar{\chi}\right)=\operatorname{tr}\left(\bar{\chi} s^{T} Y_{d} \tilde{\Theta}_{u_{f}}\right), \operatorname{tr}\left(\dot{\Theta}_{u_{f}}^{T} \tilde{\Theta}_{u_{f}}\right) \geq \gamma_{0} \operatorname{tr}\left(\bar{\chi}^{T} Y_{d} \tilde{\Theta}_{u_{f}}\right)$ and the inequality (4) have been applied. Furthermore, if the expressions $s=\left[\begin{array}{lll}-B^{T} \Lambda & I_{m}\end{array}\right] e_{o}$ and $\underline{e}=\left[\begin{array}{ll}I_{m(n-1)} & 0_{m(n-1) \times m}\end{array}\right] e_{o}$ are applied, $\dot{V}$ satisfies

$$
\dot{V}_{1} \leq e_{o}^{T}\left(\left[\begin{array}{cc}
H-\Lambda^{T} B K_{r} B^{T} \Lambda & P B+\Lambda^{T} B K_{r} \\
B^{T} P+K_{r} B^{T} \Lambda & -K_{r}
\end{array}\right]+\Psi_{e}\right) e_{o}+\frac{1}{\rho_{1}^{2}}\left\|w_{a}(t)\right\|_{2}^{2}
$$

where $H=\Lambda^{T} P+P \Lambda-\Lambda^{T} B B^{T} P-P B B^{T} \Lambda$ and $K_{r}=K-\Psi_{s}-\frac{\rho_{1}^{2}}{4}$. Therefore, the robust control result is summarized in the following theorem.

Theorem 1: Consider the highly unknown system (1) using the TS FFA-based adaptive fuzzy controller (9) with the update law (10). If there exist symmetric positive-definite matrices $P$, $K$ satisfying the following LMI problem

$$
\begin{gathered}
\text { Given } \rho_{1}>0, \Lambda_{v}>0, Q \geq 0 \\
\text { subject to } P, K>0 \\
{\left[\begin{array}{cc}
H-\Lambda^{T} B K_{r} B^{T} \Lambda & P B+\Lambda^{T} B K_{r} \\
B^{T} P+K_{r} B^{T} \Lambda & -K_{r}
\end{array}\right]+\Psi_{e}+Q \leq 0}
\end{gathered}
$$


then the closed-loop error system has the following properties: (i) all error signals and fuzzy parameters are bounded; (ii) the $H^{\infty}$ tracking performance criterion

$$
\int_{t_{0}}^{t_{f}} e_{o}^{T}(t) Q e_{o}(t) d t \leq V_{1}\left(t_{0}\right)+\frac{1}{\rho_{1}^{2}} \int_{t_{0}}^{t_{f}}\left\|w_{a}(t)\right\|_{2}^{2} d t
$$

is assured; and (iii) if $w_{a}(t) \in L_{2}$, then $e_{o}$ asymptotically converges to zero in a global manner.

Proof: From the inequality (14), a feasible solution of the LMI (15) yields

$$
\dot{V} \leq-e_{o}^{T} Q e_{o}+\frac{1}{\rho^{2}}\left\|w_{a}(t)\right\|_{2}^{2} .
$$

Since $V_{1}>0$ and $\dot{V}_{1}$ is negative semidefinite outside the compact set $\left\{\left\|e_{o}\right\|\left\|e_{o}\right\| \leq \frac{1}{\eta_{0} \rho_{1}}\left\|w_{a}\right\|<\infty\right\}$, for $\left.\eta_{0}=\lambda_{\min }(Q)\right\}$, we have $\underline{e}, s \in L_{\infty}$ and $\tilde{\Theta}_{u_{f}} \in L_{\infty}$. As a result, $\underline{\dot{e}}, \dot{s} \in L_{\infty}$ is assured from the boundedness of all terms on right-hand side of (11) and (12). In turn, $e_{o}, \dot{e}_{0} \in L_{\infty}$.

Moreover, by integrating the inequality (17), the $H^{\infty}$ tracking performance criterion (16) is assured. In other words, the disturbance $w_{a}(t)$ is attenuated to a prescribed level $1 / \rho_{1}$. Also, $e_{0} \in L_{2}$ if $w_{a}(t)$ is $L_{2}$ integrable. Due to the fact that $e_{0}, \dot{e}_{0} \in L_{\infty}$ and $e_{0} \in L_{2}$, the result $\lim _{t \rightarrow \infty} e_{0}(t)=0$ is concluded by Barbalat's lemma. In addition, since the augmented disturbance $w_{a}(t)$ is naturally bounded, all the stability is in a global sense.

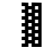

Furthermore, to avoid an unexpected transient response due to poor fuzzy approximation, the attenuation of fuzzy parameter errors is taken into consideration below.

Theorem 2: Consider the highly unknown system (1) using the TS FFA-based adaptive fuzzy controller

$$
u=\hat{u}_{f}\left(z_{d}, \Theta_{u_{f}}\right)+\left(\sum_{\kappa=1}^{p} \psi_{\kappa}\left\|e_{o}\right\|^{2 \kappa}+\frac{\rho_{2}^{2}}{4}\|\bar{\chi}\|^{2}\left\|Y_{2 d}\right\|\right) s+K s
$$

with $\rho_{2}>0, Y_{2 d}=Y_{d} Y_{d}^{T}=\operatorname{diag}\left\{\xi^{T} \xi, \ldots, \xi^{T} \xi\right\} \in R^{m \times m}$, and the update law (10). If there exist symmetric positive-definite matrices $P, K$ satisfying the LMI problem (15), then the closedloop error system achieves the $H^{\infty}$ tracking performance criterion

$$
\int_{t_{0}}^{t_{f}} e_{o}^{T}(t) Q e_{o}(t) d t \leq V_{2}\left(t_{0}\right)+\int_{t_{0}}^{t_{f}}\left(\frac{1}{\rho_{1}^{2}}\left\|w_{a}(t)\right\|_{2}^{2}+\frac{1}{\rho_{2}^{2}} \operatorname{tr}\left(\tilde{\Theta}_{u_{f}}^{T}(t) \tilde{\Theta}_{u_{f}}(t)\right)\right) d t
$$

where $V_{2}\left(t_{0}\right)$ is a quadratic term dependent on the initial values of tracking errors; and $1 / \rho_{2}>0$ is a prescribed attenuation level for the fuzzy parametric error $\tilde{\Theta}_{u_{f}}$.

Proof: Consider the Lyapunov function candidate

$$
V_{2}(t)=\frac{1}{2} s^{T} G(\underline{x}) s+\underline{e}^{T} P \underline{e}
$$


with symmetric positive-definite matrices $G(\underline{x})$ and $P$. Similar to the proof in Thm. 1 , the feasibility of the LMI (15) and the control law (18) of $u$ lead to

$$
\dot{V}_{2} \leq-e_{o}^{T} Q e_{o}+\frac{1}{\rho_{1}^{2}}\left\|w_{a}\right\|_{2}^{2}+\operatorname{tr}\left(s^{T} Y_{d} \tilde{\Theta}_{u_{f}} \bar{\chi}\right)-\frac{\rho_{2}^{2}}{4}\|\bar{\chi}\|^{2}\left\|Y_{2 d}\right\| s^{T} S
$$

From the property

$$
\operatorname{tr}\left(s^{T} Y_{d} \tilde{\Theta}_{u_{f}} \bar{\chi}\right) \leq \frac{\rho_{2}^{2}}{4} \operatorname{tr}\left(\bar{\chi}^{T} \bar{\chi}^{T}{ }^{T} Y_{2 d} s\right)+\frac{1}{\rho_{2}^{2}} \operatorname{tr}\left(\tilde{\Theta}_{u_{f}}^{T} \tilde{\Theta}_{u_{f}}\right)
$$

$\dot{V}_{2}$ further satisfies

$$
\dot{V}_{2} \leq-e_{o}^{T} Q e_{o}+\frac{1}{\rho_{1}^{2}}\left\|w_{a}\right\|_{2}^{2}+\frac{1}{\rho_{2}^{2}} \operatorname{tr}\left(\tilde{\Theta}_{u_{f}}^{T} \tilde{\Theta}_{u_{f}}\right)
$$

Integrating both sides of the above inequality, the closed-loop system guarantees the robust performance criterion (19). The gain $\rho_{2}$ is the adjustable attenuation level of fuzzy parametric errors. In addition, the boundedness of the error system is assured from the same argument in Thm. 1.

From the observation on $w_{a}$, the boundedness has been assured from the bounded fuzzy approximation output (7) and error (8) in a global sense. This implies that there exists a conservative upper bound of $w_{a}$ to be a constant $\eta$ such that $\eta \geq \max _{i=1, \ldots, m}\left\{\sup _{t}\left|w_{a i}(t)\right|\right\}$ (where $w_{a i}$ denotes the $i$-th element of the vector $w_{a}$ ). Then we are able to give an asymptotic stable result as below.

Theorem 3: Consider the highly unknown system (1) using the TS FFA-based adaptive fuzzy controller

$$
u=\hat{u}_{f}\left(z_{d}, \Theta_{u_{f}}\right)+\sum_{\kappa=1}^{p} \psi_{\kappa}\left\|e_{o}\right\|^{2 \kappa} s+K s+\eta \operatorname{sign}(s)
$$

with $\operatorname{sign}(s) \equiv\left[\begin{array}{lll}\operatorname{sign}\left(s_{1}\right) & \cdots & \operatorname{sign}\left(s_{p}\right)\end{array}\right]$ for $s_{i}$ being the $i$-th element of vector $s$ and the update law (10). If there exist symmetric positive-definite matrices $P, K$ satisfying the following LMI problem (15) for given $\rho_{1}=0$, then the tracking error asymptotically converges to zero in a global sense.

Proof: Consider the Lyapunov function candidate (13) again. Analogous to the proof of Thm. 1, the feasibility of the LMI (15) with $\rho_{1}=0$ and the control law (20) yield

$$
\begin{aligned}
\dot{V}_{1} & \leq-e_{o}^{T} Q e_{o}+s^{T} w_{a}-\eta s^{T} \operatorname{sign}(s) \\
& \leq-e_{o}^{T} Q e_{o}+\sum_{i=1}^{m}\left|s_{i}\right|\left|w_{a i}\right|-\eta \sum_{i=1}^{m}\left|s_{i}\right| \\
& \leq-e_{o}^{T} Q e_{o}
\end{aligned}
$$

where the upper boundedness of $w_{a}$ has been used. Due to $V_{1}>0$ and $\dot{V}_{1} \leq 0$, we are able to conclude the tracking error $\underline{e}$ will asymptotically converge to zero as $t \rightarrow \infty$. 
Remark 1: The proposed feedforward fuzzy system (5) has four important characteristics (a) the premise variables only consist of desired commands such that some fuzzy inference steps (e.g., calculation of $\left.Y_{d}\left(z_{d}(t)\right)\right)$ can be performed off-line; (b) an assumption on the bounded approximation error is not needed; (c) due to the naturally bounded approximation error $W_{u_{f}}$, the total number of fuzzy rules can be flexibly reduced if a large approximation error is acceptable; and (d) TS-type fuzzy rules provide more flexible approximation by using fewer rules. Therefore, the feedforward fuzzy approximator allows less computation and the synthesized controller has simpler implementation along with a globally stable manner.

\section{Application on Holonomic Systems}

\subsection{Model Descriptions of Holonomic Systems}

Consider a non-redundant holonomic system with a generalized coordinate $q \in R^{m}$ and the holonomic constraint $\phi(q)=0$ and $A(q) \dot{q}=0$, where $\phi: R^{m} \mapsto R^{p}$ and $A(q)=\frac{\partial \phi(q)}{\partial q}$. Without loss of generality, we assume that the system is operated away from any singularity with the exactly known function $\phi(q) \in C^{2}$. From investigation on well-known holonomic systems, different model descriptions exist due to the two kinds of constraints - an environmental constraint and a set of closed kinematic chains. Nevertheless, the model's general form is able to be formulated into a fully actuated system with a constraint. Referring to (Chiu et al., 2006), the general model of a holonomic system is written as

$$
M(q) \ddot{q}+C(q, \dot{q}) \dot{q}+g(q)+\tau_{d}(t)=B_{g} \tau_{g}+A^{T} \lambda_{g}
$$

where $M(q), C(q, \dot{q}) \dot{q}, g(q)$ are the inertia matrix, Coriolis/centripetal force, gravitational force, respectively (which are continuous and assumed to be poorly known); $\tau_{d}(t)$ is a bounded external disturbance; $\tau_{g} \in R^{m}$ is an applied force; $B_{g}(q)$ is an invertible input matrix; and $\lambda_{g} \in R^{p}$ physically presents a reaction force for an environmental constraint or an internal force for a set of closed kinematic chains.

Since the motion is subject to a $p$-dimensional constraint, the configuration space of the holonomic system is left with $(m-p)$ degrees of freedom. From the implicit function theorem (McClamroch \& Wang, 1988), we find a partition of $q$ as $q=\left[\begin{array}{ll}q_{1}^{T} & q_{2}^{T}\end{array}\right]^{T}$ for $q_{1} \in R^{m-p}, q_{2} \in R^{p}$, such that the generalized coordinate $q_{2}$ is expressed in terms of the independent coordinate $q_{1}$ as $q_{2}=\Omega\left(q_{1}\right)$ with a nonlinear mapping function $\Omega$. Due to the nonsingularity assumption, the terms $\frac{\partial \Omega}{\partial q_{1}}$ and $\frac{\partial^{2} \Omega}{\partial q_{1}^{2}}$ are bounded in the work space. The generalized displacement and velocity can be expressed in terms of the independent coordinates $q_{1}, \dot{q}_{1}$ as

$$
q=\left[\begin{array}{ll}
q_{1}^{T} & \left(\Omega\left(q_{1}\right)\right)^{T}
\end{array}\right]^{T}
$$




$$
\dot{q}=\left[\begin{array}{c}
I_{n-m} \\
\frac{\partial \Omega\left(q_{1}\right)}{\partial q_{1}}
\end{array}\right] \dot{q}_{1} \equiv J \dot{q}_{1} .
$$

From above equations, the constraint of velocity $A(q) \dot{q}=0$ leads to $A\left(q_{1}\right) J\left(q_{1}\right) \dot{q}_{1}=0$. Notice that here we use $A\left(q_{1}\right)$ to denote $A\left(q_{1}, \Omega\left(q_{1}\right)\right)$ for brevity. In other words, $A\left(q_{1}\right) J\left(q_{1}\right)=0$ since $A\left(q_{1}\right) J\left(q_{1}\right)$ is full column-rank and $\dot{q}_{1}$ is an independent coordinate (see (McClamroch $\&$ Wang, 1988)). Thus, there exists a reduced dynamics for the holonomic system (21). Due to the velocity transformation (23), the generalized acceleration satisfies $\ddot{q}=J \ddot{q}_{1}+J \dot{q}_{1}$. The motion equation (21) is further represented by the independent coordinates $q_{1}, \dot{q}_{1}, \ddot{q}_{1}$ as

$$
M\left(q_{1}\right) J \ddot{q}_{1}+\bar{C}\left(q_{1}, \dot{q}_{1}\right) \dot{q}_{1}+g\left(q_{1}\right)+\tau_{d}(t)=B_{g}\left(q_{1}\right) \tau_{g}+A^{T} \lambda_{g}
$$

where $\bar{C}=M \dot{J}+C J$. According to the fact $A\left(q_{1}\right) J\left(q_{1}\right)=0$, a reduced dynamics (McClamroch $\&$ Wang, 1988) is obtained after multiplying $J^{T}$ on both sides of (24):

$$
\underline{M}\left(q_{1}\right) \ddot{q}_{1}+\underline{C}\left(q_{1}, \dot{q}_{1}\right) \dot{q}_{1}+\underline{g}\left(q_{1}\right)+\underline{\tau}_{d}\left(q_{1}, t\right)=J^{T} B_{g} \tau_{g}
$$

with $\underline{M}=J^{T} M J ; \underline{C}=J^{T} \bar{C} ; g=J^{T} g ;$ and $\underline{\tau}_{d}=J^{T} \tau_{d}$. From the dynamics (25), some useful properties are addressed below.

Property 1: For the partition $I_{m}=\left[E_{1} \mid E_{2}\right]$ with $E_{1}=\left[\begin{array}{ll}I_{m-p} & 0_{(m-p) \times p}\end{array}\right]^{T} \in R^{m \times(m-p)}$ and $E_{2}=\left[0_{p \times(m-p)} I_{p}\right]^{T} \in R^{m \times p}$, the velocity transformation matrix $J$ satisfies $J^{T} E_{1}=I_{m-p}$.

Property 2: From the existence of $\Omega(\cdot)$ and the implicit function theorem, $A_{2}$ is invertible.

Property 3: The matrix $\underline{M}$ is symmetric and positive-definite while $\underline{M}^{-1} \in L_{\infty}$.

Property 4: Matrix $(\underline{M}-2 \underline{C})$ is skew-symmetric (cf. McClamroch \& Wang, 1988), i.e., $\zeta^{T}(\underline{M}-2 \underline{C}) \zeta=0, \forall \zeta \in R^{m-p}$.

\subsection{FFA-Based Adaptive Motion/Force Control}

For holonomic systems, the control objective is to track a desired motion trajectory $q_{1 d}(t) \in C^{2}$ while maintaining force $\lambda_{g}$ at a desired $\lambda_{g d}(t)$. Inspired by pure motion tracking, some notations are defined as

$$
\begin{gathered}
e_{m}=q_{1 d}-q_{1}, e_{m} \in R^{m-p} ; \\
q_{a}=\Lambda_{m} e_{m}+\dot{q}_{1 d^{\prime}} q_{a} \in R^{m-p} ; \\
s=q_{a}-\dot{q}_{1}, s \in R^{m-p} ;
\end{gathered}
$$

where $e_{m}, q_{a}, s$ are the motion error, auxiliary signal vector, error signal, respectively; and $\Lambda_{m} \in R^{(m-p) \times(m-p)}$ is a symmetric positive-definite matrix. If the system satisfies $\lim _{t \rightarrow \infty} s(t)=0$, then position and velocity tracking errors $e_{m}, \dot{e}_{m}$ exponentially converge to zero. In other 
words, the motion tracking problem is transformed to the problem of stabilizing $s(t)$. On the other hand, a force tracking error and force error filter are accordingly defined as

$$
\begin{gathered}
\tilde{\lambda}=\lambda_{g d}-\lambda_{g} \in R^{p} \\
\dot{e}_{\lambda}+\eta_{1} e_{\lambda}=\eta_{2} \tilde{\lambda}, \text { with } \eta_{1}, \eta_{2}>0 .
\end{gathered}
$$

Then, the reduced-state based scheme is to drive the motion trajectory into the stable subspace while the contact force is separately controlled maintaining a zero $e_{\lambda}$.

In order to derive the adaptive fuzzy controller, the error dynamics of $s$ along the motion equation (24) is written as

$$
\begin{aligned}
M J \dot{s} & =M J \dot{q}_{a}-M J \ddot{q}_{1} \\
& =-\bar{C} s+f+\tau_{d}-A^{T} \lambda_{g}-B_{g} \tau_{g}
\end{aligned}
$$

where $f=M\left(q_{1}\right) J\left(q_{1}\right) \dot{q}_{a}+\bar{C}\left(q_{1}, \dot{q}_{1}\right) q_{a}+g\left(q_{1}\right) \in R^{m}$. By traditional SFA-based control, we usually require to take $q_{1}, \dot{q}_{1}, q_{1 d}, \dot{q}_{1 d}, \ddot{q}_{1 d}$ as the premise variables, such that a large computational load exists on the controller processor. To avoid this situation, the FFA-based control method is used to provide the feed-forward compensation term $f_{d}\left(q_{1 d}, \dot{q}_{1 d}, \ddot{q}_{1 d}\right)=M\left(q_{1 d}\right) J\left(q_{1 d}\right) \ddot{q}_{1 d}+\bar{C}\left(q_{1 d}, \dot{q}_{1 d}\right) \dot{q}_{1 d}+g\left(q_{1 d}\right)$. Since $f_{d}$ is independent to state variables, $f_{d}(\cdot)$ is a much simpler function than $f(\cdot)$. If the effect of omitting the error $f-f_{d}$ can be coped with by feedback of tracking error, the concept of using the forward compensation $f_{d}$ is feasible. According to the FFA-based control in the above section, we closely approximate and compensate the forward term $f_{d}(\cdot)$ by a TS fuzzy system with the singleton fuzzifier and product inference. Then the fuzzy inferred output is

$$
\hat{f}_{d}\left(z_{d}(t), \Theta_{f_{d}}\right)=Y_{d}\left(z_{d}(t)\right) \Theta_{f_{d}} \bar{\chi}
$$

where $z_{d}(t), Y_{d}\left(z_{d}(t)\right)$, and $\bar{\chi}$ have the same definition as (7) being functional of $q_{1 d}(t), \dot{q}_{1 d}(t), \ddot{q}_{1 d}(t)$; and $\Theta_{f_{d}} \in R^{m r \times(g+1)}$ is a fuzzy tuning parametric vector in the consequent part of rules, with $r$ denoting the total number of rules. For the FFA (30), there exists an optimal approximation parameter

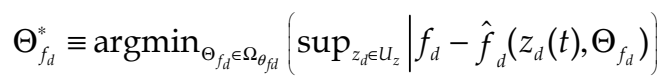

in an appropriate parametric constraint region $\Omega_{\theta_{f d}}$, which provides the most accurate approximation with the minimum error:

$$
W_{f_{d}}=f_{d}-Y_{d}\left(z_{d}(t)\right) \Theta_{f_{d}}^{*} \bar{\chi} .
$$

From the observation on the right-hand side of the above equation, the fuzzy approximation error $W_{f_{d}}$ is upper bounded for $t \geq 0$ from $f_{d} \in L_{\infty}$ and $\hat{f}_{d} \in L_{\infty}$.

Next, the overall controller is synthesized in the following. Based on the TS FFA-based fuzzy system (30), the overall control law is set in the form: 


$$
\tau_{g}=B_{g}^{-1}\left[Y_{d} \Theta_{f_{d}} \bar{\chi}+E_{1}\left(K s+\tau_{a}\right)-A^{T}\left(\lambda_{g d}+k_{f} e_{\lambda}\right)\right]
$$

where $k_{f}>0$ is a force feedback gain; $K \in R^{(m-p) \times(m-p)}$ is a symmetric positive definite matrix; $\tau_{a}$ is an auxiliary input designed later; the definition of $s$ and $e_{\lambda}$ is given in (26) and (28), respectively. Meanwhile, the fuzzy parameter $\Theta_{f_{d}}$ is adaptively adjusted by

$$
\dot{\Theta}_{f_{d}}=\left\{\begin{array}{cc}
\gamma Y_{d}^{T} J s \bar{\chi}^{T}-c\left(\Theta_{f_{d}}\right) \frac{\operatorname{tr}\left(\bar{\chi} s^{T} J^{T} Y_{d} \Theta_{f_{d}}\right)}{\operatorname{tr}\left(\Theta_{f_{d}}^{T} \Theta_{f_{d}}\right)} \Theta_{f_{d},} & \text { if }\left(c\left(\Theta_{f_{d}}\right) \geq 0\right. \text { and } \\
\left.\operatorname{tr}\left(\bar{\chi} s^{T} J^{T} Y_{d} \Theta_{u_{f}}\right)>0\right) \\
\gamma Y_{d}^{T} J s \bar{\chi}^{T}, & \text { otherwise }
\end{array}\right.
$$

with $c\left(\Theta_{f_{d}}\left(t_{0}\right)\right)<0$, where $c\left(\Theta_{f_{d}}\right)=\left(\operatorname{tr}\left(\Theta_{f_{d}}^{T} \Theta_{f_{d}}\right)-\bar{\theta}_{f_{d}}+\varepsilon_{f}\right) / \mathcal{E}_{f}$ is a projection criterion function with a tunable parameter $\varepsilon_{f}$ satisfying $\bar{\theta}_{f_{d}}>\varepsilon_{f}>0$; and $\gamma>0$ is an adaptation gain.

Furthermore, substituting the control law (32) into the dynamic equation (29) renders the closed-loop error dynamics:

$$
M J \dot{s}=-\bar{C} s-E_{1}\left(K s+\tau_{a}\right)+Y_{d} \tilde{\Theta}_{f_{d}} \bar{\chi}+\Delta f+w+A^{T}\left(\tilde{\lambda}+k_{f} e_{\lambda}\right)
$$

where $\Delta f \equiv f-f_{d} ; w \equiv W_{f_{d}}+\tau_{d}$; and the definition of approximation error $W_{f_{d}}$ in (31) and $\tilde{\lambda}$ in (27) have been applied. To analyze the convergence of motion and force tracking separately, we further multiply $J^{T}$ on both sides of (34), which leads to the motion tracking error dynamics:

$$
\underline{M} \dot{s}=-\underline{C} s-K s+J^{T} Y_{d} \tilde{\Theta}_{f_{d}} \bar{\chi}+J^{T} \Delta f+\underline{w}+\tau_{a},
$$

where Property $1\left(J^{T} E_{1}=I_{n-m}\right)$ and the fact, $J^{T}\left(q_{1}\right) A^{T}\left(q_{1}\right)=0$, have been applied; and $\underline{w} \equiv J^{T} w$. Then, replacing $\dot{s}$ of (34) by (35) and multiplying $A_{2}^{-T} E_{2}^{T}$ on both sides of (34), we obtain the force tracking error as follows:

$$
\begin{aligned}
\tilde{\lambda}+k_{f} e_{\lambda} & =A_{2}^{-T} E_{2}^{T}\left(M J \underline { M } ^ { - 1 } \left(-\underline{C} s-K s+J^{T} Y_{d} \tilde{\Theta}_{f_{d}} \bar{\chi}\right.\right. \\
& \left.\left.+J^{T} \Delta f+\underline{w}+\tau_{a}\right)+\bar{C} s-\Delta f-Y_{d} \tilde{\Theta}_{f_{d}} \bar{\chi}-w\right) \\
& \equiv \varpi\left(e_{m}, s, \tilde{\Theta}_{f_{d}}, w, t\right)
\end{aligned}
$$

where Property $2\left(A_{2}^{-T} \in L_{\infty}\right)$ and the fact, $E_{2}^{T} E_{1}=0$, have been applied above. It is a worthwhile note that the perturbed term $\Delta f$ in (35) arises from the use of the feed-forward fuzzy compensation. Nevertheless, the term $\Delta f$ is upper bounded by motion tracking errors in the following fashion:

$$
s^{T} J^{T} \Delta f \leq s^{T}\left(\Psi_{s}+\frac{1}{2 \kappa^{2}} I_{n-m}\right) s+\left\|\Lambda_{m} e_{m}\right\|^{2} s^{T} \Psi_{s e} s+e_{m}^{T}\left(\Psi_{e}+\frac{\kappa^{2}}{2} \Psi_{J}\right) e_{m}
$$


where there exist an intermediate parameter $\kappa>0$ and symmetric positive semidefinite matrices $\Psi_{s}, \Psi_{s e}, \Psi_{e}, \Psi_{J}$ dependent on the desired motion trajectory, control parameter $\Lambda_{m}$, and system parameters. This boundedness is assured for all well-known holonomic mechanical systems (cf. Appendix of (Chiu et al., 2006)).

Now, the main results of the FFA-based adaptive control of holonomic systems are stated as follows.

Theorem 4: Consider the holonomic system (21) using the TS FFA-based adaptive controller (32) tuned by the update law (33). If the auxiliary input is set as

$$
\tau_{a}=2 P_{m} e_{m}+\left\|\Lambda_{m} e_{m}\right\|^{2} \Psi_{s e} S+\frac{\rho^{2}}{4} \bar{\chi}^{T} \bar{\chi} J^{T} Y_{d} Y_{d}^{T} J s
$$

and there exist $\kappa, K, P_{m}$ satisfying the following LMI problem

$$
\begin{aligned}
& \text { Given } \rho, \Lambda_{m}>0 \text { and } Q=\left[\begin{array}{ll}
Q_{11} & Q_{21}^{T} \\
Q_{21} & Q_{22}
\end{array}\right]>0 \\
& \text { subject to } \kappa, K, P_{m}>0 \\
& {\left[\begin{array}{ccc}
K_{p}-Q_{11} & \Lambda_{m}^{T} K_{a}^{T}-Q_{21}^{T} & \kappa \Psi_{J}^{1 / 2} \\
K_{a} \Lambda_{m}-Q_{21} & K_{a}-Q_{22} & 0 \\
\kappa \Psi_{J}^{1 / 2} & 0 & 2 I_{n-m}
\end{array}\right] \geq 0}
\end{aligned}
$$

with $K_{a}=K-\left(\frac{\rho^{2}}{4}+\frac{1}{2 \kappa^{2}}\right) I_{n-m}-\Psi_{s}$ and $K_{p}=\Lambda_{m}^{T} K_{a} \Lambda_{m}+\Lambda_{m}^{T} P_{m}+P_{m} \Lambda_{m}-\Psi_{e}$, then (a) error signals $e_{m}, \dot{e}_{m}, e_{\lambda}, \tilde{\lambda}$ and fuzzy parameter $\Theta_{f_{d}}$ are bounded; (b) error vectors $e_{m}, s, \tilde{\lambda}$ have globally uniform ultimate bounds being proportional to the inversion of control gains; and (c) the closed-loop system is guaranteed with the robust motion tracking performance

$$
\int_{t_{0}}^{t_{f}} e_{a}^{T} Q e_{a} d t \leq V_{s}\left(t_{0}\right)+\frac{1}{\rho^{2}} \int_{t_{0}}^{t_{f}}\left(\|\underline{w}(t)\|^{2}+\operatorname{tr}\left(\tilde{\Theta}_{f_{d}}^{T}(t) \tilde{\Theta}_{f_{d}}(t)\right)\right) d t
$$

for $e_{a}=\left[\begin{array}{ll}e_{m}^{T} & \dot{e}_{m}^{T}\end{array}\right]^{T}$ and a nonnegative constant $V_{s}\left(t_{0}\right)$.

Proof: First, we prove the claim (a). Consider the Lyapunov function candidate

$$
V=\frac{1}{2} s^{T} \underline{M} s+e_{m}^{T} P_{m} e_{m}+\frac{1}{2 \gamma} \operatorname{tr}\left(\tilde{\Theta}_{f_{d}}^{T} \tilde{\Theta}_{f_{d}}\right)
$$

with a proper symmetric positive-definite matrix $P_{m}$. Along the error dynamics (35) and the fact $\dot{e}_{m}=-\Lambda_{m} e_{m}+s$, the time derivative of $V$ is written as follows:

$$
\begin{aligned}
\dot{V}= & \frac{1}{2} s^{T}(\underline{\dot{M}}-2 \underline{C}) s-s^{T} K s-e_{m}^{T}\left(\Lambda_{m}^{T} P_{m}+P_{m} \Lambda_{m}\right) e_{m}-\left\|\Lambda_{m} e_{m}\right\|^{2} s^{T} \Psi_{s e} s-\frac{\rho^{2}}{4} \bar{\chi}^{T} \bar{\chi} s^{T} J^{T} Y_{d} Y_{d}^{T} J s \\
& +s^{T} J^{T} Y_{d} \tilde{\Theta}_{f_{d}} \bar{\chi}-\frac{1}{\gamma} \operatorname{tr}\left(\dot{\Theta}_{f_{d}}^{T} \tilde{\Theta}_{f_{d}}\right)+s^{T} J^{T} \Delta f+s^{T} \underline{w} \\
\leq & -s^{T} K s-e_{m}^{T}\left(\Lambda_{m}^{T} P_{m}+P_{m} \Lambda_{m}\right) e_{m}-\left\|\Lambda_{m} e_{m}\right\|^{2} s^{T} \Psi_{s e} s+s^{T} J^{T} \Delta f+s^{T} \underline{w}
\end{aligned}
$$


where the definition of $\tau_{a}$, Property 4, and the update law (33) have been applied; and the above inequality is ensured by the property of the update law (i.e., $\left.s^{T} J^{T} Y_{d} \tilde{\Theta}_{f_{d}} \bar{\chi}-\frac{1}{\gamma} \operatorname{tr}\left(\dot{\Theta}_{f_{d}}^{T} \tilde{\Theta}_{f_{d}}\right) \leq 0\right)$. Due to the boundedness of $\Delta f$ as the fashion (37), we further obtain

$$
\dot{V} \leq-s^{T} K_{a} s-e_{m}^{T} \Upsilon e_{m}+\frac{1}{\rho^{2}}\|\underline{w}\|^{2}
$$

where $K_{a}=K-\left(\frac{\rho^{2}}{4}+\frac{1}{2 \kappa^{2}}\right) I_{n-m}-\Psi_{s} ; \Upsilon=\Lambda_{m}^{T} P_{m}+P_{m} \Lambda_{m}-\Psi_{e}-\frac{\kappa^{2}}{2} \Psi_{J} ;$ and $\rho>0$. Then, applying the expressions $s=\left[\begin{array}{ll}\Lambda_{m} & I_{n-m}\end{array}\right] e_{a}$ and $e_{m}=\left[\begin{array}{ll}I_{n-m} & 0_{n-m}\end{array}\right] e_{a}$, the inequality (41) is rewritten as

$$
\begin{aligned}
\dot{V} & \leq-e_{a}^{T}\left(\left[\begin{array}{cc}
\Lambda_{m}^{T} K_{a} \Lambda_{m}+\Upsilon & \Lambda_{m}^{T} K_{a} \\
K_{a} \Lambda_{m} & K_{a}
\end{array}\right]-Q\right) e_{a} \\
& -e_{a}^{T} Q e_{a}+\frac{1}{\rho^{2}}\|\underline{w}\|^{2}
\end{aligned}
$$

Thus, if the LMI (39) has a feasible solution, then the following $\dot{V}$ holds

$$
\dot{V} \leq-\alpha_{0}\left\|e_{a}\right\|^{2}+\frac{1}{\rho^{2}}\|\underline{w}\|^{2}
$$

with $\alpha_{0}=\lambda_{\min }(Q)$. Since $V$ is positive-definite and $\dot{V}$ satisfies the inequality (42), we can conclude that $s, e_{m}, \dot{e}_{m} \in L_{\infty}$ and $\tilde{\Theta}_{f_{d}}, \Theta_{f_{d}} \in L_{\infty}$. As a result, $\dot{s}, \ddot{e}_{m} \in L_{\infty}$ is assured based on the boundedness of all terms on right-hand side of (35). On the other hand, taking the force filter (28) into Eq. (36) yields that the force tracking error is expressed in the form:

$$
\tilde{\lambda}=\left(1-\frac{k_{f} \eta_{2}}{D+\eta_{1}+k_{f} \eta_{2}}\right) \varpi\left(e_{m}, s, \tilde{\Theta}_{f_{d}}, w, t\right)
$$

where $D$ is a differential operator. Since $\frac{k_{f} \eta_{2}}{D+\eta_{1}+k_{f} \eta_{2}}$ is a stable filter and all signals $e_{m}, s, \tilde{\Theta}_{f_{d}}, w$ are bounded, the bounded $\varpi(\cdot)$ implies the boundedness of $\tilde{\lambda}$ and $e_{\lambda}$. Note that since the boundedness assumption on the fuzzy approximation error $W_{f_{d}}$ is not utilized here, this proof is achieved in a global sense.

Second, consider the claim (b). Since $\dot{V}$ is negative semidefinite outside the compact set $\left\{\left\|e_{a}\right\|\left\|e_{a}\right\|<\frac{1}{\alpha_{0} \rho}\|\underline{w}\|_{\infty}\right\}$ from the inequality (42), the tracking error $e_{a}(t)$ is globally uniformly ultimately bounded with convergence to a compact residual set. To find the uniformly ultimate bound, we rewrite (42) as

$$
\dot{V} \leq-\frac{\alpha_{0}}{\alpha_{1}} V+\frac{\alpha_{0}}{\alpha_{1}} \zeta(t)
$$

where $\zeta=\frac{1}{2 \gamma} \operatorname{tr}\left(\tilde{\Theta}_{f_{d}}^{T} \tilde{\Theta}_{f_{d}}\right)+\frac{\alpha_{1}}{\alpha_{0} \rho^{2}}\|\underline{w}\|^{2}$ and $\alpha_{1}=\sup _{t} \lambda_{\max }\left(M_{a}\right)>0$ with $M_{a}=\frac{1}{2}\left[\begin{array}{ll}\Lambda_{m} & I_{n-m}\end{array}\right]^{T} \underline{M}\left[\Lambda_{m}\right.$ $\left.I_{n-m}\right]+\left[\begin{array}{ll}I_{n-m} & 0_{n-m}\end{array}\right]^{T} P_{m}\left[\begin{array}{ll}I_{n-m} & 0_{n-m}\end{array}\right]$. Then, the solution of the above inequality leads to that the error trajectory of $e_{a}(t)$ is shaped by 


$$
\left\|e_{a}\right\| \leq \sqrt{\frac{1}{\alpha_{2}} V\left(t_{0}\right)} \exp \left(-\frac{\alpha_{0}}{2 \alpha_{1}}\left(t-t_{0}\right)\right)+\sqrt{\frac{1}{\alpha_{2}}\left[1-\exp \left(-\frac{\alpha_{0}}{\alpha_{1}}\left(t-t_{0}\right)\right)\right] \sup _{t} \zeta(t)}
$$

with $\alpha_{2}=\inf _{t} \lambda_{\min }\left(M_{a}\right)>0$. In other words, the uniform ultimate bound of $e_{a}(t)$ is

$$
\left\|e_{a}\right\| \leq \sqrt{\frac{1}{\alpha_{2}} \sup _{t} \zeta(t)}=\bar{\zeta}\left(\frac{1}{\gamma}\left\|\tilde{\Theta}_{f_{d}}\right\|_{\infty}, \frac{1}{\rho}\|\underline{w}\|_{\infty}\right)
$$

which can be adjusted by tuning $\gamma$ and $\rho$. Meanwhile, the residual force tracking error is adjusted by tuning $\eta_{1}, \eta_{2}, k_{f}$ according to

$$
\lim _{t \rightarrow \infty}\|\tilde{\lambda}(t)\| \cong \frac{\eta_{1}}{\eta_{1}+k_{f} \eta_{2}} \overline{\bar{\omega}}\left(\bar{\zeta},\left\|\tilde{\Theta}_{f_{d}}\right\|\left\|_{\infty},\right\| w \|_{\infty}\right)
$$

with a nonnegative constant $\overline{\bar{\sigma}}=\sup _{t} \bar{\sigma}(t)$ dependent on $\bar{\zeta},\left\|\tilde{\Theta}_{f_{d}}(t)\right\|_{\infty}$, and $\|w(t)\|_{\infty}$.

\begin{tabular}{|c|c|c|c|}
\hline \multirow{2}{*}{\begin{tabular}{c} 
Examples \\
\cline { 2 - 4 }
\end{tabular}} & \multicolumn{3}{|c|}{ Cooperative three-link robots } \\
\hline $\begin{array}{c}\text { Approximated } \\
\text { term }\end{array}$ & $f(\cdot)$ & $f_{d}(\cdot)$ & $f_{d}(\cdot)$ \\
\hline $\begin{array}{c}\text { Premise Variables } \\
q_{1}, \dot{q}_{1} \\
q_{1 d}, \dot{q}_{1 d}, \ddot{q}_{1 d}\end{array}$ & $q_{1 d}, \dot{q}_{1 d}, \ddot{q}_{1 d}$ & $q_{1 d}$ \\
\hline $\begin{array}{c}\text { Number of } \\
\text { Premise Variables }\end{array}$ & 15 & 9 & 3 \\
\hline $\begin{array}{c}\text { Number of Rules } \\
(5 \times 3)\end{array}$ & $\begin{array}{c}32768 \\
\left(2^{15}\right)\end{array}$ & $\begin{array}{c}512 \\
\left(2^{9}\right)\end{array}$ & $\begin{array}{c}8 \\
\left(2^{3}\right)\end{array}$ \\
\hline $\begin{array}{c}\text { Number of fuzzy } \\
\text { Parameters }\end{array}$ & $\begin{array}{c}294912 \\
(32768 \times 9)\end{array}$ & $\begin{array}{c}4608 \\
(512 \times 9)\end{array}$ \\
\hline $\begin{array}{c}\text { Approximation } \\
\text { errors }\end{array}$ & $\begin{array}{c}\text { Assumedly Bounded } \\
\text { a priori }\end{array}$ & Always Bounded & Always Bounded \\
\hline
\end{tabular}

$\Delta$ : each premise variable has two fuzzy sets.

Table 1. Comparisons between SFA and FFA Based Schemes

Third, we prove the claim (c). Consider an energy function $V_{s}=\frac{1}{2} s^{T} \underline{M} s+e_{m}^{T} P_{m} e_{m}$. Analogous to the proof of Theorem 2, a feasible solution of the LMI (39) leads to

$$
\begin{aligned}
\dot{V}_{s} & \leq-e_{a}^{T} Q e_{a}+s^{T} J^{T} Y_{d} \tilde{\Theta}_{f_{d}} \bar{\chi}-\frac{\rho^{2}}{4} \bar{\chi}^{T} \bar{\chi}^{T} J^{T} Y_{d} Y_{d}^{T} J s+\frac{1}{\rho^{2}}\|\underline{w}\|^{2} \\
& \leq-e_{a}^{T} Q e_{a}+\frac{1}{\rho^{2}}\left(\|\underline{w}\|^{2}+\operatorname{tr}\left(\tilde{\Theta}_{f_{d}}^{T} \tilde{\Theta}_{f_{d}}\right)\right)
\end{aligned}
$$

where the fact that $s^{T} J^{T} Y_{d} \tilde{\Theta}_{f_{d}} \bar{\chi} \leq \frac{\rho^{2}}{4} \bar{\chi}^{T} \bar{\chi}^{T} J^{T} Y_{d} Y_{d}^{T} J s+\frac{1}{\rho^{2}} \operatorname{tr}\left(\tilde{\Theta}_{f_{d}}^{T} \tilde{\Theta}_{f_{d}}\right)$ has been applied. Therefore, integrating both sides of the above inequality, the robust performance (40) for the motion tracking objective is assured.

Remark 2: The comparison between SFA and FFA based controllers applied to typical holonomic systems is made in Table 1. From the work (Chang \& Chen, 2000), the SFA-based 
controller requires to take $q_{1}, \dot{q}_{1}, q_{1 d}, \dot{q}_{1 d}, \ddot{q}_{1 d}$ as the premise variables. In contrast, the TS FFAbased controller only needs commands $q_{1 d}$ as the premise variable. The benefits of using the FFA-based controller (fewer rules and tuned parameters) are apparent. Moreover, the fuzzy approximation error of SFA-based controllers needs to be assumedly bounded a priori.

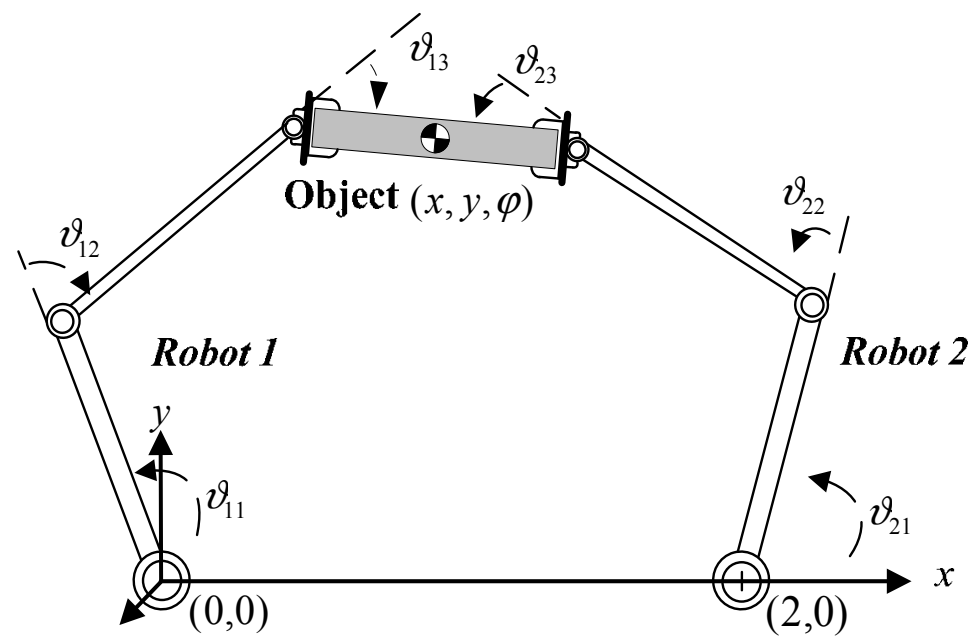

Figure 3. A two-link planer constrained robot manipulator

\section{Simulation Example}

To verify the theoretical derivations, we take a cooperative two-robot system transporting an object as an application example. This holonomic system is subject to a set of closed kinematic chains as illustration in Fig. 3. Two robots are identical in mass and length of links. The center of mass for each link is assumed at the end of each link. All the length of the first and second links $l_{1}, l_{2}$, and the held object are $1 \mathrm{M}$. The length of the third link is sufficiently short and is taken as a part of the object. Let $(x, y, \varphi)$ denote the position and orientation of the held object. Let $\vartheta_{1 \ell}, \vartheta_{2 \ell}(\ell=1,2,3)$ denote joint angles of two robots, respectively. The configuration coordinate of the system is thus denoted as $q_{1}=\left[\begin{array}{lll}x & y & \varphi\end{array}\right]^{T}$ and $q_{2}=\left[\begin{array}{llllll}\vartheta_{11} & \vartheta_{12} & \vartheta_{13} & \vartheta_{21} & \vartheta_{22} & \vartheta_{23}\end{array}\right]^{T}$. Due to the fact that all the end-effectors are rigidly attached to the common object, the holonomic constraint $\phi(q)=\left[\begin{array}{ll}\phi_{1}^{T}(q) & \phi_{2}^{T}(q)\end{array}\right]^{T} \in R^{6}$ consists of

$$
\begin{aligned}
\phi_{1}(q) & =\left[\begin{array}{c}
x-0.5 \cos \varphi \\
y-0.5 \sin \varphi \\
\varphi
\end{array}\right]-\psi_{1}=0 \\
\phi_{2}(q) & =\left[\begin{array}{c}
x+0.5 \cos \varphi-2 \\
y+0.5 \sin \varphi \\
\varphi+\pi
\end{array}\right]-\psi_{2}=0
\end{aligned}
$$




$$
\psi_{j}=\left[\begin{array}{c}
\cos \left(\vartheta_{j 1}\right)+\cos \left(\vartheta_{j 1}+\vartheta_{j 2}\right) \\
\sin \left(\vartheta_{j 1}\right)+\sin \left(\vartheta_{j 1}+\vartheta_{j 2}\right) \\
\vartheta_{j 1}+\vartheta_{j 2}+\vartheta_{j 3}
\end{array}\right], \text { for } j=1,2
$$

Therefore, the Jacobian matrix $A(q)$ is consists of $A_{1}^{T}=$ block-diag $\left\{A_{11}^{T}, A_{12}^{T}\right\}$ and $A_{2}=$ block-diag $\left\{A_{21}, A_{22}\right\}$ with:

$$
\begin{gathered}
A_{11}^{T}=\left[\begin{array}{ccc}
1 & 0 & 0 \\
0 & 1 & 0 \\
0.5 \sin \varphi & -0.5 \cos \varphi & 1
\end{array}\right] \\
A_{12}^{T}=\left[\begin{array}{ccc}
1 & 0 & 0 \\
0 & 1 & 0 \\
-0.5 \sin \varphi & 0.5 \cos \varphi & 1
\end{array}\right] \\
A_{2 j}=-\left[\begin{array}{ccc}
-\sin \left(\vartheta_{j 1}\right)-\sin \left(\vartheta_{j 12}\right) & -\sin \left(\vartheta_{j 12}\right) & 0 \\
\cos \left(\vartheta_{j 1}\right)+\cos \left(\vartheta_{j 12}\right) & \cos \left(\vartheta_{j 12}\right) & 0 \\
1 & 1 & 1
\end{array}\right]
\end{gathered}
$$

where $\vartheta_{j 12}=\vartheta_{j 1}+\vartheta_{j 2}$. The kinematic transformation matrix is written as $J=\left[\begin{array}{ll}I_{3} & -\left(A_{2}^{-1} A_{1}\right)^{T}\end{array}\right]^{T}$. In addition, the general dynamic model (21) is composed of $M=$ block-diag $\left\{M_{0}, M_{1}\right.$, $\left.M_{2}\right\}, C=$ block-diag $\left\{C_{0}, C_{1}, C_{2}\right\} ， \quad g=\left[\begin{array}{lll}g_{0} & g_{1} & g_{2}\end{array}\right]^{T}, M_{0}=\operatorname{diag}\left\{m_{o}, m_{o}, I_{0}\right\} ， C_{0}=0$ ， $g_{0}=\left[\begin{array}{lll}0 & m_{o} g & 0\end{array}\right]^{T}$,

$$
\begin{gathered}
M_{j}=\left[\begin{array}{ccc}
a_{j 1}+2 a_{j 2} \cos \left(\vartheta_{j 2}\right)+a_{j 3} & (*) & (*) \\
a_{j 2} \cos \left(\vartheta_{j 2}\right)+a_{j 3} & a_{j 3} & (*) \\
a_{j 4} & a_{j 4} & a_{j 4}
\end{array}\right] \\
C_{j}=\left[\begin{array}{ccc}
-a_{j 2} \sin \left(\vartheta_{j 2}\right) \dot{\vartheta}_{j 2} & -a_{j 2} \sin \left(\vartheta_{j 2}\right) \dot{\vartheta}_{j 12} & 0 \\
a_{j 2} \sin \left(\vartheta_{j 2}\right) \dot{\vartheta}_{j 1} & 0 & 0 \\
0 & 0 & 0
\end{array}\right] \\
g_{j}=\left[\begin{array}{c}
\left(a_{j 1} \cos \left(\vartheta_{j 1}\right)+a_{j 2} \cos \left(\vartheta_{j 1}+\vartheta_{j 2}\right)\right) g / l_{1} \\
a_{j 2} \cos \left(\vartheta_{j 1}+\vartheta_{j 2}\right) g / l_{1} \\
0
\end{array}\right]
\end{gathered}
$$

for $j=1,2$, where $\left(^{*}\right)$ represents a symmetric term; $a_{j 1}=\left(m_{j 1}+m_{j 2}+m_{j 3}\right) l_{1}^{2}$; $a_{j 2}=\left(m_{j 2}+m_{j 3}\right) l_{1} l_{2} ; a_{j 3}=\left(m_{j 2}+m_{j 3}\right) l_{2}^{2}+I_{j 3} ; a_{j 4}=I_{j 3} ;$ and $m_{j 1}, m_{j 2}, m_{j 3}, I_{j 3}, m_{o}, I_{o}$ are system parameters. The actual value of $\left(m_{0}, I_{o}, a_{11}, a_{12}, a_{13}, a_{14}, a_{21}, a_{22}, a_{23}, a_{24}\right)$ is set as $(1,0.25,5,3,3.05,0.05,5,3,3.05,0.05)$. According to the holonomic constraint $\phi(q)=0$, we 
can find $B_{g}=I_{9}, \tau_{g}=\left[\left(A_{1}^{T} \lambda_{M}\right)^{T} \quad\left(\tau+A_{2}^{T} \lambda_{M}\right)^{T}\right]^{T}$, and $\lambda_{g}=\lambda_{I}$, where $\tau=\left[\begin{array}{ll}\tau_{1}^{T} & \tau_{2}^{T}\end{array}\right]^{T} \in R^{6}$ is the applied force for the two robots; $\lambda_{M}$ denotes a motion-inducing force which has contribution to the motion of the object by $A_{1}^{T} \lambda_{M}$; and $\lambda_{I}$ denotes an internal force which lies in a nontrivial null space $Z=\left\{\lambda_{I} \in R^{m} \mid A_{1}^{T} \lambda_{I}=0\right\}$. Therefore, if the control input $\tau_{g}$ is designed according to Thm. 4 , then the actual control input is calculated by

$$
\tau=\tau_{g^{2}}-A_{2}^{T}\left(A_{1}^{T}\right)^{+} \tau_{g 1}
$$

where $\tau_{g 1} \in R^{3}, \tau_{g^{2}} \in R^{6}$ are partitioned components of $\tau_{g}$ (i.e. $\tau_{g}=\left[\begin{array}{ll}\tau_{g 1}^{T} & \tau_{g 2}^{T}\end{array}\right]^{T}$ ); and $\left(A_{1}^{T}\right)^{+}=A_{1}\left(A_{1}^{T} A_{1}\right)^{-1}$ denotes the pseudo-inverse of $A_{1}^{T}$.

For this cooperative two-robot system, the control objective is to track desired trajectories for the object and internal force as

$$
\begin{gathered}
q_{1 d}(t)=\left[\begin{array}{c}
1+0.25 \cos (t) \\
1+0.25 \sin (t) \\
0.25
\end{array}\right], \\
\lambda_{g d 1}=40\left[\begin{array}{c}
\cos \varphi \\
\sin \varphi \\
0
\end{array}\right], \lambda_{g d 2}=40\left[\begin{array}{c}
-\cos \varphi \\
-\sin \varphi \\
0
\end{array}\right]
\end{gathered}
$$

where $\lambda_{g d 1}$ and $\lambda_{g d 2}$ represent the compressed force vector.

On the other hand, since the TS FFA has a general representation capability, we are able to properly choose the basis function such that fewer premise variables are used. According to the function $f_{d}(\cdot)$, the feed-forward TS FFA-based fuzzy system (30) is constructed with $\bar{\chi}=\left[\begin{array}{lllllllllll}1 & q_{1 d 1} & q_{1 d 2} & q_{1 d 3} & \dot{q}_{1 d 1}^{2} & \dot{q}_{1 d 2}^{2} & \dot{q}_{1 d 1} \dot{q}_{1 d 2} & \ddot{q}_{1 d 1} & \ddot{q}_{1 d 2} & \ddot{q}_{1 d 3}\end{array}\right]^{T} \in R^{10}$ (where $q_{1 d \ell}$ is the $\ell$-th element of $q_{1 d}$, for $\ell=1,2,3$ ) and linguistic variables $q_{1 d \ell}$, which accordingly are classified into two fuzzy sets. From the exactly known mean and varying region, the fuzzy sets are easily characterized by the following membership functions:

$$
\begin{aligned}
& \left\{\begin{array}{l}
\mu_{\mathbf{x}_{n \ell}}\left(q_{1 d \ell}\right)=1-\mu_{\mathbf{x}_{s \ell}}\left(q_{1 d \ell}\right) \\
\mu_{\mathbf{x}_{s \ell}}\left(q_{1 d \ell}\right)=\exp \left(-2\left(q_{1 d \ell}-1\right)^{2}\right), \text { for } \ell=1,2
\end{array}\right. \\
& \left\{\begin{array}{l}
\mu_{\mathbf{x}_{n 3}}\left(q_{1 d 3}\right)=1-\mu_{\mathbf{x}_{s 3}}\left(q_{1 d 3}\right) \\
\mu_{\mathbf{x}_{s 3}}\left(q_{1 d 3}\right)=\exp \left(-2\left(q_{1 d 3}+0.25\right)^{2}\right), \text { for } \ell=3
\end{array}\right.
\end{aligned}
$$

This results in the total number of fuzzy rules to be 8 , i.e., $\Theta_{f_{d}} \in R^{72 \times 10}$. When considering the special case with $\bar{\chi}=1$ (i.e., Mamdani FFA), all of $q_{1 d \ell}, \dot{q}_{1 d \ell}$ and $\ddot{q}_{1 d \ell}$ should be utilized as linguistic variables for an admissible approximation, which needs 512 fuzzy rules and 4608 tuning parameters. This implies that the proposed approach in this paper leads to less 
numbers of fuzzy rules and tuning parameters. Furthermore, the fuzzy consequent parts are adjusted by (33), where $\gamma=50, \bar{\theta}_{f_{d}}=10^{4}, \varepsilon_{f}=10$, and $\Theta_{f_{d}}(0)=[0]_{72 \times 10}$.

Furthermore, the control parameters are chosen as: $\eta_{1}=0.1, \eta_{2}=20, k_{f}=15, \Lambda_{m}=\operatorname{diag}\{10$, $5,5\}, \rho=5$, and $Q=I_{6}$. Then, after choosing $\Psi_{s}=\operatorname{diag}\{40,20,10\}, \Psi_{s e}=\Psi_{e}=2 I_{3}, \Psi_{J}=I_{3}$ for (37), the control gains are obtained as $\kappa=7.9, K=\operatorname{diag}\{53.5,45.2,35.2\}$, and $P_{m}=$ $\operatorname{diag}\{36.5,51.7,51.7\}$, by solving the LMI (39). In this simulation, the system begins at the position $q(0)=\left[\begin{array}{lllllllll}1 & \frac{2-\sqrt{3}}{2} & 0 & \frac{\pi}{2} & -\frac{5 \pi}{6} & \frac{\pi}{3} & \frac{\pi}{2} & \frac{5 \pi}{6} & -\frac{\pi}{3}\end{array}\right]^{T}$ and all have zero initial velocities, i.e., $\dot{q}(0)=0$. The external disturbance is injected to the first joint of two robots as $\tau_{d}$ which is a square wave with amplitude 0.25 and frequency $0.5 \mathrm{~Hz}$. According to Thm. 4 , the simulation results of position and velocity tracking for the object are illustrated in Figs. 4 and 5, respectively. The internal force errors between the desired and actual internal force are shown in Fig. 6. The second joints of two robots are driven by torques illustrated in Fig. 7. For a comparison, a traditional SFA-based controller is also constructed and applied to the cooperative robots, where the Mamdani SFA takes $q_{1 \ell}, \dot{q}_{1 \ell}, q_{1 d \ell}, \dot{q}_{1 d \ell}$ and $\ddot{q}_{1 d \ell}$ as the premise variables. Furthermore, the SFA-based control is set with the same initial conditions and feedback compensation part as the proposed controller but $\Psi_{s}=0, \Psi_{e}=0, \Psi_{s e}=0$, and $\Psi_{J}=0$. Then, the position tracking results for using Mamdani SFA and TS FFA based control are made as a comparison given in Fig. 8. Obviously, the TS FFA-based controller leads to a smaller tracking error.
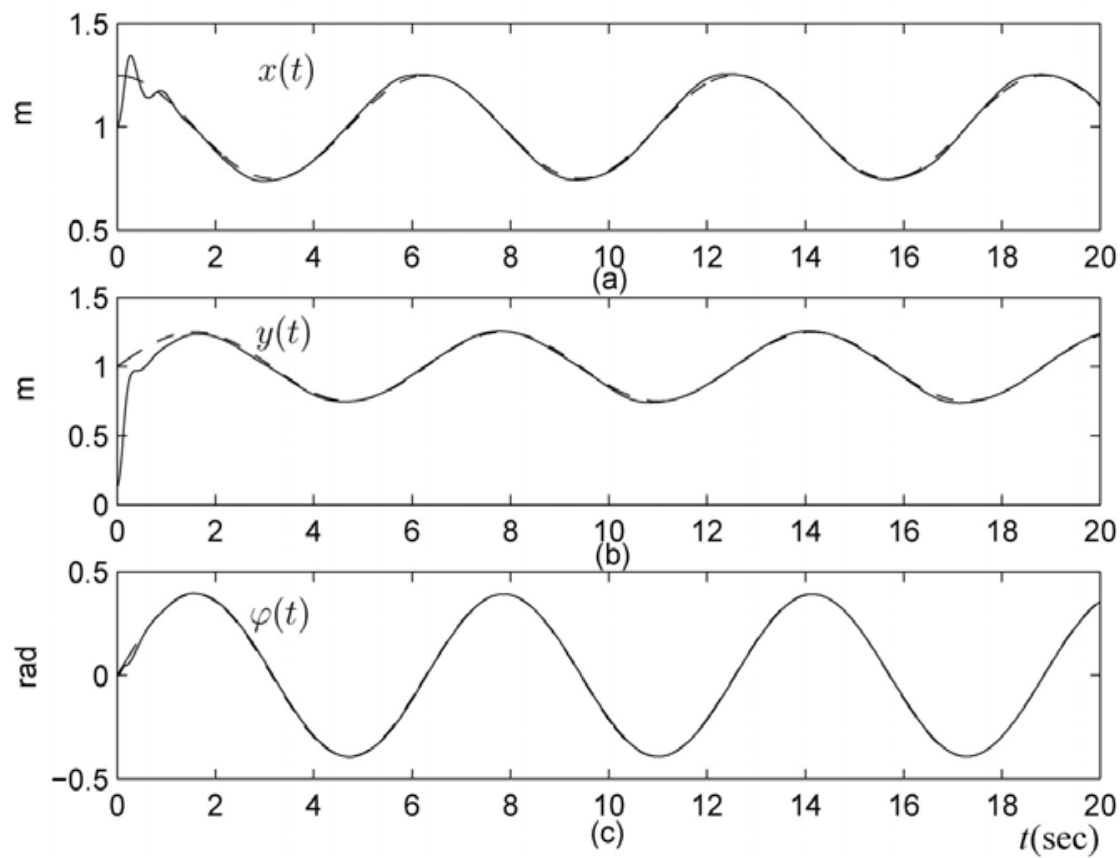

Figure 4. The position tracking results of the held object. (- object, - - reference) 

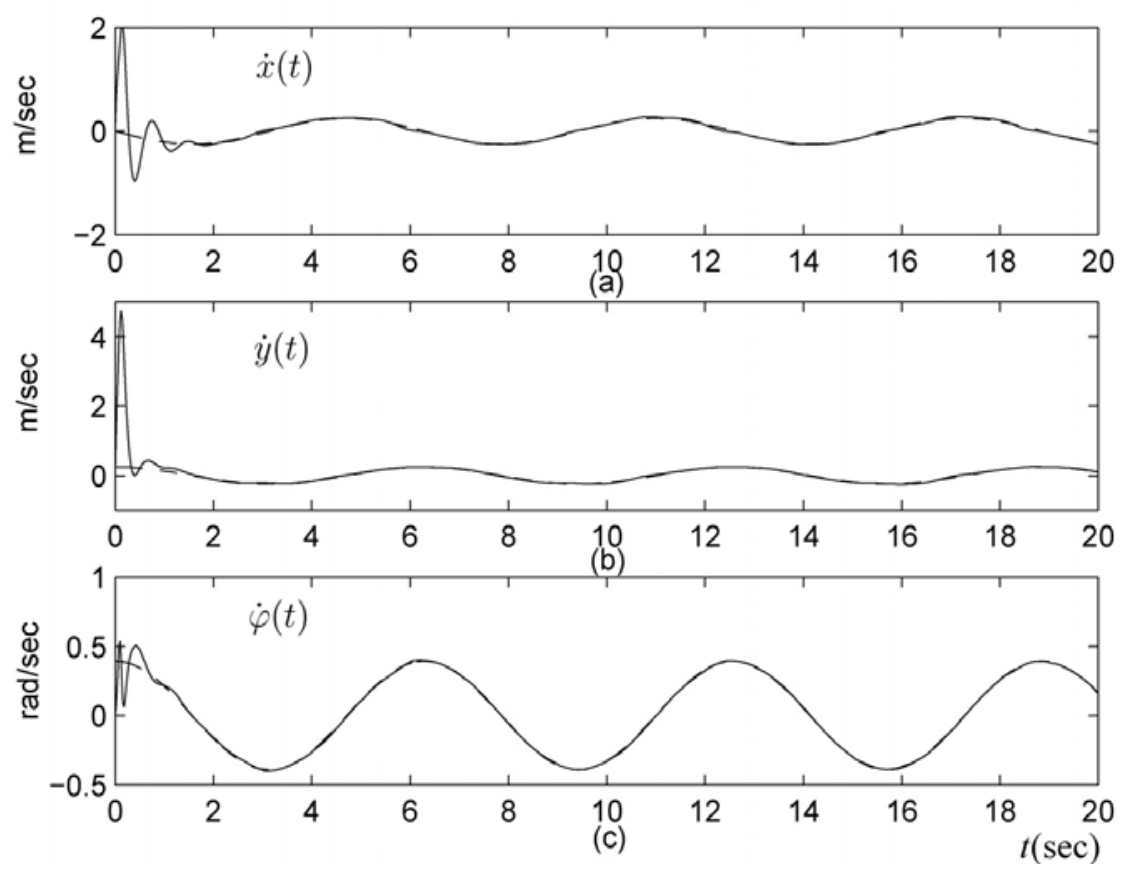

Figure 5. The velocity tracking results of the held object. (- object, - - reference)
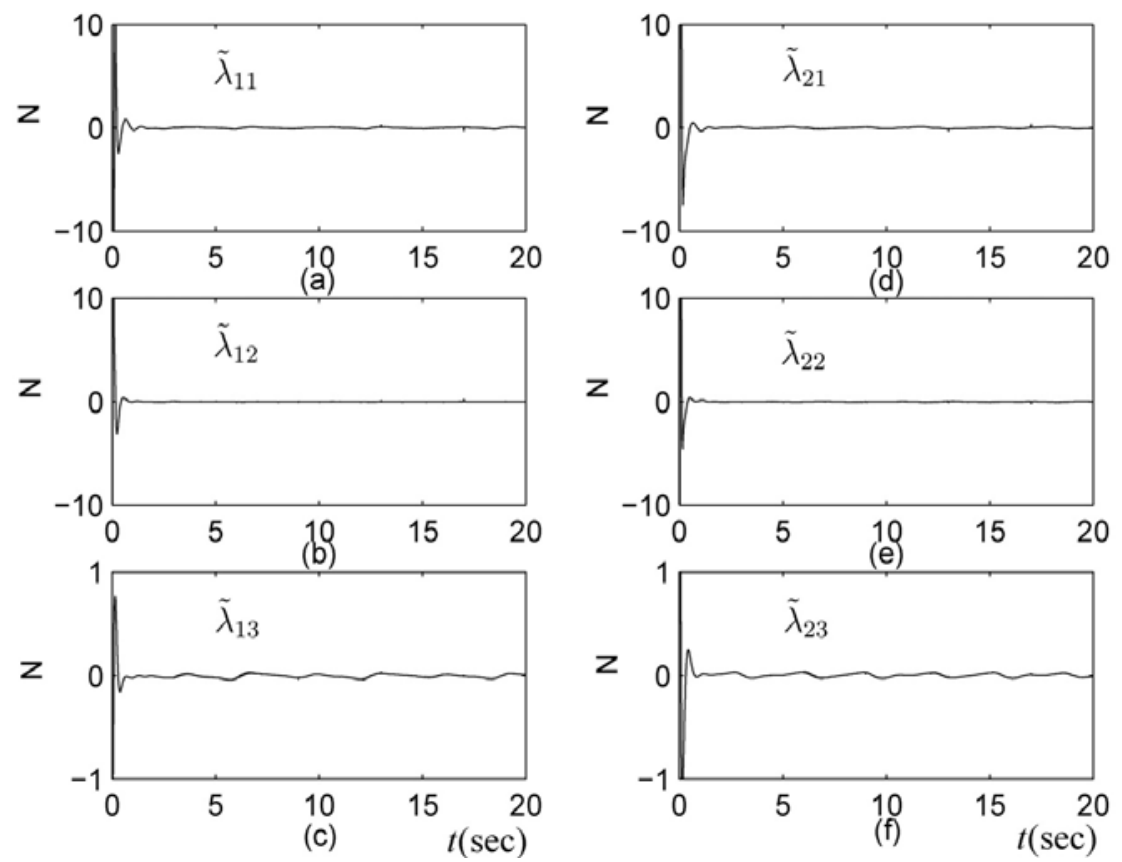

Figure 6. (a-c) Internal force tracking errors for Robot 1; and (d-f) internal force tracking errors for Robot 2 

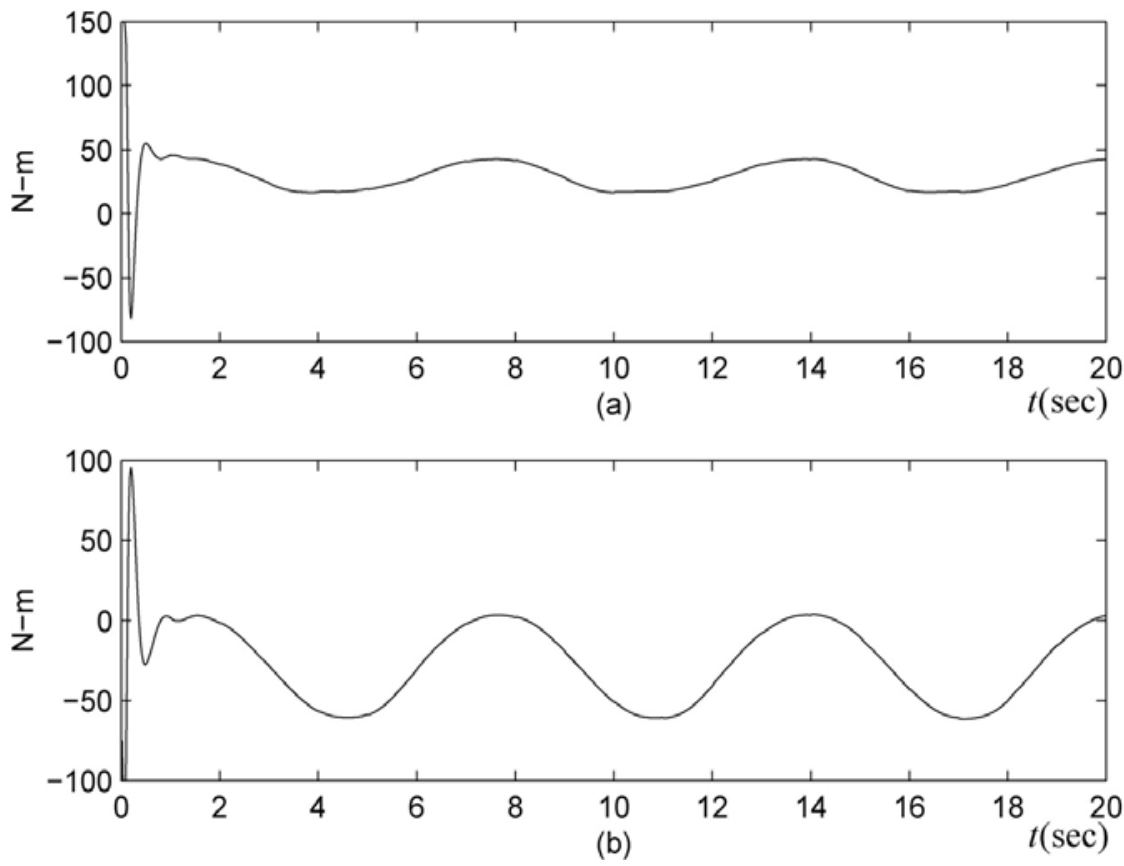

Figure 7. (a) Control input for the second joint of Robot 1; and (b) control input for the second joint of Robot 2
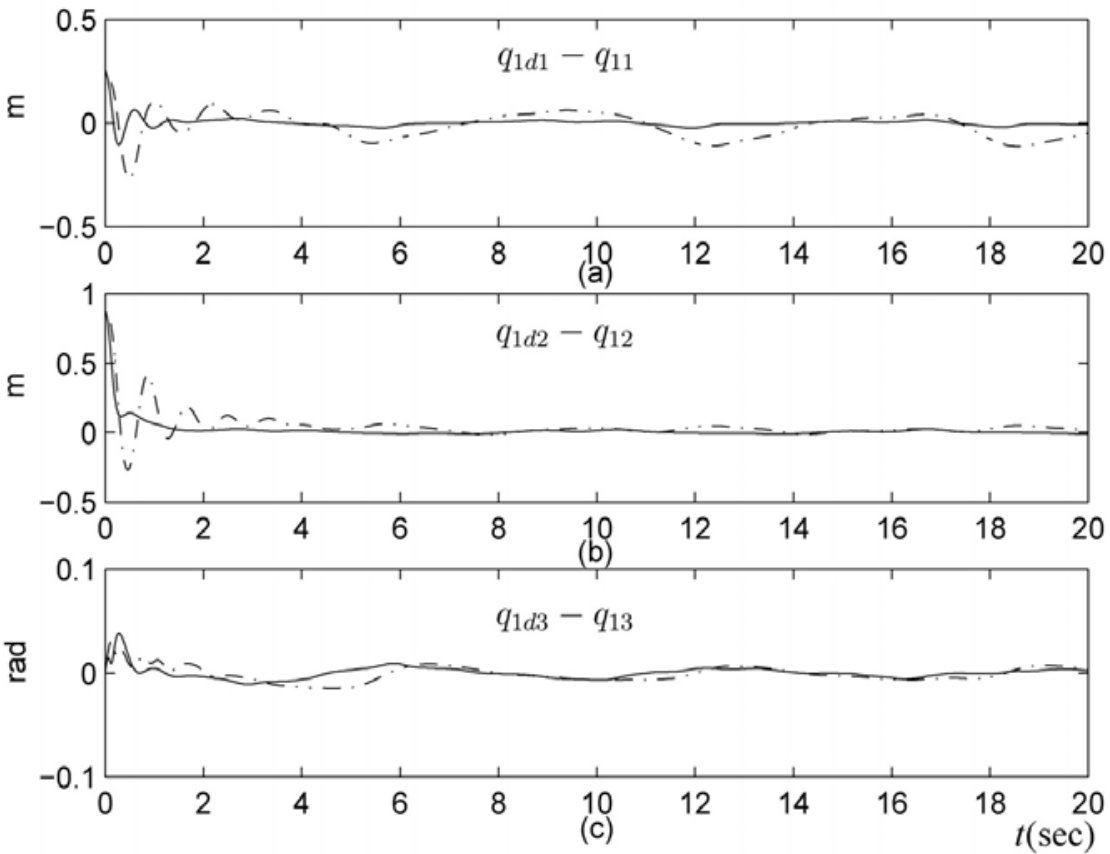

Figure 8. Comparison result of the position tracking errors of the held object. ( - TS FFA, - Mamdani SFA) 


\section{Conclusions}

In this study, a novel TS FFA-based adaptive control scheme has been proposed and applied to motion/force tracking control of holonomic systems. By integrating the feed-forward fuzzy compensation and error-feedback concepts, the proposed FFA-based control concept avoids heavy computation load and achieves global control results. In detail, the FFA-based adaptive control has removed some disadvantages of traditional adaptive fuzzy control including the boundedness assumption on fuzzy approximation errors, a vast amount of rules and tuning parameters, and complicated implementation architecture. Based on an LMI technique and nonlinear damping error-feedback, the overall controlled uncertain system further assures either robust tracking performance or asymptotic convergence. In addition, the TS FFA-based adaptive controller can straightforwardly solve the control problem of complicated and high-dimension systems - holonomic systems. As a result, $H^{\infty}$ motion tracking performance is guaranteed with the attenuation of disturbances, approximation errors, and tuned fuzzy parameter errors. Meanwhile, the residual force tracking error is confined to a small value by adjusting control gains feasibly.

\section{Acknowledgment}

This work was supported by the National Science Council, R.O.C., under Grant NSC-962221-E-033-077.

\section{References}

Wang, L. X. \& Mendel, J. M. (1992). Fuzzy basis functions, universal approximation, and orthogonal least squares learning. IEEE Trans. on Neural Networks, Vol. 3, No. 5, pp. 807-814.

Chen, B.-S.; Lee, C.-H. \& Chang, Y.-C. (1996). $H^{\infty}$ tracking design of uncertain nonlinear SISO systems: adaptive fuzzy approach. IEEE Trans. on Fuzzy Systems, Vol. 4, pp. 32-43.

Lee, H. \& Tomizuka, M. (2000). Robust adaptive control using a universal approximator for SISO nonlinear systems. IEEE Trans. on Fuzzy Systems, Vol. 8, pp. 95-106.

Lin, W.-S. \& Chen, C.-S. (2002). Robust adaptive sliding mode control using fuzzy modeling for a class of uncertain MIMO nonlinear systems. IEE Proc.-Control Theory Applications, Vol. 149, No. 3, pp. 193-201.

Ying, H. (1998). Sufficient conditions on uniform approximation of multivariate functions by general Takagi-Sugeno fuzzy systems with linear rule consequent. IEEE Trans. on Syst., Man, Cybern. Part-A, Vol. 28, No. 4, pp. 515-520.

Tsay, D.-L.; Chung, H.-Y. \& Lee, C.-J. (1999). The adaptive control of nonlinear systems using the Sugeno-type of fuzzy logic. IEEE Trans. on Fuzzy Systems, Vol. 7, pp. 225229.

Chen, J.-Y. \& Wong, C.-C. (2000). Implementation of the Takagi-Sugeno model-based fuzzy control using an adaptive gain controller. IEE Proc.-Control Theory Applications, Vol. 147, No. 5, pp. 509-514.

Alata, M.; Su, C.-Y. \& Demirli, K. (2001). Adaptive control of a class of nonlinear systems with a first-order parameterized Sugeno fuzzy approximator. IEEE Trans. on Systems, Man, Cybernetics - part C, Vol. 31, pp. 410-419. 
McClamroch, N. H. \& Wang, D. (1988). Feedback stabilization and tracking of constrained robots. IEEE Trans. on Automatic Control, Vol. 33, pp. 419-426.

Tarn, T. J.; Bejzcy, A. K. \& Yun, X. (1987). Design of dynamic control of two cooperating robot arms: closed chain formulation. Proceedings of IEEE Conf. on Robotics and Automation, pp. 7-13.

Li, Z.; Hsu, P. \& Sastry, S. (1989). Grasping and coordinated manipulation by a multifingered robot hand. Int. Journal of Robotic Research, Vol. 8, pp. 33-50.

Wang, J.; Dodds, S. J. \& Bailey, W. N. (1997). Coordinated control of multiple robotic manipulators handling a common object - theory and experiments. IEE Proceedings Control Theory and Applications, Vol. 144, pp. 103-109.

Jean, J.-H. \& Fu, L.-C. (1993). An adaptive control scheme for coordinated manipulator systems. IEEE Trans. on Robotics and Automation, Vol. 9, pp. 226-231.

Liu, Y.-H.; Arimoto, S.; Parra-Vega, V. \& Kitagaki, K. (1997). Decentralized adaptive control of multiple manipulators in cooperations. Int. Journal of Control, Vol. 67, pp. 649-673.

$\mathrm{Yu}, \mathrm{H}$. \& Lloyd, S. (1997). Combined direct and indirect adaptive control of constrained robots. Int. Journal of Control, Vol. 68, pp. 955-970.

Zhu, W.-H. \& Schutter, J. D. (1999). Control of two industrial manipulators rigidly holding an egg. IEEE Control Systems Magazine, Vol. 19, pp. 24-30.

Yuan, J. (1997). Adaptive control of a constrained robot - ensuring zero tracking and zero force errors. IEEE Trans. on Automatic Control, Vol. 42, pp. 1709-1714.

Chiu, C.-S.; Lian, K.-Y. \& Wu, T.-C. (2004). Robust adptive motion/force tracking control design for uncertain constrained manipulators. Automatica, Vol. 40, pp. 2111-2119.

Zhen, R. Y. \& Goldenberg, A. A. (1996). Variable structure hybrid control of manipulators in unconstrained and constrained motion. ASME Journal of Dynamic Systems, Measurement and Control, Vol. 118, pp. 327-332.

Gueaieb, W.; Karray, F. \& Al-Sharhan, S. (2003). A robust adaptive fuzzy position/force control scheme for cooperative manipulators. IEEE Trans. on Control Systems Technology, Vol. 11, pp. 516-528.

Chang, Y.-C. \& Chen, B.-S. (2000). Robust tracking designs for both holonomic and nonholonomic constrained mechanical systems: adaptive fuzzy approach. IEEE Trans. on Fuzzy Systems, Vol. 8, pp. 46-66.

Lian, K.-Y.; Chiu, C.-S. \& Liu, P. (2002). Semi-decentralized adaptive fuzzy control for cooperative multirobot systems with $H^{\infty}$ motion/internal force tracking performance. IEEE Trans. on Systems, Man, Cybernetics - part B, Vol. 32, pp. 269-280.

Boyd, S.; Ghaoui, L. E.; Feron, E. \& Balakrishnan, V. (1994). Linear Matrix Inequalities in System and Control Theory, SIAM, Philadelphia.

Sadegh, N. \& Horowitz, R. (1990). Stability and robustness analysis of a class of adaptive controller for robotic manipulators. Int. J. Robotic Research, Vol. 9, pp. 74-92.

Chiu, C.-S.; Lian, K.-Y. \& Liu, P. (2006). Adaptive control of holonomic constrained systems: a feedforward fuzzy approximation based approach. IEEE Transaction on Control Systems Technology, Vol. 14, No. 3, pp. 456-466.

Pomet, J.-B. \& Praly, L. (1992). Adaptive nonlinear regulation: estimation from the Lyapunov equation. IEEE Trans. on Automatic Control, Vol. 37, pp. 729-740.

Chiu, C.-S. (2006). Mixed feedforward/feedback based adaptive fuzzy control for a class of MIMO nonlinear systems. IEEE Trans. on Fuzzy Systems, Vol. 14, pp. 716-727. 


\title{
Function Approximation-based Sliding Mode Adaptive Control for Time-varying Uncertain Nonlinear Systems
}

\author{
Shuang Cong, Yanyang Liang and Weiwei Shang \\ University of Science and Technology of China \\ P. R. China
}

\section{Introduction}

Dead zone characteristics exist in many physical components of control systems. They are nonlinear features particularly in direct current (DC) motor position tracking control systems, mainly caused by the uncertain time-varying nonlinear friction. They can severely limit the control performance owing to their non-smooth nonlinearities. However, dead zone characteristics usually are not easy to be known exactly and may vary with time in practical. In addition to the uncertainties in the linear part of the plant, controllers are often required to accommodate time-varying dead zone uncertainties. In general, there are two usual methods treating the systems with uncertain time-varying dead zone characteristics caused by uncertain nonlinear frictions in DC motor position control systems. The first one is to separate the unknown dead zone from the original DC motor systems and construct an adaptive dead zone inverse, and then compensate the effects of unknown dead zone characteristics (Gang \& Kokotovic, 1994; Cho \& Bai, 1998; Wang et al., 2004; Zhou et al., 2006). The second method is to deal with both the unknown dead zone characteristics and all the other uncertainties as one uniform uncertainty, thereupon design proper compensator (Wang et al., 2004) or adaptive controller which can counteract the effects of uncertainty(Selmic \& Lewis, 2000; Tian-Ping et al., 2005). Furthermore, dead zone uncertainties' bounds remain unknown in many practical DC motor control systems. This problem can't be coped with conventional sliding mode controller (Young et al., 1999; Hung et al., 1993) and general adaptive controller (Gang \& Kokotovic, 1994; Cho \& Bai, 1998; Wang et al., 2004; Zhou et al., 2006; Wang et al., 2004; Selmic \& Lewis, 2000; Tian Ping et al., 2005; Young et al., 1999; Hung et al., 1993). In order to deal with nonlinear systems with unknown bound time-varying uncertainties, adaptive control schemes combined with sliding mode technique have been developed (Chyau-An \& Yeu-Shun, 2001; Chyau-An \& Yuan-Chih, 2004; Huang \& Chen, 2004; Chen \& Huang, 2004). These control schemes can transform the unknown bound time-varying uncertainties into finite combinations of Fourier series as long as the uncertainties satisfy Dirichlet condition, so that they can be estimated by updating the Fourier coefficients. Since the coefficients are timeinvariant, update laws are easily obtained from the Lyapunov design to guarantee output error convergence.

This chapter is devided into two parts. In the first part, for the position tracking in DC motor with unknown bound time-varying dead zone uncertainties, we'll propose a Function 
Approximation-based Sliding Mode Adaptive Controller (short for FASMAC). Firstly, we obtain a control law consisting of an unknown bound time-varying uncertain term same as An-Chyau (2001) and another compensative term through sliding mode technique and, afterwards, transform the uncertain term into a combination of a set of orthonormal basis functions with the approach of function approximation technique, where Laguerre function series are employed for their widely application in system model approximation (Wahlberg, 1991; Oliver et al., 1994; Campello et al., 2004) and adaptive controller design(Zervos \& Dumont, 1988; Wang, 2004). Then concrete expressions of uncertain term and compensative term can thus be derived based on the Lyapunov design to guarantee output error convergence. This control scheme can not only approximate the unknown bound timevarying uncertainties online but also compensate the error of approximation synchronously. Actual experiments on DC motor position tracking demonstrate the performance of the control scheme. In the second part, we'll extend the sliding mode adaptive controller for SISO system in the first part of the chapter to an adaptive controller for SIMO system with unknown bound time-varying uncertainty. The control strategy only requires that the uncertainty is the piecewise continuous or square integrable in finite time interval, and doesn't demand for the information of the uncertainty's bound and some conditions of the uncertainty, thus it is more suitable for actual SIMO uncertain nonlinear systems. The SIMOAC strategy gives a sliding function according to the sliding mode control basic principle firstly, and then transforms the time-varying uncertainty into the multiplying of a known time-varying basis function vector and an unknown time-invariant coefficient vector, and further obtains the updating law of coefficient vector and an adaptive on-line compensation of approximation error, then adaptive control law are obtained at last.

\section{Function Approximation-based Sliding Mode Adaptive Control for SISO system}

\subsection{Problem statement}

The system to be controlled is a DC motor position control system. The simplified plant model is shown in Fig.1, where $U_{f}$ stands for the equivalent voltage caused by unknown time-varying nonlinear friction, $x_{1}$ and $x_{2}$ represent the system position state and velocity state, respectively, and $T_{m}$ is the time constant value of DC motor, $K_{e}$ is the ratio of speed feedback.

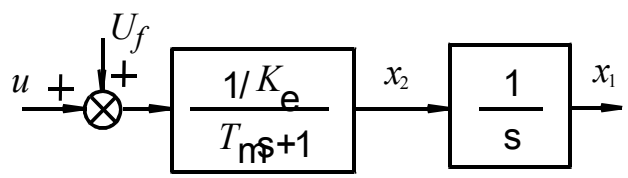

Figure 1. Simplified open-loop model of DC motor position control system

Let $U$ be the known static friction moment of $U_{f}$ in Fig.1, we obtain system state space equation as

$$
\left\{\begin{array}{l}
\dot{x}_{1}=x_{2} \\
\dot{x}_{2}=-\frac{1}{T_{m}} x_{2}+\frac{1}{T_{m} K_{e}} u(t)+\frac{1}{T_{m} K_{e}} U+\frac{1}{T_{m} K_{e}}\left(U_{f}(t)-U\right)
\end{array}\right.
$$


For the purpose of convenience of controller design procedure, let $X(t)=\left[x_{1}(t) x_{2}(t)\right]^{\mathrm{T}}$. Then (1) can be rewritten as

$$
\dot{X}(t)=\bar{A} X(t)+\bar{B} u(t)+\bar{U}+D(t)
$$

where $\bar{A}, \bar{B}$ and $\bar{U}$ are known constant vectors, while $D(t)$ is unknown time-varying uncertainty, and

$$
\bar{A}=\left[\begin{array}{cc}
0 & 1 \\
0 & -\frac{1}{T_{m}}
\end{array}\right], \bar{B}=\left[\begin{array}{c}
0 \\
\frac{1}{T_{m} K_{e}}
\end{array}\right], \bar{U}=\left[\begin{array}{c}
0 \\
\frac{1}{T_{m} K_{e}} U
\end{array}\right], D(t)=\left[\begin{array}{c}
0 \\
\frac{1}{T_{m} K_{e}}\left(U_{f}(t)-U\right)
\end{array}\right]
$$

Eq. (2) is a perturbation model of DC motor position system, where $D(t)$ denotes the unknown bound time-varying dead zone uncertainty which mainly origins from uncertain time-varying nonlinear friction. Since conventional control strategy based on precise mathematic model usually can't reach performance requirement, thus, new control scheme needs be developed to improve system performance.

Assumption 1. $\exists C=\left[c_{1} c_{2}\right] \in \mathfrak{R}^{1 \times 2}, c_{1}$ and $c 2$ are both positive or negative, for perturbation model of DC motor position system (2), guarantees that $C \bar{B} \neq 0$ and timevarying uncertain function $C D(t)$ is square integral for any finite time $T, T \in \Re$, that is $C D(t) \in L^{2}\left[R^{+}\right]$.

Under the Assumption 1, the unknown bound time-varying uncertainty $C D(t)$ can be transformed into a finite combination of Laguerre functions, and then coefficients of Laguerre functions are obtained using Lyapunov direct method.

\subsection{Function approximation-based sliding mode adaptive controller design}

In this section, we give the details of the FASMAC design. Firstly, a standard linear switch function $s(t)$ is chosen; then, the unknown bound time-varying uncertainty is transformed into a combination of series of orthonormal basis function employing Laguerre functions; thirdly, a control law including the approximation of uncertainty and it's approximation error compensation is proposed; finally, the concrete expression of the control law is obtained through Lyapunov direct method.

Given referenced position as $x_{d 1}(t)$, assume that it's not special limit for the velocity of DC motor. Let

$$
x_{d 2}(t)=\dot{x}_{d 1}(t), \quad X_{d}(t)=\left[x_{d 1}(t) \quad x_{d 2}(t)\right]^{T}
$$

Define error function as

$$
E(t)=X(t)-X_{d}(t)=\left[\begin{array}{c}
e_{1}(t) \\
e_{2}(t)
\end{array}\right]=\left[\begin{array}{c}
e_{1}(t) \\
\dot{e}_{1}(t)
\end{array}\right]
$$

where $e_{1}(t)$ denotes position error, and $e_{2}(t)$ denotes velocity error.

We choose a switch function $s(t)$ as

$$
s(t)=C\left(X(t)-X_{d}(t)\right)=C \cdot E(t)
$$


where $C=\left[c_{1} c_{2}\right]$ is the constant vector in the Assumption 1 .

According to (5) and (6), we have

$$
s(t)=C\left[\begin{array}{c}
e_{1}(t) \\
\dot{e}_{1}(t)
\end{array}\right]=c_{1} e_{1}(t)+c_{2} \dot{e}_{1}(t)=c_{1}\left(e_{1}(t)+\frac{c_{2}}{c_{1}} \dot{e}_{1}(t)\right)
$$

where $c_{1} \neq 0$. If $E(t)$ lies on the surface $s(t)=0$, it's easy to guarantee position error $e_{1}(\mathrm{t})$ asymptotically stable by holding $c_{1}$ and $c_{2}$ both positive or negative, or alternatively, switch function (6) is a sliding surface(Young et al., 1999; Hung et al., 1993). In the following we will employ the FAT and Lyapunov direct method to derivate a sliding mode adaptive control law that guarantees the stability of sliding surface $s(t)$.

Let $u_{m}(t)=C D(t), \hat{u}_{m}(t)$ is the on-line approximation of $u_{m}(t)$. Under the Assumption 1, there exists a sufficient large $N, u_{m}(t)$ can be transformed into a combination of a set of Laguerre function series as

$$
u_{m}(t)=W^{T} Z(t)+\varepsilon(t)
$$

Using the same Laguerre function series, the on-line approximation of $u_{m}(t)$ can be expressed as

$$
\hat{u}_{m}(t)=\hat{W}^{T} Z(t)
$$

where $\varepsilon(t)$ is the approximation error of Laguerre function series, $\hat{W}$ is the approximation of $W$, and

$$
\begin{gathered}
W=\left[\begin{array}{llllll}
w_{1} & w_{2} & w_{3} & \ldots & w_{N-1} & w_{N}
\end{array}\right]^{T}, \quad \hat{W}=\left[\begin{array}{llllll}
\hat{w}_{1} & \hat{w}_{2} & \hat{w}_{3} & \ldots & \hat{w}_{N-1} & \hat{w}_{N}
\end{array}\right]^{T} \\
Z(t)=\left[\begin{array}{llll}
\phi_{1}(t) & \cdots & \phi_{N}(t)
\end{array}\right]^{T}
\end{gathered}
$$

An excellent property of (8) is its linear parameterization of the time-varying uncertainty into a time-varying basis function $Z(t)$ and a time invariant coefficient vector $W$, where $Z(t)$ is known while $W$ is an unknown time invariant constant vector. With this transformation, the unknown bound time-varying uncertainty is replaced by a set of unknown constants. Therefore, the approximation of $u_{m}(t)$ turns to find the update law for $\hat{W}$ in (9) by selecting proper Lyapunov function.

Define

$$
\widetilde{W}=W-\hat{W}
$$

Generally, the bound of $\varepsilon(t)$ can be made small enough by choosing a sufficient large $N$, and there always exists an error between $W$ and $\hat{W}$ when the control system is running, that is to say, $\widetilde{W}$ only converges at a bound, but not asymptotically. Therefore, the main approximation error is $\tilde{W}^{T} Z(t)$ which is the error between $W^{T} Z(t)$ and $\hat{W}^{T} Z(t)$ in many 
practical applications. So, it is necessary to compensate this approximation error online when $\hat{W}$ is updated.

Taking the time derivative of Eq. (6) along system trajectory, we have

$$
\dot{s}(t)=C \dot{X}(t)-C \dot{X}_{d}(t)=C(\bar{A} X(t)+\bar{B} u(t)+\bar{U}+D(t))-C \dot{X}_{d}(t)
$$

On the basis of Eq. (6) and (13), An-Chyau (2001) developed a control law including a timevarying uncertain term and a signum function of sliding surface, where the uncertain term is represented by a set of Fourier series, and then the concrete expression of the control law is obtained with direct Lyapunov method. However, the proposed control scheme can't compensate the on-line approximation error. Adopting the same approach, we propose a control law consisting of an unknown bound time-varying uncertain term same as AnChyau (2001) and add another compensative term for compensating the on-line approximation error between $W^{T} Z(t)$ and $\hat{W}^{T} Z(t)$, and then employ the function approximation technique to transform the uncertain term into a combination of a set of Laguerre series. According to above idea, the form of the proposed control law can be expressed as

$$
u(t)=-(C \bar{B})^{-1} C\left(\bar{A} X(t)+\bar{U}-\dot{X}_{d}(t)\right)-(C \bar{B})^{-1} \hat{u}_{m}(t)-(C \bar{B})^{-1} k \operatorname{sgn}(s(t))+(C \bar{B})^{-1} u_{r}(t)
$$

in which, the first term $-(C \bar{B})^{-1} C\left(\bar{A} X(t)+\bar{U}-\dot{X}_{d}(t)\right)$ on the right side is the control term based on nominal system model; the second term $-(C \bar{B})^{-1} \hat{u}_{m}(t)$ is from the approximation of unknown bound time-varying uncertainty; the last term $(C \bar{B})^{-1} u_{r}(t)$ is a compensative control term and $u_{r}(t)$ is the compensation of on-line approximation error between $W^{T} Z(t)$ and $\hat{W}^{T} Z(t)$; while $-(C \bar{B})^{-1} k \operatorname{sgn}(s(t))$ which is used to compensate $\varepsilon(t)$ is a control term including the sign function of sliding surface $s(t)$, and $k$ is a positive constant. Substituting (14) into (13), yields

$$
\begin{aligned}
\dot{s}(t) & =C \bar{A} X(t)+C \bar{U}+u_{m}(t)-C \dot{X}_{d}(t)+(C \bar{B})^{-1} u(t) \\
& =C \bar{A} X(t)+C \bar{U}+u_{m}(t)-C \dot{X}_{d}(t)-C \bar{A} X(t)-C \bar{U}-\hat{u}_{m}(t)+C \dot{X}_{d}(t)-k \operatorname{sgn}(s(t))+u_{r}(t) \\
& =u_{m}(t)-\hat{u}_{m}(t)-k \operatorname{sgn}(s(t))+u_{r}(t)
\end{aligned}
$$

From (8)-(12), and (15), we have

$$
\dot{s}(t)=\tilde{W}^{T} Z(t)+\varepsilon(t)-k \operatorname{sgn}(s(t))+u_{r}(t)
$$

In the following, the on-line update law $\hat{W}$ and expression of $u_{r}(t)$ can be obtained from a Lyapunov function about $\widetilde{W}$ and $s(t)$ properly selected, and then concrete expression of $\hat{u}_{m}(t)$ also can be obtained through (9). Firstly, we propose the FASMAC using the following theorem, and prove the asymptotic stability of the system under control law (14).

Theorem 1. For DC motor position tracking control system with unknown bound timevarying uncertainty described as (2), select (6) as the sliding surface $s(t)$. There exists real 
positive constant $\eta_{1}, \eta_{2}, \eta_{3}$ and $k$, when the update law of $\hat{W}$ and compensative term $u_{r}(t)$ satisfy Eq. (17), under the control of control law (14), the sliding surface $s(t)$ converges to zero and, thus, the position tracking error $e_{1}(t)$ of uncertain system (2) is asymptotically stable. The update law can be expressed as

$$
\dot{\hat{W}}=\frac{\eta_{1}}{\eta_{2}} s(t) Z(t), \quad u_{r}(t)=-\eta_{3} \eta_{1} s(t)
$$

Proof. Let $k, \eta_{1}, \eta_{2}, \eta_{3}$ be the positive constants. We choose the Lyapunov function as

$$
V(s(t), \tilde{W})=\frac{1}{2} \eta_{1}(s(t))^{2}+\frac{1}{2} \eta_{2} \tilde{W}^{T} \tilde{W} \geq 0
$$

Take time derivative of Eq. (18), yields

$$
\begin{aligned}
\dot{V}(s(t), \widetilde{W}) & =\eta_{1} s(t) \dot{s}(t)-\eta_{2} \widetilde{W}^{T} \dot{\hat{W}} \\
& =\eta_{1} s(t)\left(\widetilde{W}^{T} Z(t)+\varepsilon(t)-k \operatorname{sgn}(s(t))+u_{r}(t)\right)-\eta_{2} \widetilde{W}^{T} \dot{\hat{W}} \\
& =\eta_{1} s(t) \tilde{W}^{T} Z(t)-\eta_{2} \tilde{W}^{T} \dot{\hat{W}}+\eta_{1} s(t) \varepsilon(t)-k \eta_{1}|s(t)|+\eta_{1} s(t) u_{r}(t) \\
& =\widetilde{W}^{T}\left(\eta_{1} s(t) Z(t)-\eta_{2} \dot{\hat{W}}\right)+\eta_{1} s(t) \varepsilon(t)-k \eta_{1}|s(t)|+\eta_{1} s(t) u_{r}(t)
\end{aligned}
$$

Substituting (17) into (19), we obtain

$$
\begin{gathered}
\dot{V}(s(t), \tilde{W})=-\eta_{3}\left(\eta_{1} s(t)\right)^{2}-k \eta_{1}|s(t)|+\eta_{1} s(t) \varepsilon(t) \\
\leq-\eta_{3}\left(\eta_{1} s(t)\right)^{2}-(k-|\varepsilon(t)|) \eta_{1}|s(t)|
\end{gathered}
$$

If the variation bound of $\varepsilon(t)$ can be estimated, that is, there exists a positive constant $\delta>0$ such that $|\varepsilon(t)| \leq \delta$, with the selection of a sufficient large positive constant $k$, such that $k \geq \delta$. Then $\dot{V}(s(t), \widetilde{W}(t))$ can be derived to be

$$
\dot{V}(s(t), \tilde{W}) \leq-\eta_{3}\left(\eta_{1} s(t)\right)^{2}-(k-\delta) \eta_{1}|s(t)| \leq 0
$$

Therefore, it can be easily shown by the Barbalat's lemma (Slotine and Li, 1991) that the sliding surface $s(t)$ converges to zero, and the velocity of convergence can be adjusted by choosing the different values of $k, \eta_{1}, \eta_{2}, \eta_{3}$. Moreover, position error $e_{1}(t)$ of the uncertain system (2) is asymptotically stablé.

Remark 1. Since the approximation error $\varepsilon(t)=\sum_{i=N+1}^{\infty} w_{i} z_{i}(t)$, if a sufficient number of basis functions are used, then $\varepsilon(t) \approx 0$ in many practical occasions. Because $\tilde{W}(t)$ is only 
bounded, the main approximation error using FAT is the error between $W^{T} Z(t)$ and $\hat{W}(t)^{T} Z(t)$. The function of $u_{r}(t)$ in control law (14) is used to compensate for this approximation error online, and it is seen in (21) that the additional term $-\eta_{3}\left(\eta_{1} s(t)\right)^{2}$ is from $u_{r}(t)$.

\subsection{Actual experiments and results analysis}

From the procedure of FASMAC design in Section 2.2, the nominal model of the DC motor position system should be identified before doing actual experiments. The DC motor position model can be seen as a combination of a speed model and an integral. The speed model is identified firstly, and then the whole position model can be easily obtained through the integral. The positive and negative speed model should be identified separately owing to their different parameters in actual DC motor system in the paper.

The nominal form of DC motor speed model can be obtained from Section 2.1 as

$$
\Omega(s)=\frac{1 / K_{e}}{T_{m} s+1}(U(s)+U)=\frac{K}{T_{m} s+1}(U(s)+U)
$$

There are three parameters needed to identify in (22), $T_{m}, K, U$, where $K=1 / K_{e}$. By testing and measuring step response of the DC motor speed system, we obtain the input and output data and further identify the three unknown parameters employing curve fitting and optimizing techniques with MATLAB. Finally, nominal model of DC motor position system with positive and negative speed are obtained, respectively, as

$$
\begin{aligned}
& \left\{\begin{array}{l}
\dot{x}_{1}=x_{2} \\
\dot{x}_{2}=-\frac{1}{3.0787} x_{2}+\frac{5.3658}{3.0787} u(t)-\frac{5.3658}{3.0757} \times 464.6780
\end{array}\right. \\
& \left\{\begin{array}{l}
\dot{x}_{1}=x_{2} \\
\dot{x}_{2}=-\frac{1}{2.7746} x_{2}+\frac{5.3169}{2.7746} u(t)+\frac{5.3169}{2.7746} \times 204.7935
\end{array}\right.
\end{aligned}
$$

Based on (9), (14) and (17), the on-line control law of FASMAC are

$$
\begin{gathered}
u(k)=-(C \bar{B})^{-1} C\left(\bar{A} X(k)+\bar{U}-\dot{X}_{d}(k)\right)-(C \bar{B})^{-1} \hat{u}_{m}(k)-(C \bar{B})^{-1} k \operatorname{sgn}(s(k))+(C \bar{B})^{-1} u_{r}(k) \\
\hat{u}_{m}(k)=(\hat{W}(k))^{T} Z(k), \quad u_{r}(k)=-\eta_{3} \eta_{1} s(k) \\
\hat{W}(k)=\hat{W}(k-1)+\frac{\eta_{1}}{\eta_{2}} s(k) Z(k)
\end{gathered}
$$

With the discussion above, the proposed on-line control strategy can be realized as

1) Choose proper series number $N$ of Laguerre function. 
2) Choose proper such parameters of controller as $C, \eta_{1}, \eta_{2}, \eta_{3}, k$.

3) Initialize coefficients $\hat{W}$ of Laguerre function series, here we let $\hat{w}_{i}(0)=0(i=1,2, \cdots, N)$.

4) In every sample step $k$ when system is running, do

(1) Read system states $X(k)$, calculate reference states $X_{d}(k)$ and $\dot{X}_{d}(k)$.

(2) Calculate the value of sliding function $s(t)$ according to (6).

(3) Calculate $\hat{W}(k)$ according to (27).

(4) Calculate $\hat{u}_{m}(k)$ and $u_{r}(k)$ according to (26).

(5) Calculate $u(k)$ according to (25).

(6) $\quad k=k+1$.

Return to step (1) in 4) and repeat the on-line operating.

Fig. 2 shows the actual DC motor device, and the control system consists of a pulse width modulation (PWM) driver, a microcomputer and a build-in card with A/D and D/A channel. The range of digital control signal in this experimental device is in [-2048 2048], and the corresponding voltage after the D/A channel ranges from -20 to 20 voltage. The digital value of position whose range is in [-180 180] degree can be read from the A/D channel. Moreover, the digital value of velocity can also be read from A/D channel directly.

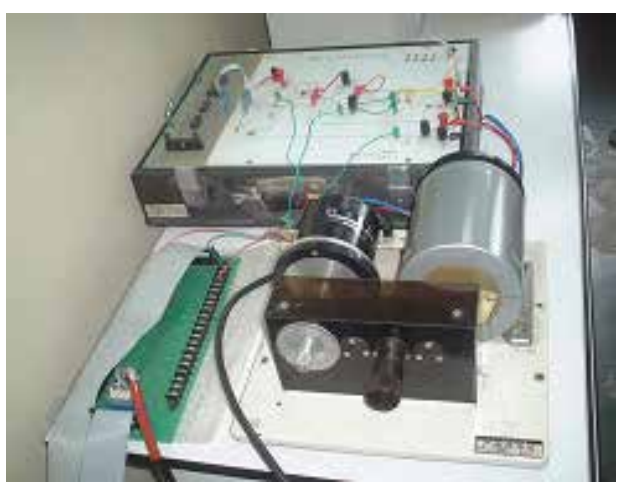

Figure 2. Actual DC motor experiment device

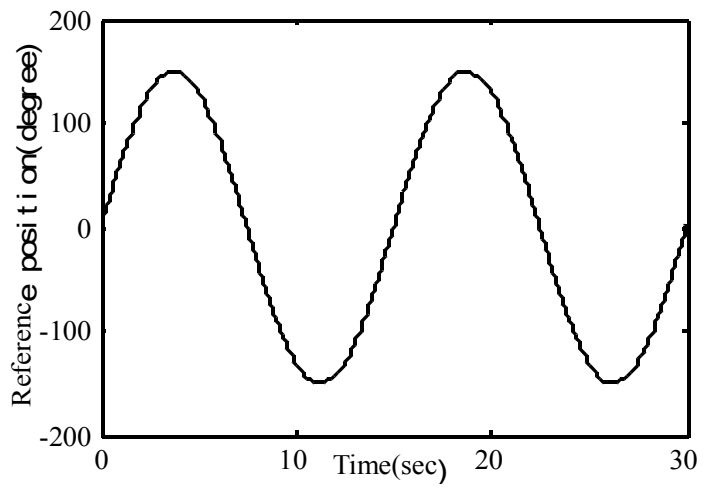

Figure 3. Reference signal 
The proposed controller is implemented in a time-interrupt service routine at $10 \mathrm{~ms}$ sampling period under MS-DOS environment. The reference trajectory is designed to be

$$
y(t)=150 \sin (2 \pi \times 0.0667 t)
$$

which is showed in Fig. 3. The number of terms of Laguerre function series is 8 , and after several adjustments, the actual parameters of FASMAC are chosen as $C=10 *\left[\begin{array}{ll}10 & 0.5\end{array}\right]$, $\eta_{1}=1, \eta_{2}=1, \eta_{3}=6$ and $k=20$. Besides, to do the comparison with the results of FASMAC, experiment of control strategy proposed by An-Chyau (2001) is also implemented on the same DC motor system.

The results of the experiments are shown in Figs. 4-9. Fig.4 shows a comparison of the system output tracking errors between the proposed FASMAC and the controller of AnChyau. It can be seen that the tracking error under the control of FASMAC mainly lies in [-2, 2], and only reach -7 or 7 when the direction of DC motor speed is changing, whereas, tracking error of An-Chyau's controller lies in [-10, 10] and arrive at peak value of -20 sometimes. Therefore, the tracking performance of the proposed FASMAC is better than the An-Chyau's controller. Fig.5 displays the sliding behaviour of the sliding surface $s(t)$ in our control scheme. Fig. 6, Fig.7 and Fig.8 show the total control value $u(t)$, the uncertain control term $-(C \bar{B})^{-1} \hat{u}_{m}(t)$ and compensative control term $(C \bar{B})^{-1} u_{r}(t)$, respectively. Fig.9 depicts the approximation of nonlinear friction $U_{f}(t)$, which can be calculated as $U+(C \bar{B})^{-1} \hat{u}_{m}(t)$. Fig.7 reveals that the uncertain term undulates at -100 or 100 in all the control period except the time of the direction of speed is changing. The main reason is that the identified linear nominal model of DC motor system is proper to the actual DC motor when the system is running at high speed, while it's not accurate at low speed because of the complicated dead zone characteristics caused by uncertain time-varying nonlinear friction, especially when the direction of DC motor speed is changing. Therefore, the model error between the identified nominal model and the actual system at low speed in peak value of uncertain control term $-(C \bar{B})^{-1} \hat{u}_{m}(t)$ when speed direction is changing. Since there is a compensative control term $(C \bar{B})^{-1} u_{r}(t)$ in control law $u(t)$ in FASMAC, which can compensate for the on-line approximation error of nonlinear friction rapidly, the proposed controller can still guarantee good performance even when the DC motor is running at low speed or its direction is changing, thus, the tracking performance of FASMAC is much better than that of An-Chyau. It should be noted that the compensative term $u_{r}(t)$ is almost the same as approximation error of nonlinear friction in simulation experiments.

From the proof of Theorem 1, we can see that the derivative of Lyapunov function (21) consists of $-\eta_{3}\left(\eta_{1} s(t)\right)^{2}$ and $-k \eta_{1}|s(t)|$, which are contributed by compensative control term $(C \bar{B})^{-1} u_{r}(t)$ and constant control term $-(C \bar{B})^{-1} k \operatorname{sgn}(s(t))$, respectively. Thus, the sliding surface converges rapidly mainly owing to the existent of compensative control term when its error is large, and when the error falls to a certain extent, the sliding surface error still converges to zero because of the constant control term. 


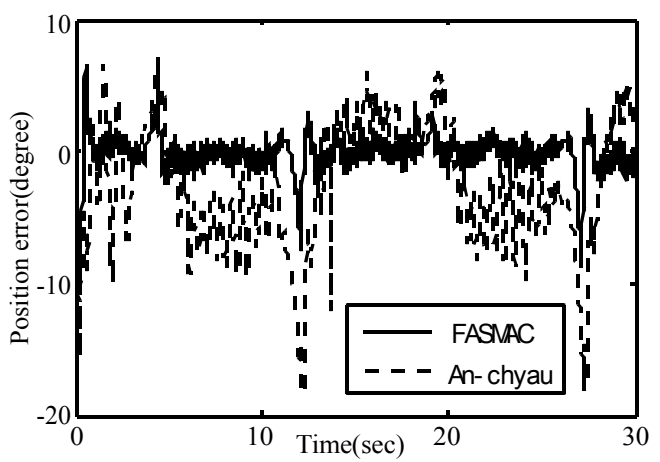

Figure 4. System output tracking error

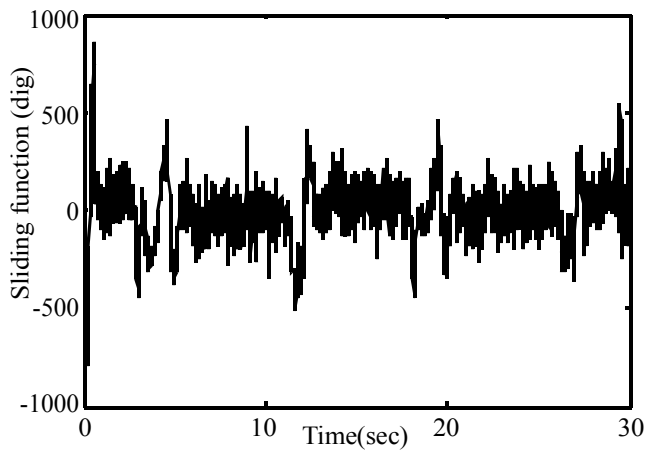

Figure 5. Behaviour of sliding surface $s(t)$

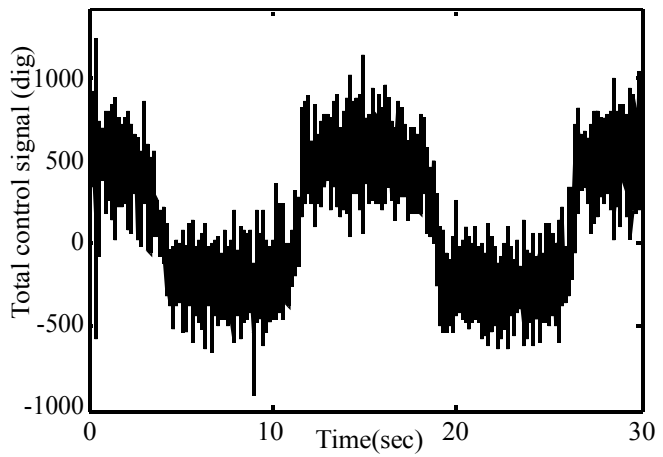

Figure 6. Total control law $u(t)$ 


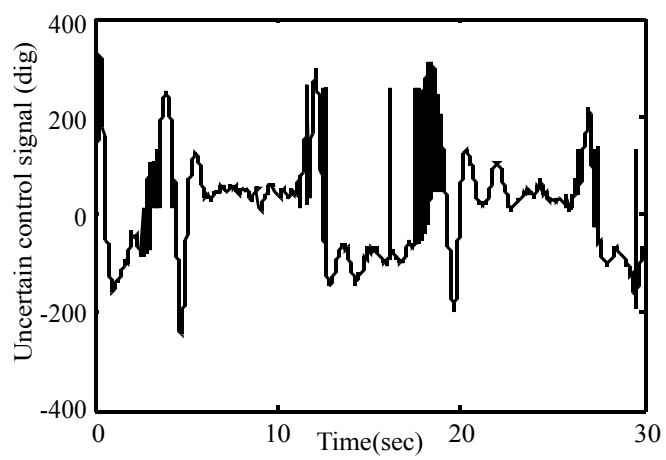

Figure 7. Uncertain term $-(C \bar{B})^{-1} \hat{u}_{m}(t)$

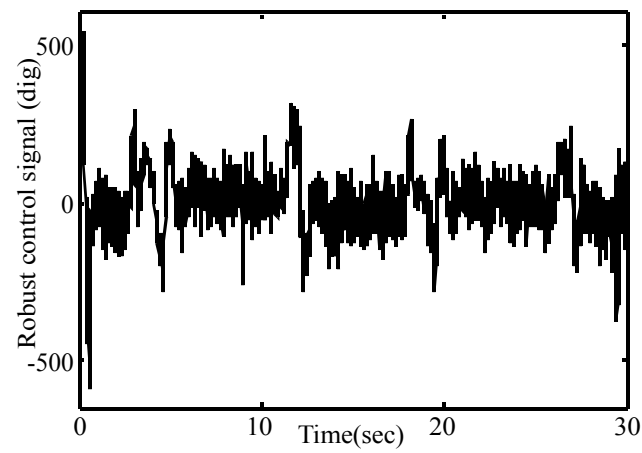

Figure 8. Compensative term $(C \bar{B})^{-1} u_{r}(t)$

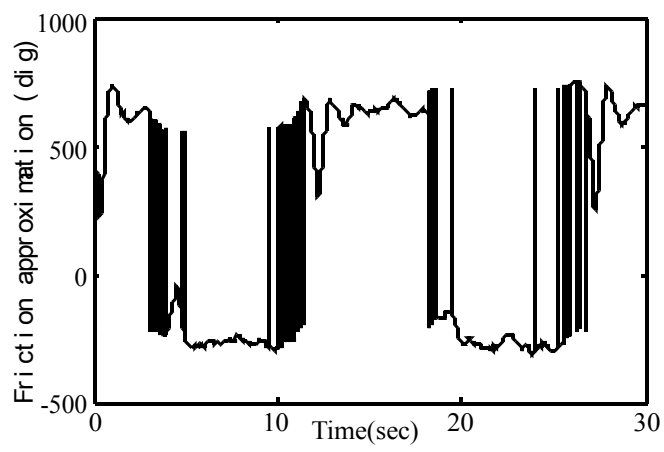

Figure 9. Nonlinear friction $U_{f}(t)$

\section{FAT-based Adaptive Sliding Mode Control of SIMO Nonlinear System with Time-varying Uncertainty}

In recent years, the usual methods for uncertain systems with specific structure have used in adaptive controller design include robust control (Zhou \& Ren, 2001; Zhou, 2004; Hu \& Liu, 2004; Wu et al., 2006), back-stepping(Do \& Jiang, 2004; Manosa et al., 2005; Wu et al., 2007), sliding mode control (Huang \& Cheng, 2004a; 2004b; Huang \& Kuo, 2001; Chu \& Tung, 
2005; Fang et al., 2006; Chiang et al., 2007), neural network technique (Yang \& Calise, 2007; Fu \& Chai, 2007; Zhou et al., 2007 Tang et al., 2007) and fuzzy method (Hsu \& Lin, 2005; Huang \& Chen, 2006; Liu \& Wang, 2007). For instance, combining a linear nominal controller with an adaptive compensator, Ruan (2007) and Hovakimyan (2006) realized the high performance stabilizing of inverted pendulum with un-modeling nonlinear dynamics. Since sliding mode control is robust to uncertainties of system structure and parameters, external disturbances and other unexpected factors, when the system lies on the sliding surface, it is obtained more and more attention in the control realm (Hung et al., 1993; Young et al., 1999).

At present, many adaptive control methods for nonlinear uncertain system whose uncertainty satisfies some conditions (Barmish \& Leitmann, 1982; Chen \& Huang, 1987) or the bound of uncertainty satisfies strict conditions have been developed (L. G. Wu et al., 2006; Z. J. Wu et al., 2007; Fang et al., 2006; Chiang et al., 2007). These research problems are hotspot in the control realm, and some results have been obtained through years of hard work of researchers (Huang \& Chen, 2004; Chen \& Huang, 2005; Huang \& Liao, 2006; Liang et al., 2008). These research works adopt a common technique named function approximation technique (FAT) despite of their different design methods. Utilizing the FAT, the nonlinear time-varying uncertainty can be transformed into a finite combination of basis functions, and Lyapunov direct method can thus be used to find adaptive laws for updating time-invariant coefficients in the approximating series. Using Fourier series, Huang proposed an adaptive sliding control strategy for a class of nonlinear system with unknown bound time-varying uncertainty satisfying the Dirichlet condition, and further obtained the updating law of coefficients in Fourier series by Lyapunov direct method (Chen \& Huang, 2005; Huang \& Liao, 2006). The Section 2 proposed a FAT-based adaptive sliding mode control method. But the above mentioned control strategies are only suitable for single input single output (SISO) nonlinear systems with certain specific structure, not for SIMO uncertain system. In the second part of the chapter we'll propose a FAT-based adaptive sliding mode control method for SIMO nonlinear contol system.

\subsection{Problem statement}

Giving the following SIMO uncertain nonlinear system

$$
\left.\begin{array}{rl}
\dot{X} & =(A(X)+\Delta A(X, t)) X+B(X) u \\
Y & =X
\end{array}\right\}
$$

where, $X, Y \in R^{n}, A(X) \in R^{n \times n}, \quad \Delta A(X, t) \in R^{n \times n}, \quad B(X) \in R^{n}, u \in R$, and $\Delta A(X, t)$ is a time-varying uncertain function matrix. Let $\Delta A(X, t) X=D(X, t) \in R^{n}$, then (29) can be rewritten as

$$
\left.\begin{array}{rl}
\dot{X} & =A(X) X+B(X) u+D(X, t) \\
Y & =X
\end{array}\right\}
$$

The mathematical model of many actual SIMO electro-mechanical nonlinear systems with un-modeling dynamics in practical engineering can be described by $(30)$, in which $D(X, t)$ is an unmodeling time-varying dynamics. Suppose system (30) satisfies the following assumption. 
Assumption 2. $\exists C \in R^{1 \times n}$, for $\forall X \in R^{n}, C B(X) \neq 0$ exists, and time-varying function $C D(t)$ is piecewise continuous or square integrable in finite time interval.

Under the Assumption 2, we give a linear sliding function about system error firstly, and then transforms the approximation problem of unknown bound time-varying function $C D(X, t)$ into the updating of constant coefficient vector by FAT, and design a control law $u(t)$ including approximation of $C D(X, t)$ and compensation of approximation error, and then obtains the concrete expression of updating law of constant coefficient vector and compensation of approximation error through Lyapunov direct method, and thus adaptive sliding control law $u(t)$ can be obtained finally.

\subsection{FAT-based sliding mode adaptive controller design}

This section gives the details of design procedure of SIMOAC. Firstly, a linear sliding function $S(t)$ is chosen, and then the sliding mode adaptive control law guaranteeing closedloop system stability can be obtained through Lyapunov direct method.

Let the expected state of system (30) is $X_{d}(t)$, and defining system state error as

$$
E(t)=X(t)-X_{d}(t)
$$

Choosing standard sliding function $S(t)$ as

$$
S(t)=C E(t)
$$

where, $C \in R^{1 \times n}$ is constant row vector.

Let $u_{m_{1}}(t)=C D(X, t)$. According to the Assumption 2 and FAT, time-varying scalar function $u_{m_{1}}(t)$ can be expressed as

$$
u_{m_{1}}(t)=W_{1}^{T} Z_{1}(t)+\varepsilon_{1}
$$

In (33), $W_{1}$ is an unknown $n$-dimension constant column vector, $Z_{1}(t)$ is a known $n$ dimension time-varying basis function column vector, $\varepsilon_{1}$ is a approximation error. The essential of FAT is that $u_{m_{1}}(t)$ is approximated by means of the approximation of coefficient column vector $W_{1}$. Let the approximation of $u_{m_{1}}(t)$ is denoted by $\hat{u}_{m_{1}}(t)$, then utilizing the same basis function vector, yields

$$
\hat{u}_{m_{1}}(t)=\hat{W}_{1}(t)^{T} Z_{1}(t)+u_{c}(t)
$$

where, $u_{c}(t)$ is the adaptive compensation of approximation error.

Taking time derivative along system trajectory, and according to (33), yields

$$
\dot{S}(t)=C(A(X) X(t)+B(X) u(t)+D(X, t))-C \dot{X}_{d}(t)
$$




$$
\begin{gathered}
=C\left(A(X) X(t)-\dot{X}_{d}(t)\right)+C D(X, t)+C B(X) u(t) \\
=C\left(A(X) X(t)-\dot{X}_{d}(t)\right)+C B(X) u(t)+W^{T}{ }_{1} Z_{1}(t)+\varepsilon_{1}
\end{gathered}
$$

According to (Liang et al., 2008), the form of the proposed control law can be chosen as

$$
\left.u(t)=-(C B(X))^{-1}\left[C\left(A(X) X(t)-\dot{X}_{d}(t)\right)+\hat{u}_{m_{1}}(t)\right)\right]
$$

in which the first term $-(C B(X))^{-1} C\left(A(X) X(t)-\dot{X}_{d}(t)\right)$ of right side of Eq. (36) is the control term based on nominal system model, the second one $-(C B(X))^{-1} \hat{u}_{m_{1}}(t)$ is the approximation of unknown bound time-varying uncertain term $u_{m}(t)=C D(t)$. Afterwards, a proper Lyapunov function about sliding function $S(t)$, the error square sum performance function and the error of coefficient vector can be constructed, and thus the updating law of coefficient vector $\hat{W}_{1}(t)$ in uncertainty approximation $\hat{u}_{m_{1}}(t)$ and concrete expression of adaptive compensation $u_{c}(t)$ for approximation error can be obtained by Lyapunov direct method.

Let $\widetilde{W}_{1}(t)=W_{1}(t)-\hat{W}_{1}(t)$, substituting Eq. (36) into Eq. (35), yields

$$
\begin{aligned}
\dot{S}(t) & =u_{m_{1}}(t)-\hat{u}_{m_{1}}(t)+u_{c}(t) \\
& =\widetilde{W}_{1}(t) Z_{1}(t)-u_{c}(t)+\varepsilon_{1}
\end{aligned}
$$

Defining system error square sum performance function $f(t)$ as

$$
f(t)=E^{T}(t) Q E(t)
$$

in which, $Q \in R^{n \times n}$ is a semi-positive definite diagonal constant matrix.

Taking the time derivative of $f(t)$ along system trajectory, yields

$$
\dot{f}(t)=2 E^{T}(t) Q\left(A(X) X(t)-\dot{X}_{d}(t)\right)+2\left(E^{T}(t) Q B(X)\right) u(t)+2 E^{T}(t) Q D(t)
$$

Let $u_{m_{2}}(t)=E^{T}(t) Q D(t)$, according to the Assumption 2 and FAT, $u_{m_{2}}(t)$ can also be expressed as

$$
u_{m_{2}}(t)=W_{2}^{T} Z_{2}(t)+\varepsilon_{2}
$$

where, $W_{2}$ is an unknown n-dimension constant column vector, $Z_{2}(t)$ is a known ndimension time-varying basis function column vector, $\varepsilon_{2}$ is the approximation error. Utilizing the same basis function, the approximation of $u_{m_{2}}(t)$ can also be expressed as

$$
\hat{u}_{m_{2}}(t)=\hat{W}_{2}(t)^{T} Z_{2}(t)
$$


Let $\widetilde{W}_{2}(t)=W_{2}(t)-\hat{W}_{2}(t)$. According to (36) and (40), (39) can be rewritten as

$$
\begin{aligned}
\dot{f}(t)= & 2\left\{E^{T}(t) Q\left(I-(C B(X))^{-1} B(X) C\right)\left(A(X) X(t)-\dot{X}_{d}(t)\right)\right. \\
& \left.-(C B(X))^{-1} E^{T}(t) Q B(X) \hat{u}_{m_{1}}(t)+u_{m_{2}}(t)\right\}
\end{aligned}
$$

Let

$$
\begin{gathered}
h(t)=E(t)^{T} Q\left(I-(C B(X))^{-1} B(X) C\right)\left(A(X) X(t)-\dot{X}_{d}(t)\right) \\
g(t)=\eta_{1} S(t)+\eta_{2} f(t)(C B(X))^{-1} E(t)^{T} Q B(X) \\
p(t)=\eta_{2} f(t)\left(h(t)-(C B(X))^{-1} E(t)^{T} Q B(X) \hat{W}_{1}(t)^{T} Z_{1}(t)+\hat{u}_{m_{2}}(t)\right)+\eta_{1}|S(t)| \delta_{1}+\eta_{2}|f(t)| \delta_{2}
\end{gathered}
$$

Theorem 2. For the SIMO nonlinear system (30) with unknown bound time-varying uncertainty, choosing sliding function $S(t)$ defined as (32) and performance function $f(t)$ defined as (38), then there exist constant scalar value $\eta_{i} \geq 0,(i=1, \cdots, 7), \delta_{1} \geq 0$ and $\delta_{2} \geq 0$, when $\dot{\hat{W}}_{1}(t), \quad \dot{\hat{W}}_{2}(t)$ and $u_{c}(t)$ satisfy (46) and (47), sliding surface $S(t)=0$ and the error square sum performance function $f(t)$ of system (2) are stable under the control of (36).

$$
\begin{aligned}
& \dot{\hat{W}}_{1}(t)=\eta_{1} / \eta_{3} S(t) Z_{1}(t), \dot{\hat{W}}_{2}(t)=\eta_{2} / \eta_{4} f(t) Z_{2}(t) \\
& u_{c}(t)=1 / g(t)\left(p(t)+\eta_{5} f(t)+\eta_{6} S(t)^{2}+\eta_{7}|S(t)|\right)
\end{aligned}
$$

Proof: Choosing the Lyapunov function as

$$
V\left(S(t), f(t), \tilde{W}_{1}(t), \tilde{W}_{2}(t)\right)=\frac{1}{2} \eta_{1} S(t)^{2}+\frac{1}{4} f(t)^{2}+\frac{1}{2} \eta_{3} \tilde{W}_{1}^{T}(t) \tilde{W}_{1}(t)+\frac{1}{2} \eta_{4} \tilde{W}_{2}^{T}(t) \tilde{W}_{2}(t) \geq 0
$$

Taking the time derivative of (48), one yields

$$
\begin{gathered}
\dot{V}(t)=\dot{V}\left(S(t), f(t), \widetilde{W}_{1}(t), \widetilde{W}_{2}(t)\right) \\
=\eta_{1} S(t)\left(\widetilde{W}_{1}^{T}(t) Z_{1}(t)-u_{c}(t)+\varepsilon_{1}\right) \\
+\eta_{2} f(t)\left\{E^{T}(t) Q\left(I-(C B(X))^{-1} B(X) C\right)\left(A(X) X(t)-\dot{X}_{d}(t)\right)\right. \\
\left.-(C B(X))^{-1} E^{T}(t) Q B(X)\left(\hat{W}_{1}(t)^{T} Z_{1}(t)-u_{c}(t)\right)+u_{m_{2}}(t)\right\} \\
-\eta_{3} \widetilde{W}_{1}^{T}(t) \dot{\hat{W}}_{1}(t)--\eta_{4} \widetilde{W}_{2}^{T}(t) \dot{\hat{W}}_{2}(t)
\end{gathered}
$$

In time-varying scalar function $u_{m_{i}}(t)(i=1,2)$ defined in (33) and (40), the approximation error $\varepsilon_{i}$ satisfies $\left|\varepsilon_{i}\right| \leq \delta_{i} \geq 0$ only if a sufficient large dimension $N$ is chosen. According to (43), (44) and (45), (49) can be rewritten as

$$
\dot{V}(t) \leq \tilde{W}_{1}(t)^{T}\left(\eta_{1} S(t) Z_{1}(t)-\eta_{3} \dot{\hat{W}}_{1}(t)\right)+\tilde{W}_{2}(t)^{T}\left(\eta_{2} f(t) Z_{2}(t)-\eta_{4} \dot{\hat{W}}_{2}(t)\right)-g(t) u_{c}(t)+p(t)
$$


Substituting (46) and (47) into (50), one yields

$$
\dot{V}(t) \leq-\eta_{5} f(t)-\eta_{6} S(t)^{2}-\eta_{7}|S(t)| \leq 0
$$

According to Lyapunov stability theorem, sliding surface $S(t)=0$ and the error square sum performance function $f(t)$ of system (30) are stable. $\square$

With the discussion above, the proposed on-line control strategy can be realized as

1. According to the characteristic of actual control plant, choosing proper basis function series and the series number $N$, such as Fourier series and Laguerre series, and then initializing the coefficient vector $W_{i}=\left[w_{1}, w_{2}, \cdots, w_{n}\right](i=1,2)$.

2. Choosing proper weight vector $C \in R^{1 \times n}$, matrix $Q=\operatorname{diag}\left(q_{11}, q_{22}, \cdots, q_{n n}\right) \in R^{n \times n}$ and learning rate $\eta_{j}>0, \quad(j=1, \cdots, 7)$, where $q_{i i}>0, \quad(i=1, \cdots, n)$.

3. In every sample step $k$ when the system is running, do

4. Reading system current states $X(k)$, and obtaining error $E(k)=X(k)-X_{d}(k)$, and then calculating $S(k)$ and $f(k)$ according to (32) and (38).

5. Calculating coefficient increment $\Delta \hat{W}_{i}(k) \quad(i=1,2)$ according to (36), that is $\Delta \hat{W}_{1}(k)=\eta_{1} / \eta_{3} S(k) Z_{1}(k) T_{s}, \quad \Delta \hat{W}_{2}(k)=\eta_{2} / \eta_{4} f(k) Z_{2}(k) T_{s}, \quad T_{s} \quad$ is sample period.

6. Calculating $\hat{u}_{m_{2}}(k)$ according to (41), that is $\hat{u}_{m_{2}}(k)=\left(\hat{W}_{2}(k-1)+\Delta \hat{W}_{2}(k)\right)^{T} Z_{2}(k)$

7. Calculating $h(k), g(k), p(k)$ according to (43), (44) and (45).

8. Calculating $u_{c}(k)$ according to (47).

9. Calculating time-varying uncertainty term $\hat{u}_{m_{1}}(k)$ according to (34), that is

$$
\hat{u}_{m_{1}}(k)=\left(\hat{W}_{1}(k-1)+\Delta \hat{W}_{1}(k)\right)^{T} Z_{i}(k)+u_{c}(k) \text {. }
$$

10. Calculating sliding mode adaptive control law $u(k)$ according to (36).

11. $k=k+1$.

Return to step 4 and repeat the on-line operating.

\subsection{Simulation experiment and result analysis on a double inverted pendulum}

This section applies the adaptive controller proposed to the stabilizing control of a double inverted pendulum simulating system, and analyzes the simulation result through the comparison with the result of the linear quadratic regulator (LQR).

Fig. 10 depicts the system diagram of the double inverted pendulum. The system is mainly composed of a car, two rods linked each other, optical-electrical encoder coders measuring displacement information, an alternating current electric motor driving the car which is linked with a belt. In actual system operatinon the real-time number control signal can be obtained according to current states of the double inverted pendulum, and then this signal can be used to drive the motor to control, and finally the car traverses along the rail. 


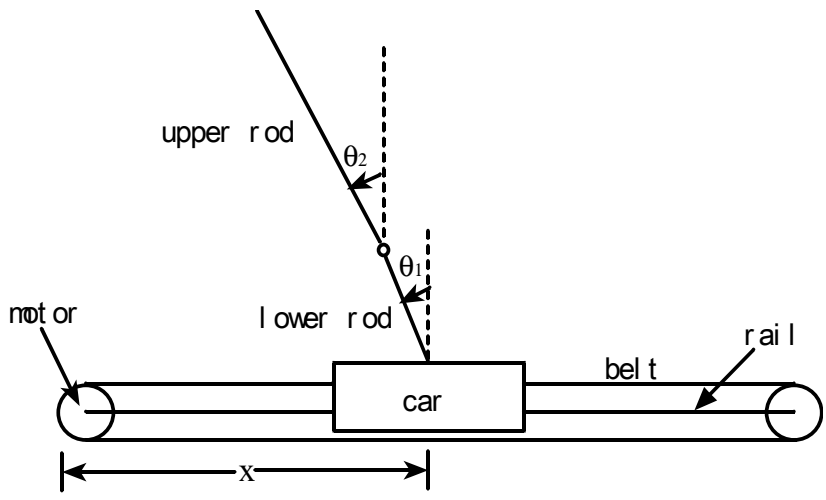

Figure 10. System diagram of double inverted pendulum

According to Fig.10, the mathematical model of the double inverted pendulum can be established by adopting Lagrange method. Choosing system states as $x_{1}=x, x_{2}=\theta_{1}$, $x_{3}=\theta_{2}, \quad x_{4}=\dot{x}, \quad x_{5}=\dot{\theta}_{1}, \quad x_{6}=\dot{\theta}_{2}$, and let $X=\left[\begin{array}{llllll}x_{1} & x_{2} & x_{3} & x_{4} & x_{5} & x_{6}\end{array}\right]^{T}$, then the linear state equation of the double inverted pendulum nearby equilibrium $X_{0}=\left[\begin{array}{llllll}0 & 0 & 0 & 0 & 0 & 0\end{array}\right]^{T}$ is

$$
\begin{aligned}
\dot{X} & =A X+B u \\
Y & =C X
\end{aligned}
$$

Where

$$
\begin{aligned}
& A=\left[\begin{array}{cccccc}
0 & 0 & 0 & 1 & 0 & 0 \\
0 & 0 & 0 & 0 & 1 & 0 \\
0 & 0 & 0 & 0 & 0 & 1 \\
0 & \frac{3\left(-2 g m_{1}-4 g m_{2}\right)}{2\left(-4 m_{1}-3 m_{2}\right) l_{1}} & 0 & 0 & 0 & 0 \\
0 & \frac{9 m_{2} g}{2\left(-4 m_{1}-3 m_{2}\right) l_{1}} & 0 & 0 & 0 \\
0 & \frac{4 g m_{2}\left(m_{1}+2 m_{2}\right) l_{1}^{2} l_{2}}{4 m_{2}^{2} l_{1}^{2} l_{2}^{2}-\frac{16}{9} m_{2}\left(m_{1}+3 m_{2}\right) l_{1}^{2} l_{2}^{2}} & -\frac{\left.4 m_{2}\right) l_{1}^{2} l_{2}}{3\left(4 m_{2}^{2} l_{1}^{2} l_{2}^{2}-\frac{16}{9} m_{2}\left(m_{1}+3 m_{2}\right) l_{1}^{2} l_{2}^{2}\right)} & 0 & 0 & 0
\end{array}\right], \\
& B=\left[\begin{array}{llllll}
0 & 0 & 0 & 1 & \frac{3\left(-2 m_{1}-m_{2}\right)}{2\left(-4 m_{1}-3 m_{2}\right) l_{1}} & \frac{2 m_{2}\left(m_{1}+2 m_{2}\right) l_{1}^{2} l_{2}-\frac{4}{3} m_{2}\left(m_{1}+3 m_{2}\right) l_{1}^{2} l_{2}}{4 m_{2}^{2} l_{1}^{2} l_{2}^{2}-\frac{16}{9} m_{2}\left(m_{1}+3 m_{2}\right) l_{1}^{2} l_{2}^{2}}
\end{array}\right]^{T}, \\
& C=\left[\begin{array}{llllll}
1 & 0 & 0 & 0 & 0 & 0 \\
0 & 1 & 0 & 0 & 0 & 0 \\
0 & 0 & 1 & 0 & 0 & 0
\end{array}\right]
\end{aligned}
$$

Applying physical parameters in Table 1, the nominal model of the pendulum for simulation can be obtained as 


$$
\dot{X}=\left[\begin{array}{cccccc}
0 & 0 & 0 & 1 & 0 & 0 \\
0 & 0 & 0 & 0 & 1 & 0 \\
0 & 0 & 0 & 0 & 0 & 1 \\
0 & 0 & 0 & 0 & 0 & 0 \\
0 & 245.00 & -147.00 & 0 & 0 & 0 \\
0 & -183.75 & 171.50 & 0 & 0 & 0
\end{array}\right] X+\left[\begin{array}{c}
0 \\
0 \\
0 \\
1.00 \\
10.00 \\
-1.25
\end{array}\right] u
$$

According to (53), using the LQR algorithm named lqr.m in MATLAB and choosing $r=1.0, Q=\operatorname{diag}(18036907.23 .690)$, the feedback control matrix $K$ can be

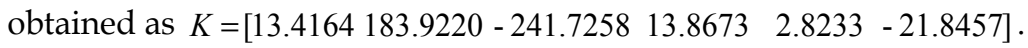

\begin{tabular}{|c|c|c|c|c|c|}
\hline Symbol & Description & Parameter & Symbol & Description & Parameter \\
\hline$m_{1}$ & $\begin{array}{c}\text { Quality of lower } \\
\text { rod }\end{array}$ & $0.1033 \mathrm{~kg}$ & $l_{1}$ & $\begin{array}{c}\text { Length of lower } \\
\text { rod }\end{array}$ & $0.06 \mathrm{~m}$ \\
\hline$m_{2}$ & $\begin{array}{c}\text { Quality of upper } \\
\text { rod }\end{array}$ & $0.2066 \mathrm{~kg}$ & $l_{2}$ & $\begin{array}{c}\text { Length of upper } \\
\text { rod }\end{array}$ & $0.12 \mathrm{~m}$ \\
\hline$M$ & Quality of car & $0.5 \mathrm{~kg}$ & $\mathrm{~g}$ & $\begin{array}{c}\text { Acceleration of } \\
\text { gravity }\end{array}$ & $\begin{array}{c}9.8000 \\
\mathrm{~N} / \mathrm{m}^{2}\end{array}$ \\
\hline
\end{tabular}

Table 1. Physical parameters of double inverted pendulum

Furthermore, the nonlinear virtual prototype of the double inverted pendulum can be established utilizing the SimMechanics toolbox in MATLAB, which depicted in Fig. 11. According to the nominal mathematical model of double inverted pendulum expressed as (53), firstly, choosing Laguerre series as basis functions with series number $N=8$, and controller parameters as $C=\left[\begin{array}{llllll}13.4164 & 183.9220 & -241.7258 & 13.8673 & 2.8233 & -21.8457\end{array}\right]$,

$$
Q=\operatorname{diag}\left(\left[\begin{array}{llllll}
1 & 2 & 2 & 0.1 & 0.1 & 0.1
\end{array}\right]\right), \eta_{1}=0.5, \eta_{2}=0.5, \eta_{3}=1, \eta_{4}=1, \eta_{5}=0.1,
$$

$\eta_{6}=5, \eta_{7}=1$, then the proposed SIMOAC can be realized in S-function form in MATLAB. Finally, nonlinear stabilizing control simulating system of the double inverted pendulum depicted in Fig. 12 can be established through the series connection of virtual prototype and S-function controller in simulink environment of MATLAB.

Afterwards, the stabilizing control simulation experiments on double inverted pendulum can be conduced applying LQR algorithm and the proposed SIMOAC strategy in the simulating system in Fig.12, respectively.

The simulation results under the same initial condition $X_{0}=\left[\begin{array}{llllll}0 & -0.0873 & -0.0873 & 0 & 0 & 0\end{array}\right]^{T}$ are depicted in Fig. 13-18, in which Fig. 13 depicts the car displacement the error of the double inverted pendulum system under the control of SIMOAC and LQR. It can be seen clearly that the steady state displacement error is about -0.0250 meter under the control of SIMOAC, while it's about -0.1115 meter under the control of LQR. This shows the predominant performance of the proposed SIMOAC. Besides, Fig. 14 and Fig. 15 depict the angular displacement error of two pendulum rods, respectively, Fig. 16 depicts the adaptive control signal, Fig. 17 depicts on-line approximation of un-modeling nonlinear dynamics of the double inverted pendulum, Fig. 18 depicts the behavior of sliding function $s(t)$. 


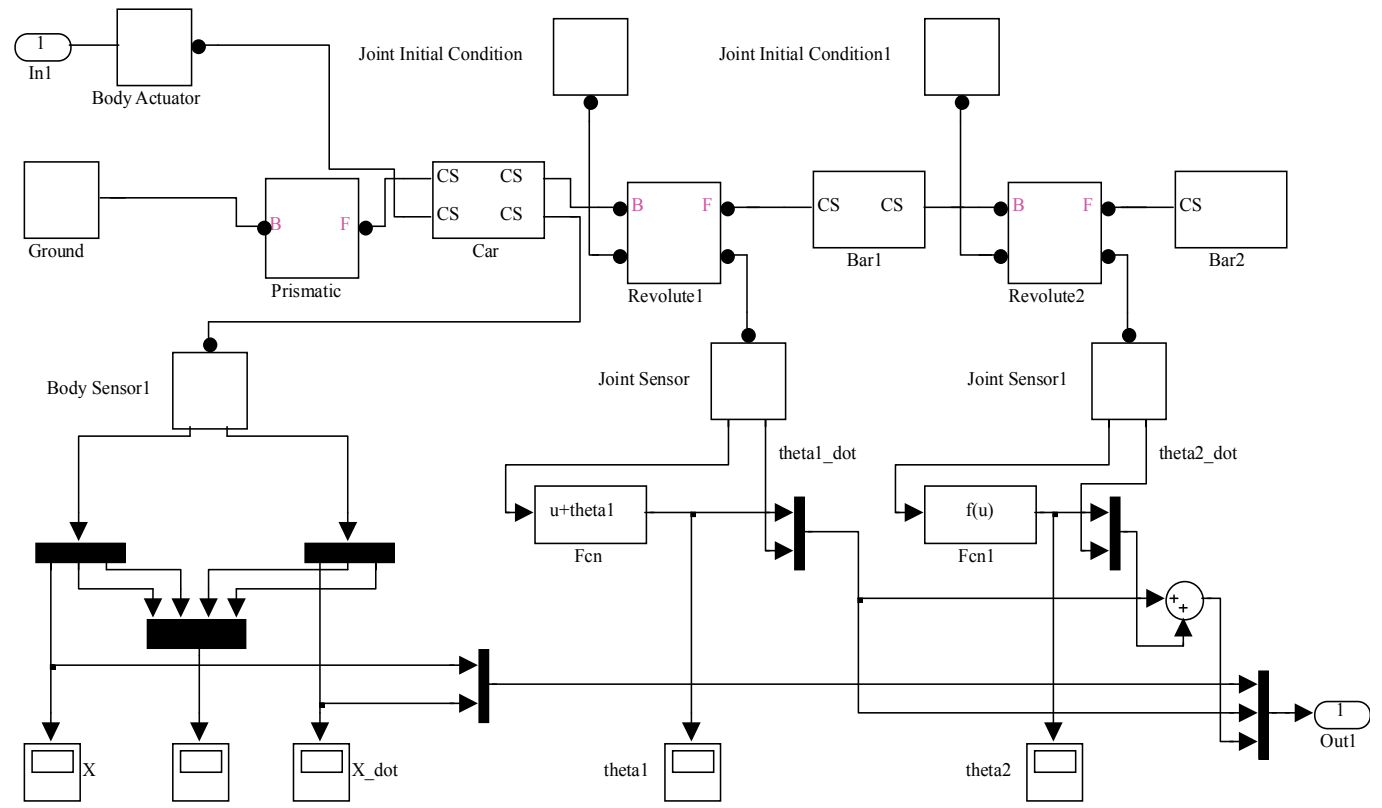

Figure 11. Virtual prototype of double inverted pendulum

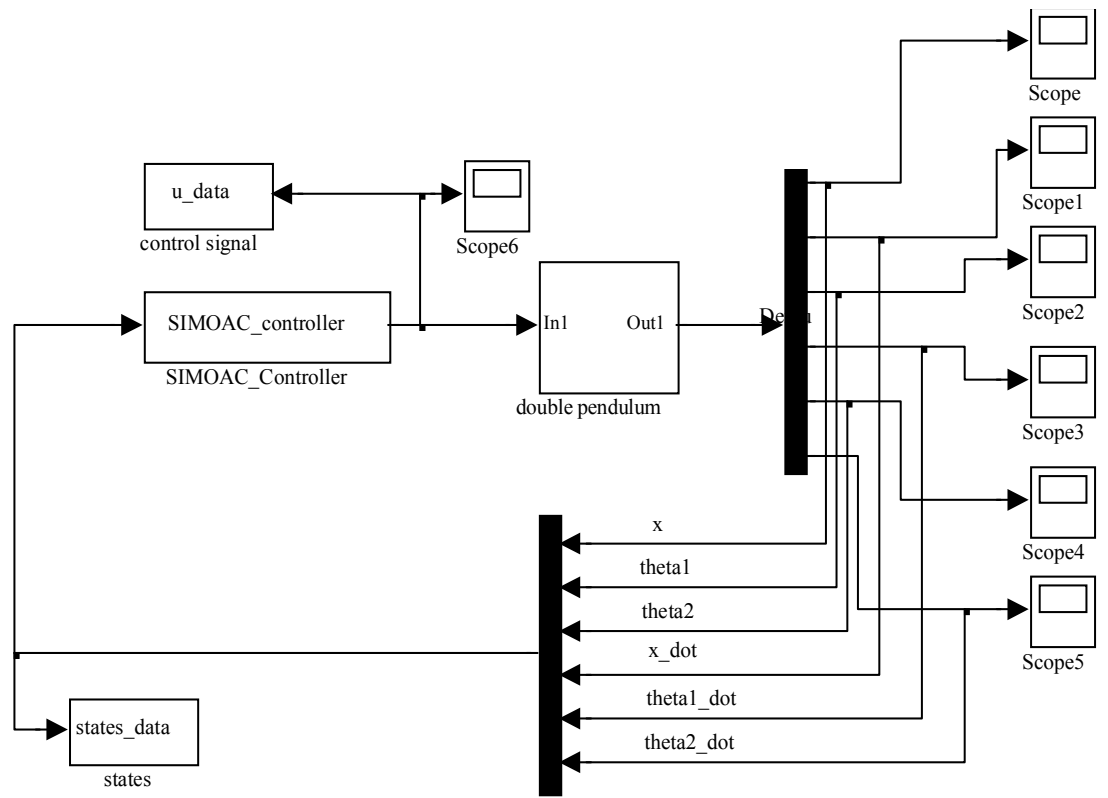

Figure12. Nonlinear stabilizing control simulating system of double inverted pendulum 


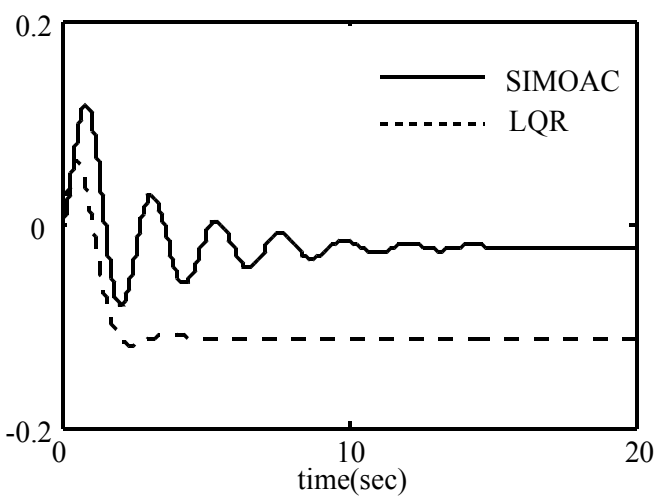

Figure 13. Displacement error of car

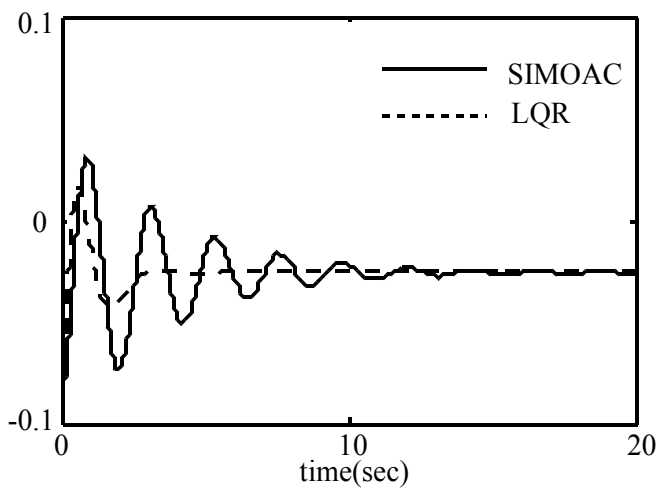

Figure 14. Angular displacement error of the lower rod

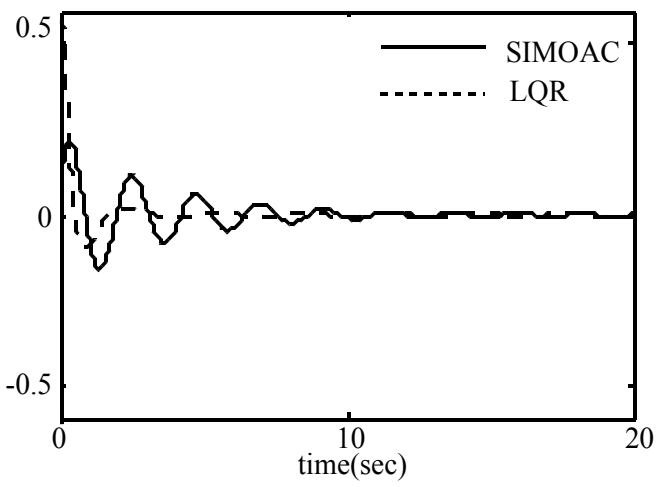

Figure 15. Angular displacement error of the upper rod 


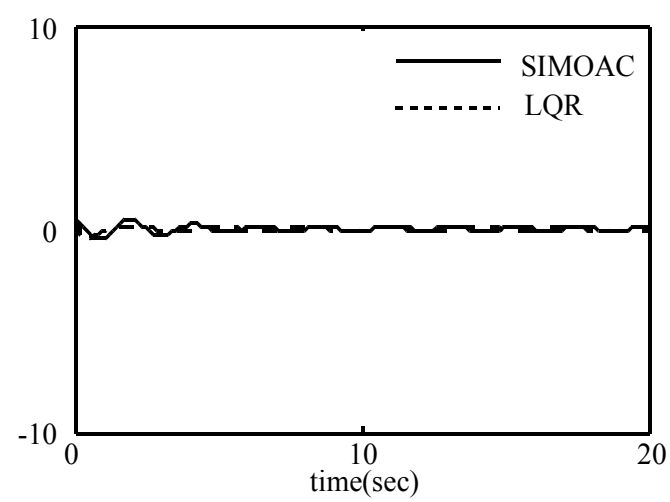

Figure 16. Adaptive control signal

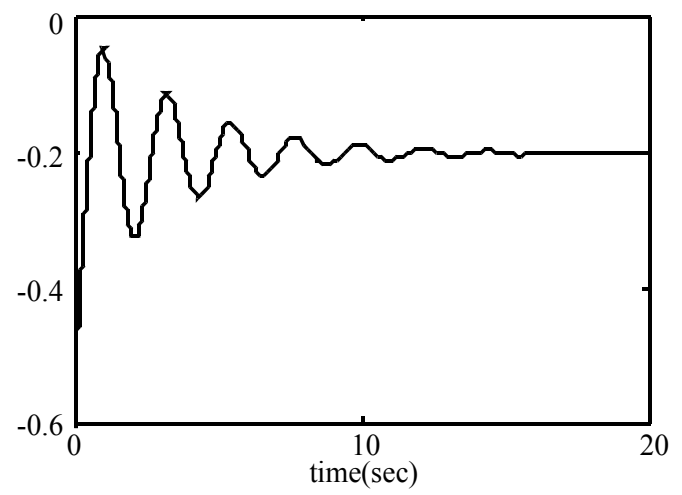

Figure 17. The approximation of uncertainty $-(C B(X))^{-1} \hat{u}_{m_{1}}(t)$

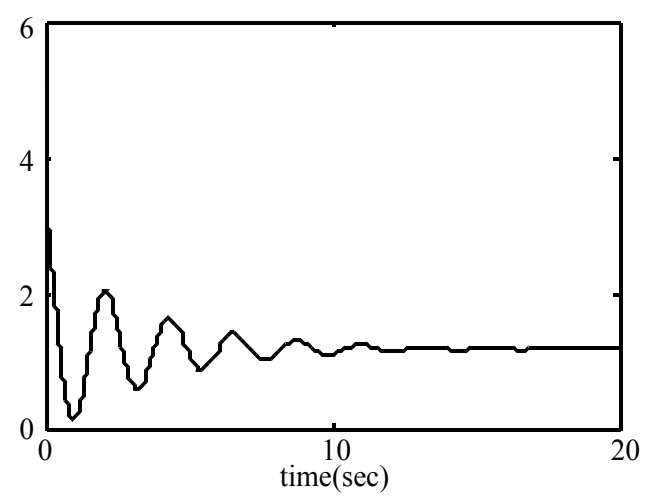

Figure 18. Behavior of sliding function $s(t)$

\section{Conclusions}

In this chapter, two sliding mode adaptive control strategies have been proposed for SISO and SIMO systems with unknown bound time-varying uncertainty respectively. Firstly, for a typical SISO system of position tracking in DC motor with unknown bound time-varying dead 
zone uncertainty, a novel sliding mode adaptive controller is proposed with the techniques of sliding mode and function approximation using Laguerre function series. Actual experiments of the proposed controller are implemented on the DC motor experimental device, and the experiment results demonstrate that the proposed controller can compensate the error of nonlinear friction rapidly. Then, we further proposed a new sliding model adaptive control strategy for the SIMO systems. Only if the uncertainty satisfies piecewise continuous condition or is square integrable in finite time interval, then it can be transformed into a finite combination of orthonormal basis functions. The basis function series can be chosen as Fourier series, Laguerre series or even neural networks. The on-line updating law of coefficient vector in basis functions series and the concrete expression of approximation error compensation are obtained using the basic principle of sliding mode control and the Lyapunov direct method. Finally, the proposed control strategy is applied to the stabilizing control simulating experiment on a double inverted pendulum in simulink environment in MALTAB. The comparison of simulation experimental results of SIMOAC with LQR shows the predominant control performance of the proposed SIMOAC for nonlinear SIMO system with unknown bound time-varying uncertainty.

\section{Acknowledgements}

This work was supported by the National Natural Science Fundation of China under Grant No. 60774098.

\section{References}

An-Chyau, H, Yeu-Shun, K. (2001). Sliding control of non-linear systems containing timevarying uncertainties with unknown bounds. International Journal of Control, Vol. 74, No. 3, pp. 252-264.

An-Chyau, H, \& Yuan-Chih, C. (2004). Adaptive sliding control for single-link flexible-joint robot with mismatched uncertainties. IEEE Transactions on Control Systems Technology, Vol. 12, No. 5, pp. 770-775.

Barmish B. R., \& Leitmann G. (1982). On ultimate boundedness control of uncertain systems in the absence of matching condition. IEEE Transactions on Automatic Control, Vol. 27, No. 1, pp. 153-158.

Campello, R.J.G.B., Favier, G., and Do Amaral, W.C. (2004). Optimal expansions of discretetime Volterra models using Laguerre functions. Automatica, Vol. 40, No. 5, pp. 815822.

Chen, P.-C. \& Huang, A.-C. (2005). Adaptive sliding control of non-autonomous active suspension systems with time-varying loadings. Journal of Sound and Vibration, Vol. 282, No. 3-5, pp. 1119-1135.

Chen Y. H., \& Leitmann G. (1987). Robustness of uncertain systems in the absence of matching assumptions. International Journal of Control, Vol. 45, No. 5, pp. 1527-1542.

Chiang T. Y., Hung M. L., Yan J. J., Yang Y. S., \& Chang J. F. (2007). Sliding mode control for uncertain unified chaotic systems with input nonlinearity. Chaos, Solitons and Fractals, No. 34, No. 2, pp. 437-442.

Chu W. H., \& Tung P. C. (2005). Development of an automatic arc welding system using a sliding mode control, International Journal of Machine Tools and Manufacture, Vol. 45, No. 7-8, pp. 933-939. 
Do K. D., \& Jiang Z. P.(2004). Robust adaptive path following of underactuated ships. Automatica, Vol. 40, No. 6, pp. 929-944.

Fang H., Fan R., Thuilot B., \& Martinet P. (2006). Trajectory tracking control of farm vehicles in presence of sliding. Robotics and Autonomous Systems, Vol. 54, No. 10, pp. 828-839.

Fu Y., \& Chai T. (2007). Nonlinear multivariable adaptive control using multiple models and neural networks. Automatica, Vol. 43, No. 6, pp. 1101-1110.

Gang, T. \& Kokotovic, P.V. (1994). Adaptive control of plants with unknown dead-zones. IEEE Transactions on Automatic Control, Vol. 39, No. 1, pp. 59-68.

Hovakimyan N., Yang B. J., \& Calise A. J. (2006). Adaptive output feedback control methodology applicable to non-minimum phase nonlinear systems. Automatica, Vol. 42, No. 4, pp. 513-522.

Hsu C. F., \& Lin C. M. (2005). Fuzzy-identification-based adaptive controller design via backstepping approach. Fuzzy Sets and Systems, Vol. 151, No. 1, pp. 43-57.

Huang S. J., \& Chen H. Y. (2006). Adaptive sliding controller with self-tuning fuzzy compensation for vehicle suspension control. Mechatronic, Vol. 16, No. 10, pp. 607622.

Huang, A. C., \& Chen, Y. C. (2004). Adaptive multiple-surface sliding control for nonautonomous systems with mismatched uncertainties. Automatica, Vol. 40, No. 11, pp. 1939-1945.

Huang, A. C., \& Chen, Y. C. (2004). Adaptive sliding control for single-link flexible-joint robot with mismatched uncertainties. IEEE Transactions on Control Systems Technology, Vol. 12, No. 5, pp. 770-775.

Huang A. C., \& Kuo Y. S. (2001). Sliding control of non-linear systems containing timevarying uncertainties with unknown bounds. International Journal of Control, Vol. 74, No. 3, pp. 252-264.

Huang A. C., \& Liao K. K. (2006). FAT-based adaptive sliding control for flexible arms: theory and experiments. Journal of Sound and Vibration, Vol. 298, No. 1-2, pp. 194205.

Hung J. Y., Gao W., \& J. C. Hung. (1993). Variable structure control: a survey. IEEE Transactions on Industrial Electronics, Vol. 40, No. 1, pp. 2-22.

Hu S., \& Liu Y. (2004). Robust $H_{\infty}$ control of multiple time-delay uncertain nonlinear system using fuzzy model and adaptive neural network. Fuzzy Sets and Systems, Vol. 146, No. 3, pp. 403-420.

Hung, J.Y., Gao, W. \& Hung J.C. (1993). Variable structure control: a survey. IEEE Transactions on Industrial Electronics, Vol. 40, No. 1, pp. 2-22.

Hyonyong, Cho \& E.-W, B. (1998). Convergence results for an adaptive dead zone inverse. International Journal of Adaptive Control and Signal Processing, Vol. 12, No. 5, pp. 451466.

Liang Y. Y, Cong S, \& Shang W. W. (2008). Function approximation-based sliding mode adaptive control. Nonlinear Dynamics, DOI 10.1007/s11071-007-9324-0.

Liu Y. J., \& Wang W. (2007). Adaptive fuzzy control for a class of uncertain nonaffine nonlinear systems. Information Sciences, Vol. 117, No. 18, pp. 3901-3917.

Manosa V., Ikhouane F., \& Rodellar J. (2005) Control of uncertain non-linear systems via adaptive backstepping, Journal of Sound and Vibration, Vol. 280, No. 3-5, pp. 657-680.

Olivier, P.D. (1994). Online system identification using Laguerre series. IEE ProceedingsControl Theory and Applications, 1994. 141(4): p. 249-254. 
Ruan X., Ding M., Gong D., \& Qiao J. (2007). On-line adaptive control for inverted pendulum balancing based on feedback-error-learning, Neurocomputing, Vol. 70, No. 4-6, pp. 770-776.

Selmic, R. R., Lewis, F.L. (2000). Deadzone compensation in motion control systems using neuralnetworks. IEEE Transactions on Automatic Control, Vol. 45, No. 4, pp. 602-613.

Slotine, J. J. E., \& Li, W. P. (1991). Applied Nonlinear Control, Prentice-Hall, Englewood Cliffs.

Tang Y, Sun F, \& Sun Z. (). Neural network control of flexible-link manipulators using sliding mode. Neurocomputing, Vol. 70, No. 13, pp. 288-295.

Tian-Ping, Z., et al. (2005). Adaptive neural network control of nonlinear systems with unknown dead-zone model. Proceedings of International Conference on Machine Learning and Cybernetics, pp. 18-21, Guangzhou, China, August 2005.

Wahlberg, B. (1991). System identification using Laguerre models. IEEE Transactions on Automatic Control, Vol. 36, No. 5, pp. 551-562.

Wang, L. (2004). Discrete model predictive controller design using Laguerre functions. Journal of Process Control, Vol. 14, No. 2, pp. 131-142.

Wang, X. S, Hong, H., Su, C. Y. (2004). Adaptive control of flexible beam with unknown dead-zone in the driving motor. Chinese Journal of Mechanical Engineering, Vol. 17, No. 3, pp. 327-331.

Wang, X.-S., Su C.-Y., \& Hong, H. (2004) Robust adaptive control of a class of nonlinear systems with unknown dead-zone. Automatica, Vol. 40, No. 3, pp. 407-413.

Wu L. G., Wang C. H., Gao H. J., \& Zhang L. X. (2006). Sliding mode $H_{\infty}$ control for a class of uncertain nonlinear state-delayed systems, Journal of Systems Engineering and electronics, Vol. 27, No, 3, pp. 576-585.

Wu Z. J., Xie, X. J., \& Zhang S. Y., (2007). Adaptive backstepping controller design using stochastic small-gain theorem. Automatica, Vol. 43, No. 4, pp. 608-620.

Yang B. J., \& Calise A. J. (2007). Adaptive Control of a class of nonaffine systems using neural networks. IEEE Transactions on Neural Networks, Vol. 18, No. 4, pp. 11491159.

Young, K.D., Utkin, V.I., \& Ozguner U. (1999). A control engineer's guide to sliding mode control. IEEE Transactions on Control Systems Technology, Vol. 7, No. 3, pp. 328-342.

Zervos, C.C., \& Dumont, G A. (1988). Deterministic adaptive control based on Laguerre series representation. International Journal of Control, Vol. 48, No. 6, pp. 2333-2359.

Zhou K. (2005). A natural approach to high performance robust control: another look at Youla parameterization. Annual Conference on Society of Instrument and Control Engineers, pp. 869-874, Tokyo, Japon, August, 2005.

Zhou J., Er M. J., \& Zurada J. M. (2007). Adaptive neural network control of uncertain nonlinear systems with nonsmooth actuator nonlinearities. Neurocomputing, Vol. 70, No. 4-6, pp. 1062-1070.

Zhou K., \& Ren Z. (2001). A new controller architecture for high performance, robust, and fault-tolerant control. IEEE Transactions on Automatic Control, Vol. 46, No. 10, pp. 1613-1618.

Zhou, J., Wen, C. \& Zhang, Y. (2006). Adaptive output control of nonlinear systems with uncertain dead-zone nonlinearity. IEEE Transactions on Automatic Control, Vol. 51, No.3: pp. 504-511. 


\title{
Model Reference Adaptive Control for Robotic Manipulation with Kalman Active Observers
}

\author{
Rui Cortesão \\ Institute of Systems and Robotics, Electrical and Computer Engineering Department, \\ University of Coimbra \\ Portugal
}

\section{Introduction}

Nowadays, the presence of robotics in human-oriented applications demands control paradigms to face partly known, unstructured and time-varying environments. Contact tasks and compliant motion strategies cannot be neglected in this scenario, enabling safe, rewarding and pleasant interactions. Force control, in its various forms, requires special attention, since the task constraints can change abruptly (e.g. free-space to contact transitions) entailing wide variations in system parameters. Modeling contact/non-contact states and designing appropriate controllers is yet an open problem, even though several control solutions have been proposed along the years. Two main directions can be followed, depending on the presence of force sensors. The perception of forces allows explicit force control (e.g. hybrid position/force control) to manage the interaction imposed by the environment, which is in general more accurate than implicit force control schemes (e.g. impedance control) that do not require force sensing. A major problem of force control design is the robustness to disturbances present in the robotic setup. In this context, disturbances include not only system and measurement noises but also parameter mismatches, nonlinear effects, discretization errors, couplings and so on. If the robot is interacting with unknown objects, "rigid" model based approaches are seldom efficient and the quality of interaction can be seriously deteriorated. Model reference adaptive control (MRAC) schemes can have an important role, imposing a desired closed loop behavior to the real plant in spite of modeling errors.

This chapter introduces the Active Observer (AOB) algorithm for robotic manipulation. The AOB reformulates the classical Kalman filter (CKF) to accomplish MRAC based on: 1) A desired closed loop system. 2) An extra equation to estimate an equivalent disturbance referred to the system input. An active state is introduced to compensate unmodeled terms, providing compensation actions. 3) Stochastic design of the Kalman matrices. In the AOB, MRAC is tuned by stochastic parameters and not by control parameters, which is not the approach of classical MRAC techniques. Robotic experiments will be presented, highlighting merits of the approach. The chapter is organized as follows: After the related work described in Section 3, the AOB concept is analyzed in Sections 4, 5 and 6, where the general algorithm and main design issues are addressed. Section 7 describes robotic 
experiments. The execution of the peg-in-hole task with tight clearance is discussed. Section 8 concludes the chapter.

\section{Keywords}

Kalman Filter, State Space Control, Stochastic Estimation, Observers, Disturbances, Robot Force Control.

\section{Related Work}

Disturbance sources including external disturbances (e.g. applied external forces) and internal disturbances (e.g. higher order dynamics, nonlinearities and noise) are always present in complex control systems, having a key role in system performance. A great variety of methods and techniques have been proposed to deal with disturbances. De Schutter [Schutter, 1988] has proposed an extended deterministic observer to estimate the motion parameters of a moving object in a force control task. In [Chen et al., 2000], model uncertainties, nonlinearities and external disturbances are merged to one term and then compensated with a nonlinear disturbance observer based on the variable structure system theory. Several drawbacks of previous methods are also pointed out in [Chen et al., 2000]. The problem of disturbance decoupling is classical and occupies a central role in modern control theory. Many control problems including robust control, decentralized control and model reference control can be recast as an almost disturbance decoupling problem. The literature is very extensive on this topic. To tackle the disturbance decoupling problem, PIDbased techniques [Estrada \& Malabre, 1999], state feedback [Chu \& Mehrmann, 2000] geometric concepts [Commault et al., 1997], tracking schemes [Chen et al., 2002] and observer techniques [Oda, 2001] have been proposed among others. In [Petersen et al., 2000], linear quadratic Gaussian (LQG) techniques are applied to uncertain systems described by a nominal system driven by a stochastic process. Safanov and Athans [Safanov \& Athans, 1977] proofed how the multi-variable LQG design can satisfy constraints requiring a system to be robust against variations in its open loop dynamics. However, LQG techniques have no guaranteed stability margins [Doyle, 1978], hence Doyle and Stein have used fictitious noise adjustment to improve relative stability [Doyle \& Stein, 1979].

In the $\mathrm{AOB}$, the disturbance estimation is modeled as an auto-regressive (AR) process with fixed parameters driven by a random source. This process represents stochastic evolutions. The AOB provides a methodology to achieve model-reference adaptive control through extra states and stochastic design in the framework of Kalman filters.

It has been applied in several robotic applications, such as autonomous compliant motion of robotic manipulators [Cortesão et al., 2000], [Cortesão et al., 2001], [Park et al., 2004], haptic manipulation [Cortesão et al., 2006], humanoids [Park \& Khatib, 2005], and mobile systems [Coelho \& Nunes, 2005], [Bajcinca et al., 2005], [Cortesão \& Bajcinca, 2004], [Maia et al., 2003].

\section{AOB Structure}

Given a discretized system with equations

$$
x_{r, k}=\Phi_{r} x_{r, k-1}+\Gamma_{r} u_{k-1}+\xi_{x_{r, k}}
$$


and

$$
y_{k}=C_{r} x_{r, k}+\eta_{k}
$$

an observer of the state $x_{r, k}, \hat{x}_{r, k}$, can be written as

$$
\hat{x}_{r, k}=\Phi_{r, n} \hat{x}_{r, k-1}+\Gamma_{r, n} u_{k-1}+K_{k}\left[y_{k}-C_{r}\left(\Phi_{r, n} \hat{x}_{r, k-1}+\Gamma_{r, n} u_{k-1}\right)\right],
$$

where $\Phi_{r, n}$ and $\Gamma_{r, n}$ are respectively the nominal state transition and command matrices (i.e., the ones used in the design). $\Phi_{r}$ and $\Gamma_{r}$ are the real matrices. $\xi_{x_{r, k}}$ and $\eta_{k}$ are Gaussian random variables associated to the system and measures, respectively, having a key role in the AOB design. Defining the estimation error as

$$
e_{r, k}=x_{r, k}-\hat{x}_{r, k}
$$

and considering ideal conditions (i.e., the nominal matrices are equal to the real ones and $\xi_{x_{r, k}}$ and $\eta_{k}$ are zero), $e_{r, k}$ can be computed from (1) and (3). Its value is

$$
e_{r, k}=\left(\Phi_{r}-K_{k} C_{r} \Phi_{r}\right) e_{r, k-1} .
$$

The error dynamics given by the eigenvalues of $\left(\Phi_{r}-K_{k} C_{r} \Phi_{r}\right)$ is function of the $K_{k}$ gain. The Kalman observer computes the best $K_{k}$ in a straightforward way, minimizing the mean square error of the state estimate due to the random sources $\xi_{x_{r, k}}$ and $\eta_{k}$. When there are unmodeled terms, (5) needs to be changed. A deterministic description of $e_{r, k}$ is difficult, particularly when unknown modeling errors exist. Hence, a stochastic approach is attempted to describe it. If state feedback from the observer is used to control the system, $p_{k}$ enters as an additional input

$$
p_{k}=L_{r} e_{r, k},
$$

where $L_{r}$ is the state feedback gain. A state space equation should be found to characterize this undesired input, leading the system to an extended state representation. Figure 1 shows the AOB.

To be able to track functions with unknown dynamics, a stochastic equation is used to describe $p_{k}$

$$
p_{k}-p_{k-1}=\xi_{p_{k}},
$$

in which $\xi_{p_{k}}$ is a zero-mean Gaussian random variable ${ }^{1}$. Equation (7) says that the first derivative (or first-order evolution) of $p_{k}$ is randomly distributed. Defining ${ }^{N} \xi_{p_{k}}$ as the $N^{t h}$ order evolution of $\xi_{p_{k}}$ (or the $(N+1)^{\text {th }}$ order evolution of $p_{k}$ ),

$$
{ }^{N} \xi_{p_{k}}={ }^{N-1} \xi_{p_{k}}-{ }^{N-1} \xi_{p_{k-1}} \text {, with }{ }^{0} \xi_{p_{k}}=\xi_{p_{k}},
$$

the general form of $(7)$ is

\footnotetext{
1 The mathematical notation along the paper is for single input systems. For multiple input systems, $p_{k}$, in (7) is a column vector with dimension equal to the number of inputs.
} 


$$
p_{k}=\sum_{j=1}^{N}(-1)^{j+1} \frac{N !}{j !(N-j) !} p_{k-j}+{ }^{N-1} \xi_{p_{k}} .
$$

$\left\{p_{n}\right\}$ is an AR process ${ }^{2}$ of order $N$ with undetermined mean. It has fixed parameters given by (9) and is driven by the statistics of $\left\{{ }^{N-1} \xi_{p_{n}}\right\}$. The properties of ${ }^{N-1} \xi_{p_{k}}$ can change on-line based on a given strategy. The stochastic equation (7) for the AOB-1 or (9) for the AOB-N is used to describe $p_{k}$. If ${ }^{N-1} \xi_{p_{k}}=0$, (9) is a deterministic model for any disturbance $p_{k}$ that has its $N^{\text {th }}$-derivative equal to zero. In this way, the stochastic information present in ${ }^{N-1} \xi_{p_{k}}$ gives more flexibility to $p_{k}$, since its evolutionary model is not rigid. The estimation of unknown functions using (7) and (9) is discussed in [Cortesão et al., 2004].

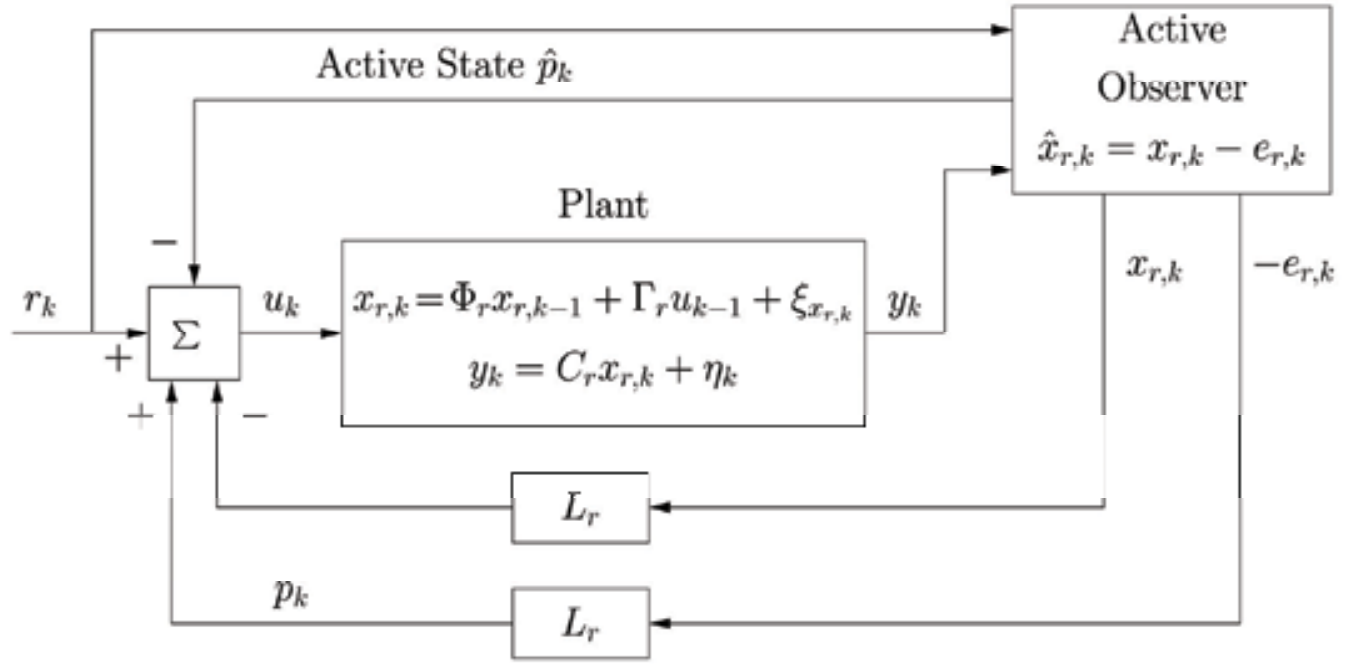

Figure 1. Active Observer. The active state $\hat{p}_{k}$ compensates the error input, which is described by $p_{k}$

\section{AOB-1 Design}

The AOB-1 algorithm is introduced in this section based on a continuous state space description of the system.

\subsection{System Plant}

Discretizing the system plant (without considering disturbances)

$$
\left\{\begin{aligned}
\frac{\mathrm{d} x_{r}(t)}{\mathrm{d} t} & =A x_{r}(t)+B u(t-\tau) \\
y(t) & =C_{r} x_{r}(t)
\end{aligned}\right.
$$

with sampling time $h$ and dead-time

$$
\tau=(d-1) h+\tau^{\prime}, \text { with } 0<\tau^{\prime} \leq h,
$$

${ }^{2} p_{k}$ is a random variable and $\left\{p_{n}\right\}$ is a random process. 
the discrete time system

$$
\left[\begin{array}{c}
x_{k}^{\prime} \\
u_{k-d} \\
\vdots \\
u_{k-2} \\
u_{k-1}
\end{array}\right]=\left[\begin{array}{ccccc}
\Phi_{1} & \Gamma_{1} & \Gamma_{0} & \cdots & 0 \\
0 & 0 & 1 & \cdots & 0 \\
\vdots & \vdots & \vdots & \ddots & \vdots \\
0 & 0 & 0 & \cdots & 1 \\
0 & 0 & 0 & \cdots & 0
\end{array}\right]\left[\begin{array}{c}
x_{k-1}^{\prime} \\
u_{k-d-1} \\
\vdots \\
u_{k-3} \\
u_{k-2}
\end{array}\right]+\left[\begin{array}{c}
0 \\
0 \\
\vdots \\
0 \\
1
\end{array}\right] u_{k-1}
$$

and

$$
y_{k}=C_{r} x_{r, k}
$$

is obtained $\Phi_{1}, \Gamma_{0}$ and $\Gamma_{1}$ are given by (14) to (16), respectively [Aström \& Wittenmark, 1997].

$$
\begin{gathered}
\Phi_{1}=e^{A h}=\phi(h), \\
\Gamma_{0}=\int_{0}^{h-\tau^{\prime}} \phi(\lambda) d \lambda B
\end{gathered}
$$

and

$$
\Gamma_{1}=\phi\left(h-\tau^{\prime}\right) \int_{0}^{\tau^{\prime}} \phi(\lambda) d \lambda B
$$

The state $x_{r, k}$ is

$$
x_{r, k}=\left[\begin{array}{lllll}
x_{k}^{\prime} & u_{k-d} & \cdots & u_{k-2} & u_{k-1}
\end{array}\right]^{T},
$$

in which $x_{k}^{\prime}$ is the system state considering no dead-time. Therefore, the $\tau$ of $(6.10)$ increases the system order.

\subsection{AOB-1 Algorithm}

From Figure 1 and knowing (1) and (7), the augmented state space representation (open loop) ${ }^{3}$ is

$$
\left[\begin{array}{c}
x_{r, k} \\
p_{k}
\end{array}\right]=\left[\begin{array}{cc}
\Phi_{r} & \Gamma_{r} \\
0 & 1
\end{array}\right]\left[\begin{array}{c}
x_{r, k-1} \\
p_{k-1}
\end{array}\right]+\left[\begin{array}{c}
\Gamma_{r} \\
0
\end{array}\right] u_{k-1}^{\prime}+\left[\begin{array}{c}
\xi_{x_{r, k}} \\
\xi_{p_{k}}
\end{array}\right]
$$

where

$$
u_{k-1}^{\prime}=r_{k-1}-\left[\begin{array}{ll}
L_{r} & 1
\end{array}\right]\left[\begin{array}{c}
x_{r, k-1} \\
\hat{p}_{k-1}
\end{array}\right]
$$

\footnotetext{
${ }^{3}$ In this context, open loop means that the state transition matrix does not consider the influence of state feedback.
} 


$$
\Phi_{r}=\left[\begin{array}{ccccc}
\Phi_{1} & \Gamma_{1} & \Gamma_{0} & \cdots & 0 \\
0 & 0 & 1 & \cdots & 0 \\
\vdots & \vdots & \vdots & \ddots & \vdots \\
0 & 0 & 0 & \cdots & 1 \\
0 & 0 & 0 & \cdots & 0
\end{array}\right]
$$

and

$$
\Gamma_{r}=\left[\begin{array}{lllll}
0 & 0 & \cdots & 0 & 1
\end{array}\right]^{T} .
$$

$L_{r}$ is obtained by any control technique applied to (12) to achieve a desired closed loop behavior. The measurement equation is

$$
y_{k}=C\left[\begin{array}{c}
x_{r, k} \\
p_{k}
\end{array}\right]+\eta_{k},
$$

with $^{4}$

$$
C=\left[\begin{array}{ll}
C_{r} & 0
\end{array}\right]
$$

The desired closed loop system appears when $\hat{p}_{k}=p_{k}$ i.e.,

$$
\left[\begin{array}{c}
x_{r, k} \\
p_{k}
\end{array}\right]=\left[\begin{array}{cc}
\Phi_{r}-\Gamma_{r} L_{r} & 0 \\
0 & 1
\end{array}\right]\left[\begin{array}{c}
x_{r, k-1} \\
p_{k-1}
\end{array}\right]+\left[\begin{array}{c}
\Gamma_{r} \\
0
\end{array}\right] r_{k-1}+\left[\begin{array}{c}
\xi_{x_{r, k}} \\
\xi_{p_{k}}
\end{array}\right] .
$$

The state $x_{r, k}$ in (24) is accurate if most of the modeling errors are merged to $p_{k}$. Hence, $\xi_{x_{r, k}}$ should be small compared to $\xi_{p_{k}}$. The state estimation ${ }^{5}$ must consider not only the influence of the uncertainty $\xi_{x_{r, k}}$, but also the deterministic term due to the reference input, the extended state representation and the desired closed loop response. It is given by ${ }^{6}$

$$
\begin{aligned}
{\left[\begin{array}{c}
\hat{x}_{r, k} \\
\hat{p}_{k}
\end{array}\right]=} & {\left[\begin{array}{cc}
\Phi_{r, n}-\Gamma_{r, n} L_{r} & 0 \\
0 & 1
\end{array}\right]\left[\begin{array}{c}
\hat{x}_{r, k-1} \\
\hat{p}_{k-1}
\end{array}\right]+\left[\begin{array}{c}
\Gamma_{r, n} \\
0
\end{array}\right] r_{k-1}+} \\
& +K_{k}\left\{y_{k}-C\left(\left[\begin{array}{cc}
\Phi_{r, n}-\Gamma_{r, n} L_{r} & 0 \\
0 & 1
\end{array}\right]\left[\begin{array}{c}
\hat{x}_{r, k-1} \\
\hat{p}_{k-1}
\end{array}\right]+\left[\begin{array}{c}
\Gamma_{r, n} \\
0
\end{array}\right] r_{k-1}\right)\right\} .
\end{aligned}
$$

$K_{k}$ is

$$
K_{k}=P_{1 k} C^{T}\left[C P_{1 k} C^{T}+R_{k}\right]^{-1}
$$

and

$$
P_{1 k}=\Phi_{n} P_{k-1} \Phi_{n}^{T}+Q_{k},
$$

\footnotetext{
4 The form of $C$ is maintained for the AOB-N, since the augmented states that describe $p_{k}$ are not measured.

5 The CKF algorithm can be seen in [Bozic, 1979].

$6 \Phi_{r, n}$ and $\Gamma_{r, n}$ represent the nominal values of $\Phi_{r_{1}, n}$ and $\Gamma_{r, n}$, respectively.
} 
with

$$
P_{k}=P_{1 k}-K_{k} C P_{1 k}
$$

$\Phi_{n}$ is the augmented open loop matrix used in the design,

$$
\Phi_{n}=\left[\begin{array}{cc}
\Phi_{r, n} & \Gamma_{r, n} \\
0 & 1
\end{array}\right] .
$$

$Q_{k}$ is the system noise matrix, ${ }^{7}$

$$
Q_{k}=\left[\begin{array}{cc}
Q_{x_{r, k}} & 0 \\
0 & Q_{p_{k}}
\end{array}\right] .
$$

$R_{k}$ is the measurement noise matrix, $R_{k}=E\left\{\eta_{k} \eta_{k}^{T}\right\} . P_{k}$ is the mean square error matrix. It should be pointed out that $P_{1 k}$ given by (6.27) uses $\Phi_{n}$. More details can be seen in [Cortesão, 2007].

\section{AOB-N Design}

The AOB-N is discussed in this section enabling stronger nonlinearities to be compensated by $\hat{p}_{k}$. Section 6.6 .1 presents the AOB-N algorithm and Section 6.2 discusses the stochastic structure of AOB matrices.

\subsection{AOB-N Algorithm}

The AOB-1 algorithm has to be slightly changed for the AOB-N. Only the equation of the active state changes, entailing minor modifications in the overall AOB design. Equation (9) has the following state space representation:

$$
\begin{gathered}
{\left[\begin{array}{c}
p_{k-(N-1)} \\
p_{k-(N-2)} \\
\vdots \\
p_{k-1} \\
p_{k}
\end{array}\right]=\left[\begin{array}{ccccc}
0 & 1 & 0 & \cdots & 0 \\
0 & 0 & 1 & \cdots & 0 \\
\vdots & \vdots & \vdots & \ddots & \vdots \\
0 & 0 & 0 & \cdots & 1 \\
a_{N} & a_{N-1} & a_{N-2} & \cdots & a_{1}
\end{array}\right]\left[\begin{array}{c}
p_{k-N} \\
p_{k-(N-1)} \\
\vdots \\
p_{k-2} \\
p_{k-1}
\end{array}\right]} \\
+\left[\begin{array}{c}
0 \\
0 \\
\vdots \\
0 \\
N-1 \xi_{p_{k}}
\end{array}\right] \text { with } a_{i}=(-1)^{i+1} \frac{N !}{i !(N-i) !}, i=1, \cdots, N .
\end{gathered}
$$

In compact form, (31) is represented by

$$
{ }^{N} p_{k}=\Phi_{2,2}{ }^{N} p_{k-1}+\xi_{{ }^{N} p_{k}} .
$$

\footnotetext{
7 Purther analysis of the $Q_{k}$ matrix is given in Section 6.2.
} 
Equation (18) is now re-written as

$$
\begin{aligned}
{\left[\begin{array}{l}
x_{r, k} \\
{ }^{N} p_{k}
\end{array}\right]=} & {\left[\begin{array}{cc}
\Phi_{r} & \Phi_{1,2} \\
0 & \Phi_{2,2}
\end{array}\right]\left[\begin{array}{l}
x_{r, k-1} \\
{ }^{N} p_{k-1}
\end{array}\right] } \\
& +\left[\begin{array}{c}
\Gamma_{r} \\
0
\end{array}\right] u_{k-1}^{\prime}+\left[\begin{array}{l}
\xi_{x_{r, k}} \\
\xi_{N_{p_{k}}}
\end{array}\right],
\end{aligned}
$$

where

$$
{ }^{N} p_{k}=\left[\begin{array}{lllll}
p_{k-(N-1)} & p_{k-(N-2)} & \cdots & p_{k-1} & p_{k}
\end{array}\right]^{T} .
$$

$\Phi_{2,2}$ is the state transition matrix of (31),

$$
\Phi_{1,2}=\left[\begin{array}{ccc}
0 & \cdots & 0 \\
\vdots & \ddots & \vdots \\
0 & \cdots & 1
\end{array}\right]
$$

and

$$
\xi_{N_{p_{k}}}=\left[\begin{array}{lllll}
0 & 0 & \cdots & 0 & { }^{N-1} \xi_{p_{k}}
\end{array}\right]^{T} .
$$

The desired closed loop of (24) is changed to

$$
\left[\begin{array}{l}
x_{r, k} \\
{ }^{N} p_{k}
\end{array}\right]=\left[\begin{array}{cc}
\Phi_{L} & 0 \\
0 & \Phi_{2,2}
\end{array}\right]\left[\begin{array}{c}
x_{r, k-1} \\
{ }^{N} p_{k-1}
\end{array}\right]+\left[\begin{array}{c}
\Gamma_{r} \\
0
\end{array}\right] r_{k-1}+\left[\begin{array}{c}
\xi_{x_{r, k}} \\
\xi_{N_{k}}
\end{array}\right],
$$

with

$$
\Phi_{L}=\left[\begin{array}{ccccc}
\Phi_{1} & \Gamma_{1} & \Gamma_{0} & \cdots & 0 \\
0 & 0 & 1 & \cdots & 0 \\
\vdots & \vdots & \vdots & \ddots & \vdots \\
0 & 0 & 0 & \cdots & 1 \\
-L_{1} & -L_{2} & -L_{3} & \cdots & -L_{M}
\end{array}\right]
$$

The $L_{r}$ components $\left(L_{1}, \ldots, L_{M}\right)$ can be obtained by Ackermann's formula, i.e.,

$$
L_{r}=\left\lceil\begin{array}{llll}
0 & \cdots & 0 & 1
\end{array}\right] W_{c}^{-1} P\left(\Phi_{r}\right) .
$$

$W_{c}$ is the reachability matrix

$$
W_{c}=\left[\begin{array}{llll}
\Gamma_{r} & \Phi_{r} \Gamma_{r} & \cdots & \Phi_{r}^{M-1} \Gamma_{r}
\end{array}\right],
$$

and $P\left(\Phi_{r}\right)$ is the desired characteristic polynomial. The state estimation is 8

$8 \Phi_{L, n}$ is the nominal value of $\Phi_{L}$ 


$$
\begin{aligned}
{\left[\begin{array}{c}
\hat{x}_{r, k} \\
{ }^{N} \hat{p}_{k}
\end{array}\right]=} & {\left[\begin{array}{cc}
\Phi_{L, n} & 0 \\
0 & \Phi_{2,2}
\end{array}\right]\left[\begin{array}{c}
\hat{x}_{r, k-1} \\
{ }^{N} \hat{p}_{k-1}
\end{array}\right]+\left[\begin{array}{c}
\Gamma_{r, n} \\
0
\end{array}\right] r_{k-1} } \\
& +K_{k}\left\{y_{k}-C\left(\left[\begin{array}{cc}
\Phi_{L, n} & 0 \\
0 & \Phi_{2,2}
\end{array}\right]\left[\begin{array}{c}
\hat{x}_{r, k-1} \\
{ }^{N} \hat{p}_{k-1}
\end{array}\right]+\left[\begin{array}{c}
\Gamma_{r, n} \\
0
\end{array}\right] r_{k-1}\right)\right\} .
\end{aligned}
$$

$K_{k}$ is given by (26) to (28), with

$$
\Phi_{n}=\left[\begin{array}{cc}
\Phi_{r, n} & \Phi_{1,2} \\
0 & \Phi_{2,2}
\end{array}\right]
$$

The state feedback gain $L$ is

$$
L=\left[\begin{array}{ll}
L_{r} & L_{N_{p_{k}}}
\end{array}\right]
$$

with

$$
L_{p_{k}}=\left[\begin{array}{llll}
0 & \cdots & 0 & 1
\end{array}\right] .
$$

\subsection{AOB-N Matrices}

$R_{k}$ is function of sensor characteristics. The form of $Q_{k}$ is

$$
Q_{k}=\left[\begin{array}{cc}
Q_{x_{r, k}} & 0 \\
0 & Q_{N_{p_{k}}}
\end{array}\right] .
$$

$Q_{x_{r, k}}$ is a diagonal matrix. The uncertainty associated with $x_{r, k}$ is low since all system disturbances should be compensated with ${ }^{N} p_{k}$. Hence, $Q_{x_{r, k}}$ should have low values compared to $Q_{N_{p_{k}}}$, which is defined as

$$
Q_{N_{p_{k}}}=E\left\{\xi_{N_{p_{k}}} \cdot \xi_{N_{p_{k}}}^{T}\right\} \text {. }
$$

From (36),

$$
Q_{N_{p_{k}}}=\left[\begin{array}{ccc}
0 & \cdots & 0 \\
\vdots & \ddots & \vdots \\
0 & \cdots & \sigma_{N-1}^{2} \xi_{p_{k}}
\end{array}\right] .
$$

$\sigma_{N-1}^{2} \xi_{p_{k}}$ represents the variance of the $N^{\text {th }}$ derivative of $p_{k}$, and is related with $\sigma_{\xi_{p_{k}}}^{2}$. Hence, the design can be done for $\sigma_{\xi_{p_{k}}}^{2}$ (see [Cortesão, 2007]).

\subsection{AOB Estimation Strategies}

An important property of the Kalman gain is introduced in Proposition 1, enabling to define different estimation strategies.

Proposition 1 The Kalman gain $K_{k}$ obtained from (26) to (28) does not have a unique solution.

Proof: Using (27) and (28), the matrix $P_{k}$ can be written in a recursive way as 


$$
P_{k}=\left(I-K_{k} C_{a}\right)\left(\Phi_{a} P_{k-1} \Phi_{a}^{T}+Q_{k}\right) .
$$

Let's consider one solution

$$
\left(Q_{k}, R_{k}, P_{0}\right) \rightarrow\left(K_{k}, P_{k}\right) .
$$

Another candidate solution

$$
\left(Q_{k}^{\prime}, R_{k}^{\prime}, P_{0}^{\prime}\right) \rightarrow\left(K_{k}, P_{k}^{\prime}\right)
$$

should be found, giving the same $K_{k}$ values. If

$$
Q_{k}^{\prime}=\alpha Q_{k},
$$

in which $a$ is a scalar', (48) is satisfied if

$$
P_{k}^{\prime}=\alpha P_{k} \text { and } P_{k-1}^{\prime}=\alpha P_{k-1} .
$$

Hence, from (27), (51) and (52),

$$
P_{1 k}^{\prime}=\alpha P_{1 k} .
$$

From (26), the new Kalman gain $K_{k}^{\prime}$ is

$$
\begin{aligned}
K_{k}^{\prime} & =P_{1 k}^{\prime} C_{a}^{T}\left[C_{a} P_{1 k}^{\prime} C_{a}^{T}+R_{k}^{\prime}\right]^{-1} \\
& =\alpha P_{1 k} C_{a}^{T}\left[C_{a} \alpha P_{1 k} C_{a}^{T}+R_{k}^{\prime}\right]^{-1} .
\end{aligned}
$$

To accomplish $K_{k}^{\prime}=K_{k}$, it is necessary that $R_{k}^{\prime}=\alpha R_{k}$. The candidate solution exists and is

$$
\left(Q_{k}^{\prime}=\alpha Q_{k}, R_{k}^{\prime}=\alpha R_{k}, P_{0}^{\prime}=\alpha P_{0}\right) \rightarrow\left(K_{k}, P_{k}^{\prime}=\alpha P_{k}\right)
$$

Corollary 1 What defines the Kalman gain, or the estimation strategy, are only the relations between the $Q_{k}$ values, the $R_{k}$ values, and both $Q_{k}$ and $R_{k}$ values.

Proof: Similar to Proposition 1.

In the $\mathrm{AOB}$, the relations between the $Q_{k}$ values are straightforward. The estimation strategy is thus based on the relation between $Q_{k}$ and $R_{k}$. If model accuracy is very good compared with measure accuracy, a model based approach (MBA) is followed. The estimation $\hat{x}_{k}$ given by (6.41) is mainly based on the model, giving little importance to measures. The Kalman gain has low values. On the other hand, if the measures are very accurate with respect to the model, a sensor based approach (SBA) is followed. The estimates are very sensitive to measures. The Kalman gain has high values. The hybrid based approach (HBA) is the general form of the AOB and establishes a trade-off between the SBA and MBA, i.e. it balances the estimates based on sensory and model information. To keep the same strategy as the $\mathrm{AOB}$ order changes, some adjustments in $Q_{k}$ or $R_{k}$ may be necessary, since the extended system matrix $\Phi_{a}$ that affects $K_{k}$ changes.

\footnotetext{
${ }^{9}$ Physically, it makes no sense a negative value of $\alpha$.
} 
Eliminating the active state and redesigning $Q$ (with new values) gives the CKF. In this situation, the $Q$ design is not straightforward. Nevertheless, the relation between $Q$ and $R$ defines the control strategy.

For complex control tasks (e.g. compliant motion control), switching between AOB and CKF may appear dynamically as a function of the task state.

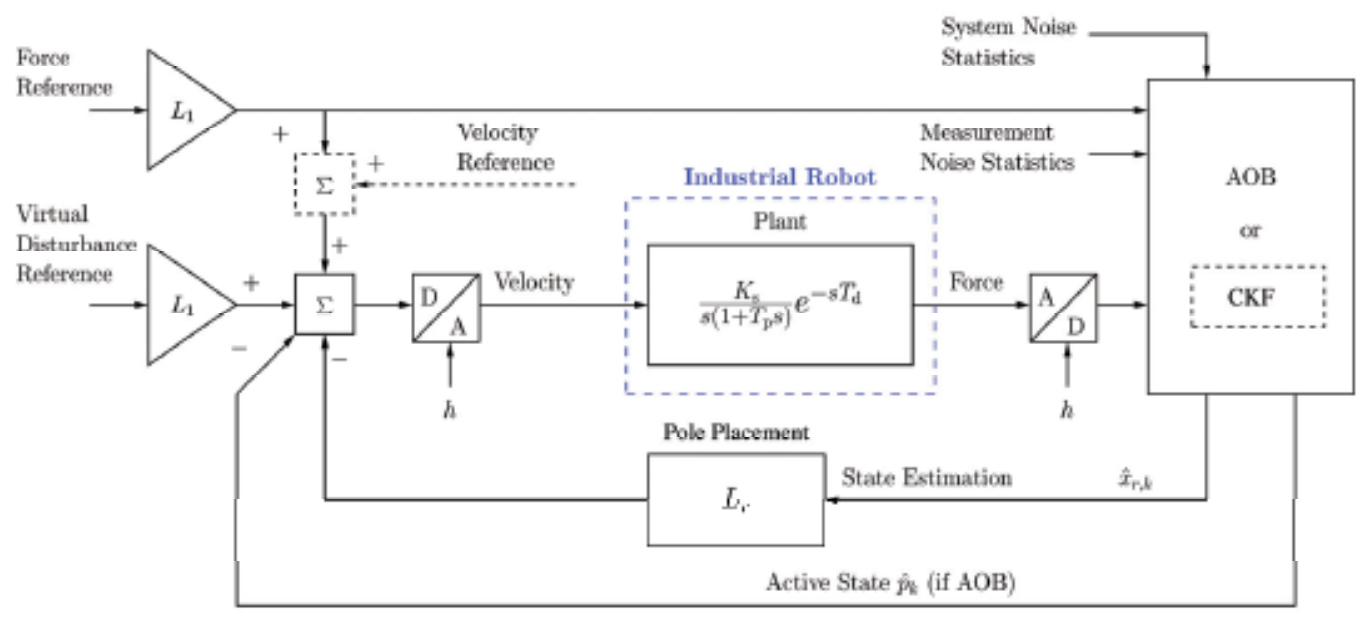

Figure 2. Global control scheme for each force/velocity dimension. $K_{s}$ is the system stiffness. $T_{p}$ is the position response time constant, and $T_{d}$ is the system time delay due to the signal processing of the cascade controller

\section{Experiments}

If a manipulator is working in free space, position or velocity control of the end-effector is sufficient. For constrained motion, specialized control techniques have to be applied to cope with natural and artificial constraints. The experiments reported in this section apply AOBs to design a compliant motion controller $(\mathrm{CMC})$ for the peg-in-hole task, guaranteeing model reinforcement in critical directions.

\subsection{Peg-in-Hole Task}

The robotic peg-in-hole insertion is one of the most studied assembly tasks. This longstanding problem has had many different solutions along the years. If the peg-hole clearance is big, position controlled robots can perform the task relatively easy. Otherwise, force control is necessary. The insertion requires position and angular constrained motion, which is a common situation in automation tasks. Several authors have tackled the peg-inhole problem. Yoshimi and Allen [Yoshimi \& Allen, 1994] performed the peg-in-hole alignment using uncalibrated cameras. Broenink and Tiernego [Broenink \& Tiernego, 1996] applied impedance control methods to peg-in-hole assembly. Itabashi and co-authors [Itabashi et al., 1998] modeled the impedance parameters of a human based peg-in-hole insertion with hidden Markov models to perform a robotic peg-in-hole task. In [Morel et al., 1998], the peg-in-hole task is done combining vision and force control, and in [Kim et al., 2000], stiffness analysis is made for peg-in/out-hole tasks, showing the importance of the 
compliance center location. Newman and co-authors [Newman et al., 2001] used a feature based technique to interpret sensory data, applying it to the peg-in-hole task.

\begin{tabular}{|l|l|}
\hline Parameter & Value \\
\hline$T_{P}$ & $0.032[\mathrm{~s}]$ \\
$T_{d}$ & $0.040[\mathrm{~s}]$ \\
$h$ & $0.008[\mathrm{~s}]$ \\
\hline & $3[\mathrm{~N} / \mathrm{mm}](\mathrm{x}$ lin.) \\
& $3[\mathrm{~N} / \mathrm{mm}](\mathrm{y}$ lin.) \\
& $20[\mathrm{~N} / \mathrm{mm}](\mathrm{z}$ lin.) \\
$K_{w}$ & $100[\mathrm{Nm} / \mathrm{rad}]$ (x rot.) \\
& $100[\mathrm{Nm} / \mathrm{rad}]$ (y rot.) \\
\hline
\end{tabular}

Table 1. Technology parameters of the Manutec R2 robot. Stiffness data

In our setup, the robotic peg-in-hole task is performed with an AOB based CMC. Figure 6.2 represents the $\mathrm{CMC}$ for each force dimension. The main goal is the human-robot skill transfer of the peg-in-hole task. The task is previously performed by a human, where the forces, torques and velocities of the peg are recorded as a function of its pose, using a teaching device. The robot should be able to perform the same task in a robust way after the skill transfer [Cortesão et al., 2004]. The CMC is an important part of the system, since it tries to accomplish a desired compliant motion for a given geometric information. This CMC may change on-line between full AOB, CKF or "pure" state feedback, based on the task state. If velocity signals are dominant, the CKF should be used since the full AOB annihilates any external inputs. If force signals are dominant, the full AOB is a good choice. Hence, the CMC can easily handle two reference inputs, $f_{d}$ (force) and $v_{d}$ (velocity). The natural constraints imply complementarity of the reference signals. When $f_{d}$ is different than zero $v_{d}$ is almost zero and vice-versa. In the sequel, the performance of the 6 DOF peg-in-hole task is analyzed with the $\mathrm{AOB}$ and $\mathrm{CKF}$ in the loop.

\subsection{Experimental Setup}

Experimental tests have been done in a robotic testbed at DLR (German Aerospace Center). The main components of this system are:

- A Manutec R2 industrial robot with a Cartesian position interface running at 8 [ms] and an input dead-time of 5 samples, equivalent to 40 [ms].

- A DLR end-effector, which consists of a compliant force/torque sensor providing force/torque measurements every 8 [ms]. The technology data of the robot and the force sensor stiffness are summarized in Table 6.1. The manipulator compliance is lumped in the force sensor.

- A multi-processor host computer running UNIX, enabling to compute the controller at each time step.

- Two cameras for stereo vision are mounted on the end-effector. A pneumatic gripper holds a steel peg of $30[\mathrm{~mm}]$ length and of $23[\mathrm{~mm}]$ diameter. The peg-hole has a clearance of $50[\mu \mathrm{m}]$, which corresponds to a tolerance with ISO quality 9 . The hole is 
chamferless. The environment is very $\operatorname{stiff}\left(K_{\mathrm{s}} \approx K_{\mathrm{w}}\right)$. Vision and pose sense belong to the data fusion architecture (see [Cortesão et al., 2004]).

- A picture of the experimental setup is depicted in Figure 3. Figure 3.a represents the peg-in-hole task with a three point contact. Figure 3.b shows the task execution with human interference.

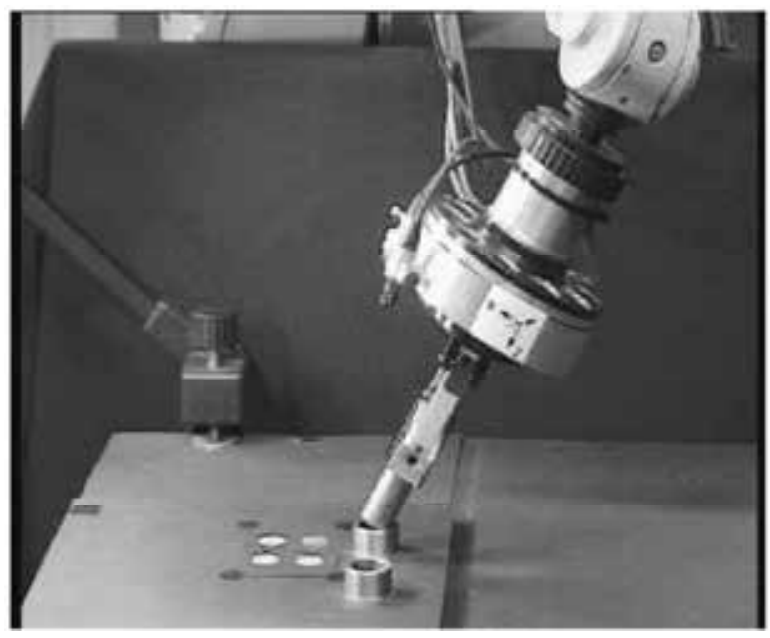

(a)

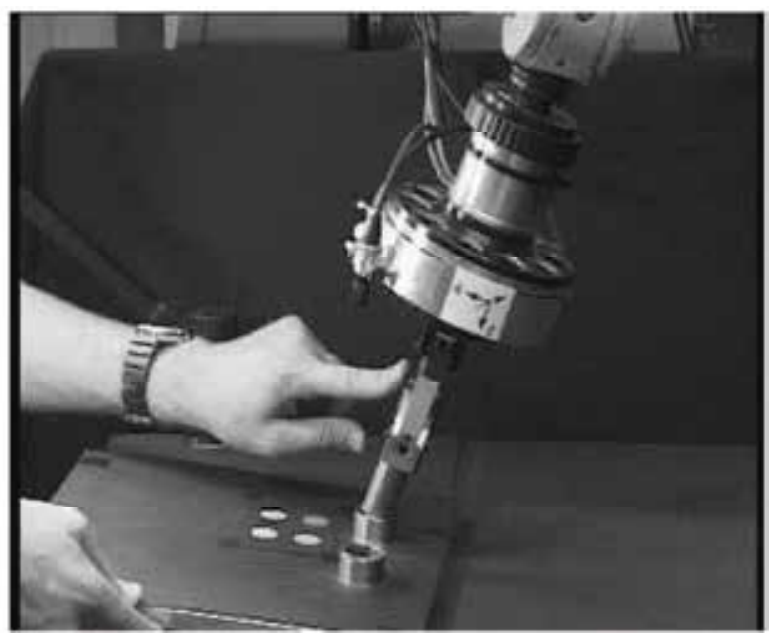

(b)

Figure 3. Experimental setup, (a) Manutec R2 robot ready to perform the peg-in-hole task, (b) Human interaction during the peg-in-hole task

\subsection{AOB Matrices}

This section discusses the AOB design for the CMC controller.

System Plant Matrices

For each DOF, the position response of the Manutec R2 robot is 


$$
G_{p}(s)=\frac{1}{1+T_{p} s} e^{-s T_{d}} .
$$

Inserting the system stiffness $K_{s}$, the system plant can be written as

$$
G(s)=\frac{k_{1}}{s\left(s+p_{1}\right)} e^{-s t_{d}},
$$

with

$$
k_{1}=K_{s} / T_{p}, \quad p_{1}=1 / T_{p} \quad \text { and } \quad t_{d}=T_{d} .
$$

Its equivalent temporal representation is

$$
\ddot{y}+p_{1} \dot{y}=k_{1} u\left(t-t_{d}\right),
$$

where $y$ is the plant output (force) and $u$ is the plant input (velocity). Defining the state variables as

$$
x_{1}=y \text { and } x_{2}=\dot{y},
$$

the state space description of (59) is given by

$$
\left[\begin{array}{l}
\dot{x}_{1} \\
\dot{x}_{2}
\end{array}\right]=\left[\begin{array}{cc}
0 & 1 \\
0 & -p_{1}
\end{array}\right]\left[\begin{array}{l}
x_{1} \\
x_{2}
\end{array}\right]+\left[\begin{array}{c}
0 \\
k_{1}
\end{array}\right] u\left(t-t_{d}\right) .
$$

Physically, $x_{1}$ represents the force at the robot's end effector and $x_{2}$ is the force derivative. Knowing (61), the AOB design described in Sections 5 and 6 can be applied in straightforward way.

\section{Stochastic Matrices}

For the first dimension $f_{x}$

$$
Q_{k}\left(f_{x}\right)=\left[\begin{array}{llll}
10^{-12} & \cdots & 0 & 0 \\
0 & \cdots & 0 & 0 \\
\vdots & \ddots & \vdots & \vdots \\
0 & \cdots & 10^{-12} & 0 \\
0 & \cdots & 0 & 10^{-5}
\end{array}\right],
$$

and

$$
P_{0}\left(f_{x}\right)=\left[\begin{array}{ccccc}
10^{-4} & 0 & \cdots & 0 & 0 \\
0 & 10^{-4} & \cdots & 0 & 0 \\
\vdots & \vdots & \ddots & \vdots & \vdots \\
0 & 0 & \cdots & 10^{-4} & 0 \\
0 & 0 & \cdots & 0 & 10^{-4}
\end{array}\right]
$$

The value of $R_{k}$ was obtained experimentally. It is given by

$$
R_{k}\left(f_{x}\right)=2.1 \times 10^{-2} .
$$


For the second dimension $f_{y}, Q_{k}$ is

$$
\begin{gathered}
Q_{k}\left(f_{y}\right)=Q_{k}\left(f_{x}\right) R_{k}\left(f_{y}\right) / R_{k}\left(f_{x}\right), \\
P_{0}\left(f_{y}\right)=P_{0}\left(f_{x}\right) R_{k}\left(f_{y}\right) / R_{k}\left(f_{x}\right),
\end{gathered}
$$

with

$$
R_{k}\left(f_{y}\right)=7.4 \times 10^{-4} .
$$

Analogous equations are obtained for the other force/torque dimensions.

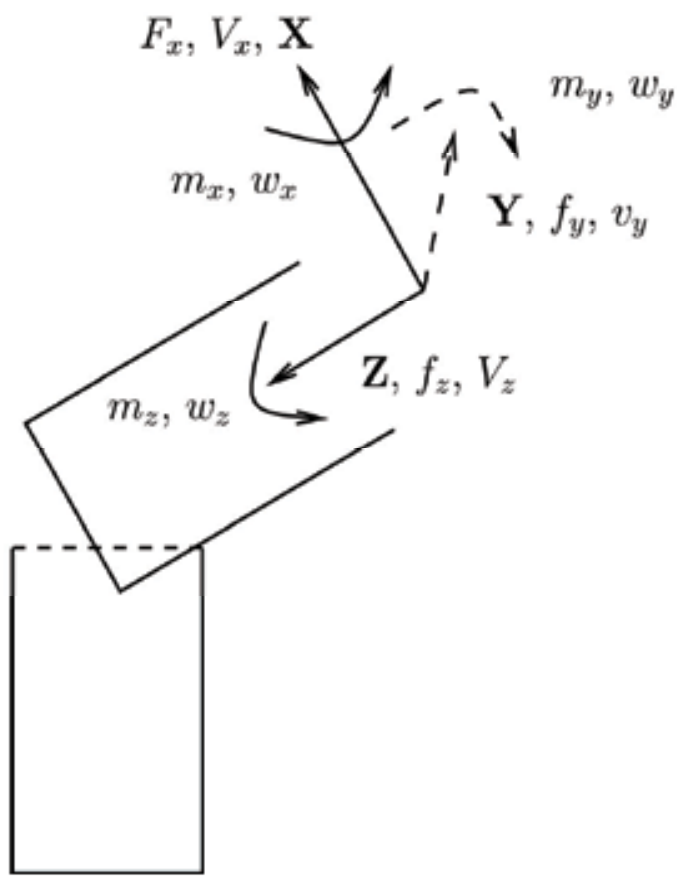

Figure 4. Peg-on-hole with a three point contact. Cartesian axes in peg coordinates.

Representation of the forces and velocities (6 DOF). $w_{y}$ represents the alignment velocity and $v_{z}$ the insertion velocity

\section{Control Matrices}

The state feedback gain $L_{r}$ is computed for a critically damped response with closed loop time constant $\tau_{r}$ equal to

$$
\tau_{c}=10 T_{p}
$$

The other poles due to dead-time are mapped in the $z$-domain at $z=0$ (discrete poles). The reachability matrix $W_{c}$ and the characteristic polynomial are described in the AOB algorithm (see Sections 5 and 6). 


\subsection{Experimental Results}

The execution of the peg-in-hole task starts with the peg-on-hole with a three point contact. The Cartesian axes and the corresponding forces and velocities are represented in Figure 6.4 in peg coordinates. If the peg is perfectly aligned, the angular velocity $w_{y}$ (negative sign) aligns the peg in vertical position, while $f_{x}$ (negative sign) and $f_{z}$ (positive sign) guarantee always a three point contact during the alignment phase. Then, only the velocity $v_{z}$ is needed to put the peg into the hole. Non-zero $m_{y}$ and $f_{x}$ reinforce contact with the hole inner surface during insertion. Ideally, the relevant signals for the alignment phase are $f_{x}, f_{z}$ and $w_{y}$, and for the insertion phase are $v_{z}, m_{y}$ and $f_{x}$. Figures 6.5 and 6.6 show force/velocity data $^{10}$ for the peg-in-hole task, applying different control strategies. An artificial neural network (ANN) trained with human data generates the desired compliant motion signals (velocity and force) from pose sense. Figures 5 and 6 present results when the AOB-1, HBA is applied to all directions except the $z$ direction that has the CKF, SBA. The moment $m_{y}$ is only function of the contact forces and of the contact geometry during the alignment phase [Koeppe, 2001]. Hence, the controller for $m_{y}$ is only active during the insertion. $f x, f y, m_{y}, m_{z \prime}$ and the corresponding velocities $v_{x}, v_{y}, w_{y}$ and $w_{z}$ follow well the reference signals (Figures 5.a, 5.b, 5.e, 5.f and Figures 6.a, 6.b, 6.e, 6.f, respectively). There is a slight misalignment in the peg during the alignment phase which originates a moment $m_{x}$ (Figure 5.d). The CMC reacts issuing an angular velocity $w_{x}$ represented in Figure 6.d. The insertion velocity $v_{z}$ is slightly biased during the alignment phase (Figure 6.c). These errors come from imperfect training of the ANN ([Cortesão et al., 2004]). Therefore, the force $f_{z}$ is seriously affected by $v_{z}$ errors. The stiffness in $\mathrm{z}$ direction is high (Table 1), magnifying even more feedforward errors. An overshoot of almost $20[\mathrm{~N}]$ is reached before insertion creating a barrier to the task execution speed due to force sensor limits (Figure 5.c). An error of $4[\mathrm{~N}]$ in $f_{z}$ (at the beginning of the alignment phase) is due to feedforward velocity errors (see Figures 5.c and 6.c.). Since no active control actions are done for the $z$ dimension, the degradation of the control performance is evident, stressing the importance of AOB actions.

\section{Conclusions}

The first part of the chapter discusses model-reference adaptive control in the framework of Kalman active observers (AOBs). The second part addresses experiments with an industrial robot. Compliant motion control with AOBs is analyzed to perform the peg-in-hole task. The AOB design is based on: 1) A desired closed loop system, corresponding to the reference model. 2) An extra equation (active state) to estimate an equivalent disturbance referred to the system input, providing a feedforward compensation action. The active state is modeled as an auto-regressive process of order $N$ with fixed parameters (evolutions) driven by a Gaussian random source. 3) The stochastic design of the Kalman matrices $\left(Q_{k}\right.$ and $R_{k}$ ) for the $\mathrm{AOB}$ context. $Q_{k}$ has a well defined structure. Most of the modeling errors are merged in the active state, therefore, the model for the other states is very accurate (the reference model has low uncertainty). The relation between $Q_{k}$ and $R_{k}$ defines the estimation strategy, making the state estimates more or less sensitive to measures.

10 The measured force/velocity signals were filtered off-line by a 6th order low-pass Butterworth filter with a $2[\mathrm{~Hz}]$ cut-off frequency. The phase distortion was compensated. 
Experiments with the peg-in-hole with tight clearance have shown the AOB importance in the control strategy. When there are relevant inconsistencies between force and velocity signals, non-active control techniques give poor results. For complex compliant motion tasks, on-line switching between AOB and classical Kalman filter (CKF) is easy, since the $\mathrm{CKF}$ is a particular form of the $\mathrm{AOB}$ which does not require extra computational power.

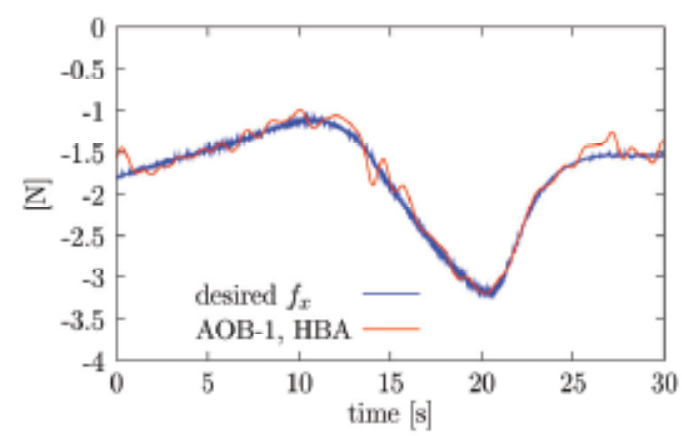

(a)

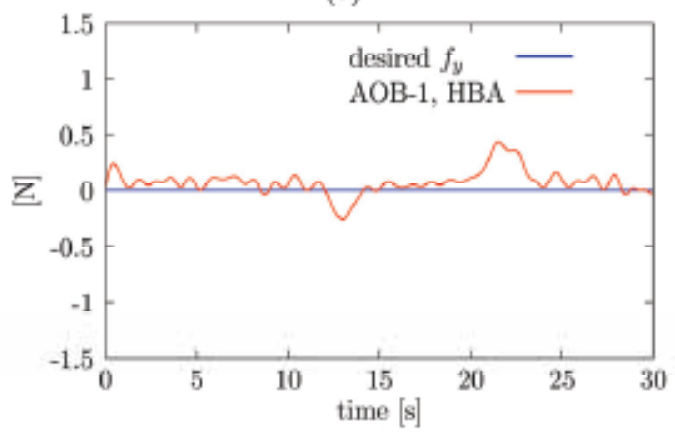

(b)

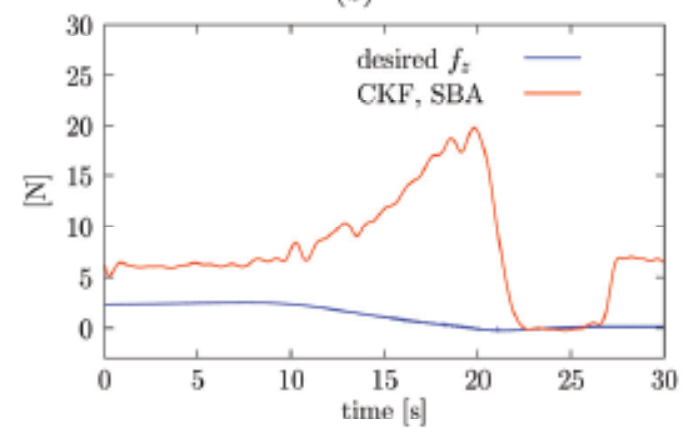

(c)

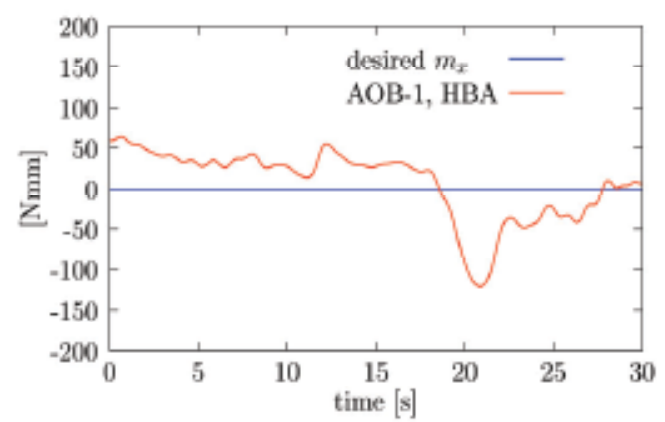

(d)

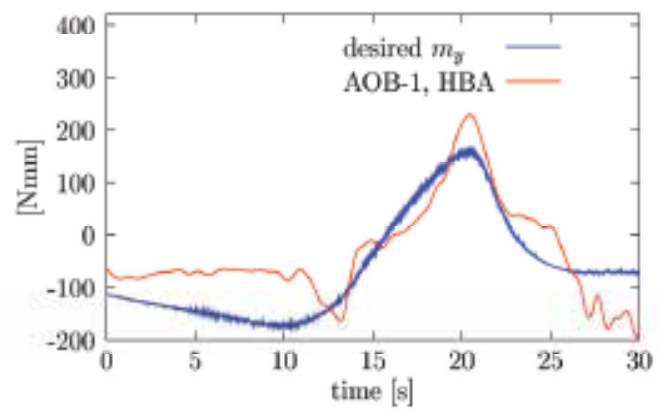

(e)

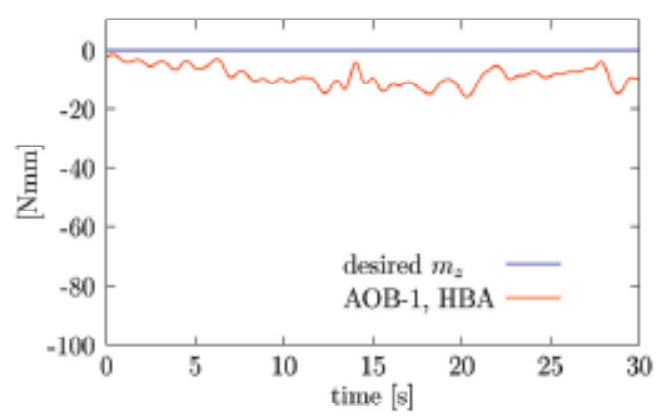

(f)

Figure 5. Experimental results for the peg-in-hole task with scale 10 (i.e. ten times slower than human demonstration). AOB-1, HBA vs. CKF, SBA. Force data. $f_{z}$ always with CKF, SBA 


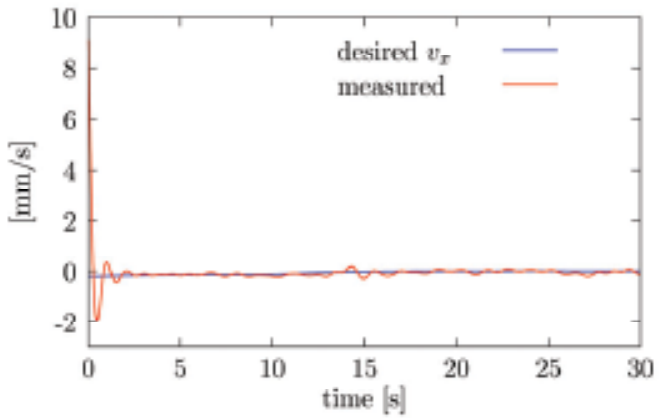

(a)

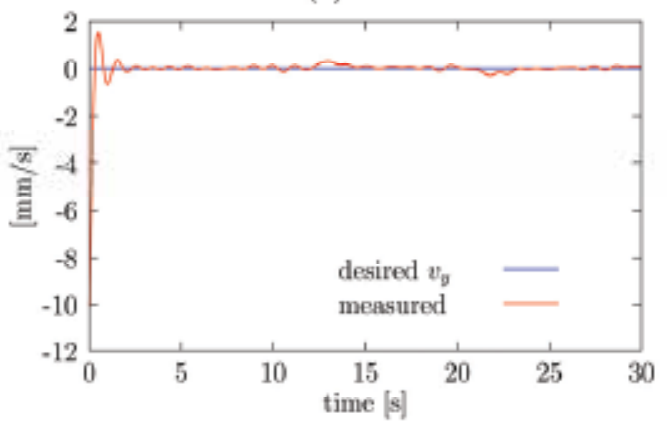

(b)

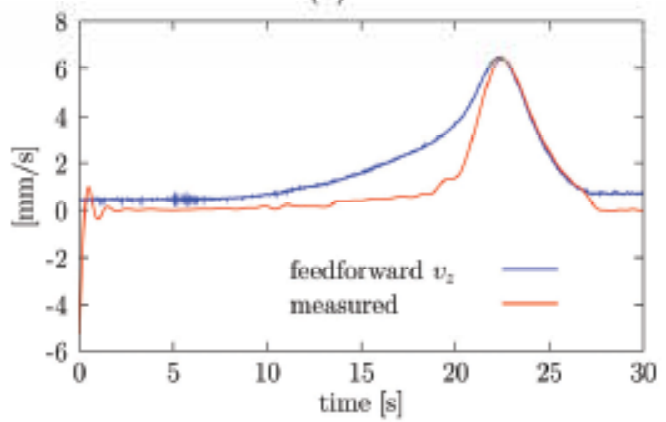

(c)

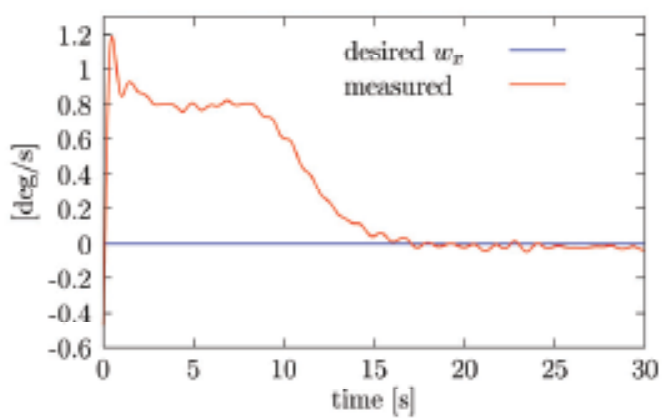

(d)

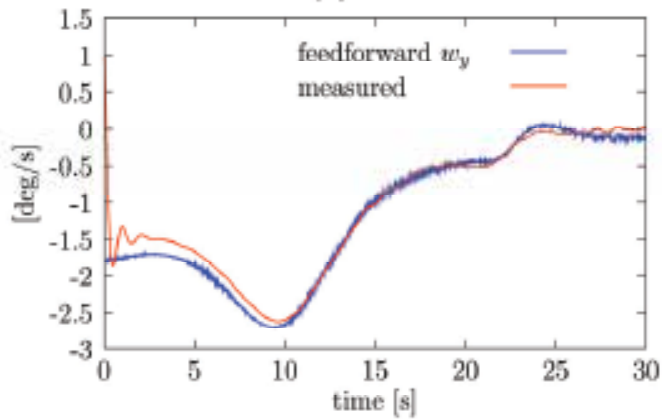

(e)

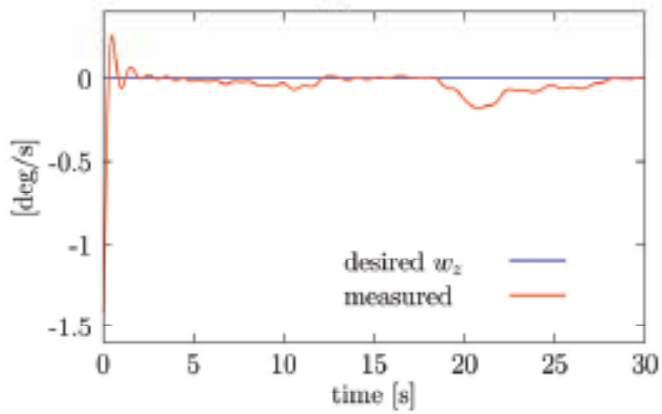

(f)

Figure 6. Experimental results for the peg-in-hole task with scale 10. AOB-1, HBA vs. CKF, SBA. Velocity data. $f_{z}$ always with CKF, SBA

\section{Acknowledgments}

This work was supported in part by the Portuguese Science and Technology Foundation (FCT) Project PTDC/EEA-ACR/72253/2006.

\section{References}

Astrom, K. J. \& Wittenmark, B. (1997). Computer Controlled Systems: Theory and Design. Prentice Hall. [Astrom \& Wittenmark, 1997] 
Bajcinca, N., Cortesão, R. \& Hauschild, M. (2005). Autonomous Robots 19, 193-214. [Bajcinca et al., 2005]

Bozic, S. M. (1979). Digital and Kalman Filtering. Edward Arnold, London. [Bozic, 1979]

Broenink, J. \& Tiernego, M. (1996). In Proc. of the European Simulation Symposium pp. 504509, Italy. [Broenink \& Tiernego, 1996]

Chen, B., Lin, Z. \& Liu, K. (2002). Automatica 38, 293-299. [Chen et al., 2002]

Chen, X., Komada, S. \& Fukuda, T. (2000). IEEE Transactions on Industrial Electronics 47, 429435. [Chen et al., 2000]

Chu, D. \& Mehrmann, V. (2000). SIAM J. on Control and Optimization 38, 1830-1858. [Chu \& Mehrmann, 2000]

Coelho, P. \& Nunes, U. (2005). IEEE Transactions on Robotics 21, 252-261. [Coelho \& Nunes, 2005]

Commault, C., Dion, J. \& Hovelaque, V. (1997). Automatica 33, 403-409. [Commault et al., 1997]

Cortesão, R. (2007). Int. J. of Intelligent and Robotic Systems 48, 131-155. [Cortesão, 2007]

Cortesão, R. \& Bajcinca, N. (2004). In Proc. of the Int. Conf. on Intelligent Robots and Systems (IROS) pp. 1148-1153, Japan. [Cortesão \& Bajcinca, 2004]

Cortesão, R., Koeppe, R., Nunes, U. \& Hirzinger, G. (2000). Int. J. of Machine Intelligence and Robotic Control (MIROC). Special Issue on Force Control of Advanced Robotic Systems 2, 59-68. [Cortesão et al., 2000]

Cortesão, R., Koeppe, R., Nunes, U. \& Hirzinger, G. (2001). In Proc. of the Int. Conf. on Intelligent Robots and Systems (IROS) pp. 1876-1881, USA. [Cortesão et al., 2001]

Cortesão, R., Koeppe, R., Nunes, U. \& Hirzinger, G. (2004). IEEE Transactions on Robotics 20, 941-952. [Cortesão et al., 2004]

Cortesão, R., Park, J. \& Khatib, O. (2006). IEEE Transactions on Robotics 22, 987-999. [Cortesão et al., 2006]

Doyle, J. (1978). IEEE Transactions on Automatic Control 23, 756-757. [Doyle, 1978]

Doyle, J. \& Stein, G. (1979). IEEE Transactions on Automatic Control 24, 607-611. [Doyle \& Stein, 1979]

Estrada, M. \& Malabre, M. (1999). IEEE Transactions on Automatic Control 44, 1311-1315. [Estrada \& Malabre, 1999]

Itabashi, K., Hirana, K., Suzuki, T., Okuma, S. \& Fujiwara, F. (1998). In Proc. of the Int. Conf. on Robotics and Automation (ICRA) pp. 1142-1147, Belgium. [Itabashi et al., 1998]

Kim, B., Yi, B., Suh, I. \& Oh, S. (2000). In Proc. of the Int. Conf. on Intelligent Robots and Systems (IROS) pp. 1229-1236, Japan. [Kim et al., 2000]

Koeppe, R. (2001). Robot Compliant Motion Based on Human Skill. PhD thesis, ETH Zurich. [Koeppe, 2001]

Maia, R., Cortesão, R., Nunes, U., Silva, V. \& Fonseca, F. (2003). In Proc. of the Int. Conf. on Advanced Robotics pp. 876-882,, Portugal. [Maia et al., 2003]

Morel, G., Malis, E. \& Boudet, S. (1998). In Proc. of the Int. Conf. on Robotics and Automation (ICRA) pp. 1743-1748,, Belgium. [Morel et al., 1998]

Newman, W., Zhao, Y. \& Pao, Y. (2001). In Proc. of the Int. Conf. on Robotics and Automation (ICRA) pp. 571-576,, Belgium. [Newman et al., 2001]

Oda, N. (2001). J. of Robotics and Mechatronics 13, 464-471. [Oda, 2001]

Park, J., Cortesão, R. \& Khatib, O. (2004). In Proc. of the IEEE Int. Conf. on Robotics and Automation, (ICRA) pp. 4789-4794,, USA. [Park et al., 2004] 
Park, J. \& Khatib, O. (2005). In Proc. of the Int. Conf. on Robotics and Automation (ICRA) pp. 3624-3629, Spain. [Park \& Khatib, 2005]

Petersen, I., James, M. \& Dupuis, P. (2000). IEEE Transactions on Automatic Control 45, 398412. [Petersen et al., 2000]

Safanov, M. \& Athans, M. (1977). IEEE Transactions on Automatic Control 22, 173-179. [Safanov \& Athans, 1977]

Schutter, J. D. (1988). In Proc. of the Int. Conf. on Robotics and Automation (ICRA) pp. 14971502, USA. [Schutter, 1988]

Yoshimi, B. \& Allen, P. (1994). In Proc. of the Int. Conf. on Robotics and Automation (ICRA) vol. 4, pp. 156-161, USA. [Yoshimi \& Allen, 1994] 


\title{
Triggering Adaptive Automation in Naval Command and Control
}

\author{
Tjerk de Greef 1,2 and Henryk Arciszewski ${ }^{2}$ \\ ${ }^{1}$ Delft University of Technology, ${ }^{2}$ TNO Defence, Security and Safety \\ The Netherlands
}

\section{Introduction}

In many control domains (plant control, air traffic control, military command and control) humans are assisted by computer systems during their assessment of the situation and their subsequent decision making. As computer power increases and novel algorithms are being developed, machines move slowly towards capabilities similar to humans, leading in turn to an increased level of control being delegated to them. This technological push has led to innovative but at the same time complex systems enabling humans to work more efficiently and/or effectively. However, in these complex and information-rich environments, task demands can still exceed the cognitive resources of humans, leading to a state of overload due to fluctuations in tasks and the environment. Such a state is characterized by excessive demands on human cognitive capabilities resulting in lowered efficiency, effectiveness, and/or satisfaction. More specifically, we focus on the human-machine adaptive process that attempts to cope with varying task and environmental demands.

In the research field of adaptive control an adaptive controller is a controller with adjustable parameters and a mechanism for adjusting the parameters (Astrom \& Wittenmark, 1994, p. 1) as the parameters of the system being controlled are slowly time-varying or uncertain. The classic example concerns an airplane where the mass decreases slowly during flight as fuel is being consumed. More specifically, the controller being adjusted is the process that regulates the fuel intake resulting in thrust as output. The parameters of this process are adjusted as the airplane mass decreases resulting in less fuel being injected to yield the same speed.

In a similar fashion a human-machine ensemble can be considered an adaptive controller. In this case, human cognition is a slowly time-varying parameter, the adjustable parameters are the task sets that can be varied between human and machine, and the control mechanism is an algorithm that 'has insight' in the workload of the human operator (i.e., an algoritm that monitors human workload). Human performance is reasonably optimal when the human has a workload that falls within certain margins; severe performance reductions result from a workload that is either too high or (maybe surprisingly) too low. Consider a situation where the human-machine ensemble works in cooperation in order to control a process or situation. Both the human and the machine cycle through an information processing loop, collecting data, interpreting the situation, deciding on actions to achieve one or more stated goals and acting on the decisions (see for example Coram, 2002; 
Parasuraman et al., 2000). If the human is getting overloaded, the control mechanisms should adjust the parameters that regulate the balance of work between human and machine and work should be reallocated to the machine in order to lower the cognitive burden of the human and optimize the performance of the human machine ensemble. Of course we must be able to automate some or all of the loop so that work can indeed be delegated to the machine. And humans must be willing to delegate the responsibility as well. The process of reallocation of the workload between man and machine is referred to as adaptive automation.

Adaptive automation is based on the idea of supporting the human only at those moments when its performance is in jeopardy. W. B. Rouse (1988) introduced adaptive aiding as an initial type of adaptive automation. Rouse stated that adaptive aiding is a human-machine system-design concept that involves using aiding/automation only at those points in time when human performance needs support to meet operational requirements (Rouse, 1988, p. 431). Whether one uses the terms adaptive automation, dynamic task allocation, dynamic function allocation, or adaptive aiding, they all reflect the dynamic reallocation of work in order to improve human performance or to prevent performance degradation. As a matter of fact, adaptive automation should scale itself down when things become quieter again and the goal of adaptive automation could be stated as trying to keep the human occupied within a band of 'proper' workload (see Endsley \& Kiris, 1995). Periods of 'underload' can have equally disastrous consequences as periods of overload due to slipping of attention and loss of situational awareness. A number of studies have shown that the application of adaptive automation enhances performance, reduces workload, improves situational awareness, and maintains skills that are deteriorating as a consequence of too highly automated systems (Bailey et al., 2006; Hilburn et al., 1997; Inagaki, 2000a; Kaber \& Endsley, 2004; Moray et al., 2000; Parasuraman et al., 1996; Scallen et al., 1995).

One of the challenging factors in the development of successful adaptive automation concerns the question of when changes in the level of automation must be effectuated. The literature repository utilizes the idea of 'the workload being too high or too low' as a reason to trigger the reallocation of work between the human and the machine. At the same time it acknowledges the fact that it remains difficult to give the concept a concrete form. We simply state that workload measurements of some sort are required in order to optimize the human-machine performance. Performance measurements are one way to operationalize such workload measurements and the next section discusses the various strategies in detail.

\section{Previous Work}

The success of the application of adaptive automation depends in part on the quality of the automation and the support it offers to the human. The other part constitute when changes in the level of automation are effectuated. 'Workload' generally is the key concept to invoke such a change of authority. Most researchers, however, have come to the conclusion that workload is a multidimensional, multifaceted concept that is difficult to define. It is generally agreed that attempts to measure workload relying on a single representative measure are unlikely to be of use (Gopher \& Donchin, 1986). The definition of workload as an intervening variable similar to attention that modulates or indexes the tuning between the demands of the environment and the capacity of the operator (Kantowitz, 1987) seems to capture the two main aspects of workload, i.e., the capacity of humans and the task demands made on them. The workload increases when the capacity decreases or the task demands increase. Both capacity and task demands 
are not fixed entities and both are affected by many factors. Skill and training, for example, are two factors that increase capacity in the long run whereas capacity decreases when humans become fatigued or have to work under extreme working conditions for a prolonged period.

If measuring workload directly is not a feasible way to trigger the adaptive automation mechanism, other ways must be found. Wilson and Russell (2007) define five strategies based on a division by Parasuraman et al (1996). They state that triggers can be based on critical events, operator performance, operator physiology, models of operator cognition, and hybrid models that combine the other four techniques. The workload perceived by the human himself or by a colleague may lead to an adaptation as well, although in such a case some papers refrain from the term adaptive automation and utilize 'adaptable automation,' as the authority shift is not instigated by the automated component. Against the first option (operator indicates a workload that is too high or too low that in turn results in work adjustments) counts the fact that he or she is already over or underloaded and the additional switching task would very likely be neglected. The second option therefore seems more feasible, but likely involves independent measurements of workload to support the supervisor's view, leading to a combination of the supervision method and other methods.

The occurrence of critical events can be used to change to a new level of automation. Critical events are defined as incidents that could endanger the goals of the mission. Scerbo (1996) describes a model where the system continuously monitors the situation for the appearance of critical events and the occurrence of such an event triggers the reallocation of tasks. Inagaki has published a number of theoretical models (Inagaki, 2000a; Inagaki, 2000b) where a probabilistic model was used to decide who should have authority in the case of a critical event.

A decline in operator performance is widely regarded as a potential trigger. Such an approach measures the performance of the human over time and regards the degradation of the performance as an indication of a high workload. Many experimental studies derive operator performance from performance measurements of a secondary task (Clamann et al., 2002; Kaber et al., 2006; Kaber \& Riley, 1999; Kaber et al., 2005). Although this approach works well in laboratory settings, the addition of an artificial task to measure performance in a real-world setting is unfeasible so extracting performance measures from the execution of the primary task seems the only way to go.

Physiological data from the human are employed in various studies (Bailey et al., 2006; Byrne \& Parasuraman, 1996; Prinzel et al., 2000; Veltman \& Gaillard, 1998; Wilson \& Russell, 2007). The capability of human beings to adapt to variable conditions, however, may distort accurate measurements (Veltman \& Jansen, 2004). There are two reasons why physiological measures are difficult to use in isolation. First of all, the human body responds to an increased workload in a reactive way. Physiological measurements therefore provide the system with a delayed cognitive workload state of the operator instead of the desired realtime measure. Second, it is possible that physiological data indicate high workload but that these not necessarily commensurate with poor performance. This is the case when operators put in extra effort to compensate for increases in task demands. At least several measurements (physiological or otherwise) are required to get rid of such ambiguities.

The fourth approach uses models of operator cognition. These models are approximations of human cognitive processes for the purpose of prediction or comprehension of human operator state and workload. The winCrew tool (Archer \& Lockett, 1997), for example, 
implements the multiple resource theory (Wickens, 1984) to evaluate function allocation strategies by quantifying the moment-to-moment workload values. Alternatively, the human's interactions with the machine can be monitored and evaluated against a model to determine when to change levels of automation. In a similar approach, Geddes (1985) and Rouse, Geddes, and Curry (1987) base adaptive automation on the human's intentions as predicted from patterns of activity.

The fifth approach follows Gopher and Donchin (1986) in that a single method to measure workload is too limited. Hybrid models therefore combine a number of triggering techniques because the combination is more robust against the ambiguities of each single model.

Each of the five described approaches has been applied more or less successfully in an experimental setting, especially models that consider the effects of (neuro)physiological triggers and critical events. Limited research is dedicated to applying a hybrid model that integrates operator performance models and models of operator cognition. We have based our trigger model on precisely such a combination because we feel our approach to adaptive automation using an object-oriented model (de Greef \& Arciszewski, 2007) offers good opportunities for an operational implementation. The cognitive model we use is based in turn on the cognitive task load (CTL) model of Neerincx (2003). In addition, we provide a separate mechanism for critical events.

\section{Naval Command and Control}

As our implementation domain concerns naval command and control (C2), we begin our discussion with a brief introduction to this subject. Specifically, command and control is characterized as focusing the efforts of a number of entities (individuals and organizations) and resources, including information, toward the achievement of some task, objective, or goal (Alberts \& Hayes, 2006, p. 50). These activities are characterized by efforts to understand the situation and subsequently acting upon this understanding to redirect it toward the intended one. A combat management system (CMS) supports the team in the command center of a naval vessel with these tasks. Among other things this amounts to the continuous execution of the stages of information processing (data collection, interpretation, decision making, and action) in the naval tactical domain and involves a number of tasks like correlation, classification, identification, threat assessment, and engagement. Correlation is the process whereby different sensor readings are integrated over time to generate a track. The term track denotes the representation of an external platform within the CMS, including its attributes and properties, rather than its mere trajectory. Classification is the process of determining the type of platform of a track and the identification process attempts to determine its identity in terms of it being friendly, neutral, or hostile. The threat assessment task recognizes entities that pose a threat toward the commanded situation. In other words, the threat assessment task assesses the danger a track represents to the own ship or other friendly ships or platforms. One should realize that hostile tracks do not necessarily imply a direct threat. The engagement task includes the decision to apply various levels of force to neutralize a threat and the execution of this decision. Because the identification process uses information about such things as height, speed, maneuvering, adherence to an air or sealane, and military formations, there is a continuous need to monitor all tracks with respect to such aspects. Therefore monitoring is also part of the duties of a command team. See Figure 1 for an overview of $\mathrm{C} 2$ tasks in relation to a track. 


\section{The Object-oriented framework}

Before describing triggering in an object-oriented framework, we summarize our previous work (Arciszewski et al., in press).

\subsection{Object-Oriented Work Allocation}

We have found it fruitful to focus on objects rather than tasks in order to distribute work among actors (compare Bolderheij, 2007, pp. 47-48). Once we have focused our attention on objects, tasks return as the processes related to the objects (compare Figure 1). For example, some of the tasks that can be associated with the all-evasive 'track' object in the C2 domain are classification, the assignment of an identity, and continuous behavioral monitoring (compare Figure 1). The major advantage of the object focus in task decomposition is that it is both very easy to formalize and comprehensible by the domain users. Partitioning work using tasks only has proven difficult. If we consider identification, for example, this task is performed for each object (track) in turn.

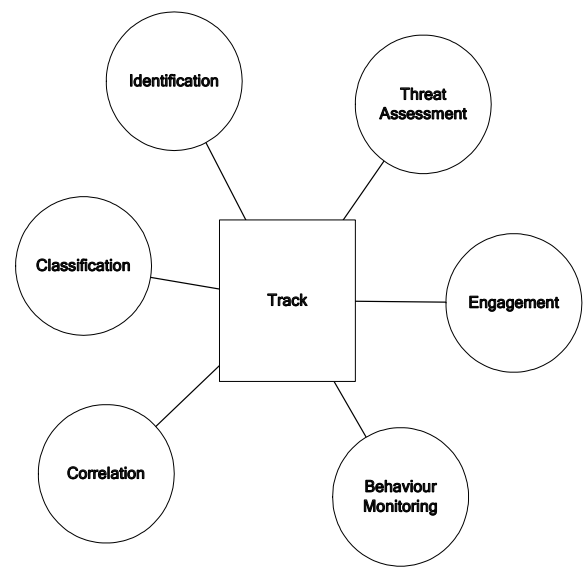

Figure 1. Some of the more important tasks a command crew executes in relation to a track

\subsection{Concurrent Execution and Separate Work Spaces}

Instead of letting a task be performed either by the human or the machine, we let both parties do their job concurrently. In this way both human and machine arrive at their own interpretation of the situation, building their respective world-views (compare Figure 2).

One important result of this arrangement is the fact that the machine always calculates its view, independent of whether the human is dealing with the same problem or not. To allow this, we have to make provisions for 'storage space' where the two parties can deposit the information pertaining to their individual view of the world. Thus we arrive at two separate data spaces where the results of their computational and cognitive efforts can be stored. This has several advantages. Because the machine view is always present, advice can be readily looked up. Furthermore, discrepancies between the two world views can lead to warnings from the machine to the human that the latter's situational awareness may no longer be up to date and that a reevaluation is advisable. Assigning more responsibility to the machine, in practice comes down to the use of machine data in situation assessment, decision making, and acting without further intervention from the human. 


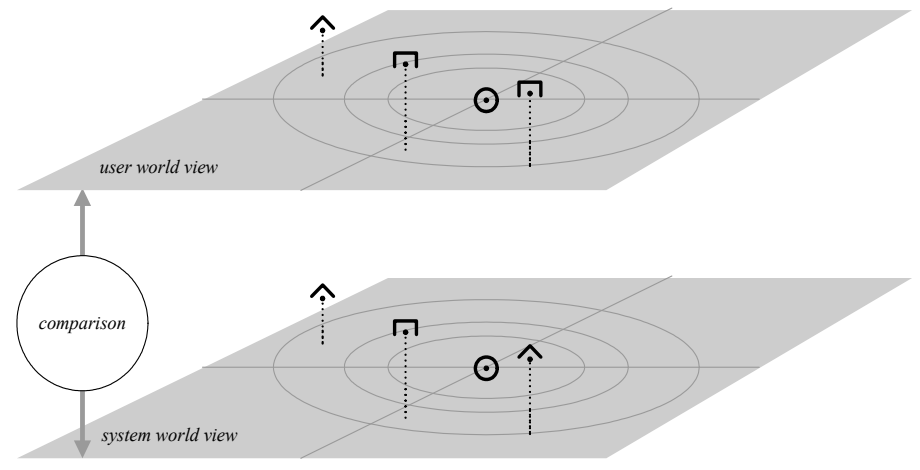

Figure 2. The two different world views and a comparison of them by the system. A difference between the interpretation of the two worlds could lead to an alert of the human

\subsection{Levels of Automation}

Proceeding from the machine and human view, levels of automation (LoA) more or less follow automatically. Because the machine view is always available, advice is only a key press or mouse click away. This readily available opinion represents our lowest LoA (ADVICE). At the next higher LoA, the machine compares both views and signals any discrepancies to the human, thus alerting the user to possible gaps or errors in his situational picture. This signalling functionality represents our second LoA (CONSENT). At the higher levels of automation we grant the machine more authority. At our highest LoA (SYSTEM), the machine entirely takes over the responsibility of the human for certain tasks. At the lower LoA (VETO), the machine has the same responsibility, but alerts the human to its actions, thus allowing the latter to intervene.

Adaptive automation now becomes adjusting the balance of tracks for each task between the human and the machine. By decreasing the number of tracks under control of the human, the workload of the human can be reduced. Increasing the number of tracks managed by the human on the other hand results in a higher workload.

\section{Global and local adaptation}

Having outlined an architectural framework for our work, we now focus on the problem of triggering. We envision two clearly different types of adaptation. The distinction between the two types can be interpreted as that between local and global aiding (de Greef \& Lafeber, 2007, pp. 68-69). Global aiding is aimed at the relief of the human from a temporary overload situation by taking over parts of the work. If on the other hand the human misses a specific case that requires immediate attention in order to maintain safety, local aiding comes to the rescue. In both cases work is shifted from the human to the machine, but during global aiding this is done in order to avoid the overwhelming of the human, whereas local aiding offers support in those cases the human misses things. As indicated before, global aiding should step back when things become quiet again in order to keep the human within a band of 'proper' workload (see Endsley \& Kiris, 1995). On the other hand, a human is not overloaded in cases where local adaptation is necessary; he or she may be just missing those 
particular instances or be postponing a decision with potentially far-reaching consequences. A further distinction is that local aiding concerns itself with a specific task or object whereas global aiding takes away from the operator that work that is least detrimental to his or her situational awareness. According to this line of reasoning a local case ought to be an exception and the resulting actions can be regarded as a safety net. The safety net can be realized in the form of separate processes that check safety criteria. In an ideal world, global adaptation would ensure that local adaptation is never necessary because the human always has enough cognitive resources to handle problems. But things are not always detected in time and humans are sometimes distracted or locked up so that safety nets remain necessary.

\section{Triggering local aiding}

Local aiding is characterized by a minimum time left for an action required to maintain safety and be able to achieve the mission goals. Activation of such processing is through triggers that are similar to the critical events defined by Scerbo (1996). The triggers are indicators of the fact that a certain predefined event that endangers mission goals is imminent and that action is required shortly. In the case of naval C2 a critical event is usually due to a predefined moment in the (timeline of the) state of an external entity and hence it is predictable to some extent.

Typically, local aiding occurs in situations where either the human misses something due to a distraction by another non-related event or entity, to tunnel vision, or to the fact that the entity has so far been unobserved or been judged to be inconsequential.

In the naval command and control domain, time left as a way to initiate a local aiding trigger can usually be translated to range from the ship or unit to be protected. In most cases therefore triggers can be derived from the crossing of some critical boundary. Examples are (hostile) missiles that have not been engaged by the crew at a certain distance or tracks that are not yet identified at a critical range called the identification safety range (ISR). The ship's weapon envelopes define a number of critical ranges as well. It is especially the minimum range, within which the weapon is no longer usable, that can be earmarked as a critical one.

\section{Triggering global aiding}

One of the advantages of the object-oriented framework outlined in section 4 is that it offers a number of hooks for the global adaptation approach. The first hook is the difference between human world-view and machine world-view (see sect. 4.2).

The second hook is based on the number and the character of the objects present and is utilized for estimating the workload imposed on the human by the environment. In the case of military $\mathrm{C} 2$ the total number of tracks provides an indication of the volume of information processing whereas the character of the tracks provides an indication of the complexity of the situation. These environmental items therefore form the basis for our cognitive model.

\subsection{The Operator Performance Model}

Performance is usually defined in terms of the success of some action, task, or operation. Although many experimental studies define performance in terms of the ultimate goal, real world settings are more ambiguous and lack an objective view of the situation (the 'ground truth') that could define whether an action, task, or operator is successful or not. Defining 
performance in terms of reaction times is another popular means although some studies found limited value in utilizing performance measures as a single way to trigger adaptive automation. This has been our experience as well (de Greef \& Arciszewski, 2007).

As explained in section 4.2, the object-oriented framework includes the principle of separate workspaces for man and machine. This entails that both the machine and the human construct their view of the world and store it in the system. For every object (i.e., track) a comparison between the two world views can then be made and significant differences can be brought to the attention of the human. This usually means that new information has become available that requires a reassessment of the situation as there is a significant chance that the human's world view has grown stale and his or her expectations may no longer be valid. We use these significant differences in two ways to model performance.

First, an increase in the number of differences between the human world view and the machine world view is viewed as a performance decrease. Although differences will inevitably occur, as the human and the machine do not necessarily agree, an increasing skew between the two views is an indication that the human has problems with his or her workload. Previous work suggested that the subjective workload fluctuated in proportion to the density of signals resulting from skew differences (van Delft \& Arciszeski, 2004). The average reaction time to these signals is used as a second measure of performance. Utilizing either skew or reaction times as the only trigger mechanism is problematic because of the sparseness of data due to the small number of significant events per time unit in combination with a wide spread of reaction times (de Greef \& Arciszewski, 2007). The combined use of skew and reaction times provides more evidence in terms of human cognitive workload. This in turn is enhanced by the operator cognitive model discussed below.

\subsection{The Operator Cognition Model}

While the operator performance model is aimed to get a better understanding of the human response to the situation, the operator cognition model aims at estimating the cognitive task load the environment exerts on the human operator. The expected cognitive task load is based on Neerincx's (2003) cognitive task load (CTL) model and is comprised of three factors that have a substantial effect on the cognitive task load.

The first factor, percentage time occupied (TO), has been used to assess workload for timeline assessments. Such assessments are based on the notion that people should not be occupied more than 70 to 80 percent of the total time available. The second load factor is the level of information processing (LIP). To address cognitive task demands, the cognitive load model incorporates the skill-rule-knowledge framework of Rasmussen (1986) where the knowledge-based component involves the highest workload. To address the demands of attention shifts, the model distinguishes task-set switching (TSS) as a third load factor. It represents the fact that a human operator requires time and effort to reorient himself to a different context. These factors present a three-dimensional space in which all human activities can be projected as a combined factor (i.e., it displays the workload due to all activities combined). Specific regions indicate the cognitive demands activities impose on a human operator. Figure 3 displays the three CTL factors and a number of cognitive states. Applying Neerincx's CTL model leads to the notion that the cognitive task load is based on the volume of information processing (reflecting time occupied), the number of different objects and tasks (task set switching), and the complexity of the situation (level of information 
processing). As the volume of information processing is likely to be proportional to the number of objects (tracks) present, the TO factor will be proportional to the total number of objects.

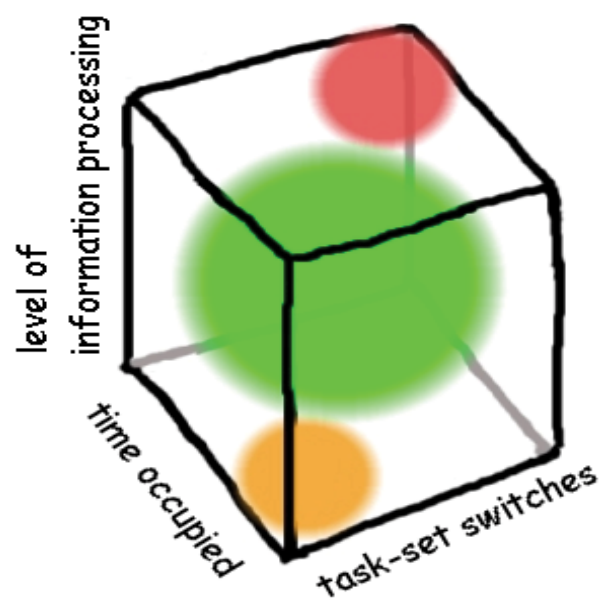

Figure 3. The three dimensions of Neerincx's (2003) cognitive task load model: time occupied, task-set switches, and level of information processing. Within the cognitive task load cube several regions can be distinguished: an area with an optimal workload displayed in the center, an overload area displayed in top vertex, and an underload area displayed in the lower vertex

The second CTL factor is the task set switching factor. We recognize two different types of task set switching, each having a different effect size $C_{x}$. The human operator can change between tasks or objects (tracks). The first switch relates to the attention shift that occurs as a consequence of switching tasks, for example from the classification task to the engagement task. The second type of TSS deals with the required attention shift as a result of switching from object to object. The latter type of task switch is probably cognitively less demanding because it is associated with changing between objects in the same task and every object has similar attributes, each requiring similar information-processing capabilities.

Finally, a command and control context can be expressed in terms of complexity (i.e., LIP). The LIP of an information element in C2, a track, depends mainly on the identity of the track. For example, 'unknown' tracks result in an increase in complexity since the human operator has to put cognitive effort in the process of ascertaining the identity of tracks of which relatively little is known. The cognitive burden will be less for tracks that are friendly or neutral.

The unknown, suspect, and hostile tracks require the most cognitive effort for various reasons. The unknown tracks require a lot of attention because little is known about them and the operator will have to ponder them more often. On the other hand, hostile tracks require considerable cognitive effort because their intent and inherent danger must be decided. Especially in current-day operations, tracks that are labeled hostile do not necessarily attack and neutralization might only be required in rare cases of clear hostile intent. Suspect tracks are somewhere between hostile and unknown identities, involving too little information to definitely identify them and requiring continuous threat assessment as well. We therefore conclude a relationship between the LIP, an effect size $C$, and the numbers of hostile, 
suspect, and unknown tracks and the other categories where the effect is larger for the hostile, suspect, and unknown tracks.

\subsection{The hybrid cognitive task load model}

The operator performance model describes a relation between performance and 1) average response time and 2) skew between the human view and the machine view of the situation. A decrease in performance, in its turn, is the result of a task load that is too high (see de Greef \& Arciszewski, 2007).

In the second place, the model of operator cognition describes a relation between the environment and the cognitive task load in terms of the three CTL factors. We therefore define a relation between cognitive task load and the number of tracks $\left(N_{T}\right)$ the number of objects $\left(N_{O}\right)$, and the number of difficult tracks $\left(N_{U, S, H}\right)$.

In all cases, a further investigation into the relation between the cognitive task load indicators and the performance measurements is worthwhile. We expect that a change in one of the workload indicators $N_{T}, N_{O}, N_{U, S, H}$ results in a change in cognitive load, leading in turn to a (possibly delayed) change in performance and hence a change in a performance measurement.

\section{Experiment I}

In order to see whether the proposed model of operator cognition is a true descriptor for cognitive workload we looked at data from an experiment. This experiment investigated the relation between the object-oriented approach and cognitive task load. More specifically, this experiment attempted to answer the question whether CTL factors properly predict or describe changes in cognitive workload.

\subsection{Apparatus \& Procedure}

The subjects were given the role of human operators of (an abstracted version of) a combat management workstation aboard naval vessels. The workstation comprised a schematic visual overview of the nearby area of the ship on a computer display, constructed from the data of radar systems. On the workstation the subject could manage all the actions required to achieve mission goals. Before the experiment, the subjects were given a clear description of the various tasks to be executed during the scenarios. Before every scenario, a description about the position of the naval ship and its mission was provided. The experiment was conducted in a closed room where the subjects were not disturbed during the task. During the experiment, an experimental leader was situated roughly two meters behind the subject to assist when necessary.

\subsection{Participants}

Eighteen subjects participated in the experiment and were paid EUR 40 to participate. The test subjects were all university students, with a good knowledge of English. The participant group consisted of ten men and eight women. They had an average age of 25, with a standard deviation of 5.1 . 


\subsection{Experimental tasks}

The goal of the human operator during the scenarios was to monitor, classify, and identify every track (i.e. airplanes and vessels) within a 38 nautical miles range around the ship. Furthermore, in case one of these tracks showed hostile intent (in this simplified case a dive toward the ship), they were mandated to protect the naval vessel and eliminate the track.

To achieve these goals, the subject was required to perform three tasks. First, the classification task gained knowledge of the type of the track and its properties using information from radar and communication with the track, air controller, and/or the coastguard. The subject could communicate with these entities using chat functionality in the CMS. The experimental leader responded to such communications. The second task was the identification process tat labeled a track as friendly, neutral, or hostile. The last task was weapon engagement in case of hostile intent as derived from certain behavior. The subject was required to follow a specific procedure to use the weapons.

\subsection{Scenarios}

There were three different scenarios, each implying a different cognitive task load. The task loads were under-load, normal load, and an overload achieved by manipulating two of the three CTL factors. First, the total number of tracks in a scenario was changed. If many tracks are in the observation range, the percentage of the total time that the human is occupied is high (see section 7.2). Second, a larger amount of tracks that show special behavior and more ambiguous properties increases the operator's workload. It forces the human operator to focus attention and to communicate more in order to complete the tasks.

We hypothesize that manipulation of these two items has an effect on the cognitive task load factors, similar to our model of operator cognition described in section 7.2. In summary:

- Time occupied: manipulated by the number of tracks in the range of the ship.

- $\quad$ Task set switches: likewise manipulated by number of tracks in the range.

- Level of information processing: manipulated by the behavior of the tracks.

Table 1 provides the values used per scenario. The scenarios were presented to the participants using a Latin square design to compensate for possible learning effects. The TO, TSS, and LIP changes were applied at the same time.

\begin{tabular}{lll}
\cline { 2 - 3 } & $\begin{array}{l}\text { Total number of track } \\
\text { within 38 nautical miles }\end{array}$ & $\begin{array}{l}\text { Track with hostile } \\
\text { behavior }\end{array}$ \\
\hline Under-load scenario & 9 & 1 \\
Normal workload scenario & 19 & 7 \\
Overload scenario & 34 & 16 \\
\hline
\end{tabular}

Table 1. Total number of tracks and the number of tracks with hostile behavior per scenario

\subsection{Results}

In order to verify whether the manipulated items affected the load factors and induced mental workload as expected, the subjects were asked to indicate their workload. Every 100 seconds subjects had to rate his or her perceived workload on a Likert scale (one to five). Level 1 indicated low workload, level 3 normal workload, and level 5 high workload. The levels in between indicate intermediate levels of workload. 


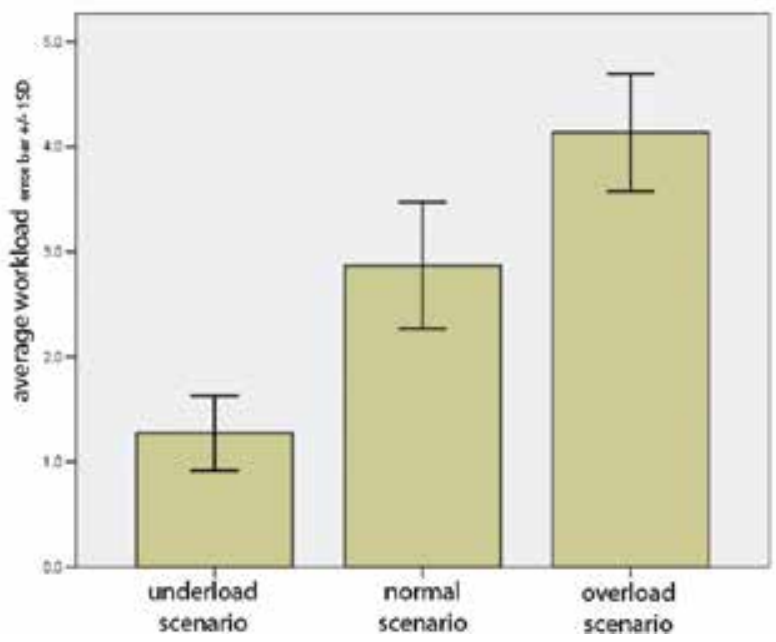

Figure 4. The subjective workload per scenario as indicated every 100 seconds on a five point Likert scale. Note: for the mental workload verification, $\mathrm{N}=17$ as the data of one subject was missing due to a failure in logging

Repeated-measures ANOVA reveals a significant effect in perceived cognitive task load between the three scenario's $(\mathrm{F}(2,32)=190.632, p<0.001$, see Figure 4$)$. Least square difference post-hoc analysis reveals that all three means were significantly different $(p<$ 0.05). Compared to the under-load scenario, the perceived mental workload was significantly higher in the normal workload scenario. In turn, the perceived mental workload in the overload scenario was significantly higher again than in the normalworkload scenario.

\subsection{Conclusion}

The data from the experiment reveal that manipulation of the CTL factors using numbers and types of domain objects has a significant effect on the subjective cognitive task load. We therefore conclude that the total number of tracks and the number of tracks with extraordinary behavior are good indicators of the difficulty the environment poses on a human operator. The data supports our model of operator cognition described in section 7.2

\section{Experiment II}

While experiment I studied the relation between the object-oriented approach and cognitive task load in a naïve setting, the second experiment investigated the performance model and the application of a hybrid cognitive task load model in a semi-realistic setting of naval operations during peace keeping and embargo missions.

Experiment II was in the first place designed to compare the efficiency and effectiveness between an adaptive and a non-adaptive mode of the CMS during high-workload situations. The results revealed a clear performance increase in the adaptive mode with no differentiation in subjective workload and trust (for a detailed review see de Greef et al., 2007). The triggers for the adaptive mode, mandated by the high-workload situations, were mainly based on performance measures and to a lesser extent on cognitive indicators. 
In spite of the different goal, the data of the non-adaptive subset of runs help investigating the claims with respect to the proposed hybrid model. In addition to the model of operator cognition, we hypothesize that the operator performance model is a predictor for workload in accordance with section 7.1 and 7.3. Experiment II therefore uses the non-adaptive subset of the data to investigate this aspect.

\subsection{Subjects, Tasks and Apparatus}

The subjects were four warfare officers and four warfare officer assistants of the Royal Netherlands Navy with several years of operational experience. All subjects were confronted with a workstation called the Basic-T (van Delft \& Schraagen, 2004) attached to a simulated combat management system. The Basic-T (see Figure 5) consists of four 19-inch touch screens arranged in a T-shaped layout driven by two heavy-duty PCs. The Basic-T functioned as an operational workstation in the command centre of a naval ship and was connected by means of a high-speed data bus to the simulated CMS running on an equally simulated naval vessel.

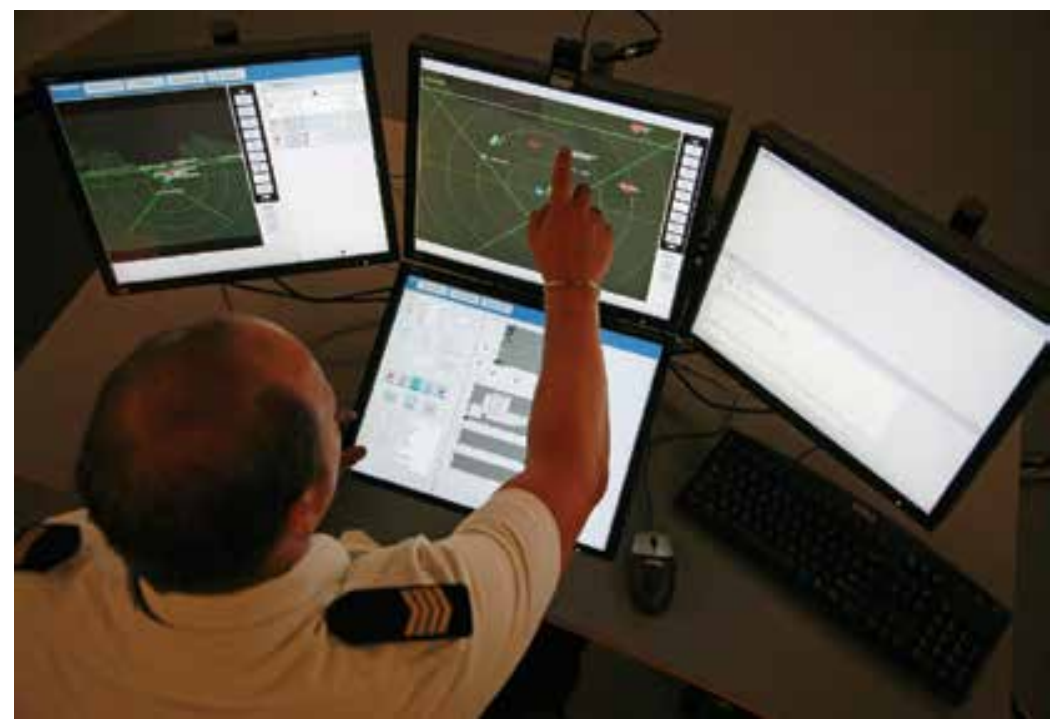

Figure 5. The Basic-T functions as a test bed for the design and evaluation of future combat management workstations

In all cases the primary mission goal for the subjects was to build a complete maritime picture of the surroundings of the ship and to defend the ship against potential threats. Building the maritime picture amounted to monitoring the operational space around the vessel and classifying and identifying contacts. The defense of the ship could entail neutralizing hostile entities. As the sensor reach of a modern naval ship extends to many miles around the ship, the mission represented a full-time job. In addition, the subjects were responsible for the short-term navigation of the ship, steering it toward whatever course was appropriate under the circumstances and had a helicopter at their disposal to investigate the surrounding surface area. Although the use of a helicopter greatly extended surveillance capabilities, it also made the task heavier because of the increased data volume and the direction and control of the helicopter. 
Each subject was offered an abstract top-down tactical view of the situation in order to support his or her situational awareness. The tactical display was amended by a second display that contained detailed information about the selected track (for example, flight profile, classification, and radar emissions). A chat console aided the subject to gather and distribute information. The subject could communicate with and assign a new task to the helicopter and contact external entities such as the coastguard and aircraft. One of the experimental leaders controlled the helicopter, generally executed commands to emulate onboard personnel (controlling the fire control radar, gunnery, etc.) and responded to the chat.

\subsection{Procedure}

The subjects participated in the experiment for two days. The first day was divided into two parts. In the first part of the day the participants were informed about the general goals of the experiment and the theoretical background of the research. The second part was used to familiarize the participants with the Basic-T and the various tasks. This stage consisted of an overall demonstration of the system and three training scenarios. The offered scenarios showed an increasing complexity and the last scenario approached the complexity of the experimental trials.

The evaluation took place on the second day. Prior to the experimental trials both subjects were offered a scenario to refresh their memory on the ins and outs of the workstation. After this warming up, the trials commenced. After each run a debriefing session with the subject was held in order to discuss his or her experiences.

\subsection{Scenarios}

A total number of four scenarios were developed in cooperation with experts of the Royal Netherlands Navy. All scenarios were intended to pose a substantial workload to the subjects and included various threats or suspicious-looking tracks that contributed to the workload. Two of the four scenarios were developed around more or less traditional air and surface warfare in a high-tension situation while the other two scenarios were situated against a civilian background where countering smuggling was the main mission objective. The latter two scenarios were made more ambiguous and threatening by the possibility of a terrorist attack. All scenarios took about 20 minutes to conclude. Because of the relative freedom of the subjects to operate their resources, differences in the actual runs of the scenario occurred. For example, by sending the helicopter to different locations, the actual time at which hostile ships were positively identified could shift by one to two minutes. Generally, however, the scenarios ran in agreement.

In order to exclude sequence effects and minimize effects of learning, increasing acquaintance with the workstation, personal experience, etc., the scenarios were allocated in a balanced way where each subject executed one of each scenario-type.

\subsection{Experimental setup}

As only the data of the non-adaptive mode were used for this investigation, three independent variables remain: scenario type, subject rank, and scenario time. Scenario type was balanced within subjects, subject rank between subjects, and the scenarios were divided into 16 equal time slots. The start of the first time slot was dependent on the first time a subject entered his or her subjective workload (thereafter every 80 seconds). The rank 
variable described whether the subject worked as a warfare officer assistant (Chief Petty officer) or a warfare officer (Lieutenant Commander).

A number of dependent variables was measured:

- The subjective workload as rated every 80 seconds during each scenario on a one dimensional Likert rating scale ranging from one to five, one meaning heavy underload and boredom, three a comfortable and sustainable workload and five an overload of the operator. Six was logged in case the subject didn't indicate his or her subjective workload and was converted to five during the analysis.

- The number of tracks and the number of signals were logged every second.

- The performance in terms of tracks handled and reaction time to signals was logged every second.

- $\quad$ The data describing the human world-view and the machine world-view was stored (logged every second). This includes the position, class, and identity of each track.

\subsection{Hypotheses}

The data from the experiment enabled us to investigate the claims with respect to the operator performance model and the hybrid model. Software, known as the cognitive task load and performance monitor, was developed both to generate the adaptation triggers during the original experiment (on-line) and to facilitate an off-line first-order analysis between performance and cognitive effects on workload. The CTL monitor visualized the reaction times, the number of tracks, the number of signals, the machine world view, the human world view, and the subjective workload (see Figure 6).

The world views were 'summarized' in numbers of friendly, assumed friendly, neutral, suspect, and hostile tracks. For the tracks designated 'assumed friendly' and 'suspect', not enough hard data are available to assign a definite identity to them, although they 'seem to be' friendly and hostile, respectively. Tracks can also be designated 'unknown', in which case so little is known about them that they can be anything. As tracks are first observed they are assigned the identity 'pending', meaning the operator has not had time to take a look at them yet. A lot of pending tracks is an indication that the user is behind with his or her work (a lack of time). A situation with a lot of 'unknown' tracks rather indicates a lack of data instead.

A first order analysis of the data from the experiments using the CTL and performance monitor resulted in the generation of three hypotheses.

1. Because all scenarios were intended to stress the subjects, the difference between the scenarios was not expected to be large. Nevertheless the smuggling scenarios seemed to contain more 'theoretically difficult' tracks (as a percentage of the total number of tracks to compensate for differences in the total number of tracks) compared to the traditional warfare scenarios. The 'theoretically difficult' tracks consist of ambiguous, suspect or unknown, tracks as discussed in sect. 7.

2. If 'theoretically difficult' tracks are experienced by subjects as difficult as well, the smuggling scenarios should show an increased workload when compared to the traditional scenarios.

3. The warfare officers seemed to show a different behavior in dealing with the situation compared to the warfare assistants. 


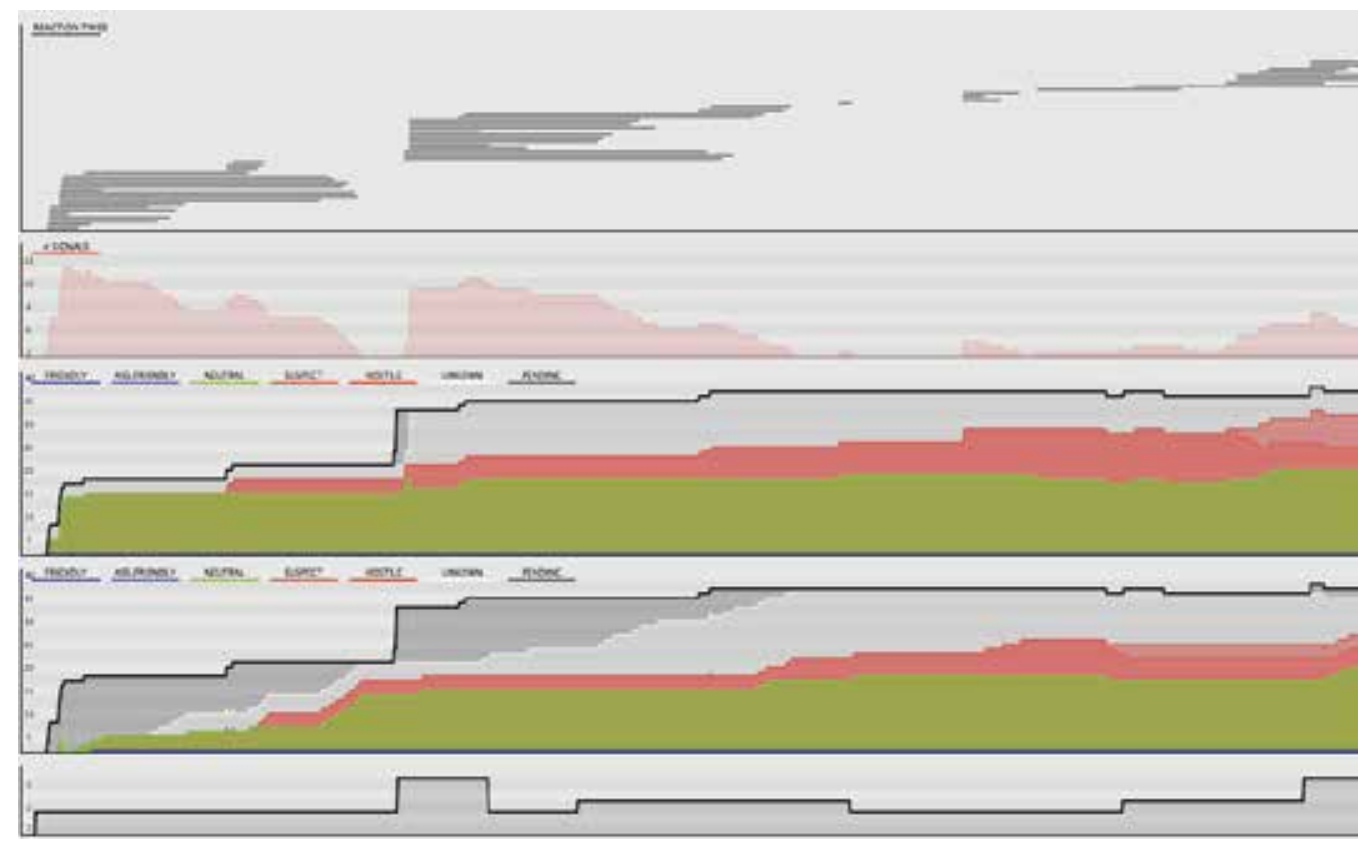

Figure 6. The cognitive task load and performance monitor describes (from lower to upper graph): the subjective workload over time, the human world view over time, the machine world view over time, the number of tracks (in gray) in combination with the number of signals (in red) over time (a performance measure), and reaction times represented using the start and end time (another performance measure)

\subsection{Statistical Results}

For each dependent variable a repeated-measures analysis MANOVA was used to analyze the data using scenario and time as a within factor and subject rank as a between factor. In all cases, an alpha level of .05 was used to determine statistical significance. Post-hoc analyses were conducted using Tukey's HSD and Fishers LSD tests.

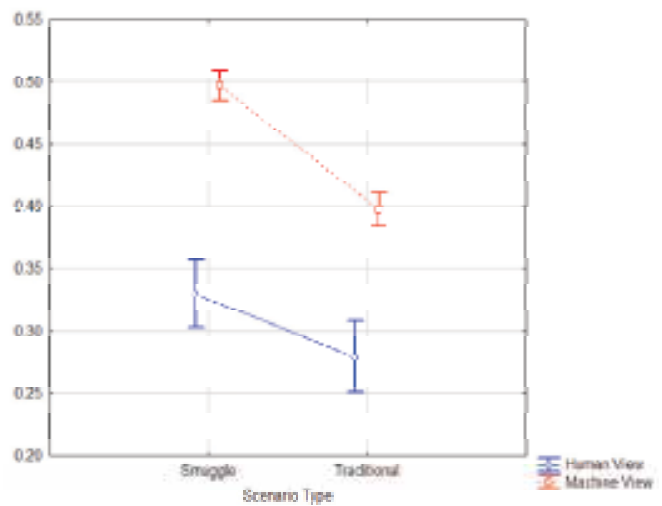

Figure 7. The plot shows a significant different number of ambiguous tracks (expressed as a percentage of the total number of tracks) per scenario type according to both the human interpretation and machine interpretation 
Analysis of the two different scenario types (smuggling vs. traditional) reveals that the smuggling scenarios contain an effect in terms of more ambiguous tracks as compared to the traditional ones $(\mathrm{F}(2,153)=59.463, p<.0001)$ according to both human and machine interpretation (see Figure 7). The value is expressed as a percentage of the total number of tracks per time unit to compensate for differences in the total number of tracks. Tukey's post-hoc analysis reveals that the smuggling scenarios have more 'difficult' tracks according to the human interpretation of the world $(p<.01)$ and the machine interpretation of the world $(p<.0001)$. Detailed analysis of the class of ambiguous tracks discloses that the increase could be mainly attributed to an increase in both unknown $(p<.0001)$ and suspect $(p<.0001)$ tracks according to machine reasoning and an increase in unknown tracks alone $(p<.0001)$ according to human reasoning. In synopsis, the data show that the smuggling scenarios are more 'difficult' than the traditional ones in terms of ambiguous tracks.
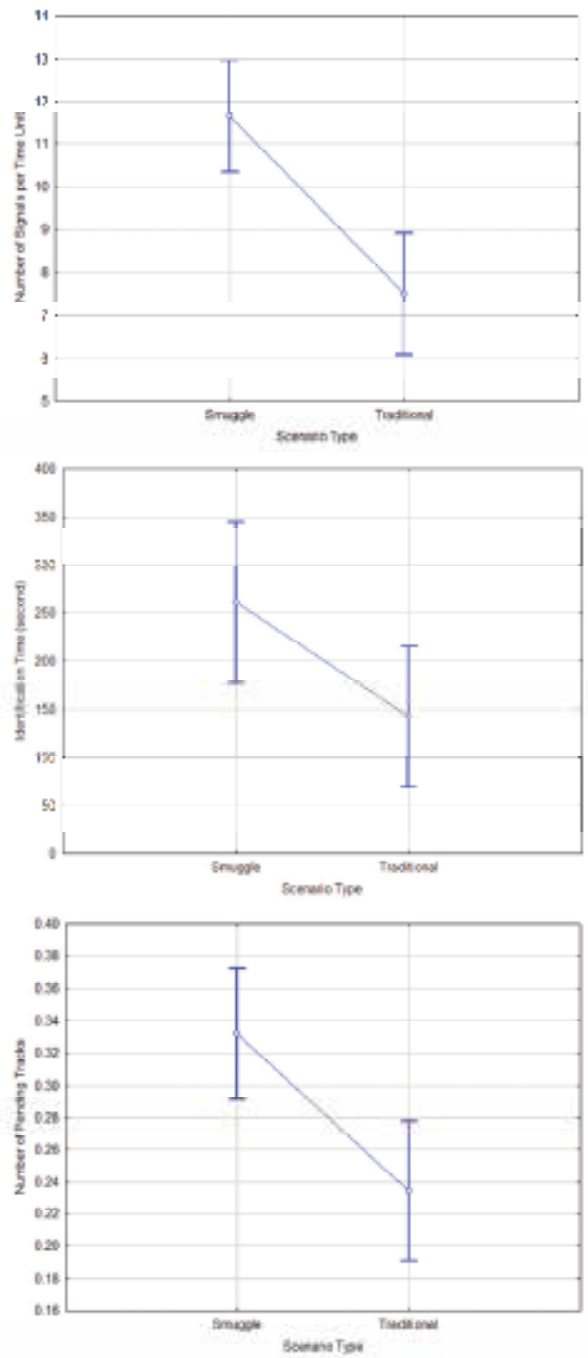

Figure 8 . The number of pending tracks, average reaction times for the identification process, and number of signals as a function of scenario type 
Furthermore, the data shows an increase in pending tracks in the smuggling scenarios $(p<$ .001) (i.e. more pending tracks per time unit) indicating that the human required more time to provide an initial identity in the smuggling scenarios as compared to traditional scenario type (see Figure 8 top). Furthermore, the averaging response times over scenarios disseminates that the response times to identification signals in the traditional scenarios is lower $(\mathrm{F}(1,12)=5.4187, p<.05)$ as compared with the smuggle scenarios (see Figure 8 middle). In addition, the number of signals per time unit was significantly higher in the smuggling scenarios $(\mathrm{F}(1,154)=18.081, p<.0001)$ as compared to the traditional scenario indicating that an increased number of tracks are awaiting attention of the human operator (see Figure 8 bottom). These signals requiring attention indicate work to be done and such an increase convey that the human operator requires more effort to get the work done. To summarize, the data reveals three indicators of declined performance in the more difficult scenarios.

A time analysis (see Figure 9 top) reveals an effect of time and scenario type on ambiguous track class $(\mathrm{F}(26,306)=1.5485, p<.05)$. Fisher's test reveals that the difference manifests itself mainly in the beginning of the scenarios as the first four times slots of the traditional scenarios show significantly less ambiguous tracks than the smuggle scenarios (all $p<.001$ ).

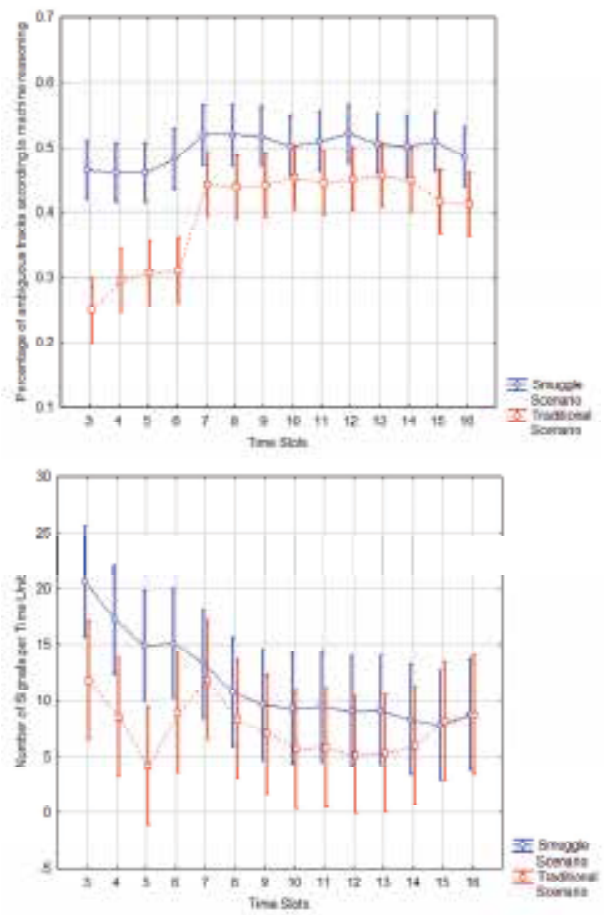

Figure 9. Top: The number of signals per time unit split by timeslot and scenario type reveal significant different in the first three time slots. Bottom: The number of difficult tracks split by timeslot and scenario type reveals that the scenarios differed mainly in the beginning

Applying the same time analysis to the number of signals shows for the first three time slots significant less signals in the traditional scenario as compared to the smuggle scenario (all $p$ $<.01$, see Figure 9 bottom). An increasing number of signals represents the fact that the human view and the machine view are increasing in skew as well. This correlation between 
number of difficult tracks and number of signals is upheld during the remains of the scenarios. As a larger number of signals requires more attention of the human this can be interpreted as work to be done. Combining the difference in ambiguous tracks with the difference in signals leads to the conclusion that we are not only able to observe overall differences in scenarios or performance, but also to pinpoint those differences in time.

With respect to the effect of scenario type on subjective workload, contrary to expectation we failed to find any subjective workload effects $(\mathrm{F}(1,154)=1.0288, p=.31)$.

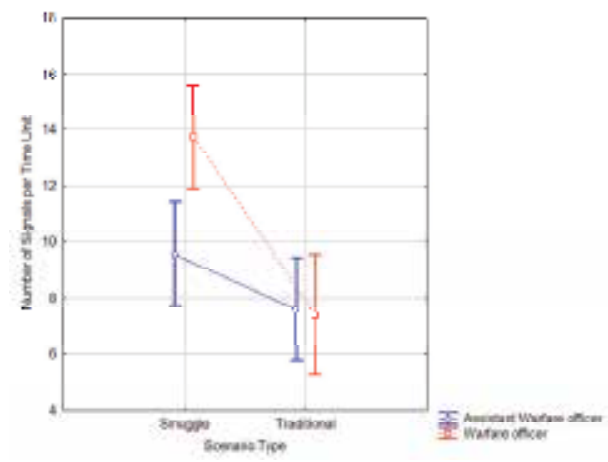

Figure 10. Analysis of the operator rank shows that behavioral differences occur and manifest mainly in the smuggling scenarios

Analysis with respect to different behavior of subject rank shows an effect $(\mathrm{F}(1,154)=4.1954, p$ $<.05$ ) of subject rank on signals per time unit in that the warfare officer has more signals per time unit when compared to the assistant. Furthermore, detailed analysis reveals an additional effect of scenario type on subject rank behavior $(\mathrm{F}(1,154)=5.0065, p<.05)$. Post hoc (Tukey) analysis learns that subject rank behavior manifests mainly in the more difficult scenarios $(p<.001)$ in that the warfare officer has more tracks per time unit (see Figure 10).

\subsection{Conclusions}

Although the experiment was not designed specifically to validate variation of the CTL variables on workload, we were nevertheless able to determine that:

1. the smuggle scenarios contain more 'theoretically difficult' (ambiguous) tracks;

2. the more 'difficult' scenarios in terms of ambiguous tracks had a lower performance in terms of pending contacts, response times, and signals awaiting attention and thus were experienced as more difficult by the subjects;

3. the difference in the two types of scenario manifested strongest at the start of the scenarios which correlated nicely with an increase in signals that conveyed the fact that the human operator required more effort to get the work done;

4. there was no effect of scenario type on the subjective workload; and

5. there existed a difference in behavior dependent on subject rank that discriminated in the more 'difficult' scenarios.

Taking these five statements into account, we conclude that two of the three hypotheses are clearly confirmed. First of all, although they were not expressly designed as such, the smuggle scenarios are more difficult in terms of ambiguous tracks. Second, there was a clear correlation with the theoretical difficulty of a scenario or situation in terms of ambiguous tracks and the performance of the subjects. We therefore conclude that describing scenarios 
in terms of 'difficult' tracks is feasible. Such an environmental description in terms of expected workload can be very useful for distilling causes of a performance decrease or an increase in workload. This knowledge, in its turn, benefits the determination of the optimal aiding strategy (i.e. optimizing the what to support question of Endsley, 1996).

In addition, we are not only able to indicate overall differences in scenarios or performance, but also to locate those differences in time due to a combination of the difference in difficult tracks with the difference in signals. This knowledge aids in determining when support is opportune.

The failure to measure subjective-workload effects due to scenario type clearly rejects hypothesis 2 (statement 4). We were, however, able to show a clear performance variation due to a variation in scenario type (statement 2), indicating a larger objective workload in terms of 'things to do'. The data show performance effects in terms of the number of pending tracks awaiting identification by the user, the number of signals indicating work to be done (objects to inspect and identify), and reaction times to these signals.

Failing to find a subjective workload effect but finding performance effects is attributed by us to a restricted focus of attention by the subjects on the more important objects with at the same time an acceptance of a larger risk due to the diminished attention to the other objects. Humans are capable of maintaining their workload at a 'comfortable' level (i.e., level three on a five-point scale) while accepting an increased risk due to not finishing tasks. This notion matches the adaptive-operator theory of Veltman \& Jansen (2004) that argues that human operators can utilize two strategies to cope with increased demands. The first strategy involves investing more mental effort and the second involves reducing the task goals. For this second strategy Veltman \& Jansen state that 'operators will slow down the task execution, will skip less relevant tasks, or accept good instead of perfect performance'. As an example, the Tenerife air crash in 1977 was partly attributed to the acceptance of increased risk (Weick, 1993). In our case the subjects seemed to accept the larger risk of not identifying all contacts by limiting their attention to a smaller area around the ship in order to maintain their mental effort at a reasonable level. This is an applied strategy for operational situations where watches take eight hours and it is not known how long an effort must be maintained and it appears the same strategy was followed during the experiments. As a matter of fact, one of the subjects stated as much in saying that 'his workload should have been five for much of the time as he did not get all the work done' (i.e., he did not identify all tracks).

Adaptive aiding strategies should consequently be cautious using human indicators of workload only and include at least some performance measures.

The third hypothesis stated that warfare officers show a different behavior in dealing with the tracks compared to warfare assistants. The data indicate evidence in support of this hypothesis (statement 5). Different capabilities, experience, and function show different behavior in that the warfare officer allowed more signals per time unit as compared to the warfare assistants. We argue that this is due to the rank and function-dependent training and background. The assistant warfare officer is trained to construct a complete maritime picture while the warfare officer is supposed to deal with the difficult cases that (potentially) represent a threat. The fact that warfare officers allowed more signals per time unit in the more difficult scenarios indicates that they focused on the more difficult cases and tended to leave the easy cases for the assistant (not present in these single-user experiments). This behavior did not manifest as strongly in the traditional scenarios as these are easier, 
resulting in an improved performance in that the warfare officers were not required to focus on the difficult cases alone.

Finding such differences that were not taken into account initially (see section 7.1, 7.2, and 7.3) shows that studies like these are very useful in order to improve cognitive modeling.

We conclude that the hybrid model is capable of triggering adaptive automation for a number of reasons. First, the operator performance model optimizes the timing of support and second the model of operator cognition indicates how much work is to be expected in the short term. Third, the model helps optimizing the type of aiding based on the cause of the increased workload.

\section{Summary}

This chapter took as a starting point that the human-machine ensemble could be regarded as an adaptive controller where both the environment and human cognition vary, the latter due to environmental and situational demands. We have described a human operator performance model and a model of human operator cognition that describe the variability of the human controller as a function of the situation. In addition, we have provided an empirical foundation for the utilization of the combined models. The data from two different experiments show either a change in subjective workload or a performance effect that correlate nicely when the environment or situation is varied.

Both the operator performance model and the model of operator cognition therefore show potential to be used as triggering mechanisms for adaptive automation, or as a measure of a human operator as a slowly changing parameter in an adaptive control system.

\section{Acknowledgement}

The work described in this chapter was supported by the MOD-NL DR\&D organization under programs 'human supervision and advanced task execution' (V055) and 'human system task integration' (V206). We like to thank Harmen Lafeber, a master student under the auspice of the University of Utrecht, whose master thesis work contributed to experiment I. In addition a number of colleagues from TNO Defence, Security and Safety are thanked for their contribution to the development of the prototype platform, the experiment, and reflective ideas: Jasper Lindenberg, Jan van Delft, Bert Bierman, Louwrens Prins, Rob van der Meer and Kees Houttuin were part of our team in these programs. The eight officers of the Royal Netherlands Navy are thanked for their contributions to experiment II. Finally, we like to thank Willem Treurniet, Erik Willemsen, and Jasper Lindenberg for their useful comments during the writing of this chapter.

\section{References}

Alberts, D.S. \& Hayes, R.E. (2006). Understanding Command and Control, CCRT Publication Series, 1-893723-17-8, Washington, D.C.

Archer, R.D. \& Lockett, J.F. (1997). WinCrew - a tool for analyzing performance, mental workload and functional allocation among operators, Proceedings of the First International Conference on Allocation and Functions (ALLFN'97), 157-165, Galway, Ireland, October 1-3, IEA Press, Louisville 
Arciszewski, H.F.R.; De Greef, T.E. \& Van Delft, J.H. (in press). Adaptive Automation in a Naval Combat Management System, IEEE Transactions on Systems, Man, and Cybernetics Part A: Systems and Humans, 1083-4427

Astrom, K.J. \& Wittenmark, B. (1994). Adaptive Control, Addison-Wesley Longman Publishing Company, 0201558661, Boston

Bailey, N.R.; Scerbo, M.W.; Freeman, F.G.; Mikulka, P.J. \& Scott, L.A. (2006). Comparison of a brain-based adaptive system and a manual adaptable system for invoking automation, Human Factors, 48, 4, 693-709, 0018-7208

Bolderheij, F. (2007). Mission-Driven sensor management analysis, design, implementation and simulation, Delft University of Technology, 978-90-76928-13-5, Delft

Byrne, E.A. \& Parasuraman, R. (1996). Psychophysiology and adaptive automation, Biological Psychology, 42, 3, 249-268, 0301-0511

Clamann, M.P.; Wright, M.C. \& Kaber, D.B. (2002). Comparison of performance effects of adaptive automation applied to various stages of human-machine system information processing, Proceedings of the Human Factors and Ergonomics Society 46th Annual Meeting, 342-346, 0-945289-20-0, Baltimore, Sept. 30-Oct. 4, Human Factors and Ergonomics Society, Santa Monica

Coram, R. (2002). Boyd: The Fighter Pilot Who Changed the Art of War, Little, Brown \& Company, 0316881465, New York

De Greef, T.E. \& Arciszewski, H.F.R. (2007). A Closed-Loop Adaptive System for Command and Control, Foundations of Augmented Cognition - Third International Conference, FAC 2007, Held as Part of HCI International 2007, 276-285, 978-3-540-73215-0, Beijing, China, July 22-27, Springer Berlin, Heidelberg

De Greef, T.E.; Arciszewski, H.F.R.; Lindenberg, J. \& Van Delft, J.H. (2007). Adaptive Automation Evaluated, TNO Defence, Security and Safety, TNO-DV 2007 A610, Soesterberg, the Netherlands

De Greef, T.E. \& Lafeber, H. (2007). Utilizing an Eye-Tracker Device for Operator Support, 4th Augmented Cognition International (ACI) Conference being held in conjunction with the HFES 51st Annual Meeting, 67-72, 0-9789812-1-9, Baltimore, Maryland, October 1-3, Strategic Analysis, Inc, Arlington

Endsley, M. (1996). Automation and situation awareness, In: Automation and human performance: Theory and applications Parasuraman, R. \& Mouloua, M., (Ed.), 163-181, Lawrence Erlbaum Associates, 080581616X, Mahwah

Endsley, M. \& Kiris, E. (1995). The Out-of-the-Loop Performance Problem and Level of Control in Automation., Human Factors, 381-394, 0018-7208

Geddes, N.D. (1985). Intent inferencing using scripts and plans, Proceedings of the First Annual Aerospace Applications of Artificial Intelligence Conference, 160-172, Dayton, Ohio, 17-19 September, U.S. Air Force, Washington

Gopher, D. \& Donchin, E. (1986). Workload - An examination of the concept, In: Handbook of Perception and Human Performance, Boff, K. \& Kaufmann, J., (Ed.), 4141-4949, WileyInterscience, 0471829579, New York

Hilburn, B.; Jorna, P.G.; Byrne, E.A. \& Parasuraman, R. (1997). The effect of adaptive air traffic control (ATC) decision aiding on controller mental workload, In: HumanAutomation Interaction: Research and Practice, Mouloua, M.; Koonce, J. \& Hopkin, V.D., (Ed.), 84-91, Lawrence Erlbaum Associates, 0805828419, Mahwah 
Inagaki, T. (2000a). Situation-adaptive autonomy for time-critical takeoff decisions, International Journal of Modelling and Simulation, 20, 2, 175-180, 0037-5497

Inagaki, T. (2000b). Situation-adaptive autonomy: Dynamic trading of authority between human and automation, Proceedings of the XIVth Triennial Congress of the International Ergonomics Association and 44th Annual Meeting of the Human Factors and Ergonomics Association, 'Ergonomics for the New Millennium', 13-16, San Diego, CA, July 30 - August 4, Human Factors and Ergonomics Society, Santa Monica

Kaber, D.B. \& Endsley, M. (2004). The effects of level of automation and adaptive automation on human performance, situation awareness and workload in a dynamic control task, Theoretical Issues in Ergonomics Science, 5, 2, 113-153, 1463 $922 X$

Kaber, D.B.; Perry, C.M.; Segall, N.; Mcclernon, C.K. \& Prinzel III, L.J. (2006). Situation awareness implications of adaptive automation for information processing in an air traffic control-related task, International Journal of Industrial Ergonomics, 36, 5, 447462, 0169-8141

Kaber, D.B. \& Riley, J.M. (1999). Adaptive Automation of a Dynamic Control Task Based on Secondary Task Workload Measurement, International Journal of Cognitive Ergonomics, 3, 3, 169-187, 1088-6362

Kaber, D.B.; Wright, M.C.; Prinzel, L.J. \& Clamann, M.P. (2005). Adaptive automation of human-machine system information-processing functions, Human Factors, 47, 4, 730-741, 0018-7208

Kantowitz, B.H. (1987). Mental Workload, In: Human factors psychology, Hancock, P.A., (Ed.), 81-121, North-Holland, 0-444-70319-5, New York

Moray, N.; Inagaki, T. \& Itoh, M. (2000). Adaptive Automation, Trust, and Self-Confidence in Fault Management of Time-Critical Tasks, Journal of experimental psychology, 44$57,0096-3445$

Neerincx, M.A. (2003). Cognitive task load design: model, methods and examples, In: Handbook of Cognitive Task Design, Hollnagel, E., (Ed.), 283-305, Lawrence Erlbaum Associates, 0805840036, Mahwah, NJ

Parasuraman, R.; Mouloua, M. \& Molloy, R. (1996). Effects of adaptive task allocation on monitoring of automated systems, Human Factors, 38, 4, 665-679, 0018-7208

Parasuraman, R.; Sheridan, T.B. \& Wickens, C.D. (2000). A model for types and levels of human interaction with automation, IEEE Transactions on Systems, Man, and Cybernetics Part A:Systems and Humans, 30, 3, 286-297, 1083-4427

Prinzel, L.J.; Freeman, F.G.; Scerbo, M.W.; Mikulka, P.J. \& Pope, A.T. (2000). A closed-loop system for examining psychophysiological measures for adaptive task allocation, International Journal of Aviation Psychology, 10, 4, 393-410, 1050-8414

Rasmussen, J. (1986). Information Processing and Human-Machine Interaction: An Approach to Cognitive Engineering, North-Holland, 0444009876, Amsterdam

Rouse, W.B. (1988). Adaptive Aiding for Human/Computer Control, Human Factors, 30, 4, 431-443, 0018-7208

Rouse, W.B.; Geddes, N.D. \& Curry, R.E. (1987). Architecture for Interface: Outline of an Approach to Supporting Operators of Complex Systems, Human-Computer Interaction, 3, 2, 87-122, 10447318 
Scallen, S.; Hancock, P. \& Duley, J. (1995). Pilot performance and preference for short cycles of automation in adaptive function allocation, Applied Ergonomics, 397-404, 0003 6870

Scerbo, M. (1996). Theoretical perspectives on adaptive automation, In: Automation and human performance: theory and applications, Parasuraman, R. \& Mouloua, M., (Ed.), pp. 37-63, Lawrence Erlbaum Assiciated, Publishes Mahwah, 080581616X, New Jersey

Van Delft, J.H. \& Arciszeski, H.F.R. (2004). Eindevaluatie automatiserings- en ondersteuningsconcepten Studie Commandovoering, TNO Human Factors, Soesterberg, The Netherlands

Van Delft, J.H. \& Schraagen, J.M. (2004). Decision Support Interfaces, Proceedings of the IEEE International Conference on Systems, Man and Cybernetics, 827-832, 0-7803-8567-5, The Hague, the Netherlands, October 10-13,

Veltman, J.A. \& Gaillard, A.W.K. (1998). Physiological workload reactions to increasing levels of task difficulty, Ergonomics, 41, 5, 656-669, 0014-0139

Veltman, J.A. \& Jansen, C. (2004). The Adaptive Operator, Human Performance, Situation Awareness and Automation Technology Conference, Daytona Beach FL, March 22-25,

Weick, K.E. (1993). The vulnerable system: an analysis of the Tenerife air disaster, In: New challenges to understanding organizations, Roberts, K.H., (Ed.), Macmillan, 0-02402052-4, New York

Wickens, C.D. (1984). Processing resources in attention, In: Varieties of attention, Parasuraman, R. \& Davies, D.R., (Ed.), 63-101, Academic Press, 0125449704, Orlando, FL

Wilson, G.F. \& Russell, C.A. (2007). Performance enhancement in an uninhabited air vehicle task using psychophysiologically determined adaptive aiding, Human Factors, 49, 6, 1005-1018, 0018-7208 


\title{
Advances in Parameter Estimation and Performance Improvement in Adaptive Control
}

\author{
Veronica Adetola and Martin Guay \\ Department of Chemical Engineering, Queen's University Kingston \\ Canada
}

\section{Introduction}

In most adaptive control algorithms, parameter estimate errors are not guaranteed to converge to zero. This lack of convergence adversely affects the global performance of the algorithms. The effect is more pronounced in control problems where the desired reference setpoint or trajectory depends on the system's unknown parameters. This paper presents a parameter estimation routine that allows exact reconstruction of the unknown parameters in finite-time provided a given excitation condition is satisfied. The robustness of the routine to an unknown bounded disturbance or modelling error is also shown.

To enhance the applicability of the finite-time (FT) identification procedure in practical situations, a novel adaptive compensator that (almost) recover the performance of the FT identifier is developed. The compensator guarantees exponential convergence of the parameter estimation error at a rate dictated by the closed-loop system's excitation. It was shown how the adaptive compensator can be used to improve upon existing adaptive controllers. The modification provided guarantees exponential stability of the parametric equilibrium provided the given PE condition is satisfied. Otherwise, the original system's closed-loop properties are preserved.

The results are independent of the control structure employed. The true parameter value is obtained without requiring the measurement or computation of the velocity state vector. Moreover, the technique provides a direct solution to the problem of removing auxiliary perturbation signals when parameter convergence is achieved. The effectiveness of the proposed methods is illustrated with simulation examples.

There are two major approaches to online parameter identification of nonlinear systems. The first is the identification of parameters as a part of state observer while the second deals with parameter identification as a part of controller. In the first approach, the observer is designed to provide state derivatives information and the parameters are estimated via estimation methods such as least squares method [19] and dynamic inversion [6]. The second trend of parameter identification is much more widespread, as it allows identification of systems with unstable dynamics. Algorithms in this area include parameter identification methods based on variable structure theory $[22,23]$ and those based on the notion of passivity [13].

In the conventional adaptive control algorithms, the focus is on the tracking of a given reference trajectory and in most cases parameter estimation errors are not guaranteed to 
converge to zero due to a lack of excitation [10]. Parameter convergence is an important issue as it enhances the overall stability and robustness properties of the closed-loop adaptive systems [14]. Moreover, there are control problems whereby the reference trajectory is not known a priori but depends on the unknown parameters of the system dynamics. For example, in adaptive extremum seeking control problems, the desired target is the operating setpoint that optimizes an uncertain cost function [8, 21].

Assuming the satisfaction of appropriate excitation conditions, asymptotic and exponential parameter convergence results are available for both linear and nonlinear systems. Some lower bounds which depends (nonlinearly) on the adaptation gain and the level of excitation in the system have been provided for some specific control and estimation algorithms [11, 17, 20]. However, it is not always easy to characterize the convergence rate. Since the performance of any adaptive extremum seeking control is dictated by the efficiency of its parameter adaptation procedure. This chapter presents a parameter estimation scheme that allows exact reconstruction of the unknown parameters in finitetime provided a given persistence of excitation (PE) condition is satisfied. The true parameter estimate is recovered at any time instant the excitation condition is satisfied. This condition requires the integral of a filtered regressor matrix to be invertible. The finite-time (FT) identification procedure assumes the state of the system $x(\cdot)$ is accessible for measurement but does not require the measurement or computation of the velocity state vector $\dot{x}(\cdot)$. The robustness of the estimation routine to bounded unknown disturbances or modeling errors is also examined. It is shown that the parameter estimation error can be rendered arbitrarily small for a sufficiently large filter gain.

A common approach to ensuring a PE condition in adaptive control is to introduce a perturbation signal as the reference input or to add it to the target setpoint or trajectory. The downside of this approach is that a constant PE deteriorates the desired tracking or regulation performance. Aside from the recent results on intelligent excitation signal design $[3,4]$, the standard approach has been to introduce such PE signal and remove it when the parameters are assumed to have converged. The fact that one has perfect knowledge of the convergence time in the proposed framework allows for a direct and immediate removal of the added PE signal. The result on finite-time identification has been published in [2].

The main drawback of the finite-time identification algorithm is the requirement to check the invertibility of a matrix online and compute the inverse matrix when appropriate. To avoid these concerns and enhance the applicability of the FT method in practical situations, the procedure was employed to develop a novel adaptive compensator that (almost) recover the performance of the FT identifier. The compensator guarantees exponential convergence of the parameter estimation error at a rate dictated by the closed-loop system's excitation. It was shown how the adaptive compensator can be used to improve upon existing adaptive controllers. The modification provided guarantees exponential stability of the parametric equilibrium provided the given PE condition is satisfied. Otherwise, the original system's closed-loop properties are preserved.

\section{Problem Description and Assumptions}

The system considered is the following nonlinear parameter affine system

$$
\dot{x}=f(x, u)+g(x, u) \theta
$$


where $x \in \mathbb{R}^{n_{x}}$ is the state and $u \in \mathbb{R}^{n_{u}}$ is the control input. The vector $\theta \in \mathbb{R}^{n_{\theta}}$ is the unknown parameter vector whose entries may represent physically meaningful unknown model parameters or could be associated with any finite set of universal basis functions. It is assumed that $\theta$ is uniquely identifiable and lie within an initially known compact set $\Theta^{0}$. The $n_{x}$-dimensional vector $f(x, u)$ and the $\left(n_{x} \times n_{\theta}\right)$-dimensional matrix $g(x, u)$ are bounded and continuous in their arguments. System (1) encompasses the special class of linear systems,

$$
\begin{aligned}
f(x, u) & =A_{0} x+B_{0} u \\
g(x, u) & =\left[A_{1} x+B_{1} u, A_{2} x+B_{2} u, \ldots A_{n_{\theta}} x+B_{n_{\theta}} u\right],
\end{aligned}
$$

where $A_{i}$ and $B_{i}$ for $i=0 \ldots n_{\theta}$ are known matrices possibly time varying.

Assumption 2.1 The following assumptions are made about system (1).

1. The state of the system $x(\cdot)$ is assumed to be accessible for measurement.

2. There is a known bounded control law $u=\alpha($.$) and a bounded parameter update law \dot{\hat{\theta}}$ that achieves a primary control objective.

The control objective can be to (robustly) stabilize the plant and/or to force the output to track a reference signal. Depending on the structure of the system (1), adaptive control design methods are available in the literature $[12,16]$.

For any given bounded control and parameter update law, the aim of this chapter is to provide the true estimates of the plant parameters in finite-time while preserving the properties of the controlled closed-loop system.

\section{Finite-time Parameter Identification}

Let $\hat{x}$ denote the state predictor for (1), the dynamics of the state predictor is designed as

$$
\dot{\hat{x}}=f(x, u)+g(x, u) \hat{\theta}+k_{w}(t) e+w \dot{\hat{\theta}}
$$

where $\hat{\theta}$ is a parameter estimate generated via any update law $\dot{\hat{\theta}}, k_{w}>0$ is a design matrix, $e=x-\hat{x}$ is the prediction error and $w$ is the output of the filter

$$
\dot{w}=g(x, u)-k_{w} w, \quad w\left(t_{0}\right)=0 .
$$

Denoting the parameter estimation error as $\tilde{\theta}=\theta-\hat{\theta}$, it follows from (1) and (2) that

$$
\dot{e}=g(x, u) \tilde{\theta}-k_{w} e-w \dot{\hat{\theta}} .
$$

The use of the filter matrix $w$ in the above development provides direct information about parameter estimation error $\tilde{\theta}$ without requiring a knowledge of the velocity vector $\dot{x}$. This is achieved by defining the auxiliary variable

$$
\eta=e-w \tilde{\theta}
$$

with $\eta$, in view of $(3,4)$, generated from

$$
\dot{\eta}=-k_{w} \eta, \quad \eta\left(t_{0}\right)=e\left(t_{0}\right) .
$$

Based on the dynamics (2), (3) and (6), the main result is given by the following theorem. 
Theorem 3.1 Let $Q \in \mathbb{R}^{n_{\theta} \times n_{\theta}}$ and $C \in \mathbb{R}^{n_{\theta}}$ be generated from the following dynamics:

$$
\begin{aligned}
\dot{Q} & =w^{T} w, & & Q\left(t_{0}\right)=0 \\
\dot{C} & =w^{T}(w \hat{\theta}+e-\eta), & C\left(t_{0}\right) & =0
\end{aligned}
$$

Suppose there exists a time $t_{c}$ and a constant $c_{1}>0$ such that $Q\left(t_{c}\right)$ is invertible i.e.

$$
Q\left(t_{c}\right)=\int_{t_{0}}^{t_{c}} w^{T}(\tau) w(\tau) d \tau \succ c_{1} I,
$$

then

$$
\theta=Q(t)^{-1} C(t) \quad \text { for all } t \geq t_{c} .
$$

Proof: The result can be easily shown by noting that

$$
Q(t) \theta=\int_{t_{0}}^{t} w^{T}(\tau) w(\tau)[\hat{\theta}(\tau)+\tilde{\theta}(\tau)] d \tau .
$$

Using the fact that $w \tilde{\theta}=e-\eta$, it follows from (10) that

$$
\theta=Q(t)^{-1} \int_{t_{0}}^{t} \dot{C}(\tau) d \tau=Q(t)^{-1} C(t)
$$

and (11) holds for all $t \geq t_{c}$ since $Q(t) \succeq Q\left(t_{c}\right)$.

The result in theorem 3.1 is independent of the control $u$ and parameter identifier $\hat{\theta}$ structure used for the state prediction (eqn 2). Moreover, the result holds if a nominal estimate $\theta^{0}$ of the unknown parameter (no parameter adaptation) is employed in the estimation routine. In this case, $\hat{\theta}$ is replaced with $\theta^{0}$ and the last part of the state predictor (2) is dropped $(\dot{\hat{\theta}}=0)$.

Let

$$
\theta^{c} \triangleq Q\left(t_{c}\right)^{-1} C\left(t_{c}\right)
$$

The finite-time (FT) identifier is given by

$$
\hat{\theta}^{c}(t)= \begin{cases}\hat{\theta}(t), & \text { if } t<t_{c} \\ \theta^{c}, & \text { if } t \geq t_{c} .\end{cases}
$$

The piecewise continuous function (13) can be approximated by a smooth approximation using the logistic functions

$$
\begin{aligned}
& \psi_{1} \triangleq \frac{\hat{\theta}(t)}{2}\left(1-\tanh \nu_{1}\left(t-t_{c}\right)\right)=\frac{\hat{\theta}(t)}{1+\exp ^{2 \nu_{1}\left(t-t_{c}\right)}} \\
& \psi_{2} \triangleq \frac{\theta^{c}}{2}\left(1+\tanh \nu_{2}\left(t-t_{c}\right)\right)=\frac{\theta^{c}}{1+\exp ^{-2 \nu_{2}\left(t-t_{c}\right)}}
\end{aligned}
$$




$$
\hat{\theta}^{\tilde{c}}=\psi_{1}+\psi_{2}
$$

where larger $\nu_{1}, \nu_{2}$ correspond to a sharper transition at $t=t_{c}$ and $\lim _{\left(\nu_{1}, \nu_{2}\right) \rightarrow \infty} \hat{\theta}^{\tilde{c}}=\hat{\theta}^{c}$. An example of such approximation is depicted in Figure 1 where the function

$$
z(t)=\left\{\begin{array}{cc}
6+t^{0.3} & \text { if } t<5 \\
4 & \text { otherwise }
\end{array}\right.
$$

is approximated by (14) with $\nu_{1}=\nu_{2}=5$.

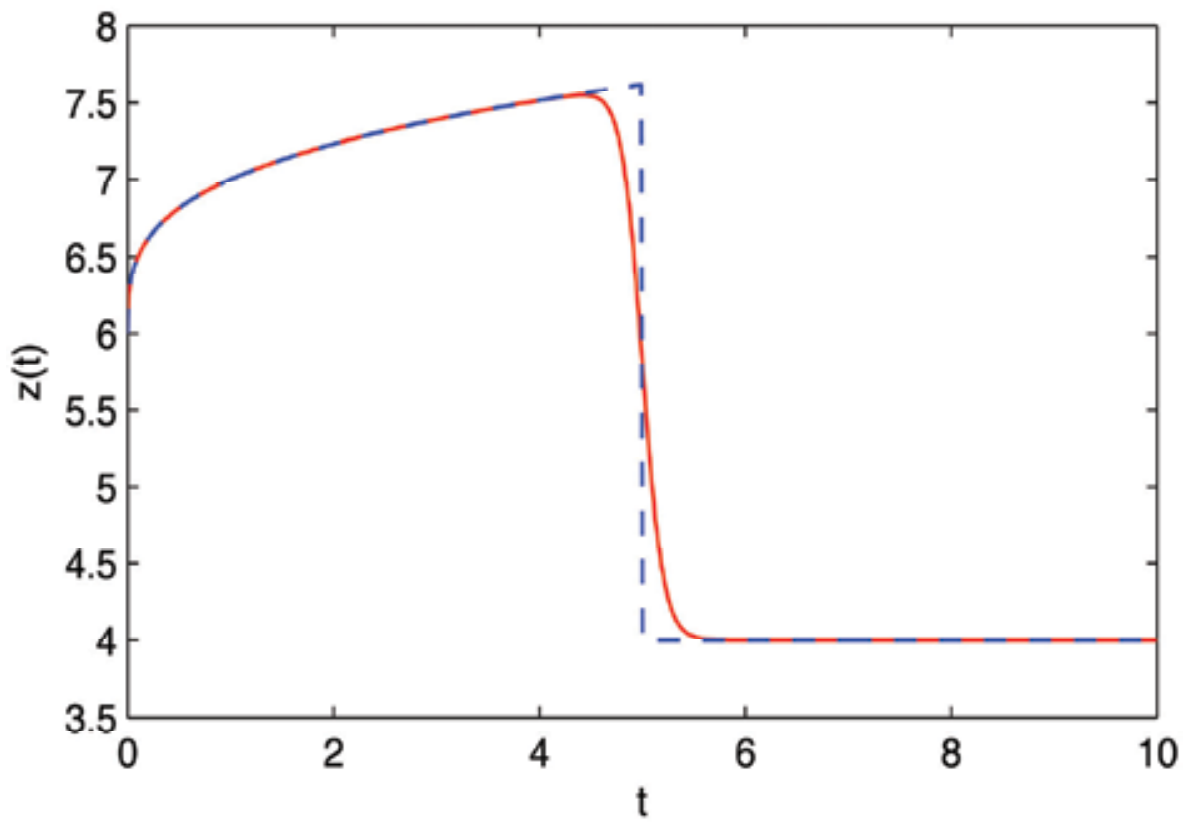

Figure 1. Approximation of a piecewise continuous function. The function $z(t)$ is given by the full line. Its approximation is given by the dotted line

The invertibility condition (8) is equivalent to the standard persistence of excitation (PE) condition required for parameter convergence in adaptive control. The condition (8) is satisfied if the regressor matrix $g$ is PE. To show this, consider the filter dynamic (3), from which it follows that

$$
w(t)=\int_{t_{0}}^{t} \exp ^{-k_{\varphi}(t-\tau)} g(\tau) d \tau=\frac{1}{s+k_{w}}[g(t)] .
$$

Since $g(t)$ is PE by assumption and the transfer function $\frac{1}{s+k_{w}}$ is stable, minimum phase and strictly proper, we know that $w(t)$ is PE [18]. Hence, there exists $t_{c}$ and a $c_{1}$ for which (8) is satisfied. The superiority of the above design lies in the fact that the true parameter value can be computed at any time instant $t_{c}$ the regressor matrix becomes positive definite and subsequently stop the parameter adaptation mechanism.

The procedure in theorem 42 involves solving matrix valued ordinary differential equations $(3,7)$ and checking the invertibility of $Q(t)$ online. For computational considerations, the 
invertibility condition (8) can be efficiently tested by checking the determinant of $Q(t)$ online. Theoretically, the matrix is invert-ible at any time $\operatorname{det}(\mathrm{Q}(t))$ becomes positive definite. The determinant of $Q(t)$ (which is a polynomial function) can be queried at pre-scheduled times or by propagating it online starting from a zero initial condition. One way of doing this is to include a scalar differential equation for the derivative of $\operatorname{det}(\mathrm{Q}(\mathrm{i}))$ as follows [7]:

$$
\frac{d}{d t} \operatorname{det}(Q)=\operatorname{Trace}\left(\operatorname{Adjugate}(Q) w^{T} w\right), \quad \operatorname{det}\left(Q\left(t_{0}\right)\right)=0
$$

where Adjugate(Q), admittedly not a light numerical task, is also a polynomial function of the elements of $Q$.

\subsection{Absence of PE}

If the PE condition (8) is not satisfied, a given controller and the corresponding parameter estimation scheme preserve the system established closed-loop properties. When a bounded controller that is robust with respect to input $(\tilde{\theta}, \hat{\hat{\theta}})$ is known, it can be shown that the state prediction error $e$ tends to zero as $t \rightarrow \infty$. An example of such robust controller is an inputto-state stable (iss) controller [12].

Theorem 3.2 Suppose the design parameter $k_{w}$ in (2) is replaced with $k_{w}(t)=$ $k_{w_{1}}+k_{w_{2}}(t), k_{w_{1}}>\frac{1}{4} I$, and $k_{w_{2}}(t)=\frac{1}{4} g \gamma g^{T}$. Then the state predictor (2) and the parameter update law

$$
\dot{\hat{\theta}}=\gamma\left(w^{T}+g^{T}\right) e
$$

with $\gamma=\gamma^{T}>0$, a design constant matrix, guarantee that

1. $(e, \eta, \tilde{\theta}) \in \mathcal{L}_{\infty}$ and $(e, \eta) \rightarrow 0$ as $t \rightarrow \infty$.

2. $\lim _{t \rightarrow \infty} \tilde{\theta}(t)=\bar{\theta}$, a constant.

Proof:

1. Consider a Lyapunov function

$$
V=\frac{1}{2}\left(e^{T} e+\tilde{\theta}^{T} \gamma^{-1} \tilde{\theta}+\eta^{T} \eta\right)
$$

It follows from equations (4), (5), (6) and (17) that

$$
\begin{gathered}
\dot{V}=-e^{T} k_{w_{1}} e-e^{T}\left(\frac{1}{4} g \gamma g^{T}+w \gamma w^{T}+w \gamma g^{T}\right) e-\tilde{\theta}^{T} w^{T} w \tilde{\theta}-\tilde{\theta}^{T} w^{T} \eta-\eta^{T} k_{w}(t) \eta \\
\leq-e^{T} k_{w_{1}} e-\lambda_{\min }(\gamma)\left\|w^{T} e+\frac{1}{2} g^{T} e\right\|^{2}-\eta^{T} k_{w_{3}} \eta-\left\|w \tilde{\theta}+\frac{1}{2} \eta\right\|^{2} \\
\leq-\left(e^{T} k_{w_{1}} e+\eta^{T} k_{w_{3}} \eta\right) .
\end{gathered}
$$

where $k_{w_{3}}=k_{w_{1}}-\frac{1}{4}$. This implies uniform boundedness of $(\eta, e, \tilde{\theta})$ as well as global asymptotic convergence of $(\eta, e)$ to zero. Hence, it follows from (5) that $\lim _{t \rightarrow \infty} w \tilde{\theta}=0$.

2. This can be shown by noting from (17) that $\tilde{\theta}(t)=\tilde{\theta}\left(t_{0}\right)-\gamma \int_{t_{0}}^{t}\left(w^{T}+g^{T}\right) e d \sigma$. Since $g($.$) and e$ are bounded signals and $e \rightarrow 0$, the integral term exists and it is finite. 


\section{Robustness Property}

In this section, the robustness of the finite-time identifier to unknown bounded disturbances or modeling errors is demonstrated. Consider a perturbation of

(1):

$$
\dot{x}=f(x, u)+g(x, u) \theta+\vartheta(t, x, \theta)
$$

where $\vartheta($.$) is a disturbance or modeling error term that satisfies \|\vartheta(t)\| \leq M_{\vartheta}(t)<\infty$. If the PE condition (8) is satisfied and the disturbance term is known, the true unknown parameter vector is given by

$$
\theta_{\vartheta}^{c} \triangleq \theta=Q(t)^{-1} \int_{t_{0}}^{t} w^{T}(\tau)\left[w(\tau) \hat{\theta}(\tau)+e(\tau)-\eta_{\vartheta}(\tau)\right] d \tau, \quad \text { for all } t \geq t_{c},
$$

with $e=x-\hat{x}$ and the signals $\hat{x}, w, \eta_{\vartheta}=e-w \theta$ generated from (2), (3) and

$$
\dot{\eta}_{\vartheta}=-k_{w} \eta_{\vartheta}+\vartheta(.), \quad \eta_{\vartheta}\left(t_{0}\right)=e\left(t_{0}\right)
$$

respectively.

Since $\vartheta($.$) is unknown, we provide a bound on the parameter identification error$ $\tilde{\theta^{c}}=\theta_{\theta}^{c}-\theta^{c}$ when (6) is used instead of (24). Considering (9) and (23), it follows that

$$
\begin{gathered}
\tilde{\theta^{c}}=Q(t)^{-1} \int_{t_{0}}^{t} w^{T}(\tau)\left(-\eta_{\vartheta}(\tau)+\eta(\tau)\right) d \tau \\
=-Q(t)^{-1} \int_{t_{0}}^{t} w^{T}(\tau) \tilde{\eta}(\tau) d \tau .
\end{gathered}
$$

where $\tilde{\eta}=\eta_{\vartheta}-\eta$ is the output of

$$
\dot{\tilde{\eta}}=-k_{w} \tilde{\eta}+\vartheta(.), \quad \tilde{\eta}\left(t_{0}\right)=0 .
$$

Since $k_{w} \geq k_{w_{1}}>0$, it follows that

$$
\|\tilde{\eta}(t)\| \leq \frac{M_{\vartheta}}{k_{w_{1}}}
$$

and hence

$$
\left\|\tilde{\theta}^{c}(t)\right\| \leq\left\|Q(t)^{-1}\right\|\left\{\frac{\bar{w} M_{\vartheta}\left(t-t_{0}\right)}{k_{w_{1}}}\right\}, \quad \text { for all } t \geq t_{c} .
$$

where $\bar{w}=\max _{\sigma \in\left[t_{0}, t\right]}\left\|w^{T}(\sigma)\right\|$.

This implies that the identification error can be rendered arbitrarily small by choosing a sufficiently large filter gain $k_{w_{1}}$. In addition, if the disturbance term $\vartheta$ and the system satisfies some given properties, then asymptotic convergence can be achieved as stated in the following theorem.

Theorem 4.1 Suppose $\vartheta \in \mathcal{L}_{p}$, for $p=1$ or 2 and $\lim _{t \rightarrow \infty} \lambda_{\min }(Q)=\infty$, then $\tilde{\theta^{c}} \rightarrow 0$ asymptotically with time. 
To proof this theorem, we need the following lemma

Lemma 4.2 [5]: Consider the system

$$
\dot{x}(t)=A x(t)+u(t)
$$

Suppose the equilibrium state $x_{e}=0$ of the homogeneous equation is exponentially stable,

1. if $u \in E_{p}$ for $1<p<\infty$, then $x \in E_{p}$ and

2. if $u \in E_{p}$ for $p=1$ or 2 , then $x \rightarrow 0$ as $t \rightarrow \infty$.

Proof of theorem 4.1. It follows from Lemma 4.2.2 that $\tilde{\eta} \rightarrow 0$ as $t \rightarrow \infty$ and therefore $\lim _{t \rightarrow \infty} \int_{t_{0}}^{t} w^{T}(\tau) \tilde{\eta}(\tau) d \tau$ is finite. So

$$
\lim _{t \rightarrow \infty} \tilde{\theta^{c}}=\lim _{t \rightarrow \infty}\left\{Q(t)^{-1} \int_{t_{0}}^{t} w^{T}(\tau) \tilde{\eta}(\tau) d \tau\right\}=0 .
$$

\section{Dither Signal Design}

The problem of tracking a reference signal is usually considered in the study of parameter convergence and in most cases, the reference signal is required to provide sufficient excitation for the closed-loop system. To this end, the reference signal $y_{r}(t) \in \mathbb{R}^{r}$ is appended with a bounded excitation signal $d(t)$ as

$$
y_{r d}(t)=y_{r}(t)+d(t)
$$

where the auxiliary signal $d(t)$ is chosen as a linear combination of sinusoidal functions with $\hbar$ distinct frequencies:

$$
d(t):=\sum_{k=1}^{\hbar} \underline{a}_{k}(t) \sin \left(\omega_{k} t\right)=\mathcal{A}(t) \zeta(t)
$$

where

$$
\mathcal{A}(t)=\left[\begin{array}{ccc}
a_{11} & \cdots & a_{1 \hbar} \\
\vdots & & \vdots \\
a_{r 1} & \cdots & a_{r \hbar}
\end{array}\right]
$$

is the signal amplitude matrix and

$$
\zeta(t)=\left[\begin{array}{lll}
\sin \omega_{1} t & \ldots & \sin \omega_{\hbar} t
\end{array}\right]^{T}, \omega_{i} \neq \omega_{j} \text { for } i \neq j
$$

is the corresponding sinusoidal function vector.

For this approach, it is sufficient to design the perturbation signal such that the regressor matrix $g$ is PE. There are very few results on the design of persistently exciting (PE) input signals for nonlinear systems. By converting the closed-loop PE condition to a sufficient richness (SR) condition on the reference signal, attempts have been made to provide verifiable conditions for parameter convergence in some classes of nonlinear systems $[3,1$, $14,15]$. 


\subsection{Dither Signal Removal}
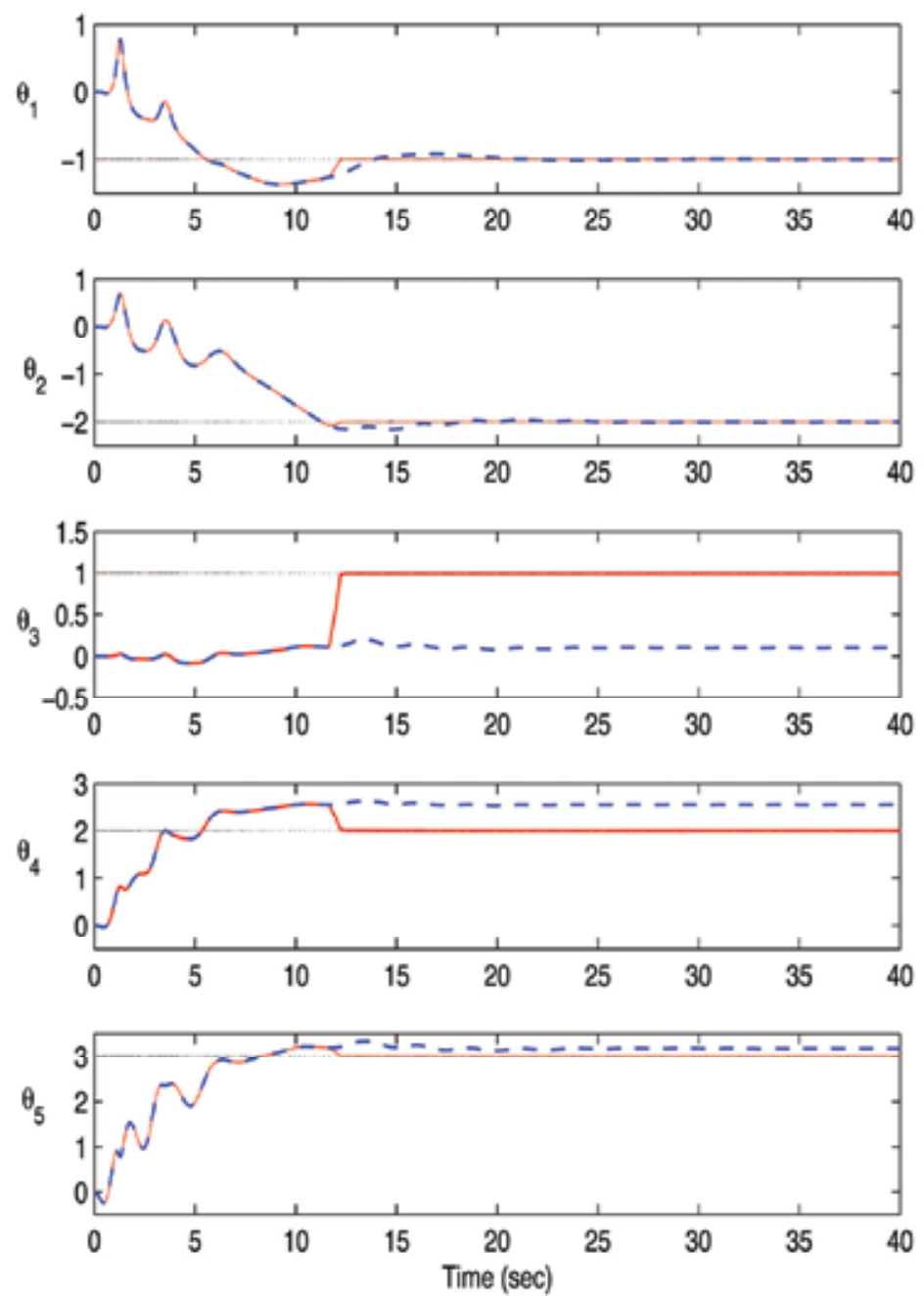

Figure 2. Trajectories of parameter estimates. Solid(-) : FT estimates $\hat{\theta}^{\tilde{c}}$ dashed(--) : standard estimates $\hat{\theta}$ [15]; dashdot(-.): actual value

Let $\mathcal{H} \leq(\hbar \times r)$ denotes the number of distinct elements in the dither amplitude matrix $\mathcal{A}(t)$ and let $a \in \mathbb{R}^{\mathcal{H}}$ be a vector of these distinct coefficients. The amplitude of the excitation signal is specified as

$$
a(t)= \begin{cases}a, & \text { if } t<t_{c} \\ 0, & \text { otherwise }\end{cases}
$$

or approximated by

$$
a(t) \approx \frac{a}{1+\exp ^{2 \nu\left(t-t_{e}\right)}}
$$

where equality holds in the limit as $\nu \rightarrow \infty$. 


\section{Simulation Examples}

\subsection{Example 1}

We consider the following nonlinear system in parametric strict feedback form [15]:

$$
\begin{aligned}
\dot{x}_{1} & =x_{2}+\theta_{1} x_{1} \\
\dot{x}_{2} & =x_{3}+\theta_{2} x_{1} \\
\dot{x}_{3} & =\theta_{3} x_{1}^{3}+\theta_{4} x_{2}+\theta_{5} x_{3}+\left(1+x_{1}^{2}\right) u \\
y & =x_{1},
\end{aligned}
$$

where $\theta^{T}=\left[\theta_{1}, \ldots, \theta_{5}\right]$ are unknown parameters. Using an adaptive backstep-ping design, the control and parameter update law presented in [15] were used for the simulation. The pair stabilize the plant and ensure that the output $y$ tracks a reference signal $y_{r}(t)$ asymptotically. For simulation purposes, parameter values are set to $\theta^{T}=[-1,-2,1,2$, $3]$ as in [15] and the reference signal is $y_{r}=1$, which is sufficiently rich of order one. The simulation results for zero initial conditions are shown in Figure 2. Based on the convergence analysis procedure in [15], all the parameter estimates cannot converge to their true values for this choice of constant reference. As confirmed in Fig. 2, only $\theta_{1}$ and $\theta_{2}$ estimates are accurate. However, following the proposed estimation technique and implementing the FT identifier (14), we obtain the exact parameter estimates at $t=17 \mathrm{sec}$. This example demonstrates that, with the proposed estimation routine, it is possible to identify parameters using perturbation or reference signals that would otherwise not provide sufficient excitation for standard adaptation methods.

\subsection{Example 2}

To corroborate the superiority of the developed procedure, we demonstrate the robustness of the developed procedure by considering system (36) with added exogeneous disturbances as follows:

$$
\begin{aligned}
\dot{x}_{1} & =x_{2}+\theta_{1} x_{1}+\left[\begin{array}{ll}
1 & 0
\end{array}\right] \vartheta \\
\dot{x}_{2} & =x_{3}+\theta_{2} x_{1}+\left[\begin{array}{ll}
1 & x_{1}
\end{array}\right] \vartheta \\
\dot{x}_{3} & =\theta_{3} x_{1}^{3}+\theta_{4} x_{2}+\theta_{5} x_{3}+\left(1+x_{1}^{2}\right) u+\left[\begin{array}{ll}
0 & 1
\end{array}\right] \vartheta \\
y & =x_{1},
\end{aligned}
$$

where $\vartheta=\left[0.1 \sin (2 \pi t / 5)\right.$ and the tracking signal remains a constant $y_{r}=1$.

The simulation result, Figure 3, shows convergence of the estimate vector to a small neighbourhood of $\theta$ under finite-time identifier with filter gain $k_{w}=1$ while no full parameter convergence is achieved with the standard identifier. The parameter estimation error $\tilde{\theta}(t)$ is depicted in Figure 4 for different values of the filter gain $k_{w}$. The switching time for the simulation is selected as the time for which the condition number of $Q$ becomes less than 20. It is noted that the time at which switching from standard adaptive estimate to FT estimate occurs increases as the filter gain increases. The convergence performance improves as $k_{w}$ increases, however, no significant improvement is observed as the gain is increased beyond 0.5 . 


\section{Performance Improvement in Adaptive Control via Finite-time Identification Procedure}

This section demonstrates how the finite-time identification procedure presented in section 3 can be employed to improve the overall performance (both transient and steady state) of adaptive control systems in a very appealing manner. Fisrt, we develop an adaptive compensator which guarantees exponential convergence of the estimation error provided the integral of a filtered regressor matrix is positive definite. The approach does not involve online checking of matrix in-vertibility and computation of matrix inverse nor switching between parameter estimation methods. The convergence rate of the parameter estimator is directly proportional to the adaptation gain and a measure of the system's excitation. The adaptive compensator is then combined with existing adaptive controllers to guarantee exponential stability of the closed-loop system.
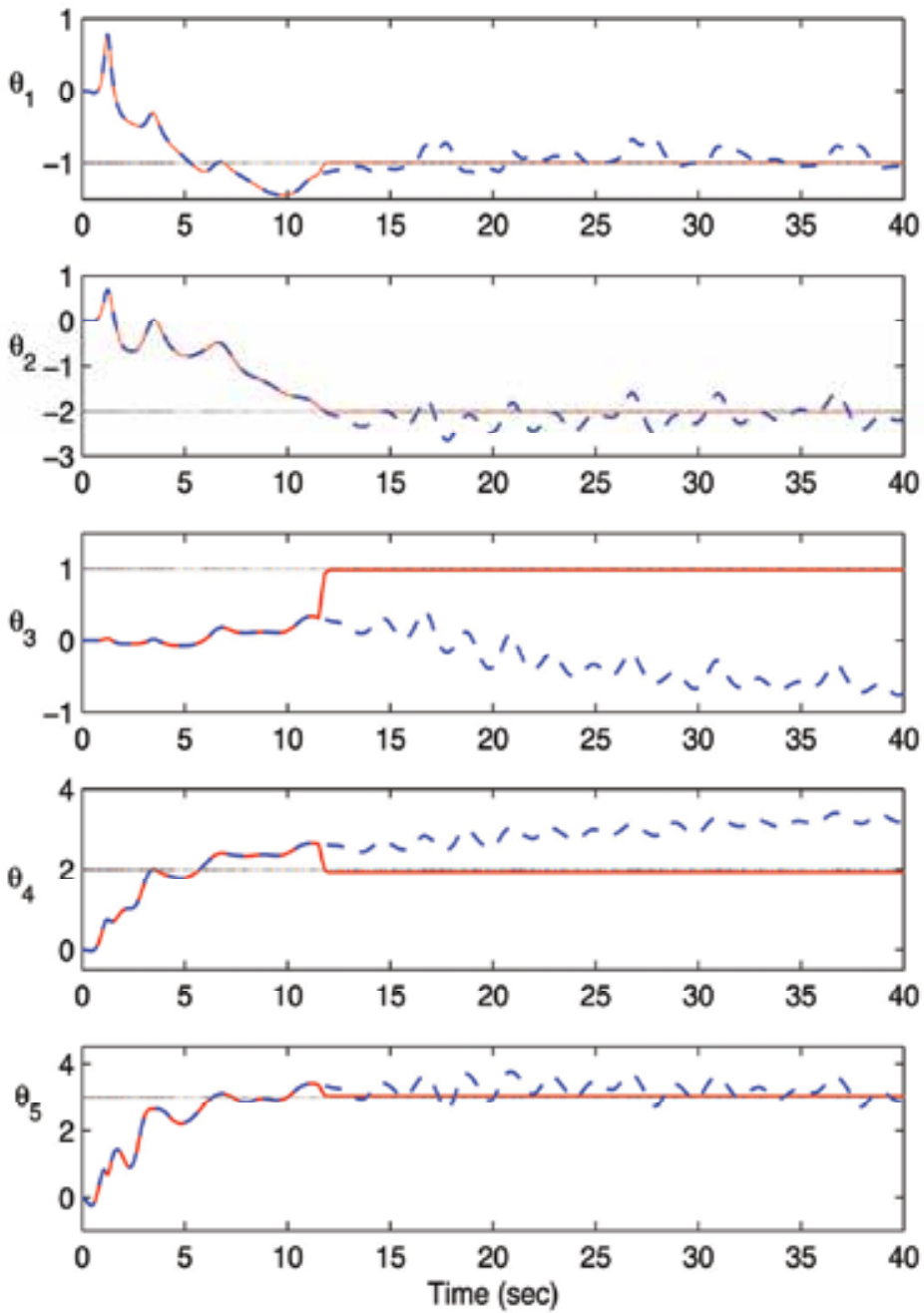

Figure 3. Trajectories of parameter estimates. Solid(-) : FT estimates for the system with additive disturbance $\hat{\theta}^{\tilde{c}}$, dashed(--): standard estimates $\hat{\theta}$ [15]; dashdot(-.): actual value 


\section{Adaptive Compensation Design}

Consider the nonlinear system 1 satisfying assumption 2.1 and the state predictor

$$
\dot{\hat{x}}=f(x, u)+g(x, u) \theta^{0}+k_{w}(x-\hat{x})
$$

where $k_{w}>0$ and $\theta^{0}$ is the nominal initial estimate of $\theta$. If we define the auxiliary variable

$$
\eta=x-\hat{x}-w\left(\theta-\theta^{0}\right)
$$

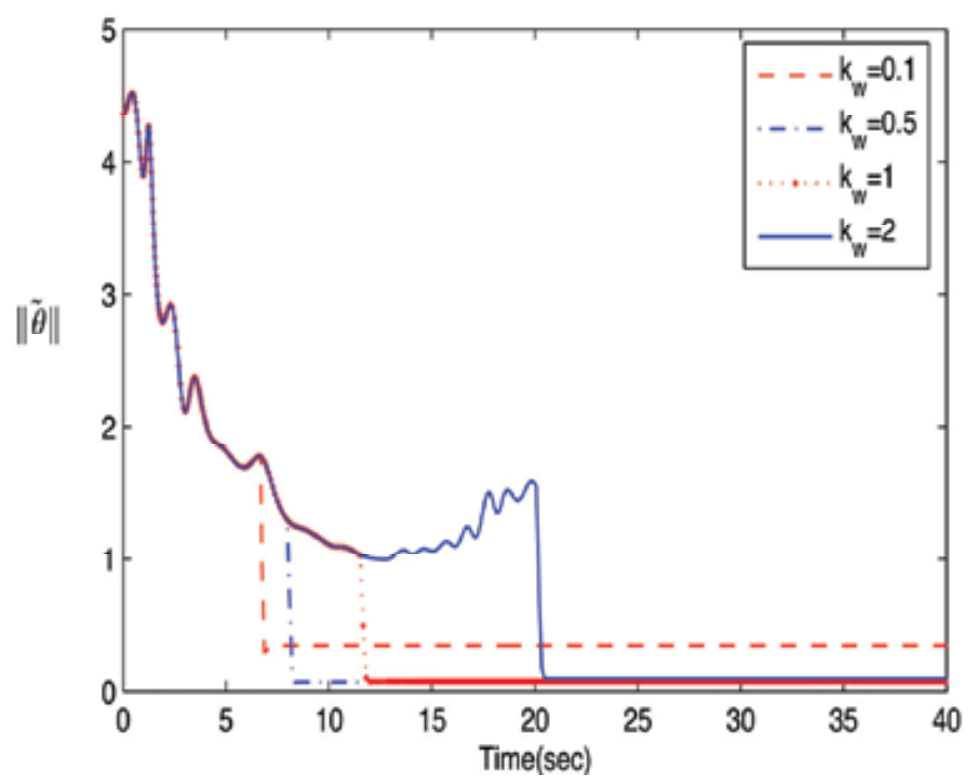

Figure 4. Parameter estimation error for different filter gains $k_{w}$ and select the filter dynamic as

$$
\dot{w}=g(x, u)-k_{w} w, \quad w\left(t_{0}\right)=0
$$

then $\eta$ is generated by

$$
\dot{\eta}=-k_{w} \eta, \quad \eta\left(t_{0}\right)=e\left(t_{0}\right) .
$$

Based on (38) to (41), our novel adaptive compensation result is given in the following theorem.

Theorem 8.1 Let $Q$ and $C$ be generated from the following dynamics:

$$
\begin{array}{rlrl}
\dot{Q} & =w^{T} w, & Q\left(t_{0}\right)=0 \\
\dot{C}=w^{T}\left(w \theta^{0}+x-\hat{x}-\eta\right), & C\left(t_{0}\right)=0
\end{array}
$$

and let $t_{c}$ be the time such that $Q\left(t_{c}\right) \succ 0$, then the adaptation law

$$
\dot{\hat{\theta}}=\Gamma(C-Q \hat{\theta}), \quad \hat{\theta}\left(t_{0}\right)=\theta^{0}
$$


with $\Gamma=\Gamma^{T} \succ 0$ guarantees that $\|\tilde{\theta}\|=\|\theta-\hat{\theta}\|$ is non-increasing for to $t_{0} \leq t \leq t_{c}$ and converges to zero exponentially fast, starting from $t_{c}$. Moreover, the convergence rate is lower bounded by $\mathcal{E}(t)=\lambda_{\min }(\Gamma Q(t))$.

Proof: Consider a Lyapunov function

$$
V_{\tilde{\theta}}=\frac{1}{2} \tilde{\theta}^{T} \tilde{\theta}
$$

it follows from (43) that

$$
\dot{V}_{\tilde{\theta}}(t)=-\tilde{\theta}^{T}(t) \Gamma(C(t)-Q(t) \hat{\theta}(t)) .
$$

Since $w \theta=w \theta^{0}+x-\hat{x}-\eta($ from (39)), then

$$
C(t)=\int_{t_{0}}^{t} \dot{C}(\tau) d \tau=\int_{t_{0}}^{t} w^{T}(\tau) w(\tau) d \tau \theta=Q(t) \theta
$$

and equation (45) becomes

$$
\begin{aligned}
\dot{V}_{\tilde{\theta}}(t) & =-\tilde{\theta}^{T}(t) \Gamma Q(t) \tilde{\theta}(t) \\
& \leq-\mathcal{E}(t) V_{\tilde{\theta}}(t)
\end{aligned}
$$

This implies non-increase of $\|\tilde{\theta}\|$ for $t \geq t_{0}$ and the exponential claim follows from the fact that $\Gamma Q(t)=\Gamma \int_{t_{0}}^{t} w(\tau)^{T} w(\tau) d \tau$ is positive definite for all $t \geq t_{c}$. The convergence rate is shown by noting that

$$
\begin{gathered}
\dot{V}_{\tilde{\theta}}(t)=-\tilde{\theta}^{T}(t) \Gamma\left(Q\left(t_{c}\right)+\int_{t_{c}}^{t} w(\tau)^{T} w(\tau) d \tau\right) \tilde{\theta}(t), \quad \forall t \geq t_{c} \\
\leq-\tilde{\theta}^{T}(t) \Gamma Q\left(t_{c}\right) \tilde{\theta}(t) \leq-\mathcal{E}\left(t_{c}\right) V(t)
\end{gathered}
$$

which implies

$$
\|\tilde{\theta}(t)\| \leq \exp ^{-\mathcal{E}\left(t_{c}\right)\left(t-t_{0}\right)}\left\|\tilde{\theta}\left(t_{0}\right)\right\|, \quad \forall t \geq t_{c}
$$

Both the FT identification (9) and the adaptive compensator (43) use the static relationship developed between the unknown parameter $\theta$ and some measurable matrix signals $C$, i.e, $Q \theta=C$. However, instead of computing the parameter values at a known finite-time by inverting matrix $Q$, the adaptive compensator is driven by the estimation error $C-Q \hat{\theta}=Q \tilde{\theta}$.

\section{Incorporating Adaptive Compensator for Performance Improvement}

It is assumed that the given control law $u$ and stabilizing update law (herein denoted as $\dot{\hat{\theta}}^{s}$ ) result in closed-loop error system

$$
\dot{Z}=A Z+\Phi^{T} \tilde{\theta}^{s}
$$




$$
\dot{\tilde{\theta}}^{s}=-\Gamma \Phi Z
$$

where the matrix $A$ is such that $A+A^{T}<-2 k_{A} I<0, \Phi$ is a bounded matrix function of the regressor vectors, $\tilde{\theta}^{s}=\theta-\hat{\theta}^{s}$ and $Z=\left[z_{1}, z_{2}, \ldots z_{n_{x}}\right]^{T}$ is a vector function of the tracking error with $z_{1}=y-y^{r}$. This implies that the adaptive controller guarantees uniform boundedness of the estimation error $\tilde{\theta}^{s}$ and asymptotic convergence of the tracking error $Z$ dynamics. Such adaptive controllers are very common in the literature. Examples include linearized control laws [16] and controllers designed via backstepping [12, 15].

Given the stabilizing adaptation law $\tilde{\theta}^{s}$, we propose the following update law which is a combination of the stabilizing update law (52b) and the adaptive compensator (43)

$$
\dot{\hat{\theta}}=\Gamma(\Phi Z+C-Q \hat{\theta}) .
$$

Since $C(t)=Q(t) \theta$, the resulting error equations becomes

$$
\left\lceil\begin{array}{c}
\dot{Z} \\
\dot{\tilde{\theta}}
\end{array}\right]=\left[\begin{array}{cc}
A & \Phi^{T} \\
-\Gamma \Phi & -\Gamma Q
\end{array}\right]\left[\begin{array}{c}
Z \\
\tilde{\theta}
\end{array}\right] .
$$

Considering the Lyapunov function $V=\frac{1}{2}\left(z^{T} z+\tilde{\theta}^{T} \Gamma^{-1} \tilde{\theta}\right)$ and differentating along (54) we have

$$
\dot{V}=\frac{1}{2} z^{T}\left(A+A^{T}\right) z-\tilde{\theta}^{T} Q \tilde{\theta} \leq-k_{A} z^{T} z-\tilde{\theta}^{T} Q \tilde{\theta}
$$

Hence $\tilde{\theta} \rightarrow 0$ exponentially for $t \geq t_{c}$ and the initial asymptotic convergence of $Z$ is strengthened to exponential convergence.

For feedback linearizable systems

$$
\begin{aligned}
\dot{x}_{i} & =x_{i+1} \quad 1 \leq i \leq n-1 \\
\dot{x}_{n} & =f_{1}(x)+f_{2}(x) u+\theta^{T} g_{n}(x) \\
y & =x_{1}
\end{aligned}
$$

the PE condition $Q\left(t_{c}\right) \succ 0$ translates to a priori verifiable sufficient condition on the reference setpoint. It requires the rows of the regressor vector $g_{n}(x)$ to be linearly independent along a desired trajectory $x^{r}(t)$ on any finite interval $t \in\left[t_{1}, t_{2}\right), t_{1}<t_{2}<\infty$. This condition is less restrictive than the one given in [9] for the same class of system. This is because the linear independence requirement herein is only required over a finite interval and it can be satisfied by a non-periodic reference trajectory while the asymptotic stability result in [9] relies on a T-periodic reference setpoint. Moreover exponential, rather than asymptotic stability of the parametric equilibrium is achieved. 


\section{Dither Signal Update}

Perturbation signal is usually added to the desired reference setpoint or trajectory to guarantee the convergence of system parameters to their true values. To reduce the variability of the closed-loop system, the added PE signal must be systematically removed in a way that sustains parameter convergence.

Suppose the dither signal $d(t)$ is selected as a linear combination of sinusoidal functions as detailed in Section 5. Let $a^{0}$ be the vector of the selected dither amplitude and let $T>0$ be the first instant for which $d(T)=0$, the amplitude of the excitation signal is updated as follows:

$$
a(t)= \begin{cases}a^{0}, & t \in[0, T) \\ \exp ^{-\gamma \bar{\varepsilon} T} a(j-1) T, & t \in[j T,(j+1) T), \quad j \geq 1\end{cases}
$$

where the gain $\gamma>0$ is a design parameter, $a(0)=a^{0}$ and

$$
\begin{aligned}
& \mathcal{E}(0)=0, \quad \mathcal{E}(\tau)=\lambda_{\min }(Q(\tau)) \\
& \overline{\mathcal{E}}=\max \{\mathcal{E}(j T), \quad \mathcal{E}((j-1) T)\} .
\end{aligned}
$$

It follows from (56) that the reference setpoint will be subject to PE with constant amplitude $a^{0}$ if $t \in[0, T)$. After which the trajectory of $a(t)$ will be dictated by the filtered regressor matrix $Q$. The amplitude vector $a(t)$ will start to decay exponentially when $Q(t)$ becomes positive definite. Note that parameter convergence will be achieved regardless of the value of the gain $\gamma$ selected as the only requirement for convergence is $Q(t) \succ 0$.

Remark 10.1 The other major approach used in traditional adaptive control is parameter estimation based design. A well designed estimation based adaptive control method achieves modularity of the controller-identifier pair. For nonlinear systems, the controller module must possess strong parametric robustness properties while the identifier module must guarantee certain boundedness properties independent of the control module. Assuming the existence of a bounded controller that is robust with respect to $(\tilde{\theta}, \dot{\hat{\theta}})$, the adaptive compensator (43) serves as a suitable identifier for modular adaptive control design.

\section{Simulation Example}

To demonstrate the effectiveness of the adaptive compensator, we consider the example in Section 6 for both the nominal system (36) and the system under additive disturbance (37). The simulation is performed for the same reference setpoint $y_{r}=1$, disturbance vector $\vartheta=[0.1 \sin (2 \pi t / 5), 0.2 \cos (\pi t)]^{T}$, parameter values $\theta=[-1,-2,1,2,3]$ and zero initial conditions.

The adaptive controller presented in [15] is also used for the simulation. We modify the given stabilizing update law by adding the adaptive compensator (43) to it. The modification significantly improve upon the performance of the standard adaptation mechanism as shown in Figures 5 and 6. All the parameters converged to their values and we recover the performance of the finite-time identifier (14). Figures 7 and 8 depict the performance of the output and the input trajectories. While the transient behaviour of the 
output and input trajectories is slightly improved for the nominal adaptive system, a significant improvement is obtained for the system subject to additive disturbances.

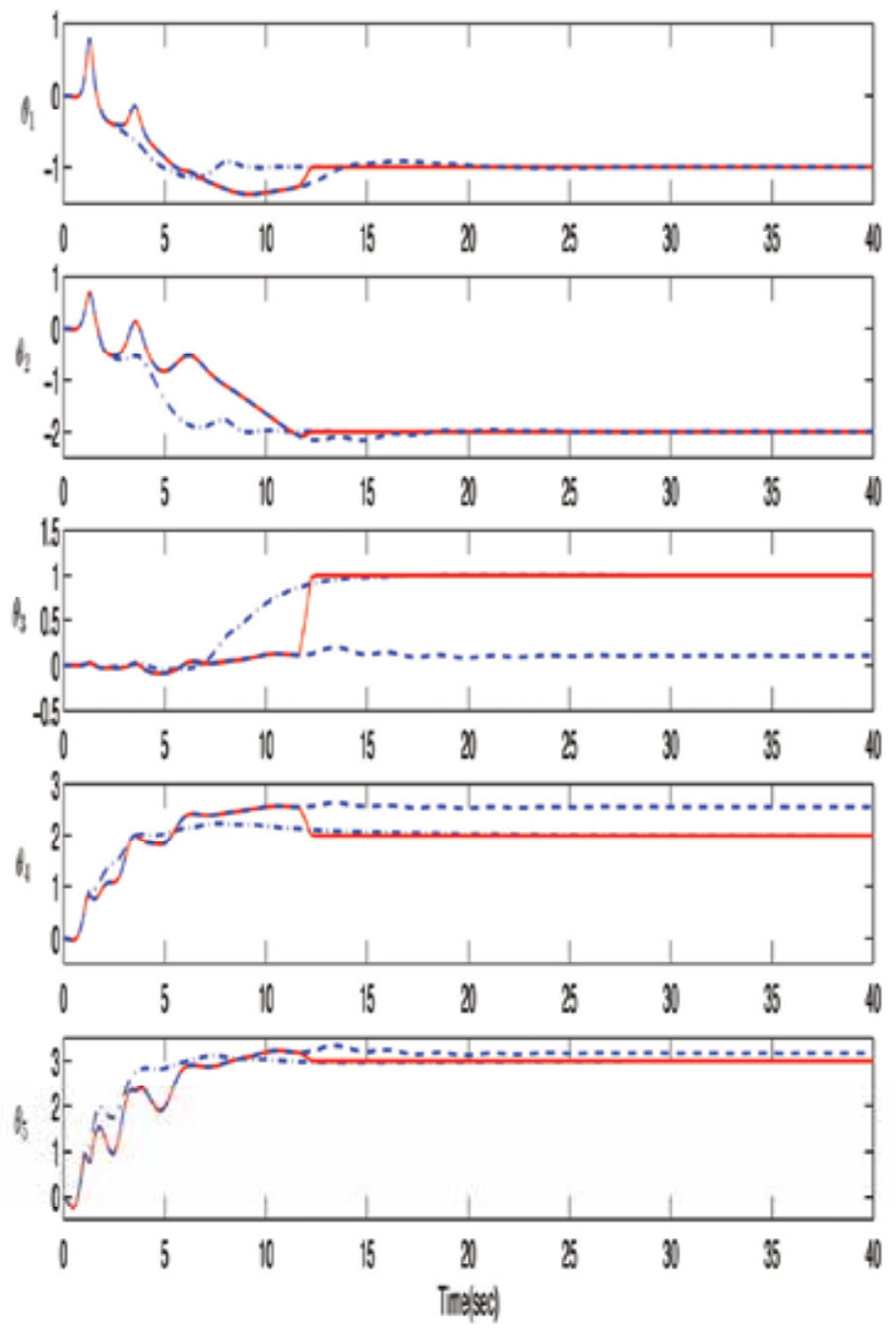

Figure 5. Trajectories of parameter estimates. Solid(-) : compensated estimates; dashdot(-.): FT estimates; dashed(--) : standard estimates [15] 

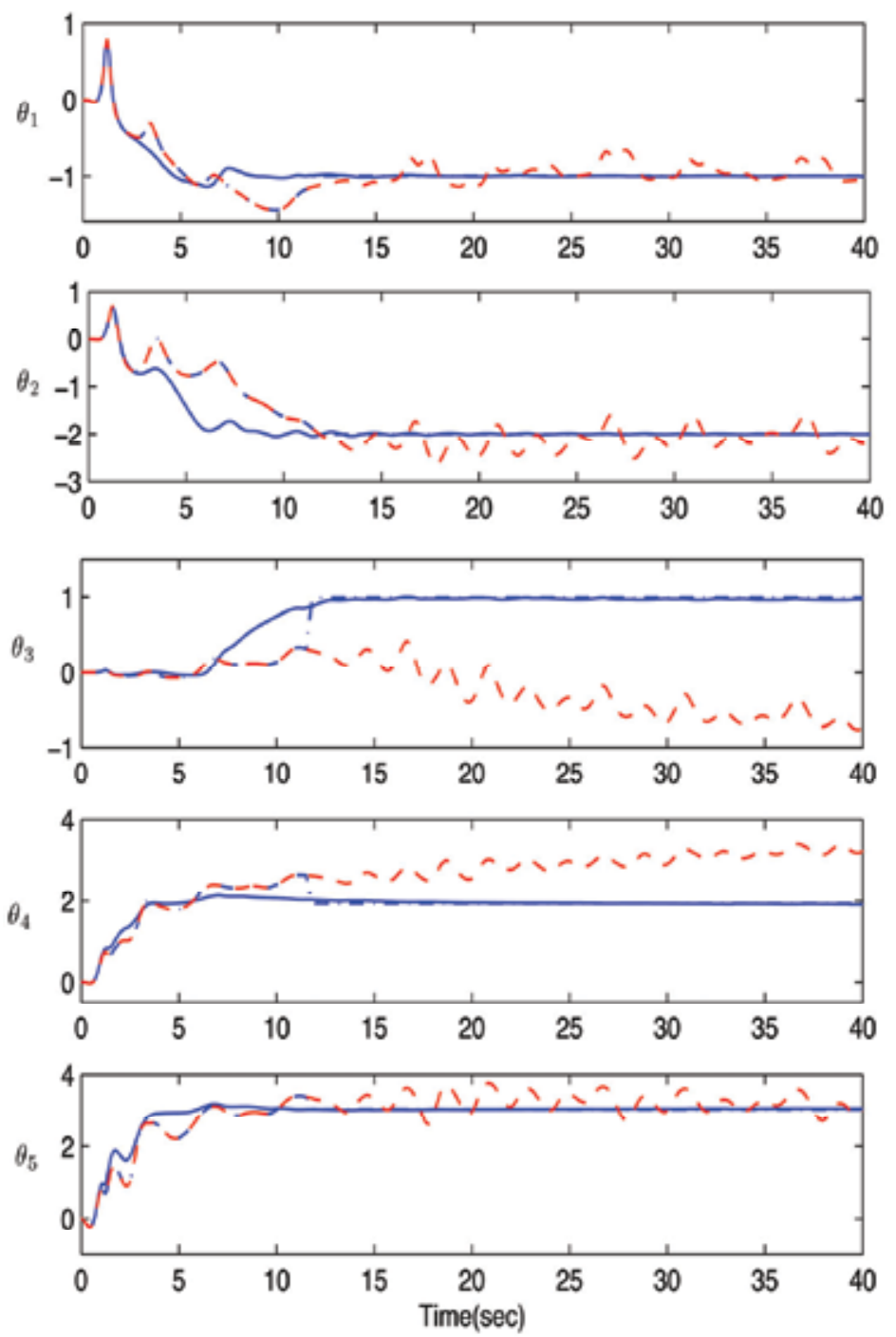

Figure 6. Trajectories of parameter estimates under additive disturbances. Solid(-): compensated estimates; dashdot(-.): FT estimates; dashed(--) : standard estimates [15] 

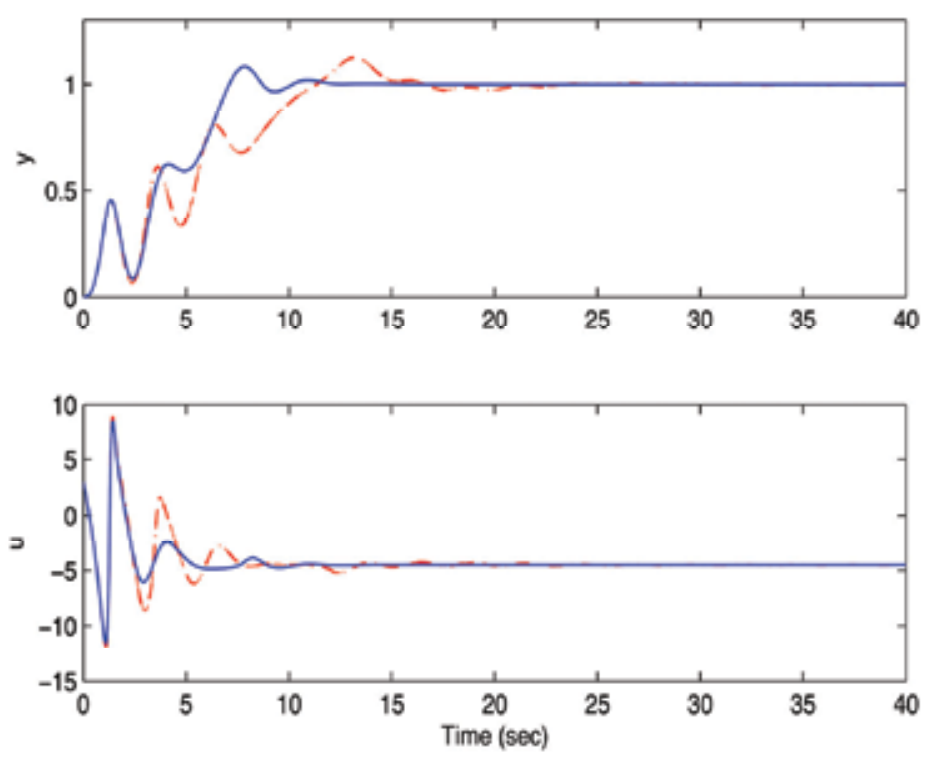

Figure 7. Trajectories of system's output and input for different adaptation laws. Solid(-): compensated estimates; dashdot(-.): FT estimates; dashed(--) : standard estimates [15]
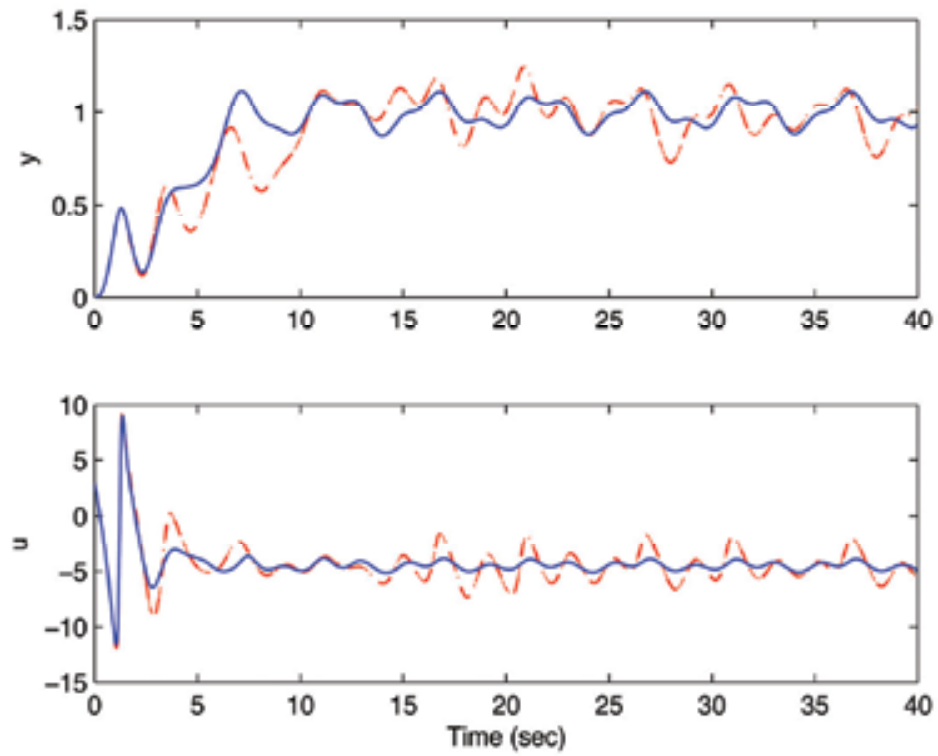

Figure 8. Trajectories of system's output and input under additive disturbances for different adaptation laws. Solid(-) : compensated estimates; dashdot(-.): FT estimates; dashed(--) : standard estimates [15] 


\section{Conclusions}

The work presented in this chapter transcends beyond characterizing the parameter convergence rate. A method is presented for computing the exact parameter value at a finite-time selected according to the observed excitation in the system. A smooth transition from a standard estimate to the FT estimate is proposed. In the presence of unknown bounded disturbances, the FT identifier converges to a neighbourhood of the true value whose size is dictated by the choice of the filter gain. Moreover, the procedure preserves the system's established closed-loop properties whenever the required PE condition is not satisfied. We also demonstrate how the finite-time identification procedure can be used to improve the overall performance (both transient and steady state) of adaptive control systems in a very appealing manner. The adaptive compensator guarantees exponential convergence of the estimation error provided a given PE condition is satisfied. The convergence rate of the parameter estimator is directly proportional to the adaptation gain and a measure of the system's excitation. The adaptive compensator is then combined with existing adaptive controllers to guarantee exponential stability of the closed-loop system. The application reported in Section 9 is just an example, the adaptive compensator can easily be incorporated into other adaptive control algorithms.

\section{References}

V. Adetola and M. Guay. Parameter convergence in adaptive extremum seeking control. Automatica, 43(1):105-110, 2007. [1]

V. Adetola and M. Guay. Finite-time parameter estimation in adaptive control of nonlinear systems. IEEE Transactions on Automatic Control, 53(3):807-811, 2008. [2]

V.A. Adetola and M. Guay. Excitation signal design for parameter convergence in adaptive control of linearizable systems. In Proceedings of the 45th IEEE Conference on Decision and Control, San Diego, CA, USA, 2006. [3]

Chengyu Cao, Jiang Wang, and N. Hovakimyan. Adaptive control with unknown parameters in reference input. In Proceedings of the 2005 IEEE International Symposium on, Mediterrean Conference on Control and Automation, pages 225-230, June 2005. [4]

C.A. Desoer and M. Vidyasagar. Feedback Systems: Input-Output Properties. Academic Press, New York, 1975. [5]

F. Floret-Pontet and F. Lamnabhi-Lagarrigue. Parameter identification and state estimation for continuous-time nonlinear systems. In American Control Conference, 2002. Proceedings of the 2002, volume 1, pages 394-399vol. 1, 8-10 May 2002. [6]

M. A. Golberg. The derivative of a determinant. The American Mathematical Monthly, 79(10):1124-1126, 1972. [7]

M. Guay, D. Dochain, and M. Perrier. Adaptive extremum seeking control of continuous stirred tank bioreactors with unknown growth kinetics. Automatica, 40:881-888, 2004. [8]

Jeng Tze Huang. Sufficient conditions for parameter convergence in linearizable systems. IEEE Transactions on Automatic Control, $48: 878$ - 880, 2003. [9]

P.A loannou and Jing Sun. Robust Adaptive Control. Pentice Hall, Upper Saddle River, New Jersey, 1996. [10] 
Gerhard Kreisselmeier. Adaptive observers with exponential rate of convergence. IEEE Transactions on Automatic Control, 22:2-8, 1977. [11]

M. Krstic, I. Kanellakopoulos, and P. Kokotovic. Nonlinear and Adaptive Control Design. John Wiley and Sons Inc, Toronto, 1995. [12]

I. D. Landau, B. D. O. Anderson, and F. De Bruyne. Recursive identification algorithms for continuous-time nonlinear plants operating in closed loop. Automatica, 37(3):469475, March 2001. [13]

Jung-Shan Lin and loannis Kanellakopoulos. Nonlinearities enhance parameter convergence in output-feedback systems. IEEE Transactions on Automatic Control, 43:204-222, 1998. [14]

Jung-Shan Lin and loannis Kanellakopoulos. Nonlinearities enhance parameter convergence in strict feedback systems. IEEE Transactions on Automatic Control, 44:89-94, 1999. [15]

Ricardo Marino and Patricio Tomei. Nonlinear Control Design. Prentice Hall, 1995. [16]

Riccardo Marino and Patrizio Tomei. Adaptive observers with arbitrary exponential rate of convergence for nonlinear systems. IEEE Transactions on Automatic Control, 40:13001304, 1995. [17]

K.S Narendra and A.M Annaswamy. Stable and Adaptive Systems. Prentice Hall New Jersey, 1989. [18]

Marc; Niethammer, Patrick Menold, and Frank Allgower. Parameter and derivative estimation for nonlinear continuous-time system identification. In 5th IFAC Symposium Nonlinear Control Systems (NOLCOS'Ol), Russia, 2001. [19]

S. Sastry and Marc Bodson. ADAPTIVE CONTROL Stability, Convergence, and Robustness. Prentice Hall, New Jersey, 1989. [20]

H.H. Wang, M Krstic, and G. Bastin. Optimizing bioreactors by extremum seeking. International Journal of Adaptive Control and Signal Processing, 13:651-669, 1999. [21]

Jian-Xin $\mathrm{Xu}$ and Hideki Hashimoto. Parameter identification methodologies based on variable structure control. International Journal of Control, 57(5):1207-1220, 1993. [22]

Jian-Xin $\mathrm{Xu}$ and Hideki Hashimoto. VSS theory-based parameter identification scheme for MIMO systems. Automatica, 32(2):279-284, 1996. [23] 


\title{
Estimation and Control of Stochastic Systems under Discounted Criterion
}

\author{
Hilgert Nadine ${ }^{1}$ and Minjárez-Sosa J. Adolfo ${ }^{2}$ \\ IUMR 729 ASB, INRA SUPAGRO, Montpellier, \\ ${ }^{2}$ Departamento de Matemáticas, Universidad de Sonora, Hermosillo \\ ${ }^{1}$ rance, ${ }^{2}$ Mexico
}

\section{Introduction}

We consider a class of discrete-time Markov control processes evolving according to the equation

$$
x_{t+1}=F\left(x_{t}, a_{t}, \xi_{t}\right), \quad t=0,1, \ldots,
$$

where $x_{t}, a_{t}$ and $\xi_{t}$ are the state, action and random disturbance at time $t$ respectively, taking values on Borel spaces. $F$ is a known continuous function. Moreover, $\left\{\xi_{t}\right\}$ is an observable sequence of independent and identically distributed (i.i.d.) random vectors with distribution $\theta_{\xi}$. This class of control systems has been widely studied assuming that all the components of the corresponding control model are known by the controller. In this context, the evolution of the system is as follows. At each stage $t$, on the knowledge of the state $x_{t}=x$ as well as the history of the system, the controller has to select a control or action $a_{t}=a$. Then a cost $c$, depending on $x$ and $a$, is incurred, and the system moves to a new state $x_{t+1}=x^{\prime}$ according to the transition probability determined by the equation (1). Once the transition to state $x^{\prime}$ occurs, the process is repeated. Moreover, the costs are accumulated throughout the evolution of the system in an infinite horizon using a discounted criterion. The actions applied at any given time are selected according to rules known as control policies, and therefore the standard optimal control problem is to determine a control policy that minimizes a discounted cost criterion.

However, assuming the knowledge of all components of the control model might be non realistic from the point of view of the applications. In this sense we consider control models that may depend on an unknown component.

Two cases are discussed in the present chapter. In the first one we assume that the disturbance distribution $\theta_{\xi}$ is unknown, whereas in the second one we consider a cost function depending on an exogenous random variable $\eta_{t}$ at time $t$, whose distribution $\theta_{\eta}$ is unknown. First situation is well documented in the literature and will be briefly described, while the second is less known (even if it is of great interest for application problems) and will be largely developed.

Thus, in contrast with the evolution of a standard system as described above, in both cases, before choosing the control $a_{t}$, the controller has to implement a statistical estimation procedure of $\theta_{\xi}$ (or $\theta_{\eta}$ ) to get an estimate $\theta_{\xi, t}$ (or $\theta_{\eta, t}$ ), and combines this with the history of 
the system to select a control $a=a_{t}^{\theta_{\xi, t}}$ (or $a_{t}^{\theta_{\eta, t}}$ ). The resulting policy in this estimation and control process is called adaptive. Therefore, the optimal control problem we are dealing with in this chapter is to construct adaptive policies that minimize a discounted cost criterion. Furthermore, we study the optimality of such policies in an asymptotic sense.

The chapter is organized as follows. The models and definitions are introduced in the Section 2, as well as an overview of adaptive Markov control processes under discounted criteria. In particular, the required sets of assumptions are introduced and commented. The Section 3 is dedicated to the adaptive control of stochastic systems in the case where the cost function depends on an exogenous random variable with unknown distribution. Here we present two approaches to construct optimal adaptive policies. Finally we conclude in Section 4 with some remarks.

Remark 1.1 Given a Borel space $X$ (that is, a Borel subset of a complete and separable metric space) its Borel sigma-algebra is denoted by $\mathcal{B}(X)$, and "measurable", for either sets or functions, means "Borel measurable". The space of probability measures on $X$ is denoted by $\mathbb{P}(X)$. Let $X$ and $Y$ be Borel spaces. Then a stochastic kernel $Q(d x \mid y)$ on $X$ given $Y$ is a function such that $Q(\cdot \mid y)$ is a probability measure on $X$ for each fixed $y \in Y$, and $Q(B \mid \cdot)$ is a measurable function on $Y$ for each fixed $B \in \mathcal{B}(X)$.

\section{Adaptive stochastic optimal control problems}

\subsection{Markov control models}

We consider a class of discrete-time Markov control models

$$
\mathcal{M}:=(X, A,\{A(x) \subset A \mid x \in X\}, c, Q)
$$

satisfying the following conditions. The state space $X$ and action space $A$ are Borel spaces endowed with their Borel $\sigma$-algebras (See Remark 1.1). For each state $x \in X, A(x)$ is a nonempty Borel subset of $A$ denoting the set of admissible controls when the system is in state $x$. The set

$$
\mathbb{K}=\{(x, a): x \in X, a \in A(x)\}
$$

of admissible state-action pairs is assumed to be a Borel subset of the Cartesian product of $X$ and $A$. In addition, the cost-per-stage $c(x, a)$ is a nonnegative measurable real-valued function, possibly unbounded, and depends on the pair $(x, a) \in \mathbb{K}$. Finally, the transition law of the system $Q(\cdot)$ is a stochastic kernel on $X$ given $\mathbb{K}$. That is, for all $t \in \mathbb{N},(x, a) \in \mathbb{K}$ and $B \in \mathcal{B}(X)$,

$$
Q(B \mid x, a):=P\left[x_{t+1} \in B \mid x_{t}=x, a_{t}=a\right] .
$$

We will consider independently the two following cases:

- $\quad 1$ st case: the stochastic kernel $Q$ is unknown, as depending on the system disturbance distribution $\theta_{\xi}$, which is unknown. We have, for all $(x, a) \in \mathbb{K}$ and $B \in \mathcal{B}(X)$,

$$
\begin{aligned}
Q(B \mid x, a) & =\int_{S} 1_{B}(F(x, a, s)) \theta_{\xi}(d s) \\
& =\theta_{\xi}(\{s \in S: F(x, a, s) \in B\}),
\end{aligned}
$$


where $S$ is the Borel space of the disturbance $\xi$ in (1). The Markov control model under consideration can also be noted $\mathcal{M}_{\xi}:=\left(X, A,\{A(x) \subset A \mid x \in X\}, S, F, c, \theta_{\xi}\right)$.

- 2nd case: the cost function $c$ is poorly known, as depending on the unknown distribution $\theta_{\eta}$, of a stochastic variable $\%$ through the relation:

$$
c(x, a):=E_{\theta_{\eta}} \bar{c}(x, a, \eta),
$$

where $\eta$ is an exogenous variable belonging to a Borel space $S$ and $E_{\theta_{\eta}}$ denotes the expectation operator with respect to the probability distribution $\theta_{\eta}$. Thus

$$
\theta_{\eta}(B)=P\left(\eta_{t} \in B\right), t \in \mathbb{N}, B \in \mathcal{B}(S) .
$$

The function $\bar{c}$ is in fact the true cost function, whose mean $c$ is unknown, which yields the following Markov control model $\mathcal{M}_{\eta}:=\left(X, A,\{A(x) \subset A \mid x \in X\}, Q, S, \theta_{\eta}, \bar{c}\right)$.

Throughout the paper we suppose that the random variables $\xi$ and $\eta$ are defined on an underlying probability space $(\Omega, \mathcal{F}, P)$, and a.s. means almost surely with respect to $P$. In addition, we assume the complete observability of the states $x_{0}, x_{1}, \ldots$, and also of the realizations $\eta_{0}, \eta_{1}, \ldots$ or $\xi_{0}, \xi_{1}, \ldots$ when their distribution is unknown.

\subsection{Set of admissible policies}

We define the spaces of admissible histories up to time $t$ by $\mathbb{H}_{0}:=X$ and $\mathbb{H}_{t}:=(\mathbb{K} \times S)^{t} \times X, t \geq 1$. A generic element of $\mathbb{H}_{t}$ is written as $h_{t}=\left(x_{0}, a_{0}, \eta_{0}, \ldots, x_{t-1}, a_{t-1}, \eta_{t-1}, x_{t}\right)$. A control policy $\pi=\left\{\pi_{t}\right\}$ is a sequence of measurable functions $\pi_{t}: \mathbb{H}_{t} \rightarrow A$ such that $\pi_{t}\left(h_{t}\right) \in A\left(x_{t}\right), h_{t} \in \mathbb{H}_{t}, t \geq 0$. Let $\Pi$ be the set of all control policies and $\mathbb{F} \subset \Pi$ the subset of stationary policies. If necessary, see for example (Dynkin \& Yushkevich, 1979); (Hernández-Lerma \& Lasserre, 1996 and 1999); (Hernández-Lerma, 1989) or (Gordienko \& Minjárez-Sosa, 1998) for further information on those policies. As usual, each stationary policy $\pi \in \mathbb{F}$ is identified with a measurable function $f: X \rightarrow A$ such that $f(x) \in A(x)$ for every $x \in X$, so that $\pi$ is of the form $\pi=\{f, f, f, \ldots\}$. In this case we denote $\pi$ by $f$, and we write

$$
c(x, f):=c(x, f(x)) \text { and } Q(B \mid x, f):=Q(B \mid x, f(x)),
$$

for all $x \in X$.

\subsection{Discounted criterion}

Once we are given a Markov control model $\mathcal{M}$ and a set $\Pi$ of admissible policies, to complete the description of an optimal control problem we need to specify a performance index, that is, a function measuring the system's performance when a given policy $\pi \in \Pi$ is used and the initial state of the system is $x_{0}=x$. This study concerns the $\alpha$-discounted cost, whose definition is as follows:

$$
V(\pi, x):=E_{x}^{\pi}\left[\sum_{t=0}^{\infty} \alpha^{t} c\left(x_{t}, a_{t}\right)\right], \quad x \in X, \pi \in \Pi .
$$

where $\alpha \in(0,1)$ is the so-called discount factor, and $E_{x}^{\pi}$ denotes the expectation operator with respect to the probability measure $P_{x}^{\pi}$ induced by the policy $\pi$, given the initial state $x_{0}$ $=x$. 
The $\alpha$-discounted criterion is one of the most famous long run criteria. Among the main motivations to study this optimality criterion are to analyze an economic or financial model as an optimal control problem (for instance optimal growth of capital model, see Stockey $\mathcal{E}$ Lucas (1989)), and the mathematical convenience (the discounted criterion is the best understood of all performance index). In fact, it is often studied before other more complicated criteria, like for example the expected average cost, which can be seen as the limit of $V(\pi, x)$ when $r$ tends to 1 .

The optimal control problem is then defined as follows: determine a policy $\pi^{*} \in \Pi$ such that:

$$
V\left(\pi^{*}, x\right)=\inf _{\pi \in \Pi} V(\pi, x) \text { for all } x \in X .
$$

The function $V^{*}$ defined by

$$
V^{*}(x):=\inf _{\pi \in \Pi} V(\pi, x), x \in X
$$

is called the value (or optimal cost) function. A policy $\pi^{*} \in \Pi$ is said to be $\alpha$-discount optimal (or simply $\alpha$-optimal) for the control model $\mathcal{M}$ if

$$
V^{*}(x)=V\left(\pi^{*}, x\right) \text { for all } x \in X .
$$

Note that, in the case of model $\mathcal{M}_{\psi}$ we are in fact interested by looking for optimal policies with respect to the general $\alpha$-discounted cost

$$
V_{\eta}(\pi, x):=E_{x}^{\pi}\left[\sum_{t=0}^{\infty} \alpha^{t} \bar{c}\left(x_{t}, a_{t}, \eta_{t}\right)\right], \quad x \in X, \pi \in \Pi .
$$

But, as $c$ is the mean cost of function $\bar{c}$, see (4), and using properties of conditional expectation, we have that $V_{\eta}(\pi, x)=V(\pi, x)$. So, looking for optimal policies for $V_{\eta}$, is equivalent to looking for optimal policies for $V$.

Since $\theta_{\xi}$ and $\theta_{\eta}$ are unknown, we combine suitable statistical estimation methods and control procedures in order to construct the adaptive policy. That is, we use the observed history of the system to estimate $\theta_{\xi}$ or $\theta_{\eta}$ and then adapt the decision or control to the available estimate. On the other hand, as the discounted cost depends heavily on the controls selected at the first stages (precisely when the information about the unknown distribution is poor or deficient), we can't ensure the existence of an $\alpha$-optimal adaptive policy (see Hernández-Lerma, 1989). Thus the $\alpha$-optimality of an adaptive policy will be understood in the following asymptotic sense:

Definition 2.1 (Schäl, 1987). A policy $\pi \in \Pi$ is said to be asymptotically discounted optimal for the control model $\mathcal{M}$ if

$$
\left|V^{(k)}(\pi, x)-E_{x}^{\pi}\left[V^{*}\left(x_{k}\right)\right]\right| \rightarrow 0 \text { as } k \rightarrow \infty, \text { for all } x \in X,
$$

where

$$
V^{(k)}(\pi, x):=E_{x}^{\pi}\left[\sum_{t=k}^{\infty} \alpha^{t-k} c\left(x_{t}, a_{t}\right)\right]
$$

is the expected total discounted cost from stage $k$ onward and $a_{t}=\pi_{t}\left(h_{t}\right)$.

In the above definition, the model $\mathcal{M}$ stands either for $\mathcal{M}_{\xi}$ or for $\mathcal{M}_{\eta}$. 
Remark 2.2 Let $\pi \in \Pi$ be a policy such that $V(\pi, x)<\infty$ for each $x \in X$, and $\left\{\left(x_{t}, a_{t}\right)\right\}$ be a sequence of state-actions pairs corresponding to application of $\pi$. In (Hernández-Lerma $\mathcal{E}$ Lasserre, 1996), it has been proved that $\pi$ is an asymptotically discounted optimal policy if, and only if, $E_{x}^{\pi} \Phi\left(x_{t}, a_{t}\right) \rightarrow 0$, as $t \rightarrow \infty$, where

$$
\Phi(x, a):=c(x, a)+\alpha \int_{X} V^{*}(y) Q(d y \mid x, a)-V^{*}(x), \quad(x, a) \in \mathbb{K},
$$

is the well-known discrepancy function, which is nonnegative from (15).

In the remainder of the paper, we fix an arbitrary discount factor $\alpha \in(0,1)$.

\subsection{Overview of adaptive Markov control processes with Borel state and action spaces, and possibly unbounded costs}

Even in the non adaptive case, handling Markov control processes with Borel state and action spaces, and possibly unbounded costs, requires much attention in the work space setting towards specific assumptions. Three types of hypotheses are usually imposed, see (Hernández-Lerma \& Lasserre, 1999). The first one is about compactness-continuity conditions for Markov control models. The second one introduces a weight function $W$ to impose a growth condition on the cost function, which will yield that the dynamic programming operator $T$ :

$$
T u(x):=\min _{A(x)}\left(c(x, a)+\alpha \int_{X} u(y) Q(d y \mid x, a)\right)
$$

is a contraction (on some space that will be specified later, see §3.1). The third type of assumptions is a further continuity condition, which combined with the previous ones, will ensure the existence of measurable minimizers for $T$. We don't detail these assumptions for the general non adaptive case. They are extended to model $\mathcal{M}_{\eta}$ in the adaptive case as follows:

Assumption 2.3 a) For each $x \in X$, the set $A(x)$ is $\sigma$-compact.

b) For each $x \in X$ the function $a \rightarrow \bar{c}(x, a, s)$ is l.s.c. on $A(x)$ for all s. Moreover, there exists $a$ measurable function $W: X \rightarrow[1, \infty)$ such that $\sup _{a \in A(x)} \sup _{s \in S} \bar{c}(x, a, s) \leq W(x)$ for all $x \in X$. (Recall that $\bar{c}$ is assumed to be nonnegative.)

c) There exist three constants $p>1, \beta_{0}<1$ and $b_{0}<+\infty$ such that for all $x \in X, a \in A(x)$,

$$
\int_{X} W^{p}(y) Q(d y \mid x, a) \leq \beta_{0} W^{p}(x)+b_{0} .
$$

d) The function $(x, a) \rightarrow \int_{X} v(y) Q(d y \mid x, a)$ is continuous and bounded on $\mathbb{K}$ for every bounded and continuous function $v$ on $X$.

e) For each $x \in X$, the function $a \rightarrow \int_{X} W(y) Q(d y \mid x, a)$ is continuous on $A(x)$.

Remark 2.4 Note that from Jensen's inequality, (11) implies

$$
\int_{X} W(y) Q(d y \mid x, a) \leq \beta W(x)+b, \quad \text { for all }(x, a) \in \mathbb{K},
$$

where $\beta=\beta_{0}^{1 / p}$ and $b=b_{0}^{1 / p}$. Moreover, a consequence for both inequalities (11) and (12), is (see (Gordienko \& Minjdrez-Sosa, 1998) or (Hernández-Lerma \& Lasserre, 1999)) 


$$
\sup _{n \geq 0} E_{x}^{\pi}\left(W^{p}\left(x_{n}\right)\right)<\infty \text { and } \sup _{n \geq 0} E_{x}^{\pi}\left(W\left(x_{n}\right)\right)<\infty,
$$

for each $\pi \in \Pi$ and $x \in X$.

We denote by $L_{W}^{\infty}$ the normed linear space of all measurable functions $u: X \rightarrow \Re$ with a finite norm $\|u\|_{W}$ defined as

$$
\|u\|_{W}:=\sup _{x \in X} \frac{|u(x)|}{W(x)} .
$$

A first consequence of Assumption 2.3 is the following proposition, which states the existence of a stationary $\alpha$-discount optimal policy in the general case:

Proposition 2.5 (Hernández-Lerma \& Lasserre, 1999) Suppose that Assumption 2.3 holds. Then: a) The function $V^{*}$ belongs to $L_{W}^{\infty}$ and satisfies the $\alpha$-discounted optimality equation

$$
V^{*}(x)=\min _{a \in A(x)}\left(c(x, a)+\alpha \int_{X} V^{*}(y) Q(d y \mid x, a)\right), x \in X
$$

Moreover, we have $0 \leq V^{*}(x) \leq W(x) /(1-\alpha), x \in X$.

b) There exists $f \in \mathbb{F}$ such that $f(x) \in A(x)$ attains the minimum in (15), i.e.

$$
V^{*}(x)=c(x, f)+\alpha \int_{X} V^{*}(y) Q(d y \mid x, f), x \in X,
$$

and the stationary policy $f$ is optimal.

As we already mentioned, our main concern is in the two cases of adaptive control we introduced in $\$ 2.1$ where the distribution $\theta_{\xi}$ or $\theta_{\eta}$ is unknown. Thus, the solution given in the Proposition 2.5 is not accessible to the controller. In fact, an estimation process has to be chosen, which depends on the knowledge we have of this distribution, for example: absolutely continuous with respect to the Lebesgue measure (and so with an unknown density). With the estimator on hand we can apply the "principle of estimation and control" proposed by Kurano (1972) and Mandl (1974). That is, we obtain an estimated optimality equation with which we can construct the adaptive policies.

The case of the model $\mathcal{M}_{\xi}$, and assuming that $\theta_{\xi}$ has a density, is described in (Gordienko $\mathcal{E}$ Minjárez-Sosa, 1998), and also in (Minjárez-Sosa, 1999) for the expected average cost. The estimation of $\theta_{\xi}$ is obtained by means of an estimator of its density function. However the unboundedness assumption on the cost $c$ makes difficult the implementation of the density estimation process. The estimator is defined by the projection (of an auxiliary estimator) on some special set of density functions to ensure good properties of the estimated model. Beyond the complexity of the estimation procedure, the assumption of absolutely continuity excludes the case of discrete distributions, which appears in some inventory-production and queuing systems. On the other hand, the case of an arbitrary distribution $\theta_{\xi}$ (without a priori assumption) has been treated in (Hilgert \& Minjárez-Sosa, 2006) and relies on the empirical distribution. It may seem an obvious choice, but this was a great improvement on what was done previously. The assumptions used are even weaker than in the non adaptive case and wouldn't be sufficient to prove the existence of a stationary optimal policy with a known distribution $\theta_{\xi}$. The extension to the expected average cost is the subject of (Minjárez-Sosa, 2008).

The case of model $\mathcal{M}_{\eta}$, is less known in the literature and is treated in detail in the following section. 


\section{Adaptive control of stochastic systems with poorly-known cost function}

The construction of the adaptive policies is based mainly on the cost estimation process which, in turns, is obtained by implementing suitable estimation methods of the probability distribution $\theta_{\eta}$. In general our approach consists in getting an estimator $c_{n}$ of the cost such that

- $\quad$ it converges to $c$ (in a sense that will be given later);

- it leads up to the convergence of the following sequence: $V_{0}^{*}=0$,

$$
V_{n}^{*}(x):=\min _{a \in A(x)}\left(c_{n}(x, a)+\alpha \int_{X} V_{n-1}^{*}(y) Q(d y \mid x, a)\right), n \geq 1, x \in X
$$

to the unknown value function $V^{*}$ given in (7).

In particular, we take

$$
c_{n}(x, a)=E_{\theta_{\eta_{,}, n}} \bar{c}(x, a, s), \quad(x, a) \in \mathbb{K},
$$

where $\left\{\theta_{\eta, n}\right\} \subset \mathbb{P}(S)$ is a sequence of "consistent" estimators of $\theta_{\eta}$.

Now, applying standard arguments on the existence of minimizers, under Assumption 2.3, we have that for each $n \in \mathbb{N}$ there exists $f_{n}^{\theta_{\eta, n}} \in \mathbb{F}$ such that,

$$
V_{n}^{*}(x)=c_{n}\left(x, f_{n}^{\theta_{\eta, n}}\right)+\alpha \int_{X} V_{n-1}^{*}(y) Q\left(d y \mid x, f_{n}^{\theta_{\eta, n}}\right), x \in X,
$$

where the minimization is done for every ' $\omega \in \Omega$. Moreover, by a result of (Schäl, 1975), there is a stationary policy $f_{\infty}^{\theta_{\eta}}$ such that for each $x \in X, f_{\infty}^{\theta_{\eta}}(x) \in A(x)$ is an accumulation point of $\left\{f_{n}^{\theta_{\eta, n}}(x)\right\}$.

We state our main result as follows:

Theorem 3.1 a) Let $\hat{\pi}=\left\{\hat{\pi}_{n}\right\}$ be the policy defined by $\hat{\pi}_{n}\left(h_{n}\right)=\hat{\pi}_{n}\left(h_{n}, \theta_{\eta, n}\right):=f_{n}^{\theta_{\eta, n}}\left(x_{n}\right), n \in \mathbb{N}$ and $\hat{\pi}_{0}$ any fixed action.

Then, under Assumption 2.3 and if $\left\{\theta_{\eta, n}\right\}$ is an appropriate sequence of "consistent" estimators of $\theta_{\eta}, \hat{\pi}$ is asymptotically discount optimal.

b) In addition, the stationary policy $f_{\infty}^{\theta_{\eta}}$ is optimal for the control model $\mathcal{M}_{\eta}$.

The remainder of this section is devoted to the proof of Theorem 3.1 for two estimators of the cost function that correspond to two different assumptions on the unknown distribution $\theta_{\eta}$. In the first one, Subsection 3.2, we suppose that $\theta_{\eta}$ is absolutely continuous with respect to the Lebesgue measure and has an unknown density function $g$. The estimator $c_{n}$ of the cost function is then based on a nonparametric estimator of $g$. Next, in Subsection 3.4, we don't make any a priori assumption on $\theta_{\eta}$. The estimator $c_{n}$ is based on the empirical distribution of $\theta_{\eta}$. We first give some preliminary definitions and developments that are useful for both situations.

\subsection{Preliminaries}

We present some preliminary facts that will be useful in the proof of our main result.

Let us define the operator $T_{n}$ in the same way as $T$ in (10):

$$
\Gamma_{n} u(x):=\min _{A(x)}\left(c_{n}(x, a)+\alpha \int_{X} u(y) Q(d y \mid x, a)\right)
$$

for all $x \in X$ and $u \in L_{W}^{\infty}$. Observe that from (15) and (17) 


$$
T V^{*}=V^{*} \quad \text { and } \quad T_{n} V_{n-1}^{*}=V_{n}^{*}, \quad n \in \mathbb{N} .
$$

In addition, from Assumption 2.3(a), $\sup _{A(x)} c_{n}(x, a) \leq W(x)$, and applying the inequality (12), a straightforward calculation shows that, for some constant $C$,

$$
V_{n}^{*}(x) \leq C W(x), n \in \mathbb{N}, x \in X .
$$

Thus, from Assumption 2.3, $T$ and $T_{n}$ maps $L_{W}^{\infty}$ into itself.

We fix an arbitrary number $\epsilon \in(\alpha, 1)$ and define the function $\bar{W}(x):=W(x)+d$ for $x \in X$, where $d:=b(\epsilon / \alpha-1)^{-1}$. Let $L_{\bar{W}}^{\infty}$ be the space of measurable functions $u: X \rightarrow \mathbb{R}$ with norm

$$
\|u\|_{\bar{W}}:=\sup _{x \in X} \frac{|u(x)|}{\bar{W}(x)}<\infty
$$

Observe that the norms $\|\cdot\|_{W}$ and $\|\cdot\|_{\bar{W}}$ are equivalent because

$$
\|u\|_{\bar{W}} \leq\|u\|_{W} \leq(1+d)\|u\|_{\bar{W}} .
$$

A consequence of Lemma 2 in (Van Nunen $\mathcal{E}$ Wessels, 1978) is that the inequality (12) implies respectively that the operators $T_{n}$ and $T, n \in \mathbb{N}$, are contractions with modulus $f$, with respect to the norm $\|\cdot\|_{\bar{W}}$, i.e. for all $u, v \in L_{\bar{W}}^{\infty}$ :

$$
\begin{gathered}
\|T v-T u\|_{\tilde{W}} \leq \epsilon\|v-u\|_{\tilde{W}}, \\
\left\|T_{n} v-T_{n} u\right\|_{\tilde{W}} \leq \epsilon\|v-u\|_{\tilde{W}} \text { a.s. }
\end{gathered}
$$

Hence, from (21), for each $n \in \mathbb{N}$, we have

$$
\begin{aligned}
\left\|V^{*}-V_{n+1}^{*}\right\|_{\bar{W}} & \leq\left\|T V^{*}-T_{n+1} V^{*}\right\|_{\bar{W}}+\left\|T_{n+1} V^{*}-T_{n+1} V_{n}^{*}\right\|_{\bar{W}} \\
& \leq\left\|T V^{*}-T_{n+1} V^{*}\right\|_{\bar{W}}+\epsilon\left\|V^{*}-V_{n}^{*}\right\|_{\bar{W} .}
\end{aligned}
$$

Now, let $\pi \in \Pi$ and $x \in X$ be arbitrary, and define

$$
l:=\lim \sup _{n \rightarrow \infty} E_{x}^{\pi}\left\|V^{*}-V_{n+1}^{*}\right\|_{\bar{W}}
$$

and

$$
l^{\prime}:=\lim \sup _{n \rightarrow \infty}\left\|V^{*}-V_{n+1}^{*}\right\|_{\bar{W}} .
$$

Observe that $l<\infty$ and $l^{\prime}<\infty$ (see Proposition 2.5, (22) and (23)). Then from (26)

$$
l \leq \frac{1}{1-\epsilon} \limsup _{n \rightarrow \infty} E_{x}^{\pi}\left\|T V^{*}-T_{n+1} V^{*}\right\|_{\bar{W}}
$$

and

$$
l^{\prime} \leq \frac{1}{1-\epsilon} \limsup _{n \rightarrow \infty}\left\|T V^{*}-T_{n+1} V^{*}\right\|_{\bar{W}} \quad \text { a.s. }
$$

\subsection{Cost estimation when $\theta_{\eta}$ has a density}

In this part, we suppose the existence of a density of $\theta_{\eta}$ as stated below. We will then start step by step the proof of Theorem 3.1. 
Assumption 3.2 a) $S=\Re^{k}$.

b) The distribution $\theta_{\eta}$ is absolutely continuous with respect to the Lebesgue measure on $\Re^{k}$ and has a density function $g$. That is,

$$
\theta_{\eta}(B)=\int_{B} g(s) d s, \quad B \in \mathcal{B}\left(\Re^{k}\right) .
$$

Under this context, from (4) we have

$$
c(x, a)=\int_{\pi^{k}} \bar{c}(x, a, s) g(s) d s, \quad(x, a) \in \mathbb{K}_{;}
$$

Let $\eta_{1}, \ldots, \eta_{t}$ be independent realizations (observed up to time $t$ ), of r.v.'s with the unknown density $g$, and $g_{n}(s):=g_{n}\left(s ; \eta_{1}, \ldots, \eta_{n}\right), s \in \Re^{k}$, be an arbitrary estimator of $g$ such that

$$
E \int_{\mathbb{R}^{k}}\left|g(s)-g_{n}(s)\right| \rightarrow 0 \text { as } n \rightarrow \infty .
$$

Defining, for each $n \in \mathbb{N}$,

$$
\theta_{\eta, n}(B)=\int_{B} g_{n}(s) d s, \quad B \in \mathcal{B}\left(\Re^{k}\right),
$$

the relation (18) becomes

$$
c_{n}(x, a):=\int_{\aleph^{k}} \bar{c}(x, a, s) g_{n}(s) d s, \quad(x, a) \in \mathbb{K} .
$$

Now, let us define the approximate discrepancy function $\Phi_{n}$, for each $n \in \mathbb{N}$ as (see (9))

$$
\Phi_{n}(x, a):=c_{n}(x, a)+\alpha \int_{X} V_{n-1}^{*}(y) Q(d y \mid x, a)-V_{n}^{*}(x),
$$

for all $(x, a) \in \mathbb{K}$, where $\left\{V_{n}^{*}\right\}$ is the sequence defined in (17) corresponding to the cost (33), and denote

$$
\Psi_{n}:=\sup _{x \in X}\left\{(W(x))^{-1} \sup _{a \in A(x)}\left|\Phi(x, a)-\Phi_{n}(x, a)\right|, \quad n \in \mathbb{N} .\right\}
$$

The following Lemma will be useful to prove Theorem 3.1.

Lemma 3.3 Assumptions 2.3, 3.2 and (31) imply that:

a) For each $\pi \in \Pi$ and $x \in X$,

$$
\lim _{n \rightarrow \infty} E_{x}^{\pi}\left\|V_{n}^{*}-V^{*}\right\|_{W}=0 .
$$

b)For each $x \in X$ and $\pi \in \Pi$,

$$
\lim _{n} E_{x}^{\pi} \Psi_{n}=0
$$

c)For each $x \in X$ and $\pi \in \Pi$, 


$$
\lim _{n} E_{x}^{\pi}\left(W\left(x_{n}\right) \Psi_{n}\right)=0 .
$$

d) For each $(x, a) \in \mathbb{K}$,

$$
\lim _{n} E_{x}^{\pi}\left|\int_{X} V_{n-1}^{*}(y) Q(d y \mid x, a)-\int_{X} V^{*}(y) Q(d y \mid x, a)\right|=0 .
$$

Proof:

a) From (23) and (27), to prove the part a), it is sufficient to show that

$$
E_{x}^{\pi}\left\|T V^{*}-T_{n+1} V^{*}\right\|_{\bar{W}} \rightarrow 0, \text { as } n \rightarrow \infty .
$$

To this end, from (10), (20), (30), and (33), and Assumption 2.3(a), for each $x \in X$ and $n \in \mathbb{N}$,

$$
\begin{aligned}
\left|T V^{*}(x)-T_{n+1} V^{*}(x)\right| & \leq \sup _{a \in A(x)}\left|c(x, a)-c_{n}(x, a)\right| \\
& \leq \sup _{a \in A(x)} \int_{\Re^{k}} \bar{c}(x, a, s)\left|g(s)-g_{n}(s)\right| d s \\
& \leq W(x) \int_{\Re^{k}}\left|g(s)-g_{n}(s)\right| d s .
\end{aligned}
$$

Hence, as $\bar{W}(\cdot)>W(\cdot)$,

$$
\left\|T V^{*}-T_{n} V^{*}\right\|_{\bar{W}} \leq \int_{\Re^{k}}\left|g(s)-g_{n}(s)\right| d s, n \in \mathbb{N} .
$$

Taking expectation $E_{x}^{\pi}$ on both sides of (41) and observing that (since $g_{n}$ does not depend on $x \in X$ and $\pi \in \Pi)$

$$
E_{x}^{\pi} \int_{\Re^{k}}\left|g(s)-g_{n}(s)\right| d s=E \int_{\Re^{k}}\left|g(s)-g_{n}(s)\right| d s, n \in \mathbb{N},
$$

relation (40) follows thanks to (31).

b) From definitions of the function $\Phi$. and $\Phi_{n}$, the norm $\|\cdot\|_{\bar{W}}$ in (14), and (12), for each $(x, a) \in \mathbb{K}$ and $n \in \mathbb{N}$,

$$
\begin{aligned}
\left|\Phi(x, a)-\Phi_{n}(x, a)\right| \leq & \left|c(x, a)-c_{n}(x, a)\right|+\left|V^{*}(x)-V_{n}^{*}(x)\right| \\
& +\alpha \int_{X}\left|V^{*}(y)-V_{n}^{*}(y)\right| Q(d y \mid x, a) \\
\leq & W(x) \int_{X}\left|g_{n}(s)-g(s)\right| d s+\left\|V^{*}-V_{n}^{*}\right\| W(x) \\
& +\left\|V^{*}-V_{n-1}^{*}\right\| W(\beta W(x)+b) .
\end{aligned}
$$

Thus, (31) and Lemma 3.3a yield (37). In addition, there exists a finite constant $M$ such that $\sup _{n} \Psi_{n} \leq M$, which, combined with (37), yields the convergence in probability

$$
\Psi_{n} \stackrel{P_{\pi}^{\pi}}{\rightarrow} 0 \text { as } n \rightarrow \infty \text {. }
$$

c) But, from (13) we have that 


$$
\sup _{n} E_{x}^{\pi}\left(W^{p}\left(x_{n}\right) \Psi_{n}\right)^{p}<M^{p} \sup _{n} E_{x}^{\pi} W^{p}\left(x_{n}\right)<\infty .
$$

We then deduce that $W\left(x_{n}\right) \Psi_{n}$ is $P_{x}^{\pi}$-uniformly integrable. Moreover, using Chebychev's inequality,

$$
\begin{gathered}
P_{x}^{\pi}\left(W\left(x_{n}\right) \psi_{n}>l_{1}\right) \leq P_{x}^{\pi}\left(\psi_{n}>\frac{l_{1}}{l_{2}}\right)+P_{x}^{\pi}\left(W\left(x_{n}\right)>l_{2}\right) \\
\leq P_{x}^{\pi}\left(\psi_{n}>\frac{l_{1}}{l_{2}}\right)+\frac{E_{x}^{\pi} W\left(x_{n}\right)}{l_{2}} .
\end{gathered}
$$

Hence,

$$
W\left(x_{n}\right) \psi_{n} \stackrel{P_{x}^{\pi}}{\rightarrow} 0 \text { as } n \rightarrow \infty,
$$

and as it is $P_{x}^{\pi}$-uniformly integrable, we get (38).

d) This result is a consequence of Lemma 3.3a and the following inequalities

$$
\begin{aligned}
\left|\int_{X} V_{n-1}^{*}(y) Q(d y \mid x, a)-\int_{X} V^{*}(y) Q(d y \mid x, a)\right| & \leq \int_{X}\left|V_{n-1}^{*}(y)-V^{*}(y)\right| Q(d y \mid x, a) \\
& \leq\left\|V^{*}-V_{n-1}^{*}\right\|_{W}(\beta W(x)+b)
\end{aligned}
$$

\subsection{Proof of Theorem 3.1}

We prove Theorem 3.1 in the specific case of $\theta_{\eta}$, absolutely continuous with respect to the Lebesgue measure, that is, with $\theta_{\eta}, c, \theta_{\eta, n}$ and $c_{n}$ given by (29), (30), (32), and (33), respectively. We will show in the Subsection 3.4 that it still holds with an arbitrary distribution $\theta_{\eta,}$.

Proof of part a) Observe that by definition of the control policy $\pi$ (see (19) and Theorem 3.1) and (34), we have $\Phi_{n}\left(., \hat{\pi}\left(h_{n}\right)\right)=0$ for all $n$. Hence,

$$
\begin{aligned}
\Phi\left(x_{n}, \hat{\pi}\left(h_{n}\right)\right) & =\left|\Phi\left(x_{n}, \hat{\pi}\left(h_{n}\right)\right)-\Phi_{n}\left(x_{n}, \hat{\pi}\left(h_{n}\right)\right)\right| \\
& \leq \sup _{a \in A(x)}\left|\Phi\left(x_{n}, a\right)-\Phi_{n}\left(x_{n}, a\right)\right| \\
& \leq W\left(x_{n}\right) \sup _{X}(W(x))^{-1} \sup _{a \in A(x)}\left|\Phi(x, a)-\Phi_{n}(x, a)\right| \\
& \leq W\left(x_{n}\right) \Psi_{n},
\end{aligned}
$$

which, combined with (38), proves the asymptotic discounted optimality of $\pi$.

Proof of part b) We fix an arbitrary $x \in X$. Since $f_{\infty}^{\theta_{\eta}}$ is an accumulation point of $\left\{f_{n}^{\theta_{\eta}, n}(x)\right\}$, there exists a subsequence $\left\{n_{i}(x)\right\}$ of $\{n\}$ such that $f_{n_{i}(x)}^{\theta_{\eta, n_{i}}} \rightarrow f_{\infty}^{\theta_{\eta^{\prime}}}(x)$ as $i \rightarrow \infty$. In addition,

$$
V_{n_{i}(x)}^{*}(x)=c_{n_{i}(x)}\left(x, f_{n_{i}(x)}^{\theta_{\eta_{i}, n_{i}}}\right)+\alpha \int_{X} V_{n_{i}(x)-1}^{*}(y) Q\left(d y \mid x, f_{n_{i}(x)}^{\theta_{\eta, n_{i}}}\right)
$$

Moreover, from (39), we deduce that

$$
\begin{aligned}
& \liminf _{i} E \int_{X} V_{n_{i}(x)-1}^{*}(y) Q\left(d y \mid x, f_{n_{i}(x)}^{\theta_{\eta, n_{i}}}\right)=\lim _{i} \inf E \int_{X} V^{*}(y) Q\left(d y \mid x, f_{n_{i}(x)}^{\theta_{\eta, n_{i}}}\right) \\
& \geq \int_{X} V^{*}(y) Q\left(d y \mid x, f_{\infty}^{\theta_{\eta}}\right) \quad \text { by Fatou's Lemma. }
\end{aligned}
$$


Then, taking the limit infimum in (45) yields

$$
c\left(x, f_{\infty}^{\theta_{\eta}}\right)+\alpha \int_{X} V^{*}(y) Q\left(d y \mid x, f_{\infty}^{\theta_{\eta}}\right) \leq V^{*}(x) .
$$

As $x$ was arbitrary, the equality holds for every $x \in X$ and so $f_{\infty}^{\theta_{\eta}}$ is optimal for the control $\operatorname{model} \mathcal{M}_{\eta}$.

\subsection{Cost estimation with the empirical distribution of $\theta_{\eta}$}

In this part, we suppose the disturbance space $S$ and the distribution $\theta_{\eta}$ arbitrary. To estimate $\theta_{\eta}$ we use the empirical distribution $\left\{\theta_{\eta, t}\right\} \subset \mathbb{P}(S)$ of the disturbance process $\left\{\eta_{t}\right\}$, defined as follows. Let $\nu \in \mathbb{P}(S)$ be a given arbitrary probability measure. Then

$$
\begin{aligned}
\theta_{\eta, 0} & :=\nu, \\
\theta_{\eta, n}(B) & :=\frac{1}{n} \sum_{i=0}^{n-1} 1_{B}\left(\eta_{i}\right), \quad \text { for all } n \geq 1 \text { and } B \in \mathcal{B}(S) .
\end{aligned}
$$

Under this context, we have (see (4) and (18))

$$
c(x, a)=\int_{S} \bar{c}(x, a, s) \theta_{\eta}(d s)
$$

and

$$
c_{n}(x, a)=\int_{S} \bar{c}(x, a, s) \theta_{\eta, n}(d s):=\frac{1}{n} \sum_{i=0}^{n-1} \bar{c}\left(x, a, \eta_{i}\right) .
$$

Clearly, from the law of large numbers, for each $(x, a) \in \mathbb{K}, c_{n}(x, a) \rightarrow c(x, a)$ a.s., as $n \rightarrow \infty$. However, to our objectives, we need uniform convergence on $(x, a)$ of the costs, for which we impose the following conditions.

Assumption $3.4 a$ ) The family of functions

$$
\mathcal{C}_{W}:=\left\{\frac{\bar{c}(x, a, \cdot)}{W(x)}:(x, a) \in \mathbb{K}\right\}
$$

is equicontinuous on $S$.

b) The function

$$
\varphi(s):=\sup _{(x, a) \in \mathbb{K}}[W(x)]^{-1} \bar{c}(x, a, s)
$$

is continuous on $S$.

Remark 3.5 a) Observe that from Assumption 2.3(b), $\varphi(s) \leq 1, s \in S$. Hence $E\left[\varphi\left(\eta_{0}\right)\right]^{r}<\infty$, for all $r>0$. Then, from Assumption 3.4 and applying Theorem 6.4 in (Ranga Rao, 1962), we get, as $n \rightarrow \infty$,

$$
\sup _{(x, a) \in \mathbb{K}}\left|\int_{S} \frac{\bar{c}(x, a, s)}{W(x)} \theta_{\eta}(d s)-\int_{S} \frac{\bar{c}(x, a, s)}{W(x)} \theta_{\eta, n}(d s)\right| \rightarrow 0 \quad a . s .
$$


b) The function $\varphi$ in Assumption 3.4 might be non continuous. In such case we replace Assumption 3.4(b) by supposing the existence of a continuous majorant $\varphi$ of $\varphi$ such that $E\left[\bar{\varphi}\left(\eta_{0}\right)\right]^{r}<\infty$ for some $r>1$.

Let $\left\{V_{n}^{*}\right\},\left\{\Phi_{n}\right\}$, and $\left\{\Psi_{n}\right\}$ be the sequences of functions defined in (17), (34), and (35), respectively, corresponding to the cost functions (47) and (48). According to Subsection 3.3 and the proof of Lemma 3.3, to prove the Theorem 3.1 under the empirical estimator, it is sufficient to state the following results.

Lemma 3.6 Under Assumptions 2.3 and 3.4,

a) $\lim _{n}\left\|V_{n}^{*}-V^{*}\right\|_{W}=0$ a.s.;

b) $\lim _{n} \Psi_{n}=0$ a.s.;

c) for each $x \in X$ and $\pi \in \Pi, \lim _{n} E_{x}^{\pi}\left(W\left(x_{n}\right) \Psi_{n}\right)=0$;

d) $\lim _{n}\left|\int_{X} V_{n-1}^{*}(y) Q(d y \mid x, a)-\int_{X} V^{*}(y) Q(d y \mid x, a)\right|=0$ a.s.

Proof. The part a) is a consequence of the following inequality. From (10), (20), (47), (48), and (49), for each $x \in X$,

$$
\left\|T V^{*}-T_{n} V^{*}\right\|_{\bar{W}} \leq \sup _{(x, a) \in \mathbb{K}}\left|\int_{S} \frac{\bar{c}(x, a, s)}{W(x)} \theta_{\eta}(d s)-\int_{S} \frac{\bar{c}(x, a, s)}{W(x)} \theta_{\eta, n}(d s)\right| \rightarrow 0 \text { a.s. }
$$

Thus, (28) and (23) yield the part a).

On the other hand, observe that (see (42))

$$
\Psi_{n} \leq \sup _{(x, a) \in \mathbb{K}}\left|\int_{S} \frac{\bar{c}(x, a, s)}{W(x)} \theta_{\eta}(d s)-\int_{S} \frac{\bar{c}(x, a, s)}{W(x)} \theta_{\eta, n}(d s)\right|+\left\|V^{*}-V_{n}^{*}\right\|_{W}+\left\|V^{*}-V_{n-1}^{*}\right\|_{W}(\beta+b) .
$$

Therefore, the part b) follows from (49) and the part a).

Finally, the parts c) and d) are obtained by applying similar arguments as for proving (38) and (39).

\section{Concluding remarks}

A general scheme to construct adaptive policies in control models as (2) is to combine statistical estimation methods of the unknown distribution with control procedures. Such policies have optimality properties provided that the estimators are consistent in an appropriate sense. In this paper we studied two cases of adaptive control models, model $\mathcal{M}_{\xi}$ who has an unknown system disturbance distribution, and model $\mathcal{M}_{\eta}$ where the cost function depends on an exogenous variable of unknown distribution. We stated two ways of estimating $\theta_{\xi}$ or $\theta_{\eta}$ which yielded two different asymptotically discounted optimal adaptive policies. In the first one, it is assumed that the distribution possesses a density function $g$ on $\Re^{k}$. The estimation process in this case is based on the estimation of $g$, which can be done in a number of nice ways (see, e.g., Devroye (1987), Devroye $\mathcal{E}$ Lugosi (2001)), but has the disadvantage of excluding the case when the distribution is discrete.

The construction of adaptive policies using the empirical distribution as estimator is very general in the sense that the disturbance space $S$ as well as the distribution $\theta_{\xi}$ or $\theta_{\eta}$ can be arbitrary. This approach has the disadvantage that it requires restrictive equicontinuity conditions (see Assumption 3.4) which is the price we have to pay for nothing assuming on the unknown distribution. However, this assumption is satisfied in important cases. For 
instance, an obvious sufficient condition for Assumption 3.4(a) is that $S$ is countable. Also, this assumption holds in the case of additive-noise cost function of the form $\bar{c}(x, a, s)=G(x, a)+s$, where $\mathrm{G}$ is a continuous function.

\section{References}

Devroye, L. (1987). A Course in Density Estimation, Birkhauser, Boston.

Devroye, L. E Lugosi, G. (2001). Combinatorial Methods in Density Estimation, Springer, New York.

Gordienko, E.I. \& Minjárez-Sosa, J.A. (1998). Adaptive control for discrete-time Markov processes with unbounded costs: discounted criterion. Kybernetika, Vol. 34, 217-234.

Hernández-Lerma, O. (1989). Adaptive Markov Control Processes, Springer-Verlag, New York.

Hernández-Lerma, O. \& Lasserre, J.B. (1996). Discrete-Time Markov Control Processes: Basic Optimality Criteria, Springer- Verlag, New York.

Hernández-Lerma, O. \& Lasserre, J.B. (1999). further Topics on Discrete-Time Markov Control Processes, Springer-Verlag, New York.

Hilgert, N. \& Minjárez-Sosa, J.A. (2006). Adaptive Control of Stochastic Systems with Unknown Disturbance Distribution: Discounted Criteria. Math. Methods Oper. Res., Vol. 63, No. 3, 443-460.

Kurano M. (1972). Discrete-time markovian decision processes with an unknown parameter - average return criterion. J. Oper. Res. Soc. Japan, Vol. 15, 67-76.

Mandl P. (1974). Estimation and control in Markov chains. Adv. Appl. Probab., Vol. 6, 40-60.

Minjárez-Sosa, J.A. (1999). Nonparametric adaptive control for discrete-time Markov processes with unbounded costs under average criterion. Appl. Math., Vol. 26, No. 3, 267-280.

Minjárez-Sosa, J.A. (2008). Empirical estimation in average Markov control processes. Applied Mathematics Letters, Vol. 21, No. 5, 459-464.

Ranga Rao, R. (1962). Relations between weak and uniform convergence of measures with applications. Ann. Math. Statistics, Vol. 33, 659-680.

Schäl, M. (1975). Conditions for optimality and for the limit of n-stage optimal policies to be optimal. Z. Wahrs. Verw. Gerb., Vol. 32,179-196.

Schäl, M. (1987). Estimation and control in discounted stochastic dynamic programming. Stochastics, Vol. 20, 51-71.

Stockey N.L.\& Lucas R.E. Jr (1989). Recursive Methods in Economic Dynamics. Harvard University Press, Cambridge, MA.

Van Nunen, J.A.E.E. E Wessels, J. (1978). A note on dynamic programming with unbounded rewards. Manag. Sci., Vol. 24, 576-580. 


\title{
Lipschitzian Parameterization-Based Approach for Adaptive Controls of Nonlinear Dynamic Systems with Nonlinearly Parameterized Uncertainties: A Theoretical Framework and Its Applications
}

\author{
N.V.Q. Hung1, H.D. Tuan ${ }^{2}$ and T. Narikiyo 3 \\ ${ }^{1}$ Technical Research Center, Toyota Motor Corporation, ${ }^{2}$ School of Electrical Engineering \\ and Telecommunications, The University of New South Wales \\ ${ }^{3}$ Department of Electrical and Computer Engineering, Toyota Technological Institute \\ 1,3Japan, ${ }^{2}$ Australia,
}

\section{Introduction}

The original and popular adaptive control theory usually deals with linear parameterizations (LP) of uncertainties, that is, it is assumed that uncertain quantities in dynamic systems are expressed linearly with respect to unknown parameters. Actually, most developed approaches such as gradient-based ones or recursive least squares $[1,2]$ rely heavily on this assumption and effective techniques have been proposed in this context [2]. However, LP is impossible in practical applications whose dynamic parameters are highly coupled with system states. Stribeck effect of frictional forces at joints of the manipulators [3] or nonlinear dynamics of space-robot in inertia space are typical examples [4].

Unfortunately, there were very few results in the literature addressing the adaptive control problem for NP in a general and direct manner. Recently, adaptation schemes for NP have been proposed $[5,6]$ with the assumption on the convexity/concavity and smoothness of the nonlinear functions in unknown parameters. In this approach, the controllers search a known compact set bounding the unknown parameters (i.e. the unknown parameter must belong to a prescribed closed and bounded set) for min-max parameter estimation. Also, the resulting controllers posse a complex structure and need delicate switching due to change of adaptation mechanism up to the convexity/concavity of the nonlinear functions. Such tasks may be hard to be implemented in a real-time manner.

In this chapter, we propose novel adaptive control technique, which is applicable to any NP systems under Lipschitzian structure. Such structure is exploited to design linear-inparameter upper bounds for the nonlinear functions. This idea enables the design of adaptive controllers, which can compensate effectively for NP uncertainties in the sense that it can guarantee global boundedness of the closed-loop system signals and tracking error within any prescribed accuracies. The structures of the resulting controllers are simple since they are designed based on the nonlinear functions' upper bound, which depends only on 
the system variables. Therefore, an important feature of the proposed technique is that the compactness of uncertain parametric sets is not required. Another interesting feature of the technique is that regardless of parametric dimension, even 1-dimension estimator-based control is available. This is an important feature from practical implementation viewpoint. This result is of course new even for traditional LP systems. As a result, the designed adaptive controls can gain a great amount of computation reduced. Also, a very broad class of nonlinearly parameterized adaptive control problems such as Lipschitzian parameterization (including convex/concave, smooth parameterizations as a particular case), multiplicative parameterization, fractional parameterization or their combinations can be solved by the proposed framework.

The chapter is organized as follows. In Section 2, we formulate the control problem of nonlinear dynamic system with NP uncertainties. Adaptive control is designed for uncertainties, which satisfy Lipschitz condition (Lipschitzian parameterization). Our formulating Lipschitzian parameterization plays a central role to convert the NP adaptive control problem to a handleable form. Adaptation laws are designed for both nonnegative unknown parameters and unknown parameters with unknown sign. With the ability to design 1-dimension-observer for unknown parameter, we also redesign the traditional adaptive control of LP uncertain plants. Next is a design of adaptive control for a difficult but popular form of uncertainties, the multiplicative parameterizations. Examples of a control design of the proposed approach is illustrated at the end of the section. Section 3 remarks our results extended to the adaptive controls in systems with indirect control inputs. In this section, we describe the control problems of the backstepping design method to control complex dynamic structures whose their control input can not directly compensate for the effect of unknown parameters (un-matching system). Section 4 is devoted to the incorporation of proposed techniques to a practical application: adaptive controller design applied to path tracking of robot manipulators in the presence of NP. A general framework of adaptive control for NP in the system is developed first. Then, adaptive control for friction compensation in tracking problem of a 2DOF planar robot is introduced together with comparative simulations and experiments. Conclusions and discussions are given in Section 5.

\section{Lipschitzian parameterization-based techniques for Adaptive Control of NP}

\subsection{Problem formulation}

We consider adaptive systems admitting a nonlinear parameterization in the form

$$
\dot{\mathbf{e}}=\mathbf{A e}+\mathbf{B}(\mathbf{f}(\mathbf{x}, \boldsymbol{\theta})+\mathbf{u}),
$$

where $\mathbf{u}$ is the control input, $\mathbf{e}$ is the state vector, $\mathbf{x}$ is the system variable, $\boldsymbol{\theta}$ is an unknown time-invariant parameter. Both the state $\mathbf{e}$ and variable $\mathbf{x}$ are available for online measurement. The function $\mathrm{f}(\mathrm{x}, \boldsymbol{\theta})$ is nonlinear in both the system variable $\mathbf{x}$ and unknown parameter $\boldsymbol{\theta}$. The problem is to design a control signal $\mathbf{u}(\mathbf{t})$ enforcing asymptotic convergence of the state, that is, $\mathbf{e}(\mathbf{t}) \rightarrow 0$ as $t \rightarrow \infty$.

Note that any general adaptive control problem where the state $\mathbf{x}_{\mathbf{m}}$ of uncertain plants (satisfying the model matching condition) is required to track the state of a reference model $\mathbf{x}_{\mathbf{p}}$ can actually be reduced to the above described problem. 
For the simplicity of description and without loss of generality, the following standard assumption is used throughout the chapter.

Assumption 1. $e \in R, u \in R$ (i.e. the state and control are scalars) and $A=-I$, whereas $x(t) \in R$ is bounded.

From this assumption, it is clear that we can set $B=1$ without loss of generality, so from now on, we are considering the system

$$
\dot{e}=-e+f(x, \theta)+u \text {. }
$$

Note that from [5, Lemma 3], it is known that indeed the vector case of the state can be easily transformed to the scalar case. Clearly, under the model matching condition, the methodology for scalar control can be easily and naturally extended to multidimensional controls.

Let us also recall that a function $f: R_{+}^{p} \rightarrow R$ is increasing (decreasing, resp.) if and only if $f(\boldsymbol{\theta}) \leq f(\overline{\boldsymbol{\theta}})\left(f(\boldsymbol{\theta}) \geq f(\overline{\boldsymbol{\theta}})\right.$, resp.) whenever $\boldsymbol{\theta} \leq \overline{\boldsymbol{\theta}}$ (i.e. $\left.\theta_{j} \leq \theta_{j}, j=1,2, \ldots, p\right)$.

We shall use the absolute value of a vector, which is defined as

$$
|\mathbf{w}|=\left[\begin{array}{llll}
\left|w_{1}\right| & \left|w_{2}\right| & \ldots & \left|w_{p}\right|
\end{array}\right]^{T}, \quad \forall \mathbf{w} \in \mathbf{R}^{\mathbf{p}} .
$$

\subsection{Lipschitzian parameterization}

We consider the case where $f(x, \theta)$ in (1) is Lipschitzian in $\boldsymbol{\theta}$. It suffices to say that any convex or concave or smooth function is Lipschitzian in their effective domain [7] . As we discuss later on, the Lipschitzian parameterization-based method allows us to solve the adaptive control problems in a very efficient and direct manner. The Lipschitz condition is recalled first.

Assumption 2. The function $f(x,$.$) is Lipschitzian in \boldsymbol{\theta}$, i.e. there are continuous functions $0 \leq L_{j}(x)<+\infty, j=1,2, \ldots, p$ such that

$$
|f(x, \overline{\boldsymbol{\theta}})-f(x, \boldsymbol{\theta})| \leq \sum_{j=1}^{p} L_{j}(x)\left|\bar{\theta}_{j}-\theta_{j}\right|
$$

Note that in literature, the Lipschitz condition is often described by

$$
|f(x, \overline{\boldsymbol{\theta}})-f(x, \boldsymbol{\theta})| \leq \mathbf{L}(x)\|\overline{\boldsymbol{\theta}}-\boldsymbol{\theta}\|
$$

which can be shown to be equivalent to (3). In what follows, we shall set

$$
\mathbf{L}(x)=\left[\begin{array}{llll}
L_{1}(x) & L_{2}(x) & \ldots & L_{p}(x)
\end{array}\right] .
$$

\subsection{Adaptation techniques for unknown parameters}

To make our theory easier to follows, let's first assume that

$$
\boldsymbol{\theta} \in R_{+}^{p} \text {, i.e. } \theta_{j} \geq 0, j=1,2, \ldots, p .
$$

The following lemma plays a key role in the subsequent developments.

Lemma 1 The function $f(x, \boldsymbol{\theta})-\mathbf{L}(x) \boldsymbol{\theta}$ is decreasing in $\boldsymbol{\theta}$ whereas the function $f(x, \boldsymbol{\theta})+\mathbf{L}(x) \boldsymbol{\theta}$ is increasing in $\boldsymbol{\theta}$.

Proof. By (3), for every $\boldsymbol{\theta} \geq \overline{\boldsymbol{\theta}}$, 


$$
\max \{f(x, \boldsymbol{\theta})-f(x, \overline{\boldsymbol{\theta}}), f(x, \overline{\boldsymbol{\theta}})-f(x, \boldsymbol{\theta})\} \leq \mathbf{L}(x)(\boldsymbol{\theta}-\overline{\boldsymbol{\theta}})
$$

which implies

$$
\begin{aligned}
& f(x, \boldsymbol{\theta})-\mathbf{L}(x) \boldsymbol{\theta} \leq f(x, \overline{\boldsymbol{\theta}})-\mathbf{L}(x) \overline{\boldsymbol{\theta}}, \\
& f(x, \boldsymbol{\theta})+\mathbf{L}(x) \boldsymbol{\theta} \geq f(x, \overline{\boldsymbol{\theta}})+\mathbf{L}(x) \overline{\boldsymbol{\theta}},
\end{aligned}
$$

i.e. function $f(x, \boldsymbol{\theta})-\mathbf{L}(x) \boldsymbol{\theta}$ is decreasing in $\boldsymbol{\theta}$, while $f(x, \boldsymbol{\theta})+\mathbf{L}(x) \boldsymbol{\theta}$ in increasing in $\boldsymbol{\theta}$. Now, take the following Lyapunov function for studying the stabilization of system (2)

$$
V(e, \hat{\boldsymbol{\theta}})=\frac{1}{2}\left[e^{2}+\|\boldsymbol{\theta}-\hat{\boldsymbol{\theta}}\|^{2}\right]
$$

where $\hat{\boldsymbol{\theta}}=\hat{\boldsymbol{\theta}}(t)$ is an "observer" of $\boldsymbol{\theta}$ to be designed with the controller $u$. Then

$$
\begin{aligned}
\dot{V}= & -e(t)^{2}+e(t)[f(x(t), \boldsymbol{\theta})+u]-\hat{\boldsymbol{\theta}}^{T}(\boldsymbol{\theta}-\hat{\boldsymbol{\theta}}) \\
= & -e(t)^{2}+e(t)[f(x(t), \boldsymbol{\theta})-\operatorname{sgn}(e(t)) \mathbf{L}(x(t)) \boldsymbol{\theta}] \\
& +e(t)[\operatorname{sgn}(e(t)) \mathbf{L}(x(t)) \boldsymbol{\theta}+u]-\hat{\boldsymbol{\theta}}^{T}(\boldsymbol{\theta}-\hat{\boldsymbol{\theta}}) .
\end{aligned}
$$

As a consequence of lemma 1 , we have

Lemma 2 The function

$$
e(t)[f(x(t), \boldsymbol{\theta})-\operatorname{sgn}(e(t)) \mathbf{L}(x(t)) \boldsymbol{\theta}]
$$

is decreasing in $\boldsymbol{\theta}$.

Proof. It suffices to show that function

$$
k(e, x, \boldsymbol{\theta}):=e[f(x, \boldsymbol{\theta})-\operatorname{sgn}(e) \mathbf{L}(x) \boldsymbol{\theta}]
$$

is decreasing in $\boldsymbol{\theta}$.

When $e>0$,

$$
k(e, x, \boldsymbol{\theta})=e[f(x, \boldsymbol{\theta})-\mathbf{L}(x) \boldsymbol{\theta}]
$$

and thus $k(e, x, \boldsymbol{\theta})$ is decreasing because $f(x, \boldsymbol{\theta})-\mathbf{L}(x) \boldsymbol{\theta}$ is decreasing (by lemma 1$)$ and $e>$ 0 . On the other hand, when $e<0$,

$$
k(e, x, \boldsymbol{\theta})=e[f(x, \boldsymbol{\theta})+\mathbf{L}(x) \boldsymbol{\theta}]
$$

and again $k(e, x, \boldsymbol{\theta})$ is decreasing because $f(x, \boldsymbol{\theta})+\mathbf{L}(x) \boldsymbol{\theta}$ is increasing (by lemma 1 ) and e $<0$. Finally, $k(e, x, \boldsymbol{\theta})$ is obviously decreasing (constant) when $e=0$, completing the proof of lemma 2.

From (7) and lemma 2, we have

$$
\dot{V} \leq-e(t)^{2}+e(t)[f(x(t), \mathbf{0})+\operatorname{sgn}(e(t)) \mathbf{L}(x(t)) \boldsymbol{\theta}+u]-\dot{\boldsymbol{\theta}}^{T}(\boldsymbol{\theta}-\hat{\boldsymbol{\theta}}) .
$$

Therefore, we design the following controller $u$

$$
u=-f(x, \mathbf{0})-\operatorname{sgn}(e) \mathbf{L}(x) \hat{\boldsymbol{\theta}}
$$

in tandem with the adaptive rule 


$$
\begin{aligned}
\dot{\hat{\boldsymbol{\theta}}} & =\operatorname{esgn}(e) \mathbf{L}(x)^{T} \\
\Leftrightarrow \dot{\hat{\theta}}_{j} & =|e| L_{j}(x), j=1,2, \ldots, p,
\end{aligned}
$$

which together lead to

$$
\dot{V} \leq-e(t)^{2} .
$$

The last inequality implies that $V$ is decreasing as a function of time, and thus is bounded by $y(0)$. Therefore, by definition $(6), e(t)$ and $\hat{\boldsymbol{\theta}}(t)$ must be bounded from which we infer the boundness of $\dot{e}(t)$ as well. Also, (11) also gives $\int_{0}^{T} e(t)^{2} d t \leq V(0), \forall T>0$, i.e. $e($.$) is in L_{2}$. Therefore, by a consequence of Barbalat's lemma [1, p. 205],

$$
\lim _{t \rightarrow+\infty} e(t)=0 \text {. }
$$

Finally, let us mention that equation (10) guarantees $\hat{\boldsymbol{\theta}}(t) \in R_{+}^{p}, \forall t>0$ provided that $\hat{\boldsymbol{\theta}}(0) \in R_{+}^{p}$. The following theorem summarizes the results obtained so far.

Theorem 1 Under the assumption 2, the control $u$ and observer $\hat{\boldsymbol{\theta}}$ defined by (9) and (10) stabilizes system (2).

The control law determined by (9) and (10) is discontinuous at $e(t)=0$. According to a suggested technique in [5], we can modify the control (9) and (10) to get a continuous one as follows

$$
\begin{gathered}
u=-f(x, \mathbf{0})-\operatorname{sat}(e / \epsilon) \mathbf{L}(x) \hat{\boldsymbol{\theta}}, \\
\dot{\hat{\boldsymbol{\theta}}}(t)=\left|e_{\epsilon}(t)\right| \mathbf{L}(x(t))^{T},
\end{gathered}
$$

where $\epsilon>0$ and

$$
\begin{aligned}
\operatorname{sat}(e / \epsilon) & = \begin{cases}e / \epsilon & \text { when }-\epsilon \leq e \leq \epsilon \\
1 & \text { when } e>\epsilon \\
-1 & \text { when } e<-\epsilon\end{cases} \\
e_{\epsilon} & =e-\epsilon \operatorname{sat}(e / \epsilon) .
\end{aligned}
$$

Note that whenever $|e|>\epsilon$,

$$
e_{\epsilon}^{2} \leq e_{\epsilon} e \& \quad \operatorname{sat}(e / \epsilon)=\operatorname{sgn}\left(e_{\epsilon}\right) .
$$

Then, instead of Lyapunov function (8), take the function

$$
V(e, \hat{\boldsymbol{\theta}})=\frac{1}{2}\left[e_{\epsilon}^{2}+\|\boldsymbol{\theta}-\hat{\boldsymbol{\theta}}\|^{2}\right]
$$

and thus

$$
\dot{V}=0 \text { when }|e(t)| \leq \epsilon
$$




$$
\begin{aligned}
\dot{V} & =-e_{\epsilon} e+e_{\epsilon}[f(x, \boldsymbol{\theta})-f(x, \mathbf{0})-\operatorname{sat}(e / \epsilon) \mathbf{L}(x) \hat{\boldsymbol{\theta}}]-\dot{\boldsymbol{\theta}}^{T}(\boldsymbol{\theta}-\hat{\boldsymbol{\theta}}) \\
& \leq-e_{\epsilon}^{2}+e_{\epsilon}\left[f(x, \boldsymbol{\theta})-f(x, \mathbf{0})-\operatorname{sgn}\left(e_{\epsilon}\right) \mathbf{L}(x) \hat{\boldsymbol{\theta}}\right]-\hat{\boldsymbol{\theta}}^{T}(\boldsymbol{\theta}-\hat{\boldsymbol{\theta}}) \\
& \leq-e_{\epsilon}^{2} \quad \text { when }|e(t)|>\epsilon .
\end{aligned}
$$

The last inequality follows from the fact that function

$$
e_{\epsilon}\left[f(x, \boldsymbol{\theta})-\operatorname{sgn}\left(e_{\epsilon}\right) \mathbf{L}(x) \boldsymbol{\theta}\right]
$$

is decreasing in $\boldsymbol{\theta}$.

Therefore, it can be proved that the control (12)-(13) guarantees that $e(t)$ asymptotically tracks 0 within a precision of $\epsilon$.

\subsection{1-dimension estimator for unknown parameters}

In controls defined by (9)-(10) and (12)-(13), the dimension of the observer $\hat{\boldsymbol{\theta}}$ is the same as that of the unknown parameter $\boldsymbol{\theta}$. We now reveal that we can design a control with new observer $\hat{\boldsymbol{\theta}}$ of even dimension 1 (!) which does not depend on the dimension of the unknown parameter $\boldsymbol{\theta}$. For that, instead of $L(x)$ defined by (4), take

$$
L(x):=\max _{j=1,2, \ldots, s} L_{j}(x)
$$

then, by (3), it is obvious that

$$
|f(x, \bar{\theta})-f(x, \boldsymbol{\theta})| \leq L(x) \sum_{j=1}^{p}\left|\bar{\theta}_{j}-\theta_{j}\right|
$$

and by an analogous argument as that used in the proof of lemmas 1,2, it can be shown that Lemma 3 The function $f(x, \boldsymbol{\theta})-L(x) \sum_{j=1}^{p} \theta_{j}$ is decreasing in $\boldsymbol{\theta}$ whereas the function $f(x, \boldsymbol{\theta})+$ $L(x) \sum_{j=1}^{p} \theta_{\mathbb{J}}$ is increasing in 9.

Consequently, the function $e(t)\left[f(x(t), \boldsymbol{\theta})-\operatorname{sgn}(e(t)) \mathbf{L}(x(t)) \sum_{j=1}^{p} \theta_{j}\right]$ is decreasing in $\boldsymbol{\theta}$.

Based on the result of this lemma, instead of the Lyapunov function defined by (6) and the estimator defined by (10), (13), taking

$$
\begin{gathered}
V(e, \hat{\theta})=\frac{1}{2}\left[e^{2}+\left(\sum_{j=1}^{p} \theta_{j}-\hat{\theta}\right)^{2}\right], \\
\dot{\hat{\theta}}(t)=|e(t)| L(x), \\
\dot{\hat{\theta}}(t)=\left|e_{\epsilon}(t)\right| L(x),
\end{gathered}
$$

it can be readily shown that 
Theorem 2 With function sat $(e / \epsilon), e_{\varepsilon}$ defined by (14), (15) and the scalar estimator $\hat{\theta}(t)$ obeys either differential equation (22) or differential equation (23), the control (9) still stabilizes the system (2) whereas the control (12) guarantees that $e(t)$ asymptotically tracks 0 within a precision of $t$.

\subsection{Estimator for unknown parameters with unknown sign}

First, every $\boldsymbol{\theta} \in R^{p}$ can be trivially expressed as

$$
\boldsymbol{\theta}=\frac{\boldsymbol{\theta}^{(1)}-\boldsymbol{\theta}^{(2)}}{2}, \boldsymbol{\theta}^{(1)}=|\boldsymbol{\theta}|+\boldsymbol{\theta} \in R_{+}^{p}, \boldsymbol{\theta}^{(2)}=|\boldsymbol{\theta}|-\boldsymbol{\theta} \in R_{+}^{p} .
$$

For the new function $\tilde{f}$ defined by $\tilde{f}\left(x, \boldsymbol{\theta}^{(1)}, \boldsymbol{\theta}^{(2)}\right): R \times R_{+}^{2 p} \rightarrow R$ by

$$
\tilde{f}\left(x, \boldsymbol{\theta}^{(1)}, \boldsymbol{\theta}^{(2)}\right)=f\left(x,\left(\boldsymbol{\theta}^{(1)}-\boldsymbol{\theta}^{(2)}\right) / 2\right) .
$$

it is immediate to check that the Lipschitz condition (3) implies

$$
\left|\tilde{f}\left(x, \overline{\boldsymbol{\theta}}^{(1)}, \overline{\boldsymbol{\theta}}^{(2)}\right)-\tilde{f}\left(x, \boldsymbol{\theta}^{(1)}, \boldsymbol{\theta}^{(2)}\right)\right| \leq \frac{1}{2} \sum_{j=1}^{p} L_{j}(x)\left[\left|\bar{\theta}_{j}^{(1)}-\theta_{j}^{(1)}\right|+\left|\bar{\theta}_{j}^{(2)}-\theta_{j}^{(2)}\right|\right] .
$$

Then according to lemma 2, the function

$$
e(t)\left[\tilde{f}\left(x(t), \boldsymbol{\theta}^{(1)}, \boldsymbol{\theta}^{(2)}\right)-\frac{1}{2} \operatorname{sgn}(e(t)) \mathbf{L}(x)\left(\boldsymbol{\theta}^{(1)}+\boldsymbol{\theta}^{(2)}\right)\right]
$$

is decreasing in $\left(\boldsymbol{\theta}^{(1)}, \boldsymbol{\theta}^{(2)}\right)$.

Note that for $\boldsymbol{\theta}^{(1)}, \boldsymbol{\theta}^{(2)}$ defined by (24),

$$
\frac{1}{2}\left(\theta^{(1)}+\theta^{(2)}\right)=|\theta| .
$$

Therefore, using the Lyapunov functions defined by

$$
\begin{gathered}
V(e, \hat{\boldsymbol{\theta}})=\frac{1}{2}\left[e^{2}+\||\boldsymbol{\theta}|-\hat{\boldsymbol{\theta}}\|^{2}\right], \\
V(e, \hat{\theta})=\frac{1}{2}\left[e^{2}+\left(\sum_{j=1}^{p}\left|\theta_{j}\right|-\hat{\theta}^{2}\right],\right.
\end{gathered}
$$

analogously to Theorems 1 and 2, it can be shown that

Theorem 3 All statements of Theorems 1 and 2 remain valid with the assumption in (5) removed. Namely,

i. With the Lyapunov function (28) used for checking the stability and $\mathbf{L}(\mathbf{x})$ defined by (4), the control (9), (10) still stabilizes system (2) while the control (12), (13) guarantees that e(t) asymptotically tracks 0 with a precision of $\mathrm{t}$.

ii. (ii) With the Lyapunov function (29) used for checking stability and $L(x)$ defined by (19), the control (9), (22) still stabilizes system (2) while the control (12), (23) still guarantees that e(t) asymptotically tracks 0 within a precision of $\mathrm{t}$. 


\subsection{New nonlinear control for linearly parameterized uncertain plants}

It is clear that when applied to linearly parameterized uncertain plants, Theorem 3 provides a new result on 1-dimension estimator for uncertain parameters as well. Let's describe this application in some details. A typical adaptive control problem for linearly parameterized uncertain plants can be formulated as follows $[1,8]$. For uncertain system

$$
\dot{\mathbf{X}}_{\mathbf{p}}=\mathbf{A}_{\mathbf{m}} \mathbf{X}_{\mathbf{p}}+\mathbf{B}\left(\boldsymbol{\alpha}^{T} \mathbf{X}_{\mathbf{p}}+u\right), \mathbf{A}_{\mathbf{m}} \in R^{n \times n}, \mathbf{B} \in R^{n}, \boldsymbol{\alpha} \in R^{n}, u \in R \text { (30) }
$$

with unknown parameter $\boldsymbol{\alpha}$, design a control to makes the state $\mathbf{X}_{\mathbf{p}}(t)$ track a reference trajectory $\mathbf{X}_{\mathbf{m}}$ described by the equation

$$
\dot{\mathbf{X}}_{\mathbf{m}}=\mathbf{A}_{\mathbf{m}} \mathbf{X}_{\mathbf{m}}+\mathbf{B} r
$$

where $\mathbf{A}_{\mathbf{m}}$ is asymptotically stable with one negative real eigenvalue $-k$.

The problem is thus to design the control $u$ such that the state $\mathbf{E}:=\mathbf{X}_{\mathbf{p}}-\mathbf{X}_{\mathbf{m}}$ of the error equation

$$
\dot{\mathbf{E}}=\mathbf{A}_{\mathbf{m}} \mathbf{E}+\mathbf{B}\left[\alpha^{T} \mathbf{X}_{\mathbf{p}}+u-r\right]
$$

is asymptotically stable. Taking $\mathbf{h} \in R^{n}$ such that $\mathbf{h}^{\mathbf{T}}\left(s \mathbf{I}-\mathbf{A}_{\mathbf{m}}\right)^{-1} \mathbf{B}=1 /(s+k)$ and defining $e=\mathbf{h}^{\mathbf{T}} \mathbf{E}$, then

$$
\dot{e}(t)=-k e(t)+\left[\boldsymbol{\alpha}^{\boldsymbol{T}} \boldsymbol{X}_{\boldsymbol{p}}+u-r\right]
$$

and it is known [5] that $\mathbf{E}(t) \rightarrow \mathbf{0}$ if and only if $e(t) \rightarrow 0$. It is obvious that the function $\alpha^{T} \boldsymbol{X}_{p}$ satisfies the Lipschitz condition

$$
\left|\alpha^{T} X_{p}-\bar{\alpha}^{T} X_{p}\right| \leq \max _{j=1, ., n}\left|X_{p j}\right| \sum_{j=1}^{n}\left|\alpha_{j}-\bar{\alpha}_{j}\right|
$$

and applying Theorem 3, we have the following result showing that 1-dimension estimator can be used for update law, instead of full $n$-dimension estimator in previously developed results of linear adaptive control.

Theorem 4 The nonlinear control

$$
\begin{gathered}
u=r-\operatorname{sgn}(e) \max _{j=1, . ., n}\left|X_{p j}\right| \hat{\alpha} \\
\dot{\hat{\alpha}}(t)=|e(t)| \max _{j=1, . ., n}\left|X_{p j}\right|
\end{gathered}
$$

makes $\mathbf{X}_{\mathbf{p}}$ track; $\mathbf{X}_{\mathbf{m}}$ asymptotically, while the nonlinear control

$$
\begin{gathered}
u=r-\operatorname{sat}(e / \epsilon) \max _{j=1, . ., n}\left|X_{p j}\right| \hat{\alpha} \\
\dot{\hat{\alpha}}(t)=|e(t)-\epsilon \operatorname{sat}(e(t) / \epsilon)| \max _{j=1, . ., n} \mid X_{p j}
\end{gathered}
$$

guarantees $\mathbf{X}_{\mathbf{p}}$ tracking $\mathbf{X}_{\mathbf{m}}$ asymptotically with a precision of $\epsilon$. 


\subsection{Case of Multiplicative parameterizations}

It is assumed in this section that

$$
f(x, \boldsymbol{\theta})=\mathbf{g}(x, \boldsymbol{\theta}) \mathbf{h}(x, \boldsymbol{\theta}),
$$

where the assumption below is made.

Assumption 3. The functions $\mathbf{g}(x, \boldsymbol{\theta}): R \times R^{p} \rightarrow\left(R^{m}\right)^{T}$ and $\mathbf{h}(x, \boldsymbol{\theta}): R \times R^{p} \rightarrow R^{m}$ are Lipschitzian in $\boldsymbol{\theta}$, i.e. there are continuous functions $L_{j}(x) \geq 0, \ell_{j}(x) \geq 0$ such that

$$
\begin{array}{ll}
\|\mathbf{g}(x, \boldsymbol{\theta})-\mathbf{g}(x, \overline{\boldsymbol{\theta}})\| \leq \sum_{j=1}^{p} L_{j}(x)\left|\theta_{j}-\bar{\theta}_{j}\right|, & \forall(x, \boldsymbol{\theta}, \overline{\boldsymbol{\theta}}) \\
\|\mathbf{h}(x, \boldsymbol{\theta})-\mathbf{h}(x, \overline{\boldsymbol{\theta}})\| \leq \sum_{j=1}^{p} \ell_{j}(x)\left|\theta_{j}-\bar{\theta}_{j}\right|, & \forall(x, \boldsymbol{\theta}, \overline{\boldsymbol{\theta}})
\end{array}
$$

true.

Let $L(x)$ be defined by (19) and

$$
\ell(x):=\max _{j=1,2, \ldots, p} \ell_{j}(x) .
$$

Then, whenever $\boldsymbol{\theta} \geq \overline{\boldsymbol{\theta}}$,

$$
\begin{aligned}
& \max \{\mathbf{g}(x, \boldsymbol{\theta}) \mathbf{h}(x, \boldsymbol{\theta})-\mathbf{g}(x, \overline{\boldsymbol{\theta}}) \mathbf{h}(x, \overline{\boldsymbol{\theta}}), \mathbf{g}(x, \overline{\boldsymbol{\theta}}) \mathbf{h}(x, \overline{\boldsymbol{\theta}})-\mathbf{g}(x, \boldsymbol{\theta}) \mathbf{h}(x, \boldsymbol{\theta})\} \leq \\
& \|\mathbf{g}(x, \boldsymbol{\theta})-\mathbf{g}(x, \overline{\boldsymbol{\theta}})\|\|\mathbf{h}(x, \boldsymbol{\theta})\|+\|\mathbf{g}(x, \overline{\boldsymbol{\theta}})\|\|\mathbf{h}(x, \boldsymbol{\theta})-\mathbf{h}(x, \overline{\boldsymbol{\theta}})\| \\
& {\left[L(x) \sum_{j=1}^{p}\left(\theta_{j}-\bar{\theta}_{j}\right)\right]\left[\ell(x) \sum_{j=1}^{p} \theta_{j}+\|\mathbf{h}(x, \mathbf{0})\|\right]+} \\
& {\left[L(x) \sum_{j=1}^{p} \bar{\theta}_{j}+\|\mathbf{g}(x, \mathbf{0})\|\right] \ell(x) \sum_{j=1}^{p}\left(\theta_{j}-\bar{\theta}_{j}\right)} \\
& {\left[L(x) \ell(x)\left(\sum_{j=1}^{p} \theta_{j}\right)^{2}+(\|\mathbf{h}(x, \mathbf{0})\| L(x)+\|\mathbf{g}(x, \mathbf{0})\| \ell(x)) \sum_{j=1}^{p} \theta_{j}\right]-} \\
& {\left[L(x) \ell(x)\left(\sum_{j=1}^{p} \bar{\theta}_{j}\right)^{2}+(\|\mathbf{h}(x, \mathbf{0})\| L(x)+\|\mathbf{g}(x, \mathbf{0})\| \ell(x)) \sum_{j=1}^{p} \bar{\theta}_{j}\right] .}
\end{aligned}
$$

As in the proof of lemma 2, the last inequality is enough to conclude:

Lemma 4 On $R_{+}^{p}$, the function

$$
\begin{aligned}
k(e, x, \boldsymbol{\theta}):= & e\left[\mathbf{g}(x, \boldsymbol{\theta}) \mathbf{h}(x, \boldsymbol{\theta})-\operatorname{sgn}(e)\left(L(x) \ell(x)\left(\sum_{j=1}^{p} \theta_{j}\right)^{2}+\right.\right. \\
& \left.\left.(\|\mathbf{h}(x, \mathbf{0})\| L(x)+\|\mathbf{g}(x, \mathbf{0})\| \ell(x)) \sum_{j=1}^{p} \theta_{j}\right)\right]
\end{aligned}
$$


is decreasing in $\boldsymbol{\theta}$.

Therefore, similarly to Sub-section 2.5, using the Lyapunov function

$$
V(e, \hat{\theta}, \hat{\alpha})=\frac{e^{2}}{2}+\frac{1}{2}\left[\left(\hat{\theta}-\sum_{j=1}^{p}\left|\theta_{j}\right|\right)^{2}+\left(\hat{\alpha}-\left(\sum_{j=1}^{p}\left|\theta_{j}\right|\right)^{2}\right)^{2}\right]
$$

we can prove the following theorem

Theorem 5 The following discontinuous control guarantees $e(t) \rightarrow 0$

$$
\begin{gathered}
u=-\mathbf{g}(x, \mathbf{0}) \mathbf{h}(x, \mathbf{0})-\operatorname{sgn}(e)[L(x) \ell(x) \hat{\alpha}+(\|\mathbf{h}(x, \mathbf{0})\| L(x)+\|\mathbf{g}(x, \mathbf{0})\| \ell(x)) \hat{\theta}], \\
\dot{\hat{\alpha}}(t)=|e| L(x) \ell(x) \\
\dot{\hat{\theta}}(t)=|e|(\|\mathbf{h}(x, \mathbf{0})|| L(x)+\| \mathbf{g}(x, \mathbf{0}) \| \ell(x))
\end{gathered}
$$

while the following continuous control with $\operatorname{sat}(e / \epsilon)$ and $e_{\epsilon}$ defined by (12), (13) guarantees the tracking of $e(t)$ to 0 with any prescribed precision $t$,

$$
\begin{gathered}
u=-\mathbf{g}(x, \mathbf{0}) \mathbf{h}(x, \mathbf{0})-\operatorname{sat}(e / \epsilon)[L(x) \ell(x) \hat{\alpha}+ \\
(\|\mathbf{h}(x, \mathbf{0})\| L(x)+\|\mathbf{g}(x, \mathbf{0})\| \ell(x)) \hat{\theta}], \\
\dot{\hat{\alpha}}(t)=\left|e_{\epsilon}\right| L(x) \ell(x), \\
\dot{\hat{\theta}}(t)=\left|e_{\epsilon}\right|(\|\mathbf{h}(x, \mathbf{0})\| L(x)+\|\mathbf{g}(x, \mathbf{0})\| \ell(x)) .
\end{gathered}
$$

Remark 1 By reseting $h_{j}(x, \boldsymbol{\theta}) \leftarrow-h_{j}(x, \boldsymbol{\theta})$ if necessarily, we can also assume without loss of generality that $g_{j}(x, 0) \geq 0$. Then, using the inequality

$$
\|\mathbf{v}\| \leq \sum_{j=1}^{p}\left|v_{j}\right| \quad \forall v \in R^{p}
$$

it can be shown that the function

$$
\begin{aligned}
k(e, x, \boldsymbol{\theta}):= & e\left[\mathbf{g}(x, \boldsymbol{\theta}) \mathbf{h}(x, \boldsymbol{\theta})-\operatorname{sgn}(e)\left(L(x) \ell(x)\left(\sum_{j=1}^{p} \theta_{j}\right)^{2}+\right.\right. \\
& \left.\left.\sum_{k=1}^{p}\left(\left|h_{j}(x, 0)\right| L(x)+g_{j}(x, 0) \ell(x)\right) \sum_{j=1}^{p} \theta_{j}\right)\right]
\end{aligned}
$$

is still decreasing in $\theta \in R_{+}^{p}$. Thus, the statement of Theorem 5 remains valid with $\|\mathbf{h}(x, \mathbf{0})\|$ and $\|\mathbf{g}(x, 0)\|$ in (40)-(45) replaced by $\sum_{j=1}^{p}\left|h_{j}(x, 0)\right|$ and $\sum_{j=1}^{p} g_{j}(x, 0)$, respectively.

Remark 2 Clearly, the statements of lemma 4 and Theorem 5 remain valid by replacing $\|\mathbf{h}(x, \mathbf{0})\|$ and $\|\mathbf{g}(x, \mathbf{0})\|$ in (38) and (40)-(42), (43)-(45) by any continuous functions $\overline{\mathbf{h}}(x) \geq\|\mathbf{h}(x, \mathbf{0})\|$ and $\overline{\mathbf{g}}(x) \geq\|\mathbf{g}(x, \mathbf{0})\|$, respectively. 


\subsection{Example of controller design}

We examine some problems of adaptive friction compensation and show that they belong to the classes considered in Sections 2.2-2.7 and thus the results there can be directly applied to solve these problems.

The model of a process with friction is given as

$$
\ddot{x}(t)=u-F
$$

where $u$ is the control force, $x$ is the motor shaft angular position, and $F$ is the frictional force that can be described in different ways depending on model types. In this discussion, we consider the Armstrong-Helouvry model [3]

$$
F=F_{C} \operatorname{sgn}(\dot{x})\left[1-e^{-\dot{x}^{2} / v_{S}^{2}}\right]+F_{S} \operatorname{sgn}(\dot{x}) e^{-\dot{x}^{2} / v_{S}^{2}}+F_{v} \dot{x},
$$

where $F_{C}, F_{S}, F_{v}$ are coefficients characterizing the Coulomb friction, static friction and viscous friction, respectively, and $v_{S}$ is the Stribeck parameter. The unknown static parameters are $F_{C}, F_{S}, F_{v}, v_{S}$.

To facilitate the developed results, we introduce the new variable

$$
e=x+\dot{x}
$$

which according to (46) obeys the equation

$$
\dot{e}=\dot{x}+u-F
$$

For (47), set $\boldsymbol{\theta}=\left(F_{C}, F_{S}, F_{v}, 1 / v_{S}^{2}\right)$. First consider $F$ defined by (47),

$$
F=f(\dot{x}, \boldsymbol{\theta})+\theta_{3} \dot{x}
$$

where $f$ has the form (36) with

$$
\mathbf{g}(\dot{x}, \boldsymbol{\theta})=\left[\begin{array}{ll}
\theta_{1} & \theta_{2}
\end{array}\right], \mathbf{h}(\dot{x}, \boldsymbol{\theta})=\operatorname{sgn}(\dot{x})\left[\begin{array}{c}
\left(1-e^{-\dot{x}^{2} \theta_{4}}\right) \\
e^{-\dot{x}^{2} \theta_{4}}
\end{array}\right] .
$$

Clearly, $\|\mathbf{h}(x, \mathbf{0})\| \leq 1$ and function $\mathbf{h}(\dot{x}, \boldsymbol{\theta})$ is Lipschitzian in $\boldsymbol{\theta}$ :

$$
\left|h_{j}(\dot{x}, \boldsymbol{\theta})-h_{j}(\dot{x}, \overline{\boldsymbol{\theta}})\right| \leq \dot{x}^{2}\left|\theta_{4}-\bar{\theta}_{4}\right|, j=1,2,
$$

Applying Theorem 5 to system (49) and taking the Remark 2 in Section 2.7 into account, the following controls are proposed for stabilizing system (46), (47),

$$
\begin{gathered}
u=-\dot{x}-e+\hat{F}_{v} \dot{x}-\operatorname{sgn}(e)\left[\dot{x}^{2} \hat{\alpha}+\hat{\theta}\right] \\
\dot{\hat{F}}_{v}=-e \dot{x}, \dot{\hat{\alpha}}=|e| \dot{x}^{2}, \dot{\hat{\theta}}=|e|
\end{gathered}
$$

and

$$
\begin{gathered}
u=-\dot{x}-e+\hat{F}_{v} \dot{x}-\operatorname{sat}(e / \epsilon)\left[\dot{x}^{2} \hat{\alpha}+\hat{\theta}\right], \\
\dot{\hat{F}}_{v}=-\dot{x}(e-\epsilon \operatorname{sat}(e / \epsilon)), \dot{\hat{\alpha}}=|e-\epsilon \operatorname{sat}(e / \epsilon)| \dot{x}^{2}, \dot{\hat{\theta}}=|e-\epsilon \operatorname{sat}(e / \epsilon)|
\end{gathered}
$$


On the other hand, (47) can be rewritten alternatively as

$$
F=\operatorname{sgn}(\dot{x}) F_{c}+F_{v} \dot{x}+\left(F_{s}-F_{c}\right) \operatorname{sgn}(\dot{x}) e^{-\dot{x}^{2} / v_{S}^{2}}
$$

with known parameters $\left(F_{c}, F_{v}, F_{s}-F_{c}, 1 / v_{S}^{2}\right)$. Again, by Theorem 5 , the following controller is proposed

$$
\begin{gathered}
u=-\dot{x}-e+\operatorname{sgn}(\dot{x}) \hat{F}_{c}+\dot{x} \hat{F}_{v}-\operatorname{sat}(e / \epsilon)\left(\dot{x}^{2} \hat{\alpha}+\hat{\theta}\right) \\
{\left[\begin{array}{c}
\dot{\hat{F}}_{c} \\
\dot{\hat{F}}_{v}
\end{array}\right]=-\left[\begin{array}{c}
\operatorname{sgn}(\dot{x}) \\
\dot{x}
\end{array}\right](e-\epsilon \operatorname{sat}(e / \epsilon)), \dot{\hat{\alpha}}=|e-\epsilon \operatorname{sat}(e / \epsilon)| \dot{x}^{2}, \dot{\hat{\theta}}=|e-\epsilon \operatorname{sat}(e / \epsilon)| .}
\end{gathered}
$$

One may guess that the term $\operatorname{sgn}(\dot{x})$ in (55) causes its nonsmooth behavior. Considering the term $\operatorname{sgn}(\dot{x}) F_{c}$ in $(54)$ as a Lipschitz function in $F_{c}$ with Lipschitz constant 1 and applying Theorem 2 to handle this term, an alternative continuous control to (55) is derived as

$$
\begin{gathered}
u=-\dot{x}-e+\operatorname{sat}(e / \epsilon) \hat{F}_{c}+\dot{x} \hat{F}_{v}-\operatorname{sat}(e / \epsilon)\left(\dot{x}^{2} \hat{\alpha}+\hat{\theta}\right) \\
{\left[\begin{array}{c}
\dot{\hat{F}}_{c} \\
\dot{\hat{F}}_{v}
\end{array}\right]=\left[\begin{array}{c}
|e-\epsilon \operatorname{sat}(e / \epsilon)| \\
-\dot{x}(e-\epsilon \operatorname{sat}(e / \epsilon))
\end{array}\right], \dot{\hat{\alpha}}=|e-\epsilon \operatorname{sat}(e / \epsilon)| \dot{x}^{2}, \dot{\hat{\theta}}=\mid e-\epsilon \operatorname{sat}(e / \epsilon)(56)}
\end{gathered}
$$

\section{Extension to the adaptive controls in systems with indirect control inputs}

\subsection{Control problems of the generalized matching system with second-order}

Without loss of generality, we describe the control problems of the generalized matching system with second-order, i.e.

$$
\begin{aligned}
& \dot{x}_{1}=x_{2}+\varphi\left(x_{1}, \boldsymbol{\theta}\right), \\
& \dot{x}_{2}=u,
\end{aligned}
$$

where $u \in R$ is the control input, $x=\left[x_{1}, x_{2}\right]^{T}$ is the system state. Function $\varphi\left(x_{1}, \boldsymbol{\theta}\right)$ is nonlinear in both the variable $x_{1}$ and the unknown parameter $\theta \in R^{p}$. The problem is to design a stabilizing state-feedback control $u$ such that the state $x_{1}(t)$ converges to 0 .

A useful methodology for designing controllers of this class is the adaptive backstepping method [9], under the assumption of a linear parameterization (LP) in the unknown parameter $\boldsymbol{\theta}$, i.e. the function $\varphi\left(x_{1}, \boldsymbol{\theta}\right)$ in $(57)$ is assumed linear. The basic idea of backstepping is to design a "stabilizing function", which prescribes a desired behavior for $x_{2}$ so that $x_{1}(t)$ is stabilized. Then, an effective control $u(t)$ is synthesized to regulate $x_{2}$ to track this stabilizing function. Very few results, however, are available in the literature that address adaptive backstepping for NP systems of the general form (57) [10]. The difficulty here is attributed to two main factors inherent in the adaptive backstepping. The first one is how to construct the stabilizing function for $x i$ in the presence of nonlinear parameterizations $[5,11,12]$. The second one arises from the fact that as the actual control $u(t)$ involves derivatives of the stabilizing function, the later must be constructed in such a way that it does not lead to multiple parameter estimates (or overparameterization) [13]. 


\subsection{Remarks on adaptive back-stepping design incorporated with Lipschitzian parameterization-based techniques}

The proposed approach in Sectionl has been extended to address the adaptive backstepping for the above general matching system. Our approach enables the design of the stabilizing function containing estimates of the unknown parameter $\boldsymbol{\theta}$ without overparameterization. The compactness of parametric sets is not required. The proposed approach is naturally applicable to smooth nonlinearities but also to the broader class of Lipschitzian functions. Interested reader can refer $[14,15,16]$ for the results in details.

\section{Adaptive controller design applied to path tracking of robot manipulators in the presence of NP.}

\subsection{Robot manipulators with NP uncertainties}

Nonlinear frictions such as Stribeck effect are very common in practical robot manipulators. However, adaptive controls for robot manipulators (see [17, 18] for a survey) cannot successfully compensate for NP frictions since they are based on the LP structure of unknown parameters. Also, most of adaptive friction compensation schemes in the literature of motion control only deal with either frictions with LP structure [19] or linearized models at the nominal values of the Stribeck friction parameters [20]. Recently, a Lyapunov-based adaptive control has been designed to compensate for the Stribeck effect under set-point control [21].

In this section, a general framework of adaptive control for NP in the system is developed. An application of adaptive control for friction compensation in tracking problem of a 2DOF planar robot is introduced together with comparative simulations and experiments.

\subsection{Problem formulation}

The dynamic model of a robot manipulator can be described by the following equation

$$
\mathbf{H}(\mathbf{q}) \ddot{\mathbf{q}}+\mathbf{C}(\mathbf{q}, \dot{\mathbf{q}}) \dot{\mathbf{q}}+\mathbf{g}(\mathbf{q})+\mathbf{f}_{N}(\mathbf{x}, \boldsymbol{\theta})=\boldsymbol{\tau}(t),
$$

where $\mathrm{q}(t) \in R^{n}$ is the joint coordinates of the manipulator, $\tau \in R^{n}$ is the torque applied to the joints, $\mathbf{H}(q) \in R^{n \times n}$ is the symmetric positive definite inertia matrix of the links, $\mathbf{C}(\mathbf{q}, \dot{\mathbf{q}}) \in R^{n \times n}$ is a matrix representing Coriolis and centrifugal effects, $\mathbf{g}(\mathbf{q}) \in R^{n}$ is the gravitational torques, $\mathbf{f}_{N}(\mathbf{x}, \boldsymbol{\theta}) \in R^{n}$ represents dynamics whose constant or slowlyvarying uncertain parameter $\boldsymbol{\theta}$

appears nonlinearly in the system. Note that $\mathbf{x}$ can be any component of the system state, for instance $\mathbf{x}=\left[\mathbf{q}^{T}, \dot{\mathbf{q}}^{T}\right]^{T}$.

We focus on the case where the uncertainties admit a general multiplicative form, i.e.,

$$
\begin{aligned}
\mathbf{f}_{N}(\mathbf{x}, \boldsymbol{\theta}) & =\left[f_{N 1}\left(\mathbf{x}, \boldsymbol{\theta}_{1}\right), \ldots, \quad, f_{N n}\left(\mathbf{x}, \boldsymbol{\theta}_{n}\right)\right]^{T} \\
f_{N i}\left(\mathbf{x}, \boldsymbol{\theta}_{i}\right) & =\mathbf{g}_{i}\left(\mathbf{x}, \boldsymbol{\theta}_{i}\right) \mathbf{h}_{i}\left(\mathbf{x}, \boldsymbol{\theta}_{i}\right), \quad i=1, \ldots, n .
\end{aligned}
$$

Here $i$ stands for the $i$-ih joint of the manipulator and functions $\mathbf{g}_{i}\left(\mathbf{x}, \boldsymbol{\theta}_{i}\right), \mathbf{h}_{i}\left(\mathbf{x}, \boldsymbol{\theta}_{i}\right)$ are assumed nonlinear and Lipschitzian in $\boldsymbol{\theta}_{i}, \boldsymbol{\theta}_{i}=\left[\theta_{i 1}, \ldots, \theta_{i p_{i}}\right]^{T} \in R^{p_{i}}$. As it will be discussed later, a typical example of uncertainty admitting this form is the Stribeck effect of frictional forces in joints of robot manipulators [3]. 
Property 2.1 The inertia matrix $\mathbf{H}(\mathbf{q})$ is positive definite and satisfies $\lambda_{\min } \mathbf{I} \leq \mathbf{H}(\mathbf{q}) \leq \lambda_{\max } \mathbf{I}$, with $0<\lambda_{\min }<\lambda_{\max }<\infty, \lambda_{\min }$, where $\lambda_{\max }$ are minimal and maximal eigenvalues of $\mathbf{H}(\mathbf{q})$.

Property 2.2 The matrix $\dot{\mathbf{H}}(\mathbf{q})-2 \mathbf{C}(\mathbf{q}, \dot{\mathbf{q}})$ is skew-symmetric.

Property 2.3 The sum of the first three terms in the LHS of equation (58) are expressed linearly with respect to a suitable set of constant dynamic parameters:

$$
\mathbf{H}(\mathbf{q}) \ddot{\mathbf{q}}+\mathbf{C}(\mathbf{q}, \dot{\mathbf{q}}) \dot{\mathbf{q}}+\mathbf{g}(\mathbf{q})=\mathbf{Y}(\mathbf{q}, \dot{\mathbf{q}}, \ddot{\mathbf{q}}) \mathbf{a},
$$

where $\mathbf{Y}(\mathbf{q}, \dot{\mathbf{q}}, \ddot{\mathbf{q}}) \in R^{n \times \omega}$ matrix function and $\mathbf{a} \in R^{\omega}$ is a vector of unknown dynamic parameters.

The following lemma will be frequently used in subsequent developments

Lemma 5 Given Lipschitzian functions $\mathbf{g}_{i}\left(\mathbf{x}, \boldsymbol{\theta}_{i}\right), \mathbf{h}_{i}\left(\mathbf{x}, \boldsymbol{\theta}_{i}\right)$, let $L_{i}(\mathbf{x})$ and $\ell_{i}(\mathbf{x})$ be defined as

$$
\begin{aligned}
& L_{i}(\mathbf{x}):=\max _{j=1,2, \ldots, p} L_{i j}(\mathbf{x}), \\
& \ell_{i}(\mathbf{x}):=\max _{j=1,2, \ldots, p} \ell_{i j}(\mathbf{x}),
\end{aligned}
$$

then, for $\boldsymbol{\theta}_{i} \in R_{+}^{p_{i}}$ the following inequalities

$$
\begin{aligned}
e(t) \mathbf{g}_{i}\left(\mathbf{x}, \boldsymbol{\theta}_{i}\right) \mathbf{h}_{i}\left(\mathbf{x}, \boldsymbol{\theta}_{i}\right) \leq & e(t) \mathbf{g}_{i}(\mathbf{x}, 0) \mathbf{h}_{i}(\mathbf{x}, 0)+|e(t)|\left\{L_{i}(\mathbf{x}) \ell_{i}(\mathbf{x})\left(\sum_{j=1}^{p_{i}} \theta_{i j}\right)^{2}\right. \\
& \left.+\left[\left\|\mathbf{h}_{i}(\mathbf{x}, 0)\right\| L_{i}(\mathbf{x})+\left\|\mathbf{g}_{i}(\mathbf{x}, 0)\right\| \ell_{i}(\mathbf{x})\right] \sum_{j=1}^{p_{i}} \theta_{i j}\right\},
\end{aligned}
$$

hold true for any $e(t) \in R$.

Proof. Since,

$$
e(t)[\mathbf{g}(\mathbf{x}, \boldsymbol{\theta}) \mathbf{h}(\mathbf{x}, \boldsymbol{\theta})-\mathbf{g}(\mathbf{x}, 0) \mathbf{h}(\mathbf{x}, 0)] \leq|e(t) \| \mathbf{g}(\mathbf{x}, \boldsymbol{\theta}) \mathbf{h}(\mathbf{x}, \boldsymbol{\theta})-\mathbf{g}(\mathbf{x}, 0) \mathbf{h}(\mathbf{x}, 0)|,
$$

it is sufficient to prove that

$$
|\mathbf{g}(\mathbf{x}, \boldsymbol{\theta}) \mathbf{h}(\mathbf{x}, \boldsymbol{\theta})-\mathbf{g}(\mathbf{x}, 0) \mathbf{h}(\mathbf{x}, 0)| \leq\left\{L(\mathbf{x}) \ell(\mathbf{x})\left(\sum_{j=1}^{p} \theta_{j}\right)^{2}+[\|\mathbf{h}(\mathbf{x}, 0)\| L(\mathbf{x})+\|\mathbf{g}(\mathbf{x}, 0)\| \ell(\mathbf{x})] \sum_{j=1}^{p} \theta_{j}\right\},
$$

where $L(\mathbf{x}), \ell(\mathbf{x})$ are defined in (61) and note that the subscripts $i$ is neglected lor simplicity. Actually,

$$
\begin{aligned}
|\mathbf{g}(\mathbf{x}, \boldsymbol{\theta}) \mathbf{h}(\mathbf{x}, \boldsymbol{\theta})-\mathbf{g}(\mathbf{x}, 0) \mathbf{h}(\mathbf{x}, 0)| & =\mid \mathbf{g}(\mathbf{x}, \boldsymbol{\theta})-\mathbf{g}(\mathbf{x}, 0)] \mathbf{h}(\mathbf{x}, \boldsymbol{\theta})+\mathbf{g}(\mathbf{x}, 0)[\mathbf{h}(\mathbf{x}, \boldsymbol{\theta})-\mathbf{h}(\mathbf{x}, 0)] \mid \\
& \leq\|\mathbf{g}(\mathbf{x}, \boldsymbol{\theta})-\mathbf{g}(\mathbf{x}, 0)\|\|\mathbf{h}(\mathbf{x}, \boldsymbol{\theta})\|+\|\mathbf{g}(\mathbf{x}, 0)\|\|\mathbf{h}(\mathbf{x}, \boldsymbol{\theta})-\mathbf{h}(\mathbf{x}, 0)\| \\
& \leq\left(\sum_{j=1}^{p} L_{j}(\mathbf{x}) \theta_{j}\right)\|\mathbf{h}(\mathbf{x}, \boldsymbol{\theta})\|+\|\mathbf{g}(\mathbf{x}, 0)\|\left(\sum_{j=1}^{p} l_{j}(x) \theta_{j}\right) \\
& \leq L(\mathbf{x})\left(\sum_{j=1}^{p} \theta_{j}\right)\|\mathbf{h}(\mathbf{x}, \boldsymbol{\theta})\|+\|\mathbf{g}(\mathbf{x}, 0)\| l(\mathbf{x})\left(\sum_{j=1}^{p} \theta_{j}\right)
\end{aligned}
$$


and

$$
\begin{aligned}
\|\mathbf{h}(\mathbf{x}, \boldsymbol{\theta})\| & \leq\|\mathbf{h}(\mathbf{x}, \boldsymbol{\theta})-\mathbf{h}(\mathbf{x}, 0)\|+\|\mathbf{h}(\mathbf{x}, 0)\| \\
& \leq l(\mathbf{x})\left(\sum_{j=1}^{p} \theta_{j}\right)+\|\mathbf{h}(\mathbf{x}, 0)\| .
\end{aligned}
$$

leads to (63).

Our goal is to control the rigid manipulator to track a given trajectory $\mathbf{q}_{d}(t)$ by designing a nonlinear adaptive control to compensate for all uncertainties which are either LP uncertain dynamics according to Property 2.3 or NP as defined by (59), in system (58). For simplicity of the derivations throughout the paper, it is assumed that $\theta_{i} \in R_{+}^{p_{i}}$, i.e. $\theta_{i j} \geq 0, j=1,2,3$, $\ldots, p_{i}$. At the end of Section 4.3.2, we will see that the general case $\boldsymbol{\theta}_{i} \in R^{p_{i}}$ can be easily retrieved from our results. While traditional adaptive controls can be effectively applied only in the context of LP [2], lemma 5 reveals an ability to approximate the NP by its certain part plus a part of LP. We will use the key property (62) to design a novel nonlinear adaptive control for the system.

\subsection{A framework for adaptive control design}

Define vector $\mathbf{s}(t) \in R^{n}$ as a "velocity error" term

$$
\mathbf{s}(t)=\dot{\tilde{\mathbf{q}}}(t)+\boldsymbol{\Lambda} \tilde{\mathbf{q}}(t)=\dot{\mathbf{q}}(t)-\dot{\mathbf{q}}_{r}(t),
$$

where $\boldsymbol{\Lambda}=\operatorname{diag}\left[\lambda_{1}, \lambda_{2}, \ldots, \lambda_{n}\right] \in R^{n \times n}$ is an arbitrary positive definite matrix, $\tilde{\mathbf{q}}(t)=\mathbf{q}(t)-\mathbf{q}_{d}(t)$ is the position tracking error, and $\dot{\mathbf{q}}_{r}(t)=\dot{\mathbf{q}}_{d}(t)-\boldsymbol{\Lambda} \tilde{\mathbf{q}}(t)$, called the "reference velocity". According to Property 2.3, the dynamics of the system (58) can be rewritten in terms of the "velocity error" $s(t)$ as

$$
\mathbf{H}(\mathbf{q}) \dot{\mathbf{s}}(t)+\mathbf{C}(\mathbf{q}, \dot{\mathbf{q}}) \mathbf{s}(t)=\boldsymbol{\tau}(t)-\mathbf{Y}\left(\mathbf{q}, \dot{\mathbf{q}}, \dot{\mathbf{q}}_{r}, \ddot{\mathbf{q}}_{r}\right) \mathbf{a}-\mathbf{f}_{N}(\mathbf{x}, \boldsymbol{\theta}),
$$

with the identity $\mathbf{Y}\left(\mathbf{q}, \dot{\mathbf{q}}, \dot{\mathbf{q}}_{r}, \ddot{\mathbf{q}}_{r}\right) \mathbf{a}=\mathbf{H}(\mathbf{q}) \ddot{\mathbf{q}}_{r}+\mathbf{C}(\mathbf{q}, \dot{\mathbf{q}}) \dot{\mathbf{q}}_{r}+\mathbf{g}(\mathbf{q})$ used.

By definition (66), the tracking error $\tilde{q}_{i}(t)$ obtained from $S i(t)$ through the above designed first-order low pass filter is

$$
\tilde{q}_{i}(t)=\tilde{q}_{i}\left(t_{0}\right) e^{-\lambda_{i}\left(t-t_{0}\right)}+\int_{t_{0}}^{t} s_{i}(\zeta) e^{\lambda_{i}(\zeta-t)} d \zeta,
$$

where $\tilde{q}_{i}\left(t_{0}\right)$ is the tracking error of joint $i^{i h}$ of the robot manipulator at the time to. If $\left|s_{i}(t)\right| \leq \rho, \forall t \geq t_{0}$ then

$$
\begin{aligned}
\left|\tilde{q}_{i}(t)\right| & \leq\left|\tilde{q}_{i}\left(t_{0}\right)\right| e^{-\lambda_{i}\left(t-t_{0}\right)}+\int_{t_{0}}^{t}\left|s_{i}(\zeta)\right| e^{\lambda_{i}(\zeta-t)} d \zeta \\
& \leq\left(\left|\tilde{q}_{i}\left(t_{0}\right)\right|-\frac{\rho}{\lambda_{i}}\right) e^{-\lambda_{i}\left(t-t_{0}\right)}+\frac{\rho}{\lambda_{i}}
\end{aligned}
$$

The relation (68) means that $\lim _{t \rightarrow \infty}\left|\tilde{q}_{i}(t)\right| \leq \frac{\rho}{\lambda_{i}}$ whenever $\lim _{t \rightarrow \infty}\left|s_{i}(t)\right| \leq \rho$. Therefore, in the next development, the model (67) is used for designing a control input $\tau(t)$ which 
guarantees the velocity error $s(t) \rightarrow 0$ under LP uncertainty $a$ and NP uncertainty $\theta$. As shown above, such performance of $s(t)$ ensures the convergence to 0 of tracking error $\tilde{q}(t)$ when $t \rightarrow \infty$.

\subsubsection{Discontinuous adaptive control design}

Consider a quadratic Lyapunov function candidate

$$
V_{1}(t):=\frac{1}{2} \mathbf{s}^{T}(t) \mathbf{H}(\mathbf{q}) \mathbf{s}(t) .
$$

By Property 2.2, its time derivative can be written as

$$
\dot{V}_{1}(t)=\mathbf{s}^{T}\left(\boldsymbol{\tau}-\mathbf{Y a}-\mathbf{f}_{N}(\mathbf{x}, \boldsymbol{\theta})\right)=\mathbf{s}^{T}(\boldsymbol{\tau}-\mathbf{Y a})-\sum_{i=1}^{n} s_{i} f_{N i}\left(\mathbf{x}, \boldsymbol{\theta}_{i}\right) .
$$

where the notations on $t, \mathbf{q}, \dot{\mathbf{q}}, \dot{\mathbf{q}}_{r}, \ddot{\mathbf{q}}_{r}$ are neglected for simplicity. In view of relation (62), it follows that

$$
\begin{aligned}
\dot{V}_{1}(t) \leq & \mathbf{s}^{T}(\boldsymbol{\tau}-\mathbf{Y a})+\left(\sum_{i=1}^{n} s_{i} \mathbf{g}_{i}(\mathbf{x}, 0) \mathbf{h}_{i}(\mathbf{x}, 0)\right) \\
& +\sum_{i=1}^{n}\left|s_{i}\right|\left\{L_{i}(\mathbf{x}) \ell_{i}(\mathbf{x})\left(\sum_{j=1}^{p_{i}} \theta_{i j}\right)^{2}+\left[\left\|\mid \mathbf{h}_{i}(\mathbf{x}, 0)\right\| L_{i}(\mathbf{x})+\left\|\mathbf{g}_{i}(\mathbf{x}, 0)\right\| \ell_{i}(\mathbf{x})\right] \sum_{j=1}^{p_{i}} \theta_{i j}\right\} .
\end{aligned}
$$

With the definitions

$$
\begin{aligned}
& \mathbf{W}(\mathbf{x}) \quad:=\operatorname{diag}\left[\mathbf{w}_{1}(\mathbf{x}), \mathbf{w}_{2}(\mathbf{x}), \ldots, \mathbf{w}_{n}(\mathbf{x})\right] \in R^{n \times 2 n}, \\
& \boldsymbol{\Phi}(\mathbf{s}, \mathbf{x}):=\operatorname{diag}\left[\operatorname{sgn}\left(s_{1}\right) \mathbf{w}_{1}(\mathbf{x}), \ldots, \operatorname{sgn}\left(s_{n}\right) \mathbf{w}_{n}(\mathbf{x})\right] \in R^{n \times 2 n}, \\
& \boldsymbol{\beta} \quad:=\left[\begin{array}{llll}
\boldsymbol{\beta}_{1}^{T} & \boldsymbol{\beta}_{2}^{T} & \ldots & \boldsymbol{\beta}_{n}^{T}
\end{array}\right]^{T} \in R^{2 n}, \\
& \mathbf{w}_{i}(\mathbf{x})=\left[\begin{array}{ll}
w_{i 1} & w_{i 2}
\end{array}\right] \\
& :=\left[L_{i}(\mathbf{x}) \ell_{i}(\mathbf{x}) \quad\left\|\mathbf{h}_{i}(\mathbf{x}, 0)\right\| L_{i}(\mathbf{x})+\left\|\mathbf{g}_{i}(\mathbf{x}, 0)\right\| \ell_{i}(\mathbf{x})\right], \\
& \boldsymbol{\beta}_{i} \quad=\left[\begin{array}{ll}
\beta_{i 1} & \beta_{i 2}
\end{array}\right]^{T} \\
& :=\left[\begin{array}{ll}
\left(\sum_{j=1}^{p_{i}} \theta_{i j}\right)^{2} & \sum_{j=1}^{p_{i}} \theta_{i j}
\end{array}\right]^{T} \text {, }
\end{aligned}
$$

the inequality (69) can be rewritten as

$$
\dot{V}_{1}(t) \leq \mathbf{s}^{T}(\boldsymbol{\tau}-\mathbf{Y a})+\mathbf{s}^{T} \mathbf{f}_{N}(\mathbf{x}, 0)+\mathbf{s}^{T} \boldsymbol{\Phi}(\mathbf{s}, \mathbf{x}) \boldsymbol{\beta},
$$

Therefore, the control input

$$
\boldsymbol{\tau}=-\mathbf{K}_{D} \mathbf{s}+\mathbf{Y} \hat{\mathbf{a}}-\mathbf{f}_{N}(\mathbf{x}, 0)-\boldsymbol{\Phi}(\mathbf{s}, \mathbf{x}) \hat{\boldsymbol{\beta}},
$$

results in

$$
\dot{V}_{1}(t) \leq-\mathbf{s}^{T} \mathbf{K}_{D} \mathbf{s}+\mathbf{s}^{T}[\mathbf{Y} \tilde{\mathbf{a}}-\boldsymbol{\Phi}(\mathbf{s}, \mathbf{x})] \tilde{\boldsymbol{\beta}}
$$


where $\tilde{\mathbf{a}}=\hat{\mathbf{a}}-\mathbf{a}$ and $\tilde{\boldsymbol{\beta}}=\hat{\boldsymbol{\beta}}-\boldsymbol{\beta}$ are parameter errors and $\mathbf{K}_{D} \in R^{n \times n}$ is an arbitrary positive definite matrix.

To derive update laws for the parameter estimates, we employ the following Lyapunov function

$$
V(t)=V_{1}(t)+\frac{1}{2}\left(\tilde{\mathbf{a}}^{T} \boldsymbol{\Gamma}_{a}^{-1} \tilde{\mathbf{a}}+\tilde{\boldsymbol{\beta}}^{T} \boldsymbol{\Gamma}_{\boldsymbol{\beta}}^{-1} \tilde{\boldsymbol{\beta}}\right),
$$

where $\boldsymbol{\Gamma}_{a}, \boldsymbol{\Gamma}_{\beta}$ are arbitrary positive definite matrices. It follows form (73) that

$$
\dot{V}(t) \leq-\mathbf{s}^{T} \mathbf{K}_{D} \mathbf{s}+\mathbf{s}^{T}[\mathbf{Y} \tilde{\mathbf{a}}-\boldsymbol{\Phi}(\mathbf{s}, \mathbf{x})] \tilde{\boldsymbol{\beta}}+\dot{\hat{\mathbf{a}}}^{T} \boldsymbol{\Gamma}_{a}^{-1} \tilde{\mathbf{a}}+\dot{\hat{\boldsymbol{\beta}}}^{T} \boldsymbol{\Gamma}_{\boldsymbol{\beta}}^{-1} \tilde{\boldsymbol{\beta}}
$$

Therefore, the following update laws

$$
\dot{\hat{\mathbf{a}}}=-\boldsymbol{\Gamma}_{a} \mathbf{Y}^{\mathbf{T}} \mathbf{s}, \dot{\hat{\boldsymbol{\beta}}}=\boldsymbol{\Gamma}_{\beta} \mathbf{W}^{T}(\mathbf{x})|\mathbf{s}|, \quad|\mathbf{s}|=\left[\begin{array}{llll}
\left|s_{1}\right| & \left|s_{2}\right| & \ldots & \left|s_{n}\right|
\end{array}\right]^{T}
$$

yield

$$
\dot{V}(t) \leq-\mathbf{s}^{T} \mathbf{K}_{D} \mathbf{s} .
$$

The last inequality implies that $V(t)$ is decreasing, and thus is bounded by $V(0)$. Consequently, $\mathbf{s}(t)$ and $\tilde{\mathbf{a}}(t), \tilde{\boldsymbol{\theta}}(t)$ must be bounded quantities by virtue of definition (74). Given the boundedness of the reference trajectory $\mathbf{q}_{d}, \dot{\mathbf{q}}_{d,}, \overrightarrow{\mathbf{q}}_{d y}$ one has $\dot{\mathbf{s}}(t) \in L_{\infty}$ from the system dynamics (67). Also, relation (77) gives $\lambda_{\min }\left(\mathbf{K}_{D}\right) \int_{0}^{T}\|\mathbf{s}(t)\|^{2} d t \leq V(0), \forall T>0$, i.e. $\mathbf{s}(t) \in L_{2}$, where $\lambda_{\min }\left(\mathbf{K}_{D}\right)$ denotes the minimum eigenvalue of $\mathbf{K}_{D}$. Applying Barbalat's lemma [2] yields $\lim _{t \rightarrow \infty} \mathbf{s}(t)=0$. However, the control (72) is still discontinuous at $\mathbf{s}(t)=0$, and thus is not readily implemented. As a next stage, we make the control action continuous by a standard modification technique which leads to a practically implementable control law.

\subsubsection{Continuous adaptive control design}

A continuous control action can be derived by modifying the velocity error $\mathbf{s}(t)$. First, introduce a new variable $\mathbf{s}_{\varepsilon}(t)$ by setting

where

$$
\mathbf{s}_{\varepsilon}=\mathbf{s}-\frac{1}{\sqrt{3}} \mathbf{c}(\mathbf{s}),
$$

$$
\begin{gathered}
\mathbf{c}(\mathbf{s})=\left[\begin{array}{ll}
c_{1}\left(s_{1}\right) \quad \ldots & c_{n}\left(s_{n}\right)
\end{array}\right]^{T}, \\
c_{i}\left(s_{i}\right)= \begin{cases}b_{i}+\sqrt{r_{i}^{2}-\left(s_{i}-\varepsilon_{i}\right)^{2}}, & \frac{\sqrt{3}-1}{2} \varepsilon_{i} \leq s_{i} \leq \varepsilon_{i} \\
\sqrt{3} s_{i}, & \left|s_{i}\right| \leq \frac{\sqrt{3}-1}{2} \varepsilon_{i} \\
-b_{i}-\sqrt{r_{i}^{2}-\left(s_{i}+\varepsilon_{i}\right)^{2}}, & -\varepsilon_{i} \leq s_{i} \leq-\frac{\sqrt{3}-1}{2} \varepsilon_{i} \\
\varepsilon_{i} \operatorname{sgn}\left(s_{i}\right), & \left|s_{i}\right|>\varepsilon_{i}\end{cases} \\
r_{i}=(\sqrt{3}-1) \varepsilon_{i}, b_{i}=(2-\sqrt{3}) \varepsilon_{i}, \text { for } \varepsilon_{i}>0, \forall i=1, \ldots, n .
\end{gathered}
$$


It is standard to show that such $\mathbf{s}_{\varepsilon}(t)$ is continuously differentiable in time $t$ (see also Figure 1). Using Property 2.3, the dynamics of system (58) in terms of the modified "velocity error" $\mathbf{s}_{\varepsilon}(t)$ is expressed by

$$
\mathbf{H}(\mathbf{q}) \dot{\mathbf{s}}_{\varepsilon}+\mathbf{C}(\mathbf{q}, \dot{\mathbf{q}}) \mathbf{s}_{\varepsilon}=\boldsymbol{\tau}-\mathbf{Y}_{\varepsilon} \mathbf{a}-\mathbf{f}_{N}(\mathbf{x}, \boldsymbol{\theta}),
$$

where $\mathbf{Y}_{\varepsilon}=\mathbf{H}(\mathbf{q})\left(\ddot{\mathbf{q}}_{r}+\frac{1}{\sqrt{3}} \dot{\mathbf{c}}(\mathbf{s})\right)+\mathbf{C}(\mathbf{q}, \dot{\mathbf{q}})\left(\dot{\mathbf{q}}_{r}+\frac{1}{\sqrt{3}} \mathbf{c}(\mathbf{s})\right)+\mathbf{g}(\mathbf{q})$ -

Now, take the following Lyapunov function

$$
V_{\varepsilon}(t)=\frac{1}{2} \mathbf{s}_{\varepsilon}^{T} \mathbf{H}(\mathbf{q}) \mathbf{s}_{\varepsilon}+\frac{1}{2}\left(\tilde{\mathbf{a}}^{T} \boldsymbol{\Gamma}_{a}^{-1} \tilde{\mathbf{a}}+\tilde{\boldsymbol{\beta}}^{T} \boldsymbol{\Gamma}_{\boldsymbol{\beta}}^{-1} \tilde{\boldsymbol{\beta}}\right)
$$

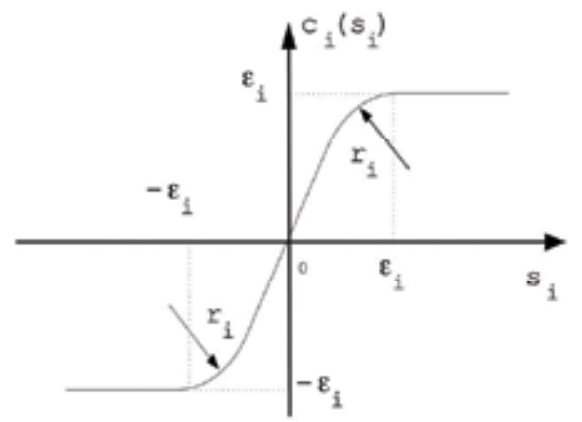

Figure 1. Smooth function $c_{i}\left(s_{i}\right)$

Like (71), it is clear that

$$
\dot{V}_{\epsilon}(t) \leq \mathbf{s}_{\varepsilon}^{T}\left(\boldsymbol{\tau}-\mathbf{Y}_{\varepsilon} \mathbf{a}\right)+\mathbf{s}_{\varepsilon}^{T} \mathbf{f}_{N}(\mathbf{x}, 0)+\mathbf{s}_{\varepsilon}^{T} \boldsymbol{\Phi}\left(\mathbf{s}_{\varepsilon}, \mathbf{x}\right) \boldsymbol{\beta}+\dot{\hat{\mathbf{a}}}^{T} \boldsymbol{\Gamma}_{a}^{-1} \tilde{\mathbf{a}}+\dot{\hat{\boldsymbol{\beta}}}^{T} \boldsymbol{\Gamma}_{\boldsymbol{\beta}}^{-1} \tilde{\beta}
$$

where let us recall that $\Phi$ is already defined by formula (70).

Note that whenever $\left|s_{i}\right| \leq \frac{\sqrt{3}-1}{2} \varepsilon_{i}$, one has

$$
s_{i \varepsilon}=0
$$

and for $\left|s_{i}\right|>\frac{\sqrt{3}-1}{2} \varepsilon_{i}$

$$
\begin{aligned}
& s_{i \varepsilon}^{2} \leq s_{i \varepsilon} s_{i}, \\
& \operatorname{sgn}\left(s_{i}\right)=\operatorname{sgn}\left(s_{i \varepsilon}\right) .
\end{aligned}
$$

Hence, introducing the saturated function

$$
\operatorname{sat}_{\varepsilon_{i}}\left(s_{i}\right)=\left\{\begin{array}{ccc}
\frac{s_{i}}{\sqrt{3}-1} \varepsilon_{i} & \text { when } & \left|s_{i}\right| \leq \frac{\sqrt{3}-1}{2} \varepsilon_{i} \\
\operatorname{sgn}\left(s_{i}\right) & \text { when } & \left|s_{i}\right|>\frac{\sqrt{3}-1}{2} \varepsilon_{i},
\end{array}\right.
$$

and taking (82), (83) into account, the following continuous control input 


$$
\boldsymbol{\tau}=-\mathbf{K}_{D} \mathbf{s}+\mathbf{Y}_{\varepsilon} \hat{\mathbf{a}}-\mathbf{f}_{N}(\mathbf{x}, 0)-\boldsymbol{\Phi}_{\varepsilon}(\mathbf{s}, \mathbf{x}) \hat{\boldsymbol{\beta}}
$$

with

$$
\boldsymbol{\Phi}_{\varepsilon}(\mathbf{s}, \mathbf{x}):=\operatorname{diag}\left[\operatorname{sat}_{\varepsilon_{1}}\left(s_{1}\right) \mathbf{w}_{1}(x), \ldots, \operatorname{sat}_{\varepsilon_{n}}\left(s_{n}\right) \mathbf{w}_{n}(x)\right] \in R^{n \times 2 n},
$$

together with the update laws

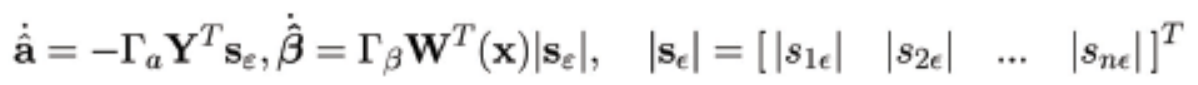

yield

$$
\dot{V}_{\varepsilon}(t) \leq-\mathbf{s}_{\varepsilon}^{T} \mathbf{K}_{D} \mathbf{s}_{\varepsilon} .
$$

Finally, by a similar analysis as done in Section 4.3.1, the error $\mathbf{s}_{\varepsilon}(t)$ of the system converges to 0 , or equivalently $\lim _{t \rightarrow \infty}\left|s_{i}\right| \leq \frac{\sqrt{3}-1}{2} \varepsilon_{i}, i=1, \ldots, n$. From relation (68), the tracking error $\tilde{q}_{i}(t)$ converges to $\frac{\sqrt{3}-1}{2 \lambda_{i}} \varepsilon_{i}$ as $t \rightarrow \infty$. We are now in a position to sum up our results.

Theorem 6 The adaptive controller defined by equations (78),(79),(84)-(86) enables system to asymptotically track a desired trajectory $\mathbf{q}_{d}(t)$ within a precision of $\frac{\sqrt{3}-1}{2 \lambda_{i}} \varepsilon_{i}, i=1, \ldots, n$.

Remark 1 In the general case where $\boldsymbol{\theta}_{i} \in R^{p_{i}}$, it follows in a straightforward manner from lemma 5 that

$$
\begin{aligned}
e(t) \mathbf{g}_{i}\left(\mathbf{x}, \boldsymbol{\theta}_{i}\right) \mathbf{h}_{i}\left(\mathbf{x}, \boldsymbol{\theta}_{i}\right) \leq & e(t) \mathbf{g}_{i}(\mathbf{x}, 0) \mathbf{h}_{i}(\mathbf{x}, 0)+|e(t)|\left\{L_{i}(\mathbf{x}) \ell_{i}(\mathbf{x})\left(\sum_{j=1}^{p_{i}}\left|\theta_{i j}\right|\right)^{2}\right. \\
& \left.+\left[\left\|\mathbf{h}_{i}(\mathbf{x}, 0)\right\| L_{i}(\mathbf{x})+\left\|\mathbf{g}_{i}(\mathbf{x}, 0)\right\| \ell_{i}(\mathbf{x})\right] \sum_{j=1}^{p_{i}}\left|\theta_{i j}\right|\right\} .
\end{aligned}
$$

Therefore, with a Lyapunov function defined in (81) where

$$
\boldsymbol{\beta}_{i}=\left[\begin{array}{ll}
\beta_{i 1}, & \beta_{i 2}
\end{array}\right]^{T}=\left[\left(\sum_{j=1}^{p}\left|\theta_{i j}\right|\right)^{2}, \quad \sum_{j=1}^{p}\left|\theta_{i j}\right|\right]^{T},
$$

Theorem 6 remains valid for $\boldsymbol{\theta}_{i} \in R_{i}^{p}$.

Remark 2 The new variable (78) and the function (79) are properly designed to make the stabilizing control (72) continuous. Of course, there are other appropriate choices other than the variable (78) and the function (79), which also make the stabilizing control (72) continuous, too.

\subsubsection{1-dimension estimator}

In the design of sections 4.3.1 and 4.3.2, the dimensions of estimators are equal to the number of unknown parameters in the system, i.e. $\hat{\mathbf{a}} \in R^{\nu}, \hat{\boldsymbol{\beta}} \in R^{2 n}$. Thus, increasing the 
number of links may result in estimators of excessively large dimension. Tuning updating gains $\boldsymbol{\Gamma}_{a}, \boldsymbol{\Gamma}_{\beta}$ for those estimators then becomes a very laborious task. In this section, we show that it is possible to design an adaptive controller for system (58) with simple 1dimension estimators $\hat{a}, \hat{\beta}$ independently of the dimensions of the unknown parameters a and $\beta$.

For that purpose, first consider the term $\mathbf{Y a}$ in (69) where $\mathbf{Y} \in R^{n \times \omega}, \mathbf{a} \in R^{\omega}$. It is clear that

$$
\sum_{j=1}^{\omega} Y_{i j} a_{j} \leq\left(\max _{j=1, \ldots, \omega}\left|Y_{i j}\right|\right) \sum_{j=1}^{\omega}\left|a_{j}\right|, i=1, \ldots, n .
$$

Also note from (70) that

$$
\mathbf{w}_{i}(\mathbf{x}) \boldsymbol{\beta}_{i} \leq \max _{j=1,2}\left|w_{i j}(\mathbf{x})\right|\left(\left|\beta_{i 1}\right|+\left|\beta_{i 2}\right|\right), i=1, \ldots, n .
$$

As a result, the inequality (71) can be rewritten as follows

$$
\dot{V}_{1}(t) \leq \mathbf{s}^{T} \boldsymbol{\tau}+\mathbf{s}^{T} \mathbf{f}_{N}(\mathbf{x}, 0)+\left|\mathbf{s}^{T}\right|\left(\mathbf{y}_{\max } \sum_{j=1}^{\omega}\left|a_{j}\right|+\mathbf{w}_{\max }(\mathbf{x}) \sum_{i=1}^{n}\left(\left|\beta_{i 1}\right|+\left|\beta_{i 2}\right|\right)\right),
$$

where

$$
\begin{aligned}
\mathbf{y}_{\max } & :=\left[\max _{j=1, \ldots, \omega}\left|Y_{1 j}\right|, \ldots, \max _{j=1, \ldots, \omega}\left|Y_{n j}\right|\right]^{T} \in R^{n}, \\
\mathbf{w}_{\max }(\mathbf{x}) & :=\left[\max _{j=1,2}\left|w_{1 j}(\mathbf{x})\right|, \ldots, \max _{j=1,2}\left|w_{n j}(\mathbf{x})\right|\right]^{T} \in R^{n} .
\end{aligned}
$$

Note that $\mathbf{y}_{\max }$ is the function whose notations on variables $\mathbf{q}, \dot{\mathbf{q}}, \dot{\mathbf{q}}_{r}, \ddot{\mathbf{q}}_{r}$ are neglected for simplicity. Therefore, with the definitions

$$
\begin{aligned}
\psi_{\max }(\mathbf{s}) & :=\operatorname{diag}\left[\operatorname{sgn}\left(s_{1}\right), \ldots, \operatorname{sgn}\left(s_{n}\right)\right] \mathbf{y}_{\max } \in R^{n}, \\
\phi_{\max }(\mathbf{s}, \mathbf{x}) & :=\operatorname{diag}\left[\operatorname{sgn}\left(s_{1}\right), \ldots, \operatorname{sgn}\left(s_{n}\right)\right] \mathbf{w}_{\max }(\mathbf{x}) \in R^{n},
\end{aligned}
$$

the following control input

$$
\begin{aligned}
\boldsymbol{\tau} & =-\mathbf{K}_{D} \mathbf{s}-\mathbf{f}_{N}(\mathbf{x}, 0)-\left(\boldsymbol{\psi}_{\max }(\mathbf{s}) \hat{a}+\phi_{\max }(\mathbf{s}, \mathbf{x}) \hat{\beta}\right), \\
\dot{\hat{a}} & =\gamma_{a} \mathbf{y}_{\max }^{T}|\mathbf{s}|, \dot{\hat{\beta}}=\gamma_{\beta} \mathbf{w}_{\max }^{T}(\mathbf{x})|\mathbf{s}|,
\end{aligned}
$$

where $\gamma_{a}$ and $\gamma_{a}$ are arbitrary positive scalars, together with the following Lyapunov function

$$
V_{1 D}(t)=V_{1}(t)+\frac{1}{2} \gamma_{a}^{-1}\left(\sum_{j=1}^{\omega}\left|a_{j}\right|-\hat{a}\right)^{2}+\frac{1}{2} \gamma_{\beta}^{-1}\left(\sum_{i=1}^{n}\left(\left|\beta_{i 1}\right|+\left|\beta_{i 2}\right|\right)-\hat{\beta}\right)^{2},
$$

yield

$$
\dot{V}(t)_{1 D} \leq-\mathbf{s}^{T} \mathbf{K}_{D} \mathbf{s} .
$$


Therefore, the discontinuous control (87) results in the convergence to 0 of velocity error $\mathbf{s}(t)$, which ensures the convergence to 0 of tracking error $\tilde{\mathbf{q}}(t)$ when $t \rightarrow \infty$. As in section 4.3.2, we can alter the discontinuous control (87) into a continuous one as follows

$$
\begin{aligned}
\boldsymbol{\tau} & =-\mathbf{K}_{D} \mathbf{s}-\mathbf{f}_{N}(\mathbf{x}, 0)-\left(\boldsymbol{\psi}_{\max \varepsilon}(\mathbf{s}) \hat{a}+\phi_{\max \varepsilon}(\mathbf{s}, \mathbf{x}) \hat{\beta}\right) \\
\dot{\hat{a}} & =\gamma_{a} \mathbf{y}_{\max }^{T}\left|\mathbf{s}_{\varepsilon}\right|, \dot{\hat{\beta}}=\gamma_{\beta} \mathbf{w}_{\max }^{T}(\mathbf{x})\left|\mathbf{s}_{\varepsilon}\right|
\end{aligned}
$$

where

$$
\begin{aligned}
\psi_{\max \varepsilon}(\mathbf{s}) & :=\operatorname{diag}\left[\operatorname{sat}_{\varepsilon_{1}}\left(s_{1}\right), \ldots, \operatorname{sat}_{e_{n}}\left(s_{n}\right)\right] \mathbf{y}_{\max } \in R^{n} \\
\phi_{\max \varepsilon}(\mathbf{s}, \mathbf{x}) & :=\operatorname{diag}\left[\operatorname{sat}_{\varepsilon_{1}}\left(s_{1}\right), \ldots, \operatorname{sat}_{\varepsilon_{n}}\left(s_{n}\right)\right] \mathbf{w}_{\max }(\mathbf{x}) \in R^{n}
\end{aligned}
$$

Then the continuous control (89) ensures the convergence to $\frac{\sqrt{3}-1}{2 \lambda_{i}} \varepsilon_{i}, i=1, \ldots, \mathrm{n}$ of the tracking error $\tilde{\mathbf{q}}(t)$ when $t \rightarrow \infty$.

\subsection{Example of nonlinear friction compensation}

In this section, we examine how effectively our designed adaptive controllers can compensate for the frictional forces in joints of robot manipulators.

\subsubsection{Friction model and friction compensators}

Frictional forces in system (58) can be described in different ways. Here, we consider the well-known Amstrong-Helouvry model [3]. For joint $i$, the frictional force is described as

$$
\begin{aligned}
f_{i}= & F_{c i} \operatorname{sgn}\left(\dot{q}_{i}\right)\left[1-\exp \left(-\frac{\dot{q}_{i}^{2}}{v_{s i}^{2}}\right)\right] \\
& +F_{s i} \operatorname{sgn}\left(\dot{q}_{i}\right) \exp \left(-\frac{\dot{q}_{i}^{2}}{v_{s i}^{2}}\right)+F_{v i} \dot{q}_{i}
\end{aligned}
$$

where $F_{c i}, F_{s i}, F_{v i}$ are coefficients characterizing the Coulomb friction, static friction and viscous friction, respectively, and $v_{s i}$ is the Stribeck parameter. Note that the friction term (90) can be decomposed into a linear part $f_{L i}$ and a nonlinear part $f_{\mathrm{Ni}}$ as

$$
f_{i}=f_{L i}+f_{N i}
$$

where

$$
f_{L i}=F_{c i} \operatorname{sgn}\left(\dot{q}_{i}\right)+F_{v i} \dot{q}_{i}=\mathbf{z}_{i} \boldsymbol{\alpha}_{i}
$$

with $\alpha_{i}=\left[\begin{array}{ll}F_{c i} & F_{v i}\end{array}\right]^{T}, \mathbf{z}_{i}=\left[\operatorname{sgn}\left(\dot{q}_{i}\right) \quad \dot{q}_{i}\right]$, and

$$
f_{N i}=\left(F_{s i}-F_{c i}\right) \operatorname{sgn}\left(\dot{q}_{i}\right) \exp \left(-\frac{\dot{q}_{i}^{2}}{v_{s i}^{2}}\right) .
$$


Practically, the frictional coefficients are not exactly known. In such case, the frictional force $f_{L i}$ can be compensated by a traditional adaptive control for LP. However, the situation becomes non trivial when there are unknown parameters appearing nonlinearly in the model of $f_{N i}$.

The NP friction term of joint $i, f_{N i}$, can be expressed in the form (59) with

$$
f_{N i}=g_{i}\left(\dot{q}_{i}, \boldsymbol{\theta}_{i}\right) h_{i}\left(\dot{q}_{i}, \boldsymbol{\theta}_{i}\right),
$$

where

$$
\begin{aligned}
\boldsymbol{\theta}_{i} & =\left[\begin{array}{ll}
\left(F_{s i}-F_{c i}\right) & \frac{1}{v_{s i}^{2}}
\end{array}\right]^{T}=\left[\begin{array}{ll}
\theta_{i 1} & \theta_{i 2}
\end{array}\right]^{T}, \\
g_{i}\left(\dot{q}_{i}, \theta_{i}\right) & =\left[\begin{array}{ll}
1 & 0
\end{array}\right] \theta_{i}, h_{i}\left(\dot{q}_{i}, \theta_{i}\right)=\operatorname{sgn}\left(\dot{q}_{i}\right) \exp \left(-\dot{q}_{i}^{2} \theta_{i 2}\right) .
\end{aligned}
$$

Clearly, $g_{i}$ and $h_{i}$ are Lipschitzian in $\theta_{i}$ with Lipschitzian coefficients $l_{i}(\dot{q})=\dot{q}_{i}^{2}, L_{i}(\dot{q})=1$. Also, we have $g_{i}\left(\dot{q}_{i}, 0\right)=0, h_{i}\left(\dot{q}_{i}, 0\right)=1$. Therefore, by Theorem 6 , the following adaptive controller enables the system (58), (90), (94) to asymptotically track a desired trajectory $q_{d i}(t)$ within a precision of $\frac{\sqrt{3}-1}{2 \lambda_{i}} \varepsilon_{q^{\prime}} i=1, \ldots, n$.

$$
\begin{aligned}
\boldsymbol{\tau} & =-\mathbf{K}_{D} \mathbf{S}+\mathbf{Y}_{\varepsilon} \hat{\mathbf{a}}+\mathbf{Z} \hat{\boldsymbol{\alpha}}-\mathbf{\Phi}_{\varepsilon}(\mathbf{s}, \mathbf{x}) \hat{\boldsymbol{\beta}} \\
\dot{\hat{\mathbf{a}}} & =-\boldsymbol{\Gamma}_{a} \mathbf{Y}^{T} \mathbf{s}_{\varepsilon}, \dot{\hat{\boldsymbol{\alpha}}}=-\boldsymbol{\Gamma}_{\alpha} \mathbf{Z}^{T} \mathbf{s}_{\varepsilon}, \hat{\hat{\boldsymbol{\beta}}}=\boldsymbol{\Gamma}_{\beta} \mathbf{W}^{T}(\mathbf{x})\left|\mathbf{s}_{\varepsilon}\right|,
\end{aligned}
$$

where

$$
\begin{array}{rc}
\mathbf{Z}= & \operatorname{diag}\left[\mathbf{z}_{1}\left(q_{1}\right), \ldots, \mathbf{z}_{n}\left(q_{n}\right)\right] \in R^{n \times 2 n}, \\
\mathbf{w}_{i}(\dot{\mathbf{q}}) & = \\
{\left[\dot{q}_{i}^{2},\right.} & 1] .
\end{array}
$$

Note that with the control (95), the term $\mathbf{Z} \hat{\alpha}$ compensates for the LP frictions $f_{\mathrm{Li}}$.

\subsubsection{Simulations}

A prototype of a planar $2 \mathrm{DOF}$ robot manipulator is built to assess the validity of the proposed methods (Figure 2) . The dynamic model of the manipulator and its linearized dynamics parameter are given in Section 6 (Appendix).

The manipulator model is characterized by a real parameter $\mathrm{a}$, which is identified by a standard technique (See Table 3 in Section 6). The parameters of friction model (90) are chosen such that the effect of the NP frictions $f_{N i}$ are significant, i.e.

$$
F_{c i}=0.49, F s i=3.5, F_{v i}=0.15, v_{s i}=0.189, \forall i=1,2 .
$$

In order to focus on the compensation of nonlinearly parameterized frictions, we have selected the objective of low-velocity tracking. The manipulator must track the desired trajectory $q_{d 1}(t)=\frac{\pi}{6}(1-\cos (t)), q_{d 2}(t)=\frac{\pi}{4}(1-\cos (t))$. Clearly, the selected trajectory contains various zero velocity crossings.

For comparison, we use 2 different controllers to accomplish the tracking task. 


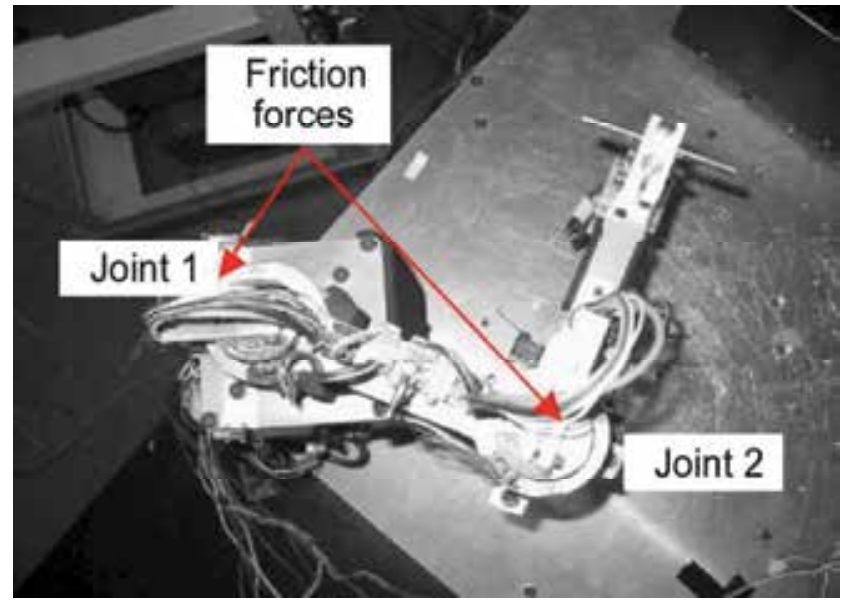

Figure 2. Prototype of robot manipulator

\begin{tabular}{|l|l|l|l|}
\hline $\mathbf{K}_{D}$ & $\boldsymbol{\Lambda}$ & $\boldsymbol{\Gamma}_{a}$ & $\boldsymbol{\Gamma}_{\alpha}$ \\
\hline \hline $10 \mathbf{I}(2,2)$ & $5 \mathbf{I}(2,2)$ & $\operatorname{diag}(5,5,5)$ & $\operatorname{diag}(3,3,3,3)$ \\
\hline
\end{tabular}

Table 1. Parameters of the controllers for simulations

- A traditional adaptive control based on the LP structure to compensate for uncertainty in dynamic parameter a of the manipulator links and the linearly parameterized frictions $f_{L i}(92)$ in joints of motors.

$$
\begin{aligned}
& \boldsymbol{\tau}=-\mathbf{K}_{D} \mathbf{s}+\mathbf{Y} \hat{\mathbf{a}}+\mathbf{Z} \hat{\mathbf{\alpha}} \\
& \dot{\hat{\mathbf{a}}}=-\boldsymbol{\Gamma}_{a} \mathbf{Y}^{T} \mathbf{s}, \dot{\hat{\alpha}}=-\boldsymbol{\Gamma}_{\alpha} \mathbf{Z}^{T} \mathbf{s},
\end{aligned}
$$

The gains of the controller are chosen as in Table $1, \hat{\mathbf{a}} \in R^{3}, \hat{\boldsymbol{\alpha}} \in R^{4}$.

- Our proposed controller (95) with the same control parameters for LP uncertainties. Additionally, $\mathbf{\Gamma}_{\beta}=\operatorname{diag}(50,50,50,50), \epsilon=0.05$ for NP friction compensation, $\hat{\boldsymbol{\beta}} \in R^{4}$.

Both controllers start without any prior information of dynamic and frictional parameters, i.e. $\hat{\mathbf{a}}(0)=0, \hat{\boldsymbol{\alpha}}(0)=0, \hat{\boldsymbol{\beta}}(0)=0$.

Tradition LP adaptive control vs. proposed control

It can be seen that the position error is much smaller with the proposed control (Figure 3), especially at points where manipulator velocities cross the value of zero. Indeed, the position error of joint 1 decreases about 20 times. The position tracking of joint 2 is improved in the sense that our proposed control obtains a same level of position error as the one of LP, but the bound of control input is reduced about 3 times. This means that the nonlinearly parameterized frictions are effectively compensated by our method.

1-dimension estimators

The performances of the controller with 1-dimension estimators (89) is shown in Figure 4. One estimate is designed for the manipulator dynamics a $a \in R^{3}$, one is for the LP friction parameters a $\boldsymbol{\alpha} \in R^{4}$, and one is for the NP friction parameters $\boldsymbol{\beta} \in R^{4}$. Thus, by using 1dimension estimators, the estimates dimension reduces from 11 to 3 . The resulting controller benefits not only from a simpler tuning scheme, but also from a minimum amount of on-line calculation since the regressor matrices $\mathbf{Y}, \mathbf{W}$ reduce to the vectors $\mathrm{y}_{\max }, \mathrm{w}_{\max }$ in this case. 
Position orrors (rad)
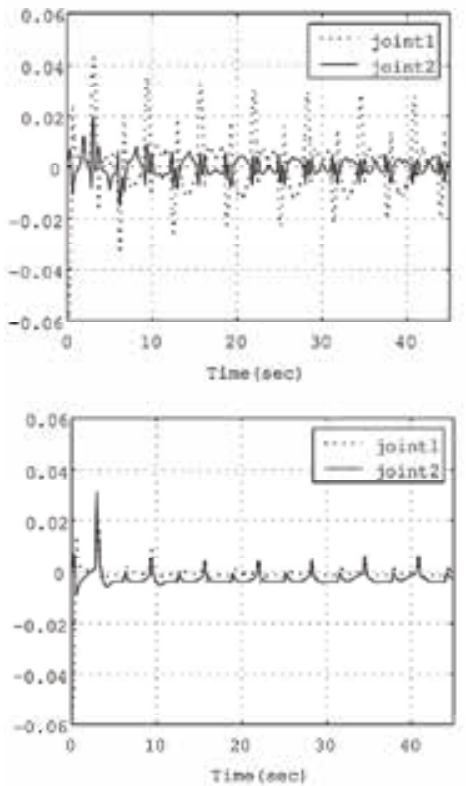

(a)

Control torques (Nm)

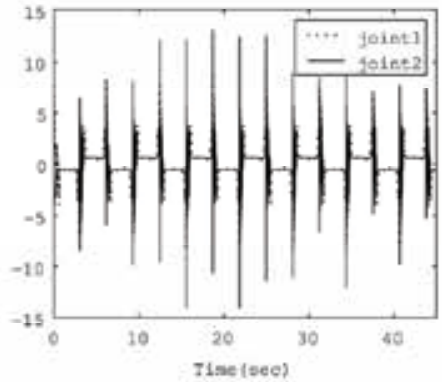

(b)

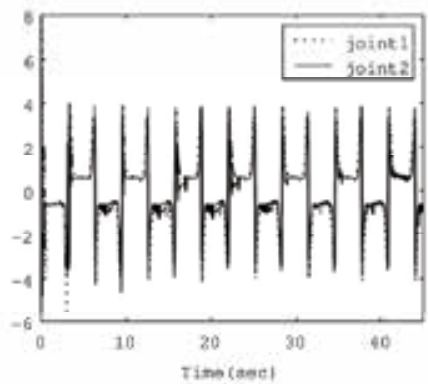

Figure 3. Simulation results: Tracking errors of joints (left) and characteristics of control inputs (right), (a): Traditional LP adaptive controller (97), (b): proposed controller (95)
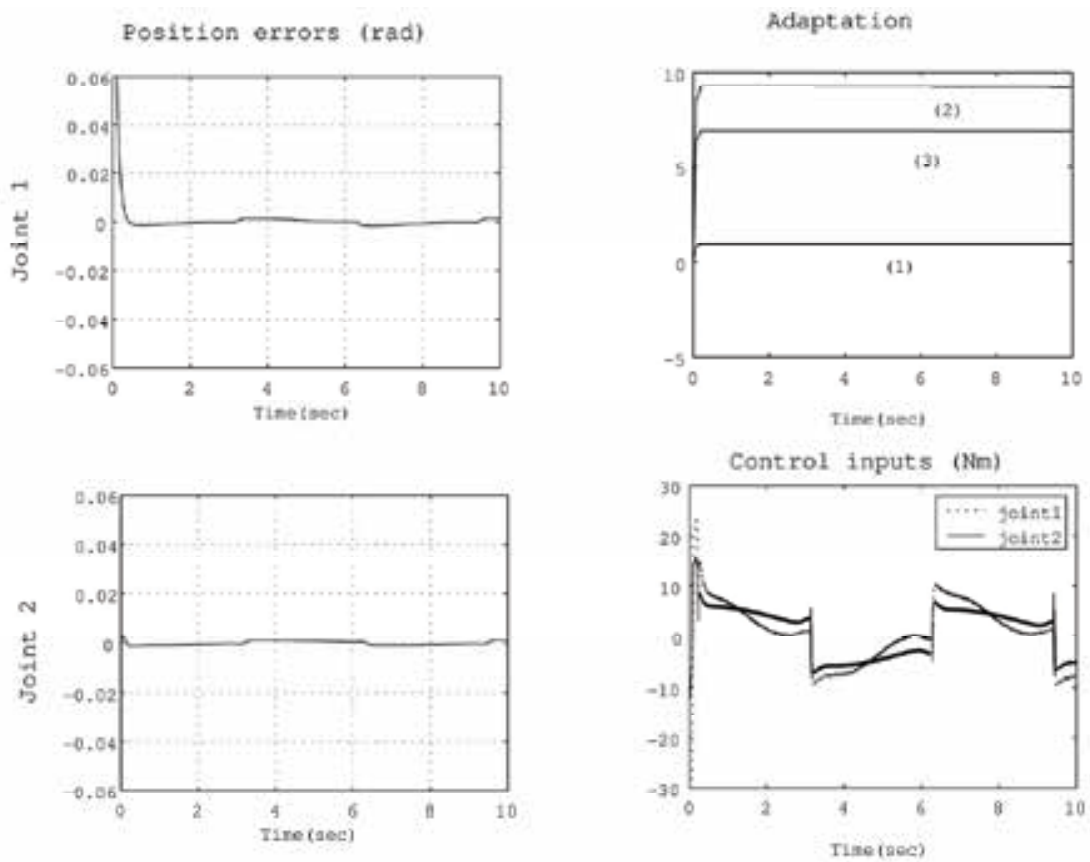

Figure 4. Simulation results for proposed 1-dimension estimators (89): Tracking errors of joints (left), the adaptation of the estimates and characteristics of control inputs (right) . (1) $-\hat{\mathbf{a}},(2)-\hat{\alpha},(3)-\hat{\beta}$ 


\begin{tabular}{|l|l|l|l|}
\hline $\mathbf{K}_{D}$ & $\boldsymbol{\Lambda}$ & $\boldsymbol{\Gamma}_{a}$ & $\boldsymbol{\Gamma}_{\alpha}$ \\
\hline \hline $3 \mathbf{I}(2,2)$ & $3 \mathbf{I}(2,2)$ & $\operatorname{diag}(.05, .05, .05)$ & $\operatorname{diag}(2.5,5,2.5,5)$ \\
\hline
\end{tabular}

Table 2. Parameters of the controllers for experiments

Indeed, under the current simulation environment (WindowsXP/Matlab Simulink), controller (89) requires a computation load 0.7 time less than the one of controller (95) and only 1.2 time bigger than the one of tradition LP adaptive control (97). Also, it can be seen in Figure 4 that these advantages result in a faster convergence (just few instants after the initial time) of the tracking errors to the designed value (0.0035 (rad) in this simulation). Note that the estimates converge to constant values since the adaptation mechanism in controller (89) becomes standstill whenever the tracking errors become less than the design value. However, it is worth noting that the maximum value of control inputs of controller (89), which is required only at the adaptation process of the estimates, is about 6 times bigger than the one of controller (95). It can be learnt from the simulation result that controller (89) can effectively compensates the NP uncertainties in the system provided that there is no limitation to the control inputs. Therefore, controller (95) can be a good choice for practical applications whose the power of actuators are limited.

\subsubsection{Experiments}

All joints of the manipulator are driven by YASKAWA DC motors UGRMEM-02SA2. The range of motor power is $[-5,5](\mathrm{Nm})$. The joint angles are detected by potentiometers $\left(350^{\circ}, \pm 0.5\right)$. Control input signals are sent to each DC motor via a METRONIX amplifier $( \pm 35 \mathrm{~V}, \pm 3 \mathrm{~A})$. The joint velocities are also calculated from the derivation of joint positions with low-pass niters. Designed controller is implemented on ADSP324-OOA, 32bit DSP board with SOMhz CPU clock. I/O interface is ADSP32X-03/53, 12bit A/D, D/A card. The DSP and the interface card are mounted on Windows98-based PC. The sampling time is $2 \mathrm{~ms}$.

Here again, the performances of controller (97) and the proposed control (95) are compared. The gains of the controllers are chosen as in Table 2. The additional control parameters for NP friction compensation with (95) are $\boldsymbol{\Gamma}_{\beta}=\operatorname{diag}(1,1,1,1), \epsilon=.1$.

Figure 5 depicts the performances of LP adaptive controller (97). The fact that the trajectory tracking error of joint 2 become about twice smaller as shown by Figure 6 highlights how effectively the NP frictions are compensated by the proposed controller. The estimates of unknown parameters with adaptation mechanisms in LP adaptive controller (97) and proposed controller (95) are shown by Figure 7 and Figure 8, respectively. Since the adaptation mechanism of LP adaptive controller (97) can not compensate for the NP friction terms, its estimates can not converge to any values able to make the trajectory tracking errors converge to 0 . For the proposed controller, a better convergence of the estimates can be observed. That the motion of the manipulator has lower frequencies in case of the proposed control (see Figure 9) shows its more robustness in face of noisy inputs. These results can be obtained because the NP frictions are compensated effectively. 
Position errors (rad)
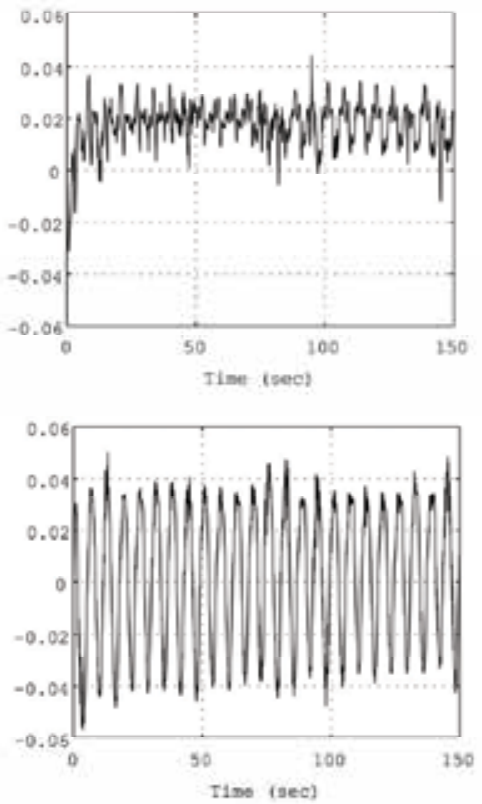

Control torques $(\mathrm{Nm})$

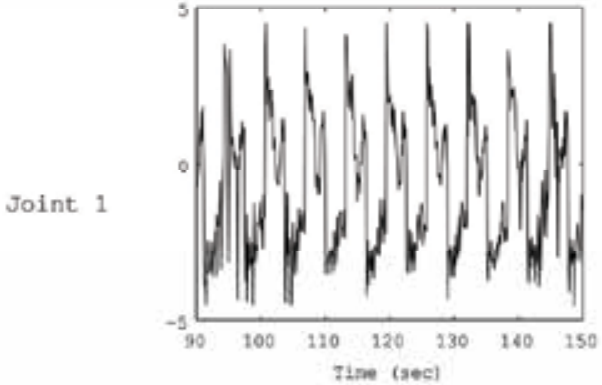

Joint. 2

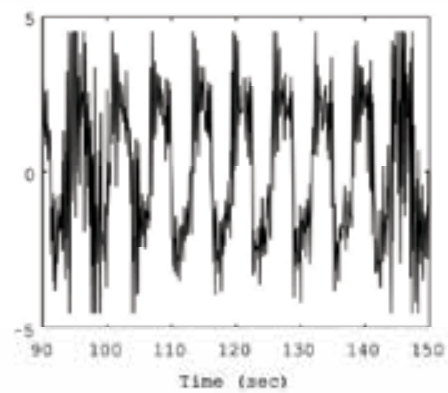

Figure 5. Experimental results for traditional LP adaptive controller (97): Tracking errors of joints (left) and characteristics of control inputs (right)

Position errors (rad)
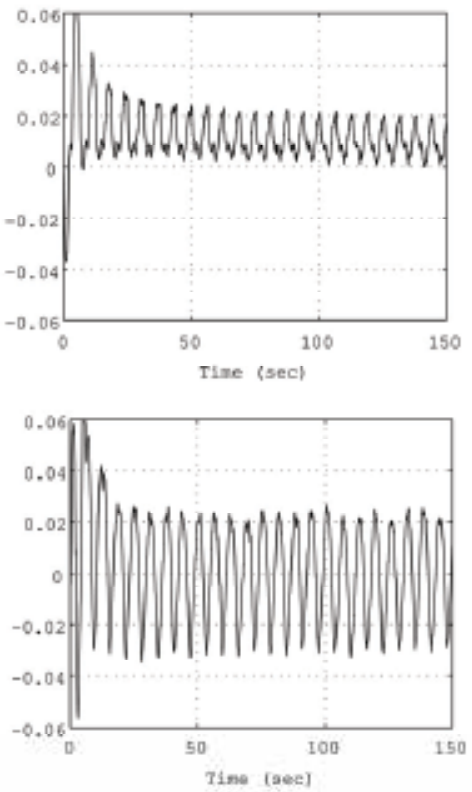

Control torques (Nm)
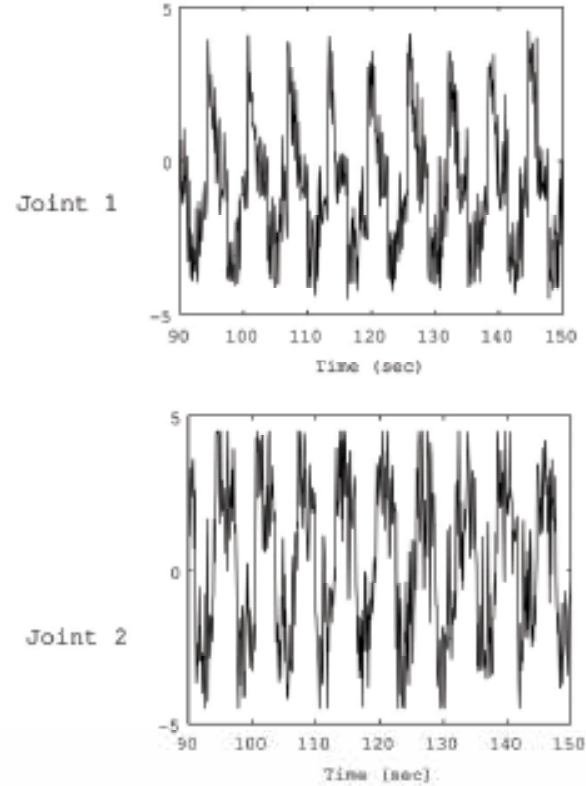

Figure 6. Experimental results for proposed controller (95): Tracking errors of joints (left) and characteristics of control inputs (right) 
Estimates of unknown parameters

(a)

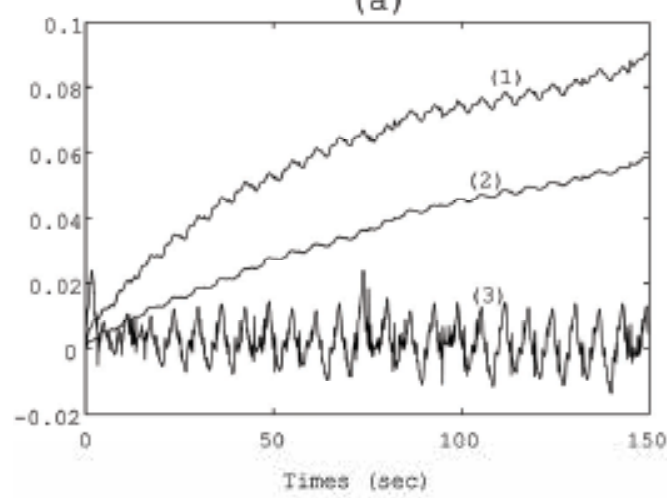

(b)

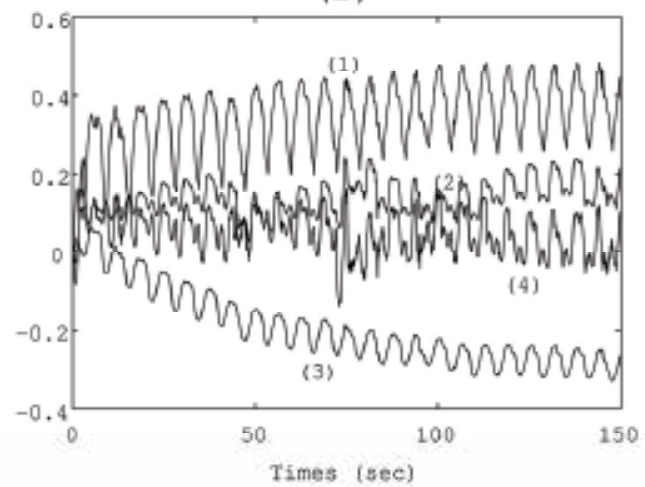

Figure 7. Experimental results: Estimates of unknown parameters with traditional LP adaptive controller (97). (a)-estimate â: (1)- $\hat{a}_{1},(2)-\hat{a}_{2},(3)-\hat{a}_{3}$. (b)-estimate $\hat{\alpha}:(1)-\hat{\alpha}_{1},(2)-\hat{\alpha}_{2},(3)-\hat{\alpha}_{3}(4)-\hat{\alpha}_{4}$

Estimates of unknown parameters

(a)

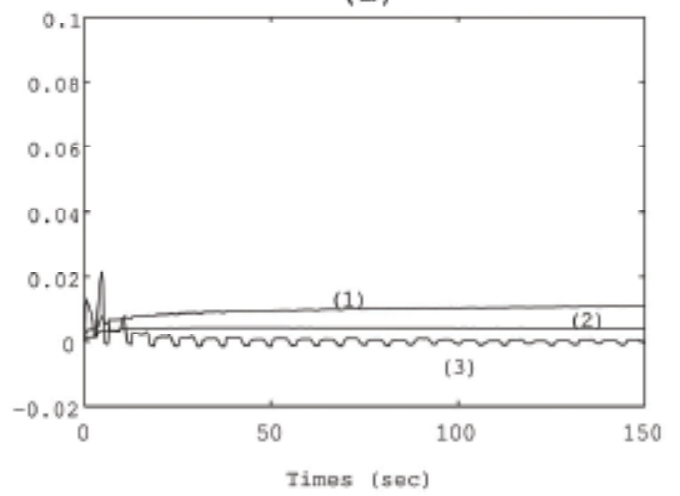

(b)

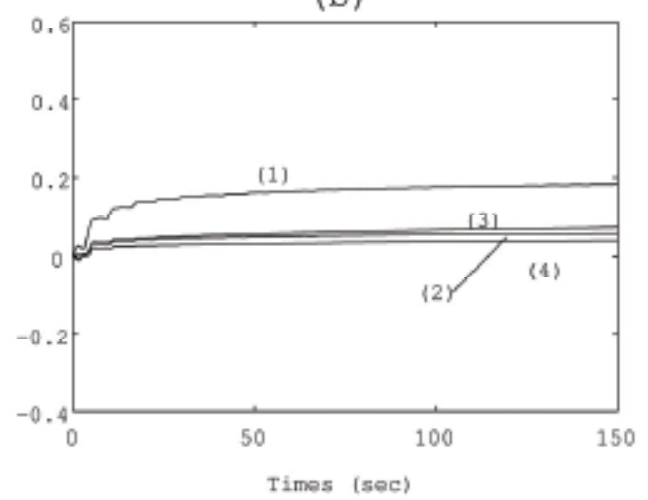

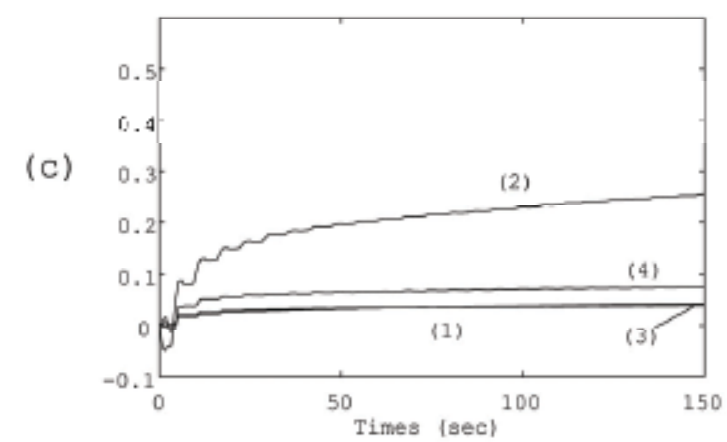

Figure 8. Experimental results: Estimates of unknown parameters with proposed controller (95). (a)-estimate $\hat{\mathbf{a}}:(1)-\hat{a}_{1},(2)-\hat{a}_{2},(3)-\hat{a}_{3}$. (b)-estimate $\hat{\alpha}$ : (1)- $\hat{\alpha}_{1},(2)-\hat{\alpha}_{2},(3)-\hat{\alpha}_{3},(4)-\hat{\alpha}_{4}$ estimate $\hat{\boldsymbol{\beta}}$ : (1)- $\hat{\beta}_{1},(2)-\hat{\boldsymbol{\beta}}_{2},(3)-\hat{\beta}_{3},(4)-\hat{\beta}_{4}$ 

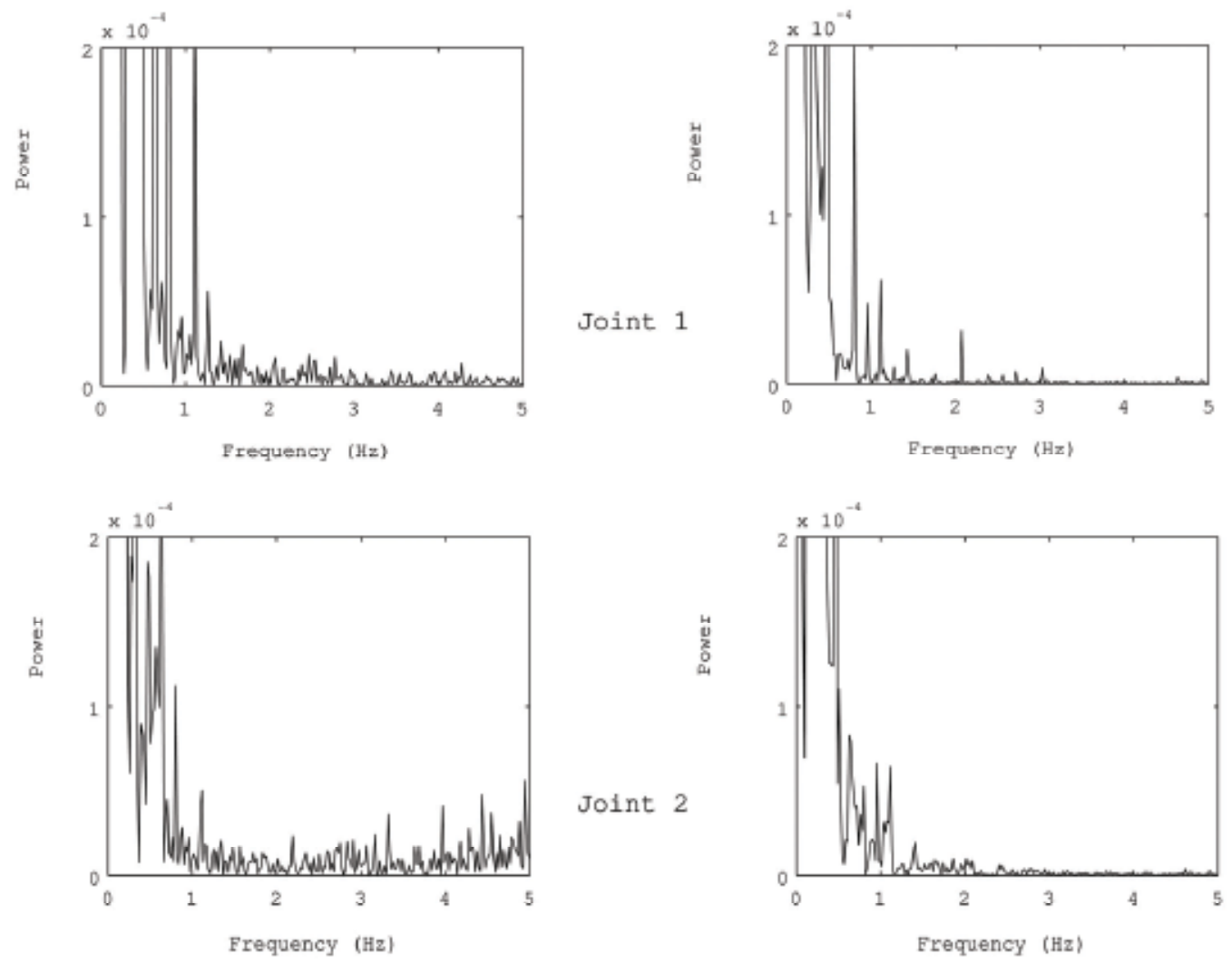

Figure 9. Experimental results: FFT of trajectory tracking errors for traditional LP adaptive controller (97) (left) and proposed controller (95) (right)

\section{Conclusions}

We have developed a new adaptive control framework which applies to any nonlinearly parameterized system satisfying a general Lipschitzian property. This allows us to extend the scope of adaptive control to handle very general control problems of NP since Lipschitzian parameterizations include as special cases convex/concave and smooth parameterizations. As byproducts, the approach permits also to treat uncertainties in fractional form, multiplicative form and their combinations thereof. Moreover, the proposed control approach allows a flexibility in the design of adaptive control system. This is because the ability of designing 1-dimension estimators provides system designers with more freedom to to balance the dimension of the design estimators and the power required by system control inputs. Otherwise, when it is necessary, simple structure is a key factor enabling the extension of the proposed adaptive controls to more complex control structures. Our next efforts are directed to the following research in order to integrate the proposed adaptive control technique to industrial control systems.

- Mechanisms to control the convergence time of the designed tracking errors. In this context, Lyapunov stability analysis incorporated with dynamic models of signals in the system can be used as an effective synthesis tool. 
- Improvement on the robustness of the adaptive schemes toward noise in the system due to un-modeled dynamics or unknown disturbances. In this context, sensing and monitoring the level of noise, and incorporating on-line noise compensation schemes will play an important role.

- Incorporation of the below system's actual working conditions in to the adaptive control system (i) Constrains on the limitation of actuators outputs (ii) Requirement of human-friendly interface (easy-to-tune interface and failure-safe). In this context, control systems need more complex control structure with more intelligent adaptation rules for dealing with wider range of system operation.

\section{Appendix}

\section{Model and parameters of the manipulator}

The equation of motion in joint space for a planar 2DOF manipulator is

$$
\mathbf{H}(\mathbf{q}) \ddot{\mathbf{q}}+\mathbf{C}(\mathbf{q}, \dot{\mathbf{q}}) \dot{\mathbf{q}}=\boldsymbol{\tau},
$$

or,

$$
\left[\begin{array}{cc}
b_{11}\left(q_{2}\right) & b_{12}\left(q_{2}\right) \\
b_{21}\left(q_{2}\right) & b_{22}
\end{array}\right]\left[\begin{array}{c}
\ddot{q}_{1} \\
\ddot{q}_{2}
\end{array}\right]+\left[\begin{array}{ll}
c_{11} & c_{12} \\
c_{21} & c_{22}
\end{array}\right]\left[\begin{array}{l}
\dot{q}_{1} \\
\dot{q_{2}}
\end{array}\right]=\tau
$$

where,

$$
\begin{aligned}
& b_{11}=\underbrace{I_{l_{1}}+m_{l_{1}} l_{1}^{2}+k r_{1}^{2} I_{m_{1}}+m_{l_{2}} a_{1}^{2}+m_{m_{2}} a_{1}^{2}}_{d_{11}^{*}}+I_{l_{2}}+I_{m_{2}}+m_{l_{2}} l_{2}^{2}+2 m_{l_{2}} a_{1} l_{2} \cos \left(q_{2}\right), \\
& b_{12}=b_{21}=\underbrace{I_{l_{2}}+k r_{2} I_{m_{2}}+m_{l_{2}} l_{2}^{2}}_{d_{12}}+m_{l_{2}} a_{1} l_{2} \cos \left(q_{2}\right), \\
& b_{22}=\underbrace{I_{l_{2}}+m_{l_{2}} l_{2}^{2}+k r_{2}^{2} I_{m_{2}}}_{d_{22}}, h=-m_{l_{2}} a_{1} l_{2} \sin \left(q_{2}\right), \\
& c_{11}=h \dot{q}_{2}, c_{12}=h\left(\dot{q}_{1}+\dot{q}_{2}\right), c_{21}=-h \dot{q}_{1}, c_{22}=0 .
\end{aligned}
$$

$m_{l i}, m_{m i}$ are the masses of link $i$ and motor $i$, respectively. $I_{l i}, I_{m i}$ are the moment of inertia relative to the center of mass of link $i$ and the moment of inertia of motor $i$. $l_{i}$ is the distance from the center of the mass of link $i$ to the joint axis. $a_{i}$ is the length of link $i . k_{r i}$ is the gear reduction ratio of motor $i$.

A constant vector $\mathbf{a} \in R^{3}$ of dynamic parameters can be defined as follows:

$$
\mathbf{a}=\left[\begin{array}{lll}
m_{l_{2}} a_{1} l_{2} & d_{11}^{*} & d_{22}
\end{array}\right]^{T}
$$

\begin{tabular}{|l|l|l|l|l|l|l|}
\hline$a_{1}$ & $a_{2}$ & $k r_{1}$ & $k r_{2}$ & $m_{l_{2}} a_{1} l_{2}$ & $d_{11}^{*}$ & $d_{22}$ \\
\hline \hline 0.15 & 0.15 & 1 & 1 & 0.0043 & 0.2602 & 0.0188 \\
\hline
\end{tabular}

Table 3. Parameters of the 2DOF manipulator

\section{References}

K.J. Astrom, B. Wittenmark, Adaptive control, Addison-Wesley, 1995. [I]

J.J.E. Slotine, W. Li, Applied nonlinear control, Prentice-Hall, 1992. [2] 
B. Armstrong-Helouvry, P. Dupont, C. Canudas de Wit, A Survey of Models, Analysis Tools and Compensation Methods for Control of Machines with Friction, Automatica pp. 1083-1138, 30(1994). [3]

Y. Xu, H.Y.Shum, T. Kanade, J.J. Lee Parameterization and Adaptive Control of Space Robot Systems, IEEE Trans, on Aerospace and Electronic Systems Vol. 30, No. 2, pp. 435-451, April 1994. [4]

A.M Annaswamy, F.P. Skantze,A.P. Loh Adaptive control of continuous time systems with convex/concave parameterization, Automatica Vol. 34, pp. 33-49, 1998[5]

C. Cao, A.M Annaswamy, A. Kojic, Parameter Convergence in Nonlinearly Parameterized Systems, IEEE Trans, on Automatic Control Vol. 48, No. 3, pp. 397 - 412, March 2003[6]

H. Tuy, Convexity and monotonicity in global optimization Advances in Convex Analysis and Global Optimization, Kluwer Academic, 2000. [7]

K.S. Narendra, A.M. Annaswamy, Stable adaptive systems, Prentice-Hall, 1989. [8]

M. Krstic, I. Kanellakopoulos, P. Kokotovic, Nonlinear and Adaptive Control Design, John Wiley \& Sons, 1995. [9]

A. Kojic, A.M. Annaswamy, A.P. Loh, R. Lozano, Adaptive control of a class of nonlinear systems with convex/concave parameterization, Systems $\mathcal{E}$ Control Letters, pp. 67274, Vol. 37, 1992. [10]

A.L. Fradkov, I.V. Miroshnik, V.O. Nikiforov, Nonlinear and adaptive control of complex systems, Kluwer Academic, 1999. [11]

A.P. Loh, A.M. Annaswamy, F.P. Skantze, Adaptation in the presence of a general nonlinear parameterization: an error model approach, IEEE Trans. Automatic Control, Vol. 44, pp. 1634-1652, 1999. [12]

P. Kokotovic, M. Arcak, Constructive nonlinear control: a historical perspective, Automatica, Vol. 37, pp. 637-662, 2001. [13]

H.D. Tuan, P. Apkarian, H. Tuy, T. Narikiyo and N.V.Q. Hung, Monotonic Approach for Adaptive Controls of Nonlinearly Parameterized Systems, Proc. of 5th IFAC Symposium on Nonlinear Control Design, pp. 116-121, July 2001. [14]

K.Yokoi, N. V. Q. Hung, H. D. Tuan and S. Hosoe, Adaptive Control Design for Nonlinearly Multiplicative with a Triangular Structure, Asian Journal of Control, Vol. 9, No. 2, pp. 121-132, June 2007[15]

K.Yokoi, N. V. Q. Hung, H. D. Tuan and S. Hosoe, Adaptive Control Design for n-th order Nonlinearly Multiplicative Parameterized Systems with Triangular Structure and Application, Transactions of SICE, Vol.39, No.12, pp. 1099-1107, December 2003[16]

R. Ortega, M.W. Spong, Adaptive motion control of rigid robots: A tutorial, Automatica, Vol. 25, pp. 877-888, 1989. [17]

P. Tomei, Adaptive PD controller for robot manipulators, IEEE Trans. Robotics Automat., Vol. 7, pp. 565-570, 1991. [18]

B. Friedland, and Y.J. Park, On Adaptive Friction Compensation, IEEE Trans. Automat. Contr., Vol. 37, No. 10, pp. 1609-1612, October 1992. [19]

G. Liu, Decomposition-Based Friction Compensation Using a Parameter Linearization Approach, Proc. IEEE Int.Conf.Robot. Automat., pp. 1155-1160, May 2001. [20]

M. Feemster, P. Vedagarbha, D.M. Dawson and D. Haste, Adaptive Control Techniques for Friction Compensation, Mechatronics - An International Journal, Vol. 9, No. 2, pp. 125-145, February 1999. [21] 


\title{
Model-free Adaptive Control in Frequency Domain: Application to Mechanical Ventilation
}

\author{
Clara Ionescu and Robin De Keyser \\ Ghent University, Department of Electrical energy, Systems and Automation \\ Belgium
}

\section{Introduction}

Looking back at the history of control engineering, one finds that technology and ideas combine themselves until they reach a successful result, over the timeline of several decades (Bernstein, 2002). It is such that before the computational advances during the so-called Information Age, a manifold of mathematical tools remained abstract and limited to theory. A recent trend has been observed in combining feedback control theory and applications with well-known, but scarcely used in practice, mathematical tools. The reason for the failure of these mathematical tools in practice was solely due to the high computational cost. Nowadays, this problem is obsolete and researchers have grasped the opportunity to exploit new horizons.

During the development of modern control theory, it became clear that a fixed controller cannot provide acceptable closed-loop performance in all situations. Especially if the plant to be controlled has unknown or varying dynamics, the design of a fixed controller that always satisfies the desired specifications is not straightforward. In the late 1950s, this observation led to the development of the gain-scheduling technique, which can be applied if the process depends in a known or measurable way on some external, measurable condition (Ilchmann \& Ryan, 2003). The drawback of this simple solution is that only static (steady state) variations can be tackled, so the need for dynamic methods of controller (re)tuning was justified.

One can speak of three distinct features of the standard PID controller tuning: auto-tuning, gain scheduling and adaptation. Although they use the same basic ingredients, controller auto-tuning and gain scheduling should not be confused with adaptive control, which continuously adjusts controller parameters to accommodate unpredicted changes in process dynamics. There are a manifold of auto-tuning methods available in the literature, based on input-output observations of the system to be controlled (Bueno et al., 1991; Åström \& Hagglund, 1995; Gorez, 1997).

The tuning methods can be classified twofold:

- direct methods, which do not use an explicit model of the process to be controlled; these can then be either based on tuning rules ( ström \& Hagglund, 1995), either on iterative search methods (Åström \& Wittemark, 1995; Gorez, 1997).

- $\quad$ indirect methods, which compute the controller parameters from a model of the process to be controlled, requiring the knowledge of the process model; these can be based on 
either models: transient-response models (step response model), frequency response models, or transfer function models.

Although many adaptive control methods are available in the literature, their implementation in practice is challenging and prone to failures (Anderson, 2005). If the process parameters are not known and not necessary, direct adaptive methods can be derived based on specifications of the closed loop performance, using explicit reference models. A relatively large gap exists between theoretical and practical model-reference adaptive control, initiated from the unknown process (Butler, 1990). Nevertheless, direct adaptive control with model reference has proved successful for a variety of applications, some of which will be presented in this contribution.

This chapter will present a simple and straightforward adaptive controller strategy from the class of direct methods, based on reference models. The algorithm will offer an alternative solution to the burden of process identification, and will present possibilities to tune both integer- and fractional- order controllers. Three examples will illustrate the simplicity of the approach and its results. A discussion section will provide advantages and dis-advantages of the proposed algorithm and some implementation issues. A conclusion section will summarize the outcome of this investigation.

\section{Methods}

\subsection{The DIRAC principle}

The DIRAC (DIrect Adaptive Controller) algorithm belongs to the class of model-free tuning methods, since it does not require the knowledge of the process, nor it needs to identify it during the tuning procedure (De Keyser, 1989; De Keyser, 2000). The most important feature in this model reference adaptive control strategy is the design of the adaptive laws, which take place directly, without an explicit process identification procedure. The aim of making the closed loop response approximately equal to a specified response is the key ingredient of DIRAC, and the design of this reference model plays a decisive role. The perfect model matching condition places some requirements on the reference model, which results in the following rule-of-thumb: the relative degree (pole excess) must be equal to the relative degree of the process; however, as shown here, this condition can be avoided. In addition to the stability and minimum-phase demands on the reference model, these are just theoretical aspects. It should be noted that in practice, where some theoretical requirements may not be satisfied, the reference model should be chosen reasonably, in the sense that the process output can actually follow the reference model output. For example, if the reference model is chosen too fast (compared to the process dynamics), the control signal needs to be extremely high, causing input saturation effects or nonlinear dynamics which may disturb the overall closed loop behaviour.

Because the actual process capabilities may be unknown or varying, the choice of the reference model is not always obvious. Choosing a conservative performance may be more robust, but it may also lead to slower closed loop behaviour than necessary.

In the standard control loop, where $C(s)$ denotes the controller, $P(s)$ denotes the (unknown) process, $w(t)$ is the reference signal $y(t)$ is the output, $e(t)=y(t)-w(t)$ is the error, the closed loop transfer function is given by:

$$
y(t)=\frac{C(s) P(s)}{1+C(s) P(s)} w(t)
$$


The desired closed loop performance will be then specified by a reference model $R(s)$, apriori user-defined, which can be used to specify the desired characteristics of the loop, for instance, the speed (bandwidth). It follows that the tuning task can be summarized as follows: find the corresponding controller's parameters (PID or any other transfer function) such that the closed-loop transfer function is more or less equal to the reference model:

$$
\frac{C(s) P(s)}{1+C(s) P(s)} \cong R(s)
$$

The trivial solution arising from solving (2) for the unknown controller will lead to undesired results, such as: i) identification of the unknown process (which is not aimed), and ii) the result will lead to a transfer function for $C(s)$ and not to a $2^{\text {nd }}$ order polynomial, which is required for obtaining a PID controller in the form:

$$
C(s)=\frac{1}{s} \underbrace{\left(c_{0}+c_{1} s+c_{2} s^{2}\right)}_{C^{*}(s)}
$$

explicitly containing an integrator to ensure zero steady state error. In order to avoid this dead-end solution, one can extract the controller from (2) taking into account the measurable signals $\mathrm{u}(\mathrm{t})$ and $\mathrm{y}(\mathrm{t})$ and the relation $P \cdot u(t)=y(t)$ :

$$
C^{*} \cdot y_{f}(t)+\varepsilon(t)=u_{f}(t)
$$

with $u_{f}(t)$ and $y_{f}(t)$ obtained as in schematically depicted in figure 1.

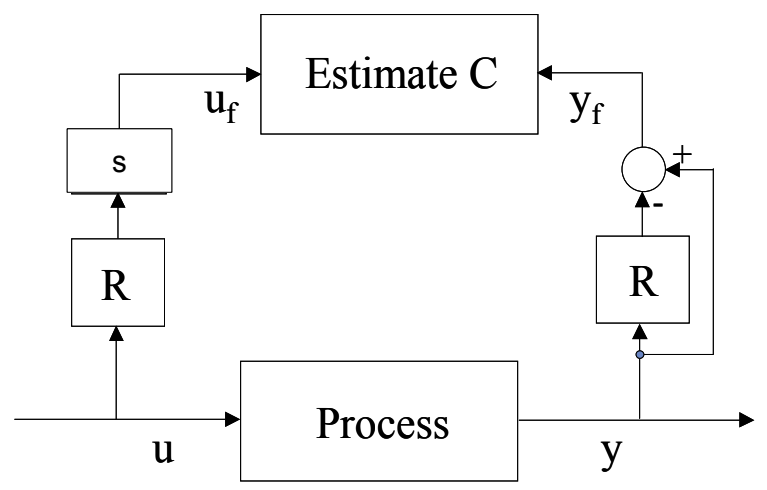

Figure 1. Block-scheme of the DIRAC strategy

Relation (4) becomes then a standard identification problem which can be solved either offline (tuning), either online (adaptation) using any parameter estimation method (Ljung, 1987). It should be noted that the least squares gives unbiased estimates even in the case of coloured noise, since (4) does not contain recursion of $u_{f}(t)$. Some guidelines to define the reference model for various classes of processes are given in (De Keyser, 2000), discussing the implementation aspects in discrete time, along with some typical examples.

\subsection{Controller's structure}

From the previous section we have concluded that the controller has to satisfy the equality: 


$$
R(s) \cong \frac{C(s) P(s)}{1+C(s) P(s)} \cong \frac{C^{*}(s) P(s)}{s+C^{*}(s) P(s)}
$$

from which the utopic controller can be extracted as:

$$
C^{*}(s) \cong \frac{s R(s)}{[1-R(s)] P(s)}
$$

Obviously, the standard 'textbook' PID transfer function $K_{p}\left(1+\frac{1}{T_{i} s}+T_{d} s\right)$ consisting of the proportional, integral and derivative terms is the most used in practice, yielding satisfactory results. If the PID controller is written in the form required by (6), it results in estimating a 2nd order polynomial:

$$
C^{*}(s) \cong c_{0} s+c_{1}+c_{2} s^{2}
$$

with $c_{0}=K_{p}, c_{1}=K_{p} / T_{i}$ and $c_{2}=K_{p} T_{d}$ the three unknown parameters to be identified.

Yet, the end of the $20^{\text {th }}$ century has brought numerous advances in technology, with visible improvements in the computational aspects and pushing onward the limits of numerical complexity. As a result, mathematical tools which were abstract and numerically too complex for practical usefulness, were enabled as powerful tools for identification and control. As a result, the control engineering research community has oriented its attention to the possibility of using non-integer order controllers, namely $P I^{\lambda} D^{\mu}$ (Monje et al., 2008).

Such a controller is in fact a generalization of the standard PID, $K_{p}\left(1+\frac{1}{T_{i} \mathrm{~s}^{\lambda}}+T_{d} \mathrm{~s}^{\mu}\right)$, and (7) can be re-written as:

$$
C^{*}(s) \cong c_{0} s+c_{1} s^{1-\lambda}+c_{2} s^{1+\mu}
$$

with $c_{0}, c_{1}, c_{2}, \lambda, \mu$ five unknown parameters to be identified. To estimate the parameters in (7), a linear identification algorithm suffices to obtain good results, such as the linear least squares method (Ljung, 1987). For (8), however, we are dealing with a polynomial which is nonlinear in the parameters and it is necessary to use nonlinear identification methods, such as nonlinear least squares (Ljung, 1987).

Simulation in time domain for fractional order controllers (FOC) such as the one described by (3) with the controller structure from (8), may be challenging. There are several definitions of the differ-integral in time domain, of which two commonly used are the Grünwald-Letnikov and Rieman-Liouville definitions (Podlubny, 1999):

$$
\text { GL: }{ }_{\mathrm{a}} D_{t}^{\alpha} f(t)=\lim _{h \rightarrow 0} h^{-\alpha} \sum_{j=0}^{[(t-a) / h]}(-1)^{j}\left(\begin{array}{c}
\alpha \\
j
\end{array}\right) f(t-j h)
$$

where [ ] denotes the integer part, respectively:

$$
\mathbf{R L}:{ }_{\mathrm{a}} D_{t}^{\alpha} f(t)=\frac{1}{\Gamma(n-\alpha)} \frac{d^{n}}{d t^{n}} \int_{a}^{t} \frac{f(\tau)}{(1-\tau)^{\alpha-n+1}} d \tau
$$


for $n-1<\alpha<n$ and $\Gamma$ is the Euler's Gamma function; a denotes the initial conditions, $\mathrm{t}$ is the differ-integration time, $a$ is the fractional order. The Laplace transform of the RiemanLiouville fractional derivative/integral (10) under zero initial conditions can be written as:

$$
£\left\{D_{t}^{ \pm \alpha} f(t)\right\}=s^{ \pm \alpha} F(s)
$$

With (11) at hand, the interpretation of fractional order derivative/integral can be simplified using the complex plane representation and Bode characteristics, the latter defined by its magnitude and phase. In this line-of-thought, $s^{ \pm \alpha}$ becomes $(j \omega)^{ \pm \alpha}$ in frequency domain, with $j=\sqrt{-1}$ and $\omega(\mathrm{rad} / \mathrm{s})$ the angular frequency. The Bode plot can be then defined as:

$$
\begin{aligned}
& \left|s^{ \pm \alpha}\right|= \pm \alpha \cdot 20 \mathrm{~dB} / \mathrm{dec} \\
& \angle\left(s^{ \pm \alpha}\right)= \pm \alpha \cdot \frac{\pi}{2} \mathrm{rad}
\end{aligned}
$$

It is now easy to understand why FOC is so interesting from identification/control standpoint: its intrinsic capability to capture variations in frequency domain which are not limited to integer-multiples of $20 \mathrm{~dB} / \mathrm{dec}$, or $\Pi / 2$, respectively (such as for integer order systems).

If the controller is in the form of (7), it results directly in the transfer function of the PID with the integrator added explicitly to the $2^{\text {nd }}$ order polynomial from (7). If the controller is in the form of (8), namely fractional order controller FOC, then an extra step is necessary to be implemented before being able to simulate the closed-loop behavior. The reason is that fractional order controllers cannot be yet implemented in practice since there are no direct analogue components available. However, it is possible to obtain the equivalent frequency response of a fractional order transfer function using high order integer-order approximations. Various methods for integer-order approximations of FOC have been proposed and successfully implemented in practice (Oustaloup et al., 2000; Melchior et al., 2002; Monje et al., 2008). Nevertheless, the burden of this extra step remains necessary in the case of FOC, in which the proper implementation is not trivial.

\subsection{Frequency domain approach}

For simplicity in formulation of the FOC, the frequency domain will be used to illustrate the determination of the utopic controller frequency response $C^{*}(j \omega)$. Recalling (6), the equivalent frequency domain formulation can be written as:

$$
C^{*}(j \omega)=\frac{(j \omega) \cdot R(j \omega)}{[1-R(j \omega)] P(j \omega)}
$$

Since we do not want to identify the process transfer function $P(j \omega)$, we introduce the signals $u_{f}(t)$ and $y_{f}(t)$. Supposing the input signal $u(t)$ is a sine-sweep with $n$ samples, in the form:

$$
u(n)=\sin \left[K\left(e^{-n / L f_{s}}-1\right)\right]
$$


which is in fact a sinusoid whose frequency is exponentially increased from the lower bound to the higher bound of frequency range $\left(\omega_{1}, \omega_{2}\right)$ over $T$ seconds, with $f s$ the sampling frequency, $K=\frac{\omega_{1} T}{\ln \frac{\omega_{2}}{\omega_{1}}}$ and $L=\frac{T}{\ln \frac{\omega_{2}}{\omega_{1}}}$. This formulation of the excitation signals allows us excite one frequency at a time, in a single trial and re-formulate (13) in function of the inputoutput signals:

$$
A_{C^{*}} e^{j \varphi_{C^{*}}}=e^{j \varphi} \frac{A_{y} e^{j \varphi_{y}}}{A_{u} e^{j \varphi_{u}}} \frac{A_{R} e^{j \varphi_{R}}}{1-A_{R} e^{j \varphi_{R}}}
$$

where $A_{u} e^{j \varphi_{u}}$ and $A_{y} e^{j \varphi_{y}}$ are available through measurements and $A_{R} e^{j \varphi_{R}}$ through calculations at each excited frequency (14) of the desired $R(j \omega)$. Finally, we obtain the frequency response of the utopic controller $C^{*}(j \omega)$ in its Bode characteristic representation, namely magnitude and phase, at the desired frequency points of interest. The frequency range in which the controller is evaluated with (15) and then identified in the form given by (7) or (8), depends on the characteristics of the process to be controlled $P$ and the desired closed-loop performance defined by $R$. Once (15) is available, a linear or nonlinear optimization problem must be solved, identifying the unknown parameters of the controller.

Global optimization is the task of finding the absolutely best set of admissible conditions to achieve an objective under given constraints, assuming that both are formulated in mathematical terms. Some large-scale global optimization problems have been solved by current methods, and a number of software packages are available that reliably solve most global optimization problems in small (and sometimes larger) dimensions. However, finding the global minimum, if one exists, can be a difficult problem (very dependant on the initial conditions). Superficially, global optimization is a stronger version of local optimization, whose great usefulness in practice is undisputed. Instead of searching for a locally feasible point one wants the globally best point in the feasible region. However, in many practical applications finding the globally best point, though desirable, is not essential, since any sufficiently good feasible point is useful and usually an improvement over what is available without optimization (this particular case). Besides, sometimes, depending on the optimization problem, there is no guarantee that the optimization functions will return a global minimum, unless the global minimum is the only minimum and the function to minimize is continuous (Pinterr, 1996). Taking all these into account, and considering that the set of functions to minimize in this case is continuous and can only present one minimum in the feasible region, any of the optimization methods available could be effective, a priori. For this reason, and taking into account that Matlab is a very appropriate tool for the analysis and design of control systems, the optimization toolbox of Matlab has been used to reach out the best solution with the minimum error. The lsqnonlin nonlinear least-squares function has been used which returns the set of parameters from either (7), either (8), depending on the desired structure of the controller (Mathworks, 2000a). 
An elegant solution to avoid this extra step is to fit directly the frequency response of the controller $C^{*}(j \omega)$ with a properly chosen order transfer function. The fact that $C^{*}(j \omega)$ is a polynomial, instead of a transfer function, does not present any particular difficulty. One may choose to use the Matlab function fitfrd which delivers a state space representation of a fitted transfer function to the given frequency response of the controller (Mathworks, 2000b). For example, in the case of the standard PID form (7), it is necessary to set the specifications to a $2^{\text {nd }}$ order transfer function, with a relative degree equal to 2 (excess poles). This will result in a transfer function of the form $C_{f i t}(s)=\frac{k}{c_{0} s+c_{1}+c_{2} s^{2}}$, from which the controller transfer function becomes:

$$
C(s)=\frac{1}{s} \cdot \frac{1}{C_{f i t}(s)}
$$

\subsection{Adaptation procedure}

Once the controller's parameters have been found, these parameters can be adapted if the changes in the process require another tuning values for fulfilling the specified closed loop performance.

The adaptation procedure can be summarized in few steps as following:

- $\quad$ perform an input-output test measurement in the practical frequency range of interest;

- calculate the magnitude-phase frequency response using frequency domain analysis techniques;

- $\quad$ calculate the frequency response of the utopic controller with (13)-(15);

- fit the controller structure from (7) with linear least squares or (8) with nonlinear least squares identification procedure;

- $\quad$ apply the controller using (16).

\section{Illustrative examples}

In this section, two typical examples which are considered of academic interest, will be presented. Both integer and fractional order controllers will be developed based on closed loop specifications given by the reference model.

\subsection{A typical position servo system}

A typical position servo system contains a first order plant with an integrator, for example:

$$
P(s)=\frac{0.25}{s(s+1)}
$$

The difficulty in this case arises from the presence of a double integrator in the closed loop, namely the one from the plant and the one from the controller. The unit impulse response of the system from (17) and the corresponding frequency response is given in figure 2, along with the frequency responses of the two reference models, namely:

$$
R(s)=\frac{1+4 \tau s}{(1+\tau s)^{4}}
$$


with $\tau=0.05$ and 0.01 , respectively. The frequency band of interest for tuning the utopic controller is $\omega \in\left(10^{-1}, 10^{1}\right)$. Figures 3-4 present the optimisation result in fitting the controller frequency response, and the closed loop unit step response in the two design cases.
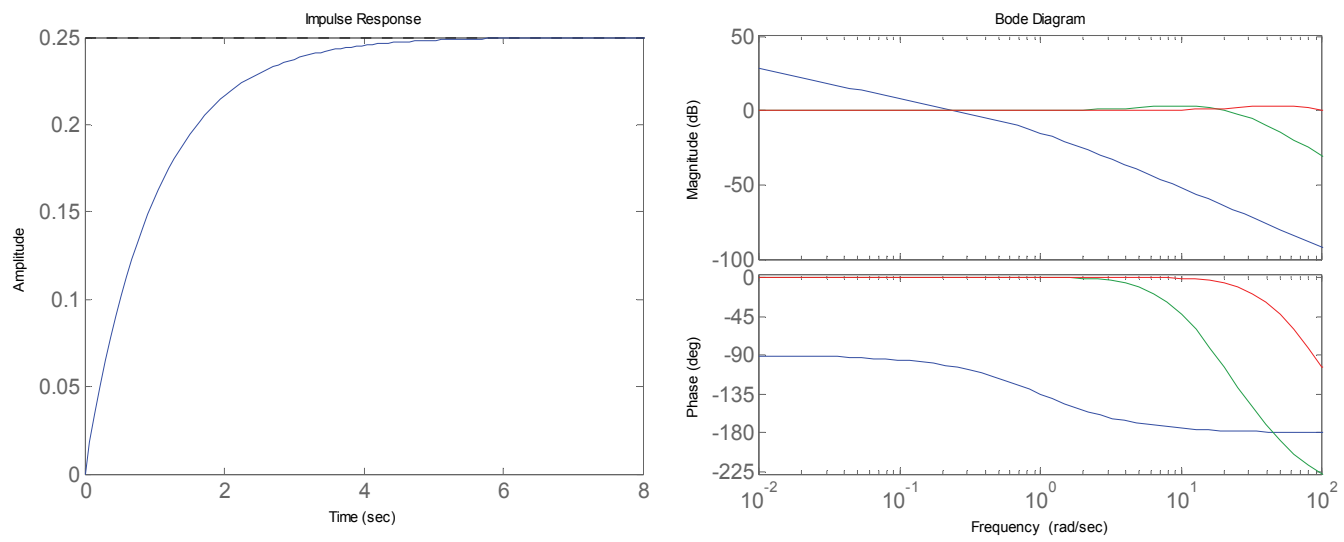

Figure 2. Left: open loop unit impulse response of the process; Right: Bode characteristics of the process (blue) and the reference models for $\tau=0.05$ (green) and for $\tau=0.01$ (red)

The corresponding controller parameters are given in Table 1.

\begin{tabular}{|l|l|l|l|l|l|c|}
\hline & $\tau$ & $c_{0}$ & $c_{1}$ & $c_{2}$ & $\lambda$ & $\mu$ \\
\hline IO_PID & 0.05 & 266.635 & 313.144 & 42.608 & 1 & 1 \\
\hline FO_PID & 0.05 & 273.332 & 298.938 & 73.356 & 0.969 & 0.759 \\
\hline & & & & & & \\
\hline OO_PID & 0.01 & 6666.7 & 6891.6 & 220.5 & 1 & 1 \\
\hline FO_PID & 0.01 & 6743.9 & 6678.7 & 267.2 & 1 & 0.9 \\
\hline
\end{tabular}

Table 1. Controller parameters for the position servo system
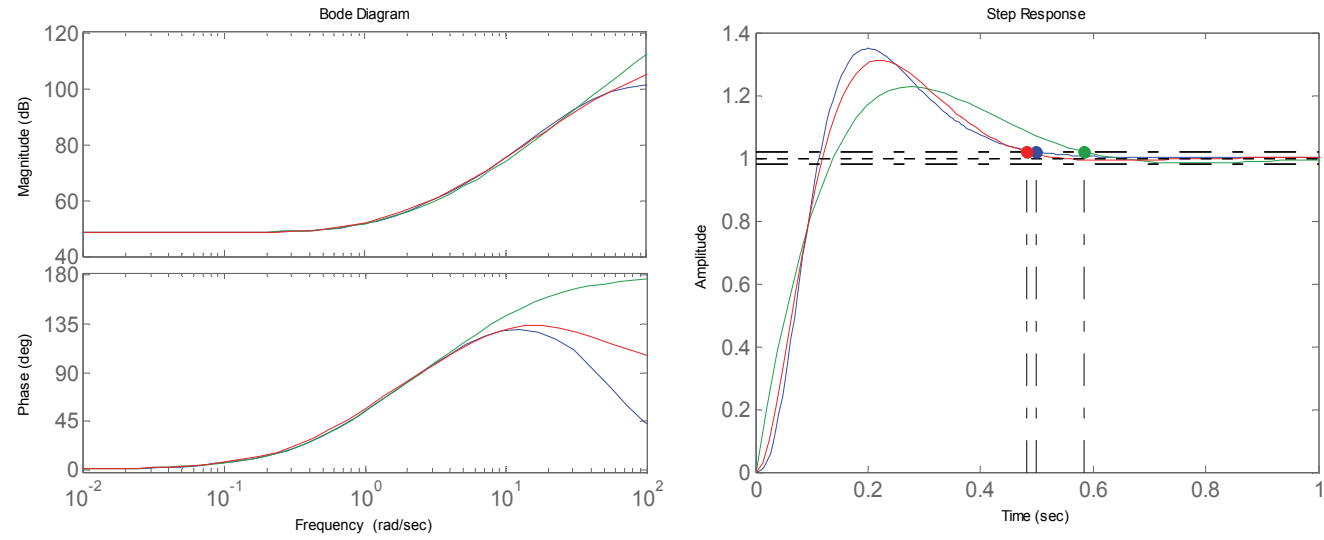

Figure 3. Left: frequency domain approximation and Right: unit step responses for $\tau=0.05$; reference (blue), IO_PID (green) and FO_PID (red). Circles denote settling times 

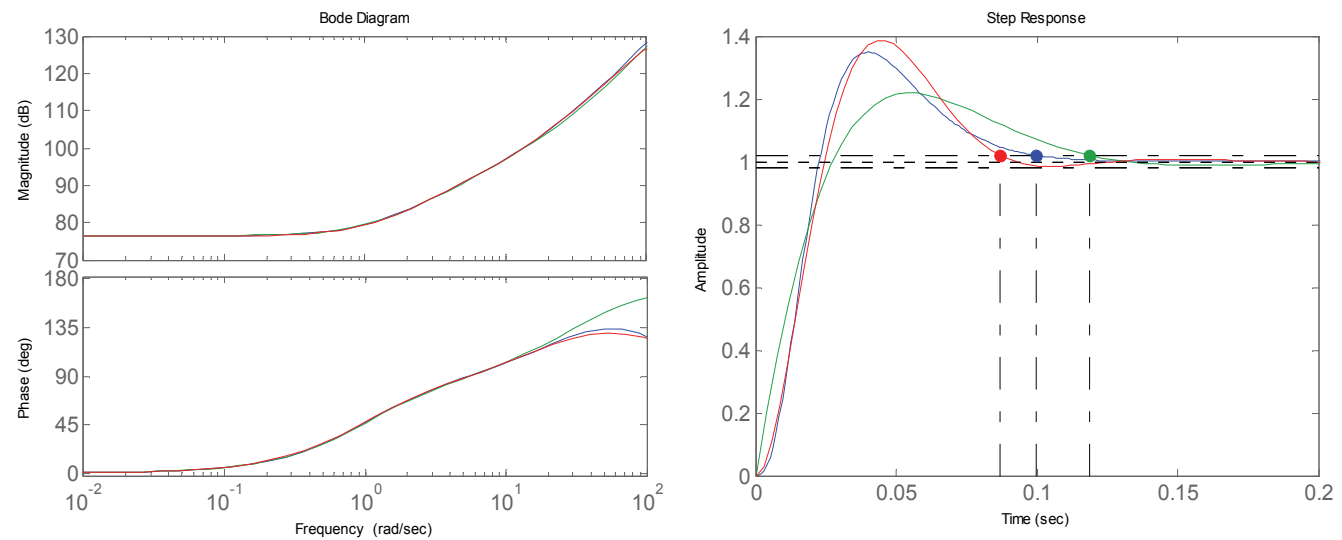

Figure 4. Left: frequency domain approximation and Right: unit step responses for $\tau=0.01$; reference (blue), IO_PID (green) and FO_PID (red). Circles denote settling times

\subsection{Highly oscillatory system}

The process in this example is highly oscillatory due to the low damping factor $\xi$ :

$$
P(s)=K \frac{\omega_{n}^{2}}{s^{2}+2 \xi \omega_{n} s+\omega_{n}^{2}} \frac{1}{(1+a s)^{2}}
$$

with $K=0.3, \omega_{n}=0.04 \pi, \xi=0.1$ and $a=5$. The reference model has been chosen as in:

$$
R(s)=\frac{1}{(1+\tau s)^{4}}
$$

with $\tau=15$ and $\tau=10$. The open loop unit step response is depicted in figure 5 , clearly showing the oscillatory behaviour of the system, making it difficult to control. The frequency characteristics of the process and the two reference models are given in figure 5, right. From these, the useful frequency range of the controller is taken as $\omega \in\left(10^{-2.8}, 10^{-0.8}\right)$. Figures 6-7 present the optimisation result in fitting the controller frequency response, and the closed loop unit step response in the two design cases, while Table 2 summarizes the corresponding controller parameters.

\begin{tabular}{|l|c|c|c|c|c|c|}
\hline & $\tau$ & $c_{0}$ & $c_{1}$ & $c_{2}$ & $\lambda$ & $\mu$ \\
\hline IO_PID & 15 & 0.056 & 0.011 & 2.809 & 1 & 1 \\
\hline FO_PID & 15 & 0 & 0.051 & 2.891 & 1 & 1 \\
\hline & & & & & & \\
\hline IO_PID & 10 & 0.083 & 0.071 & 4.426 & 1 & 1 \\
\hline FO_PID & 10 & 0 & 0.082 & 3.623 & 1 & 0.921 \\
\hline
\end{tabular}

Table 2. Controller parameters for the highly oscillatory system 

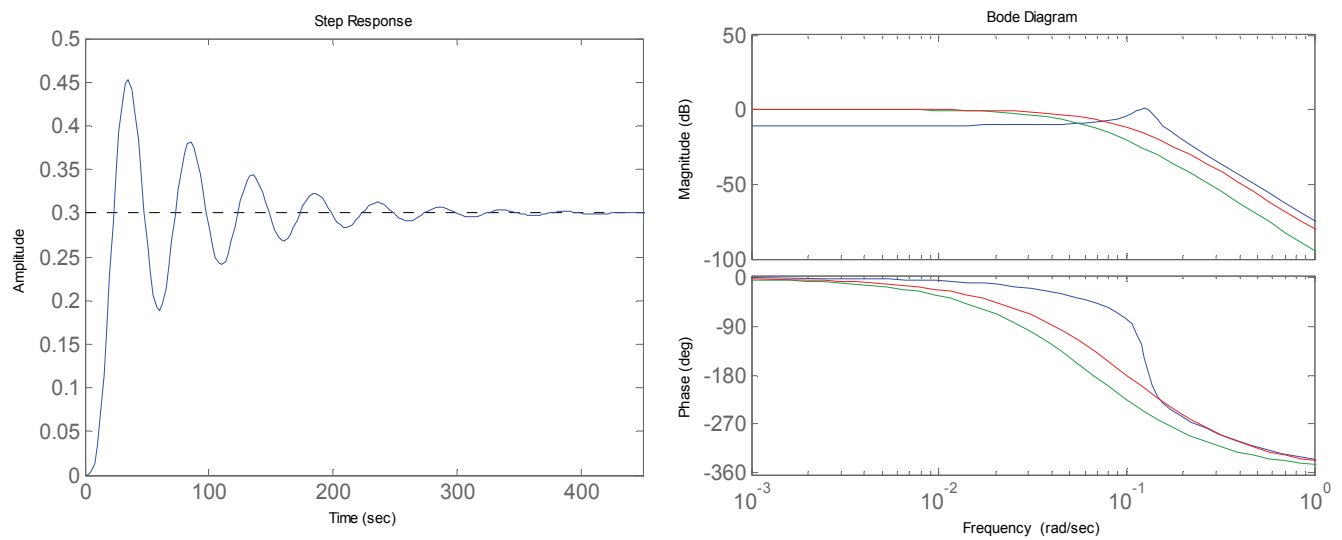

Figure 5. Left: open loop unit step response of the process; Right: Bode characteristics of the process (blue) and the reference models for $\tau=15$ (green) and for $\tau=10$ (red)
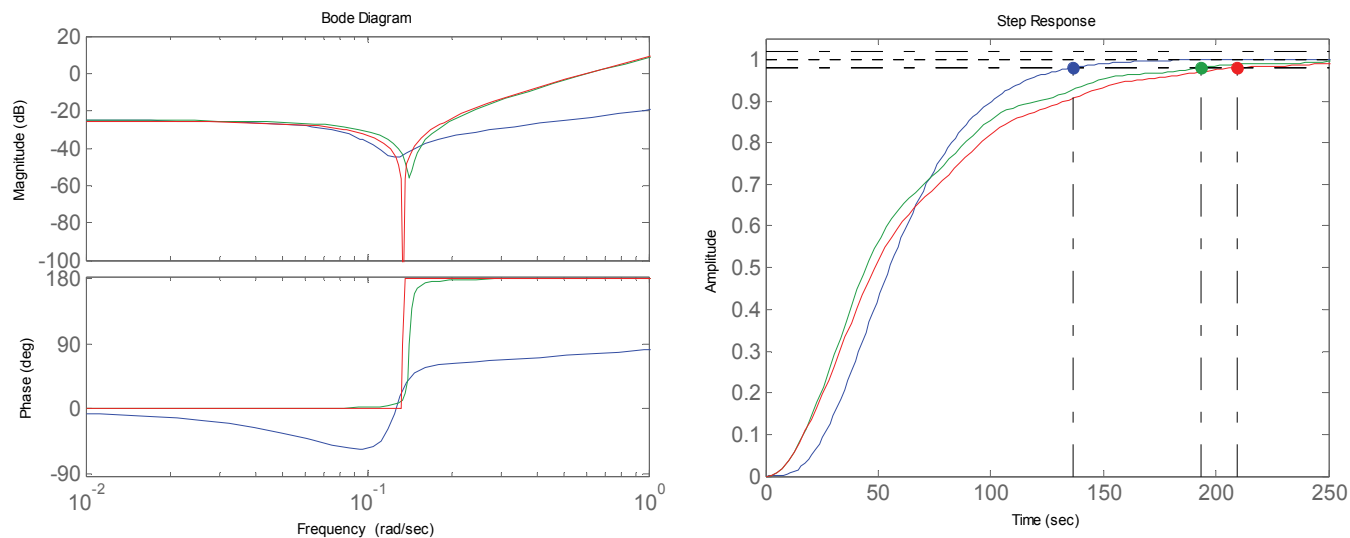

Figure 6. Left: frequency domain approximation and Right: unit step responses for $\tau=15$; reference (blue), IO_PID (green) and FO_PID (red). Circles denote settling times
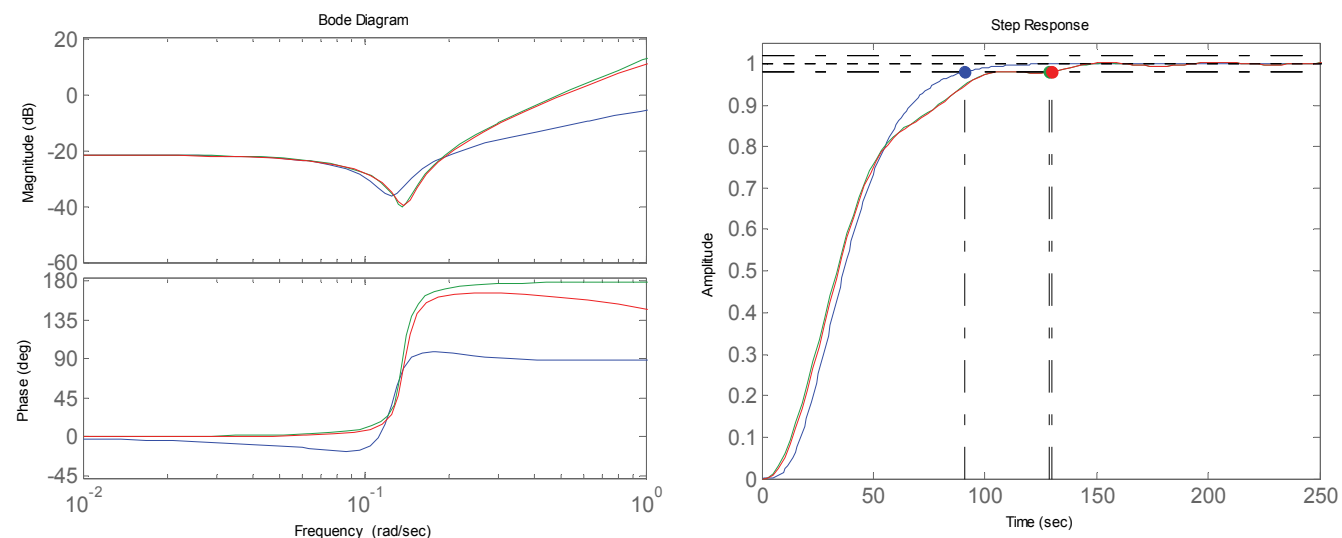

Figure 7. Left: frequency domain approximation and Right: unit step responses for $\tau=10$; reference (blue), IO_PID (green) and FO_PID (red). Circles denote settling times 


\section{Practical Application: Mechanical Ventilation}

One of the novel concepts in control engineering is that of fractals, self-similarity in geometrical structures (Weibel, 2005). Although originally applied in mathematics and chemistry, the signal processing community introduced the concept of fractional order modelling in technical and non-technical areas. A perfect example of fractal structure is that of the lungs. Observations support the claim that dependence exists between the viscoelasitcity and the air-flow properties in the presence of airway mucus with disease and that fractional orders appear intrinsically in viscoelastic materials (i.e. soft lung tissue) (Suki et al., 1994). These mechanical properties are captured in the input impedance, which gives insight upon airway and tissue resistance and compliance.

The respiratory input impedance can be measured non-invasively at the mouth during quiet breathing of the patient, without requiring any special manoeuvres. In this lung function test, forced oscillations are superimposed on the breathing pattern of the patient, in the form of a multisine signal, exciting frequencies in the $4-48 \mathrm{~Hz}$ range. An I2M (Input Impedance Measurement) device produced by Chess Medical Technologies, The Netherlands (2000) has been used for pulmonary testing. The specifications of the device are those of commercially available i2m devices: $11 \mathrm{~kg}$, 50x50×60 cm, $8 \mathrm{sec}$ measurement time, European Directive 93/42 on Medical devices and safety standards EN60601-1. The subject is connected to the typical setup from figure 8 via a mouthpiece, suitably designed to avoid flow leakage at the mouth and dental resistance artifact. The oscillation pressure is generated by a loudspeaker (LS) connected to a chamber (Oostveen et al., 2003). The LS is driven by a power amplifier fed with the oscillating signal generated by a computer $(U)$. The movement of the LS cone generates a pressure oscillation inside the chamber, which is applied to the patient's respiratory system by means of a tube connecting the LS chamber and the bacterial filter (bf). A side opening of the main tubing (BT) allows the patient to have fresh air circulation. Ideally, this pipeline will have high impedance at the excitation frequencies to avoid the loss of power from the LS pressure chamber. It is advisory that during the measurements, the patient wears a nose clip and keeps the cheeks firmly supported. Before starting the measurements, the frequency response of the transducers (PT) and of the pneumotachograph (PN) are calibrated. The measurements of air-pressure $P$ and air-flow $Q$ (= $\dot{V}$, with $V$ - air volume) during the forced oscillations lung function test is done at the mouth of the patient. Using electrical analogy, whereas the $P$ corresponds to voltage and $Q$ corresponds to current, the respiratory impedance $Z_{r}$ can be defined as their spectral (frequency domain) ratio relationship:

$$
Z_{r}(j \omega)=\frac{S_{P U}(j \omega)}{S_{Q U}(j \omega)}
$$

where $S_{i j}(j \omega)$ denotes the cross-correlation spectra between the various input-output signals, $\omega$ is the angular frequency and $j=(-1)^{1 / 2}$, resulting a complex variable. This nonparametric representation can be further identified with parametric models, quantifying some of the mechanical properties of the lung tissue, such as: resistance, compliance and inertance. Depending on the values of these parameters, clinicians can distinguish between healthy and pathologic cases, as well as between various types of lung disease. 
Recently, it has been shown that fractional order model characterizing impedance provide better identification results due to their intrinsic nature of capturing variations in frequency domain which are not dependent on the integer multiples of $20 \mathrm{~dB} / \mathrm{dec}$ and $\Pi / 2$ for magnitude and phase, respectively (Ionescu \& De Keyser, 2008a). Such a fractional order impedance model can be represented in the form:

$$
Z(s)=L s^{\alpha}+\frac{1}{C s^{\beta}}
$$

with $Z$ the impedance, $L$ the inductance and $C$ the compliance of the total respiratory system and $a, \beta$ fractional. In this example, the impedance of a patient diagnosed with chronic obstructive pulmonary disease has been used, where $L=0.00166 \mathrm{kPa} \mathrm{s} / 1, C=2.0451 / \mathrm{kPa}$, $a=0.5524$ and $\beta=0.5395$ (Ionescu et al., 2008b; Ionescu and De Keyser, 2008c). The parameters of the mechanical properties of the lung tissue in these patients can vary during several stages of the treatment applied by clinicians, including medication and ventilatory support. These patients are under mechanical ventilation, to ensure optimal conditions for gas exchange in the body (Behbehani, 2006). The efficiency of the ventilator depends on the optimal matching of the ventilator settings to the mechanical properties of the respiratory system, which may vary significantly in time. The ventilator can be approximated by a $3^{\text {rd }}$ order transfer function of the form:

$$
V(s)=\frac{1}{(10 s+1)^{3}}
$$

and the total process to be controlled is given by $P(s)=Z(s) \cdot V(s)$.

Due to the fact that (22) is a fractional order model, we evaluate the frequency domain of the process in order to decide upon the frequency band of the controller. Since the reference model can be used to specify the speed of the closed loop, one needs to attain insight on the speed of the process in open loop. For this, integer order approximation is performed using the method described in (Oustaloup et al., 2000) and the step response of the total process $P(s)$ is given in figure 9, left. Based on this information, the reference model has been chosen in the form:

$$
R(s)=\frac{1}{(\tau s+1)^{4}}
$$

with $\tau=10$ and 5 , respectively. The Bode characteristics of the process and the reference models are given in figure 9, right. Using (13), the controller transfer function is obtained and the problem of nonlinear optimization is solved using lsqnonlin for the unknown parameters in (8), in the frequency range $\omega \in\left(10^{-3}, 10^{-1}\right)$. Notice that in practice the process is unknown, so based on the known input and output signals, one may find the frequency response of the controller using (15). After fitting the frequency response with minimum errors (see figures 10-11 left), the resulted set of parameters for the integer-order controller IO_PID from (7) and for the fractional-order controller FO_PID from (8) are those given in Table 3. The corresponding closed loop responses with the respective controllers implemented in the form given by (16) are depicted in figures 10-11, right. 


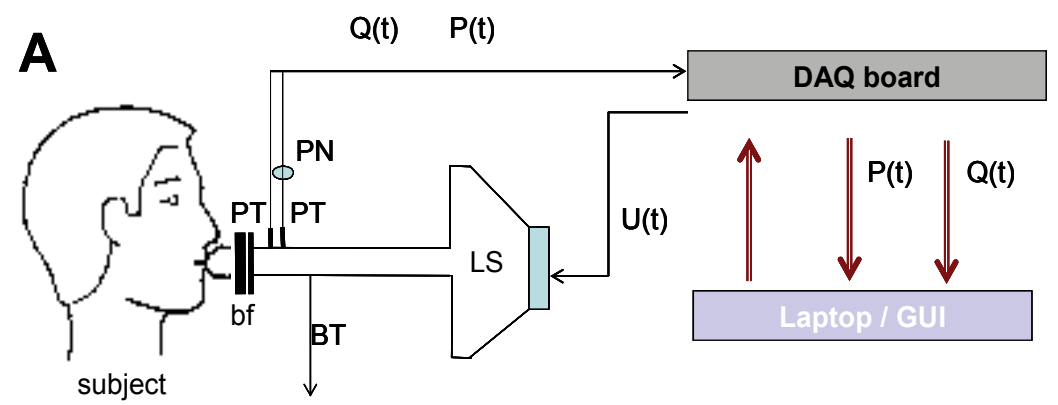

Figure 8. Schematic representation for the forced oscillation lung function testing device; see text for symbol explanation
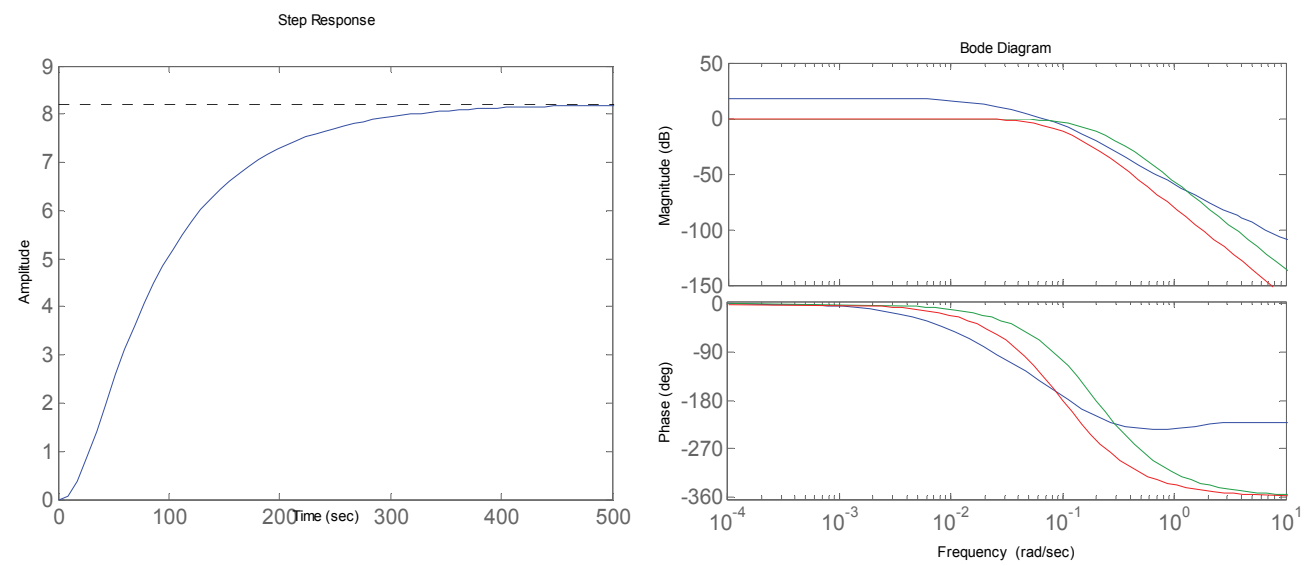

Figure 9. Left: open loop unit step response of the process; Right: Bode characteristics of the process (blue) and the reference models for $\tau=10$ (green) and for $\tau=5$ (red)
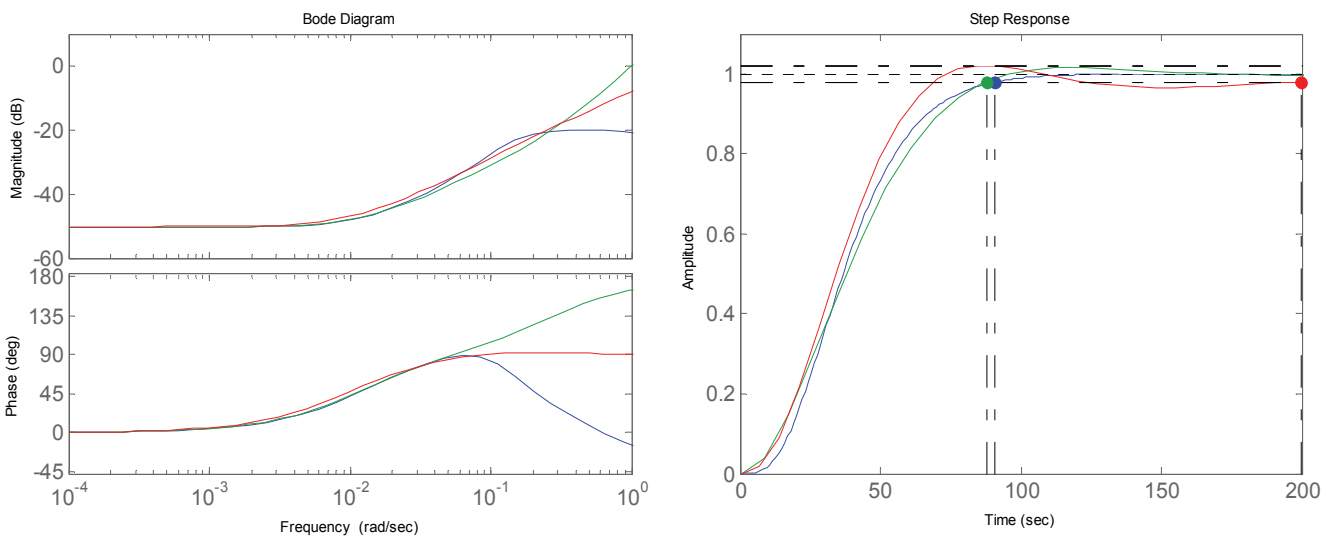

Figure 10. Left: frequency domain approximation and Right: unit step responses for $\tau=10$; reference (blue), IO_PID (green) and FO_PID (red). Circles denote settling times 


\begin{tabular}{|l|l|l|l|l|l|c|}
\hline & $\tau$ & $c_{0}$ & $c_{1}$ & $c_{2}$ & $\lambda$ & $\mu$ \\
\hline IO_PID & 10 & 0.003 & 0.267 & 0.971 & 1 & 1 \\
\hline FO_PID & 10 & 0.048 & 0.004 & 0.386 & 0.956 & 0.089 \\
\hline & & & & & & \\
\hline IO_PID & 5 & 0.006 & 0.576 & 6.381 & 1 & 1 \\
\hline FO_PID & 5 & 0.229 & 0.021 & 3.615 & 0.797 & 0.623 \\
\hline
\end{tabular}

Table 3. Controller parameters for the mechanically ventilated respiratory system
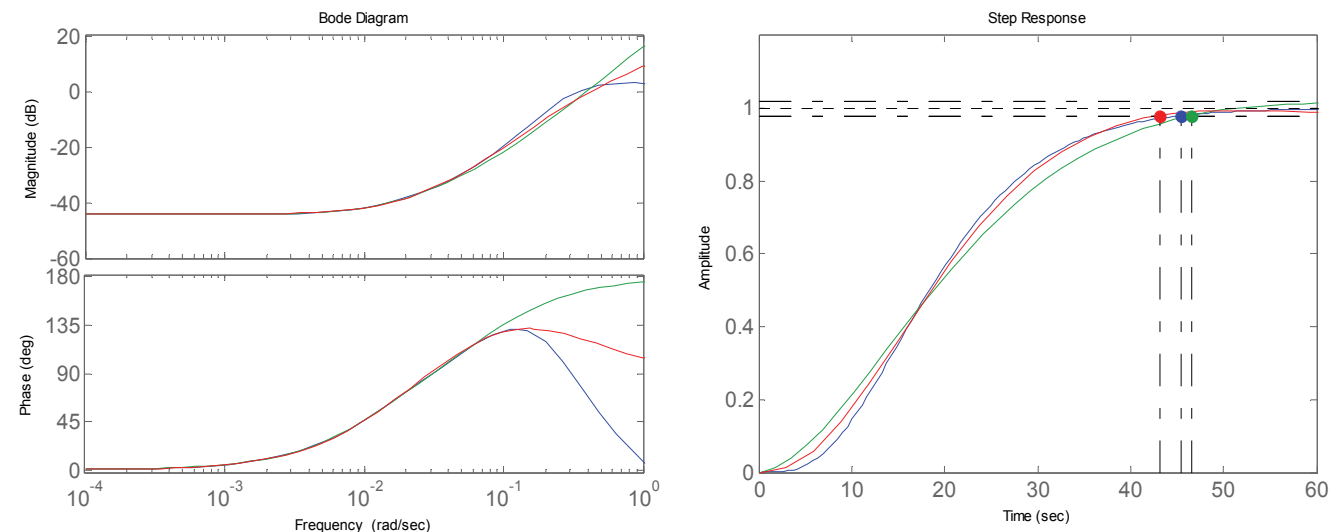

Figure 11. Left: frequency domain approximation and Right: unit step responses for $\tau=5$; reference (blue), IO_PID (green) and FO_PID (red). Circles denote settling times

\section{Discussion}

\subsection{Tuning aspects}

Obviously, the first and crucial step in DIRAC is the choice of the reference model $R(s)$. This can be done if some knowledge on the process is available. Some of the rule-ofthumb guidelines can be summarized in the following list:

- if the controller contains integral action, it ensures zero steady state error, which must be reflected in the closed loop gain; the latter should be 1, i.e. $R(1)=1$;

- if both process and controller contain an integrator, it is the case of type 2 control loop (double integrator); this means that the closed loop can track a ramp-setpoint without error and this should be reflected by the choice of the reference model (see, for example, (18));

- if the process contains a dead-time, the closed loop will also be affected by it, therefore in the reference model the presence of the dead-time is necessary and an approximate value suffices to obtain good results;

- if the process is non-minimum phase, the closed loop will also have this property; therefore the reference model should also be non-minimum phase.

In the previous sections it has been stated that the choice of the time constant in the reference model affects the speed of the closed loop. When defining the time constant of the reference model, the actual time constant of the process has to be taken into account. In other words, the desired closed loop speed should be in the same order of magnitude as the open loop settling time of the process. If this condition is not fulfilled, the reference 
model will ask the closed-loop to behave in an un-realistic way and the process will not be able to follow the actions of the controller, perhaps leading to poor robustness and even instability. Apart from this, the control effort required to fulfil the specifications imposed by the reference model should also be within realistic limits.

As a general observation, the choice of the reference model is not in all situations a 'best' choice. Especially in direct adaptive methods, in which the knowledge of the process is not required, there are uncertainties on the system behaviour. In order to overcome this problem, it is possible to adjust both the reference model as well as the controller parameters. This adaptation must be based on some capability sensing parameters from the process, which would then re-define the reference model to adapt controller parameters to the new achievable specifications. However, the baseline observation is that the reference model is specified such that its output yields a desired, as well as an achievable response.

The second step in the DIRAC algorithm presented here is related to the fact that the reasoning is transposed from time domain (or discrete time domain) to frequency domain. It is clear that a frequency band of interest must be defined, in order to fit the controller's parameters. By definition, it is not possible with a single, linear and simple model to capture the entire frequency response of the desired controller. It is important to choose meaningfully the frequency interval over which the fitting will be done. In this case, one can obtain the actual frequency response of the plant, from the input-output measurements, as from (14)-(15). In this case, the choice of the excitation signal and its frequencies is significant. By looking at the cross-over frequency of the plant and the desired frequency bandwidth of the reference model, one can reason upon the effective frequency interval. Notice that the low frequencies are not important to be perfectly modelled, because the presence of the integrator in the controller ensures steady state error zero (16).

Finally, whether the controller structure is the standard integer order PID from (7) or the more 'flexible' fractional order PID from (8) is a choice of the user. From the presented examples, it appears that there is no guarantee that a fractional-order PID outperforms an integer order PID. Further research will be necessary before a classification can be made upon processes in which FOC is better suitable than standard integer order control.

\subsection{Implementation aspects}

It is necessary to include here some of the important settings dealing with the implementation of the DIRAC scheme. The fact that in this paper we chose to work in frequency domain is solely due to the fractional order derivatives/integrals which are present. Of course, from a practical standpoint, a discrete time controller is necessary and the discrete-time DIRAC algorithm has been presented in (De Keyser, 1989; De Keyser, 2000).

Firstly, since the representation is in frequency domain, all the necessary transfer functions to calculate the utopic controller from (13) have to be dealt with in function of the chosen frequency interval of interest, from which (15) is calculated. Secondly, if the choice of the controller structure is that of an integer order PID, then the Matlab function fitfrd can be applied directly to obtain a $2^{\text {nd }}$ order transfer function with relative degree 2 (number of excess poles) and the final controller results as in (16) (MathWorks, 2000b). If the choice of the controller structure is that of a fractional order PID, the nonlinear least 
squares function lsqnonlin is employed, since the function to be minimized (8) is nonlinear in the parameters. Since the choice of the initial values is a critical step in nonlinear optimization, these have been set to the parameters resulted from the integer order PID. This choice is regarded as the best guess upon the final (optimal) values of the parameters to be estimated by the nonlinear estimator. After providing the fitting in the frequency domain with (8), the next step is to convert this polynomial to a stable, integer order transfer function. Again, the use of Matlab functions is not an obvious solution, and care must be taken when choosing the function parameters. To achieve acceptable results, the function invfreqs has been employed, delivering the transfer function fitted to the given frequency response (MathWorks, 2000c). The advantage over the fitfrd function consists in options parameters, which may be chosen such that the algorithm guarantees stability of the resulting linear system and searches for the best fit using a numerical, iterative scheme. The superior ("output-error") algorithm uses the damped Gauss-Newton method for iterative search (MathWorks, 2000b).

\section{Conclusions}

A simple and straightforward to understand direct adaptive control algorithm (DIRAC) has been presented in this chapter, from a frequency domain perspective, based on previous work derived for discrete-time DIRAC. Both integer order and fractional order PID controllers have been presented and discussed. Three typical examples have been simulated: i) a fractional order process; ii) a double integrator in the closed loop; and iii) a highly oscillatory process with low damping factor. Although the fractional order controller did not prove to outperform the standard PID controller in the presented examples, the DIRAC method remains available to the control engineering community for further research. It should be noted that the controller structure is not limited to PID; in fact, any transfer function can be fitted to the desired frequency response of the controller, as calculated based on the reference model of the closed loop performance.

Further research may be focused towards the following aspects: i) the relationship between DIRAC and other auto-tuning/adaptive methods; ii) stability and convergence analysis; iii) guidelines on the choice of the reference model; iv) the effect of noise and disturbance on the controller's parameter estimation.

\section{References}

Åström, K.J. \& Wittemark, B. (1995). Adaptive Control (2nd ed). Addison-Wesley, ISBN: 0201-55866-1

Åström, K.J \& Hägglund, T. (1995). PID Controllers: Theory, design and tuning. Instrument Society of America, Research Triangle Park, NC, USA, ISBN 1556175167

Anderson, B.D.O. (2005). Failures of adaptive control theory and their resolution. Communications in Information and Systems, 5(1), pp 1-20

Behbehani, K. (2006). Mechanical Ventilation. Biomedical Engineering Fundamentals, J. D. Bronzino, B. D. Bronzino (Eds)CRC Press, ISBN 0849321220

Bernstein, D. (2002). Feedback control: an invisible thread in the history of technology. IEEE Ctrl Syst Mag, 22(2), pp 53-68

Bueno, S., De Keyser, R., \& Favier, G. (1991). Auto-tuning and adaptive tuning of PID controllers. Journal A, 32(1), pp 28-34. 
Butler, H. (1990). Model Reference Adaptive Control. PhD Thesis, Technical University of Delft, Delft, The Netherlands.

De Keyser, R. (1989). DIRAC: A Finite Impulse Response Direct Adaptive Controller. Invited chapter in: S.L. Shah, G. Dumont (Eds), Adaptive Control Strategies for Industrial Use, Lecture Notes in Control and Information Sciences, ISBN 354051869X, Springer-Verlag, Berlin, pp 65-88

De Keyser, R. (2000). DIRAC: A Direct Adaptive Controller, IFAC Conference on Digital Control: Past, Present and Future of PID Control, pp. 199-204, Terassa, Spain, April 5-7 2000

Gorez, R. (1997). A survey of PID auto-tuning methods. Journal A, 38(1), pp. 3-10

Ionescu, C. \& De Keyser, R. (2008a). Parametric models for characterizing the respiratory input impedance. Journal of Medical Engineering $\mathcal{E}$ Technology, Taylor \& Francis, 32(4), pp 315-324

Ionescu, C., Nour, B., De Keyser, R., Dugan, V., (2008b). Respiratory pressure level regulation based on a fractional-order model for subjects with chronic obstructive pulmonary disease, IEEE Proc. 11 th Int Conf on Optimization of electrical and electronic equipment (OPTIM08), IEEE Cat. Nr 08EX1996C, ISBN 1-4244-1545-4, Vol ID-04, 6pages, May 19-21

Ionescu, C., \& De Keyser, R., (2008c). Time domain validation of a fractional order model for human respiratory system, 14th IEEE Mediterranean Electrochemical Conf (MELECON08), Ajaccio, Corsica, IEEE Cat Nr CFP08MEL-CDR, ISBN 978-1-42441633-2, pp. 89-95, May 3-5

Ilchmann, A. \& Ryan, E.P. (2003). On gain adaptation in adaptive control. IEEE Transactions on Automatic control, 48(5), pp 895-899

Ljung, L. (1987). System Identification, Theory for the user. Prentice Hall, Upper Saddle River, NJ (1987) ISBN 0-13-881640-9

MathWorks. (2000a). Matlab optimisation toolbox., User's guide. The MathWorks, Inc.

MathWorks. (2000b). Matlab robust control toolbox., User's guide. The MathWorks, Inc.

MathWorks. (2000c). Matlab signal processing toolbox, User's guide. The Mathworks, Inc.

Melchior, P., Lanusse, P., Cois, O., Dancla, F., \& Oustaloup, A. (2002). Crone Toolbox for Matlab. Tutorial Workshop on Fractional Calculus Applications in Automatic Control and Robotics, 41st IEEE Conf on Decision and Control CDC'02, Las Vegas, Nevada, USA, December 9-13.

Monje, C., Vinagre, B., Feliu, V., \& Chen Y. (2008). Tuning and auto-tuning of fractional order controllers for industry applications. Control Engineering Practice, 16, pp. 798-812

Oostveen, E., Macleod, D., Lorino, H., Farré, R., Hantos, Z., Desager, K., Marchal, F, (2003). The forced oscillation technique in clinical practice: methodology, recommendations and future developments, Eur Respir J, 22, pp 1026-1041

Oustaloup, A., Levron, F., Mathieu B. \& Nanot F. (2000). Frequency-band complex noninteger differentiatior: characterization and synthesis. IEEE Transactions of circuits and systems - I: Fundamental theory and applications, 47(1), pp 25-39

Pintér, J. (1996). Global optimization in action. Dordrecht, The Netherlands, Kluwer Academic Publishers, ISBN 0792337573

Podlubny, I. (1999). Fractional Differential Equations--Mathematics in Sciences and Engineering, vol. 198, Academic Press, ISBN 0125588402, New York. 
Suki, B., Barabasi, A.L., \& Lutchen, K. (1994). Lung tissue viscoelasticity: a mathematical framework and its molecular basis. J Applied Physiology, 76, pp. 2749-2759

Weibel, E.R. (2005). Mandelbrot's fractals and the geometry of life: a tribute to Benoît Mandelbrot on his 80 th birthday, in Fractals in Biology and Medicine, vol IV, Eds: Losa G., Merlini D., Nonnenmacher T., Weibel E.R., ISBN 9-783-76437-1722, Berlin: Birkhaüser, pp 3-16 


\title{
Adaptive Control Design for Uncertain and Constrained Vehicle Yaw Dynamics
}

\author{
Nazli E. Kahveci \\ Ford Research and Advanced Engineering \\ United States
}

\section{Introduction}

Nonlinear models describing vehicle yaw dynamics are considered in inevitably simplified forms using certain assumptions to serve relevant control design purposes. The corresponding modeling errors, however, might have adverse effects on the lateral performance of ground vehicles operating under conditions where these simplifying assumptions are no longer valid. The variations in operating conditions are seldom trivial to monitor and likely to result in significant compromises in the overall performance of the vehicle if the uncertain model parameters are not properly taken into account during the control design phase. In particular, vehicle yaw dynamics might demonstrate unexpected behavior in the presence of unusual external conditions, different side friction coefficients, and steering steps necessary to avoid obstacles. (Canale et al., 2007) Mastering vehicle yaw motions becomes a challenging task while driving on icy road or running on a flat tire. (Ackermann, 1994) Yaw dynamics control problem is additionally complicated in the presence of control input saturation constraints which are in most cases physically inherent acting to limit the magnitude or the rate of change of the effective control signal. In this work we consider a simplified model for vehicle yaw dynamics with steering angle constraints. A nominal control design is developed for the yaw rate tracking performance of the vehicle in Section 2. In order to account for potential uncertainties in the lateral dynamics an adaptive control design is proposed and presented in detail in Section 3 . The performance of our yaw rate control strategy is examined through simulations where the road adhesion factor, the vehicle velocity and the vehicle mass are unknown. Our simulation results for several scenarios are demonstrated in Section 4. Finally, our conclusions appear in Section 5.

\section{Vehicle Dynamics and Nominal Control Design}

We consider linear vehicle yaw dynamics and impose magnitude saturation nonlinearities on the steering angle which is introduced as the control input. One can also handle vehicle yaw dynamics with control inputs subject to rate constraints using an extension of our design if a rate-limited actuator is modeled as a first-order lag and a symmetric rate-limiting nonlinearity. (Kahveci \& Ioannou, 2008) We investigate several variations in the environmental conditions and unknown changes in the vehicle mass and velocity as 
parametric uncertainties which can be shown to be efficiently addressed by our adaptive control design approach. We begin our design using the simplified vehicle dynamics:

$$
\begin{gathered}
\dot{x}=A x+B \operatorname{sat}\left(\delta_{f}\right) \\
z=C_{p} x
\end{gathered}
$$

where $x=[\beta r]^{T}$ is the measurable state vector, $\beta$ is the side-slip angle, $r$ is the yaw rate, $z=r$ is the performance output, $\delta_{f}$ is the steering angle on which magnitude constraints are imposed through a scalar input saturation function defined as:

$$
\operatorname{sat}\left(\delta_{f}\right)=\operatorname{sign}\left(\delta_{f}\right) \min \left(\left|\delta_{f}\right|, \bar{\delta}_{f}\right), \quad \bar{\delta}_{f} \in R, \quad \bar{\delta}_{f}>0
$$

with $\bar{\delta}_{f}$ and $-\bar{\delta}_{f}$ representing the upper and lower saturation limits respectively. We consider the following system matrices and the performance output matrix:

$$
A=\left[\begin{array}{ll}
a_{11} & a_{12} \\
a_{21} & a_{22}
\end{array}\right], \quad B=\left[\begin{array}{l}
b_{1} \\
b_{2}
\end{array}\right], \quad C_{p}=\left[\begin{array}{ll}
0 & 1
\end{array}\right]
$$

and define the system parameters accordingly as discussed in (Ackermann \& Sienel, 1993; Ackermann et al., 1995; Mammar, 1996) :

$$
\begin{gathered}
a_{11}=-\left(c_{r}+c_{f}\right) /\left(\tilde{m}_{v} v\right) \\
a_{12}=-1+\left(c_{r} l_{r}-c_{f} l_{f}\right) /\left(\tilde{m}_{v} v^{2}\right) \\
a_{21}=\left(c_{r} l_{r}-c_{f} l_{f}\right) / \widetilde{J} \\
a_{22}=-\left(c_{r} l_{r}^{2}+c_{f} l_{f}^{2}\right) /(\widetilde{J} v) \\
b_{1}=c_{f} /\left(\tilde{m}_{v} v\right) \\
b_{2}=c_{f} l_{f} / \widetilde{J}
\end{gathered}
$$

where $c_{r}$ and $c_{f}$ are the rear and front cornering stiffness coefficients, $v$ is the magnitude of the velocity vector, $l_{f}$ and $l_{r}$ are the distances between the center of gravity and the front and rear axles respectively. Using the distances, $l_{f}$ and $l_{r}$, and the total vehicle mass, $m_{v}$, we formulate the vehicle's moment of inertia, $J$ as: 


$$
J=m_{v} l_{r} l_{f}
$$

One can also normalize the moment of inertia of the vehicle into:

$$
\widetilde{J}=J / \mu
$$

and the normalized mass of the vehicle can be represented by $\widetilde{m}_{v}$ :

$$
\tilde{m}_{v}=m_{v} / \mu
$$

where $\mu$ is the common road adhesion factor equal to 1 for dry and 0.5 for wet road. We use the data for the city bus $\mathrm{O} 305$ which is provided in (Ackermann et al., 1995) with $l_{f}=3.67 \mathrm{~m}, l_{r}=1.93 \mathrm{~m}, c_{f}=198000 \mathrm{~N} / \mathrm{rad}, c_{r}=470000 \mathrm{~N} / \mathrm{rad}, J=10.85 m_{v} \mathrm{kgm}^{2}$. The steering angle limits are $\pm \pi / 8 \mathrm{rad}$. The uncertainties in the yaw dynamics are mainly due to:

$$
\begin{gathered}
v \in[1,20] \mathrm{m} / \mathrm{s} \\
m_{v} \in[9950,16000] \mathrm{kg} \\
\mu \in[0.5,1]
\end{gathered}
$$

which represent the ranges for the vehicle velocity, the vehicle mass, and the road adhesion factor. The tools of stability analysis have been recently used to investigate the control design with anti-windup augmentation in the adaptive context, and upon combining the control structure with an adaptive law, the closed-loop system stability has been established. (Kahveci \& Ioannou, 2007) The design has been employed in aircraft control applications with unknown parameters. (Kahveci et al., 2008) We follow the corresponding control design method and evaluate compatible states, $x_{r}$ for desired yaw rate. The state tracking error is hence defined as:

$$
e=x-x_{r}
$$

and can be regulated by first augmenting the state vector in the form:

$$
x_{\text {aug }}=\left[\begin{array}{ll}
\dot{e}^{T} & C_{p} e
\end{array}\right]^{T}
$$

Using controllability and observability assumptions we consider the following Algebraic Riccati Equation (ARE):

$$
A_{\text {aug }}^{T} P+P A_{\text {aug }}+Q_{z}-P B_{\text {aug }} R_{z}^{-1} B_{\text {aug }}^{T} P=0
$$




$$
\begin{gathered}
A_{\text {aug }}=\left[\begin{array}{ll}
A & 0 \\
C_{p} & 0
\end{array}\right], \quad B_{\text {aug }}=\left[\begin{array}{l}
B \\
0
\end{array}\right] \\
Q_{z}=\left[\begin{array}{ll}
0 & 0 \\
0 & I
\end{array}\right] \times Q, \quad Q>0, \quad R_{z}>0
\end{gathered}
$$

The solution of the above ARE can then be used to obtain a PI controller as:

$$
\begin{aligned}
& u=-K_{1} e-K_{2} \int_{0}^{t} e(\tau) d \tau \\
& {\left[\begin{array}{ll}
K_{1} & K_{2}
\end{array}\right]=R_{z}^{-1} B_{\text {aug }}^{T} P}
\end{aligned}
$$

Using $A_{c}=0, B_{c}=I, C_{c}=K_{2}, D_{c}=K_{1}$ we represent the controller in state space form:

$$
\begin{gathered}
\dot{x}_{c}=A_{c} x_{c}+B_{c}(r-x) \\
u=C_{c} x_{c}+D_{c}(r-x)
\end{gathered}
$$

Given $N(s) M^{-1}(s)$ as a full-order right coprime factorization of $G(s)=(s I-A)^{-1} B$, the anti-windup compensator can be described by its transfer function matrix:

$$
K_{a w}(s)=\left[\begin{array}{c}
M(s)-I \\
N(s)
\end{array}\right]
$$

whereas it can also be represented in its state space form through the respective system matrices, $\left(A_{a w}, B_{a w}, C_{a w}, D_{a w}\right)$ which can be defined as:

$$
\begin{gathered}
A_{a w}=A+B L Q^{-1} \\
B_{a w}=B \\
C_{a w}=\left[\begin{array}{cl}
\left(L Q^{-1}\right)^{T} & I
\end{array}\right]^{T} \\
D_{a w}=0
\end{gathered}
$$

One needs to generate the term, $L Q^{-1}$ to implement the two anti-windup compensation matrices, $A_{a w}$ and $C_{a w}$ such that $A+B L Q^{-1}$ is Hurwitz. This term can possibly be evaluated by solving the following set of Linear Matrix Inequalities (LMIs): 


$$
\left[\begin{array}{ccccc}
Q A^{T}+A Q+L^{T} B^{T}+B L & B U-L^{T} & 0 & Q & L^{T} \\
U^{T} B^{T}-L & -2 U & I & 0 & U \\
0 & I & -\mu I & 0 & -I \\
Q^{T} & 0 & 0 & -W_{p}^{-1} & 0 \\
L & U^{T} & -I & 0 & -W_{r}^{-1}
\end{array}\right]<0
$$

The selection of weighting matrices, $W_{p}>0$ and $W_{r}>0$ is discussed in (Turner et al., 2004) for system performance and robustness. As a result, the anti-windup is augmented as:

$$
\begin{aligned}
& \dot{x}_{a w}=A_{a w} x_{a w}+B_{a w}\left(\delta_{f}-\operatorname{sat}\left(\delta_{f}\right)\right) \\
& y_{a w}=C_{a w} x_{a w}+D_{a w}\left(\delta_{f}-\operatorname{sat}\left(\delta_{f}\right)\right)
\end{aligned}
$$

and the term, $y_{a w}=\left[\begin{array}{ll}y_{a w 1} & y_{a w 2}^{T}\end{array}\right]^{T}, y_{a w 2} \in R^{2}, y_{a w 1} \in R$ modifies the controller into:

$$
\begin{gathered}
\dot{x}_{c m}=A_{c} x_{c m}+B_{c}\left(x_{r}-x\right)-B_{c} y_{a w 2} \\
\delta_{f}=C_{c} x_{c m}+D_{c}\left(x_{r}-x\right)-D_{c} y_{a w 2}-y_{a w 1}
\end{gathered}
$$

\section{Adaptive Control Design}

In order to avoid high frequency sensor noise amplification by the derivative term we employ a prefilter, $1 /(s+\lambda), \lambda>0$, and for any set of fixed plant parameters we obtain:

$$
\frac{s}{s+\lambda}=A \frac{1}{s+\lambda} x+B \frac{1}{s+\lambda} \operatorname{sat}\left(\delta_{f}\right)
$$

At any particular time instant, $t$ we estimate the vectors, $\theta_{1}^{*}(t)$ and $\theta_{2}^{*}(t)$ which are defined as:

$$
\theta_{1}^{*}(t)=\left[\begin{array}{lll}
a_{11} & a_{12} & b_{1}
\end{array}\right]^{T}, \quad \theta_{2}^{*}(t)=\left[\begin{array}{lll}
a_{21} & a_{22} & b_{2}
\end{array}\right]^{T}
$$

and denote these estimates by $\theta_{1}(t)$ and $\theta_{2}(t)$. The estimation model consists of:

$$
\hat{z}_{1}=\theta_{1}^{T} \phi, \quad \hat{z}_{2}=\theta_{2}^{T} \phi
$$




$$
\phi=\frac{1}{s+\lambda}\left[\begin{array}{lll}
\beta & r & \operatorname{sat}\left(\delta_{f}\right)
\end{array}\right]^{T}
$$

As a next step we construct the normalized estimation errors, $\varepsilon_{1}$ and $\varepsilon_{2}$ :

$$
\begin{gathered}
\varepsilon_{1}=\left(z_{1}-\hat{z}_{1}\right) / m^{2} \\
\varepsilon_{2}=\left(z_{2}-\hat{z}_{2}\right) / m^{2} \\
m^{2}=1+\phi^{T} \phi
\end{gathered}
$$

We use the discrete version of the Least-Squares Algorithm (LSA) given in (Ioannou \& Sun, 1996) along with a relevant orthogonal term-by-term projection as described in (Kahveci et al., 2008). Based on the Certainty Equivalence Principle we can implement the control input as:

$$
\delta_{f}=-\hat{K}_{2} \frac{1}{S}\left(e+y_{a w 2}\right)-\hat{K}_{1}\left(e+y_{a w 2}\right)-y_{a w 1}
$$

where $\left[\begin{array}{ll}\hat{K}_{1} & \hat{K}_{2}\end{array}\right]$ is evaluated through the ARE and $\hat{L} \hat{Q}^{-1}$ is calculated through the LMI solution using the parameter estimates. The modification terms can be explicitly written as:

$$
\begin{gathered}
y_{a w 1}=\hat{L} \hat{Q}^{-1}\left(s I-\hat{A}-\hat{B} \hat{L} \hat{Q}^{-1}\right)^{-1} \hat{B}\left(\delta_{f}-\operatorname{sat}\left(\delta_{f}\right)\right) \\
y_{a w 2}=\left(s I-\hat{A}-\hat{B} \hat{L} \hat{Q}^{-1}\right)^{-1} \hat{B}\left(\delta_{f}-\operatorname{sat}\left(\delta_{f}\right)\right)
\end{gathered}
$$

The overall adaptive control scheme is summarized in Figure 1.

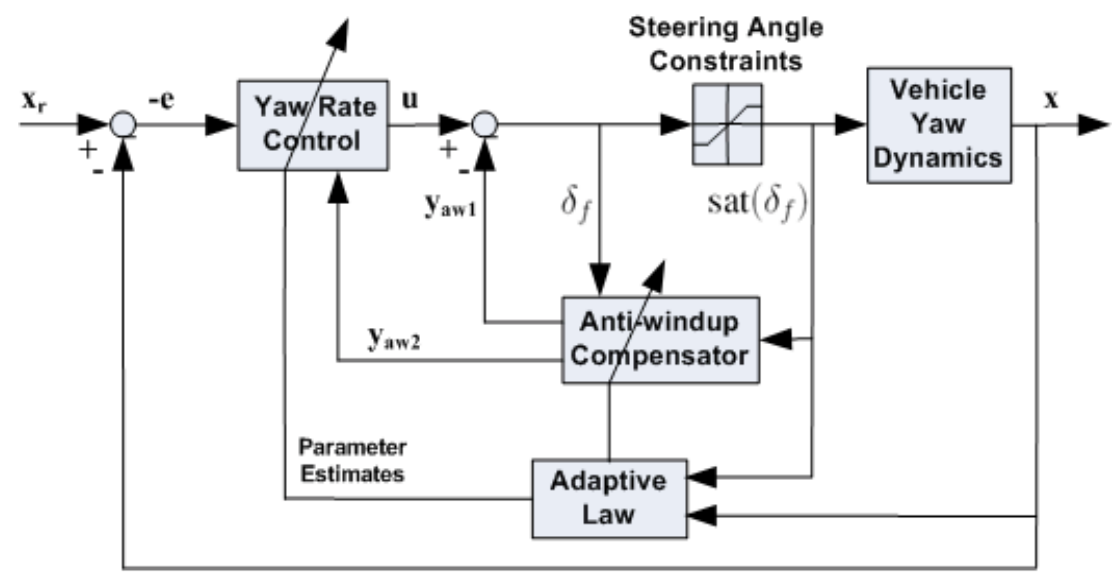

Figure 1. Adaptive control design for constrained vehicle yaw dynamics 


\section{Simulations}

The first set of simulations is conducted using the adaptive control design with no antiwindup compensation. The approximate ranges for the road adhesion factor, the vehicle mass, and the vehicle velocity are provided although the specific values of these parameters are unknown. The commanded input signal is demonstrated in Figure 2. The reference signal for the system response and the observed vehicle yaw rate are shown in Figure 3. The adaptive control design with anti-windup compensation is implemented in the second set of simulations where the target for the yaw rate of the vehicle is the same as before. The steering angle, the system response, and the anti-windup modification terms are presented in Figure 4, Figure 5, and Figure 6. When the adaptive anti-windup compensator design is included in the overall system, the overshoots in the system response are observed to be eliminated despite the parametric uncertainties in vehicle dynamics and unknown variations in external driving conditions.

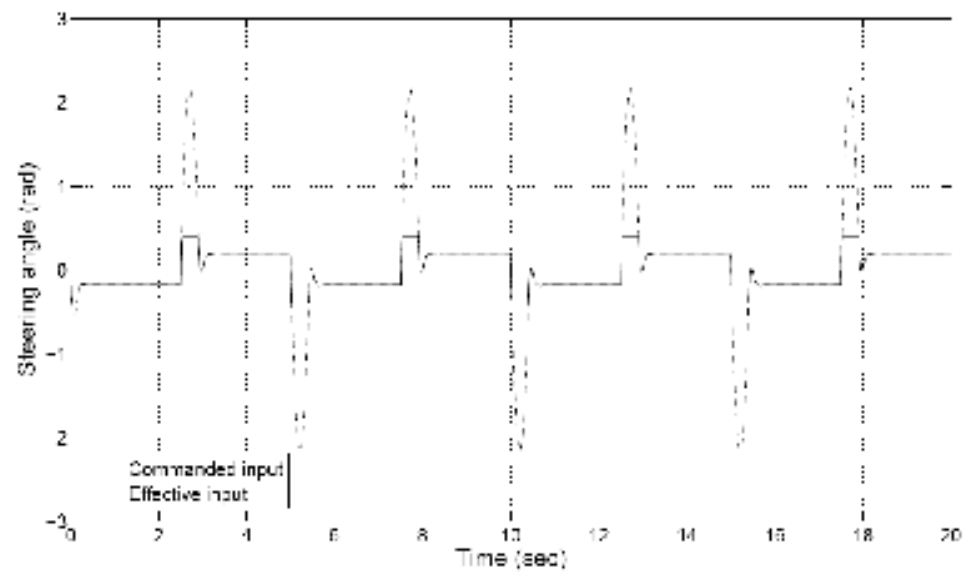

Figure 2. The commanded steering angle and the effective control signal within limits

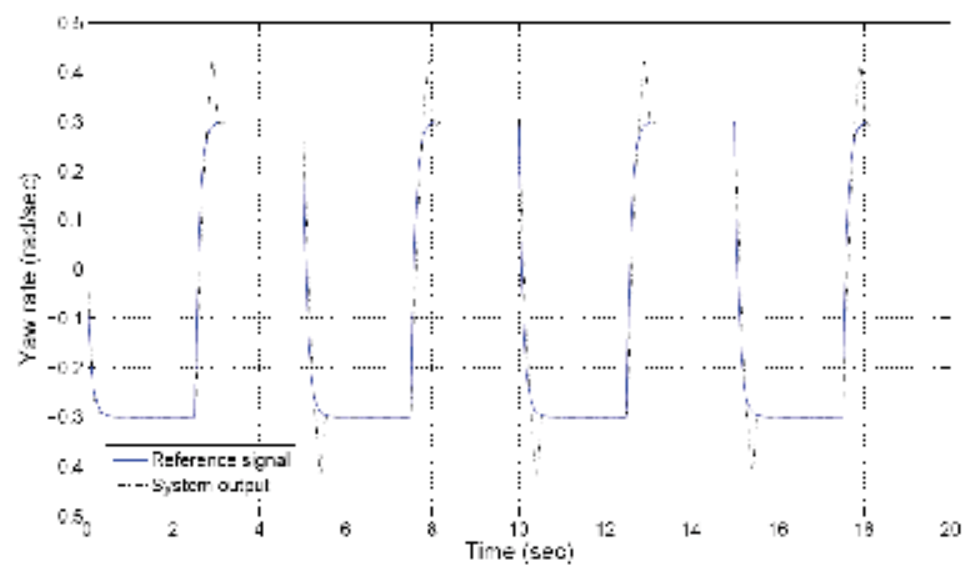

Figure 3. The system response compared with the desired yaw rate 


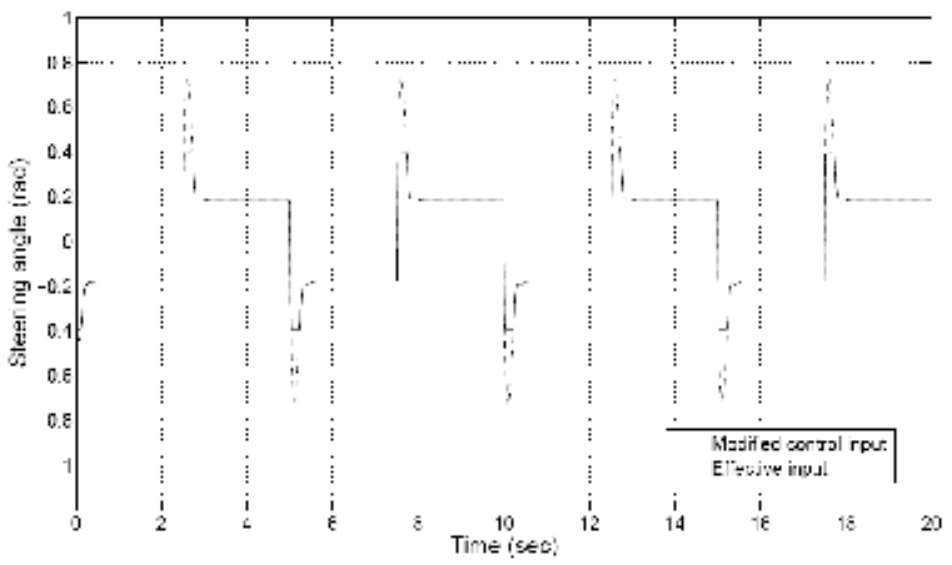

Figure 4. The modified control input subject to saturation and the effective control signal

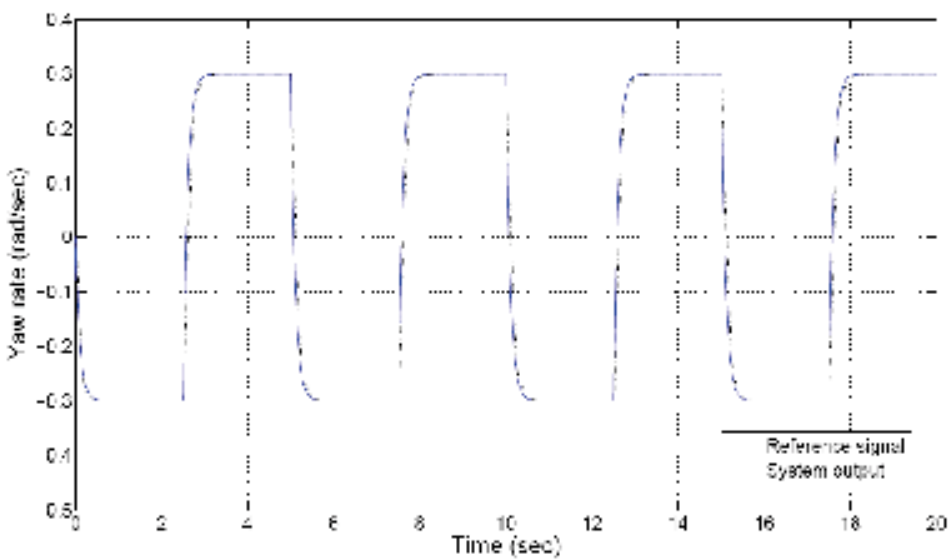

Figure 5. The system response tracking the desired reference signal

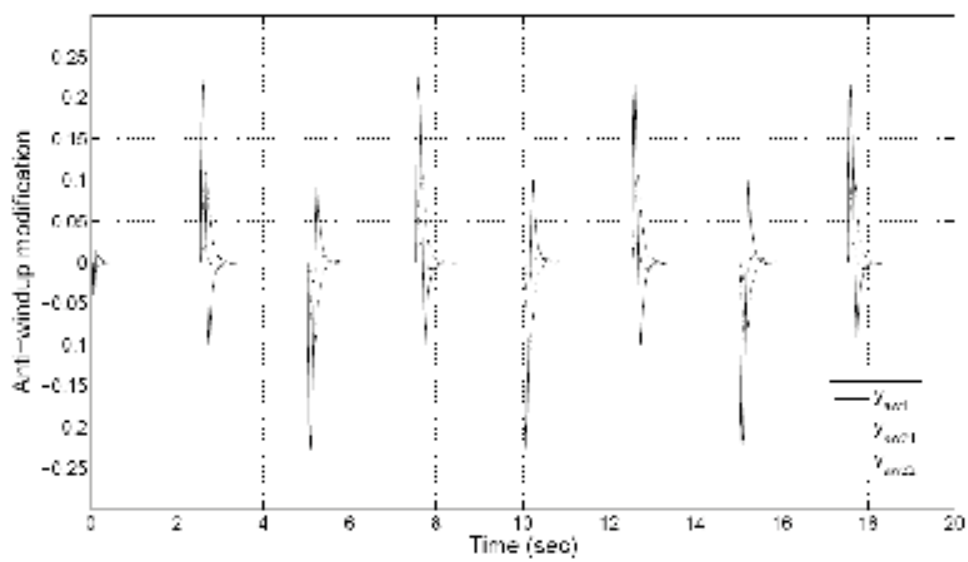

Figure 6. The adaptive anti-windup modification terms 
Interested reader might also refer to (Kahveci, 2008) for extensive simulations with an adaptive steering controller under various road conditions, crosswind effects acting on the vehicle dynamics as lateral disturbances, and actuator failure scenarios in addition to more restrictive magnitude saturation constraints imposed on the steering angle of the vehicle.

\section{Conclusion}

We consider yaw dynamics of a vehicle operating under uncertain road conditions with unknown road adhesion factor, vehicle velocity and mass. We develop an adaptive control design technique motivated by the demand for a system capable of adjusting to deviations in vehicle parameters with almost negligible performance compromises despite the changes in environmental conditions. Our simulations with the adaptive control scheme display significant enhancements in the performance of the vehicle in case the steering angle experiences saturation. The adaptive control design methodology can hence be used to address modeling uncertainties in vehicle yaw dynamics with steering angle constraints.

\section{References}

Ackermann J. (1994). Yaw rate and lateral acceleration feedback for four-wheel steering, Proceedings of the International Symposium on Advanced Vehicle Control, pp. 165-170, Tokyo, Japan, Oct. 1994.

Kahveci N. E. \& Ioannou P. A. (2008). Indirect adaptive control for systems with input rate saturation, Proceedings of the 2008 American Control Conference, pp. 3396-3401, Seattle, WA, June 2008.

Ackermann J. \& Sienel W. (1993). Robust yaw damping of cars with front and rear wheel steering, IEEE Transactions on Control Systems Technology, vol. 1, no. 1, pp. 15-20, Mar. 1993.

Ackermann J., Guldner J., Sienel W., Steinhuaser R. \& Utkin V. I. (1995). Linear and nonlinear controller design for robust automatic steering, IEEE Transactions on Control Systems Technology, vol. 3, no. 1, pp.132-143, Mar. 1995.

Mammar S. (1996). H-infinity robust automatic steering of a vehicle, Proceedings of the 1996 IEEE Intelligent Vehicles Symposium, pp. 19-24, Tokyo, Japan, Sept. 1996.

Kahveci N. E., Ioannou P. A. \& Mirmirani M. D. (2008). Adaptive LQ control with antiwindup augmentation to optimize UAV performance in autonomous soaring applications, IEEE Transactions on Control Systems Technology, vol. 16, no. 4, pp. 691-707, July 2008.

Kahveci N. E. \& Ioannou P. A. (2007). An indirect adaptive control design with antiwindup compensation: Stability analysis, Proceedings of the 46th IEEE Conference on Decision and Control, pp. 1294-1299, New Orleans, LA, Dec. 2007.

Turner M. C., Herrmann G. \& Postlethwaite I. (2004). Accounting for uncertainty in antiwindup synthesis, Proceedings of the 2004 American Control Conference, pp. 52925297, Boston, MA, June 2004.

Ioannou P. A. \& Sun J. (1996). Robust Adaptive Control, Upper Saddle River, NJ: Prentice Hall, 1996. 
Kahveci N. E. (2008). Adaptive steering control for uncertain vehicle dynamics with crosswind effects and steering angle constraints, Proceedings of the 2008 IEEE International Conference on Vehicular Electronics and Safety, pp. 168-173, Columbus, $\mathrm{OH}$, Sept. 2008. 


\title{
A Design of Discrete-Time Indirect Multivariable MRACS with Structural Estimation of Interactor
}

\author{
Wataru Kase and Yasuhiko Mutoh \\ Osaka Institute of Technology / Sophia University \\ Japan
}

\section{Introduction}

An interactor matrix introduced by Wolovich \& Falb (1976) has an important role in the design of model reference adaptive control systems (MRACS) for a class of multi-input multi-output (MIMO) plants. In the early stage of the research, the interactor was supposed to be diagonal matrix and thus there were no unknown parameters (Goodwin et al., 1980). But, there exist many plants which require non-diagonal interactors (Chan \& Goodwin, 1982). And the design of MIMO MRACS with non-diagonal interactors had been discussed, where all elements of the interactor are assumed to be known (Elliott \& Wolovich, 1982; Goodwin \& Long, 1980). However, this assumption is not adequate for adaptive control systems since the parameters of the interactor depend on the unknown parameters of the plant, i.e., the parameter values and the relative degree of each element of the plant must be used to determine the interactor. Furthermore, even we know all of these information, the structure of the interactor is not determined uniquely.

In order to remove the assumption, the MRACS design has been proposed where the degree of diagonal elements and the upper bound of the highest degree of the lower triangular interactor matrix are assumed to be known (Elliott \& Wolovich, 1984; Dugard et al., 1984). Under these assumptions, off-diagonal elements of the lower triangular interactor are estimated, and the method seemed suitable for adaptive controller design. However, it is not reasonable to assume the diagonal degrees in MRACS since the determination of the degrees depends on the relative degree and parameter values of each element of a transfer matrix of a given plant. From this view point, an interactor in generic sense was considered under the assumption that the relative degrees of all elements of the transfer function matrix are known (Kase \& Tamura, 1990; Mutoh \& Ortega, 1993). The method covers almost of all classes of MIMO plants having the same numbers of inputs and outputs generally. But there still exist some rare plants.

By the way, there exists an idea of the certainty equivalence principle for the indirect MRACS design, i.e., estimate the unknown parameters of a plant first, then design the controllers on-line, using those estimated parameters. However, the design was seemed very difficult especially for MIMO plants, since large amount of calculation is needed to solve so-called Diophantine equation, beside the derivation of the interactor. In other words, there did not exist a suitable method to solve the Diophantine equation or to derive the interactor matrix. In this chapter, an indirect approach to MIMO MRACS will be shown. For 
this purpose, it will be presented a simple derivation of the interactor matrix and preferable solution of the Diophantine equation. Both methods are based on the state space representation for the (estimated) transfer function matrix of the plant. Unlike direct calculation of polynomial matrices, it is preferable to compute via the state space representation.

This chapter is organized as follows. In the next section, the basic controller design with known parameters will be shown. The derivation of the interactor matrix and the solution to the Diophantine equation will be also presented. Then, the indirect MRACS will be shown in section 3 using the results in the previous sections. Some simulation results will be presented in section 4 to confirm the validity of the proposed method. Concluding remarks will be presented in section 5 .

Notations (See Wolovich, 1974)

$\boldsymbol{R}^{p \times m}[z]$ : Set of $p \times m$ polynomial matrices with real coefficients.

$\partial_{r i}[D(z)]:$ The $i$-th row degree of polynomial matrix $D(z)$.

$\Gamma_{r}[D(z)]$ : The row leading coefficient matrix of $D(z)$.

$$
S_{I}^{v}(z)=\left[\begin{array}{c}
I \\
z I \\
\vdots \\
z^{v} I
\end{array}\right], \quad \boldsymbol{O}_{v}=\left[\begin{array}{c}
C \\
C A \\
\vdots \\
C A^{v}
\end{array}\right], \quad T_{V}=\left[\begin{array}{cccc}
C B & 0 & \cdots & 0 \\
C A B & C B & \cdots & 0 \\
\vdots & \vdots & \ddots & \vdots \\
C A^{v} B & C A^{v-1} B & \cdots & C B
\end{array}\right]
$$

\section{Design for Plant with Known Parameters}

\subsection{Problem Statement}

Assume that a plant to be controlled is given by

$$
y(t)=N(z) D^{-1}(z) u(t)=\tilde{D}^{-1}(z) \tilde{N}(z) u(t)
$$

where $D(z), N(z), \tilde{D}(z), \tilde{N}(z) \in \boldsymbol{R}^{m \times m}[z]$ such that

$$
\begin{aligned}
& D(z)=D_{0}+z D_{1}+\cdots+z^{\mu} D_{\mu}=\left[\begin{array}{llll}
D_{0} & D_{1} & \cdots & D_{\mu}
\end{array}\right]\left[\begin{array}{c}
I \\
z I \\
\vdots \\
z^{\mu} I
\end{array}\right]=D S_{I}^{\mu}(z), \\
& N(z)=N_{0}+z N_{1}+\cdots+z^{\mu-1} N_{\mu-1}=\left[\begin{array}{llll}
N_{0} & N_{1} & \cdots & N_{\mu}
\end{array}\right]\left[\begin{array}{c}
I \\
z I \\
\vdots \\
z^{\mu-1} I
\end{array}\right]=N S_{I}^{\mu-1}(z),
\end{aligned}
$$




$$
\begin{aligned}
& \widetilde{D}(z)=\widetilde{D}_{0}+z \widetilde{D}_{1}+\cdots+z^{v} \widetilde{D}_{v}=\left[\begin{array}{llll}
I & z I & \cdots & z^{v} I
\end{array}\right]\left[\begin{array}{c}
\widetilde{D}_{0} \\
\widetilde{D}_{1} \\
\vdots \\
\widetilde{D}_{v}
\end{array}\right]=\left\{S_{I}^{v}(z)\right\}^{T} \widetilde{\boldsymbol{D}} \\
& \tilde{N}(z)=\tilde{N}_{0}+z \tilde{N}_{1}+\cdots+z^{v-1} \tilde{N}_{v-1}=\left[\begin{array}{llll}
I & z I & \cdots & z^{v-1} I
\end{array}\right]\left[\begin{array}{c}
\tilde{N}_{0} \\
\tilde{N}_{1} \\
\vdots \\
\tilde{N}_{v}
\end{array}\right]=\left\{S_{I}^{v-1}(z)\right\}^{T} \tilde{N}, \\
& \boldsymbol{D}=\left[\begin{array}{llll}
D_{0} & D_{1} & \cdots & D_{\mu}
\end{array}\right], \quad \boldsymbol{N}=\left[\begin{array}{llll}
N_{0} & N_{1} & \cdots & N_{\mu-1}
\end{array}\right], \tilde{\boldsymbol{D}}=\left[\begin{array}{c}
\widetilde{D}_{0} \\
\widetilde{D}_{1} \\
\vdots \\
\widetilde{D}_{v}
\end{array}\right], \quad \tilde{\boldsymbol{N}}=\left[\begin{array}{c}
\tilde{N}_{0} \\
\tilde{N}_{1} \\
\vdots \\
\tilde{N}_{v-1}
\end{array}\right] \text {. }
\end{aligned}
$$

Without loss of generality, assume that $D(z)$ is column proper and $\widetilde{D}(z)$ is row proper. The purpose of control is to generate the uniformly bounded input signals $u(t)$ which cause the output signals $y(t)$ of the transfer function matrix $G(z)=N(z) D^{-1}(z)$ to follow the reference output signals $y_{m}(t)$ asymptotically. For this purpose, the following assumptions are made: 1. $\operatorname{det} N(z)$ is a Hurwitz polynomial.

2. $y_{m}(t+1), y_{m}(t+2), \ldots, y_{m}(t+w)$ are available at the time instant $t$, where $w$ is the degree of the interactor for $G(z)$, which will be discussed later.

The control input to achieve the above objective is given by

$$
u(t)=\frac{X(z)}{z^{v-1}} u(t)+\frac{Y(z)}{z^{\nu-1}} y(t)+L(z) y_{m}(t)
$$

where $X(z), Y(z) \in \boldsymbol{R}^{m \times m}[z]$ satisfy the following Diophantine equation:

$$
X(z) D(z)+Y(z) N(z)=z^{\nu-1}\{D(z)-L(z) N(z)\}
$$

and $L(z) \in \boldsymbol{R}^{m \times m}[z]$ satisfies the following relation:

$$
\lim _{z \rightarrow \infty} L(z) G(z)=I .
$$

$L(z) y_{m}(t)$ in eqn.(3) is available from assumption 2. $L(z)$ is known as an interactor matrix. Substituting eqn.(3) to eqn.(1),

$$
\begin{aligned}
y(t) & =N(z)\left\{z^{v-1} D(z)-X(z) D(z)-Y(z) N(z)\right\}^{-1} z^{\nu-1} L(z) y_{m}(t) \\
& =N(z)\left\{z^{\nu-1} L(z) N(z)\right\}^{-1} z^{\nu-1} L(z) y_{m}(t)
\end{aligned}
$$

Thus from assumption 1 , the purpose will be achieved if 
1. det $L(z)$ is a Hurwitz polynomial, and

2. The degrees of $X(z)$ and $Y(z)$ are, at most, $v-2$ and $v-1$ respectively.

In the following subsections, simple calculation methods of $L(z), X(z)$ and $Y(z)$ satisfying the above constraints will be shown.

\subsection{A Simple Derivation of Interactor (Kase \& Mutoh, 2008)}

Consider the problem to find an interactor $L(z)$ for a given $m \times m$ non-singular transfer function matrix $G(z)$. In the direct MRACS, an interactor with lower triangular structure is useful to insure the global stability of the overall system. But, in this chapter, the interactor is not assumed to have any special form.

Let $(A, B, C)$ denote a realization of $G(z)$. Then, using the Markov parameters, $G(z)$ can be expressed by

$$
G(z)=z^{-1} C B+z^{-2} C A B+z^{-3} C A^{2} B+\cdots
$$

If we set $L(z)$ by

$$
L(z)=z L_{1}+z^{2} L_{2}+\cdots+z^{w} L_{w}=\left[\begin{array}{llll}
L_{1} & L_{2} & \cdots & L_{w}
\end{array}\right]\left[\begin{array}{c}
z I \\
z^{2} I \\
\vdots \\
z^{w} I
\end{array}\right]=L z S_{I}^{w-1}(z) .
$$

Then,

$$
\begin{aligned}
& L(z) G(z)=\left[\begin{array}{llll}
L_{1} & L_{2} & \cdots & L_{w}
\end{array}\right]\left[\begin{array}{c}
z I \\
z^{2} I \\
\vdots \\
z^{w} I
\end{array}\right]\left(z^{-1} C B+z^{-2} C A B+z^{-3} C A^{2} B+\cdots\right) \\
& =L\left[\begin{array}{c}
C B+z^{-1} C A B+z^{-2} C A^{2} B+\cdots \\
z C B+C A B+z^{-1} C A^{2} B+\cdots \\
\vdots \\
z^{w-1} C B+z^{w-2} C A B+\cdots+C A^{w-1} B+\cdots
\end{array}\right] \\
& =L\left[\begin{array}{cccccc}
\cdots & C A B & C B & 0 & \cdots & 0 \\
\cdots & C A^{2} B & C A B & C B & \cdots & 0 \\
& \vdots & \vdots & & \ddots & \vdots \\
\cdots & C A^{w} B & C A^{w-1} B & \cdots & C A B & C B
\end{array}\right]\left[\begin{array}{c}
\vdots \\
z^{-1} I \\
I \\
z I \\
\vdots \\
z^{w-1} I
\end{array}\right]
\end{aligned}
$$

If eqn.(5) holds, then

$$
\mathbf{L T}_{w-1}=\boldsymbol{J}_{w-1}, \quad \boldsymbol{J}_{w-1}:=\left\lfloor\begin{array}{ll}
I & 0_{m \times m(w-1)}
\end{array}\right\rfloor
$$


must hold. Conversely, the identity interactor $L(z)$ can be obtained by solving this equation and the solvability of this is guaranteed if and only if

$$
\operatorname{rank}\left[\begin{array}{c}
\boldsymbol{T}_{w-1} \\
\boldsymbol{J}_{w-1}
\end{array}\right]=\operatorname{rank} \boldsymbol{T}_{w-1},
$$

and define the integer $w$ by the least integer satisfying the above equation. Thus, using Moore-Penrose pseudo-inverse $T_{w-1}^{+}$of $T_{w-1}, L$ can be calculated if and only if

$$
L=J_{w-1} T_{w-1}^{+} .
$$

All of $L$ that satisfy eqn.(11) are coefficient matrices of the identity interactor, which form a subset of coefficient matrices of the interactor defined by Mutoh \& Ortega (1993). In the paper, a certain calculating algorithm was used to obtain $L$ and to assign stable zeros of the interactor as well. But, for the identity interactor, as shown above, it is quite natural to solve eqn.(11) using Moore-Penrose pseudo-inverse, because $J_{w-1}$ is a fixed matrix and the pseudo-inverse can be calculated easily using some standard softwares in these days. Then, since we need a stable interactor in control design problems, the remaining problem is to check the location of zeros of the identity interactor given by eqn.(12). For $T_{w-1}^{+}$, the following Lemma holds.

Lemma 1. For the integer $k \geq w-1$, the following equation holds:

$$
\mathbf{T}_{k}^{+}=\left[\begin{array}{c}
\boldsymbol{M}_{k} \\
\mathbf{Z}_{k}-\boldsymbol{T}_{k-1}^{+} \boldsymbol{O}_{k-1} A B M_{k}
\end{array}\right]
$$

where

$$
\boldsymbol{M}_{k}=\left[\begin{array}{ll}
\boldsymbol{L} & \boldsymbol{0}_{k}
\end{array}\right], \quad \mathbf{Z}_{k}=\left[\begin{array}{ll}
0_{k m \times m} & \boldsymbol{T}_{k-1}^{+}
\end{array}\right], \mathbf{0}_{k}=0_{m \times m(k-w+1)} .
$$

Proof. Let

$$
\boldsymbol{T}_{k}^{+}=\left[\begin{array}{c}
\boldsymbol{M}_{k} \\
\boldsymbol{P}_{k}
\end{array}\right], \quad \boldsymbol{P}_{k} \in \boldsymbol{R}^{k m \times(k+1) m} .
$$

Since $T_{k}^{+}$is the pseudo-inverse of $T_{k}$,

$$
T_{k} T_{k}^{+}=\left(T_{k} T_{k}^{+}\right)^{T} .
$$

Substituting the above equation into eqn.(15),

$$
\left[\begin{array}{c}
C B \\
\boldsymbol{O}_{k} A B
\end{array}\right] \boldsymbol{M}_{k}+\left[\begin{array}{c}
0_{m \times k m} \\
\boldsymbol{T}_{k-1}
\end{array}\right] \boldsymbol{P}_{k}=\left[\begin{array}{ll}
\boldsymbol{M}_{k}^{T} & \boldsymbol{P}_{k}^{T}
\end{array}\right] \boldsymbol{T}_{k}^{T} .
$$

By post-multiplying the above equation by $\boldsymbol{M}_{k}^{T}$ and then using eqn.(10), it follows that

$$
\left[\begin{array}{c}
C B \\
\boldsymbol{O}_{k} A B
\end{array}\right] \boldsymbol{M}_{k} \boldsymbol{M}_{k}^{T}+\left[\begin{array}{c}
0_{m \times k m} \\
\boldsymbol{T}_{k-1}
\end{array}\right] \boldsymbol{P}_{k} \boldsymbol{M}_{k}^{T}=\boldsymbol{M}_{k}^{T} .
$$


From the existence of $\boldsymbol{P}_{k}$,

$$
\left(I_{m k}-\boldsymbol{T}_{k-1} \boldsymbol{T}_{k-1}^{T}\right)\left(\left[\begin{array}{c}
L_{2}^{T} \\
L_{3}^{T} \\
\vdots \\
L_{w}^{T} \\
\mathbf{0}_{k}^{T}
\end{array}\right]-\boldsymbol{O}_{k} \boldsymbol{L} \boldsymbol{L}^{T}\right)=0
$$

holds. Using the singular value decomposition, $T_{k-1}$ can be written as

$$
T_{k-1}=U_{k-1}\left[\begin{array}{cc}
\Sigma_{k-1} & 0 \\
0 & 0
\end{array}\right] V_{k-1}^{T}
$$

for some unitary matrices $U_{k-1}$ and $V_{k-1}$. Since eqn.(10) implies

$$
\left[\begin{array}{lllll}
L_{2} & L_{3} & \cdots & L_{w} & \mathbf{0}_{k}
\end{array}\right] \boldsymbol{T}_{k-1}=0,
$$

post-multiplying the above equation by $V_{k-1}\left[\begin{array}{cc}\Sigma^{-2} & 0 \\ 0 & 0\end{array}\right] V_{k-1}^{T}$ gives

$$
\left[\begin{array}{lllll}
L_{2} & L_{3} & \cdots & L_{w} & \boldsymbol{0}_{k}
\end{array}\right]\left(\boldsymbol{T}_{k-1}^{T}\right)^{T}=0 .
$$

Thus,

$$
\left[\begin{array}{c}
L_{2}^{T} \\
L_{3}^{T} \\
\vdots \\
L_{w}^{T} \\
\mathbf{0}_{k}^{T}
\end{array}\right]=\left(I_{m k}-T_{k-1} T_{k-1}^{+}\right) \boldsymbol{O}_{k} A B L L^{T}
$$

is obtained from eqns.(18) and (19). Using a free parameter matrix $Z_{k} \in \boldsymbol{R}^{m k \times m(k+1)}$, the general solution of eqn.(17) is given by

$$
\boldsymbol{P}_{k}=Z_{k}-T_{k-1}^{+} T_{k-1} Z_{k} M_{k}^{T}\left(M_{k}^{T}\right)^{+}-T_{k-1}^{+} O_{k} M_{k} .
$$

Finally, by choosing $Z_{k}$ as in eqn.(14), eqn.(13) is obtained from eqns.(21) and (23). It is easy to verify that the above $T_{k}^{+}$satisfies the rest of conditions for the pseudo-inverse, i.e.

$$
\left(\boldsymbol{T}_{k}^{+} \boldsymbol{T}_{k}\right)^{T}=\boldsymbol{T}_{k}^{+} \boldsymbol{T}_{k,}, \boldsymbol{T}_{k} \boldsymbol{T}_{k}^{+} \boldsymbol{T}_{k}=\boldsymbol{T}_{k,}, \boldsymbol{T}_{k}^{+} \boldsymbol{T}_{k} \boldsymbol{T}_{k}^{+}=\boldsymbol{T}_{k}^{+} .
$$

Therefore the Lemma has been proved. $\quad \nabla \nabla \nabla$ 
In MRACS case, it can not be assumed to know the exact value of $w$ in eqn.(11). Lemma 1 shows that non-zero parameters in the interactor are not changed, if the upper bound of $w$ is known. This is a nice property of the proposed method for MRACS. The following Theorem shows that all zeros of the interactor by proposed method lie at the origin. So, the stability of the interactor is clear although it does not have a lower triangular form. Moreover, the proposed interactor is optimal for the LQ cost with singular weightings. See Kase et al. (2004) for the proof.

Theorem 1. If the interactor is given by

$$
L(z)=\boldsymbol{J}_{w-1} \boldsymbol{T}_{w-1}^{+} z S_{I}^{w-1}(z),
$$

then the following properties hold:

$$
\begin{aligned}
& \text { P1 } L(z) L^{\sim}(z)=\boldsymbol{L L}^{T}, \\
& \text { P2 }\left[\begin{array}{c}
C B \\
C A_{F} B \\
\vdots \\
C A_{F}^{w-1} B
\end{array}\right]=\mathbf{L}^{+} \text {, } \\
& \text { P3 } C A_{F}^{w}=0
\end{aligned}
$$

where $L^{+}$is the pseudo-inverse of $L$, and

$$
L^{\sim}(z)=L^{T}\left(z^{-1}\right)=z^{-1} L_{1}^{T}+z^{-2} L_{2}^{T}+\cdots+z^{-w} L_{w}^{T}, \quad A_{F}=A-B L \boldsymbol{O}_{w-1} A .
$$

\subsection{A Solution of Diophantine Equation (Kase, 1999; 2008)}

There are many methods to solve eqn.(4). In this subsection, a method using state space parameters is presented. First, the following lemma holds.

Lemma 2. Let $P(z), N(z), D(z) \in \boldsymbol{R}^{m \times m}[z]$, where $D(z)$ is non-singular. Then, there exist polynomial matrices $Q(z), R(z) \in \boldsymbol{R}^{m \times m}[z]$ such that

$$
\begin{aligned}
& P(z) N(z)=Q(z) D(z)+R(z), \\
& R(z) D^{-1}(z) \text { is strictly proper. }
\end{aligned}
$$

Furthermore, let $(A, B, C)$ denote any realization of $N(z) D^{-1}(z)$. Then, $Q(z)$ and $R(z) D^{-1}(z)$ are given by the following equations:

$$
\begin{aligned}
& Q(z)=\boldsymbol{P}\left[\begin{array}{c}
0_{m \times f m} \\
\boldsymbol{T}_{f-1}
\end{array}\right] S_{I}^{f-1}(z) \\
& R(z) D^{-1}(z)=\mathbf{P O}_{f}(z I-A)^{-1} B
\end{aligned}
$$

where $f$ denotes the degree of $P(z)=P_{0}+z P_{1}+\cdots+z^{f} P_{f}$, and $\boldsymbol{P}$ is defined by $\boldsymbol{P}=\left[\begin{array}{llll}P_{0} & P_{1} & \cdots & P_{f}\end{array}\right]$. 
Proof. Since

$$
\begin{aligned}
& P(z) N(z) D^{-1}(z)=\boldsymbol{P}\left[\begin{array}{c}
I \\
z I \\
\vdots \\
z^{f} I
\end{array}\right]\left(z^{-1} C B+z^{-2} C A B+z^{-3} C A B+\cdots\right) \\
& =\boldsymbol{P}\left[\begin{array}{c}
z^{-1} C B+z^{-2} C A B+z^{-3} C A B+\cdots \\
C B+z^{-1} C A B+z^{-2} C A B+\cdots \\
\vdots \\
z^{f-1} C B+z^{f-2} C A B+\cdots+C A^{f-1} B+z^{-1} C A^{f} B+\cdots
\end{array}\right] \\
& =\boldsymbol{P}\left[\begin{array}{cccc}
0 & 0 & \cdots & 0 \\
C B & 0 & \cdots & 0 \\
C A B & C B & \cdots & 0 \\
\vdots & \vdots & \ddots & \vdots \\
C A^{f-1} B & C A^{f-2} B & \cdots & C B
\end{array}\right]\left[\begin{array}{c}
I \\
z I \\
\vdots \\
z^{f-1} I
\end{array}\right]+\boldsymbol{P}\left[\begin{array}{c}
z^{-1} C B+z^{-2} C A B+\cdots \\
z^{-1} C A B+z^{-2} C A^{2} B+\cdots \\
z^{-1} C A^{2} B+z^{-2} C A^{3} B+\cdots \\
\vdots \\
z^{-1} C A^{f} B+z^{-2} C A^{f+1} B+\cdots
\end{array}\right] \\
& =\boldsymbol{P}\left[\begin{array}{c}
0_{m \times f m} \\
\boldsymbol{T}_{f-1}
\end{array}\right] S_{I}^{f-1}(z)+z^{-1} \boldsymbol{P} \boldsymbol{O}_{f} B+z^{-2} \boldsymbol{P} \boldsymbol{O}_{f} A B+z^{-3} \boldsymbol{P} \boldsymbol{O}_{f} A^{2} B+\cdots
\end{aligned}
$$

the results can be obtained. $\quad \nabla \nabla \nabla$

Theorem 2. Let $(A, B, C)$ denote any realization of $N(z) D^{-1}(z)$. Then, there exist $X(z)$ and $Y(z)$ which satisfy

1) Diophantine equation (4).

2) $X(z) / z^{\nu-1}$ is strictly proper.

3) $Y(z) / z^{v-1}$ is proper.

If and only if the following relation holds:

$$
Y O_{v-1}=-L O_{w-1} A^{v}
$$

where $\boldsymbol{Y}$ is defined by $\boldsymbol{Y}=\left[\begin{array}{llll}Y_{0} & Y_{1} & \cdots & Y_{\nu-1}\end{array}\right]$ for $Y(z)=Y_{0}+z Y_{1}+\cdots+z^{\nu-1} Y_{\nu-1}$.

Proof. If eqn.(4) holds, then from Lemma 2,

$$
Y \boldsymbol{O}_{v-1}=-\left[0_{m \times m v} \quad L\right] \boldsymbol{O}_{v+w-1}=-\mathbf{L} \boldsymbol{O}_{w-1} A^{v} .
$$

Conversely, if eqn.(29) holds, multiply the both sides of eqn.(29) by $\sum_{i=1}^{\infty} z^{-i} A^{i-1} B$, it follows that 


$$
\begin{aligned}
& \boldsymbol{Y O}_{v-1} \sum_{i=1}^{\infty} z^{-i} A^{i-1} B=\boldsymbol{Y}\left(\left[\begin{array}{c}
N(z) D^{-1}(z) \\
z N(z) D^{-1}(z) \\
z^{2} N(z) D^{-1}(z) \\
\vdots \\
z^{v-1} N(z) D^{-1}(z)
\end{array}\right]-\left[\begin{array}{c}
0 \\
C B \\
C A B+z C B \\
\vdots \\
C A^{v-2} B+z C A^{v-3} B+\cdots+z^{v-1} C B
\end{array}\right]\right) \\
& =Y(z) N(z) D^{-1}(z)-Y\left[\begin{array}{c}
0_{m \times m(v-1)} \\
\mathbf{T}_{\nu-2}
\end{array}\right] S_{I}^{\nu-2}(z) \\
& =-\left[0_{m \times m v} \quad \boldsymbol{L}\right] \boldsymbol{O}_{\boldsymbol{v}+w-1} \sum_{i=1}^{\infty} z^{-i} A^{i-1} B \\
& =-\left[\begin{array}{ll}
0_{m \times m v} & L
\end{array}\right]\left(\left[\begin{array}{c}
N(z) D^{-1}(z) \\
z N(z) D^{-1}(z) \\
z^{2} N(z) D^{-1}(z) \\
\vdots \\
z^{v+w-1} N(z) D^{-1}(z)
\end{array}\right]-\left[\begin{array}{c}
0 \\
C B \\
C A B+z C B \\
\vdots \\
C A^{v-2} B+z C A^{v-3} B+\cdots+z^{v+w-1} C B
\end{array}\right]\right) \\
& =-z^{\nu-1} L(z) N(z) D^{-1}(z)+z^{v-1} I+L\left[\begin{array}{cccc}
C A^{v-1} B & \cdots & C A B \\
\vdots & & \vdots \\
C A^{v+w-2} B & \cdots & C A^{w} B
\end{array}\right] S_{I}^{v-2}(z) .
\end{aligned}
$$

Defining $X(z)$ by

$$
X(z)=-\boldsymbol{L}\left[\begin{array}{ccc}
C A^{v-1} B & \cdots & C A B \\
\vdots & & \vdots \\
C A^{v+w-2} B & \cdots & C A^{w} B
\end{array}\right] S_{I}^{\nu-2}(z)-Y\left[\begin{array}{c}
0_{m \times m(v-1)} \\
T_{\nu-2}
\end{array}\right] S_{I}^{\nu-2}(z),
$$

then the Diophantine equation (4) can be obtained. It is clear that $X(z) / z^{\nu-1}$ and $Y(z) / z^{\nu-1}$ are proper from the above discussion. $\quad \nabla \nabla \nabla$

It is worth noting that Theorem 2 holds for any realization of $N(z) D^{-1}(z)$. So, this method is easy to apply for the indirect adaptive control.

\section{Indirect Adaptive Controller Design}

Using some suitable parameter estimation algorithm, such as the least squares algorithm, obtain the estimated values of $\tilde{D}$ and $\tilde{N}$. Then, obtain the observability canonical realization $\hat{A}(t), \hat{B}(t)$ and $\hat{C}(t)$ from these estimated values. After that, the control input is generated by calculating $\hat{L}(z, t), \hat{X}(z, t)$ and $\hat{Y}(z, t)$ recursively. Before the discussions of the adaptive controller design, the following assumptions are imposed:

1. The upper bound degree $\bar{w}$ of the interactor is known.

2. $y_{m}(t+1), y_{m}(t+2), \ldots, y_{m}(t+\bar{w})$ are available at the time instant $t$. 
3. $\operatorname{det} \tilde{N}(z)$ is a Hurwitz polynomial.

4. $\partial_{r i}[\widetilde{D}(z)]=v_{i}$ is known and $\Gamma_{r}[\widetilde{D}(z)]$ is a lower triangular with ones in the diagonal positions.

5. $\hat{A}(t), \hat{B}(t)$ and $\hat{C}(t)$ converge to their true values.

6. A lower bound of the minimum singular decomposition value $\varepsilon_{\min }$ of $T_{\bar{w}-1}$ is known.

In the adaptive controller design, it will be carried out by the recursive calculation of the previous section. That is, based on the $\hat{A}(t), \hat{B}(t)$ and $\hat{C}(t)$, set

$$
\hat{O}_{\bar{w}-1}(t)=\left[\begin{array}{c}
\hat{C}(t) \\
\hat{C}(t) \hat{A}(t) \\
\vdots \\
\hat{C}(t) \hat{A}^{\bar{w}-1}(t)
\end{array}\right], \quad \hat{T}_{\bar{w}-2}(t)=\left[\begin{array}{cccc}
\hat{C}(t) \hat{B}(t) & 0 & \cdots & 0 \\
\hat{C}(t) \hat{A}(t) \hat{B}(t) & \hat{C}(t) \hat{B}(t) & \cdots & 0 \\
\vdots & \vdots & \ddots & \vdots \\
\hat{C}(t) \hat{A}^{\bar{w}-2} \hat{B}(t) & \hat{C}(t) \hat{A}^{\bar{w}-3} \hat{B}(t) & \cdots & \hat{C}(t) \hat{B}(t)
\end{array}\right] .
$$

Then, solve

$$
\hat{\mathbf{L}}(t) \hat{\boldsymbol{T}}_{\bar{w}-1}(t)=J_{\bar{w}-1}
$$

using pseudo-inverse of $\hat{T}_{\bar{w}-1}(t)$. Assume that $\hat{T}_{\bar{w}-1}(t)$ is given by

$$
\begin{aligned}
& \hat{T}_{\bar{w}-1}(t)=\hat{U}_{\bar{w}-1}(t)\left[\begin{array}{cc}
\hat{\Sigma}_{\bar{w}-1}(t) & 0 \\
0 & 0
\end{array}\right] \hat{V}_{\bar{w}-1}^{\frac{T}{1}}(t), \\
& \hat{\Sigma}_{\bar{w}-1}(t)=\operatorname{diag}\left\{\hat{\lambda}_{1}(t), \quad \hat{\lambda}_{2}(t), \quad \ldots, \quad \hat{\lambda}_{r}(t)\right\}, \quad \hat{\lambda}_{i}(t)>0
\end{aligned}
$$

for some unitary matrices $\hat{U}_{\bar{w}-1}(t)$ and $\hat{V}_{\bar{w}-1}(t)$. If

$$
\hat{\lambda}_{i}(t)<\varepsilon_{\min }
$$

for some integers $i$, then modify

$$
\hat{\lambda}_{i}(t)=\varepsilon_{\min }
$$

and calculate $\hat{T}_{\bar{w}-1}^{+}(t)$ by

$$
\hat{\boldsymbol{T}}_{\bar{w}-1}^{+}(t)=\hat{V}_{\bar{w}-1}(t)\left[\begin{array}{cc}
\hat{\Sigma}_{\bar{w}-1}^{-1} & 0 \\
0 & 0
\end{array}\right] \hat{U}_{\bar{w}-1}^{T}(t) .
$$

Next, solve

$$
\hat{Y}(t) \hat{\boldsymbol{O}}_{v-1}(t)=-\hat{L}(t) \hat{\boldsymbol{O}}_{\bar{w}-1}(t) \hat{A}^{v}(t) .
$$

Note that $\hat{O}_{v-1}(t)$ always has full column rank since $(\hat{C}(t), \hat{A}(t))$ is in the observability canonical form. So eqn.(35) is easy to solve. In general, $\hat{O}_{v-1}(t)$ is a tall matrix. For the solution to eqn.(35), the method employing the pseudo-inverse is effective for the plant with measurement noise (Kase \& Mutoh, 2000). It may be also useful for the improvement of the transit response of MRACS. 
Finally, calculate $\hat{X}(t)$ by

$$
\hat{X}(t)=-\hat{Y}(t)\left[\begin{array}{c}
0_{m \times m(v-1)} \\
\hat{T}_{v-2}(t)
\end{array}\right]-\hat{L}(t) \hat{V}(t)
$$

where

$$
\hat{V}(t)=\left[\begin{array}{ccc}
\hat{C}(t) \hat{A}^{v}(t) \hat{B}(t) & \cdots & \hat{C}(t) \hat{A}(t) \hat{B}(t) \\
\vdots & & \vdots \\
\hat{C}(t) \hat{A}^{2 v+\bar{w}-2}(t) \hat{B}(t) & \cdots & \hat{C}(t) \hat{A}^{v+\bar{w}-2}(t) \hat{B}(t)
\end{array}\right] .
$$

Then, using $\hat{L}(t), \hat{X}(t)$ and $\hat{Y}(t)$, the adaptive control input is given by

$$
u(t)=\hat{X}(t)\left[\begin{array}{c}
u(t-1) \\
\vdots \\
u(t-v+1)
\end{array}\right]+\hat{\boldsymbol{Y}}(t)\left[\begin{array}{c}
y(t) \\
\vdots \\
y(t-v+1)
\end{array}\right]+\hat{\boldsymbol{L}}\left[\begin{array}{c}
y_{m}(t+1) \\
\vdots \\
y_{m}(t+\bar{w})
\end{array}\right]
$$

The global stability of the over-all system may be proved under the assumption 5 . However, the details are under studying.

\section{Numerical Examples}

Consider the following plant:

$$
\begin{aligned}
G(z) & =\left[\begin{array}{cc}
\frac{a}{z+0.1} & \frac{1}{z+0.2} \\
\frac{1}{z+b} & \frac{1}{z+1.2}
\end{array}\right] \\
& =\left[\begin{array}{cc}
z^{2}+0.3 z+0.02 & 0 \\
0 & z^{2}+(b+1.2) z+1.2 b
\end{array}\right]^{-1}\left[\begin{array}{cc}
a(z+0.2) & z+0.1 \\
z+1.2 & z+b
\end{array}\right]
\end{aligned}
$$

Although the plant seems simple enough, there exist three variations of the interactor depend on the values of $a$ and $b$.

[Case 1] $a=1.5, b=0.9$

In this case, the interactor of the plant is

$$
L(z)=z\left[\begin{array}{cc}
2 & -2 \\
-2 & 3
\end{array}\right]
$$

[Case 2] $a=1, b=0.9$

In this case, the interactor of the plant is

$$
L(z)=z\left[\begin{array}{cc}
-10 z+5 & 10 z+5 \\
10 z-4 & -10 z-4
\end{array}\right] .
$$


[Case 3] $a=1, b=1.1$

In this case, the interactor of the plant is

$$
L(z)=z\left[\begin{array}{cc}
10 z^{2}-3.6 z+2.8 & -10 z^{2}-6.4 z+2.8 \\
-10 z^{2}+3.8 z-2.4 & 10 z^{2}+6.2 z-2.4
\end{array}\right] .
$$

For the above three cases, the interactor can be obtained by solving $\hat{L}(t) \hat{T}_{2}(t)=\hat{J}_{2}$. Using the above $\hat{L}(t), \hat{Y}(t)$ can be obtained by solving

$$
\hat{Y}(t)\left[\begin{array}{c}
C \\
C \hat{A}(t)
\end{array}\right]=-\hat{L}(t)\left[\begin{array}{c}
C \hat{A}^{2}(t) \\
C \hat{A}^{3}(t) \\
C \hat{A}^{4}(t)
\end{array}\right] .
$$

Since $C$-matrix does not depend on $t$ in this example and $(C, A)$ is observable independent on $t, \hat{O}_{1}(t)$ always has column-full rank and thus the above equation has a solution.

Finally, $\hat{X}(t)$ can be calculated by

$$
\hat{\boldsymbol{X}}(t)=-\hat{\boldsymbol{Y}}\left[\begin{array}{c}
0 \\
C \hat{B}(t)
\end{array}\right]-\hat{\boldsymbol{L}}(t)\left[\begin{array}{c}
C \hat{A}(t) \hat{B}(t) \\
C \hat{A}^{2}(t) \hat{B}(t) \\
C \hat{A}^{3}(t) \hat{B}(t)
\end{array}\right] .
$$

The reference signal vector was given by

$$
y_{m}(t)=G_{m}(z) r(t)=\left[\begin{array}{c}
\sin \pi t / 15 \\
\sin 2 \pi t / 25
\end{array}\right] .
$$

The least square algorithm with constant trace was used to estimate the parameters where the initial values of the covariance matrix is $10^{6}$, and $\varepsilon_{\min }=0.02$. Fig. 1,3 and 5 show the tracking errors of the proposed indirect MRACS for Case 1-3 respectively. Fig.2, 4 and 6 show the tracking errors of the proposed indirect MRACS for Case 1-3 without modification respectively. The results show the effectiveness of the proposed method.

\section{Conclusion}

In this chapter, an indirect MIMO MRACS with structural estimation of the interactor was proposed. By using indirect method, unreasonable assumptions such as assuming the diagonal degrees of interactor can be avoided. Since the controller parameters are calculated based on the observability canonical realization of the estimated values, the proposed method is suitable for on-line calculations. In the proposed method, the degree of the controllers do not depend on the estimated structure of the interactor. The global stability of the overall system is under studying. 

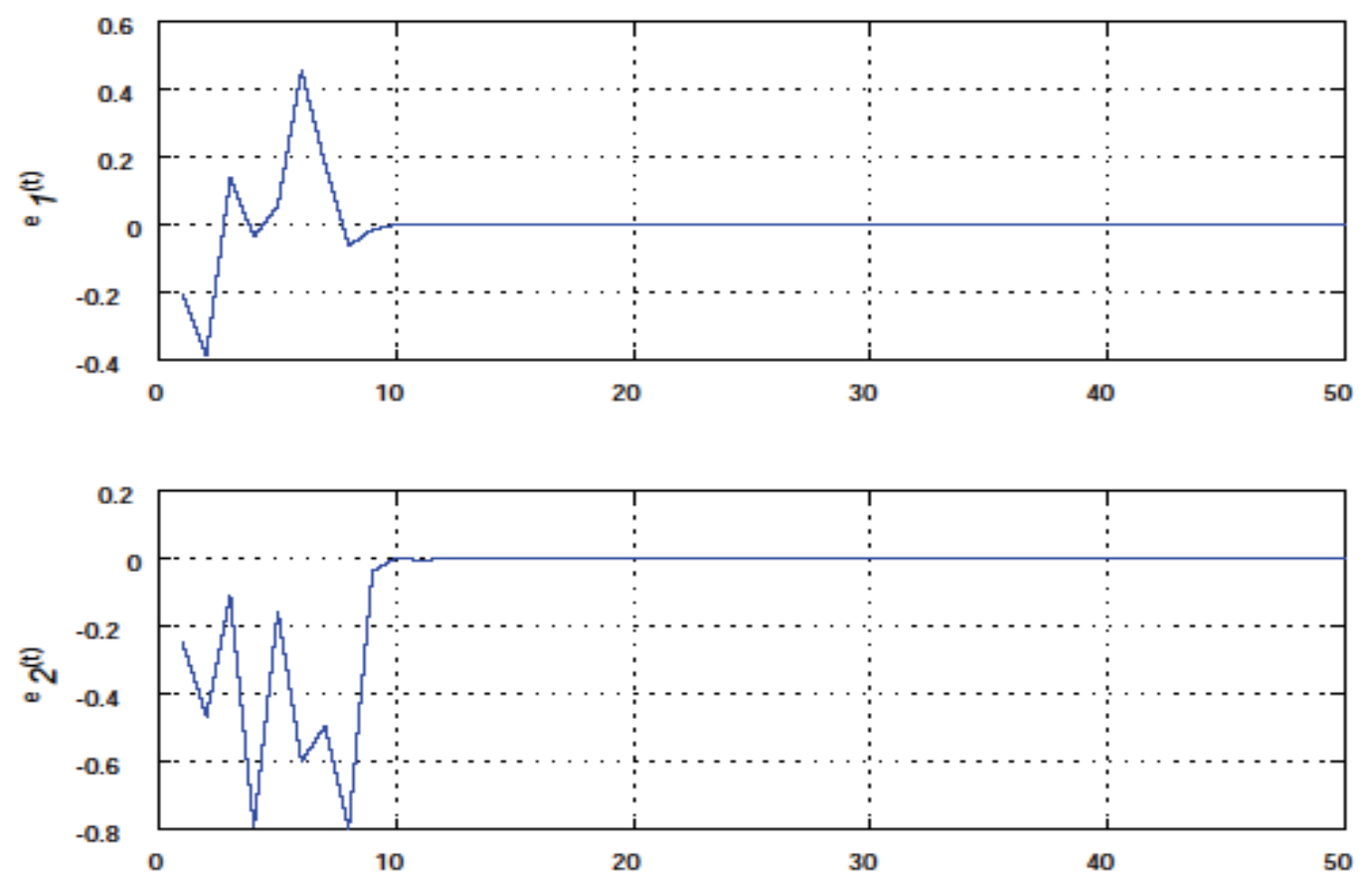

Figure 1. Output tracking error of the proposed MRACS (Case 1; with modifications)
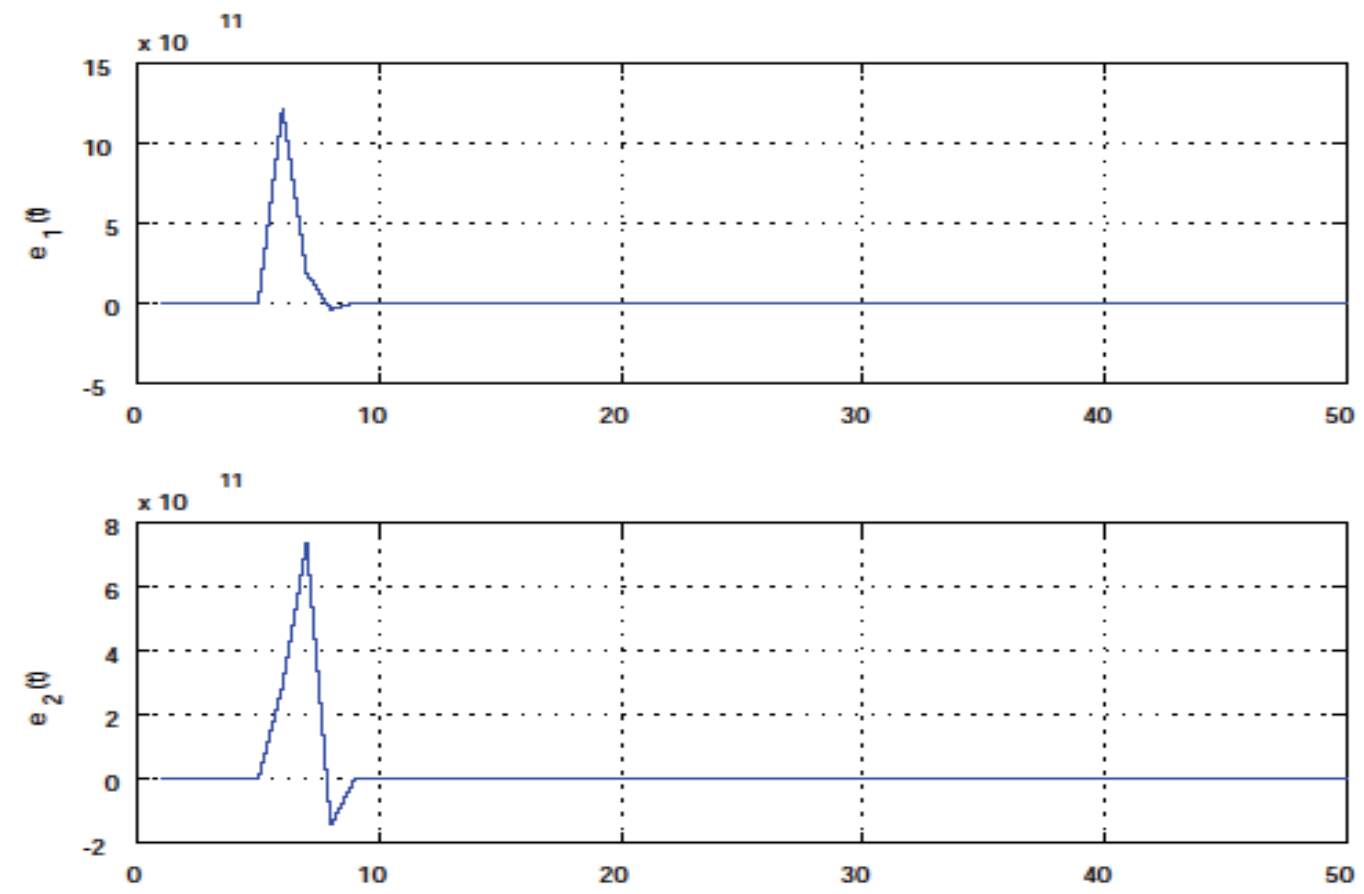

Figure 2. Output tracking error of the proposed MRACS (Case 1; without modifications) 

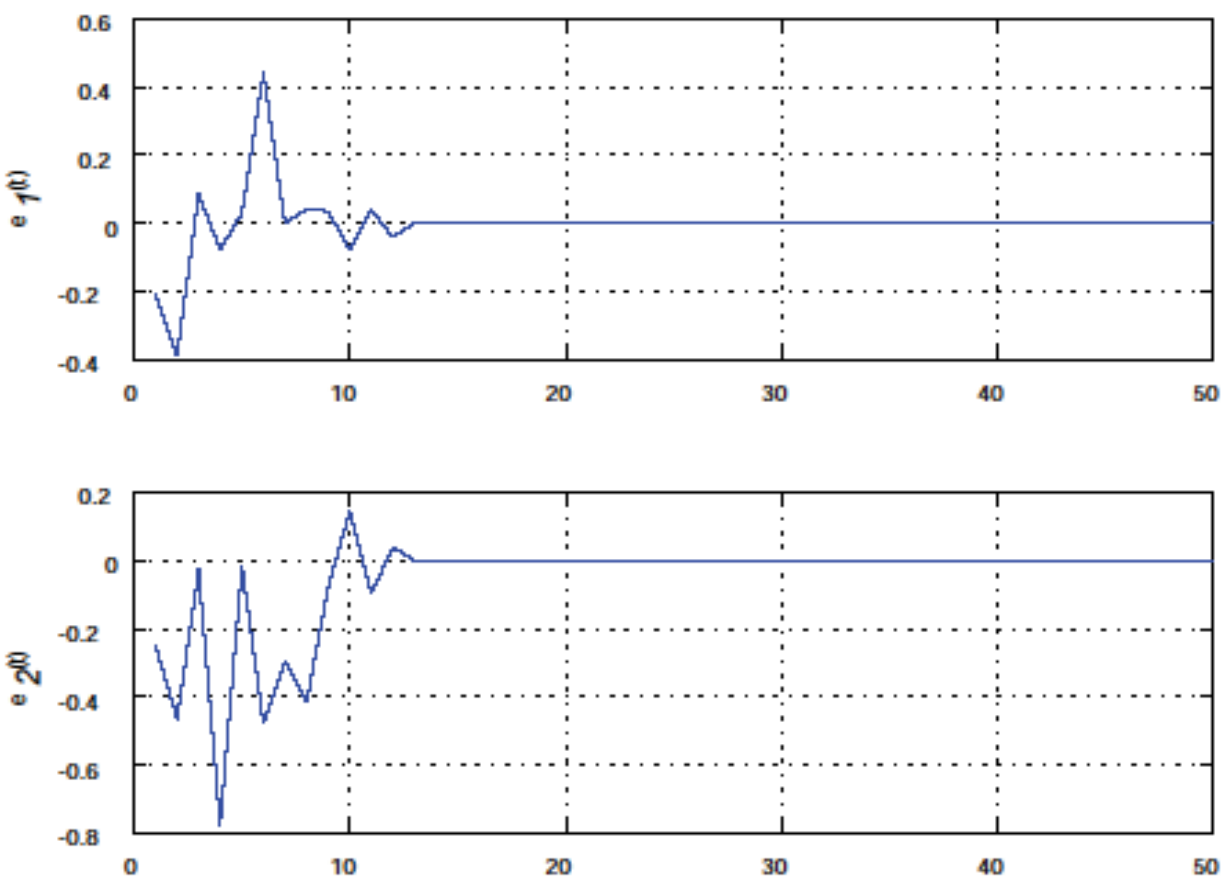

Figure 3. Output tracking error of the proposed MRACS (Case 2; with modifications)
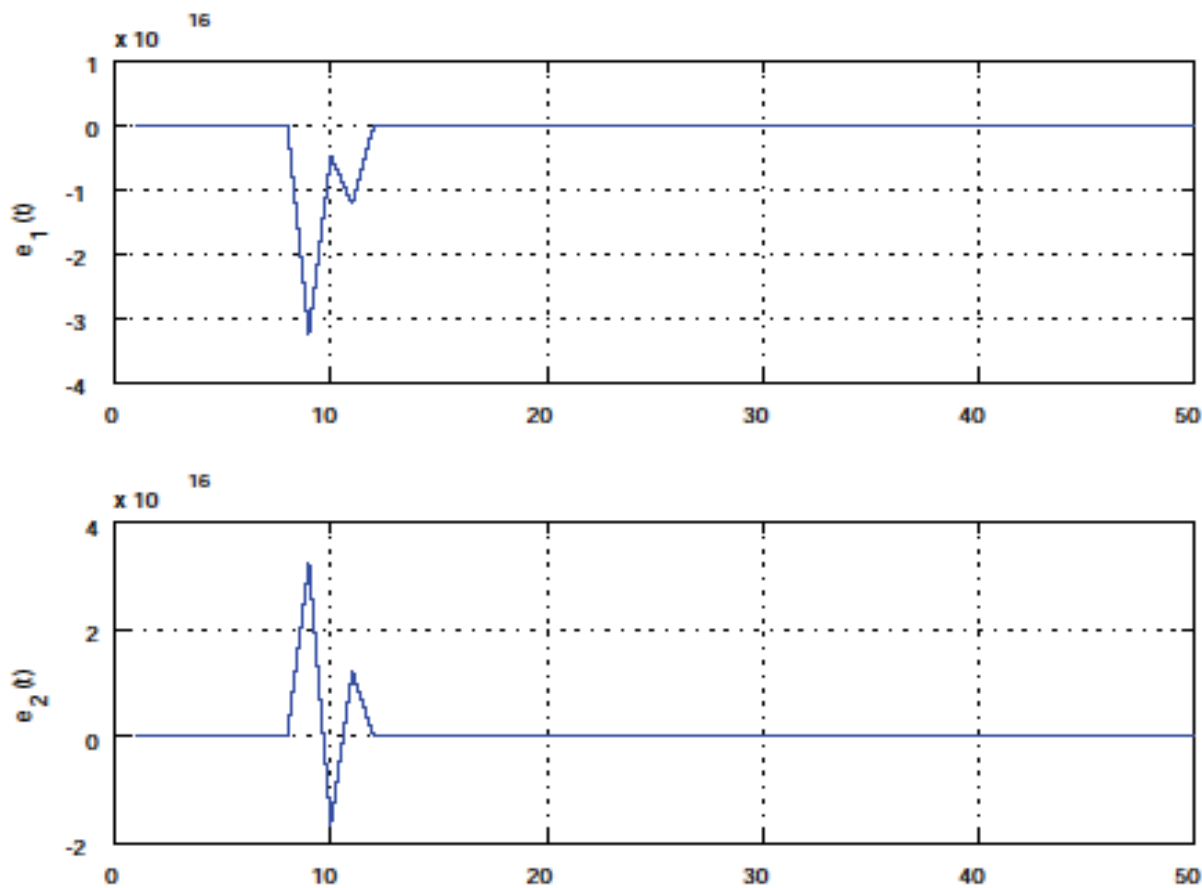

Figure 4. Output tracking error of the proposed MRACS (Case 2; without modifications) 

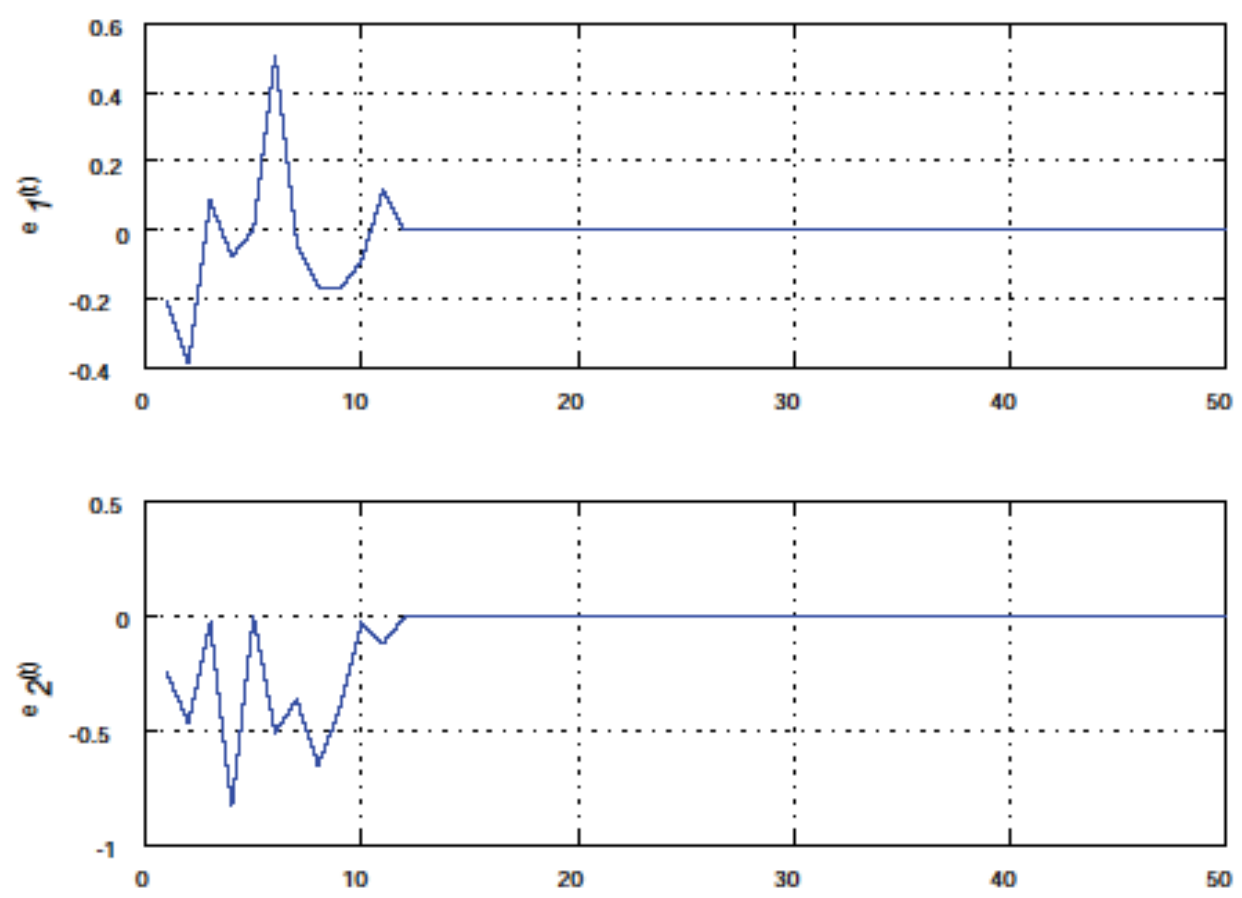

Figure 5. Output tracking error of the proposed MRACS (Case 3; with modifications)
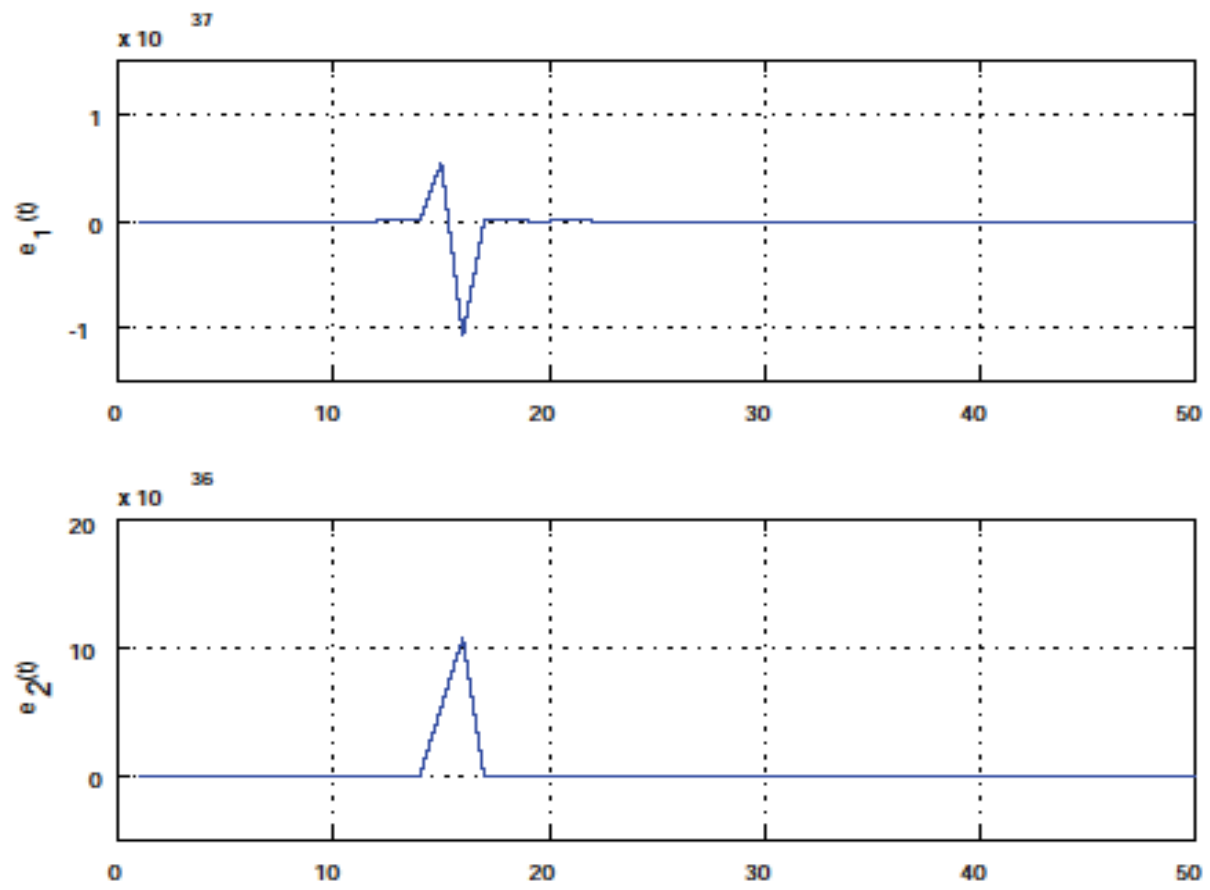

Figure 6. Output tracking error of the proposed MRACS (Case 3; without modifications) 


\section{References}

Wolovich, W. A. \& Falb, P. L. (1976). Invariants and canonical forms under dynamic compensations, SIAM Journal of Control and Optimization, Vol.14, No.6, pp.996-1008.

Goodwin, G. C.; Ramadge, R. J. \& Caines, P. E. (1980). Discrete-time multivariable adaptive control, IEEE Transactions on Automatic Control, Vol.25, No.3, pp.449-456.

Chan, S. \& Goodwin, G. C. (1982). On the role of the interactor matrix in multiinputmultioutput adaptive control, IEEE Transactions on Automatic Control, Vol.27, No.3, pp.713-714.

Elliott, H. \& Wolovich, W. A., (1982). A parameter adaptive control structure for linear multivariable systems, IEEE Transactions on Automatic Control, Vol.27, No.2, pp.340352.

Goodwin, G. C. \& Long, R. S., (1980). Generalization of results on multivariable adaptive control, IEEE Transactions on Automatic Control, Vol.25, No.6, pp.1241-1245.

Elliott, H. \& Wolovich, W. A., (1984). Parametrization issues in multivariable adaptive control, Automatica, Vol.20, No.5, pp.533-545.

Dugard, L.; Goodwin, G. C. \& de Souza, C. E., (1984). Prior knowledge in model reference adaptive control of multiinput multioutput systems, IEEE Transactions on Automatic Control, Vol.29, No.8, pp.761-764.

Kase, W. \& Tamura, K., (1990). Design of G-interactor and its application to direct multivariable adaptive control, International Journal of Control, Vol.51, No.5, pp.1067-1088.

Mutoh, Y. \& Ortega, R., (1993). Interactor structure estimation for adaptive control of discrete-time multivariable nondecouplable systems, Automatica, Vol.29, No.3, pp.635-647.

Wolovich, W. A., (1974). Linear multivariable systems, Springer-Varlag, Berlin.

Kase, W. \& Mutoh, Y., (2009). A simple derivation of interactor matrix and its applications, International Journal of Systems Science (to appear).

Kase, W.; Miyoshi, R. \& Mutoh, Y., (2004). An explicit solution to the discrete-time singular LQ regulation problem for a plant having more inputs than the outputs, Proceedings of IEEE Conference on Decision and Control, pp.2273-2278, ISBN 0-7803-8683-3, Paradise Island, Bahamas, December.

Kase, W., (1999). A solution of polynomial matrix equations using extended division algorithm and another description of all-stabilizing controllers, International Journal of Systems Science, Vol.30, No.1, pp.95-104, ISSN 0020-7721.

Kase, W., (2008). A revision of extended division algorithm for polynomial matrices and its application, Proceedings of 2008 IEEE International Symposium on Industrial Electronics, pp.1103-1107, ISBN 978-1-4244-1666-0, Cambridge, UK, June/July.

Kase, W. \& Mutoh, Y. (2000). Suboptimal exact model matching for multivariable systems with measurement noise, IEEE Transactions on Automatic Control, Vol.45, No.6, pp.1170-1175, ISSN 0018-9286. 


\title{
16
}

\section{Adaptive Control in Wireless Networks}

\author{
Thomas D. Lagkas, Pantelis Angelidis and Loukas Georgiadis \\ Informatics and Telecommunications Engineering Dpt., University of Western Macedonia
}

Greece

\section{Introduction}

In wireless networks, where all mobile stations share the same unreliable medium (the air), channel access control is crucial. The role of the medium access control mechanism becomes even more important nowadays, since the mobile users' network demands have greatly increased. Initially, the wireless networks were employed as simple extensions of the wired ones, thus, the requirements for this new kind of computer networks were not high. The first wireless computer communications were mainly involving traditional data transmissions of low demands. Modern wireless networks are expected to serve "heavy" multimedia transmissions as well. Now, the medium access control has to efficiently adapt to the different network conditions, the different demands and characteristics of the served transmissions.

There is a great number of medium access control methods proposed in the literature. A categorization of these methods is provided in this chapter. Some of the proposed methods are quite generic and can be theoretically employed in a large number of networks, while others are more specialized and target, for example, exclusively on wireless local area networks. The type and the specific features of the used access control mechanism significantly defines the overall network behavior. Modern wireless network access control is expected to efficiently provide QoS.

Obviously, different types of traffic have different transmission requirements. This chapter discusses on the traffic classification and the special characteristics of each class. Supporting real-time traffic of strict QoS requirements (low delay-jitter, high throughput) in a wireless local area environment is quite challenging. There are some relevant protocols proposed, but the most significant one is the Hybrid Control Channel Access (HCCA), which is part of the IEEE 802.11e standard (IEEE 802.11e - Amendment 8, 2005). Here, an operation overview of HCCA is provided.

This chapter presents an alternative proposal in wireless network adaptive control, which is called POAC-QG (Priority Oriented Adaptive Control with QoS Guarantee). POAC-QG is a complete centralized channel access mechanism, it is able to guarantee QoS for all types of multimedia network applications, it enhances the parameterized traffic with priorities, and it supports time division access using slots. Furthermore, it instantly negotiates the quality levels of the Traffic Streams (TSs) according to their priorities, supporting multiple streams to the best quality it can achieve. POAC-QG compared to HCCA, provides higher channel utilization, adapts better to the characteristics of the different traffic types, differentiates the traffic streams more efficiently using priorities, and generally exhibits superior performance. 


\section{Medium Access Control and QoS Support for Wireless LANs}

The protocols that control channel access are responsible for ensuring efficient and fair sharing of the available bandwidth. There are various relative proposals in the literature for different kinds of network conditions (Nicopolitidis et al., 2003; a. Papadimitriou et al., 2003; Chlamtac et al., 2003; Issariyakul et al., 2003). In wireless networks, the role of the MAC protocol is crucial. The available resources are limited, so there is a great need for efficient control of the transmissions. QoS support is also strongly related with the access control mechanism. A QoS supportive MAC protocol is able to distinguish different types of traffic and treat them accordingly. Usually, traffic is prioritized and high priority data is favored by the access control mechanism.

\subsection{Classification of Traffic}

Traffic can be categorized according to the transmission requirements (Chandra et al., 2000). Various ways to classify traffic have been proposed. First of all, we can distinguish between non-real-time (such as background data) and real-time traffic (such as voice and video). Background data traffic is not time-critical. It does not require low delay or jitter, but it demands reliable packet delivery. Usually, it is considered as low priority traffic and an acknowledgement mechanism is used to ensure reliability. On the other hand, real-time traffic mainly concerns digital voice and video transmission and is time-bounded. Low packet delay is required in order to have qualitative audio and video reproduction. Jitter must be also kept at low values, because the packet buffer size is limited and the lifetime of the packets is small. For these reasons, high jitter increases the packet drop ratio. Live voice and video transmissions are even more demanding, because they involve extra delay caused by the real-time digital encoding at the source. However, some packet losses or bit errors can be allowed, because high reliability is not essential.

Traffic is also classified according to the way packets are generated (Akyildiz et al., 1999). When packets of the same size are generated at constant time intervals, then traffic is characterized as Constant Bit Rate (CBR). Numerous real-time voice and video digital encoders, such as G.711 and MPEG-4 respectively, produce CBR traffic. The advantage of this kind of traffic generation is that transmission time intervals can be reserved at the beginning of the communication and remain unchangeable and sufficient for its whole duration. The disadvantage is that usually this type of encoders are not bandwidth optimized, although they are rather fast. Variable Bit Rate (VBR) traffic is produced when the generated packets are not of the same size or the generation time interval is not constant. VBR traffic is common in both background data and real-time transmissions. Background data VBR traffic is usually called nrt-VBR (non-real-time Variable Bit Rate), while rt-VBR (real-time Variable Bit Rate) traffic mainly concerns compressed voice and video transmission. VBR voice-audio and video encoders, such as MPEG Audio Layer 3 and H.261 respectively, are not particularly bandwidth demanding, but the encoding time is rather long. Furthermore, the initially reserved average bandwidth for a rt-VBR communication is usually not capable to provide sufficient QoS, because the transmission requirements change dynamically. Since bandwidth is limited, particularly in wireless networks, the use of efficient VBR digital coding techniques is necessary. For this reason, adaptive control mechanisms that can efficiently support both CBR and VBR traffic seem nowadays quite useful. A summary of this traffic classification is given in Table 1. 


\begin{tabular}{|c|c|c|}
\hline Traffic Type & Examples & Characteristics \\
\hline $\begin{array}{c}\text { CBR } \\
\text { Constant Bit } \\
\text { Rate) }\end{array}$ & $\begin{array}{c}\text { real-time } \\
\text { voice-video }\end{array}$ & $\begin{array}{c}\text { efficient bandwidth } \\
\text { reservation } \\
\text { fast digital encoding } \\
\text { increased produced data }\end{array}$ \\
\hline $\begin{array}{c}\text { nrt-VBR } \\
\text { (non-real-time } \\
\text { Variable Bit } \\
\text { Rate) }\end{array}$ & $\begin{array}{c}\text { background } \\
\text { data } \\
\text { transmission }\end{array}$ & $\begin{array}{c}\text { high reliability required } \\
\text { delay-jitter tolerant }\end{array}$ \\
$\begin{array}{c}\text { rt-VBR } \\
\text { (real-time } \\
\text { Variable Bit } \\
\text { Rate) }\end{array}$ & audio-video & $\begin{array}{c}\text { real-time } \\
\text { changeable bandwidth } \\
\text { requirements } \\
\text { increased encoding delay }\end{array}$ \\
\hline
\end{tabular}

Table 1. Traffic classification

\subsection{Medium Access Control Protocols for WLANs}

QoS support in ad-hoc WLANs is definitely a hard objective. The absence of central control is the reason why QoS cannot be guaranteed. However, the use of packet priorities can partially provide QoS, thus, there are some distributed MAC protocols that favor high priority packets. In decentralized WLANs, the level of QoS support depends on the network characteristics, such as load and number of stations. Specifically, distributed access mechanisms are contention based, thus, high load and increased number of stations cause high collision rate and low channel utilization. Under these conditions, packet delay and jitter are increased. Thus, QoS cannot be really guaranteed in ad-hoc WLANs. The EYNPMA (Elimination Yield - Non Preemptive Multiple Access) protocol (HIPERLAN - ETSI Functional Specification, 1998; b. Papadimitriou et al., 2003), used in HIPERLAN (HIgh PERformance Local Area Network), which is standardized by ETSI (European Telecommunications Standards Institute), and the EDCA (Enhanced Distributed Channel Access) protocol used by IEEE 802.11e provide partial QoS for ad-hoc WLANs.

Infrastructure wireless networks, where central control is employed, are more suitable for supporting QoS. The access control and the schedule mechanism are implemented in the $\mathrm{AP}$, which is responsible for giving transmission permissions to the wireless stations. One of the centralized access methods that provide QoS involves station polling according to the previous or following packet priorities. This method does not include bandwidth reservation. The AP analyzes the feedback and decides which station should be allowed to transmit, taking into account packet priorities. These polling schemes usually ensure low collision rate and high channel utilization, and they can provide QoS but with no guarantees. The POAP (Priority Oriented Adaptive Control) (Lagkas et al., 2008), the QAP (QoS supportive Adaptive Polling) (Lagkas et al., 2006) and the GRAP (Group Randomly Addressed Polling) (Chen \& Lee, 1994) protocols belong to this class of access mechanisms.

The MAC protocols that can actually guarantee QoS in a WLAN are the reservation centralized protocols. The access mechanisms of this class give the ability to the different TSs to reserve bandwidth. According to this model, the stations send transmission requests to the AP asking for transmission intervals, usually using a contention based scheme. The scheduling algorithm implemented in the AP decides the bandwidth distribution in the 
contention free period according to the stations' requests, the priorities, the available resources etc. This type of channel access method guarantees QoS by ensuring that the packet delay of a TS will not exceed an agreed maximum limit, however the values of the actual packet delay and jitter vary and depend on the specific MAC protocol. The usual drawbacks of this model include the waste of bandwidth at the contention based period, because of the high collision probability, and the inability to efficiently support all types of real-time traffic. Specifically, if the assigned transmission periods remain constant for the whole duration of the communication, then VBR traffic cannot be efficiently supported. Representative reservation centralized WLAN MAC protocols are: DQRUMA (DistributedQueuing Request Update Multiple Access) (Karol et al., 1995), MASCARA (Mobile Access Scheme based on Contention and Reservation for ATM) (Bauchot et al., 1996), DSA++ (Dynamic Slot Assignment) (Petras \& Kramling, 1996), DTDMA (Dynamic Time Division Multiple Access) (Raychaudhuri et al., 1997), and PRMA (Packet Reservation Multiple Access) (Kim \& Widjaja, 1996; Dyson \& Haas, 1999; Bianchi et al., 1997). Variants of these protocols have also been proposed in literature. The general concept of the previously mentioned protocols is focusing on the real-time traffic and the use of a simple contention based scheme, like Slotted ALOHA, for the transmission of the requests and the non-realtime data. The hybrid solution proposed by the IEEE 802.11e workgroup is examined in the next section. This classification of the QoS supportive MAC protocols is presented in Table 2.

\begin{tabular}{|c|c|c|c|}
\hline \multicolumn{2}{|c|}{ Protocol Type } & \begin{tabular}{|l|} 
Examples \\
\end{tabular} & Characteristics \\
\hline \multicolumn{2}{|c|}{ Distributed } & EY-NPMA & $\begin{array}{l}\text { no infrastructure } \\
\text { required }\end{array}$ \\
\hline \multirow[b]{2}{*}{ Centralized } & $\begin{array}{c}\text { Random } \\
\text { Access }\end{array}$ & $\begin{array}{c}\text { POAP } \\
\text { QAP } \\
\text { GRAP }\end{array}$ & $\begin{array}{c}\text { high performance } \\
\text { not guaranteed QoS } \\
\text { support } \\
\text { low feedback } \\
\text { requirements } \\
\end{array}$ \\
\hline & $\begin{array}{c}\text { Reserved } \\
\text { Access }\end{array}$ & \begin{tabular}{|c|} 
DQRUMA \\
MASCARA \\
DSA++ \\
DTDMA \\
PRMA \\
HCCA \\
POAC-QG
\end{tabular} & $\begin{array}{c}\text { increased QoS } \\
\text { guarantee } \\
\text { not optimal channel } \\
\text { utilization } \\
\text { high feedback } \\
\text { requirements }\end{array}$ \\
\hline
\end{tabular}

Table 2. A classification of medium access control protocols that support QoS

\section{The IEEE 802.11e Hybrid Control Function}

The WLAN standard that has dominated the market is IEEE 802.11, which provides data rates up to $100 \mathrm{Mbps}$ (802.11n). Currently, the majority of the deployed 802.11 products support data rates up to $54 \mathrm{Mbps}(802.11 \mathrm{a} / \mathrm{g})$. The employed MAC protocol does not 
support QoS. However, some modifications that enhance partial QoS support have been proposed (Ni et al., 2004).

\subsection{The Operation of HCF}

IEEE formed the 802.11e workgroup, because of the increased need for QoS in modern WLANs. The 802.11e channel access mechanism is called HCF and it comprises a contention based scheme (EDCA) and a contention free scheme (HCCA). HCCA is able to guarantee QoS to some degree. It operates in infrastructure mode and its role is to efficiently support real-time voice and video communications. EDCA is designed to support prioritized traffic similar to DiffServ, whereas HCCA supports parameterized traffic similar to IntServ.

The basic concept of HCF is the transmission opportunity (TXOP), that is the time interval in which a station (also called quality enhanced station in 802.11e) is allowed to transmit. In HCCA, the TXOP is decided by the AP according to the QoS request. Specifically, the Hybrid Coordinator ( $\mathrm{HC}$ ) is responsible for the central control and it is co-located with the AP. However, here, we never refer particularly to the HC, but generally to the AP.

The superframe of HCF is defined as the beacon interval. It is composed of alternated modes of Contention Period (CP) and optional Contention-Free Period (CFP), as it can be seen in Figure 1. EDCA operates only in $\mathrm{CP}$ while HCCA can operate both during $\mathrm{CP}$ and CFP. HCCA mode can be started by the AP several times during a $\mathrm{CP}$ and these periods are called Controlled Access Periods (CAPs). The beacon transmitted by the AP at the start of every superframe contains control information, such as the maximum duration of CFP, the maximum duration of TXOP et al. The end of CFP is signaled by the AP using a CFP-End message. When the AP wants to initiate a CAP, it occupies the channel and uses a CF-Poll message to grant a HCCA-TXOP to a station.

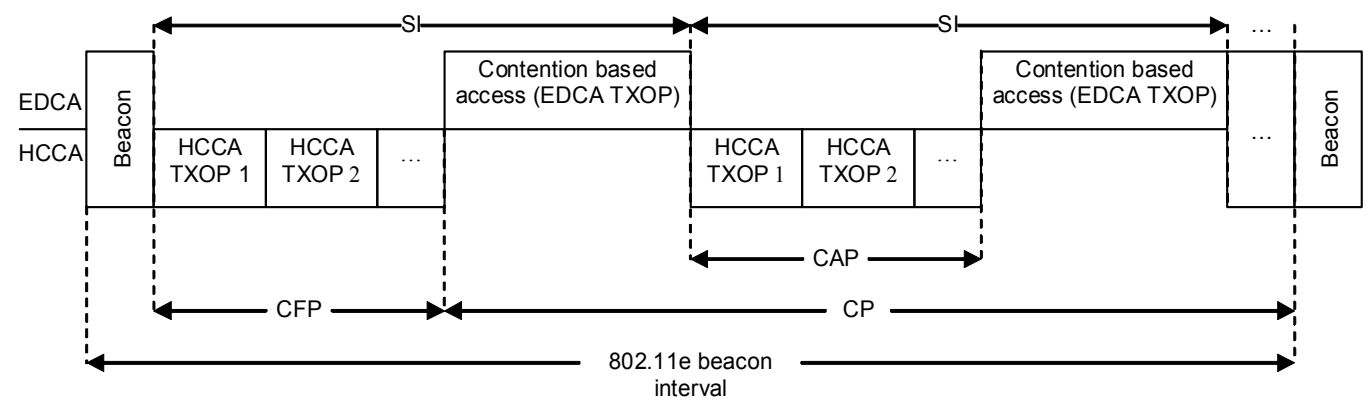

Figure 1. The HCF superframe of the IEEE 802.11e standard

In HCCA, every TS has its own packet buffer. The traffic specification (TSPEC) is responsible for the TS management. It provides the management link between higher layer QoS protocols such as IntServ or DiffServ with the 802.11e channel access functions (HCCA or EDCA, respectively). TSPEC describes characteristics of TSs, such as the mean data rate, the MAC Service Data Unit (MSDU) size and the maximum Required Service Interval (RSI). Each TS first sends a QoS request to the AP containing these characteristics. The scheduling algorithm calculates first the minimum value of all the RSIs, and then chooses the highest submultiple value of the beacon interval duration as the selected Service Interval (SI), which is less than the minimum of all the maximum RSIs. SI is the time interval between any two successive TXOPs allocated to a station. 


\subsection{Scheduling in HCCA}

The simple scheduling algorithm used in HCCA calculates the TXOPs allocated to the different TSs as follows. The TXOP corresponds to the duration required to transmit all packets generated during a SI in a TS buffer. The mean number of packets $\left(N_{i j}\right)$ generated in the TS buffer $(j)$ for a station (i) during a SI is:

$$
N_{i j}=\left\lceil\frac{\bar{r}_{i j} S I}{M_{i j}}\right\rceil
$$

where $\bar{r}_{i j}$ is the application mean data rate and $M_{i j}$ is the nominal MSDU size. The TXOP $\left(T_{i j}\right)$ is finally as follows:

$$
T_{i j}=\max \left(\frac{N_{i j} M_{i j}}{R}+2 \operatorname{SIFS}+T_{A C K}, \frac{M_{\max }}{R}+2 \operatorname{SIFS}+T_{A C K}\right)
$$

where $R$ is the transmission rate supported by the physical layer and $M_{\max }$ is the maximum MSDU size. The time interval corresponds to the overhead during a TXOP. Equation (2) guarantees that the TXOP will be long enough for the transmission of at least one packet with maximum size. The total TXOP assigned to a station is the sum of the TXOPs assigned to the different TSs of this station, that is:

$$
T X O P_{i}=\sum_{j=1}^{F i} T_{i j}
$$

where $F_{i}$ is the number of TSs in station $i$. The admission control algorithm checks for available bandwidth before assigning TXOP to a new TS. The fraction of total time assigned to a station $i$ is: $T X O P_{i} / S I$. If the total number of QoS stations that are assigned TXOPs is $K$, then the scheduler needs to check if the new request of $T X O P_{K+1}$ will keep the fraction of time allocated for TXOPs lower than the maximum fraction of time that can be used by HCCA:

$$
\frac{T X O P_{K+1}}{S I}+\sum_{i=1}^{K} \frac{T X O P i}{S I} \leq \frac{T_{\text {CAPLimit }}}{T_{\text {Beacon }}}
$$

where $T_{\text {CAPLimit }}$ is the maximum duration of HCCA in a beacon interval ( $\left.T_{\text {Beacon }}\right)$.

There are some drawbacks concerning the operation of HCCA. Regarding the polling mechanism, some valuable bandwidth is spent because of the polling packets sent to the stations. The use of acknowledgements is bandwidth costly, too. Since, the target is to attain high throughput rather than reliability, acknowledging the real-time traffic packets seems useless. Also, all the stations have to stay constantly fully awake waiting for data packets or polls, so there is increased power consumption. Concerning the scheduling algorithm, a major drawback is the fact that the allocated TXOPs are fixed. Thus, VBR traffic cannot be supported efficiently, because possible sudden increases in the bit generation rates would cause increased delays and packet drops. Furthermore, the scheduling algorithm does not take into account prioritized TSs. It just uses the quality requirements in order to assign TXOPs. This means that the traffic is not efficiently differentiated according to the demands 
for QoS support. These issues and the solutions given by the proposed POAC-QG protocol are detailed in the next sections.

\section{Priority Oriented Adaptive Control with QoS Guarantee}

The new protocol introduced in this chapter is POAC-QG. The specific access scheme is able to operate in infrastructure wireless local area networks and can be used in a $802.11 \mathrm{e}$ network in place of HCCA. The need that has led to the development of this protocol is the necessity for bandwidth saving, strict QoS with efficient VBR traffic support, and traffic type distinction. POAC-QG is presented analytically in this section.

\subsection{POAC-QG Overview}

POAC-QG adopts a superframe structure, according to which there are consecutive Realtime Traffic (RT) periods and Background Traffic (BT) periods. The POAC-QG protocol operates during the RT periods, which are contention free. During the BT periods a contention based access mechanism can be used. The 802.11e superframe is suitable for adapting POAC-QG into it. The CFPs and CAPs correspond to the RT periods, and the CPs during which EDCA takes place correspond to the BT periods.

The POAC-QG access mechanism is not based on polling, but on a TDMA scheme. The concept is to reduce the bandwidth waste due to the polling model, keep the stations synchronized by dividing the RT period into time slots, and keep them informed of the time interval, source and destination of the coming transmissions. Thus, a potential power saving model could be used, since stations can stay in "sleep" mode during the RT period and "wake" only to transmit or receive data. The AP uses the beacon signal to inform the stations of the assigned slots for real-time traffic transmissions and the SI duration for the current superframe. In the beginning of every SI, except from the first one in the superframe, the AP broadcasts a SI_Start message which carries the same information with the initial beacon signal. If a station fails to receive the beacon signal, it defers, until it successfully receives a SI_Start (or a new beacon signal).

Beacon Interval (super-frame) [default duration: $500 \mathrm{~ms}$ ]

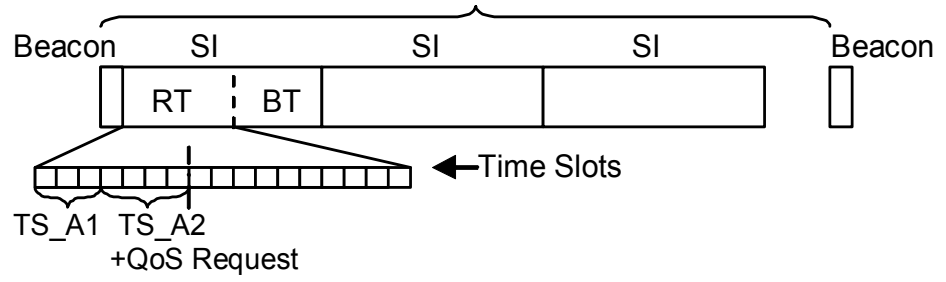

Figure 2. The superframe structure adopted by POAC-QG

When a station becomes aware of the beacon information, it ignores all subsequent SI_Start messages in the current superframe. We assume that the stations send their QoS requests for every TS during the BT periods or the last RT slots assigned to them. An overview of the superframe is shown in Figure 2.

It is known that a multimedia application can be carried out with different quality levels (depending on the codec, the audio-video quality etc). The admission control negotiates instantly multiple quality levels that can be supported by the requesting TS. The corresponding algorithm tries to serve the higher priority TSs with maximum quality level, 
but it can lower the provided quality levels in order to allocate slots for lower priority TSs, as well. It is of course assumed that the higher the quality level is, the higher are the resource requirements (bandwidth, delay). The main purpose of the protocol is to serve as many TSs as possible, favor the higher priority TSs, and provide the higher possible quality levels. When a station sends a QoS request to ask for slots for its TSs, it includes the traffic specifications of the different quality levels (traffic rate, maximum inter-transmission interval, maximum and nominal packet size).

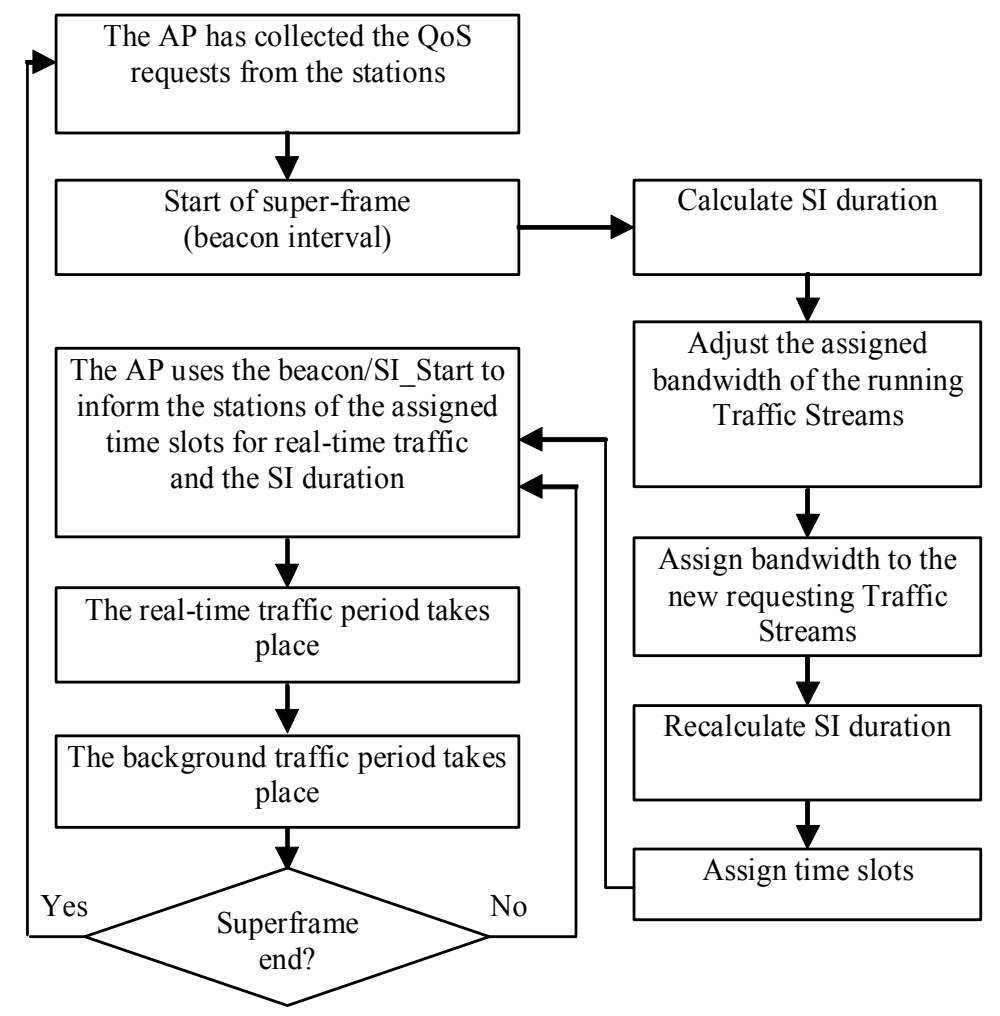

Figure 3. Operation overview of the POAC-QG protocol

Every running TS can ask for a different number of RT slots, according to its current traffic rate and the total size of its buffered packets. So, the QoS request frame that can be sent at the end of the assigned slots or during the BT periods, includes traffic specifications for both running and new TSs. This way VBR traffic can be efficiently supported. The algorithm calculates first the minimum value of all the maximum inter-transmission intervals required by the running and the new TSs, and then chooses the highest submultiple value of the beacon interval duration as the selected SI, which is less than the minimum of all the maximum inter-transmission intervals. Then, the AP allocates slots for the running TSs according to their latest requests. The reason why the running TSs are examined first is the effort of the protocol to keep the quality of the existing communications steady. After all, a new requested voice call can wait for admission, but it is unacceptable for a running call to be suddenly terminated or experience increased delays. The rest of the bandwidth is then assigned to the new TSs, according to the admission control mechanism. The new SI duration is calculated, based on the requests of the accepted TSs and finally the time slots 
are assigned to the running and the new accepted TSs. In Figure 3, an overview of the processes that take place according to POAC-QG is presented.

\section{Accepting New TS Method}

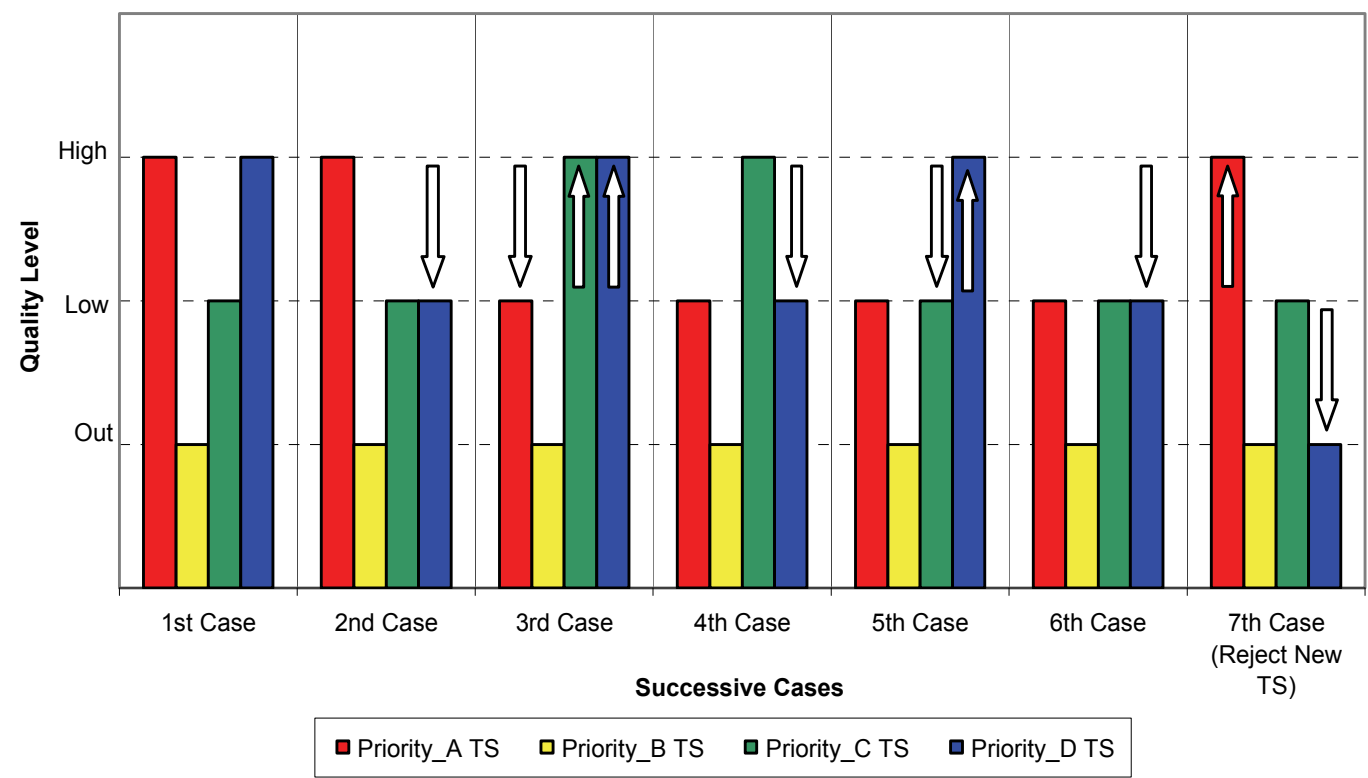

Figure 4. Example of the quality levels negotiation when examining the admission of new traffic streams

\subsection{Traffic Streams Admission Control}

The new TSs that request bandwidth allocation are sorted according to their priorities (highest priority first). The corresponding algorithm starts with the highest priority TS and checks if there is enough available bandwidth in order to serve the specific TS with maximum quality level. Otherwise, the QoS requirements of the lower quality level are checked. If neither the minimum quality level can be supported, then the TS is rejected and the next priority TS is examined. When there is no bandwidth left to serve a TS with minimum quality, then the quality levels of the previously examined higher priority TSs are lowered in order to save some bandwidth for the new TS. When the quality levels of the high priority TSs are lowered, then we also check if it becomes possible to increase the quality of the low priority TSs. This way, the best combination of supported quality levels is provided. An example of this process is described in Figure 4, where we assume two available Quality Levels (High QL, Low QL) and four new TSs with different priorities (Piority_A is the highest, while Priority_D is the lowest). The first three TSs are already examined. Let us assume that, so far, Priority_A TS has been accepted with High QL, Priority_B TS has been rejected, Priority_C TS has been accepted with Low QL, and Priority_D TS is now examined for admission. This means that we are looking for the best quality levels combination of these four TSs, which can be served using the current available bandwidth. In this example there are seven possible cases. Each time, the algorithm checks if there is enough available bandwidth in order to serve the TSs providing the corresponding quality levels combination. If there is not, then we proceed to the next best quality levels 
combination (case). The final case is the rejection of the examined TS (quality level: OUT). A simple form of the code is presented in Figure 5.

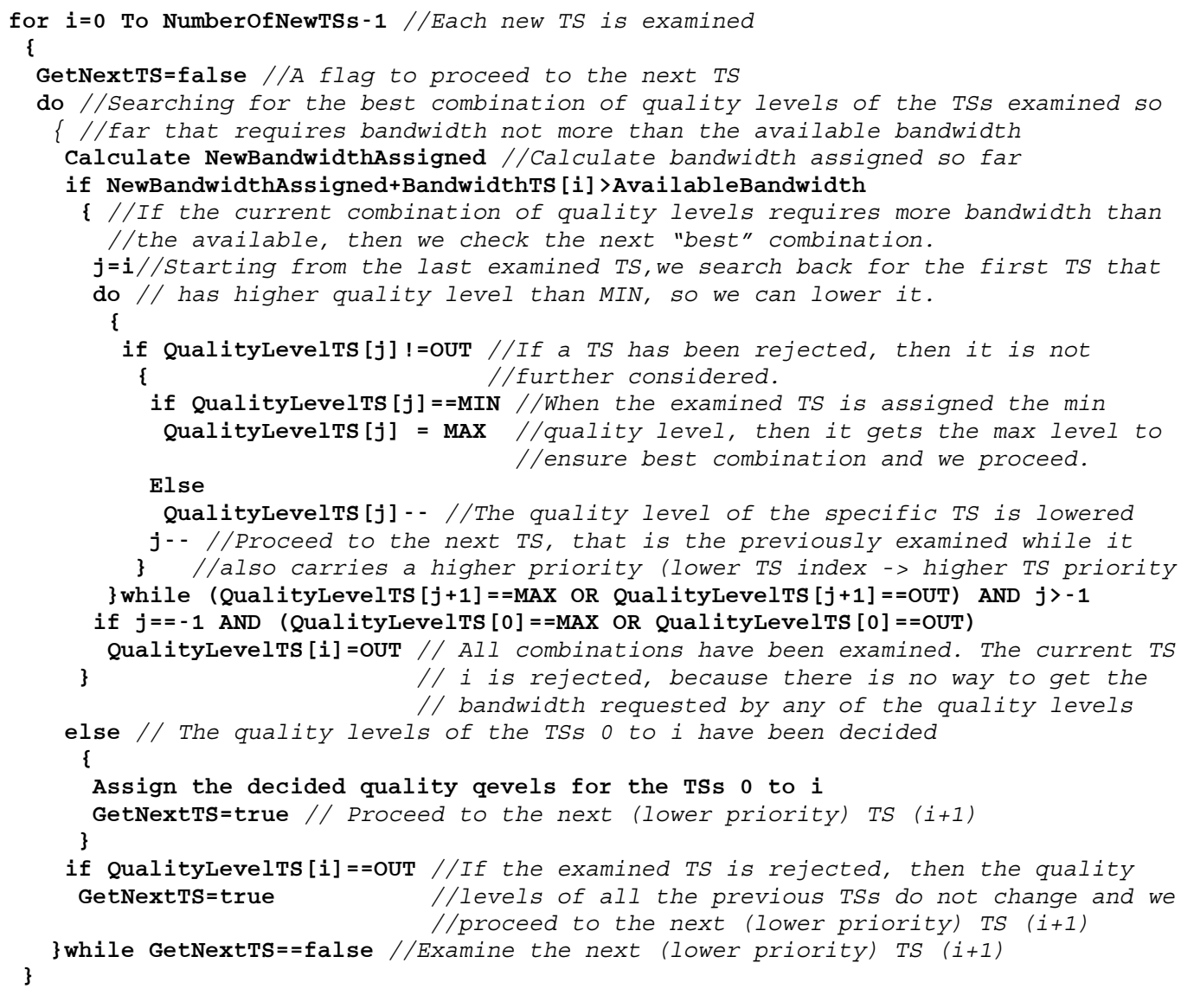

Figure 5. The code form of the TS admission control algorithm

\subsection{Dynamic Control Adapted to Resource Requirements}

POAC-QG implements a dynamic control mechanism which efficiently supports VBR realtime traffic by adapting to the changing requirements of the running TSs. When the AP assigns RT slots to a station, it provides some extra slots allocated for its QoS request frame transmission. The station uses this frame to send the TSPECs both of the new requesting and the running TSs. Before sending a QoS request, the station calculates the current traffic rate of all the running TSs by counting the generated bits for a short time interval (default value is $2 \mathrm{sec}$ ). It also includes in the QoS request the size of the corresponding packet buffer. At the start of every superframe, the AP assigns slots to the running TSs according to their new QoS requests. The rest of the RT bandwidth is then assigned to the new TSs as we have already discussed. The quality level initially provided to a TS remains static, because our aim is to have steady and reliable transmissions. 
The algorithm that assigns time slots to the running TSs tries to adapt to the variable traffic rate without sudden alterations of the allocated bandwidth. When there is not enough RT bandwidth, it assigns a proportion of the requested bandwidth to each TS according to its priority. It is considered that all the generated and buffered packets of a TS can be transmitted during a SI, if the allocated bandwidth corresponds to the theoretical traffic rate:

$$
\text { TheoreticalTR }=\text { CurrentTR }+ \text { BufferedBits } / S I
$$

where CurrentTR is the current traffic rate defined in the QoS request. Since we try to avert sudden and continuous alterations of the allocated bandwidth, a proportion of the requested bandwidth accession or reduction is considered to be the target. Specifically, the considered target traffic rate is:

$$
\text { TargetTR }=\text { PreviousTR }+ \text { BW_DifPercent } \times(\text { TheoreticalTR }- \text { PreviousTR })
$$

where PreviousTR is the traffic rate corresponding to the bandwidth assigned during the previous superframe, and BW_DifPercent (default value is 0.8 ) is the percentage of the requested bandwidth accession or reduction which is considered to be the target. We also use a down limit for the target traffic rate related to the initial traffic rate requested, in order to avoid packet drops in cases of sharp increase of the generated packets after a long silent interval.

Obviously, when a TS requests to give back some of its assigned bandwidth because it doesn't need it anymore, this is done with no further consideration. An issue arises when there is not enough bandwidth to cover all the extra requests of the running TSs. For this reason, an algorithm that distributes the available bandwidth taking into account the traffic priorities has been developed. It initially calculates the percentage of the available bandwidth that each requesting TS deserves (eligible bandwidth). The available bandwidth corresponds to the slots left in the maximum RT period, after assigning to all the running TSs the slots that already occupied in the previous beacon interval and freeing the returned slots. The eligible bandwidth percentage depends on the traffic priority and the amount of extra bandwidth requested by the TS. Specifically, we use the weights $W_{-} P R$ (default value is 5) and $W_{-} B W$ (default value is 1) to control the contribution of the traffic priority and the extra bandwidth requested, respectively, to the eligible extra bandwidth. It is obviously assumed that the traffic priority is clearly the most significant factor. The equation that gives the non-normalized eligible bandwidth percentage for the TS $i$ is:

$$
\operatorname{Per}[i]=W_{-} P R \times P e r P R[i]+W_{-} B W \times P e r B W[i]
$$

where $\operatorname{PerPR}$ is the normalized traffic priority:

$$
\operatorname{PerPR}[i]=\frac{\text { PriorityWeight }[i]}{\sum_{j=0}^{\text {NumberOfRequestingTSs-1 }} \text { PriorityWeight }[j]}
$$

and PerBW is the normalized extra bandwidth requested: 


$$
\operatorname{Per} B W[i]=\frac{\text { ExtraB } W \_ \text {Requested }[i]}{\sum_{j=0}^{\text {NumberOfRequestingTSs }-1} \text { ExtraBW_Requested }[j]}
$$

We use the term "priority weight" instead of just "priority", because the weight of a traffic priority might be considered to be different than the index of the specific priority. We assume that it holds:

$$
\text { PriorityWeight }=\text { Priority }+1
$$

(e.g. priority: $0 \rightarrow$ weight: 1). Since the AP is the "heart" of the WLAN and it often interconnects the WLAN with the backbone wired network, any traffic coming from the AP should be served with definitely higher priority. In order to favor the AP TSs, we use the $W \_A P$ (default value is 5) factor to calculate the non-normalized eligible bandwidth percentage. So, for every TS $i$ transmitted by the AP it stands:

$$
\operatorname{Per}[i]=W_{-} A P \times\left(W_{-} P R \times P e r P R[i]+W_{-} B W \times P e r B W[i]\right)
$$

We finally normalize:

\begin{tabular}{|c|c|c|c|c|c|c|}
\hline Step & TS & Priority & $\begin{array}{l}\text { Requested } \\
\text { Bandwidth }\end{array}$ & \begin{tabular}{|l} 
Available \\
Bandwidth
\end{tabular} & $\begin{array}{c}\text { Eligible } \\
\text { Bandwidth }\end{array}$ & $\begin{array}{l}\text { Assigned } \\
\text { Bandwidth }\end{array}$ \\
\hline \multirow{3}{*}{1} & $\mathrm{~A}$ & 6 & $5 \mathrm{Mbps}$ & \multirow{3}{*}{$10 \mathrm{Mbps}$} & 5.6 Mbps & $5 \mathrm{Mbps}$ \\
\hline & B & 3 & $3 \mathrm{Mbps}$ & & 2.9 Mbps & - \\
\hline & C & 1 & $4 \mathrm{Mbps}$ & & $1.5 \mathrm{Mbps}$ & - \\
\hline \multirow{2}{*}{2} & B & 3 & $3 \mathrm{Mbps}$ & \multirow{2}{*}{$5 \mathrm{Mbps}$} & 3.3 Mbps & $3 \mathrm{Mbps}$ \\
\hline & C & 1 & $4 \mathrm{Mbps}$ & & 1.7 Mbps & - \\
\hline 3 & C & 1 & $4 \mathrm{Mbps}$ & $2 \mathrm{Mbps}$ & $2 \mathrm{Mbps}$ & $2 \mathrm{Mbps}$ \\
\hline
\end{tabular}

$$
n \operatorname{Per}[i]=\frac{\operatorname{Per}[i]}{\sum_{j=0}^{\text {NumberOfRequesting } T S-1} \operatorname{Per}[j]}
$$

Table 3. Example of dynamic control adapted to requirements: Assigning extra requested bandwidth to three running traffic streams

At each step, if the eligible bandwidth of a TS is higher than its requested bandwidth, then the latter is immediately granted to this TS. Finally, a proportion of the requested bandwidth is assigned to the TSs that cannot be fully served. The algorithm that calculates the extra bandwidth that would be assigned to every requesting TS is presented in pseudocode form in Figure 6. An example is given in Table 3. This method of continuous and dynamic bandwidth assignment completes the support provided by POAC-QG to VBR traffic. 


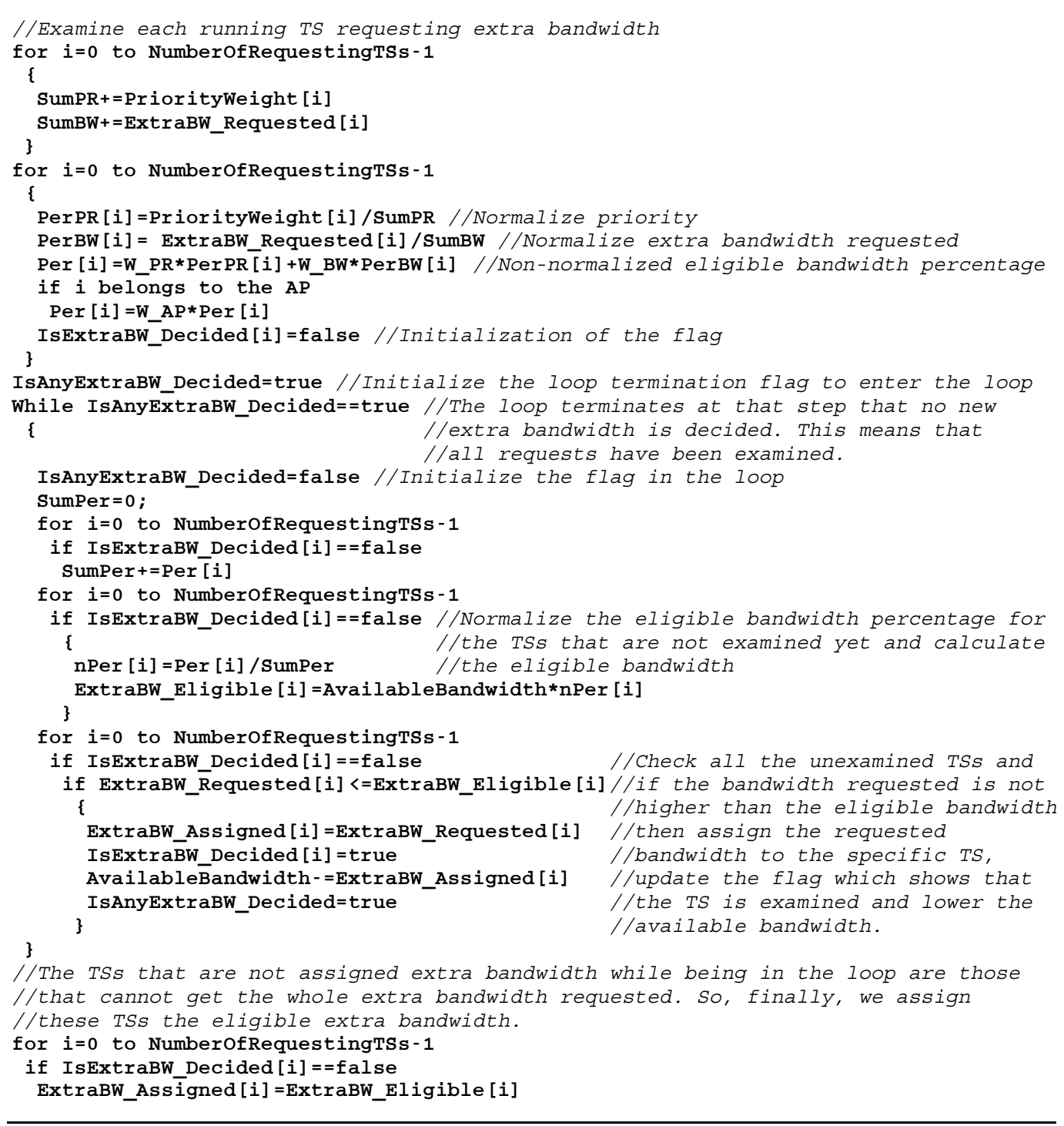

Figure 6. The code form of the dynamic resource allocation algorithm

\section{Evaluating Performance Using Simulation}

It must be clarified at this point that it is not feasible to model channel access in POAC-QG based on the concept of the classical Bianchi two-state Markov chain (Bianchi, 2000). The access scheme of POAC-QG is deterministic, since the AP is informed of the stations' transmission needs by the QoS request frames. POAC-QG, does not actually involve any idle time during the RT slots. However, we do use a three-state Markov process to simulate the link status between each pair of stations, as it is explained later. Regarding the proposed mechanism for TS admission control and dynamic adjustment of the allocated resources, the 
algorithmic complexity and heuristic nature makes further theoretical analysis impossible and actually unnecessary. In related work, it can be seen that this is a common concept. In (Grilo et al., 2003), the proposed SETT-EDD scheduling algorithm for HCCA is evaluated via simulation and no theoretical analysis is performed. The authors state that the typical two-state Markov chain used to model the channel does not accurately represent a WLAN with link adaptation. In (Larcheri \& Cigno, 2006), there is no theoretical analysis of the openloop and closed-loop scheduling proposals for HCCA. It is stated that the authors are not particularly concerned in finding a theoretical optimal scheduler, since it could turn out to be computationally complex or lose its optimality properties due to implementation impairments. In (Ni et al., 2003), no Markov modeling is used for the analysis of the proposed FHCF scheduling scheme for HCCA. Similarly to our approach, the authors propose a formula for resource allocation based on the queue length. Lastly, in (Chou et al., 2005), a TS admission control is proposed for HCCA, employing a sequence of computations and checks which involve the traffic specifications and the available resources. However, no asymptotic analysis is performed to validate the efficiency of the mechanism, instead, simulation comparison is used.

\subsection{Simulation Features}

In order to evaluate the examined protocols, a specialized simulator was developed, which models the condition of any wireless link using a finite-state machine with three states. These are the following (Gilbert, 1960; Zorzi et al., 1995):

- State G denotes that the wireless link is in a relatively "clean" condition and is characterized by a small BER, which is given by the parameter G_BER.

- State B denotes that the wireless link is in a condition characterized by increased BER, which is given by the parameter $B \_B E R$.

- State $\mathrm{H}$ denotes that the pair of communication stations is out of range (hidden stations).

We assume that the background noise is the same for all stations, and thus, the principle of reciprocity stands for the condition of any wireless link. Therefore, for any two stations $A$ and $B$, the BER of the link from $A$ to $B$ and the BER of the link from $B$ to $A$ are the same. The time spent by a link in states $G, B$ and $H$ is exponentially distributed, but with different average values, given by the parameters $T G, T B, T H$, respectively. The status of a link probabilistically changes between the three states. When a link is in state $G$ and its status is about to change, the link transits either to state $\mathrm{H}$, with probability given by the parameter $P_{h}$, or to state $\mathrm{B}$, with transition probability $1-P_{h}$. When a link is in state B and its status is about to change, the link transits either to state $\mathrm{H}$, with probability given by the parameter $P_{h}$, or to state $\mathrm{G}$, with transition probability $1-P_{h}$. Finally, when a link spent its time in state $\mathrm{H}$, it transits either to state $\mathrm{G}$ or $\mathrm{B}$, with the same probability (0.5). It can be easily seen that by setting the parameter $P_{h}$ to zero, a fully connected network topology can be assumed, whereas for values of $P_{h}$ greater than zero, the effect of the well-known "hidden station" problem on protocol performance can be studied.

In a "clean" network, it stands for the inter-station links: $T G=3 \mathrm{sec}, T B=1 \mathrm{sec}, T H=0.5 \mathrm{sec}$, $G \_B E R=0, B \_B E R=0, P_{h}=0$. Similarly, for the AP-station links it stands: TG_AP=6 sec, $T B \_A P=0.5 \mathrm{sec}, T H \_A P=0.25 \mathrm{sec}, G \_B E R \_A P=0, B \_B E R \_A P=0, P_{h} A P=0$. The links among the $\mathrm{AP}$ and the stations are considered to be more reliable than the inter-station links, because the range of the AP is usually greater than the stations' range, its emitted signal is 
usually stronger, and its default position is the center of the cell. In a rather not "clean" wireless environment, it stands for the inter-station links: $T G=3 \mathrm{sec}, T B=1 \mathrm{sec}, T H=0.5 \mathrm{sec}$, $G \_B E R=0, B \_B E R=0.00001, P_{h}=0.05$. For the AP-station links it stands: TG_AP=6 sec, $T B \_A P=0.5 \mathrm{sec}, T H \_A P=0.25 \mathrm{sec}, G \_B E R \_A P=0, B \_B E R \_A P=0.000001, P P_{h} A P=0.01$. The BERs are assumed to be resulted after the application of the standard's predefined coding techniques.

The default values of the network parameters used in our simulation scenarios are presented here. The medium bit rate is $36 \mathrm{Mbps}$, the signal propagation delay is $0.0005 \mathrm{~ms}$ corresponding to distances among the stations of $150 \mathrm{~m}$, the maximum percentage of the superframe reserved for RT transmissions is 0.95 , and the maximum allowed packet size is $10 \mathrm{~KB}$. According to the specifications of 802.11e, we consider the following total packet sizes: "POLL" $=34$ bytes, "BEACON" $=124$ bytes, and "QoS_Request" $=44$ bytes. The total overhead of every traffic packet is 106 bytes, including physical, MAC, RTP, UDP, IP, and SNAP headers.

Regarding the simulation engine, the random number generator used by our simulator is a classic multiplicative congruential random number generator with period $2^{32}$ provided by ANSI C. The simulation results presented in this section are produced by a statistical analysis based on the "sequential simulation" method [35]. We perform simulations in a sequential way, until the relative statistical error of the estimated mean value falls below an acceptable threshold. When the relative statistical error is low, the confidence interval is narrow, since the relative statistical error is defined as the ratio of the half-width of the given confidence interval at the point estimate. For this statistical analysis we used $95 \%$ confidence intervals. The relative statistical error threshold varies depending on the meaning of the metric and the magnitude of its value. However, this threshold was usually assumed to be lower than $2 \%$ and never exceeded $5 \%$.

\subsection{First Simulation Scenario}

In order to compare the performance and the general behavior of the HCCA and the POAC-QG protocols, two simulation scenarios were used. We consider only real-time traffic streams, because the background traffic access mechanism (EDCA) is the same for the two cases. In the first scenario, we have live voice and video communications (bidirectional transmissions) between the adjacent wireless stations (station 1 communicates with station 2, station 3 communicates with station 4 and so on), and a video on demand traffic stream transmitted by the AP to each station. In Figure 7, we have a representation of the transmissions taking place in the first simulation scenario. Our aim is to compare the QoS provided by the two protocols, when there is just one quality level, that is there is no QoS negotiation. So, in this case, the proposed QoS negotiation mechanism of POAC-QG does not affect the simulation results. The characteristics of the network traffic can be found in Table 4 . The simulation duration is 30 $\mathrm{sec}$, every communication lasts for $20 \mathrm{sec}$, a new set of transmissions (voice, live video, video on demand) are generated every second, and the simulated WLAN consists of 10 wireless stations (that is 30 traffic streams). Also, we consider "clean" links, so we used the respective network parameters' values mentioned earlier. It should be noticed that in both scenarios, we do not drop the packets that exceed their delay bound, so as to get results from all transmissions. 


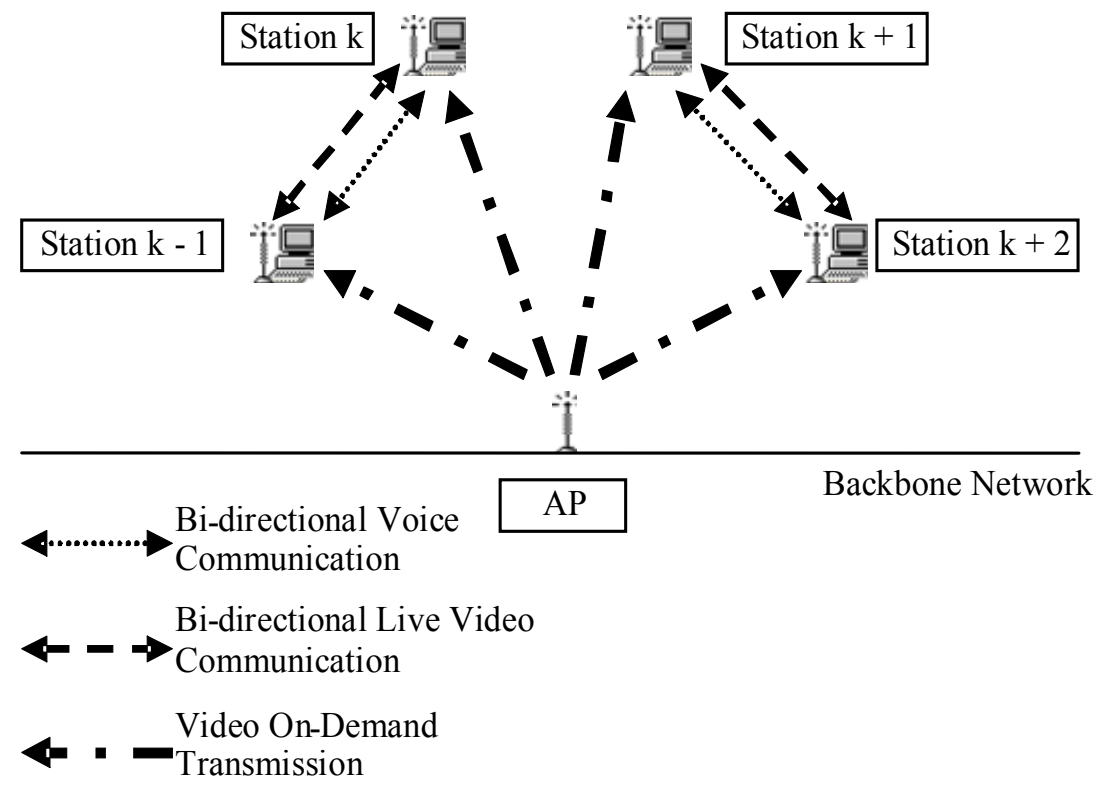

Figure 7. Traffic transmissions according to the first simulation scenario

\begin{tabular}{|c|c|c|c|c|c|}
\hline Application & Coding & $\begin{array}{c}\text { Packet Data Size } \\
\text { (bytes) }\end{array}$ & $\begin{array}{c}\text { Packet } \\
\text { Interarrival } \\
\text { Time (ms) }\end{array}$ & Data Bit Rate & $\begin{array}{c}\text { Packet } \\
\text { Delay } \\
\text { Bound } \\
\text { (ms) }\end{array}$ \\
\hline $\begin{array}{c}\text { Voice } \\
\text { (Priority: 6) }\end{array}$ & G.711 (PCM) & 160 & 20 & 64 Kbps (CBR) & 50 \\
\hline $\begin{array}{c}\text { Live Video } \\
\text { (Priority: 5) }\end{array}$ & H.261 [QCIF] & $\begin{array}{c}\text { Exponential } \\
{[20-1024] \text { Mean: 660 }}\end{array}$ & $\begin{array}{c}\text { Exponential } \\
\text { Mean: 26 }\end{array}$ & $\begin{array}{c}\sim 200 \mathrm{Kbps} \\
\text { (VBR) }\end{array}$ & 100 \\
\hline $\begin{array}{c}\text { Video } \\
\text { on Demand } \\
\text { (Priority: 4) }\end{array}$ & $\begin{array}{c}\text { MPEG-4 } \\
{[4 \mathrm{CIF}]}\end{array}$ & 800 & 2 & $\begin{array}{c}3.2 \mathrm{Mbps} \\
\text { (CBR) }\end{array}$ & 200 \\
\hline
\end{tabular}

Table 4. Traffic characteristics of the communications in the first simulation scenario

In the first simulation scenario, we get measurements of the packet jitter and the TS buffer size. These two metrics are representative of the capability of the MAC protocol to efficiently provide QoS. In Figure 8, we have plotted the results regarding packet jitter. It is obvious that in all cases POAC-QG exhibits much lower jitter than HCCA. The jitter of the voice packets is always kept below $50 \mathrm{~ms}$. The graph that concerns live video, shows that POAC-QG can efficiently support VBR traffic by providing significantly low jitter values. Furthermore, it is capable of successfully serving high bit-rate CBR traffic streams, like video on demand. This superior performance of POAC-QG is partially owed in its ability to adapt to the special requirements of every TS and continuously provide the bandwidth actually needed. 
a)
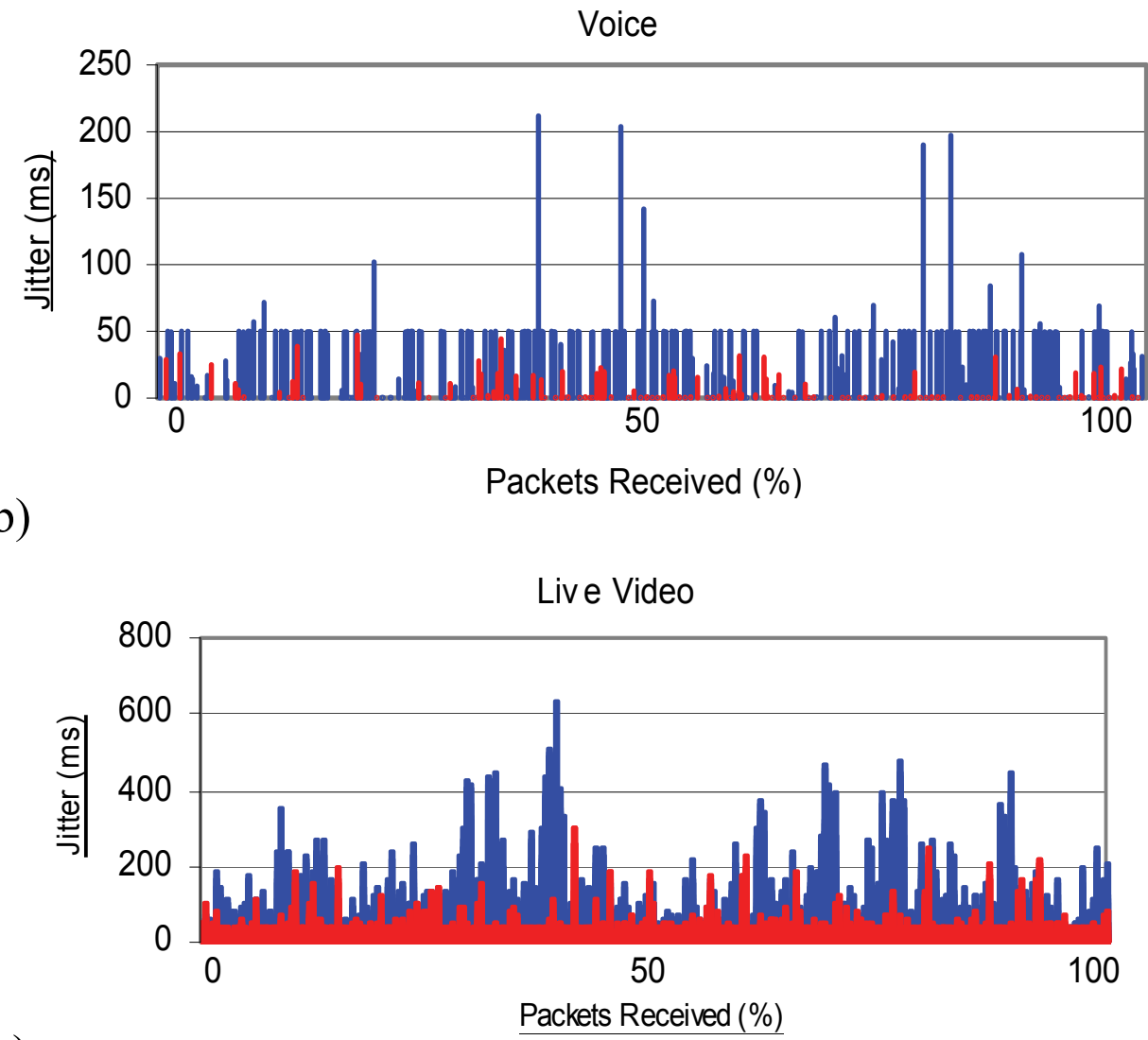

c)

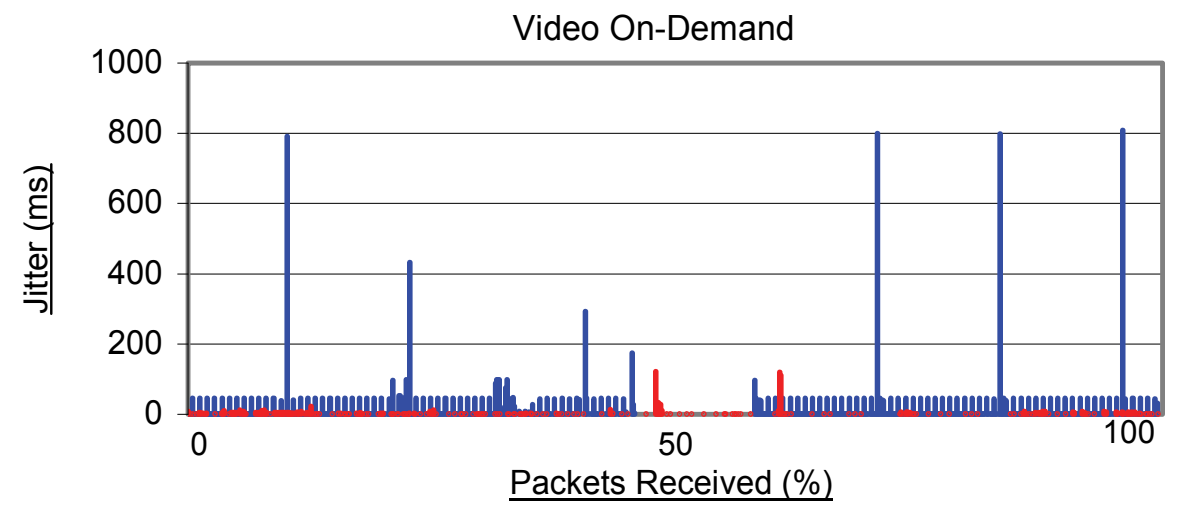

DCCA $\quad$ POAC-QG

Figure 8. Packet jitter measurements concerning a) voice, b) live video, and c) video on demand traffic 
The buffer size of the source station was also measured when a packet was transmitted. It is important for the source to be able to transmit on time the RT packets that arrive at the TS buffer. The ideal case would be the constant counterbalance of the transmission rate and the packet generation rate. However, in a real situation, it is quite difficult to adapt the transmission rate to the packet generation rate. This is particularly true when dealing with VBR traffic, where the packet generation rate changes continuously. In such cases, when a large number of packets suddenly arrive at the buffer, the station might be unable to transmit all packets on time, so there could be packet drops due to lack of buffer space or excess of packet lifetime. The results (which are relative to the jitter results) showed that in all cases POAC-QG manages to "unload" the buffers more efficiently than HCCA. This happens because of the proposed adaptive bandwidth assignment mechanism which continuously provides transmission rates according to the current packet generation rates. Also, the optimized access mechanism, which provides resources saving, significantly contributes to the superior performance of POAC-QG.

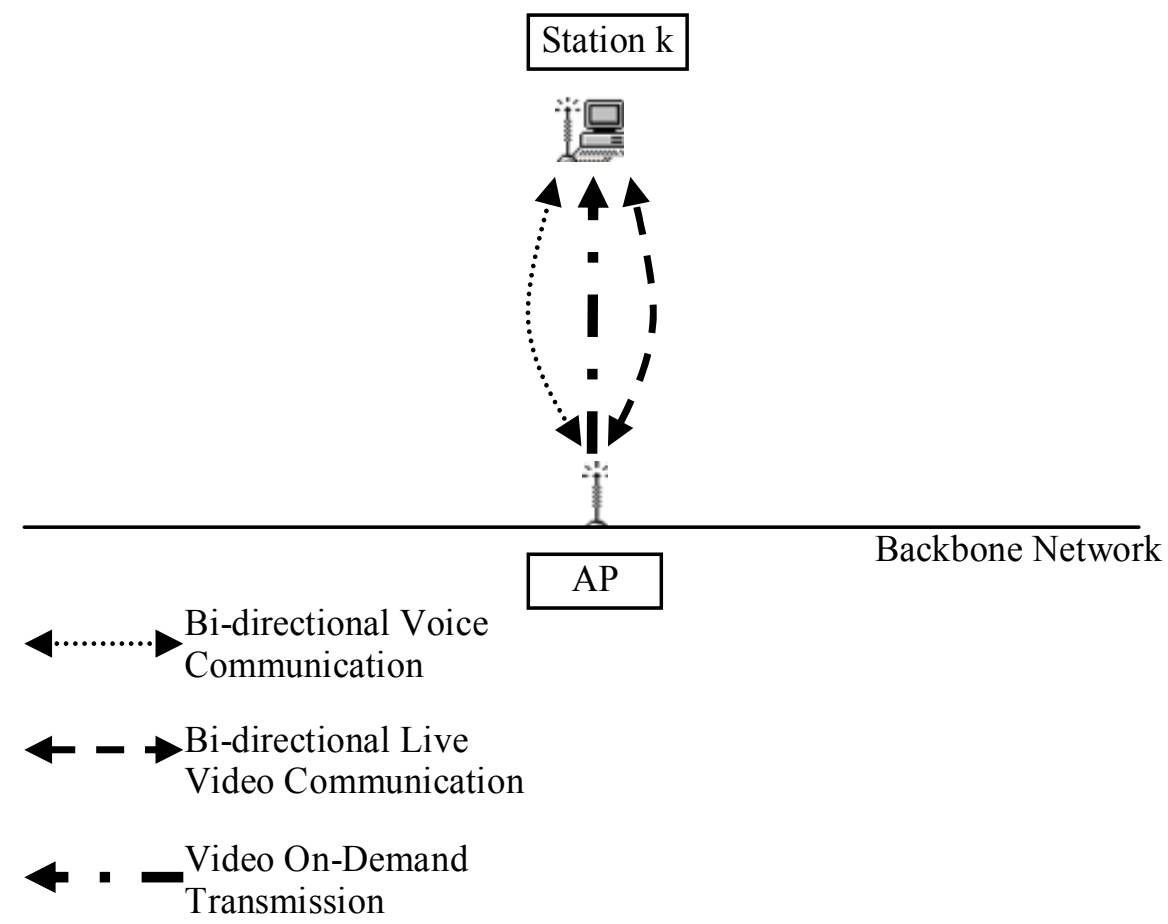

Figure 9. Traffic transmissions according to the second simulation scenario

\subsection{Second Simulation Scenario}

The packet delay and the QoS negotiation efficiency of POAC-QG in a rather not "clean" environment are studied in the second simulation scenario. For these reasons, we used two quality levels (MIN, MAX) and we set the network parameters to the earlier mentioned values that correspond to links of decreased reliability. The employed traffic model involves only AP-station communications. We have live voice and video communications (bidirectional transmissions) between the AP and each station, while the 
AP transmits a video on demand TS to each station. In Figure 9, we have a representation of the transmissions taking place in the second simulation scenario. The traffic characteristics can be found in Table 5. We notice that voice and live video traffic support two quality levels, while video on demand traffic actually supports a single quality level. This is not a problem for the operation of POAC-QG, since it does not require that all TSs support all the provided quality levels. The simulation duration is $60 \mathrm{sec}$, every communication lasts for $30 \mathrm{sec}$, half of the transmission sets (voice, live video, video on demand) start at the beginning of the simulation and the other half start $30 \mathrm{sec}$ later, and we simulated 15 WLAN topologies consisting of 2 to 30 stations (that is 10 to 150 TSs).

In Figure 10, we have plotted the average packet delay versus the number of the total offered TSs. In all cases (voice, live video, video on demand traffic), POAC-QG provides lower packet delays than HCCA, while the latter some times fails to provide delays lower than the maximum tolerable value. It should be noticed, that the total number of offered streams corresponds to the streams scheduled to take place during each simulation. However, some of them may not get permission to start at all due to limited available bandwidth. Also, the served TSs are assigned different quality levels with different bandwidth requirements, and not all of the accepted streams are served for the same time. For these reasons, we need a new metric in order to get a clear and fair view of the comparison of POAC-QG and HCCA.

\begin{tabular}{|c|c|c|c|c|c|c|c|}
\hline Application & QL & Coding & $\begin{array}{c}\text { Packet } \\
\text { Data } \\
\text { Size } \\
\text { (bytes) } \\
\end{array}$ & $\begin{array}{c}\text { Packet } \\
\text { Inter- } \\
\text { arrival } \\
\text { Time (ms) } \\
\end{array}$ & $\begin{array}{c}\text { On/Off } \\
\text { Periods } \\
\text { (sec) }\end{array}$ & $\begin{array}{l}\text { Data Bit } \\
\text { Rate }\end{array}$ & $\begin{array}{c}\text { Packet } \\
\text { Delay } \\
\text { Bound } \\
(\mathrm{ms})\end{array}$ \\
\hline \multirow{2}{*}{$\begin{array}{c}\text { Voice } \\
\text { (Priority: 6) }\end{array}$} & MAX & $\begin{array}{l}\text { G.711 } \\
\text { (PCM) }\end{array}$ & 160 & \multirow{2}{*}{20} & \multirow{2}{*}{$\begin{array}{l}\text { Expo. } \\
\text { (mean) } \\
\text { On: } 1.5 \\
\text { Off: } 1.8\end{array}$} & $\begin{array}{c}64 \mathrm{Kbps} \\
\text { (CBR) }\end{array}$ & \multirow{2}{*}{50} \\
\hline & MIN & $\begin{array}{c}\text { G.729_A } \\
\text { (CS- } \\
\text { ACELP) }\end{array}$ & 20 & & & $\begin{array}{c}8 \mathrm{Kbps} \\
(\mathrm{CBR})\end{array}$ & \\
\hline \multirow{2}{*}{$\begin{array}{l}\text { Live Video } \\
\text { (Priority: 5) }\end{array}$} & MAX & $\begin{array}{l}\text { H. } 261 \\
{[\mathrm{CIF}]}\end{array}$ & $\begin{array}{c}\text { Expo. } \\
{[40-2048]} \\
\text { Mean: } 1320\end{array}$ & $\begin{array}{c}\text { Expo. } \\
\text { Mean: } 13\end{array}$ & \multirow{2}{*}{$\begin{array}{c}\text { Always } \\
\text { On }\end{array}$} & $\begin{array}{c}\sim 800 \mathrm{Kbps} \\
\text { (VBR) }\end{array}$ & \multirow{2}{*}{100} \\
\hline & MIN & $\begin{array}{l}\text { H.261 } \\
\text { [QCIF] }\end{array}$ & $\begin{array}{c}\text { Expo. } \\
{[20-1024]} \\
\text { Mean: } 660\end{array}$ & $\begin{array}{c}\text { Expo. } \\
\text { Mean: } 26\end{array}$ & & $\begin{array}{c}\sim 200 \mathrm{Kbps} \\
\text { (VBR) }\end{array}$ & \\
\hline \multirow{2}{*}{$\begin{array}{l}\text { Video } \\
\text { On-Demand } \\
\text { (Priority: 4) }\end{array}$} & MAX & \multirow{2}{*}{$\begin{array}{c}\text { MPEG-4 } \\
{[4 \mathrm{CIF}]}\end{array}$} & \multirow{2}{*}{800} & \multirow{2}{*}{2} & \multirow{2}{*}{$\begin{array}{c}\text { Always } \\
\text { On }\end{array}$} & \multirow{2}{*}{$\begin{array}{c}\text { 3.2 Mbps } \\
\text { (CBR) }\end{array}$} & \multirow{2}{*}{200} \\
\hline & MIN & & & & & & \\
\hline
\end{tabular}

Table 5. Traffic characteristics of the communications in the second simulation scenario

We call this new metric "Q_Score". It depends on the priority of each served TS, its quality level, the number of served TSs, and the network's throughput. First of all, we define the factor "Q_Factor" which concerns the assigned quality level. Q_Factor is higher when a TS is 
assigned the MAX quality level. However, we want to get a clearly higher score when serving two MIN TSs than one MAX TS. Since it is more important to serve multiple low quality TSs than one with high quality, we decided to set Q_Factor=1 when the TS is assigned the MIN quality level, and $Q \_$Factor=1.1 when it is assigned the MAX quality level. First, we calculate the score for each TS:

$$
\text { Stream } Q \_ \text {Score }=Q_{-} \text {Factor } \times \text { PriorityWeight } \times \text { TimeServedRatio }
$$

where the PriorityWeight depends on the stream's traffic priority and the TimeServedRatio is the ratio of the time interval the TS was served to the total time it was scheduled to last. At this point, it should be reminded that according to our simulation settings all TSs are scheduled to last no more than the simulation duration. So, in an ideal situation, all the TSs would be completed before the simulation termination. The IdealStreamQ_Score is the score of a MAX quality TS that is completed before the simulation termination (TimeServedRatio=1). It stands:

$$
\text { IdealStream } Q_{-} \text {Score }=\text { MaxQ_Factor } \times \text { PriorityWeight }
$$

The RatioNetQ_Score, which concerns the total offered streams, is defined as:

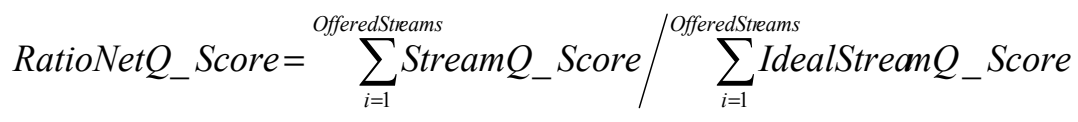

Finally, we calculate each simulated network's Q_Score in relation to the score of the same network when using a different protocol. It stands:

$$
Q_{-} \text {Score }=\text { RatioNetQ_Score } \times \frac{\text { Throughput }}{\text { HigherThroughput }}
$$

for the network with the lower throughput and Q_Score=RatioNetQ_Score for the network with the higher throughput. For example, if a HCCA network has RatioNetQ_Score=1 and Throughput=0.6, and the same network using POAC-QG has RatioNetQ_Score=1 and Throughput $=0.8$, then the Q_Score for the HCCA network is 0.75 while for the POAC-QG network is 1 . Thus, $Q \_S c o r e$ as it is formed in equation (16), can only be used to compare the performance of two networks and not as an individual metric.

The statistical results concerning the Q_Score of 15 network topologies (2 to 30 mobile stations) are depicted in Figure 11. Obviously, POAC-QG always exhibits higher Q_Score than HCCA. This is a definite indication of the efficiency of the QoS negotiation mechanism employed by POAC-QG. In all cases, the proposed protocol ensures a better combination of MAX and MIN quality level TSs, as shown in Figure 12. It appears that POAC-QG always serves as many TSs as possible to the best quality it can achieve. 

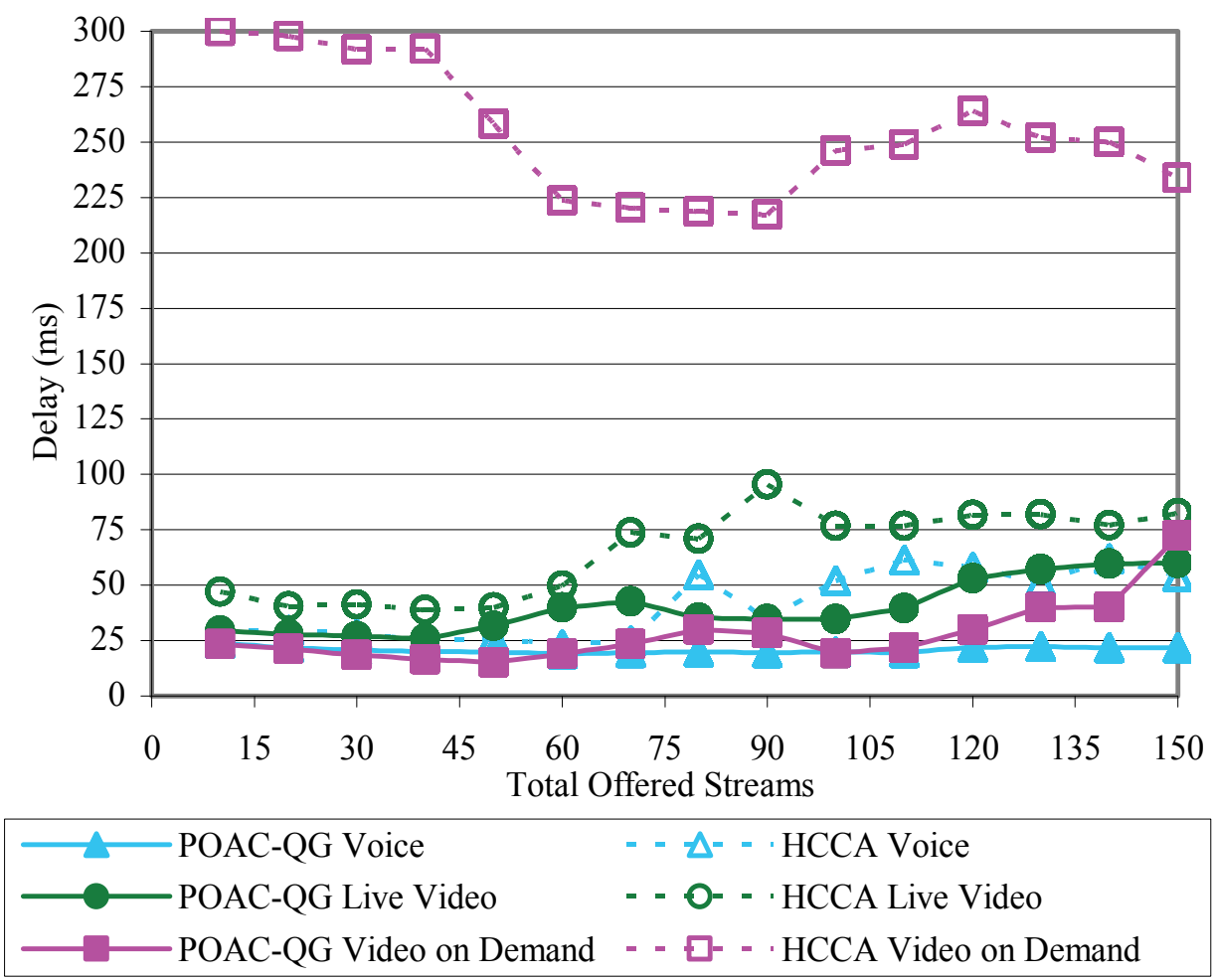

Figure 10. The average packet delay as a function of the total offered traffic streams

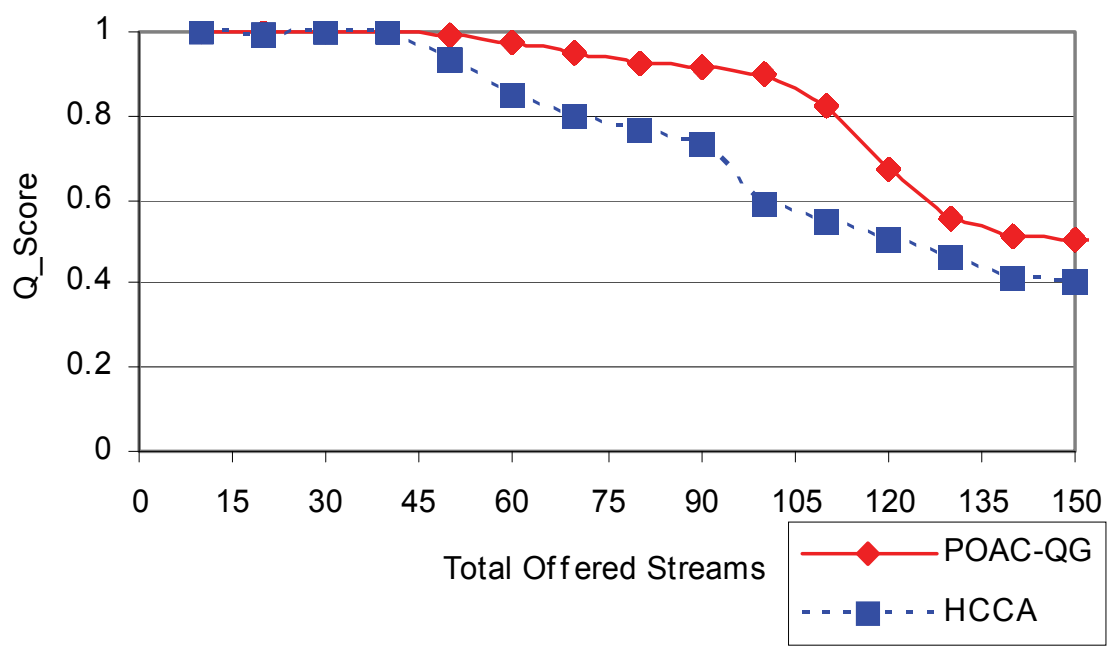

Figure 11. The Q_Score as a function of the total offered traffic streams 


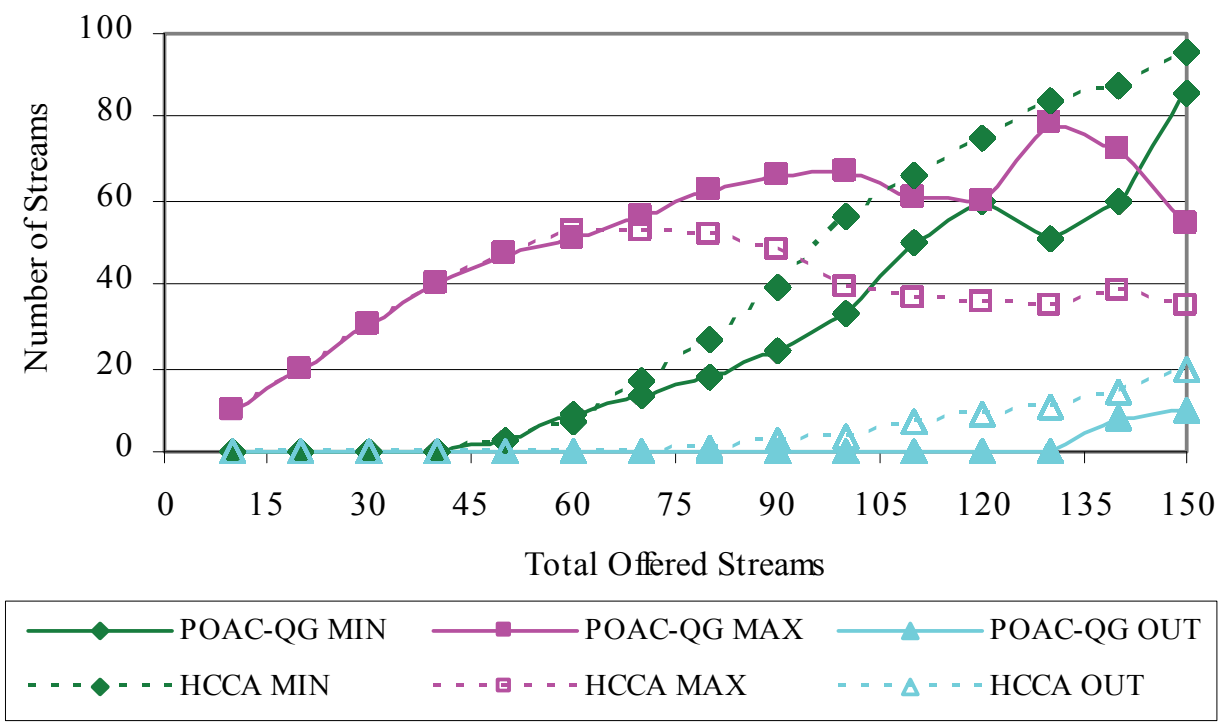

Figure 12. The number of the traffic streams assigned the minimum-maximum quality level quality level or they were rejected versus the number of the total offered traffic streams

\section{Conclusion}

This chapter discoursed on adaptive control for wireless local area networks introducing the Priority Oriented Adaptive Control with QoS Guarantee (POAC-QG) protocol for WLANs. It can be adapted into the HCF protocol of the IEEE 802.11e standard in place of HCCA. A TDMA scheme is adopted for the access mechanism. POAC-QG is designed to efficiently support all types of real-time traffic. It guarantees QoS both for CBR and VBR traffic, by continuously adapting to their special requirements. Since numerous network multimedia applications produce VBR traffic, it is essential to support it with high quality. HCCA, on the other hand, appears to be unable to efficiently support VBR traffic. POAC-QG makes extended use of traffic priorities in order to differentiate the TSs according to their application. The proposed superframe using slots decreases the total overhead, provides better synchronization, since every station is informed by the beacon of the exact time slots assigned to each station, and thus it potentially allows the use of an efficient power saving mechanism. POAC-QG employs a direct QoS negotiation mechanism that supports multiple quality levels for the TSs. This mechanism and the dynamic bandwidth allocation provide support to multiple TSs to the best quality the protocol can achieve. The simulation results reveal this behavior and show that POAC-QG always performs superiorly than HCCA when comparing the packet jitter, TS buffer size and packet delay. As future work, POACQG can be enhanced with a power saving mechanism and it can be combined with an efficient background traffic protocol in place of EDCA in order to form a complete high performance protocol for infrastructure WLANs. 


\section{References}

Akyildiz, I. F.; McNair, J.; Carrasco, L. \& Puigjaner, R. (1999). Medium Access Control Protocols for Multimedia Traffic in Wireless Networks, IEEE Network Magazine, Vol.13, No.4, pp.39-47

Bauchot, F.; Decrauzat, S.; Marmigere, G.; Merakos, L. \& Passas, N. (1996). MASCARA, a MAC Protocol for Wireless ATM, Proceedings of ACTS Mobile Summit 1996, Granada

Bianchi, G. (2000). Performance Analysis of the IEEE 802.11 Distributed Coordination Function, IEEE Journal on Selected Areas in Communications, Vol.18, No.3, pp.535-547

Bianchi, G.; Borgonovo, F.; Fratta, L.; Musumeci, L. \& Zorzi, M. (1997). C-PRMA: A centralized packet multiple access for local wireless communications, IEEE Transactions on Vehicular Technology, Vol.46, No.2, pp.422-436

Chandra, A. ; Gumalla, V. \& Limb, J. O. (2000). Wireless Medium Access Control Protocols, IEEE Communications Surveys and Tutorials, Vol.3, No.2, pp.2-15

Chen, Kwang-Cheng \& Lee, Cheng-Hua (1994). Group randomly addressed polling for wireless data networks, Proceedings of IEEE ICC 1994, pp.1713-1717, New Orleans

Chlamtac, I.; Conti, M. \& Liu, J. J. N. (2003). Mobile ad hoc networking: imperatives and challenges, ELSEVIER Ad Hoc Networks, Vol.1, No.1, pp.13-64

Chou, C.-T.; Shankar, S. N. \& Shin, K. G. (2005). Achieving per-stream QoS with distributed airtime allocation and admission control in IEEE 802.11e wireless LANs, Proceedings IEEE INFOCOM 2005, pp. 1584-1595, Miami

Dyson, D. A. \& Haas, Z. J. (1999). A dynamic packet reservation multiple access scheme for wireless ATM, ACM/Baltzer Journal of Mobile Networks $\mathcal{E}$ Applications, Vol.4, pp.8799

Gilbert, E. (1960). Capacity of a burst noise channel. Bell Syst. Tech. Journal, Vol.39, pp.12531265

Grilo, A.; Macedo, M. \& Nunes, M. (2003). A scheduling algorithm for QoS support in IEEE 802.11e networks, IEEE Communication Magazine, pp.36-43

HIPERLAN, EN 300652 V1.2.1 (1998), ETSI, Broadband Radio Access Network (BRAN); HIgh PErformance Radio Local Area Network (HIPERLAN) Type 1; Functional Specification

IEEE 802.11e WG, IEEE Standard for Information Technology--Telecommunications and Information Exchange Between Systems--LAN/MAN Specific Requirements--Part 11 Wireless Medium Access Control and Physical Layer specifications, Amendment 8: Medium Access Control Quality of Service Enhancements, (2005)

Issariyakul, T.; Hossain, E. \& Kim, D. I. (2003). Medium access control protocols for wireless mobile ad hoc networks: issues and approaches, Wiley Journal of Wireless Communication and Mobile Computing, Vol.3, No.8, pp.935-958

Karol, M. J.; Liu, Z. \& Eng, K. Y. (1995). An Efficient Demand- Assignment Multiple Access Protocol for Wireless Packet (ATM) Networks, ACM/Baltzer Journal of Wireless Networks, Vol.1, No.3, pp.267-279

Kim J. G. \& Widjaja, I. (1996). PRMA/DA: A New Media Access Control Protocol for Wireless ATM, Proceedings of ICC 1996, pp.1-19, Dallas

Lagkas, T. D.; Papadimitriou, G. I. \& Pomportsis, A. S. (2006). QAP: A QoS supportive Adaptive Polling Protocol for Wireless LANs, Elsevier Computer Communications, Vol.29, No.5, pp.618-633 
Lagkas, T. D.; Papadimitriou, G. I.; Nicopolitidis, P. \& Pomportsis, A. S. (2008). A Novel Method of Serving Multimedia and Background Traffic in Wireless LANs, IEEE Transaction on Vehicular Technology, forthcoming.

Larcheri, P. \& Cigno, R. Lo (2006). Scheduling in 802.11e: Open-Loop or Closed-Loop?, Proceedings of WONS 2006, Les Menuires

Ni, Q.; Ansel, P. \& Turletti, T. (2003). A Fair Scheduling Scheme for HCF, IEEE 802.11e Working Group Document, IEEE 802.11-03-0577-01-000e

Ni, Q.; Romdhani, L. \& Turletti, T. (2004). A Survey of QoS Enhancements for IEEE 802.11 Wireless LAN, Wiley Journal of Wireless Communication and Mobile Computing, Vol.4, No.5, pp.547-566

Nicopolitidis, P. ; Obaidat, M. S.; Papadimitriou G. I. \& Pomportsis, A. S. (2003). Wireless Networks, Wiley, ISBN 0-470-84529-5, England

Papadimitriou, G. I. \& Pomportsis, A. S. (2003)a. Adaptive MAC protocols for broadcast networks with bursty traffic, IEEE Transactions on Communications, Vol.51, No.4, pp.553-557

Papadimitriou, G. I. ; Lagkas, T. D. \& Pomportsis, A. S. (2003)b. HIPERSIM: A Sense Range Distinctive Simulation Environment for HiperLAN Systems, Simulation, Transactions of The Society for Modelling and Simulation International, Vol.79, No.8, pp.462-481

Pawlikowski, K.; Jeong, H. D. J. \& Lee, J. S. R. (2002). On Credibility of Simulation Studies of Telecommunication Networks, IEEE Communications Magazine, Vol.40, No.1, pp.132-139

Petras, D. \& Kramling, A. (1996). MAC protocol with polling and fast collision resolution for an ATM air interface, Proceedings of IEEE ATM Workshop 1996, San Francisco

Raychaudhuri, D.; French, L. J.; Siracusa, J.; Biswas, S. K.; Yuan, R.; Narasimhan, P. \& Johnston, C. A. (1997). WATMnet: A Prototype Wireless ATM System for Multimedia Personal Communication, IEEE Journal on Selected Areas in Communications, Vol.15, No.1, pp.83-95

Zorzi, M.; Rao, R. R. \& Milstein, L. B. (1995) On the accuracy of a first-order Markov model for data transmission on fading channels, Proceedings of ICUPC 1995, pp.211-215, Tokyo 


\title{
Adaptive Control Methodology for High-performance Low-power VLSI Design
}

\author{
Se-Joong Lee \\ Texas Instruments Inc. \\ U.S.A.
}

\section{Introduction}

Very Large Scale Integrated chip design consists of several steps including modeling characteristics of transistors, profiling circuit level behaviors, abstracting into gate level parameters, synthesizing logic based on timing constraints, and so on. The common concept that runs through all these sequence of design flow is modeling. Traditional VLSI design highly replies on the modeling process, and the percentage that a design chip is successful, or say yield, is determined by how much the modeling is done accurately and precisely. In order to increase the yield, designers put margins while they design. A designer assumes worst cases in design parameters like transistor speed, supply voltage, temperature, and operation frequency. Even though sufficient margin on those design parameters leads higher chance of success in chip design, the margins imply overhead on the other hand. The overhead costs additional power consumption, which should be conquered in this lowpower era.

Another cause of such over-design is variable workload in a system. Most of traditional VLSI systems are designed to support the maximum possible workload and the system becomes to have headroom in terms of performance when the workload is not that high.

Modern VLSI designs deploy the concept of adaptive control schemes to manage the costs caused by the over-designs. A chip embeds transistor speed meter, and temperature sensors to monitor actual environment that the chip is operating in, and adjusts the margins to be minimal in order to minimize the additional cost. According to the workload offered to the system, the system controls its supply voltage dynamically thus the system keeps its performance just as enough. In this chapter, we introduce the design cases that use adaptive control schemes to manage the overhead while reducing power consumption.

One another factor causing over-design is uncertainty, which is emerged in recent VLSI design area. Huge complexity of system-on-chips and ever shrinking transistor size has brought the uncertainty issue in modern VLSI design. One example is clock synchronization issue. As the clock frequency goes beyond Giga-hertz, the chip area is no longer bounded within a single clock cycle, thus, clock synchronization in a chip became extremely challenging, and even impossible to achieve. The terminology, Network-on-Chip has begun to widely spread in the VLSI design field as a chip becomes a set of systems interconnected through a network. In such a big system, transmitting and receiving data signals involves timing uncertainty because it is no longer a synchronous system. Such uncertainty incurs 
timing overhead in the signal transactions and requires additional circuitry resources. In this chapter, we also introduce design examples utilizing adaptive control schemes to address the issues in Network-on-chip design.

\section{Adaptive Control on Design Margins}

As hand-held devices became the ones driving the electronic market, demand on low-power consumption became very aggressive. And, the chip designers are requested to devise lowpower schemes in entire design flow from architecture-level to process-level. While innovating the designs for such low-power constraint, one trend was featured: Minimizing the design margins. The design margins are re-considered as a source of power reduction. In this Section, some examples of design margins are introduced and efforts to minimize them with adaptive control schemes are addressed.

\subsection{Process Variation}

The very first agenda placed in VLSI chip design flow is modeling of transistor characteristics. Based on measurements on actual transistors implemented on Silicon, electrical behavior of transistors is modeled as a function of voltages supplied to the devices, temperature in which the device is running, etc. Based on the modeling, a VLSI chip designer can simulate system behavior using computer-assisted tools.

The issue in this process is that the transistors have different characteristics whenever they are implemented on Silicon. Due to many practical reasons, transistors are not implemented identically per implementation. To come up with the phenomena, transistor characteristics are modeled with Gaussian distribution curve, rather than a fixed parameter. Based on statistics obtained from extensive measurements, designers use transistor models representing very worst case scenario thus the fabricated chips are operational unless they belong to even worse cases. In other words, designers put margins to their designs to make it operational for any condition of chip fabrication'1. And, such margins are overhead in terms of performance and power of the chip.

If the chip fabrication process is well under control, the distribution of the transistor characteristics will be narrow enough to neglect such margin overhead. However, as the process technology scales down its minimum feature size lower than tens of nanometers, just small errors in transistor etching or doping on Silicon introduces larger deviation of its characteristics than ever (Figure 1) [1,15]. This implies that designers should put more percentage margins to their designs due to the increased uncertainty of the chip fabrication process.

\footnotetext{
${ }^{1}$ Even though the author uses the term, 'any', to emphasize the importance of design margin, there is no perfect process, therefore, there will be non-zero failure probability.
} 


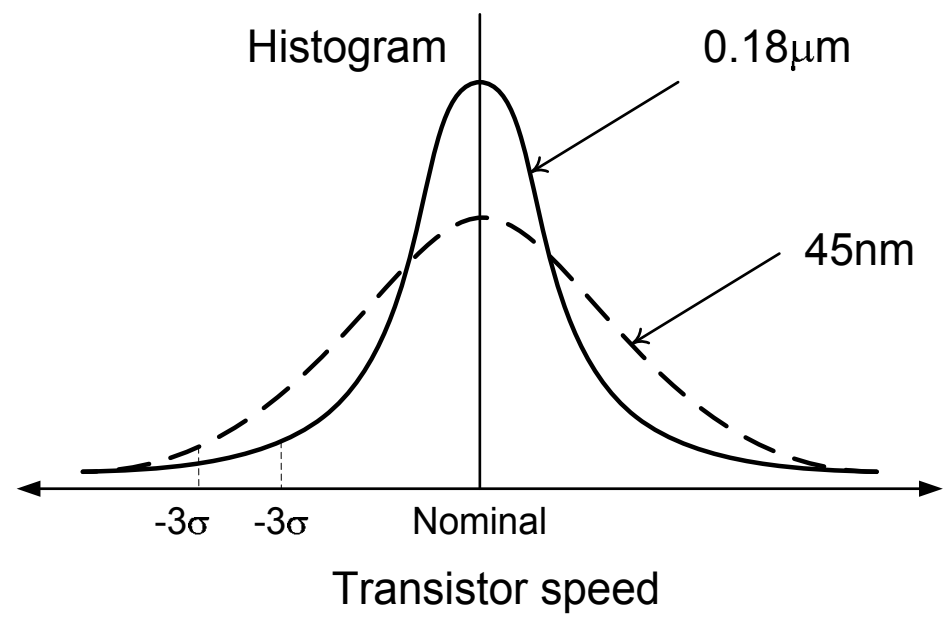

Figure 1. Distribution of transistor characteristics for different feature sizes

Putting voltage margins is trade-off between stability/productivity and powerconsumption. As the pressure for low-power consumption gets so strong in hand-held device market, such margins are re-considered as headroom for power reduction. In Figure 1 , the additional voltage margin is required by only the chips that belong to the low three sigma profile. If we can measure the quality of transistors of a chip, so that determine the proper supply voltage for that specific chip, it is possible to reduce the power consumption of most of the chips having good and even typical quality transistors.

The SmartReflex technology from Texas Instruments [2] is one practical example exploiting this type of technology. The Adaptive Voltage Scaling (AVS) scheme, used in the SmartReflex technology suite, implements a monitoring circuit in the chip, so that the circuit measures on-chip transistors' speed and temperature during run-time, and determines the quality of them. If the chip has higher quality than the chip designer assumed to be, the chip either commands the external power IC to reduce the supply voltage or decrease the internal supply voltage if embedded. In other words, the chip changes its supply voltage adaptively according to the demand of the chip itself.

The fundamental reason that this type of adaptive voltage control is successful is that the transistor quality is measurable by practical means, so that, the design margin is no longer simply insurance for safe operation, but, it is a controllable parameter. In addition to such known, or we can say measurable, distribution of the process parameters, designers consider another type of margin, which is there to protect the designs from unknown uncertainties either we haven't recognized yet or we weren't able to model properly even if we recognized. And, such unknown parameters will be discovered to turn them into useful headroom for further enhancement in the future.

\subsection{Dynamic Voltage Frequency Scaling}

Power consumption of VLSI digital systems is represented by Equation (1).

$$
P=\alpha \cdot f \cdot V^{2} \text {, where } \alpha=\text { coefficient, } f=\text { clock frequency, } \mathrm{V}=\text { supply voltage }
$$


As the equation tells explicitly, reducing the supply voltage is the most effective way to save the power. The supply voltage limits the maximum clock frequency achievable. The clock frequency, $f$, determines the performance of the system. Higher clock frequency implies faster operation, so that, executes more workload within given time. Except some high-end VLSI systems, the supply voltage is a static value which is fixed during entire operation of the chip. Therefore, the supply voltage is set to support the maximum clock frequency.

Even though a system's workload is less than the maximum for which the supply voltage is determined, the chip operates just as fast as possible and goes back to idle mode when it completes, until next task is called. However, this is not an optimal solution from the energy perspective. If we can scale down the supply voltage, thus, the operating clock frequency, we can reduce the total energy consumption while completing the task just on time. The technology scaling the supply voltage and frequency according to the workload is called Dynamic Voltage Frequency Scaling (DVFS). The underlying concept of the DVFS is turning the system performance margin into useful energy so that reduce the overall energy and power consumption $[3,4,5]$.

One of most challenging issue in this adaptive voltage control is estimating the future workload to change the supply voltage prior to arrival of the workload. The supply voltage cannot be changed frequently due to many practical limitations: Once the voltage is changed it should be kept for a while, i.e. a few msec which corresponds to 1 million cycles when it comes to $1 \mathrm{GHz}$ clock. Therefore, the change of supply voltage must guarantee that it is enough to support the workload of next, let's say 1 million cycles.

The prediction of future workload is one of open research area. Current system information like CPU utilization, Bus and Memory activity, OS scheduling, and protocol states, can be used to statistically predict the future workload. And, more accurate estimation will result in better power saving with less performance hit problems. If the system is not running hard-deadline tasks, the system is allowed to catch up any residue workload during next time frame, if its current workload estimation was incorrect so the performance setting was not enough to complete the current workload on time. Even if such catch up mechanism is allowed, it may cause sluggishness in user experience, which must be minimized.

\subsection{Range of Operational Condition}

Another design margin existing in chip design flow occurs due to environmental conditions. Some major parameters considered as the environmental conditions of a chip are supply voltage and temperatures. The speed of a circuit is a linear function of supply voltage, and leakage current of the circuit is an exponential function of temperature. Chip designers want their chips are operational in wider conditions. For example, they want their chips are operational for $0^{\circ} \mathrm{C}$ to $125^{\circ} \mathrm{C}$, rather than $30^{\circ} \mathrm{C}$ to $50^{\circ} \mathrm{C}$, because wider temperature range implies larger market and more reliable operation in unknown situations.

However, satisfying wider specification brings additional overhead in terms of power and area of the chip. One good example can be found in Dynamic Random Access Memory (DRAM) design. The DRAM is developed to store very large amount of digital information. A main physical mechanism in DRAM operations is charging electrons into a capacitor, which is a write operation, and detecting the charge, which is a read operation. And, the DRAM designer wants the electrons in the capacitor remain thus they can be detected later whenever needed. However, the charges leak out from the capacitor slowly, which is called leakage current. Eventually, most of the charges are gone so the capacitor does not have 
enough charges to be detected. In other words, the information stored in the DRAM capacitor is volatile, therefore, the charges need to be detected within certain limited time, and re-charged in order to extend the life time of information. This operation is called, refresh. The refresh operation of a DRAM is one of major factor consuming power when the system is idle. For example, when a lap-top computer is in standby-mode, the main CPU can sleep to reduce the power consumption, but, the DRAM should perform refresh operation regularly in order to retain the data stored in. Therefore, the DRAM designers want their DRAMs get refreshed as less frequently as possible. The refresh frequency is determined based on the leakage current, and it increases exponentially according to the temperature. If the temperature of the DRAM is high, the leakage current is also high; therefore, refresh must be done more frequently. Therefore, if the temperature specification of the DRAM is $30^{\circ} \mathrm{C} \sim 100^{\circ} \mathrm{C}$, which looks typical, the DRAM designer determines the refresh frequency based on the highest temperature, say $100^{\circ} \mathrm{C}$.

For advanced power management, some designers had started to embed a temperature sensor circuitry to monitor the temperature and determine decent refresh frequency based on the temperature [6,7]. Figure 2 shows an example block diagram of this type of DRAM architecture. Around the DRAM cells which are the source of leakage current drawing, temperature sensor circuits are placed, and the adaptive refresh controller monitors the highest temperature. Based on pre-programmed temperature-to-refresh conversion curve, the adaptive refresh controller commands the refresh circuit with minimum frequency necessary.

This adaptive refresh frequency control is very useful for extending the standby time of our lap-top computers, because, the lap-top computers are not that literally hot during standby mode, and the DRAM will adaptively set the refresh frequency low, and decrease the power consumption while retaining the data properly.

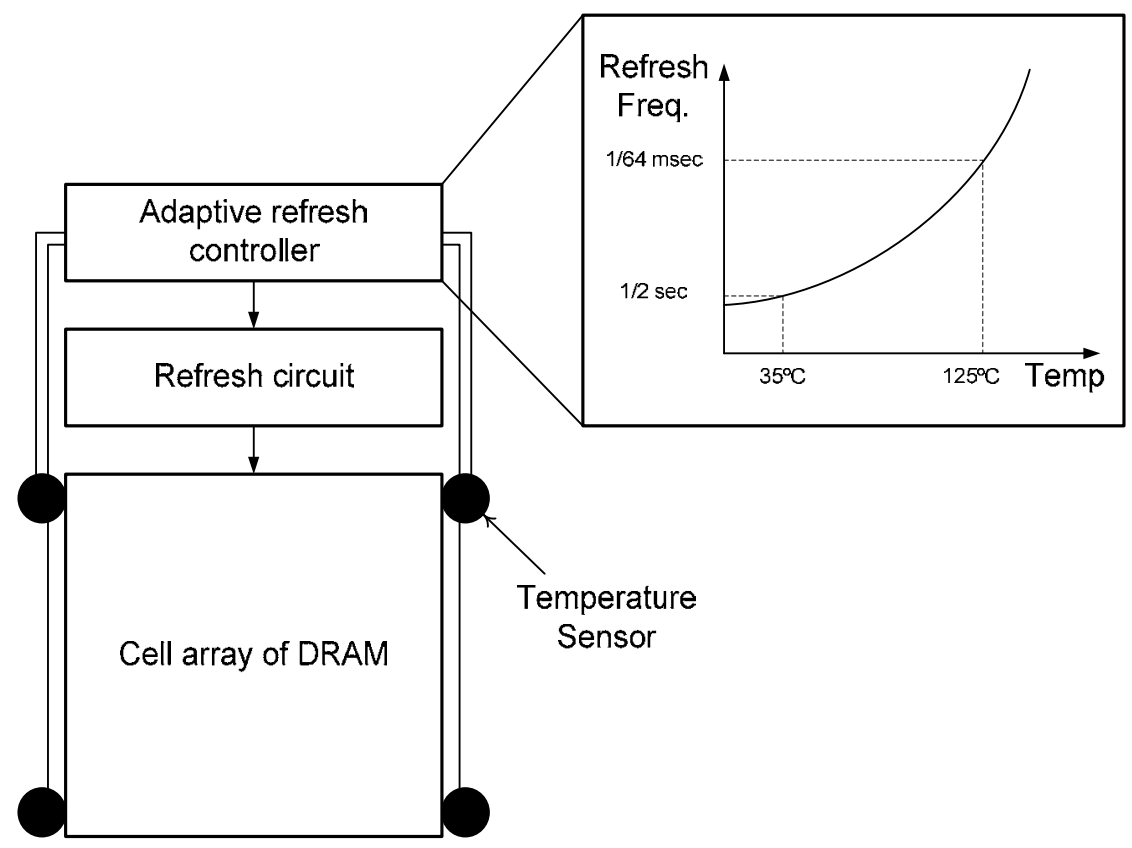

Figure 2. An example of DRAM block diagram with adaptive refresh scheme 


\section{Adaptive Technologies in Network-on-Chips}

A modern VLSI chip is no longer a simple functional unit, but, it consists of tens of processing elements, constituting a system in the chip, and we call it as a System-on-Chip or SoC. As the number of processing elements in an SoC increases, the interconnection gets longer and more complex. It is intuitive that the complexity of an interconnect system for the number of $\mathrm{N}$ processing elements is $\mathrm{N}^{2}$, which implies that the interconnect complexity will dominate the SoC design as the $\mathrm{N}$ increases. The Network-on-Chip, which is a new concept representing the most complex SoC in which the interconnect system is even considered as a network $[8,9,10,11]$.

Such an on-chip network consists of sophisticated circuitry to comply with complex data transaction pattern in the VLSI system. This Section introduces some adaptive control schemes making the on-chip network more flexible, stable, and power-efficient.

\subsection{Adaptive Topology Configuration}

On-chip network topology has impacts on chip performance, power, and area of the network, and choosing optimal topology depends on the traffic pattern traversing through the network [14]. For example, Mesh topology shown in Figure 3-(a) is a widely used network architecture for parallel computing systems, because of its beauty of symmetric shape, unique link length, and scalability with low cost. However, the Mesh topology suffers from long latency for long-distance packet transmission. In Figure 3-(a), a packet from the left-top switch takes 7 intermediate switches to arrive at the right-bottom one. Each switch is a packet-switching switch, and a packet-switching operation requires a series of pipeline activities like packet header parsing, arbitration, and routing. As a result, each packet switch takes at least a couple of clock cycles to transfer a packet from an input port to an output port, thus, passing through several packet switches takes tens of cycles. Due to the high latency issue for long-distance packet transmission, it is known that Mesh network is useful for traffics with high locality. Figure 3-(b) shows another topology, Star, which has different characteristics against Mesh. A Star network shows the minimum packet latency2, because the switch hop count between two nodes is 1. In case of Tree-topology, the number of switch hops is given by logarithmic function of the distance of the two nodes, whereas that of Mesh network is linear function of the distance.

\footnotetext{
2 The Star network may have longer interconnect wires, which will contribute additional propagation delay thus increases the end-to-end packet latency. The author, however, points out that the propagation delay is not yet considerable compared to the packet switch's pipeline latency in a few Gigahertz clock frequency era.
} 


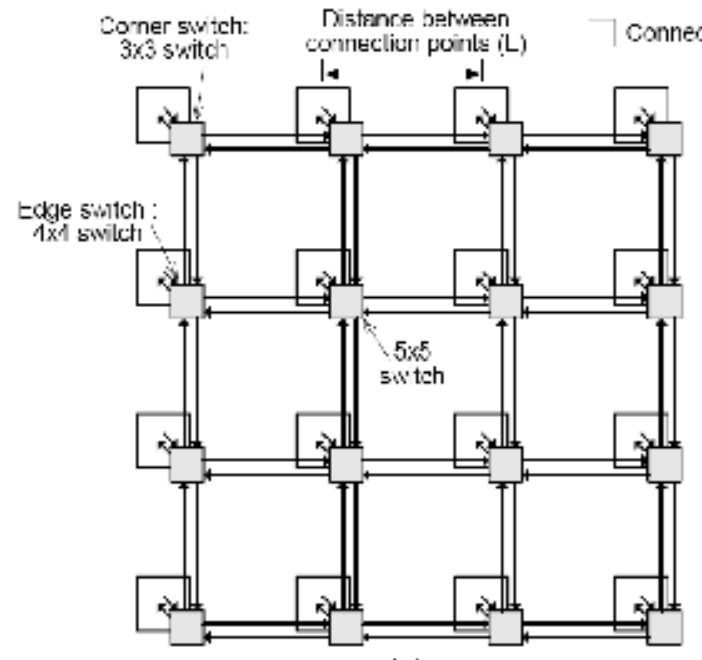

(a)
Sistcen

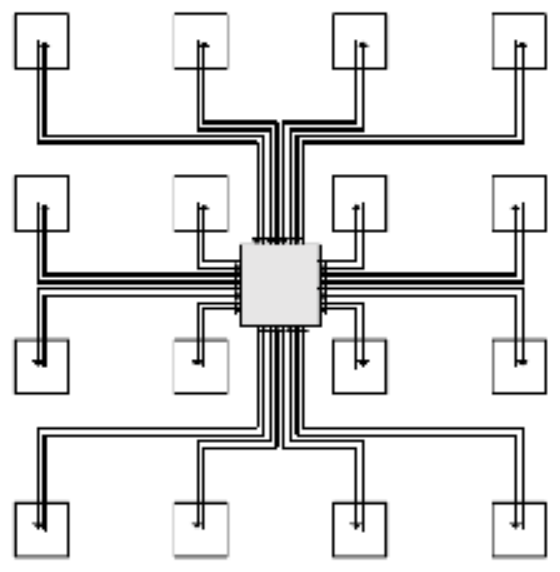

(b)

Figure 3. (a) Mesh and (b) Start topology configurations

The issue of choosing a best topology for a VLSI chip design is that the optimal topology is determined by traffic pattern and the traffic pattern is either 1. diversified per situations or 2. not determined until the system is actually used. Therefore, a configurable topology which changes adaptively according to the situations or system usage is definitely demanded.

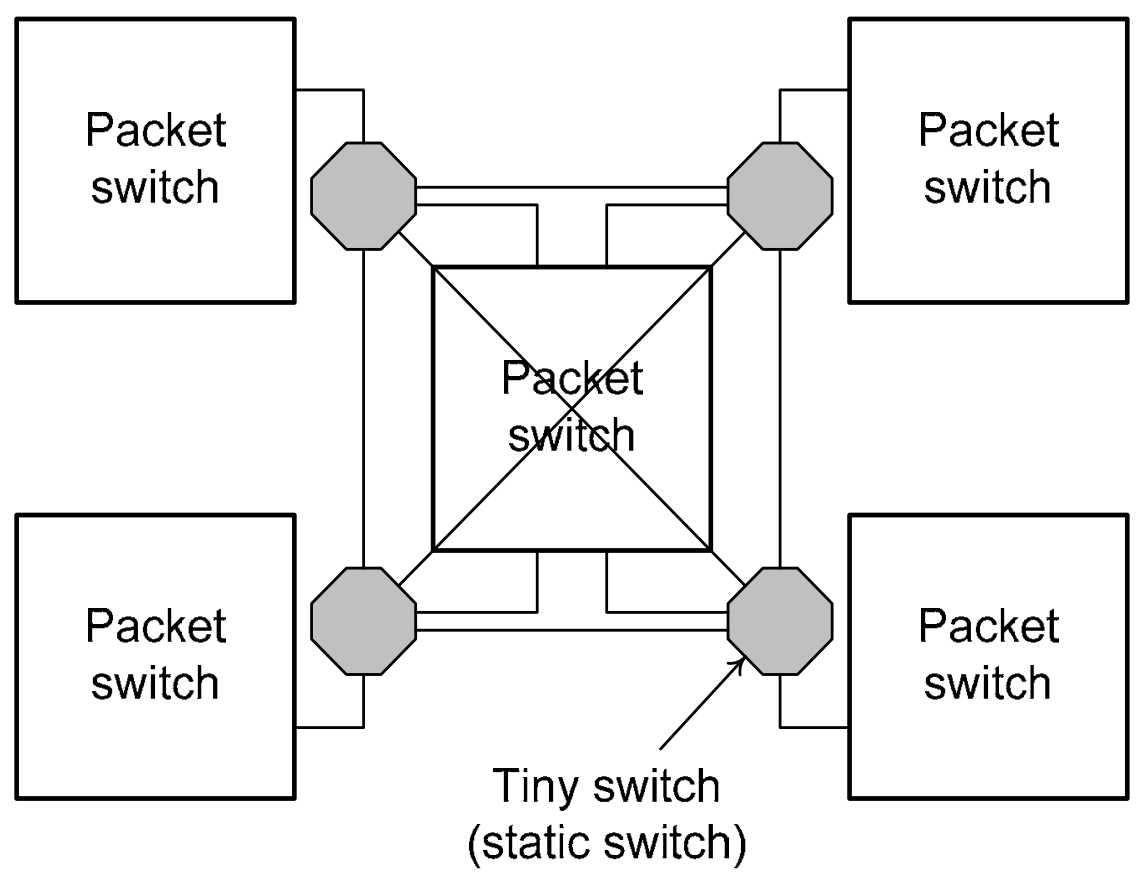

Figure 4. Configurable network topology with tiny switches 
One interesting evolution found in the VLSI chip [12] is the adaptive topology configuration. Figure 4 illustrates the overall block diagram of the architecture. Using tiny switches consisting of simple switch transistors and wires, the way the main packet network switches are connected is changed according to the control signals. The tiny switch is not a packet switch, therefore, it does not incur cycle-level latency. Instead, it takes propagation delay time for signals passing through the tiny switches.

In order to utilize the adaptive topology configuration scheme, an efficient mechanism detecting the system situation is required. A traffic monitoring unit can be deployed to inspect the average packet delivery distance, and feedbacks the information to a central network controller. The network controller changes the network topology based on the traffic information in order to tune the network performance or reduce power consumption. This type of research will be continued in Network-on-Chip area.

\subsection{Adaptive Voltage Scaling in Interconnect}

In spite of ever shrinking chip size as the process technology scales down the minimum feature size aggressively, the relative chip size that the interconnect experiences gets increasing as the integration level of the chip does. And, it is well-known that the portion that the power-consumption of the interconnect occupies in total power consumption of a VLSI chip is considerable. And, among the power-consuming sources in the interconnect architecture, link wire, which is physical conductive and capacitive connection to propagate electrical signal, is one of major current drawer. Therefore, energy minimization in link wires is highly demanded.

As described in Section 2.2, the DVFS technology can be applied to a processing element having diversified workload profile, so that, the supply voltage is adaptively changed to let the processing element consume decent energy which is just enough to perform the processing within a given time constraint. This concept can be applied to the interconnect wires, too.

Figure 5 explains the correlation between the supply voltage and bandwidth of a wire. The bandwidth implies how many peak-to-peak switching digital signal symbols can propagate within a unit time. For a given capacitance of the wire, higher supply voltage results in higher bandwidth, thus, the wire can transfer the digital signal more frequently.
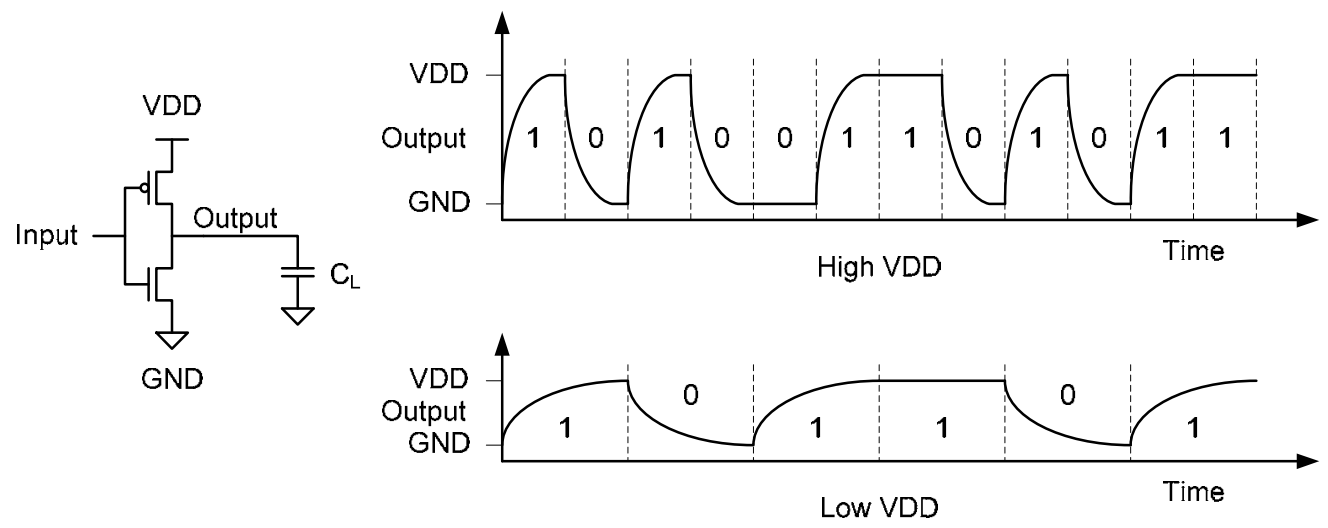

Figure 5. The correlation between the supply voltage and link bandwidth 
The maximum bandwidth of a wire required in a VLSI system is determined by maximum data transfer rate that the designer intended to have. However, as same as the motivation of DVFS in the processing elements, the data rate required is time-varying according to the system state. Therefore, if we can detect the requirement, we can adjust the supply voltage of the wire to support just enough bandwidth required.

Figure 6 illustrates an example of adaptive supply voltage control in link wires used in Network-on-Chip [13]. The queue is a FIFO buffer storing data to be transferred. If the back$\log$ of the queue exceeds a certain threshold, the adaptive supply voltage controller decides that the transmitter unit is demanding higher data transfer rate than the one currently supported by the link. Therefore, the controller chooses to use higher supply voltage. In case of the other way around, the controller selects lower supply voltage.

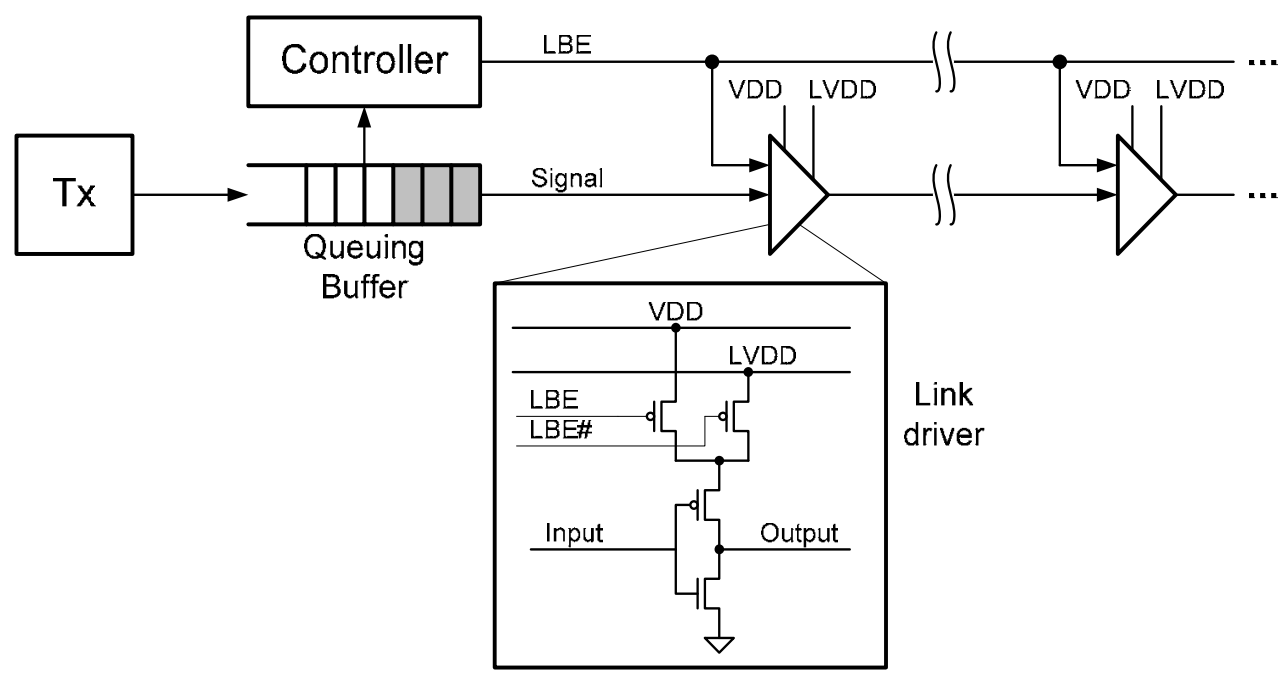

Figure 6. Adaptive bandwidth adjustment circuit

How promptly the wire's bandwidth can be changed, or the response time of the voltage scaling command, is an important parameter determining overall behavior of this adaptive control scheme. The change of bandwidth is the same operation as the change of supply voltage. And, the time required for scaling the supply voltage differs design by design. Many design solutions for voltage scaling in interconnect wires are feasible, and, one of costeffective solutions adopted in [13] is illustrated in Figure 6. The two different voltage supply lines, VDD and low VDD, are placed along with the interconnect wires, the driver circuits are connected to both of the supplies, and the control signal selects one of the two supplies. In other words, the number of available voltage levels is two. We can say this is selective voltage scheme rather than voltage scaling. The parameter determining the time for voltage change is the propagation delay time of the control signal from the transmitter to the receiver, and the actuation time required by the power switch. The actuation of the power swtich and the propagation of the control signal occur in parallel, and, the propagation delay is typically much higher than the switch enable time, so that the response time of the voltage change control is dominated by the propagation time of the control signal.

The control signal is placed along with the link wire together, therefore, the propagation delay is substantial when it comes to global interconnect. And, the power consumption of 
global interconnect is the issue of interest due to its large capacitive load, therefore, the long propagation delay is not evitable. The long propagation time, or the response time, determines the performance of the adaptive control. As the response time is shorter, the link bandwidth can be controlled more closely to follow the actual performance requirement, thus, optimize energy-consumption.

In order to overcome the long propagation delay problem, we can use more aggressive circuit technique called wave-pipelining. Underlying idea of wave-pipelining in general circuit implementation is to utilize propagation delay to split two sequential signal symbols. Figure 7 depicts how this mechanism is used to hide the propagation delay time. Once the control signal is considered to be propagated enough length, the controller circuit treats the link as if its bandwidth change has been completed. Even though this assumption is not valid yet and only a part of the link has been changed, we know that the signals from the transmitter will take the same path and experience similar propagation delay, therefore, the link bandwidth at a certain link point will be updated prior to the signal's arrival to the point. As long as we confirm that the control signal propagates at the higher or same speed compared to the data signal, this wave-pipelining scheme works properly while minimizing the response time in the adaptive control loop.

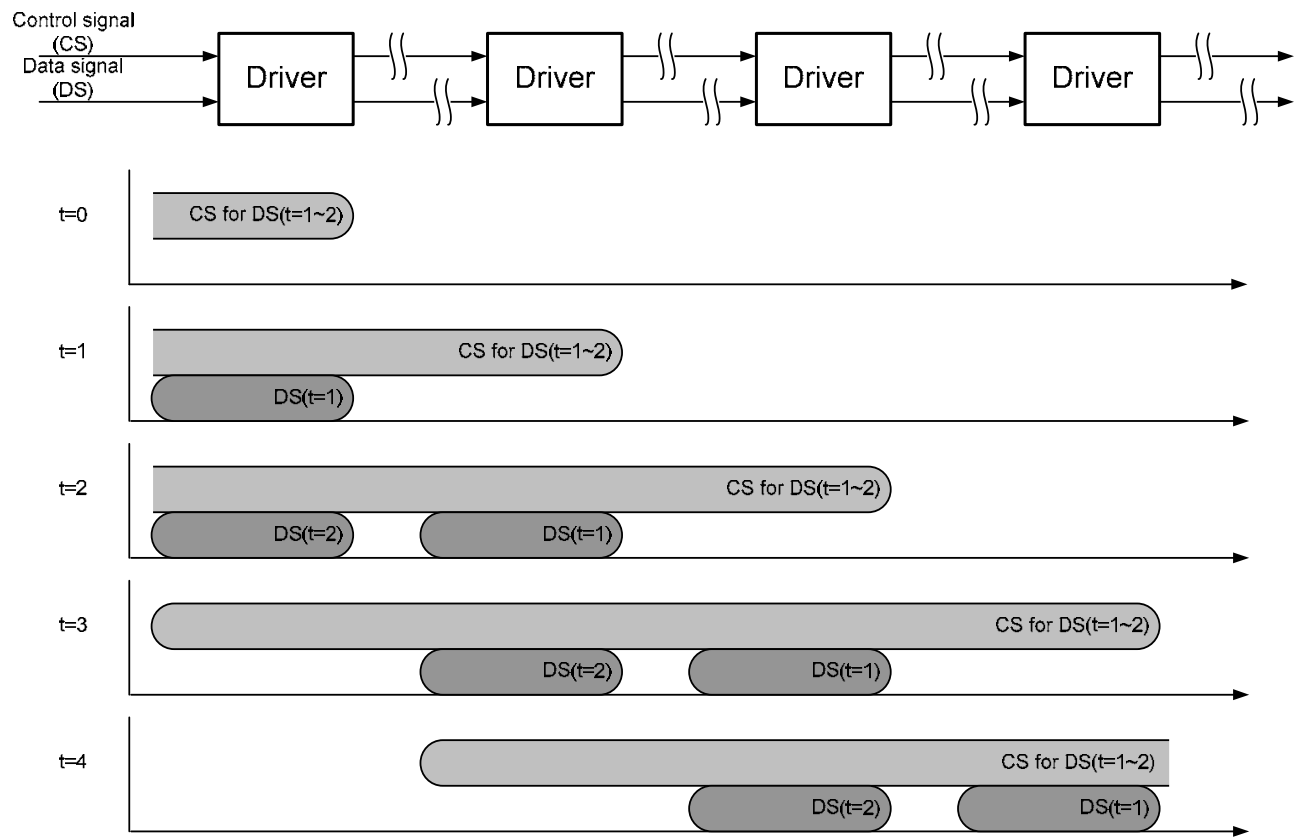

Figure 7. Wave-pipelining scheme for the control signal

\subsection{Adaptive Fetching Point Control}

One of on-chip network $(\mathrm{OCN})$ roles in an SoC design is to lessen the burden of global synchronization using mesochronous communication in which network blocks share the same clock but the phase difference is not compensated. In general, mesochronous communication implies clock phase difference between clock domains is unknown. Due to 
such uncertainty in clock phase, clock synchronization circuit typically deploys multiple stages of flip-flops incurring synchronization latency and power consumption.

In a hard-wired chip, however, the phase difference is bounded to unknown but deterministic difference. This implies that adaptive adjustment of synchronization clock with an architecture of simple pipeline synchronizer is enough to resolve the phase difference.

According to the circumstance in which a chip is working, the deterministic phase difference can vary. Below, three situations are listed, in which the phase difference between two communicating blocks can vary:

- Packet route variation (physical distance variation)

- Supply voltage variation (electrical distance variation)

- Network clock frequency variation (reference timing variation)

If the dynamic topology adaptation scheme described in Section 3.1 is applied, a switch receives packets not only from a certain neighborhood switch but also from somewhere else. This means that the physical distance between two communicating blocks can change. Supply voltage control is used as a mean of power management as described in the previous Section. In case of supply voltage variation, electrical distances ${ }^{3}$ between blocks vary, so that phase difference is changed. The network clock frequency variation also changes clock phase difference. The clock frequency variation is useful technique to manage overall power consumption based on network bandwidth requirement: For example, the OCN [9] controls network frequency with 4 steps for low-power application. When the clock frequency is changed, reference timing is changed, which shifts phase.

Due to the reasons stated above, the phase difference can be varied. In other words, if we have information about clock frequency, supply voltage, and network configuration, we can estimate phase variation. Since the combinations of the phase-varying factors are limited to several cases, the phase difference can be thought unknown but quantized.

To deal with the quantized phase differences, a programmable delay is used as shown in Figure 8. A variable delay (VD) is connected with a simple pipeline synchronizer, and the $\mathrm{VD}$ is controlled according to the network circumstance. The appropriate VD setting for a certain circumstance is obtained through calibration process. Once the adaptive clock-phase selection unit gathered the appropriate settings for every combination of the system states, it adaptively controls the delay time of the VD, therefore, it avoids hazardous situations in synchronization.

Measured waveforms of the adaptive clock-phase selection mechanism are shown in Figure 9. The initial problem is that the EN signal may be not synchronized with the clock so that the rising edge of the EN could be very close to the "rising edge minus setup time margin". In Figure 9-(a), the mode $C$ represents such a system situation that the rising edge of the EN signal is very close to the clock timing. When the system state, mode $C$, is detected, the controller expects that fetching the EN signal is very unstable so that the EN would be fetched at the next clock cycle as shown in Figure 9-(a). This phenomenon is captured more clearly in Figure 10. The EN signal should be fetched at the clock rising edge $\mathrm{T}_{\mathrm{A}}$. However, the signal is fetched at the timing $\mathrm{T}_{\mathrm{B}}$ with some finite probability. Such an unstable behavior may produce synchronization failure or meta-stable state, which is undesirable phenomenon in signal transmission.

${ }^{3}$ The propagation delay time between nodes. 


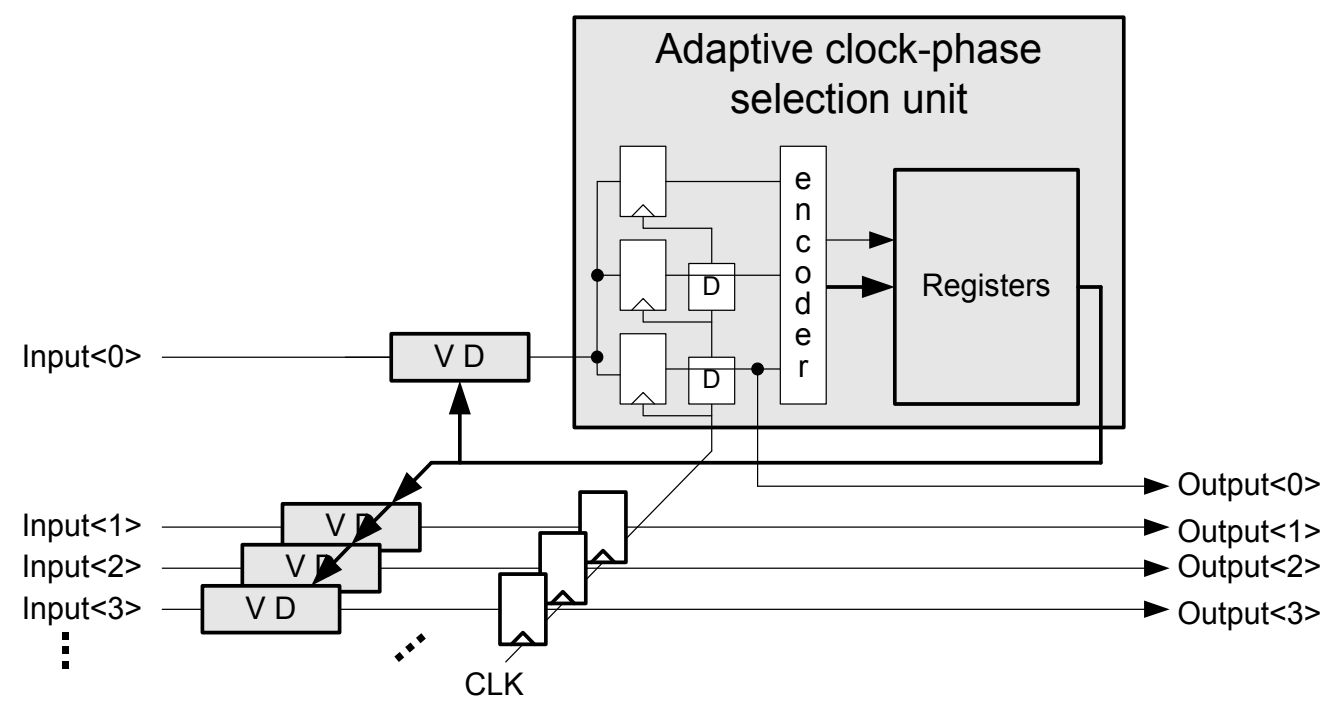

Figure 8. Adaptive clock-phase selection with variable delay circuit

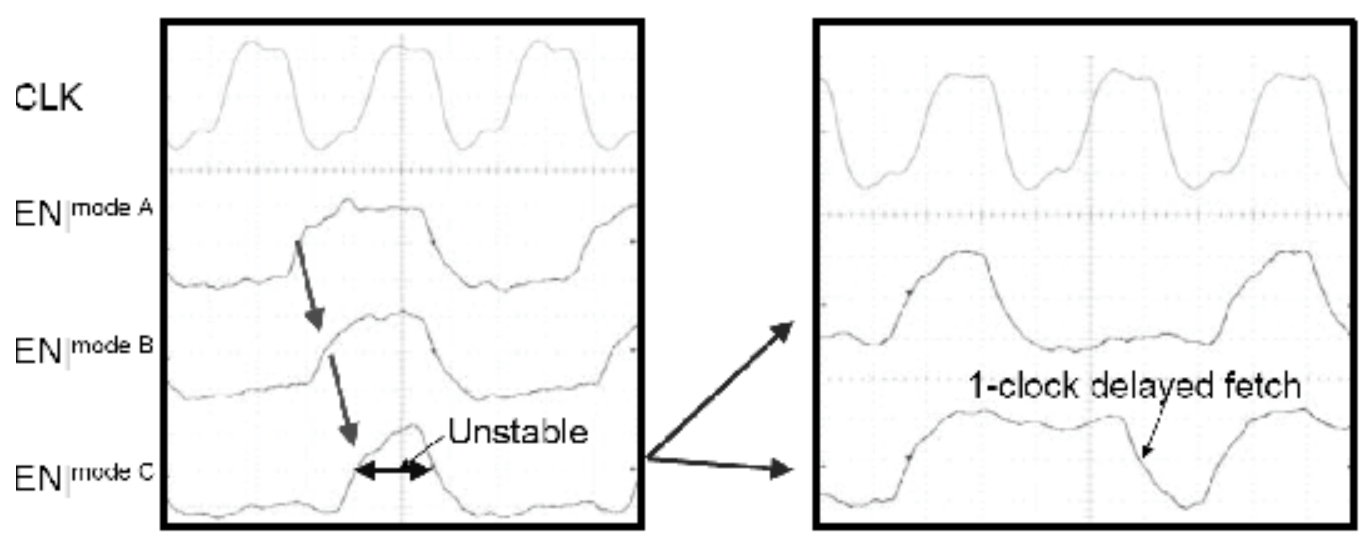

(a)

(b)

Figure 9. Uncertainty in non-synchronized global interconnect

Figure 11 shows the measured waveform when the adaptive clock-phase delay synchronization is enabled. When the mode $C$ is detected, delay time applied to the EN signal is reduced so that the fetching timing is effectively delayed compared to normal operation, which ensures sufficient timing margin to fetch the EN signal. As a result, the deassertion of the EN signal is delayed as shown in the figure. 


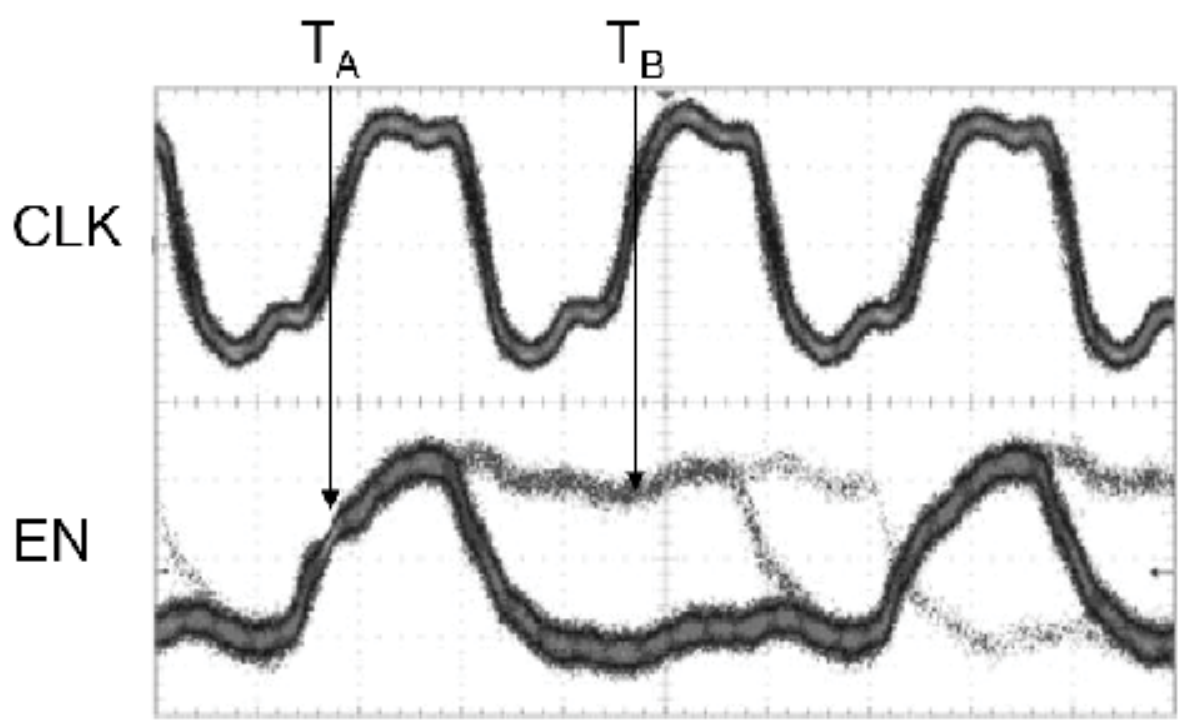

Figure 10. Accumulative display of synchronized EN signal with finite probability of delayed synchronization
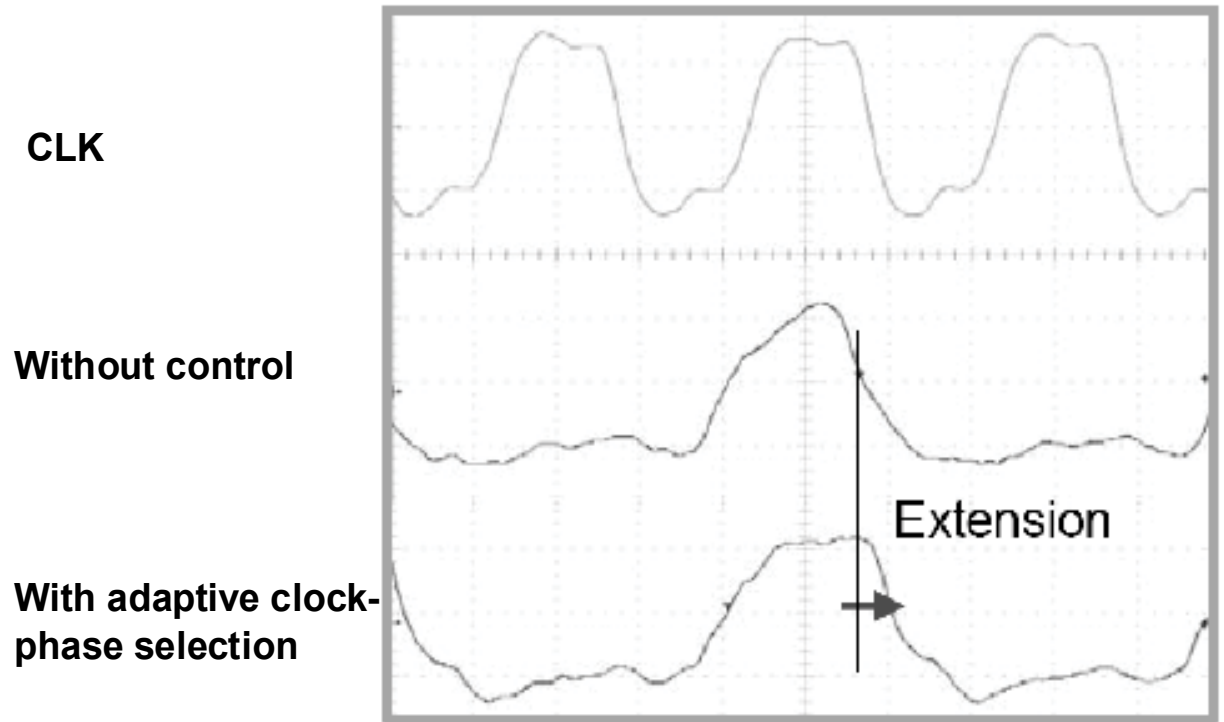

Figure 11. Stable synchronization when adaptive clock-phase shifting is applied

\section{Summary}

In this Chapter, we surveyed various topics in VLSI technology in adaptive control perspective: The design margins in process and circuit level are considered to be headroom for power savings, and adaptive control schemes are used to figure out the margins automatically and to make adjustment without harming the system operation. The margin is also found in system performance, and dynamic voltage frequency scaling can be thought as 
an adaptive control based on estimated workload. An adaptive control is also used to optimize the circuit operation for time-varying circumstances. This type of scheme enables the chip to operate always in optimal condition for wide range of operation conditions. We found the application of adaptive controls in the most advanced SoC architecture called Network-on-Chips. To comply with such a high-performance aspect of the modern VLSI systems as well as low-power requirement, the NoC utilized adaptive schemes in topology selection and link bandwidth control. And, adaptive clock-phase shifting was a solution to address uncertainty in such a large scale designs.

There is no doubt that the VLSI designer will seek adaptive control schemes more and more, as the VLSI system grows with higher complexity and wider application spectrum.

\section{References}

S. Bokar, et al., Parameter variations and impact on circuits and microarchitecture, in Proc. DAC, pp. 338-342, 2003. [1]

http:// focus.ti.com/pdfs/wtbu/smartreflex_whitepaper.pdf [2]

D. Liu, et al., Trading speed for low power by choice of supply and threshold voltages, IEEE J. Solid-State Circuits, vol. 28, pp. 10-17, Jan. 1993. [3]

T. Kuroda, et al., Low-Power CMOS Digital Design with Dual Embedded Adaptive Power Supplies, IEEE J. Solid-State Circuits, vol. 35, Apr. 2000. [4]

Jeabin Lee, et al., Dynamic Voltage Frequency Scaling Scheme for Multi-Domains Power Management, IEEE Asian Solid-State Circuits Conference, 2007. [5]

Temperature based DRAM refresh, US patent 7295484 [6]

Dynamic DRAM refresh rate adjustment based on cell leakage monitoring, US patent 6483764. [7]

Se-Joong Lee, et al., An 800MHz Star-Connected On-Chip Network for Application to Systems on a Chip, IEEE International Solid-State Circuits Conference, 2003. [8]

Kangmin Lee, et al., A 51mW 1.6GHz On-Chip Network for Low-Power Heterogeneous SoC Platform, IEEE International Solid-State Circuits Conference, 2004. [9]

Donghyun Kim, et al., An 81.6 GOPS Object Recognition Processor Based on NoC and Visual Image Processing Memory, IEEE Custom Integrated Circuits Conference, 2007. [10]

Kwanho Kim, et al., A 125GOPS 583mW Network-on-Chip Based Parallel Processor with Bio-inspired Visual Attention Engine, IEEE International Solid-State Circuits Conference, 2008. [11]

Se-Joong Lee, Cost-Optimization and Chip Implementation of On-Chip Network, Ph.D. Dissertation, 2005. [12]

Se-Joong Lee, et al., Adaptive Network-on-Chip with Wave-Front Train Serialization Scheme, IEEE Symposium on VLSI circuits, 2005. [13]

Se-Joong Lee, et al., Analysis and Implementation of Practical Cost-Effective Network-onChips, IEEE Design and Test of Computers, pp. 422-433, Sep-Oct 2005. [14]

G. De Micheli, Designing Robust Systems with Uncertain Information, Invited talk, AsiaPacific Design Automation Conference, 2003. [15] 



\section{Edited by Shuang Cong}

The objective of this book is to provide an up-to-date and state-of-the-art coverage of diverse aspects related to adaptive control theory, methodologies and applications.

These include various robust techniques, performance enhancement techniques, techniques with less a-priori knowledge, nonlinear adaptive control techniques and intelligent adaptive techniques. There are several themes in this book which instance both the maturity and the novelty of the general adaptive control. Each chapter is introduced by a brief preamble providing the background and objectives of subject matter. The experiment results are presented in considerable detail in order to facilitate the comprehension of the theoretical development, as well as to increase sensitivity of applications in practical problems 